
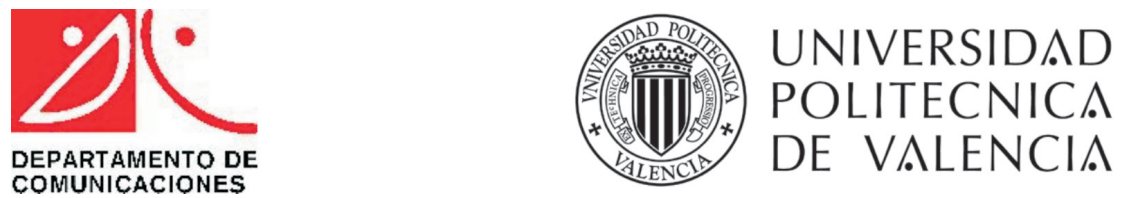

Universidad Politécnica de Valencia

Departamento de Comunicaciones

\title{
Algoritmos para Sistemas de Comunicaciones Multi-Usuario con Múltiples Antenas
}

\author{
Tesis Doctoral
}

presentada por

Carmen Botella Mascarell

dirigida por

Dra. Gema Piñero Sipán

Valencia, abril de 2008

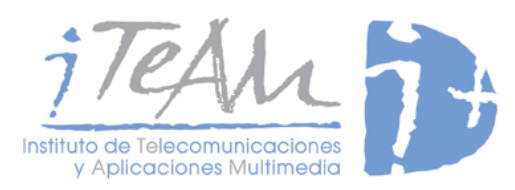



Als meus huelos, Pepe, Bernarda, Vicente i Pepica. 



\section{Agradecimientos}

Es difícil resumir en unas pocas líneas todo el apoyo que he recibido a lo largo de estos años. Sin embargo, y en un lugar destacado, me gustaría agradecerle a la directora de esta Tesis, la doctora Gema Piñero, todo el tiempo que le ha dedicado a la Tesis y el hecho de que confiara en mí para llevarla a cabo. Alguien me dijo que al finalizar una Tesis, tú eres el mayor experto en el tema. En este caso, creo que es justo afirmar que Gema es tan experta como yo.

Por otro lado, también me gustaría agradecer a Narcís Cardona, Alberto González y Gema Piñero la financiación recibida durante este tiempo, que me ha permitido dedicarme de forma exclusiva al desarrollo de la Tesis.

Al doctor David Gesbert, del Instituto Eurecom en Sophia-Antipolis, Niza, tengo que agradecerle la magnífica oportunidad que representó para mí el hecho de realizar una estancia en su grupo de investigación. Sólo fueron tres meses, pero ese tiempo me proporcionó la visión de conjunto que necesitaba para finalizar la Tesis.

A mi familia y amigos. A los que han esperado pacientemente que les explicara qué estoy haciendo y a los que me han escuchado cuando necesitaba hablar.

A mis compañeros del GTAC y del iTEAM, los más veteranos, los más nuevos, y los que ya no están. A mis otros compañeros, los de Eurecom, en Niza. Agradecerles los momentos de risas y la ayuda recibida durante todo este tiempo.

Y por último, pero no por eso menos importante, me gustaría agradecerle a Joaquín todo el apoyo, la ayuda y la comprensión recibidas a lo largo de este tiempo. Gracias Joaquín por obligarme a sonreír. 



\section{Resumen}

Esta Tesis surge en el contexto de un proyecto de investigación del Ministerio de Educación y Ciencia centrado en el análisis de una nueva arquitectura de acceso radio para los sistemas UMTS, la arquitectura Multinodo B. La idea fundamental de esta nueva propuesta para UMTS era distribuir espacialmente las antenas de la estación base sobre el área de servicio de la celda, con el objetivo de reducir el nivel de interferencias y aumentar al mismo tiempo la capacidad del sistema.

Por otro lado, los sistemas de comunicaciones con múltiples antenas o sistemas MIMO (Multiple-Input Multiple-Output), han generado en la última década un gran interés de la comunidad investigadora. Inicialmente propuestos para enlaces punto a punto, estos sistemas se perfilan como posible solución tecnológica para los futuros sistemas de comunicaciones, debido a la alta eficiencia espectral teórica que prometen.

En esta Tesis, la arquitectura Multinodo B se analiza desde el punto de vista de un sistema MIMO multi-usuario multi-celda coordinado. En esta configuración avanzada y cooperativa de sistema MIMO, varias estaciones base cooperan perfectamente en datos a través de un enlace de alta capacidad y transmiten de forma coordinada a todos los usuarios de una determinada área. Además, cada estación base del sistema está sujeta a una restricción en la potencia total que puede transmitir.

El estudio de los sistemas MIMO multi-usuario multi-celda coordinados se encuentra todavía en su fase inicial. En la actualidad, se pueden distinguir claramente dos líneas de investigación; por una parte se está tratando de caracterizar desde un punto de vista de teoría de la información la región de capacidad de estos sistemas y por otra, se está desarrollando la algorítmica necesaria para tratar distintos problemas de optimización.

En la Tesis, se plantea el problema de minimización de potencia en el enlace downlink de un sistema W-CDMA MIMO multi-usuario multi- 
celda coordinado. El problema de minimización de potencia es un problema de optimización que busca minimizar la potencia total transmitida en el sistema, entendida como la suma de las potencias que transmiten todas las estaciones base del sistema. Al mismo tiempo, todos los usuarios tienen que alcanzar un determinado nivel de calidad de servicio.

En un primer paso, se presenta un modelo de sistema donde se omite el proceso que tiene lugar en el receptor del usuario. En este escenario, se propone un primer algoritmo JPCOB o Joint Power Control and Optimal Beamforming, modificando una de las referencias clásicas de los problemas de minimización de potencia. Este primer algoritmo, el JPCOB-VUL o Virtual uplink-based JPCOB, utiliza la dualidad entre el problema de minimización de potencia en el enlace downlink y un equivalente uplink virtual para simplificar el diseño de los beamformers en transmisión de cada usuario. Además, la estructura de las matrices involucradas en el mecanismo de control de potencia del algoritmo JPCOB-VUL permite obtener una versión computacionalmente eficiente de este algoritmo.

En el análisis de los aspectos prácticos relacionados con la implementación del algoritmo JPCOB-VUL, se propone un modelo de las interferencias presentes en el sistema y se plantean esquemas de asignación de códigos de canalización que permiten disminuir el nivel de interferencia que perciben los usuarios del sistema. Al mismo tiempo, se plantea una versión robusta del JPCOB-VUL, para las situaciones en las que las estaciones base disponen de un conocimiento parcial del canal en la forma de hybrid channel knowledge. Este modelo de información de canal se corresponde con un escenario en el que existe algún tipo de restricción en la cantidad de información que las estaciones base pueden intercambiar entre ellas a través del enlace de alta capacidad.

En un segundo paso, se avanza en el modelo analítico del sistema y se propone una formulación matricial compacta que caracteriza completamente el funcionamiento de un sistema W-CDMA MIMO multi-usuario multi-celda coordinado, teniendo en cuenta la naturaleza asíncrona de las señales que recibe cada usuario y el proceso que tiene lugar en el receptor del mismo. En este punto de la Tesis, se propone un algoritmo JPCOB que mejora las prestaciones del JPCOB-VUL, el JPCOB-DL o Downlink-based JPCOB. Al mismo tiempo, se propone una versión simplificada de este algoritmo, el algoritmo JPCOB-SDL o Simplified Downlink-based JPCOB, para aquellas situaciones en las que existe algún tipo de restricción en la cantidad de información que cada usuario puede realimentar a la estación 
base, pero donde las estaciones base pueden intercambiar perfectamente datos entre ellas. Ambos algoritmos se denominan downlink-based porque abandonan la dualidad entre el problema downlink y el equivalente uplink virtual.

Por último, y aprovechando la estructura de las matrices que intervienen en el mecanismo de control de potencia, se proponen dos esquemas que mejoran las propiedades de convergencia del algoritmo JPCOB-DL en sistemas MIMO multi-usuario multi-celda coordinados mal condicionados. Las simulaciones demuestran que estos esquemas se pueden interpretar en realidad como criterios eficientes y computacionalmente sencillos para eliminar usuarios en un sistema MIMO multi-usuario multi-celda coordinado, en el caso en que no se alcance la convergencia del algoritmo. 



\section{Resum}

Aquesta Tesi sorgeix en el marc d'un projecte d'investigació del Ministeri d'Educació i Ciència centrat en l'anàlisi d'una nova arquitectura d'accés ràdio per als sistemes UMTS, l'arquitectura Multinodo $B$. La idea fonamental d'aquesta nova proposta per a UMTS era distribuir espacialment les antenes de l'estació base al llarg de l'àrea de servei de la cel·la, amb l'objectiu de reduir el nivell d'interferències i augmentar al mateix temps la capacitat del sistema.

D'altra banda, els sistemes de comunicacions amb múltiples antenes o sistemes MIMO (Multiple-Input Multiple-Output), han generat en l'última dècada un gran interés per part de la comunitat investigadora. Aquests sistemes van ser proposats inicialment per enllaços punt a punt, però a hores d'ara es presenten com una possible solució tecnològica per als futurs sistemes de comunicacions, en base a l'alta eficiència espectral que prometen.

En aquesta Tesi, l'arquitectura Multinodo B s'analitza des de'l punt de vista d'un sistema MIMO multi-usuari multi-cel-la coordinat. En aquesta configuració avançada i cooperativa de sistema MIMO, vàries estacions base cooperen perfectament en dades per mitjà d'un enllaç d'alta capacitat i transmeten de forma coordinada a tots els usuaris d'una determinada àrea. A més a més, cada estació base disposa d'una restricció en la potència total que pot transmetre.

L'estudi dels sistemes MIMO multi-usuari multi-cel-la coordinats es troba encara en la fase inicial. En l'actualitat, es poden distingir clarament dues línees d'investigació; per una banda s'està intentant caracteritzar la regió de capacitat d'aquests sistemes, i per l'altra, s'està desenvolupant l'algorítmica necessària per a tractar diversos problemes d'optimització.

En la Tesi, es planteja el problema de minimització de potència en l'enllaç downlink d'un sistema MIMO multi-usuari multi-cel-la coordinat. El problema de minimització de potència és un problema d'optimització que 
busca minimitzar la potència total transmesa en el sistema, entesa com la suma de les potències que transmeten totes les estacions base del sistema. $\mathrm{Al}$ mateix temps, tots els usuaris deuen obtindre un determinant nivell de qualitat de servei.

En un primer pas, es presenta un model de sistema on s'omet el procés que es duu a terme al receptor de l'usuari. En aquest context, es proposa un primer algoritme JPCOB o Joint Power Control and Optimal Beamforming, modificant una de les referències clàssiques dels problemes de minimització de potència. Aquest primer algoritme, el JPCOB-VUL o Virtual uplink-based JPCOB, utilitza la dualitat entre el problema de minimització de potència en l'enllaç downlink i un equivalent uplink virtual per a simplificar el disseny dels beamformers en transmissió de cada usuari. A més a més, l'estructura de les matrius que intervenen en el mecanisme de control de potència permet obtindre una versió computacionalment eficient d'aquest algoritme.

En l'anàlisi dels aspectes pràctics relacionats amb la implementació de l'algoritme JPCOB-VUL, es proposa un model de les interferències presents en el sistema i es plantegen diversos esquemes d'assignació de còdigs de canalització que permeten disminuir el nivell d'interferència que reben els usuaris del sistema. Al mateix temps, es proposa una versió robusta del JPCOB-VUL, per a aquelles situacions en les que les estacions base disposen d'un coneixement parcial del canal en la forma de hybrid channel knowledge. Aquest model d'informació de canal es correspon amb un escenari en el que existeix algun tipus de restricció en la quantitat d'informació que les estacions base poden intercanviar entre elles per mitjà de l'enllaç d'alta capacitat.

En un segon pas, s'avança en el model analític del sistema i es proposa una formulació matricial compacta que caracteritza completament el funcionament d'un sistema W-CDMA MIMO multi-usuari multi-cel-la coordinat, tenint en compte la naturalesa asíncrona dels senyals que arriben a l'usuari i el procés que es duu a terme al receptor del mateix. En aquest punt de la Tesi, es proposa un algoritme JPCOB que millora les prestacions del JPCOB-VUL, el JPCOB-DL o Downlink-based JPCOB. Al mateix temps, es proposa una versió més simple d'aquest algoritme, el JPCOB-SDL o Simplified Downlink-based JPCOB, per a aquelles situacions en les quals existeix algun tipus de restricció en la quantitat d'informació que un usuari pot realimentar a l'estació base, però a on les estacions base poden intercanviar dades entre elles sense cap problema. Aquests algoritmes es defineixen 
com a downlink-based en base a l'abandonament de la dualitat entre el problema downlink i l'equivalent uplink virtual.

Per últim, i aprofitant l'estructura de les matrius que intervenen en el mecanisme de control de potència, es proposen dos esquemes que milloren les propietats de convergència de l'algoritme JPCOB-DL en sistemes MIMO multi-usuari multi-cel-la coordinats mal condicionats. Les simulacions demostren que aquests esquemes es poden interpretar com a criteris eficients i computacionalment senzills per a eliminar usuaris d'un sistema MIMO multi-usuari multi-cel-la coordinat, en aquelles situacions en les quals no s'arriba a la convergència de l'algoritme. 



\section{Abstract}

This Thesis starts in the framework of a research project supported by the Ministerio de Educación y Ciencia. This research project analyzed a new radio access architecture for UMTS systems, the Multinodo $B$ architecture. The key idea for this new proposal for UMTS systems was to spatially distribute the antennas from the base station around the service area, with the aim of reducing the interference of the system and thus, increasing its capacity.

On the other hand, communication systems with multiple antennas or MIMO systems (Multiple-Input Multiple-Output), have received increasing attention from the research community over the last ten years. Initially proposed for point-to-point links, MIMO systems are seen as an interesting option for future communication systems due to their high spectral efficiency.

In this Thesis, the proposed Multinodo $B$ architecture is analyzed and characterized from the point of view of a coordinated multi-cell multi-user MIMO system. In this advanced and cooperative MIMO configuration, base stations achieve perfect data cooperation through a high speed backbone and hence, there is a chance for transmitting coordinatedly to all users in the area. Moreover, per-base station power constraints are taken into account.

The analysis of coordinated multi-cell multi-user MIMO systems is still in its first stages. Nowadays, it is possible to differentiate between two research lines; on one hand, some progress is taking place in order to obtain the capacity region of this MIMO configuration and on the other hand, several algorithms are being proposed to solve different optimization problems.

In the Thesis, the power minimization problem is stated for the downlink of a coordinated multi-cell multi-user MIMO system. The power mini- 
mization problem is an optimization problem that tries to minimize the total transmitted power in the system, which can be defined as the sum of the transmitted power from all the base stations in the system. At the same time, all the users are subject to a certain quality of service requirement.

Firstly, a system model is proposed, where the process carried out at each receiver is omitted. In this context, a first Joint Power Control and Optimal Beamforming (JPCOB) algorithm is stated by modifying one of the classical references dealing with the power minimization problem. This first algorithm, the JPCOB-VUL or Virtual uplink-based JPCOB, makes use of the duality between the power minimization problem in the downlink and an equivalent virtual uplink formulation for simplifying the transmit beamformer design. Moreover, the structure of the matrices involved in the power control mechanism allows to formulate an efficient version of the JPCOB-VUL algorithm.

In the analysis of some practical concerns dealing with the JPCOB-VUL algorithm, a model for the interferences present in the system is proposed. Moreover, several spreading sequence assignment schemes are presented in order to minimize the interference power received by the users in the system. On the other hand, a robust version of the JPCOB-VUL is obtained for the cases in which base stations have partial channel state information in the form of hybrid channel knowledge. This model of channel information stands for the case in which there is a constraint in the amount of information that base stations can exchange through the high speed backbone.

Secondly, a complete and matricial system model is proposed, which totally characterizes the main features of a coordinated multi-cell multiuser MIMO system, including realistic system parameters such as the asynchronous nature of the signals arriving at each user and the process carried out by the receivers. At this point, a JPCOB algorithm is proposed which outperforms the JPCOB-VUL algorithm, the JPCOB-DL or Downlinkbased JPCOB. Moreover, a simplified version of this proposal, the JPCOBSDL or Simplified Downlink-based JPCOB, is obtained for limited feedback scenarios, where there is a constraint in the amount of information that each user can feedback to the base stations. JPCOB-DL and JPCOB-SDL algorithms are regarded as downlink-based algorithms because both algorithms do not make use of the duality between the downlink and virtual uplink problem.

Finally, two schemes are proposed in order to improve the convergence properties of the JPCOB-DL algorithm in ill-conditioned coordinated 
multi-cell multi-user MIMO systems. Simulation results show that the proposed schemes can be seen as efficient and computationally simple criteria for removing users from a coordinated system in case the system is not feasible. 



\section{Lista de acrónimos}

$\begin{array}{ll}\text { AP } & \text { Access Point. } \\ \text { AS } & \text { Active Set. } \\ \text { ASW } & \text { Active Set Window. } \\ \text { AWGN } & \text { Additive White Gaussian Noise. } \\ \text { BC } & \text { Broadcast Channel. } \\ \text { BD } & \text { Block Diagonalization. } \\ \text { BER } & \text { Bit Error Rate. } \\ \text { BLAST } & \text { Bell Labs Layered Space-Time. } \\ \text { BS } & \text { Base Station. } \\ \text { CCI } & \text { Co-channel Interference. } \\ \text { CDF } & \text { Cumulative Distribution Function. } \\ \text { CDI } & \text { Channel Distribution Information. } \\ \text { CM3 } & \text { Coordinated Multi-cell Multi-user MIMO. } \\ \text { CSI } & \text { Channel State Information. } \\ \text { DL } & \text { Downlink. } \\ \text { DOA } & \text { Direction of Arrival. } \\ \text { DPC } & \text { Dirty Paper Coding. } \\ \text { EGC } & \text { Equal Gain Combining. } \\ \text { FDD } & \text { Frequency Division Duplex. } \\ \text { GEVP } & \text { Generalized Eigenvalue Problem. } \\ \text { IFI } & \text { Inter-finger Interference. } \\ \text { i.i.d. } & \text { Independent identically distributed. } \\ \text { ISI } & \text { Inter-symbol Interference. } \\ \text { JPCOB } & \text { Joint Power Control and Optimal Beamforming. } \\ \text { JPCOB-DL } & \text { Downlink-based JPCOB. } \\ \text { JPCOB-SDL } & \text { Simplified Downlink-based JPCOB. } \\ \text { JPCOB-VUL } & \text { Virtual uplink-based JPCOB. } \\ \text { KKT } & \text { Karush-Kuhn-Tucker. } \\ \text { LOS } & \text { Line of Sight. }\end{array}$




\begin{tabular}{|c|c|}
\hline MAC & Multiple Access Channel. \\
\hline MAI & Multiple Access Interference. \\
\hline MIMO & Multiple-Input Multiple-Output. \\
\hline MISO & Multiple-Input Single-Output. \\
\hline ML & Maximum Likelihood. \\
\hline MMSE & Minimum Mean Square Error. \\
\hline MRC & Maximum Ratio Combining. \\
\hline MSE & Mean Square Error. \\
\hline NLOS & Non-Line of Sight. \\
\hline OVSF & Orthogonal Variable Spreading Factor. \\
\hline PDP & Power Delay Profile. \\
\hline QoS & Quality of Service. \\
\hline QPSK & Quadrature Phase Shift Keying. \\
\hline SDMA & Spatial Division Multiple Access. \\
\hline SDP & Semidefinite Program. \\
\hline SIMO & Single-Input Multiple-Output. \\
\hline SINR & Signal-to-Interference-plus-Noise Ratio. \\
\hline SISO & Single-Input Single-Output. \\
\hline SNR & Signal-to-Noise Ratio. \\
\hline SOCP & Second-order Cone Program. \\
\hline SOI & Signal Of Interest. \\
\hline STBC & Space-Time Block Codes. \\
\hline STC & Space-Time Codes. \\
\hline STTC & Space-Time Trellis Codes. \\
\hline SVD & Singular Value Decomposition. \\
\hline TDD & Time Division Duplex. \\
\hline UL & Uplink. \\
\hline UMTS & Universal Mobile Telecommunication System. \\
\hline UTRA & Universal Terrestrial Radio Access. \\
\hline V-BLAST & Vertical - Bell Labs Layered Space-Time. \\
\hline W-CDMA & Wideband Code Division Multiple Access. \\
\hline WF & Waterfilling. \\
\hline $\mathbf{Z F}$ & Zero Forcing. \\
\hline
\end{tabular}




\section{Notación}

$\mathrm{X}$

X

$x$

$[\mathbf{X}]_{(j,:)}$

$[\mathbf{X}]_{(:, j)}$

$[\mathbf{X}]_{(i, j)}$

$[\mathbf{x}]_{j}$

$(\cdot)^{T}$

$(\cdot)^{*}$

$(\cdot)^{H}$

$\mathbf{I}_{M}$

$\mathbf{0}_{N}$

$\mathbf{1}_{N}$

$\mathbf{e}_{n}$

$\otimes$

$\odot$

$\|\cdot\|$

$\|\cdot\|_{F}$

$|\cdot|$

$\operatorname{det}(\cdot)$

$\operatorname{Tr}[\cdot]$

$\operatorname{vec}\{\cdot\}$

$\operatorname{diag}\left\{\left[\mathbf{X}_{1} \ldots \mathbf{X}_{N}\right]\right\}$

$\operatorname{diag}\left\{\left[x_{1} \ldots x_{N}\right]\right\}$

K

$N_{t}$

$M$
Matriz.

Vector.

Escalar.

Fila $j$-ésima de la matriz $\mathbf{X}$.

Columna $j$-ésima de la matriz $\mathbf{X}$.

Elemento $(i, j)$ de la matriz $\mathbf{X}$.

Elemento $j$-ésimo del vector $\mathbf{x}$.

Traspuesto.

Conjugado.

Traspuesto conjugado.

Matriz identidad $[M \times M]$.

Vector columna todo ceros $[N \times 1]$.

Vector columna todo unos $[N \times 1]$.

Columna $n$-ésima de la matriz identidad.

Operador de Kronecker.

Producto elementwise.

Norma vectorial o matricial.

Norma de Frobenius.

Valor absoluto.

Determinante.

Traza de una matriz.

Vectorización de una matriz.

Matriz diagonal a bloques.

Matriz diagonal.

Número de transmisores/estaciones base.

Número de antenas en el transmisor/estación base.

Número de receptores/usuarios en el sistema. 



\section{Índice general}

Lista de acrónimos $\quad$ XV

$\begin{array}{lll}\text { Notación } & \text { XVII }\end{array}$

1. Introducción 1

1.1. Objetivos de la Tesis . . . . . . . . . . . . . . . . 4

1.2. Descripción de los contenidos . . . . . . . . . . . 4

I Introducción a los sistemas MIMO 9

2. Introducción a los sistemas MIMO 11

2.1. Sistemas SISO, SIMO y MISO . . . . . . . . . . . . 13

2.2. Sistemas single-user MIMO . . . . . . . . . . . . . . . . 16

2.2.1. Modelo del sistema . . . . . . . . . . . . . . . 16

2.2.2. Capacidad del sistema . . . . . . . . . . . . . 19

2.2.3. Técnicas single-user MIMO . . . . . . . . . . . . 22

2.3. Sistemas MIMO multi-usuario . . . . . . . . . . . . 26

2.3.1. Capacidad del sistema . . . . . . . . . . . 28

2.3.2. Criterios de diseño . . . . . . . . . . . . . 35

2.3.3. Técnicas MIMO multi-usuario: enlace UL . . . . . . 38

2.3.4. Criterios de optimización: enlace UL . . . . . . . . . 39

2.3.5. Técnicas MIMO multi-usuario: enlace DL . . . . . . 41

2.3.6. Criterios de optimización: enlace DL . . . . . . . . 46

2.3.7. Tendencias . . . . . . . . . . . . . 52

2.4. Sistemas MIMO multi-usuario multi-celda . . . . . . . . 53

2.4.1. Aspectos teóricos . . . . . . . . . . . . . . 54

2.4.2. Aspectos prácticos . . . . . . . . . . . 55 
2.5. Sistemas MIMO multi-usuario multi-celda coordinados . . . 60

2.5.1. Capacidad del sistema . . . . . . . . . . . . 61

2.5.2. Soluciones algorítmicas en DL . . . . . . . . . . . 64

2.5.3. Criterios de diseño . . . . . . . . . . . . 75

2.5.4. Tendencias . . . . . . . . . . . . . 78

II Solución Virtual uplink-based $\quad 81$

3. Algoritmos clásicos para sistemas sin coordinación 83

3.1. Aproximación de Schubert y Boche . . . . . . . . . . 84

3.1.1. Problema max-min . . . . . . . . . . . . . 86

3.1.2. Problema de minimización de potencia . . . . . . . 93

3.1.3. Caracterización del óptimo . . . . . . . . . . . 95

3.2. Aproximación de Bengtsson y Ottersten . . . . . . . . . 97

3.2.1. Problema de minimización de potencia . . . . . . . . 97

3.3. Aproximación de Rashid-Farrokhi et al. . . . . . . . . . . . 102

3.3.1. Problema de minimización de potencia en UL . . . . 102

3.3.2. Problema de minimización de potencia en DL . . . . 106

3.3.3. Convergencia del algoritmo JPCOB en DL . . . . . 109

3.3.4. Caracterización del óptimo . . . . . . . . . . . . . 111

3.4. Comparativa entre las distintas aproximaciones . . . . . . . 112

4. Algoritmo JPCOB-VUL para un sistema CM3 117

4.1. Modelo del sistema CM3 . . . . . . . . . . . . . . . . . . 118

4.1.1. Modelo de canal . . . . . . . . . . . . . . . 120

4.1.2. Enlace DL del sistema . . . . . . . . . . . . . . . . 121

4.1.3. Modelo matricial del sistema . . . . . . . . . . . . 123

4.2. Algoritmo JPCOB-VUL . . . . . . . . . . . . . 126

4.2.1. Beamforming en transmisión . . . . . . . . . . . . 128

4.2.2. Control de potencia . . . . . . . . . . . . . 129

4.2.3. Solución algorítmica . . . . . . . . . . . . . . 131

4.2.4. Versión eficiente . . . . . . . . . . . . . . . . . . . 134

4.2.5. Convergencia del algoritmo . . . . . . . . . . 135

4.2.6. Caracterización del óptimo . . . . . . . . . . . . 137

4.3. Aspectos prácticos del algoritmo JPCOB-VUL . . . . . . . 141

4.4. Prestaciones del algoritmo JPCOB-VUL . . . . . . . . . . 145

4.4.1. Modelo de sistema . . . . . . . . . . . . . . . . . 145

4.4.2. Modelo de canal . . . . . . . . . . . . . . . 150 
4.4.3. Algoritmo JPCOB-VUL: entorno Coordinado I . . . 153

4.4.4. Algoritmo JPCOB-VUL: entorno Coordinado II . 168

5. Algoritmo JPCOB-VUL: aplicaciones prácticas $\quad 177$

5.1. Sistema W-CDMA CM3: modelado de interferencias . . . . 179

5.1.1. Modelo de sistema . . . . . . . . . . . . . . . . 179

5.1.2. Modelo tradicional de correlación entre códigos . . . 184

5.1.3. Estudio de la correlación entre códigos . . . . . . . . 188

5.1.4. Modelo de las interferencias . . . . . . . . . . . . . . 190

5.2. Esquemas de asignación de códigos OVSF . . . . . . . . . 198

5.2.1. Entorno flat fading . . . . . . . . . . . . . . . 199

5.2.2. Entorno frequency selective fading . . . . . . . . . . 215

5.3. Control robusto de potencia . . . . . . . . . . . . . 227

III Soluciones Downlink-based 237

6. Algoritmos JPCOB-DL y JPCOB-SDL para un sistema CM3 239

6.1. Modelo matricial del sistema . . . . . . . . . . . . . . 241

6.2. Algoritmos Downlink-based . . . . . . . . . . . . . . 244

6.2.1. Beamforming en transmisión: JPCOB-DL . . . . . . 246

6.2.2. Beamforming en transmisión: JPCOB-SDL . . . . . 249

6.2.3. Control de potencia . . . . . . . . . . . . 250

6.2.4. Solución algorítmica . . . . . . . . . . . . 253

6.2.5. Convergencia de los algoritmos . . . . . . . . . 255

6.3. Prestaciones de los algoritmos Downlink-based . . . . . . . 256

6.3.1. Requerimiento de QoS: estricto . . . . . . . . . . . . 259

6.3.2. Requerimiento de QoS: tolerante . . . . . . . . . . 266

6.3.3. Estabilidad de los algoritmos . . . . . . . . . . . 270

7. Algoritmo JPCOB-DL: aplicaciones prácticas 275

7.1. Problema de minimización de potencia . . . . . . . . . . 277

7.1.1. Beamforming en transmisión . . . . . . . . . . 278

7.1.2. Control de potencia . . . . . . . . . . . . 279

7.1.3. Solución algorítmica . . . . . . . . . . . . . . 281

7.2. Esquemas de selección de enlaces activos . . . . . . . . . . . 282

7.2.1. Esquema F-based . . . . . . . . . . . . . . . 282

7.2.2. Esquema D-based . . . . . . . . . . . . . . . . 284 
7.2.3. Prestaciones de los esquemas $\boldsymbol{F}$-based y $\boldsymbol{D}$-based . . 285

8. Conclusiones y líneas futuras 291

8.1. Conclusiones . . . . . . . . . . . . . . . . . . 291

8.2. Líneas futuras . . . . . . . . . . . . . . . . . . . 301

A. Teorema de Perron-Frobenius e Iteración de Jacobi 305

A.1. Teorema de Perron-Frobenius . . . . . . . . . . . . . 305

A.1.1. Teorema de Perron . . . . . . . . . . . . . . . 306

A.1.2. Teorema de Perron-Frobenius . . . . . . . . . . . 307

A.1.3. Problema de minimización de potencia . . . . . . . 308

A.2. Iteración de Jacobi . . . . . . . . . . . . . . . . . . 311

A.2.1. Problema de minimización de potencia . . . . . . . 312

B. Optimización convexa 313

$\begin{array}{ll}\text { C. Configuraciones de celda } & 317\end{array}$

$\begin{array}{ll}\text { Bibliografía } & 321\end{array}$

$\begin{array}{ll}\text { Publicaciones relacionadas con la Tesis } & 335\end{array}$ 


\section{Índice de figuras}

1.1. Configuración Multinodo B. . . . . . . . . . . . . 2

2.1. Evolución de los sistemas MIMO. . . . . . . . . . . . . 12

2.2. Enlace UL de un sistema MIMO multi-usuario. . . . . . . . 27

2.3. Enlace DL de un sistema MIMO multi-usuario. . . . . . . . 27

2.4. Región de capacidad MAC, $M=2, N_{m}=1 . \ldots \ldots . \quad 30$

2.5. Región de capacidad MAC, $M=2, N_{m}>1$. . . . . . 31

2.6. Región de capacidad BC con DPC. . . . . . . . . . . . 34

2.7. Dualidad MAC-BC. . . . . . . . . . . . . . 35

2.8. Probabilidad de error de símbolo para distintas técnicas de transmisión DL en función de $P_{\max } . \ldots \ldots$. . . . . 45

2.9. Sistema MIMO Interferente. . . . . . . . . . . . . . . 53

2.10. Sistema MIMO Cooperativo. . . . . . . . . . . . 54

2.11. Sistema ad hoc con cooperative diversity. . . . . . . . . 57

2.12. Sistema celular convencional y sistema CM3. . . . . . . . 59

2.13. Sistema de antenas distribuidas. . . . . . . . . . . . 61

2.14. Eficiencia espectral con y sin coordinación. . . . . . . . 70

2.15. Modelo lineal de Wyner. . . . . . . . . . . . . . . . . . 77

2.16. Modelo circular de Wyner. . . . . . . . . . . . . . . . . 77

2.17. Modelo celular realista de un sistema CM3. . . . . . . . 78

2.18. Sistema CM3 modelado como un grafo. . . . . . . . . . . 79

3.1. Función $C^{D L}\left(\tilde{\mathbf{W}}, P_{\max }\right) \ldots \ldots \ldots \ldots \ldots$. . . . . . . 87

3.2. Diagrama de bloques: beamforming en recepción. . . . . . . 103

3.3. Diagrama de bloques: beamforming en transmisión. . . . . . 107

4.1. Arquitectura UMTS convencional. . . . . . . . . . . . . 118

4.2. Sistema MIMO multi-usuario multi-celda coordinado. . . . 119 
4.3. Diagrama de bloques de un sistema CM3. . . . . . . . . . 124

4.4. Sistema CM3 y sistema central equivalente. . . . . . . . . . 146

4.5. Sistema CM3, $K=3$ BSs. . . . . . . . . . . . . . . 147

4.6. Celda sectorizada convencional, $K=3$ BSs centrales. . . . . 147

4.7. División en subsectores para el cálculo de las DOAs en una celda sectorizada convencional con $K=3$ BSs centrales. . .

4.8. División en subsectores para el cálculo de las DOAs en un sistema CM3 con $K=3$ BSs. . . . . . . . . . . . . . . . 149

4.9. Número medio de usuarios co-canal admitidos por el algoritmo JPCOB-VUL para distintos valores de ventana de AS. Comparativa con un algoritmo de asignación óptima de usuarios a BS.

4.10. Tasa de fallo para una SINR umbral de 12 y $10 \mathrm{~dB} . \quad$. . . . 158

4.11. Tasa de fallo para una SINR umbral de 8 y 6 dB. . . . . . . 159

4.12. Distribución de la potencia total transmitida en DL para todas las configuraciones de sistema y tamaños de ventana de AS para la SINR umbral de 12 y $6 \mathrm{~dB} . \ldots . . . . .$.

4.13. Porcentaje de enlaces activos para el conjunto de todas las simulaciones y configuraciones de sistema de $K=2$ y $K=3$ BSs. . . . . . . . . . . . . . . . . . 161

4.14. Tasa de fallo para una SINR umbral de 12 y $10 \mathrm{~dB} . \quad$. . . . 164

4.15. Tasa de fallo para una SINR umbral de 8 y 6 dB. . . . . . . 165

4.16. Distribución de la potencia total transmitida en DL para las configuraciones de sistema central y CM3 con $K=3$ BSs, $N_{t}=3$. Resultados para la SINR umbral de 12 y $6 \mathrm{~dB} . \quad$. 167

4.17. Número medio de usuarios co-canal admitidos y potencia media total transmitida en el sistema por tres algoritmos JPCOB para distintos valores de SINR umbral. . . . . . . . 170

4.18. Número medio de iteraciones necesarias para converger para distintos valores de SINR umbral. . . . . . . . . . . . . . 171

4.19. Diagrama de bloques de la simulación que evalúa el número medio de usuarios co-canal admitidos por el algoritmo JPCOBVUL. . . . . . . . . . . . . . . . . . . . . 174

4.20. Diagrama de bloques de la simulación que evalúa la tasa de fallo del algoritmo JPCOB-VUL. . . . . . . . . . . . . . . 175

5.1. Histograma de correlaciones entre códigos OVSF. . . . . . . 189

5.2. Detalle del histograma de correlaciones entre códigos OVSF. 190 
5.3. Ejemplo de desplazamiento de los perfiles de retardo para los canales de propagación entre el usuario $m$ y las BSs $k=1$ y $k=2$.

5.4. Función de distribución estimada de la probabilidad de admisión, $K=3, N_{t}=3 \ldots \ldots \ldots \ldots \ldots$. . . . . 206

5.5. Función de distribución estimada de la probabilidad de ad-

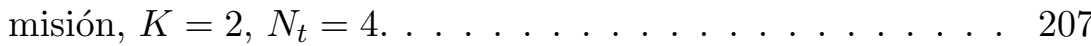

5.6. Esquema MT o Minimum Total. . . . . . . . . . . . . . 209

5.7. Esquema MP o Minimum Partial. . . . . . . . . . . . . . 209

5.8. Suma acumulada de la desviación estándar para los cuatro órdenes de asignación de códigos. . . . . . . . . . . . . . . . 210

5.9. Función de distribución estimada de la probabilidad de admisión del algoritmo JPCOB-VUL, para $K=3, N_{t}=3 \mathrm{y}$ $\mathrm{ASW}=0 \mathrm{~dB}$

5.10. Función de distribución estimada de la probabilidad de admisión del algoritmo JPCOB-VUL, para $K=3, N_{t}=3 \mathrm{y}$ $\mathrm{ASW}=2 \mathrm{~dB} \ldots \ldots \ldots \ldots \ldots \ldots \ldots \ldots \ldots$

5.11. Función de distribución estimada de la probabilidad de admisión del orden Natural, para $K=3, N_{t}=3$.

5.12. Función de distribución estimada de la probabilidad de admisión del orden MP, para $K=3, N_{t}=3 \ldots \ldots \ldots$

5.13. Función de distribución estimada de la probabilidad de admisión del orden Natural y MP, para configuraciones de sistema CM3 y centrales con $K=3$ y $N_{t}=3$.

5.14. Función de distribución estimada de la probabilidad de admisión del orden Natural y MP, para un sistema CM3 con $K=3$ y $N_{t}=2 \ldots \ldots \ldots \ldots \ldots \ldots \ldots$

5.15. Función de distribución estimada de la probabilidad de admisión del orden Natural y MP, para un sistema CM3 con $K=3$ y $N_{t}=3$.

5.16. Valor medio de la mínima SINR real obtenido por el control robusto de potencia, en función del valor de $\epsilon \ldots$. . . . . .

5.17. Valor medio de la mínima SINR real obtenido por el control no robusto de potencia, en función del valor de $\epsilon$. . . . .

5.18. Potencia media total transmitida en el sistema CM3 para el control robusto y no robusto de potencia. ASW $=70 \mathrm{~dB}$.

5.19. Probabilidad de feasibility práctica del control robusto de potencia para distintos valores de ventana de AS. . . . . . . 
6.1. Diagrama de bloques de un sistema CM3.

6.2. Función distribución estimada del número de enlaces activos en el sistema CM3 para un requerimiento de QoS de SINR = $7 \mathrm{~dB}$ y $M=3$ usuarios co-canal.

6.3. Probabilidad de feasibility práctica del algoritmo JPCOBVUL para $M=3$ usuarios co-canal.

6.4. Probabilidad de feasibility práctica de los algoritmos JPCOBDL y JPCOB-SDL para $M=4$ usuarios co-canal. . . . . . . 263

6.5. Potencia media total transmitida en el sistema por los algoritmos JPCOB-DL y JPCOB-SDL para $M=3$ usuarios co-canal. . . . . . . . . . . . . . . . . . . . . . . 264

6.6. Probabilidad de feasibility práctica del algoritmo JPCOBVUL para $M=3$ usuarios co-canal.

6.7. Probabilidad de feasibility práctica de los algoritmos JPCOBDL, JPCOB-SDL y JPCOB-VUL para $M=4$ usuarios cocanal.

6.8. Potencia media total transmitida en el sistema por los algoritmos JPCOB-DL y JPCOB-VUL para $M=4$ usuarios co-canal.

6.9. Distribución de la probabilidad de feasibility práctica del algoritmo JPCOB-VUL para $M=3$ usuarios co-canal. . . . .

6.10. Distribución de la probabilidad de feasibility práctica del algoritmo JPCOB-DL para $M=4$ usuarios co-canal. . . . . .

7.1. Probabilidad de feasibility práctica del algoritmo JPCOBDL para los esquemas $\boldsymbol{D}$-based, $\boldsymbol{F}$-based y conservative $\boldsymbol{F}$ based. . . . . . . . . . . . . . .

7.2. Evolución del radio espectral de la matriz de ganancia DL para los esquemas $\boldsymbol{D}$-based, $\boldsymbol{F}$-based y conservative $\boldsymbol{F}$-based.

7.3. Probabilidad de feasibility práctica del algoritmo JPCOBDL para los esquemas $\boldsymbol{D}$-based y $\boldsymbol{F}$-based. Posibilidad de eliminar hasta dos usuarios del sistema. . . . . . . . . . . 289

C.1. Sistema CM3, $K=2$ BSs. . . . . . . . . . . . . . 317

C.2. Celda sectorizada convencional, $K=2$ BSs centrales. . . . . 318

C.3. División en subsectores para el cálculo de las DOAs en una celda sectorizada convencional con $K=2$ BSs centrales. . . 318

C.4. División en subsectores para el cálculo de las DOAs en un sistema CM3 con $K=2$ BSs. . . . . . . . . . . . . . 319 


\section{Capítulo 1}

\section{Introducción}

La presente Tesis, Algoritmos para sistemas de comunicaciones multiusuario con múltiples antenas, se ha realizado en el Instituto de Telecomunicaciones y Aplicaciones Multimedia (iTEAM) de la Universidad Politécnica de Valencia. Más concretamente, se incluye en la línea de investigación de Procesado de Señal en Comunicaciones del Grupo de Tratamiento de Audio y Comunicaciones (GTAC).

Esta línea de investigación, que se inició en el año 2003, abarca tanto aspectos teóricos como aspectos prácticos de los sistemas de transmisión y recepción en el ámbito de las comunicaciones, fundamentalmente digitales.

Esta Tesis empezó a desarrollarse dentro de un proyecto de investigación del Ministerio de Educación y Ciencia, denominado "Mejora de Arquitecturas de Acceso Radio UMTS Mediante Multinodos B" ${ }^{1}$, que incluía una colaboración entre el Grupo de Comunicaciones Móviles y el GTAC de la Universidad Politécnica de Valencia. Paralelamente, la Tesis ha recibido financiación parcial de la empresa Telefónica Móviles de España, a través del "Plan de Promoción Tecnológica de UMTS", y de la Generalitat Valenciana, mediante el proyecto "Algoritmos de Procesado de Señal para entornos de Antenas Distribuidas en Comunicaciones Móviles"2.

El sistema UMTS [1], se basa en el empleo de una interfaz radio UTRA (Universal Terrestrial Radio Access) que utiliza la tecnología W-CDMA (Wideband Code Division Multiple Access) [2], donde los usuarios se canalizan mediante la superposición de dos secuencias código. Esta interfaz radio dispone de dos modos de operación, el modo FDD (Frequency Division

\footnotetext{
${ }^{1}$ Código del Proyecto: TIC2002-02678.

${ }^{2}$ Código del Proyecto: GV04B-427.
} 


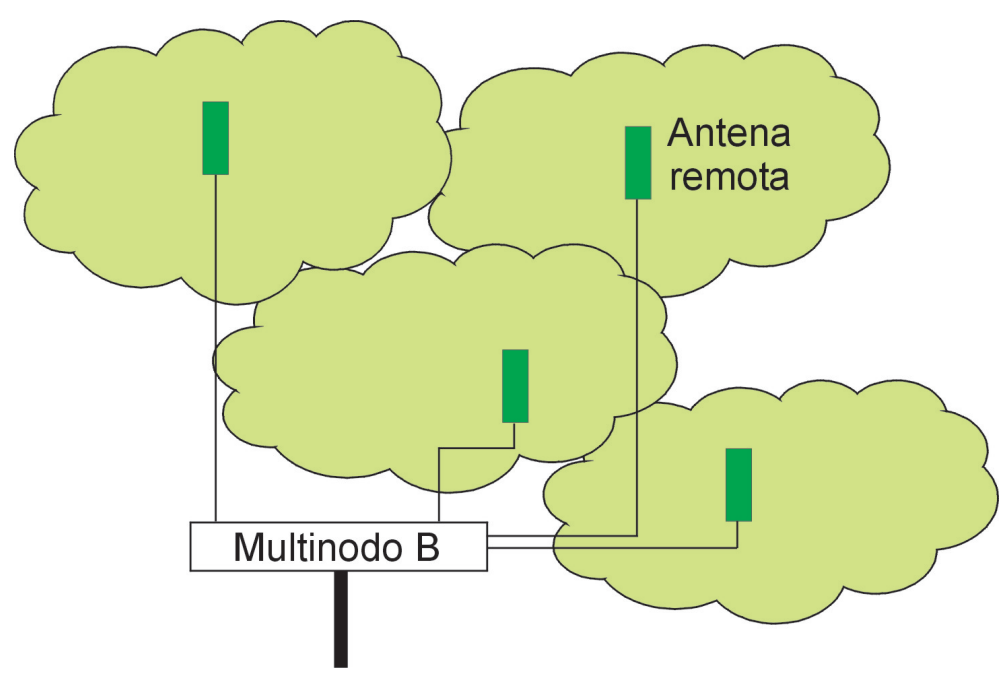

Figura 1.1: Ejemplo de la configuración Multinodo B.

Duplex) y el modo TDD (Time Division Duplex).

La idea inicial del proyecto era estudiar las prestaciones de una nueva arquitectura de acceso radio para el sistema UMTS, denominada Multinodo B. Un nodo B múltiple o multinodo, se consigue distribuyendo varias antenas en diferentes puntos del área de servicio, como muestra la figura 1.1. Estas antenas distribuidas se conectan a un mismo nodo B UMTS mediante enlaces de alta capacidad, conformando el equivalente a un array distribuido espacialmente.

Sin embargo, UMTS es un sistema limitado por interferencias. El hecho de distribuir más antenas en el área de servicio, va a afectar al nivel de interferencias que perciben los usuarios, y por extensión, también afectará a la capacidad del sistema. El objetivo de la línea de investigación en la que se enmarca esta Tesis era, precisamente, el estudio de técnicas de filtrado espacial o beamforming que contribuyeran a eliminar parte de la interferencia introducida por la nueva arquitectura de acceso radio.

La Tesis se centra en el estudio del modo FDD de un sistema W-CDMA UMTS, basado en el empleo de portadoras diferentes para el enlace uplink y downlink. Concretamente, se analiza el enlace downlink del sistema, puesto que tradicionalmente es el enlace que se considera más problemático a la hora de mejorar la capacidad del sistema.

En la Tesis pueden distinguirse claramente dos partes. La primera parte 
se desarrolla en el contexto del proyecto de investigación. En un primer paso, se define un modelo de sistema adecuado y realista para la arquitectura Multinodo B. A partir de este modelo de sistema, se consideran soluciones basadas en beamforming que permitan reducir el nivel de interferencias que perciben los usuarios del sistema. En concreto, se propone un algoritmo JPCOB o Joint Power Control and Optimal Beamforming, que combina control de potencia y beamforming óptimo, para resolver el problema de optimización clásico de minimización de potencia, donde el objetivo es minimizar la potencia total transmitida en el sistema, cumpliendo al mismo tiempo unas restricciones de calidad de servicio en los usuarios.

La segunda parte de la Tesis empieza tras la finalización del proyecto y coincide con una estancia breve en el instituto Eurecom (Sophia-Antipolis, Niza), bajo la supervisión del doctor David Gesbert. En la estancia, se revisa el trabajo realizado hasta ese momento y se llega a la conclusión de que el modelo de sistema elegido para caracterizar la arquitectura Multinodo $\mathrm{B}$, pertenece a una configuración avanzada de sistema MIMO, denominada de forma general, sistema MIMO multi-usuario multi-celda coordinado (coordinated multi-cell multi-user multiple-input multiple-output system).

Un sistema MIMO multi-usuario multi-celda coordinado está formado por varias estaciones base que cooperan perfectamente en datos a través de un enlace de alta capacidad y que transmiten de forma coordinada a todos los usuarios de una determinada área. Además, cada estación base del sistema está sujeta a una restricción en la potencia total que puede transmitir.

Bajo este nuevo punto de vista, se abandona el concepto de Multinodo B y se integra todo el trabajo realizado bajo la óptica de la configuración MIMO multi-usuario multi-celda. Paralelamente, se plantean nuevas soluciones algorítmicas para resolver el problema de minimización de potencia, que mejoran las prestaciones del algoritmo propuesto en la primera parte de la Tesis. Por continuidad con el trabajo anterior, el entorno considerado sigue siendo un sistema de comunicaciones W-CDMA.

Los sistemas MIMO multi-usuario multi-celda coordinados, se corresponden con una línea de investigación muy reciente que trata de mejorar las prestaciones de los sistemas de comunicaciones convencionales. De hecho, ni siquiera existe un nombre comúnmente aceptado para referirse a estos sistemas. Desde el inicio de esta Tesis, el modelo planteado para representar la arquitectura Multinodo B se corresponde perfectamente con una configuración MIMO multi-usuario multi-celda. Por este motivo, todo el 
trabajo desarrollado se presenta en este documento bajo la perspectiva de los sistemas MIMO.

\subsection{Objetivos de la Tesis}

Tal y como se ha planteado en el Proyecto de Tesis doctoral, aprobado por el departamento de Comunicaciones de la Universidad Politécnica de Valencia, los objetivos de la Tesis son:

- Realizar un estudio del estado del arte de los sistemas MIMO, empezando con los esquemas básicos de sistemas MIMO punto a punto hasta llegar a la configuración más reciente, el sistema MIMO multiusuario multi-celda.

Este estudio se enfoca tanto hacia a aspectos de teoría de la información como hacia técnicas y algoritmos ya propuestos para resolver los problemas de optimización clásicos de capacidad o potencia.

- Revisar las diferentes alternativas algorítmicas propuestas hasta el momento para los sistemas MIMO multi-usuario multi-celda coordinados.

- Desarrollar nuevas estrategias que mejoren las prestaciones de los algoritmos ya planteados para sistemas MIMO multi-usuario multicelda coordinados. En un primer paso, este desarrollo se realiza analíticamente, para pasar después a evaluar las prestaciones de los algoritmos propuestos mediante simulaciones.

- Realizar un análisis de aspectos prácticos de los sistemas MIMO multi-usuario multi-celda coordinados con el objetivo de mejorar aún más las prestaciones de los algoritmos propuestos (aspectos de fairness, esquemas de asignación de códigos de canalización en downlink, soluciones robustas según el grado de conocimiento del canal...). El sistema se modela de forma realista siguiendo las especificaciones del estándar UMTS.

\subsection{Descripción de los contenidos}

Los principales contenidos de la Tesis se estructuran en tres partes diferenciadas, tras las que se incluye el Capítulo de conclusiones y líneas futuras, los apéndices y las referencias bibliográficas. 
Parte 1. Introducción a los sistemas MIMO.

Esta Parte contiene un único Capítulo que revisa el estado del arte de las distintas configuraciones MIMO, empezando con la configuración básica punto a punto y finalizando con la configuración más compleja y reciente, los sistemas MIMO multi-usuario multi-celda. Se revisan los sistemas MIMO desde el punto de vista de teoría de la información y desde el punto de vista de técnicas y algoritmos propuestos para cada configuración.

El objetivo de esta Parte es presentar la evolución de los sistemas MIMO hasta la configuración MIMO multi-usuario multi-celda, y comprobar como, en general, las técnicas y algoritmos que se han propuesto para esta última configuración son una mera extensión de las ya propuestas para sistemas MIMO multi-usuario. Al mismo tiempo, esta revisión de las distintas configuraciones MIMO permite ubicar el trabajo presentado en esta Tesis en un punto concreto del estado del arte de los sistemas MIMO multi-usuario multi-celda coordinados.

Parte 2. Solución Virtual uplink-based para requerimiento de QoS en el enlace downlink de un sistema MIMO multi-usuario multi-celda coordinado.

El Capítulo 3 revisa las tres soluciones clásicas del problema de minimización de potencia sobre sistemas MIMO multi-usuario convencionales. Estas tres soluciones representan las tres formas básicas de abordar el problema de minimización de potencia mediante algoritmos JPCOB que combinan técnicas de control de potencia y de beamforming óptimo.

En el Capítulo 4, se plantea el modelo de sistema elegido para caracterizar la arquitectura Multinodo B, que coincide con la configuración habitual de un sistema MIMO multi-usuario multi-celda coordinado. En un primer paso se desarrolla analíticamente un modelo simplificado de sistema W-CDMA MIMO multi-usuario multi-celda coordinado, donde se ignora el proceso que tiene lugar en el receptor de cada usuario. A partir de este primer modelo de sistema, se plantea el problema de minimización de potencia en el enlace downlink y se propone un primer algoritmo, el algoritmo JPCOB-VUL o Virtual uplink-based JPCOB, modificando uno de los algoritmos clásicos presentados en el Capítulo 3. Además, la estructura de las matrices 
involucradas en el mecanismo de control de potencia del algoritmo JPCOB-VUL permite obtener una versión computacionalmente eficiente de este algoritmo.

En el Capítulo 5, se estudian las prestaciones del algoritmo JPCOBVUL y se analizan más en detalle algunos aspectos relacionados con una implementación práctica de este algoritmo. En primer lugar, se realiza un modelado de las interferencias presentes en un sistema W-CDMA MIMO multi-usuario multi-celda coordinado, a partir del cual se proponen distintos esquemas de asignación de códigos de canalización con el objetivo de reducir el nivel de interferencias que perciben los usuarios del sistema. Posteriormente, se propone una versión robusta del algoritmo JPCOB-VUL para los escenarios en los que las estaciones base del sistema disponen de un conocimiento parcial del canal en la forma de hybrid channel knowledge. Este modelo de información de canal se corresponde con aquellas situaciones en las que las estaciones base del sistema disponen perfectamente de sus canales locales pero sólo disponen de información estadística del resto de canales del sistema, lo que puede interpretarse como que existe algún tipo de restricción en la cantidad de información que las estaciones base pueden intercambiar entre ellas a través del enlace de alta capacidad.

Parte 3. Soluciones Downlink-based para requerimiento de QoS en el enlace downlink de un sistema MIMO multi-usuario multi-celda coordinado.

En el Capítulo 6, se avanza en el modelo analítico del sistema y se plantea una formulación matricial compacta que caracteriza completamente el funcionamiento del sistema W-CDMA MIMO multiusuario multi-celda coordinado, incorporando la naturaleza asíncrona de las señales que reciben los usuarios del sistema y el proceso que tiene lugar en el receptor de cada usuario. A partir de este modelo de sistema, se plantea de nuevo el problema de minimización de potencia en el enlace downlink y se deriva un nuevo algoritmo JPCOB que mejora las prestaciones del algoritmo JPCOB-VUL, el algoritmo JPCOB-DL o Downlink-based JPCOB. Este algoritmo requiere una implementación totalmente centralizada, por lo que se propone una versión simplificada, el algoritmo JPCOB-SDL o Simplified Downlinkbased JPCOB, para aquellos casos en los que existe algún tipo de 
restricción en la cantidad de información que cada usuario puede realimentar a las estaciones base, pero donde las estaciones base siguen intercambiando datos a través del enlace de alta capacidad sin ningún tipo de problema.

El Capítulo 7 analiza en detalle algunos aspectos que afectan a las propiedades de convergencia del algoritmo JPCOB-DL. En este análisis, se proponen dos esquemas para mejorar la probabilidad de convergencia del algoritmo JPCOB-DL en sistemas MIMO multi-usuario multi-celda coordinados mal condicionados, que indirectamente resultan ser técnicas eficientes y sencillas para eliminar usuarios del sistema en los casos en los que no se alcanza la convergencia del algoritmo.

\section{Conclusiones y líneas futuras.}

En este Capítulo, se presentan las principales conclusiones del trabajo presentado en esta Tesis. Además, se comentan algunas líneas de investigación que siguen abiertas tras la finalización de la Tesis.

\section{Apéndices.}

En el primer apéndice, se introducen brevemente el Teorema de PerronFrobenius y la iteración de Jacobi, puesto que ambos constituyen la base matemática que demuestra la convergencia de los algoritmos JPCOB presentados en la Tesis.

En el segundo apéndice, se presenta la solución del problema de minimización de potencia en un sistema MIMO multi-usuario convencional cuando se aplica optimización convexa. Este planteamiento está generando un interés creciente por parte de los grupos de investigación que estudian el problema de minimización de potencia, debido a las relaciones que presenta con las aproximaciones clásicas presentadas en el Capítulo 3.

Por último, en el tercer apéndice, se incluyen las figuras correspondientes a los sistemas MIMO multi-usuario multi-celda coordinados formados por dos estaciones base.

\section{Bibliografía.}

Finalmente, se recogen todas las referencias bibliográficas referidas a lo largo del texto. 



\section{Parte I}

\section{Introducción a los sistemas MIMO}





\section{Capítulo 2}

\section{Introducción a los sistemas MIMO}

Los sistemas de comunicaciones con múltiples antenas o sistemas MIMO (Multiple-Input Multiple-Output), han generado en la última década un gran interés de la comunidad investigadora. Inicialmente propuestos para enlaces punto a punto entre un transmisor y un receptor, estos sistemas se perfilan como una posible solución tecnológica para los futuros sistemas de comunicaciones inalámbricas o celulares, debido a la alta eficiencia espectral que presentan bajo determinadas condiciones [3,4].

En grandes líneas, un sistema MIMO punto a punto o single-user se define como un enlace donde tanto el transmisor, que podría ser una estación base o un access point, como el receptor, que sería el usuario móvil o inalámbrico, disponen de varias antenas y por tanto es posible combinar de alguna forma las múltiples muestras en transmisión y recepción. De esta forma se introduce una dimensión espacial en el procesado de señal asociado, que pasa a ser espacio-temporal. Aunque en este Capítulo se presentan los sistemas MIMO en un contexto de sistemas de comunicaciones inalámbricas, los canales MIMO son una representación matemática bastante adecuada de diversos sistemas de comunicaciones.

Este Capítulo revisa el estado del arte de las distintas configuraciones de sistemas MIMO, empezando con la configuración básica punto a punto y terminando con la configuración más avanzada, el sistema MIMO multiusuario multi-celda. Cada configuración MIMO se presenta desde el punto de vista de teoría de la información y desde el de las técnicas y algoritmos propuestos para resolver distintos criterios de optimización. La figura 2.1 


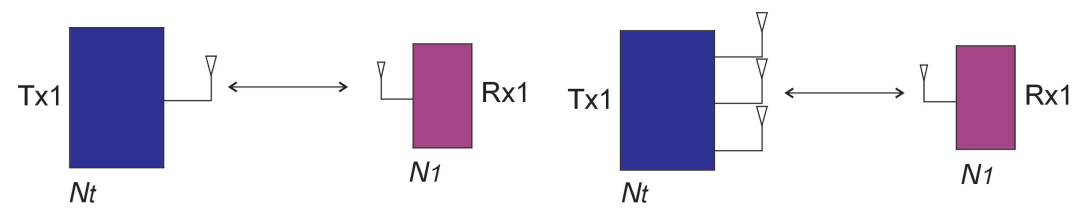

(a) Sistema SISO de una celda.

(b) Sistema SIMO/MISO de una celda.
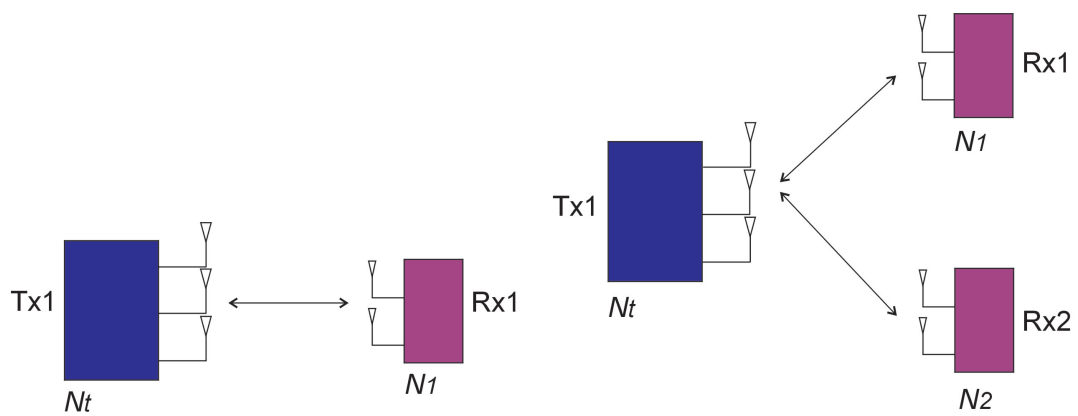

(c) Sistema MIMO de una celda.

(d) Sistema MIMO multi-usuario de una celda.
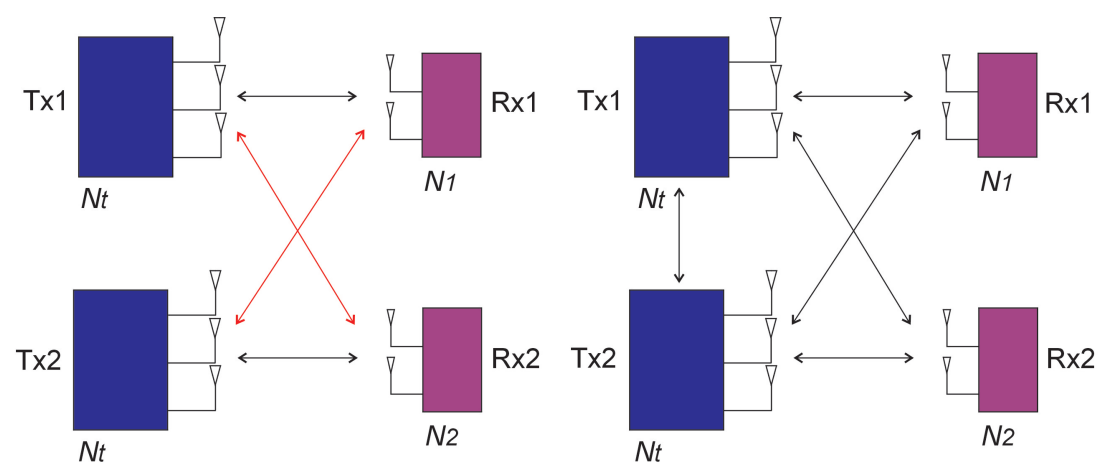

(e) Sistema MIMO multi-usuario

(f) Sistema MIMO multi-usuario multi-celda coordinado.

Figura 2.1: Evolución de los sistemas MIMO.

muestra la evolución que han seguido los sistemas MIMO hasta llegar a la configuración avanzada MIMO multi-usuario multi-celda [5]. 


\subsection{Sistemas SISO, SIMO y MISO}

Un sistema SISO (Single-Input Single-Output) es un enlace donde el transmisor y el receptor disponen de una sola antena. La capacidad de estos sistemas en (bit/seg./Hz) viene dada por [6]:

$$
C=\log _{2}\left\{1+\rho|h|^{2}\right\},
$$

donde $h$ es el canal normalizado entre las dos antenas y $\rho$ es la relación señal a ruido (SNR, Signal-to-Noise Ratio) en la antena receptora.

Cuando el transmisor o el receptor disponen de $N_{t}$ o $N_{r}$ antenas, respectivamente, aparecen los sistemas MISO (Multiple-Input Single-Output) o SIMO (Single-Input Multiple-Output). La tecnología de antenas inteligentes o smart antennas [7], considerada la precursora de los sistemas MIMO, también se incluye dentro de los sistemas SIMO o MISO. En esta tecnología, se utilizaban arrays de antenas para mejorar las prestaciones de los sistemas inalámbricos (cobertura, calidad del enlace o capacidad) en presencia de interferencias o efectos adversos de propagación.

Básicamente, la tecnología de antenas inteligentes utilizaba dos técnicas, la conformación de haz o beamforming y la diversidad espacial.

Beamforming. Un beamformer es un filtro espacial que opera sobre las salidas de un array de antenas, con el objetivo de estimar la señal procedente de una determinada dirección deseada en presencia de ruido e interferencias. El beamformer separa, mediante filtrado espacial, señales que se superponen en frecuencia pero que tienen fuentes espaciales distintas.

Los criterios típicos a la hora de diseñar un beamformer se basan en optimizar la señal a la salida del array con respecto a un criterio determinado, que puede ser potencia máxima, máxima SNR, mínima interferencia o máxima relación señal a ruido más interferencias (SINR, Signal-to-Interference-plus-Noise Ratio).

Físicamente, un beamformer está formado por un conjunto de pesos, que se encargan de modificar en módulo y fase las componentes de señal recibidas que se corresponden con la fuente en cuya dirección se quiere apuntar el haz principal, con el objetivo de sumarlas en fase. El número de antenas del array determina los cambios permitidos al diagrama de radiación. 
En el caso de un beamformer funcionando en recepción (sistema SIMO), cuando el array de $N_{r}$ antenas es uniforme y lineal, la señal a la salida de las antenas y antes del beamformer podría expresarse como un vector de $N_{r}$ muestras:

$$
\mathbf{x}=\mathbf{v} s+\mathbf{n},
$$

donde $s$ es la señal transmitida por una fuente, $\mathbf{n}$ es el ruido introducido por el array, y el vector $\mathbf{v}$ es el steering vector o respuesta del array:

$$
\mathbf{v}=\left[\begin{array}{llll}
1 & e^{-j 2 \pi \sin (\theta) \frac{d}{\lambda}} & \ldots & e^{-j 2 \pi \sin (\theta) \frac{(M-1) d}{\lambda}}
\end{array}\right]^{T} .
$$

La variable $d$ representa el espaciado entre antenas, $\lambda$ es la longitud de onda y $\theta$ es el ángulo de incidencia del frente de ondas con respecto a la perpendicular al array.

Tras pasar por el beamformer, la señal recibida quedaría:

$$
y=\mathbf{w}^{H} \mathbf{x},
$$

donde $\mathbf{w}$ representa el vector de pesos del beamformer.

En un sistema SIMO, un beamformer de $N_{r}$ antenas puede aumentar en un factor $N_{r}$ la SNR de una fuente (ganancia de array o beamforming), puede extraer una fuente y cancelar $N_{r}-1$ simultáneamente, o bien puede extraer $N_{r}$ fuentes simultáneamente, suponiendo que las restantes $N_{r}-1$ son interferentes, mediante el diseño y superposición de beamformers diseñados específicamente para cada usuario.

Diversidad espacial. Esta técnica se basa en el hecho de que en un entorno con propagación multi-camino, una misma señal que llega a ubicaciones distintas sufre desvanecimientos incorrelados. De esta forma la probabilidad de detectar un desvanecimiento del canal disminuye si se dispone de varias antenas en transmisión o recepción, obteniendo así una ganancia por diversidad.

En un sistema SIMO, el receptor puede estimar el canal mediante secuencias de entrenamiento, dando lugar a distintas técnicas de diversidad espacial en recepción.

Las técnicas óptimas de diversidad espacial en recepción se dividen en lineales y no lineales. Dentro de las técnicas óptimas lineales, se 
encuentra la combinación MRC (Maximum Ratio Combining), que trata de maximizar la SNR y la combinación MMSE (Minimum Mean Square Error), que minimiza el error cuadrático medio. Dentro de las técnicas óptimas no lineales se encuentra la combinación ML (Maximum Likelihood).

Las técnicas subóptimas más comunes son la combinación EGC (Equal Gain Combining), donde las señales se ajustan en fase y simplemente se suman, y la selección de antena o switching, donde se selecciona la salida de una antena según un criterio determinado (potencia de señal deseada, potencia total o SNR en cada antena). Esta última técnica es la más sencilla de implementar.

Por otro lado, los sistemas MISO necesitan conocer de alguna forma el canal para poder implementar técnicas de beamforming. Cuando no se dispone de un conocimiento perfecto o parcial del canal, las técnicas más utilizadas son las de diversidad espacial en transmisión, que se comentarán en el siguiente apartado de sistemas single-user MIMO.

En un sistema SIMO con $N_{r}$ antenas en recepción, la capacidad en (bit/seg./Hz) (2.1) se modifica:

$$
C=\log _{2}\left\{1+\rho \sum_{i=1}^{N_{r}}\left|h_{i}\right|^{2}\right\},
$$

donde $h_{i}$ es el canal para la antena receptora $i$. Analizando la expresión anterior, se observa que aumentar el número de antenas receptoras sólo produce un aumento logarítmico de la capacidad media.

En el caso de un sistema MISO con $N_{t}$ antenas transmisoras, cuando no se dispone de conocimiento de canal en transmisión y se opta por un esquema de diversidad espacial, la capacidad en (bit/seg./Hz) se expresa:

$$
C=\log _{2}\left\{1+\frac{\rho}{N_{t}} \sum_{i=1}^{N_{t}}\left|h_{i}\right|^{2}\right\} .
$$

El hecho de dividir la SNR por $N_{t}$ asegura que la potencia total transmitida es fija. De la expresión anterior se deduce que se pierde la ganancia de array (con respecto a (2.5)) y que la capacidad mantiene una relación logarítmica con $N_{t}$. 


\subsection{Sistemas single-user MIMO}

Siguiendo la figura 2.1, la siguiente configuración en la evolución de los sistemas MIMO es la de un sistema single-user MIMO, donde un transmisor equipado con $N_{t}$ antenas se comunica con un único receptor que dispone de $N_{r}$ antenas. La idea fundamental es la de explotar la dimensión espacial que introducen las múltiples antenas en transmisión y recepción.

En un sistema MIMO, el canal pasa a ser una matriz. En los sistemas SIMO y MISO, determinadas técnicas de beamforming mejoran sus prestaciones en entornos LOS (Line of Sight) de propagación, mientras que algunas técnicas de diversidad espacial mejoran en condiciones NLOS (Non-Line of Sight) de propagación. La diferencia fundamental de los sistemas MIMO con respecto a los sistemas SIMO o MISO es que ahora, se aprovecha la propagación multi-camino en lugar de mitigarla, mediante la técnica de multiplexado espacial.

La técnica de multiplexado espacial permite mandar señales independientes de forma simultánea sobre el canal MIMO. Esto es debido a que en un escenario con propagación multi-camino, el canal MIMO puede ser espacialmente selectivo y los distintos canales entre antenas transmisoras y receptoras pueden ser incorrelados entre sí.

Aunque actualmente los sistemas soportan múltiples usuarios, los resultados de los sistemas de un único usuario siguen siendo interesantes puesto que la forma más habitual de analizar un sistema MIMO multi-usuario es reducirlo a un sistema de un único usuario, asignando a los usuarios recursos ortogonales en tiempo, espacio, frecuencia o código.

\subsubsection{Modelo del sistema}

El modelo del sistema single-user MIMO es el siguiente:

$$
\mathbf{y}=\mathbf{H s}+\mathbf{n},
$$

donde $\mathbf{s}=\left[\begin{array}{llll}s_{1} & s_{2} & \ldots & s_{N_{t}}\end{array}\right]^{T}$ es la señal transmitida, $\mathbf{y}=\left[\begin{array}{lll}y_{1} & y_{2} \ldots y_{N_{r}}\end{array}\right]^{T}$ es la señal recibida y $\mathbf{n}$ es el vector de ruido blanco y aditivo en recepción, cuyos componentes son variables i.i.d. (independent identically distributed) complejas Gaussianas, circularmente simétricas de media nula y varianza unidad. 
La matriz de canal, de dimensiones $\left[N_{r} \times N_{t}\right]$, presenta la siguiente estructura:

$$
\mathbf{H}=\left[\begin{array}{cccc}
h_{11} & h_{12} & \cdots & h_{1 N_{t}} \\
h_{21} & h_{22} & \cdots & h_{2 N_{t}} \\
\vdots & & \ddots & \vdots \\
h_{N_{r} 1} & h_{N_{r} 2} & \cdots & h_{N_{r} N_{t}}
\end{array}\right]
$$

donde $h_{i j}$ representa el canal entre la antena $j$ en transmisión y la antena $i$ en recepción. La distribución de la matriz de canal $\mathbf{H}$ depende del entorno de propagación.

\section{Modelado del canal con CDIT y CSIR perfecta}

El comportamiento de un sistema MIMO depende directamente de las propiedades de la matriz de canal MIMO, de forma que el modelado del canal es fundamental a la hora de evaluar las prestaciones de un sistema MIMO. Por ejemplo, la técnica de multiplexado espacial requiere ciertas condiciones de propagación multi-camino para que los canales entre antenas sean independientes. Sin embargo, la condición de la matriz de canal se puede degradar, por ejemplo, debido a aspectos de correlación entre antenas.

Cuando tanto transmisor como receptor disponen de información del canal instantáneo, se dice que ambos poseen CSI perfecta (Channel State Information). La situación en la que sólo el transmisor dispone del canal instantáneo se denomina CSIT, mientras que si es el receptor el que dispone del canal instantáneo, se denomina CSIR. Si sólo la distribución del canal es conocida, se dice que se dispone de CDI (Channel Distribution Information).

A la hora de estimar el canal, el receptor utiliza secuencias de entrenamiento. El transmisor, sin embargo, necesita un canal de realimentación dedicado o bien puede obtener cierto conocimiento parcial a partir de las componentes recíprocas del canal.

A partir del modelo (2.7), el escenario de CSI perfecta se corresponde con sistemas donde la matriz de canal $\mathbf{H}$ se conoce perfectamente en el transmisor (CSIT) y en el receptor (CSIR), que es una situación muy poco realista. El escenario en el que se dispone de CDIT y CSIR, se corresponde con un sistema donde el receptor sigue perfectamente las variaciones del canal y realimenta la CDI al transmisor. La distribución del canal depen- 
derá entonces de la estimación del canal del receptor y de los errores asociados a estas estimas.

Suponiendo que los coeficientes de canal son variables complejas Gaussianas, la mayor parte de los trabajos de investigación se centran en los siguientes modelos de canal [8]:

Zero-mean spatially white (ZMSW). La media del canal es nula y los elementos de la matriz de canal son variables i.i.d.:

$$
E[\mathbf{H}]=0, \mathbf{H}=\mathbf{H}_{\mathbf{w}} .
$$

Este modelo se corresponde con una distribución donde los coeficientes del canal se promedian sobre distintos escenarios de propagación.

Channel mean information (CMI). La media del canal es no nula. La matriz de canal contiene un factor de escala que se corresponde con errores en la estima del canal:

$$
E[\mathbf{H}]=\overline{\mathbf{H}}, \mathbf{H}=\overline{\mathbf{H}}+\sqrt{\alpha} \mathbf{H}_{\mathbf{w}},
$$

donde $\overline{\mathbf{H}}$ y $\alpha$ son constantes que se interpretan como la estima del canal obtenida a partir de la información realimentada por el receptor y el error en esta estima, respectivamente.

Channel covariance information (CCI). En este modelo se supone que el canal varía demasiado rápido para poder determinar su media, así que se asume una media nula y se incluye información sobre la correlación de las antenas transmisoras $\left(\right.$ matriz $\mathbf{R}_{t}$ ) y receptoras (matriz $\mathbf{R}_{r}$ ):

$$
E[\mathbf{H}]=\overline{\mathbf{0}}, \mathbf{H}=\left(\mathbf{R}_{r}\right)^{(1 / 2)} \mathbf{H}_{\mathbf{w}}\left(\mathbf{R}_{t}\right)^{(1 / 2)} .
$$

La matriz $\mathbf{H}_{\mathbf{w}}$ es una matriz de dimensiones $\left[N_{r} \times N_{t}\right]$ con entradas que son variables i.i.d. complejas Gaussianas, circularmente simétricas de media nula y varianza unidad. 


\subsubsection{Capacidad del sistema}

La capacidad para un canal invariante con el tiempo, y para un único usuario, se define como la máxima información mutua entre la entrada del canal y la salida. Por el teorema de la capacidad de Shanon, se sabe que ésta es la máxima tasa de transmisión de datos que puede transmitirse por el canal con una probabilidad de error arbitrariamente baja.

A finales de los años noventa, los trabajos de Foschini [3] y Telatar [4] demostraron las enormes capacidades que teóricamente podían alcanzar los sistemas single-user MIMO bajo unas determinadas condiciones. Las referencias [8] y [9] resumen los principales resultados que se disponen sobre la capacidad de un sistema single-user MIMO.

\section{Capacidad del sistema single-user MIMO sin CSIT}

Si no se dispone de CSIT y se mantiene una restricción de potencia máxima en transmisión, la capacidad del sistema MIMO en (bit/seg./Hz) viene dada por $[3,4]$ :

$$
C=\log _{2}\left\{\operatorname{det}\left(\mathbf{I}_{N_{r}}+\frac{\rho}{N_{t}} \mathbf{H} \mathbf{H}^{H}\right)\right\},
$$

donde la potencia de transmisión se divide equitativamente entre las antenas transmisoras.

Foschini [3] y Telatar [4], demostraron que bajo determinadas condiciones, la capacidad (2.12) crece linealmente con $m=\operatorname{mín}\left(N_{t}, N_{r}\right)$ con respecto a (2.1) y no logarítmicamente como en (2.6). Hay que resaltar, sin embargo, que los primeros estudios sobre la capacidad de los sistemas MIMO se encuentran en [10].

En (2.12), la capacidad se presenta como una variable aleatoria. Habitualmente, para caracterizarla se utiliza la capacidad media o ergódica, que es un promedio de la capacidad sobre todos los estados posibles del canal, o la capacidad de fallo u outage. La capacidad de outage es una medida que indica la fiabilidad del sistema, es decir, los valores que soporta el sistema un determinado tanto por ciento de tiempo.

A la hora de interpretar (2.12), hay que tener en cuenta que el modelo de canal es determinante e influye en la capacidad del sistema. Generalmente, la capacidad de un sistema MIMO se analiza en un entorno casi-estático, donde se asume que el canal permanece fijo el tiempo suficiente para que se transmitan los símbolos necesarios para que tengan sentido los límites 
de teoría de la información. Asimismo, la expresión (2.12) se derivó para un canal i.i.d. Rayleigh con desvanecimiento plano en frecuencia, donde los $h_{i j}$ eran variables i.i.d. complejas Gaussianas con parte real e imaginaria independientes, de media cero e idéntica varianza.

\section{Capacidad del sistema single-user MIMO con CSIT y CSIR}

La expresión (2.12) se derivó para un sistema single-user MIMO sin CSIT. Desde el punto de vista de teoría de la información, es posible obtener una expresión de la capacidad de un sistema single-user MIMO donde el transmisor disponga de algún tipo de conocimiento de canal.

El modelo de señal (2.7) representa un sistema MIMO de un único usuario en un entorno de desvanecimiento plano en frecuencia y con ruido blanco Gaussiano aditivo (AWGN, Additive White Gaussian Noise). La única interferencia es la que se produce entre los símbolos que entran al sistema MIMO.

Si la matriz de correlación $\mathbf{Q}=E\left[\mathbf{s s}^{H}\right]$ cumple una restricción de potencia total $\operatorname{Tr}[\mathbf{Q}] \leq \rho$, la capacidad del sistema representado por (2.7) en (bit/seg./Hz) sería [4]:

$$
C=\log _{2}\left\{\operatorname{det}\left(\mathbf{I}_{N_{r}}+\mathbf{H Q H} \mathbf{H}^{H}\right)\right\} .
$$

Hay que tener en cuenta que cuando se asigna la misma potencia a todas las antenas transmisoras, $\mathbf{Q}=\left(\rho / N_{t}\right) \mathbf{I}_{N_{t}}$, las capacidades (2.12) y (2.13) coinciden. Esta distribución es óptima cuando no se dispone de CSIT [4]. Sin embargo, cuando el canal es conocido en transmisión, la $\mathbf{Q}$ óptima no tiene por qué ser proporcional a la identidad.

Telatar demostró que la expresión (2.12) puede reescribirse como (bit/seg./Hz) [4]:

$$
C=\sum_{i=1}^{m} \log _{2}\left\{1+\frac{\rho}{N_{t}} \lambda_{i}\right\},
$$

donde $\lambda_{i}$ son los valores propios no nulos de $\mathbf{W}$ :

$$
\mathbf{W}=\left\{\begin{array}{l}
\mathbf{H H}^{H}, N_{r} \leq N_{t}, \\
\mathbf{H}^{H} \mathbf{H}, \quad N_{t}>N_{r},
\end{array}\right.
$$

y $m=\min \left(N_{t}, N_{r}\right)$. 
La expresión anterior se corresponde con una descomposición del canal MIMO en $m$ canales SISO paralelos, a partir de la descomposición SVD (Singular Value Decomposition) de la matriz de canal H. Si la descomposición SVD de $\mathbf{H}$ se escribe:

$$
\mathbf{H}=\mathbf{U D V}^{H},
$$

con $\mathbf{U}$ y $\mathbf{V}$ matrices unitarias y $\mathbf{D}$ diagonal, el modelo (2.7) se reescribe:

$$
\tilde{\mathbf{y}}=\mathbf{D} \tilde{\mathbf{s}}+\tilde{\mathbf{n}},
$$

donde $\mathbf{D}=\operatorname{diag}\left\{\left[\sqrt{\lambda_{1}} \ldots \sqrt{\lambda_{m}} 0 \ldots 0\right]\right\}, \tilde{\mathbf{y}}=\mathbf{U}^{H} \mathbf{y}, \tilde{\mathbf{s}}=\mathbf{V}^{H} \mathbf{s}$ y $\tilde{\mathbf{n}}=\mathbf{U}^{H} \mathbf{n}$. Esta ecuación representa el sistema como $m$ canales SISO, cada uno con una potencia de transmisión dada por los valores propios $\lambda_{i}$. Por tanto, la capacidad puede expresarse en función de los valores propios de la matriz W.

Si se dispone de CSIT y CSIR, la expresión (2.13) se puede optimizar sobre la matriz de correlación $\mathbf{Q}$, manteniendo la restricción total en potencia. En este caso, la matriz $\mathbf{Q}$ óptima es conocida y consiste en obtener las potencias de transmisión para los canales SISO paralelos equivalentes mediante la técnica de waterfilling (WF) [4]. Teniendo en cuenta que $m=\operatorname{mín}\left(N_{t}, N_{r}\right)$, la capacidad en (bit/seg./Hz) se obtiene según:

$$
C=\sum_{i=1}^{m} \log _{2}\left\{\mu \lambda_{i}\right\}^{+},
$$

donde el parámetro $\mu$ o waterlevel se elige de forma que la potencia total transmitida cumpla:

$$
\rho=\sum_{i=1}^{m}\left(\mu-\lambda_{i}^{-1}\right)^{+},
$$

con $x^{+}=\operatorname{máx}(0, x)$.

Puesto que $\mu$ sigue una función no lineal con los valores propios $\lambda_{i}$, la capacidad (2.17) se obtiene numéricamente a partir de cualquier $\mathbf{W}$ siguiendo las ecuaciones (2.17) y (2.18).

\section{Comparativa entre las capacidades con y sin CSI}

Comparando las capacidades (2.12) y (2.17), hay que destacar que el hecho de diseñar una matriz de correlación óptima $\mathbf{Q}$ gracias al conocimiento 
del canal en transmisión, aporta una ganancia que podría denominarse de realimentación o feedback. Esta ganancia de realimentación es significativa cuando la SNR es baja, pero tiende a cero a medida que ésta aumenta. Este hecho puede explicarse de la siguiente forma: el hecho de conocer el canal en transmisión proporciona una ganancia de array, mientras que las ganancias por diversidad y por multiplexado espacial no requieren conocimiento del canal. Puesto que la ganancia de array disminuye para SNRs altas, la ganancia de realimentación también disminuye.

\subsubsection{Técnicas single-user MIMO}

Las capacidades presentadas en el apartado anterior se corresponden con límites teóricos, donde la única restricción práctica es la potencia total transmitida. Sin embargo, en un escenario MIMO realista, donde el transmisor es una estación base o access point y el receptor es un usuario móvil o inalámbrico, se requieren técnicas de transmisión y recepción de complejidad razonable.

Actualmente, las técnicas de transmisión sobre canales single-user MIMO se dividen en dos categorías, las orientadas a maximizar la diversidad (STC, Space-Time Codes) y las orientadas a maximizar la tasa de transmisión de datos (técnica de multiplexado espacial).

Las técnicas que maximizan la tasa de transmisión de datos intentan mejorar el comportamiento medio de la capacidad. Básicamente, funcionan gracias a la técnica de multiplexado espacial de los canales MIMO, donde se pueden mandar tantas señales independientes como antenas en transmisión se dispongan.

Sin embargo, estas señales independientes se codifican de forma conjunta para evitar errores de transmisión. Si el nivel de redundancia aumenta hasta el punto en que cada antena transmisora dispone de una versión totalmente redundante de la misma señal, el hecho de tener múltiples antenas en transmisión sólo sirve para aumentar la diversidad espacial y no para mejorar la tasa de transmisión de datos.

Las técnicas que realizan la codificación conjunta de las señales a transmitir son los códigos STC. Generalmente, cuando se diseñan estos códigos, se asume que una combinación ponderada de los símbolos se transmite desde una antena determinada en un instante determinado; se generan tantas combinaciones como antenas transmisoras y se transmiten simultáneamente, una por cada antena. 
Las técnicas STC y de multiplexado espacial no necesitan de CSIT. Sin embargo, el análisis teórico de las capacidades presupone que se pueden mejorar las prestaciones del sistema si se dispone de información del canal en transmisión, por ejemplo, mediante la técnica de waterfilling. Por otro lado, puede que esta técnica, óptima desde el punto de vista de teoría de la información, no lo sea en la práctica cuando se dispone de CSIT.

\section{Técnicas STC}

Las técnicas STC se plantearon originalmente para sistemas MISO, aunque son ampliables al caso MIMO. Requieren CSIR, que puede obtenerse mediante secuencias de entrenamiento.

Las técnicas STC empezaron con los códigos de Trellis (STTC, SpaceTime Trellis Codes) [11], donde se utilizaba un algoritmo de Viterbi en recepción. Los códigos STTC proporcionan una ganancia por diversidad igual al número de antenas transmisoras y una ganancia de codificación que depende de la complejidad del código. Sin embargo, cuando el número de antenas es fijo, la complejidad de decodificación de los STTC aumenta exponencialmente con parámetros como el orden de diversidad o la tasa de transmisión de datos requerida.

Posteriormente, Alamouti [12] introdujo los códigos de bloque espaciotiempo (STBC, Space-Time Block Codes), que podían decodificarse con un sencillo procesado lineal en el receptor. Los códigos STBC proporcionan la misma ganancia por diversidad que los códigos STTC, sin embargo, la ganancia por codificación que presentan es prácticamente nula. Para sistemas con $N_{t}=2$ antenas transmisoras, estos códigos STBC son códigos de tasa plena o full rate, tanto para modulaciones reales como complejas. Si el número de antenas transmisoras aumenta a $N_{t}=4$ o $N_{t}=8$, es necesario utilizar modulaciones reales para mantener la característica full rate. Sin embargo, se sabe que no es posible obtener códigos STBC de full rate para un mayor número de antenas transmisoras.

\section{Técnica de multiplexado espacial}

La técnica de multiplexado espacial puede interpretarse como un caso particular de los códigos STBC, donde conjuntos de datos independientes se transmiten en antenas distintas, mejorando la tasa de transmisión de datos media del sistema MIMO. 
En recepción, el objetivo es separar los distintos conjuntos de datos. Una posibilidad es utilizar técnicas de ZF (Zero Forcing), que dependen de la condición de la matriz de canal, y donde ésta simplemente se invierte:

$$
\hat{\mathbf{s}}=\mathbf{H}^{\dagger} \mathbf{y}
$$

donde $\mathbf{H}^{\dagger}=\left(\mathbf{H}^{H} \mathbf{H}\right)^{-1} \mathbf{H}^{H}$, es la pseudo-inversa del canal.

Otra opción es utilizar un receptor MMSE (Minimum Mean Square Error) que proporciona una mejora limitada, optimizando el criterio:

$$
\mathbf{W}=\arg \operatorname{mín}\left\{E\left[\left\|\mathbf{W}^{H} \mathbf{y}-\mathbf{s}\right\|^{2}\right]\right\},
$$

con lo que se obtiene:

$$
\hat{\mathbf{s}}=\mathbf{H}^{H}\left(\mathbf{H} \mathbf{H}^{H}+\mathbf{R}_{n}\right)^{-1} \mathbf{y},
$$

donde $\mathbf{R}_{n}$ es la matriz de correlación del ruido y las interferencias.

La técnica óptima de decodificación es la técnica ML (Maximum Likelihood), donde el receptor compara todas las posibles combinaciones de símbolos que podrían haberse transmitido con lo que recibe. La complejidad es alta, puesto que se realiza una búsqueda exhaustiva, creciente con $N_{t}$ y con el orden de la modulación:

$$
\hat{\mathbf{s}}=\arg \min _{\mathbf{s} \in \mathcal{A}}\left\{\|\mathbf{y}-\mathbf{H s}\|^{2}\right\},
$$

donde $\mathcal{A}$ es el alfabeto de la modulación.

V-BLAST (Vertical - Bell Labs Layered Space-Time) [13] también se incluye dentro de las técnicas de multiplexado espacial. En esta técnica, el proceso de invertir la matriz de canal se realiza en capas y de forma sucesiva. El primer paso es implementar un método lineal para conseguir una primera estima $\left(\hat{s}_{1}\right)$ y elegir el símbolo más cercano del alfabeto $\left(\left[\hat{s}_{1}\right]\right)$. A continuación, la contribución de esta primera estima se elimina y se continúa iterando:

$$
\begin{aligned}
\hat{s}_{1} & =\mathbf{w}_{1}^{H} \mathbf{y}, \\
\hat{s}_{1} & =\left[\hat{s}_{1}\right], \\
\mathbf{y}_{1} & =\mathbf{y}-\mathbf{h}_{1} \hat{s}_{1}, \\
\hat{s}_{2} & =\mathbf{w}_{2}^{H} \mathbf{y}_{1} .
\end{aligned}
$$


La técnica V-BLAST presenta una complejidad lineal con $N_{t}$. Su principal desventaja es que presenta un comportamiento desigual en cada capa, por lo que generalmente se ordenan los símbolos de entrada de mayor a menor SNR para mejorar las prestaciones y minimizar los errores de propagación entre etapas.

Comparando los distintos receptores, el receptor MMSE mejora las prestaciones del ZF, sin embargo, el orden de diversidad que proporciona, $N_{r}-N_{t}+1$, provoca que no sea muy adecuado para sistemas con el mismo número de antenas transmisoras que receptoras.

El receptor ML es un receptor óptimo, pero su complejidad aumenta con el orden de la modulación y $N_{t}$, mientras que V-BLAST ofrece un compromiso entre las prestaciones óptimas del ML y la baja complejidad de los receptores lineales.

Por último, cuando no se dispone de CSIT ni de CSIR, se utilizan las llamadas técnicas ciegas, que suelen aumentar la complejidad computacional.

\section{Multiplexado espacial o STC}

Cuando se estudian las prestaciones de los sistemas single-user MIMO, una de las cuestiones más interesantes es decidir entre la implementación de una técnica de multiplexado espacial o una STC.

La técnica de multiplexado espacial permite que las antenas se utilicen de forma independiente, sin embargo, no proporciona ganancia por diversidad y no es el mejor esquema de transmisión para un requerimiento de BER (Bit Error Rate) determinado. Por otro lado, cuando los símbolos se codifican con STC, se introduce una ganancia por diversidad y por codificación. Al mismo tiempo, las ganancias relacionadas con estas técnicas varían en función del número de antenas del sistema MIMO.

Algunos autores proponen que existe un punto óptimo o sweet point, donde en función de las características del sistema MIMO concreto, se combina de forma óptima el multiplexado espacial con STC, de forma que se maximiza la tasa de transmisión de datos y al mismo tiempo se garantiza una mínima ganancia por diversidad [14]. 


\subsection{Sistemas MIMO multi-usuario}

Los antecedentes de estos sistemas se encuentran también en la tecnología de antenas inteligentes, donde un array de antenas permitía, mediante SDMA (Spatial Division Multiple Access), comunicarse con múltiples usuarios que estaban separados únicamente por su firma espacial.

La configuración básica de un sistema MIMO multi-usuario (ver figura 2.1), comprende un transmisor (estación base o access point) equipado con $N_{t}$ antenas que se comunica con $M$ usuarios, que disponen de múltiples antenas en recepción.

Un sistema MIMO multi-usuario también puede interpretarse como un sistema single-user MIMO donde las $N_{r}$ antenas receptoras se distribuyen espacialmente en $M$ usuarios, cada uno con $N_{m}$ antenas disponibles, de forma que $N_{r}=\sum_{m} N_{m}$. Las $N_{m}$ antenas de cada usuario permiten mitigar la interferencia entre usuarios y aumentan los grados de libertad disponibles para separarlos espacialmente.

Sin embargo, existen diferencias esenciales entre los sistemas single-user MIMO y los MIMO multi-usuario. El hecho de distribuir espacialmente las $N_{r}$ antenas receptoras, implica que no sea posible coordinar las muestras que reciben los distintos usuarios. Al mismo tiempo, aparecen múltiples restricciones de potencia, una por usuario.

Por otro lado, esta distribución de las antenas provoca que el problema downlink (DL), o el enlace transmisor-usuarios, sea distinto al problema uplink (UL), o enlace usuarios-transmisor. En el enlace UL, el transmisor tiene que separar las señales de los distintos usuarios, mientras que en el enlace DL, los usuarios experimentan interferencias entre ellos. Las figuras 2.2 y 2.3 ilustran el enlace UL y DL, respectivamente, de un sistema MIMO multi-usuario. Es necesario remarcar que $N_{t}$ designa el número de antenas en el transmisor, independientemente de si está transmitiendo o recibiendo.

En el enlace UL, las técnicas MIMO multi-usuario son una generalización de las utilizadas en el caso single-user MIMO. Por el contrario, se sabe por teoría de la información que la estrategia de transmisión óptima en DL para MIMO multi-usuario, consiste en combinar la técnica de Dirty Paper Coding (DPC) con un algoritmo de scheduling y de asignación de potencia [15].

Las ventajas de los sistemas MIMO multi-usuario comprenden, entre otras, una mayor robustez frente a limitaciones como el condicionamiento de la matriz de canal, o la posibilidad de utilizar la técnica de multiplexado 


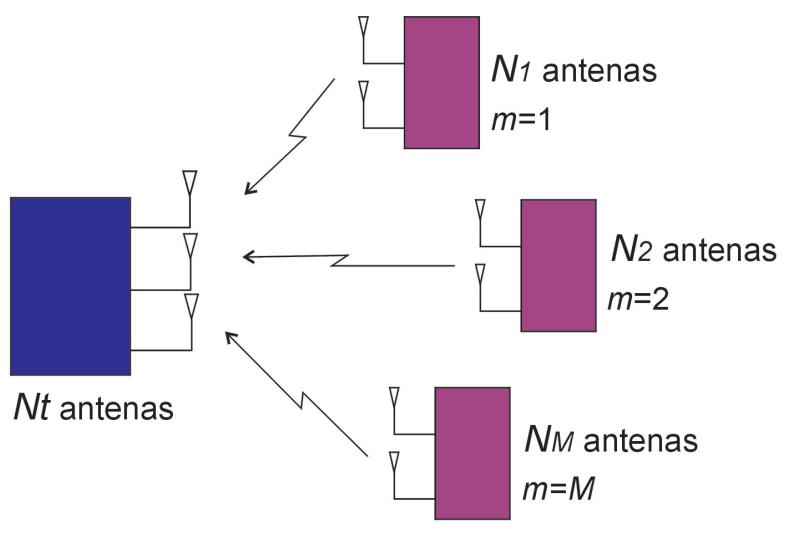

Figura 2.2: Enlace UL de un sistema MIMO multi-usuario.

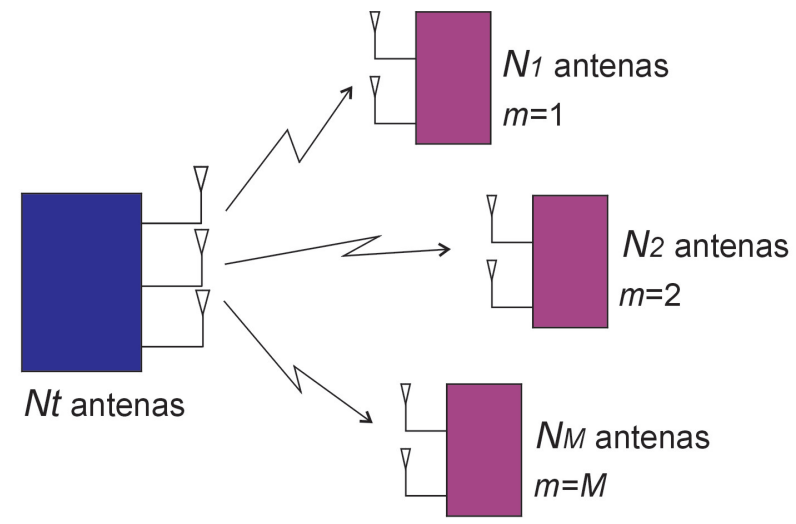

Figura 2.3: Enlace DL de un sistema MIMO multi-usuario.

espacial incluso si los usuarios disponen de una única antena, con lo que el coste de diseño de los receptores puede reducirse.

El gran problema de los sistemas MIMO multi-usuario es que necesitan de CSIT para diseñar técnicas o esquemas de transmisión en DL. En los sistemas que obtienen la CSIT mediante un canal de realimentación dedicado, la eficiencia espectral del sistema disminuye, especialmente cuando aumenta el número de usuarios $M$. Esto es especialmente crítico en sistemas de banda ancha o de alta movilidad como WiMax.

Los sistemas MIMO multi-usuario presentan también problemas de fairness, puesto que los usuarios presentan distintas condiciones de canal, y la dificultad añadida de diseñar el algoritmo de scheduling, mediante el cual 
se selecciona un grupo de $M$ usuarios a los que se les va a dar servicio simultáneamente, de entre todos los usuarios presentes en el sistema.

Los esquemas óptimos de scheduling requieren una búsqueda exhaustiva, de forma que la complejidad aumenta con el número de usuarios a seleccionar, que a su vez depende de las técnicas de transmisión, recepción y del tipo de realimentación utilizada para proporcionar CSIT. La referencia [16] presenta un resumen del estado actual de las estrategias de scheduling y de las técnicas de realimentación de CSI para los sistemas MIMO multi-usuario.

\subsubsection{Capacidad del sistema}

Consideramos un sistema MIMO multi-usuario con un transmisor equipado con $N_{t}$ antenas y $M$ usuarios equipados cada uno con $N_{m}$ antenas. El conjunto de usuarios activos, $M$, está formado por un subconjunto de los usuarios presentes en el sistema.

El estudio de los sistemas MIMO multi-usuario desde el punto de vista de teoría de la información proporciona los límites fundamentales del sistema, por ejemplo, indica cuál es la mayor tasa de transmisión de datos que va a poder recibir un usuario en el enlace DL del sistema. En el contexto de teoría de la información, el enlace DL del sistema se denomina Broadcast Channel (BC) mientras que el enlace UL es el Multiple Access Channel (MAC). La matriz $\mathbf{H}_{m}$ es la matriz del canal DL entre el transmisor y el usuario $m$; asumiendo el mismo canal en el enlace UL y DL, la matriz de canal UL del usuario $m$ sería $\mathbf{H}_{m}^{H}$.

En los sistemas single-user MIMO, la capacidad del sistema es un escalar. En los sistemas MIMO multi-usuario, sus límites vienen definidos por una región de capacidad, que es una región de $M$-dimensiones, donde cada punto representa un vector formado por el conjunto de tasas de transmisión que alcanzan los $\mathrm{M}$ usuarios simultáneamente, $\left(R_{1}, \ldots, R_{M}\right)$. Una zona importante del borde de la región de capacidad son los llamados sumrate points, sum-rate capacity o sum-capacity, que representan los puntos donde el sum-rate o la suma de las tasas de transmisión de datos entre el transmisor y todos los usuarios es máxima $\left(\operatorname{máx} \sum_{m} R_{m}\right)$.

La referencia [8] resume los principales resultados sobre la capacidad de un sistema MIMO multi-usuario desde el punto de vista de teoría de la información. 


\section{Región de capacidad MAC}

En el canal MAC (figura 2.2), la señal recibida $\mathbf{v}$, de dimensiones $\left[N_{t} \times 1\right]$, quedaría:

$$
\mathbf{v}=\sum_{m=1}^{M} \mathbf{H}_{m}^{H} \mathbf{u}_{m}+\mathbf{w}=\mathbf{H}^{H}\left[\begin{array}{c}
\mathbf{u}_{1} \\
\vdots \\
\mathbf{u}_{M}
\end{array}\right]+\mathbf{w}
$$

donde

$$
\mathbf{H}^{H}=\left[\begin{array}{lll}
\mathbf{H}_{1}^{H} & \cdots & \mathbf{H}_{M}^{H}
\end{array}\right],
$$

$\mathbf{H}_{m}$ es la matriz de canal de dimensiones $\left[N_{m} \times N_{t}\right], \mathbf{u}_{m}$ es la señal $\left[N_{m} \times 1\right]$ transmitida por el usuario $m$-ésimo y w es el ruido aditivo Gaussiano en el transmisor con componentes i.i.d variables complejas Gaussianas, circularmente simétricas de media nula y varianza unidad. Se asume que un usuario $m$ conoce perfectamente la matriz de canal $\mathbf{H}_{m}$.

En el canal MAC, cada usuario está sujeto a una restricción de potencia individual $P_{m}$. La matriz de correlación de cada usuario, $\mathbf{Q}_{m}=E\left[\mathbf{u}_{m} \mathbf{u}_{m}^{H}\right]$ define la restricción en potencia, $\operatorname{Tr}\left[\mathbf{Q}_{m}\right] \leq P_{m}, \forall m$.

La región de capacidad del canal MAC es conocida, tanto para un canal constante como para canales con desvanecimientos, y bajo distintas suposiciones de CSI y CDI. Para un conjunto de potencias UL, $\mathbf{P}=$ $\left(P_{1}, \ldots, P_{M}\right)$, y suponiendo que el usuario $m$-ésimo transmite una señal Gaussiana de media nula y matriz de correlación $\mathbf{Q}_{m}$, cada conjunto de matrices $\left(\mathbf{Q}_{1}, \ldots, \mathbf{Q}_{M}\right)$ define una región de $M$ dimensiones:

$$
\left\{\left(R_{1}, \ldots, R_{M}\right): \sum_{m=1}^{M} R_{m} \leq \log _{2}\left\{\operatorname{det}\left(\mathbf{I}+\sum_{m=1}^{M} \mathbf{H}_{m}^{H} \mathbf{Q}_{m} \mathbf{H}_{m}\right)\right\}\right\} .
$$

La región de capacidad para una realización determinada del canal es la unión, sobre todas las matrices $\mathbf{Q}_{m}$ que cumplen la restricción en potencia, de todas las regiones resultantes:

$$
\begin{aligned}
& C_{M A C}\left(\mathbf{P}, \mathbf{H}^{H}\right)= \\
& =\bigcup_{\substack{\operatorname{Tr}\left[\mathbf{Q}_{m}\right] \leq P_{m}, \forall m \\
\sum_{m=1}}}\left\{\begin{array}{l}
\left(R_{1}, \ldots, R_{M}\right): \\
\sum_{m=1}^{M} R_{m} \leq \log _{2}\left\{\operatorname{det}\left(\mathbf{I}+\sum_{m=1}^{M} \mathbf{H}_{m}^{H} \mathbf{Q}_{m} \mathbf{H}_{m}\right)\right\}
\end{array}\right\} .
\end{aligned}
$$

Esta región de capacidad es la unión de muchos pentágonos. Los puntos de las esquinas de los pentágonos se obtienen mediante successive decoding, 


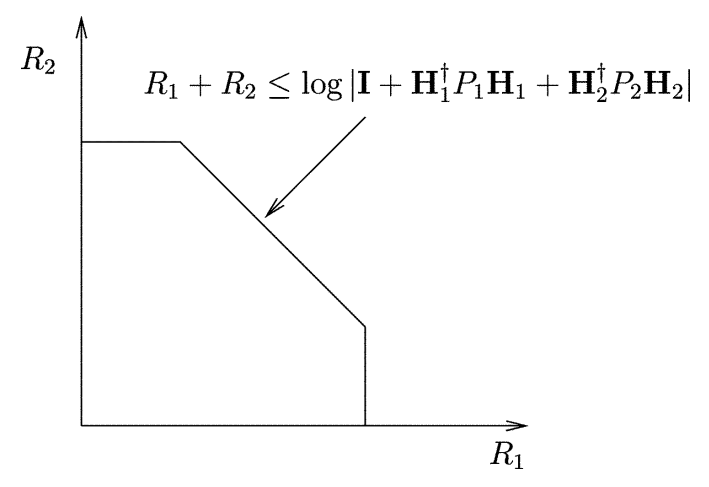

Figura 2.4: Región de capacidad MAC, $M=2, N_{m}=1$.

donde las señales de cada usuario se decodifican y se restan de la señal recibida de forma sucesiva. Para el caso concreto de un sistema MIMO con 2 usuarios, cada conjunto de matrices de correlación se corresponde con un pentágono.

La figura 2.4, muestra la región de capacidad del canal MAC para un sistema MIMO con 2 usuarios si ambos disponen de $N_{m}=1$ antenas [8]. En este caso, la matriz de correlación de cada usuario es un escalar que coincide con $P_{m}$. Obviamente, cada usuario debe transmitir a potencia máxima.

La figura 2.5 muestra la región de capacidad MAC cuando los usuarios tienen más de una antena, $N_{m}>1$ [8]. La región es ahora la unión de varios pentágonos, donde cada pentágono se corresponde con un conjunto de matrices de correlación en transmisión. El límite de la región de capacidad es una línea curva, excepto en la zona de sum-capacity, donde es una línea recta (zona entre los puntos B y C de la figura).

En [17] se propone una técnica eficiente para obtener las matrices de correlación $\mathbf{Q}_{m}$ óptimas para maximizar el sum-rate del sistema, denominada waterfilling iterativo: la matriz de correlación óptima de un usuario se obtiene aplicando la técnica de waterfilling sobre el canal del mismo usuario, incluyendo en el término de ruido la interferencia de los $M-1$ usuarios restantes. 


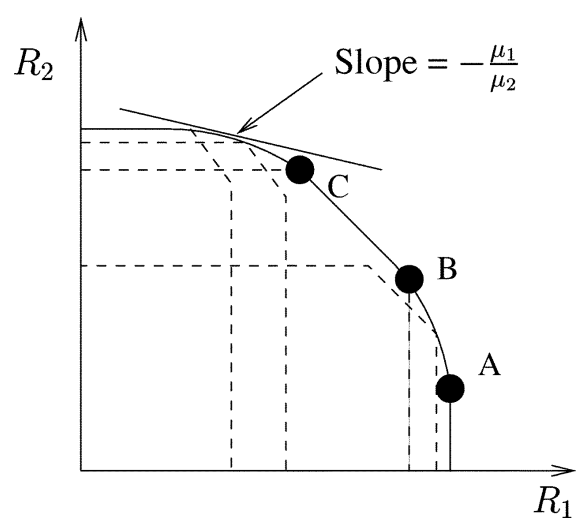

Figura 2.5: Región de capacidad MAC, $M=2, N_{m}>1$.

\section{Región de capacidad BC}

En el canal BC (figura 2.3), la señal recibida por el usuario $m$-ésimo, $\mathbf{y}_{m}$, de dimensiones $\left[N_{m} \times 1\right]$, quedaría:

$$
\mathbf{y}_{m}=\mathbf{H}_{m} \mathbf{x}+\mathbf{n}_{m}, m=1, \ldots, M
$$

donde $\mathbf{H}_{m}$ es la matriz $\left[N_{m} \times N_{t}\right]$ de canal del enlace DL, $\mathbf{x}$ es la señal $\left[N_{t} \times 1\right]$ transmitida en DL y $\mathbf{n}_{m}$, de dimensiones $\left[N_{m} \times 1\right]$ es el ruido aditivo Gaussiano en el usuario $m$, con componentes i.i.d variables complejas Gaussianas, circularmente simétricas de media nula y varianza unidad. Nuevamente, se asume que un usuario $m$ conoce perfectamente su propia matriz de canal $\mathbf{H}_{m}$.

La señal transmitida en DL, $\mathbf{x}$, es una combinación de las señales transmitidas a todos los usuarios, que puede por ejemplo, tomar la siguiente forma:

$$
\mathbf{x}=\sum_{m=1}^{M} \mathbf{x}_{m} .
$$

La potencia con la que se transmite a cada usuario viene dada por $p_{m}=$ $\operatorname{Tr}\left[\boldsymbol{\Sigma}_{m}\right]$, donde $\boldsymbol{\Sigma}_{m}$ es la matriz de correlación de la señal para el usuario $m, \boldsymbol{\Sigma}_{m}=E\left[\mathbf{x}_{m} \mathbf{x}_{m}^{H}\right]$. Asumiendo que existe una restricción en la potencia total transmitida, $\sum_{m} p_{m} \leq P_{\max }$.

Determinar la región de capacidad de un canal BC general es, actualmente, uno de los problemas más importantes en teoría de la información. 
Cuando los usuarios de un canal pueden ordenarse del más fuerte al más débil, se dice que el canal es degradado (por ejemplo, el canal SISO Gaussiano es degradado, puesto que los usuarios se pueden ordenar según la SNR recibida). En estos casos, la región de capacidad BC ya es conocida.

Sin embargo, los canales MIMO multi-usuario se caracterizan por tener una matriz de canal entre el transmisor y cada usuario. Los usuarios no se pueden ordenar, puesto que no existe un orden natural matricial, y por tanto, el canal MIMO multi-usuario es no degradado.

El canal MIMO BC Gaussiano de (2.26), aunque es no degradado, ofrece una estructura que permite caracterizar su región de capacidad, si se asume CSI perfecta y un canal constante: Caire y Shamai [15] demuestran que la técnica de DPC consigue alcanzar la sum-capacity de la región de capacidad.

Con CSIT perfecta, el transmisor conoce la interferencia que va a recibir el usuario, puesto que el transmisor genera todas las señales y al mismo tiempo conoce las distintas matrices de canal. Por tanto, teóricamente, el transmisor podría eliminar la interferencia antes de transmitir. Sin embargo, esta cancelación de interferencia no podría hacerse sin exceder la restricción en potencia $P_{\max }$.

Costa [18], demostró que cuando la interferencia y el ruido son Gaussianos, si el transmisor conoce perfectamente la interferencia en el canal, la capacidad del canal es la misma que si no hubiera interferencia aditiva, o lo que es lo mismo, se obtiene la misma capacidad que en el caso en el que el receptor también conociese la interferencia. De esta forma, se puede eliminar la interferencia antes de transmitir, sin aumentar con ello la potencia de transmisión.

En [15], Caire y Shamai demostraron que con la técnica de DPC se conseguía alcanzar la sum-capacity del canal MIMO BC con $M=2$ usuarios y $N_{t}=2$ antenas. Posteriormente se demostró que la técnica de DPC alcanza este límite con cualquier número de usuarios y de antenas transmisoras y receptoras [19-21] y finalmente, en [22] se concluye que la técnica de DPC consigue caracterizar toda la región de capacidad de un canal MIMO BC Gaussiano, cuando el canal es constante y se dispone de CSI perfecta.

En el canal BC, la técnica de DPC puede aplicarse utilizando distintas palabras código para cada usuario. El transmisor elige la palabra para el primer usuario $\left(\mathbf{x}_{1}\right)$. A continuación, la palabra para el segundo usuario, $\left(\mathbf{x}_{2}\right)$, se elige conociendo $\mathbf{x}_{1}$. Por tanto, la palabra del primer usuario puede eliminarse antes de transmitir de forma que el segundo usuario no la interprete como interferencia. De forma similar, la palabra para el ter- 
cer usuario se elige de forma que éste no interprete las señales de los dos primeros usuarios, $\mathbf{x}_{1}+\mathbf{x}_{2}$, como interferencia. Este proceso continúa para los $M$ usuarios.

Asumiendo una determina ordenación en los usuarios, si $\pi(1)$ es el primer usuario que se codifica, seguido de $\pi(2)$, hasta llegar al usuario $\pi(M)$, el vector de tasas de transmisión que puede alcanzarse para una determinada realización del canal sería:

$$
R_{\pi(m)}=\log _{2}\left\{\frac{\operatorname{det}\left(\mathbf{I}+\mathbf{H}_{\pi(m)}\left(\sum_{j \geq m} \boldsymbol{\Sigma}_{\pi(j)}\right) \mathbf{H}_{\pi(m)}^{H}\right)}{\operatorname{det}\left(\mathbf{I}+\mathbf{H}_{\pi(m)}\left(\sum_{j>m} \boldsymbol{\Sigma}_{\pi(j)}\right) \mathbf{H}_{\pi(m)}^{H}\right)}\right\}, m=1, \ldots, M,
$$

donde $\boldsymbol{\Sigma}_{\pi(j)}$ se corresponde con la matriz de correlación de la señal transmitida al usuario $\pi(j)$.

La región de capacidad definida por la técnica de DPC se define como la unión de todos los posibles vectores de tasas de transmisión de datos, sobre todas las posibles matrices de correlación $\boldsymbol{\Sigma}_{m}$ positivas, que cumplen la restricción en potencia:

$$
\operatorname{Tr}\left[\boldsymbol{\Sigma}_{1}+\cdots+\boldsymbol{\Sigma}_{M}\right] \leq P_{\max }
$$

y sobre todas las posibles permutaciones, $(\pi(1), \ldots, \pi(M))$, en el orden de los usuarios:

$$
C_{D P C}\left(P_{\max }, \mathbf{H}\right)=\bigcup_{\pi, \boldsymbol{\Sigma}_{m}} \mathbf{R}\left(\pi, \boldsymbol{\Sigma}_{m}\right),
$$

donde $\mathbf{R}\left(\pi, \boldsymbol{\Sigma}_{m}\right)$ viene dada por (2.28). La señal transmitida se corresponde con la expresión (2.27) y las matrices de correlación siguen la forma $\boldsymbol{\Sigma}_{m}=$ $E\left[\mathbf{x}_{m} \mathbf{x}_{m}^{H}\right]$.

La figura 2.6 muestra la región de capacidad BC utilizando la técnica de DPC para un sistema MIMO con 2 usuarios, cuando el transmisor dispone de $N_{t}=2$ y ambos usuarios de $N_{m}=1$ antenas [8].

En la práctica, encontrar la región de capacidad mediante la técnica de DPC es numéricamente complejo, puesto que requiere una búsqueda exhaustiva sobre todas las posibles matrices de correlación en transmisión que cumplen la restricción de potencia. A partir de los trabajos de Jindal [23] y Viswanath [19], la región de capacidad dada por (2.29) se calcula utilizando la dualidad que existe entre los problemas BC y MAC: aprovechando que la región de capacidad del problema $\mathrm{MAC}$ es mucho más sencilla de resolver, la región $\mathrm{DPC}$ del $\mathrm{BC}$ con múltiples antenas y con una restricción $P_{\max }$ 


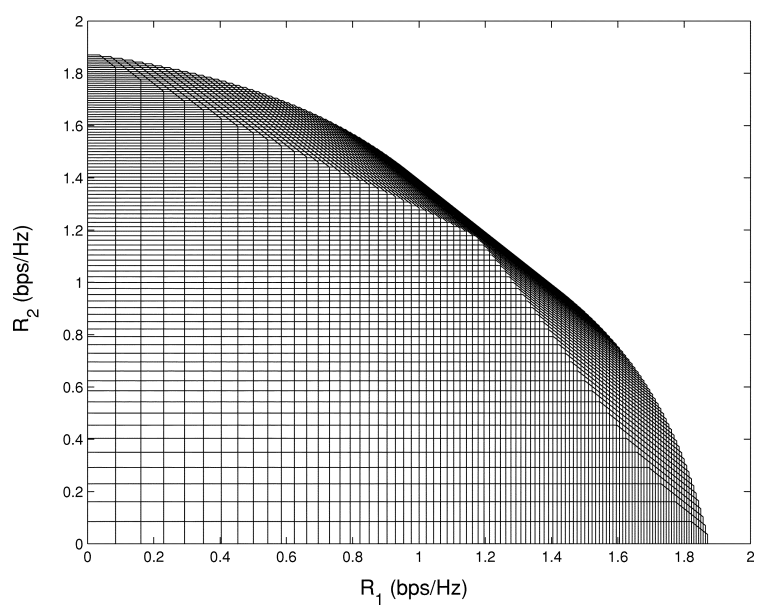

Figura 2.6: Región de capacidad BC con DPC, $M=2, N_{t}=2, N_{m}=1$.

de potencia es igual a la unión de las regiones de capacidad del problema dual MAC, donde la unión considera que la suma de las $P_{m}$ potencias individuales es igual a $P_{\max }$ :

$$
C_{D P C}\left(P_{\max }, \mathbf{H}\right)=\bigcup_{\mathbf{P}: \sum_{m} P_{m}=P_{\max }} C_{M A C}\left(P_{1}, \ldots, P_{M}, \mathbf{H}^{H}\right) .
$$

La unión de las regiones de capacidad MAC en (2.30) coincide con (2.25) si se aplica una restricción de potencia total sobre todos los usuarios en lugar de imponer restricciones de potencia individuales a cada usuario, es decir, ambas regiones de capacidad coinciden si en (2.25) se sustituye la restricción $\operatorname{Tr}\left[\mathbf{Q}_{m}\right] \leq P_{m}, \forall m$, por $\sum_{m} \operatorname{Tr}\left[\mathbf{Q}_{m}\right] \leq P_{\max }$. En este caso, las matrices de correlación óptimas en transmisión se obtienen a partir de transformar las matrices óptimas en el canal MAC, bien mediante algoritmos específicos o a partir de técnicas basadas en el algoritmo de waterfilling iterativo [24].

La figura 2.7 representa la relación (2.30), donde las regiones de capacidad MAC, $C_{M A C}\left(P_{1}, P_{\max }-P_{1}, h_{1}, h_{2}\right)$, se representan en función del valor de $P_{1}$ [23]. El límite de la región de capacidad $\mathrm{BC}$ se representa sombreado. Hay que destacar que los límites de cada región MAC coinciden con los del BC.

La región de capacidad del canal $\mathrm{BC}$ se obtiene en la práctica mediante simulaciones de Monte-Carlo. Recientemente, [25] repasa los límites del 


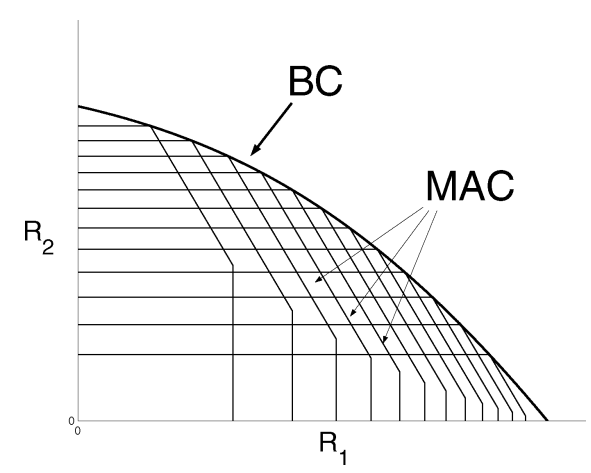

Figura 2.7: Dualidad MAC-BC.

canal MIMO BC desde un punto de vista práctico, estudiando cómo varía en función de distintos parámetros del sistema, como por ejemplo, $M, N_{t}$, $N_{m}$ y $P_{\max }$, y analizando también la influencia de aspectos como errores en la estimación de canal o efectos de la correlación espacial del canal.

\subsubsection{Criterios de diseño}

Los resultados obtenidos desde el punto de vista de la teoría de la información sugieren que en un sistema MIMO multi-usuario lo más conveniente sería servir simultáneamente a los usuarios mediante SDMA y un esquema de transmisión adecuado.

En los sistemas MIMO multi-usuario, la mayor parte del procesado de señal y la inteligencia se traslada al transmisor, estación base o access point. Esto provoca que en general, los esquemas de transmisión para sistemas MIMO multi-usuario requieran de CSIT.

El hecho de disponer de CSIT es bastante complejo en sistemas de comunicaciones reales donde la cantidad de información que se puede transmitir en el canal de realimentación UL es limitada. Además, la suposición de CSIT perfecta es poco realista, y existe una gran diferencia entre la capacidad que se puede conseguir con o sin CSIT. Por ejemplo, si $N_{m}, N_{t}$ y $P_{\max }$ son parámetros fijos, y la estación base dispone de CSIT perfecta, se sabe que cuando el número de usuarios activos $M$ es grande [26]:

$$
\lim _{M \rightarrow \infty} \frac{C_{D P C}}{\log _{2} \log _{2}\{M\}}=N_{t} .
$$


Este factor se interpreta como una ganancia de multiplexado o por diversidad multi-usuario: dado que el número de usuarios es elevado, la estación base puede seleccionar los $N_{t}$ mejores usuarios. En el enlace UL, esta ganancia es más fácil de extraer porque se puede asumir que el transmisor dispone de conocimiento del canal en UL y simplemente implementa una técnica clásica de recepción multi-usuario para separar las señales de los distintos usuarios.

Por el contrario, cuando la estación base no dispone de CSIT, y el canal es un canal i.i.d. Rayleigh con desvanecimiento plano en frecuencia [25]:

$$
\lim _{M \rightarrow \infty} \frac{C_{D P C}}{\log _{2} \log _{2}\{M\}}=0,
$$

la ganancia por diversidad multi-usuario es nula y la estrategia óptima es transmitir a un único usuario en cada instante de tiempo, mientras que para SNR elevadas, sin CSIT [25]:

$$
\lim _{P_{\max } \rightarrow \infty} \frac{C_{D P C}}{\log _{2}\left\{P_{\max }\right\}}=\min \left(N_{t}, N_{m}\right),
$$

con lo que el sistema MIMO multi-usuario se comporta como un sistema single-user MIMO. La excepción viene dada por los sistemas en los que los usuarios disponen de un número de antenas tales que $N_{m} \geq N_{t}$, donde cada usuario puede eliminar por sí solo la interferencia entre los datos transmitidos a cada usuario. Esta opción necesita que los canales individuales a cada usuario sean de rango completo.

La gran diferencia entre las capacidades obtenidas con y sin CSIT, motivan la investigación en la línea de esquemas o técnicas de transmisión que utilizan un conocimiento parcial de la CSIT. Por CSIT parcial se entiende el disponer de cierta información, pero incompleta, del canal, que puede obtenerse mediante diversos métodos.

Recientemente, se ha demostrado que el disponer de CSIT parcial permite obtener cierta ganancia con respecto a las situaciones en las que no se conoce el canal en transmisión [26]. Sin embargo, encontrar la estrategia óptima para diseñar un canal de realimentación que proporcione CSIT parcial es todavía una línea de investigación abierta, denominada de forma general técnicas de limited feedback [16]. Básicamente, estas técnicas se proponen en los sistemas en los que la CSIT se obtiene mediante un canal de realimentación en el enlace UL, para reducir la cantidad de información que el usuario necesita realimentar a la estación base. 
Según (2.31), la ganancia por diversidad multi-usuario está acotada por el número de antenas transmisoras $N_{t}$, mientras que el número de usuarios $M$ a los que se puede servir es en principio arbitrario. Cuántos y qué usuarios deberían ser servidos en cada instante de tiempo es una cuestión que resuelve el algoritmo de scheduling.

Por último, la configuración MIMO multi-usuario, por el hecho de distribuir espacialmente a los usuarios, permite mitigar los efectos adversos del canal como pueden ser una componente LOS dominante o un mal condicionamiento de la matriz de canal. En el caso multi-usuario, la matriz de canal que resulta de concatenar las matrices individuales $\mathbf{H}_{m}$, es prácticamente en todos los casos de rango completo gracias a la separación espacial de los usuarios. Esta separación espacial permite además implementar el multiplexado espacial de distintos conjuntos de datos incluso cuando los usuarios disponen de una única antena receptora, permitiendo alcanzar las capacidades asociadas a los sistemas MIMO para equipos de menor coste.

\section{Gestión de recursos y scheduling}

Las técnicas de gestión de recursos ayudan a conseguir las ganancias teóricas asociadas a los sistemas MIMO. Desde el punto de vista de la teoría de la información, el límite de la región de capacidad se consigue dando servicio a $M$ usuarios simultáneamente, donde $M$ puede ser un número elevado de usuarios. Por tanto, la asignación de recursos a cada usuario depende de las condiciones instantáneas del canal y puede variar mucho entre usuarios.

El hecho de que la ganancia por diversidad multi-usuario esté acotada por $N_{t}$ sugiere que el número de usuarios que realmente se sirven en cada instante de tiempo está relacionado con $N_{t}$, aunque $N_{t}$ siempre sea mucho menor que $M$. Por ejemplo, cuando se utiliza una técnica de ZF en transmisión, el número de usuarios seleccionado viene determinado por $N_{t}$. Esto motiva que el algoritmo de gestión de recursos intente seleccionar el conjunto más adecuado de usuarios entre los $M$ activos.

En concreto, el algoritmo de scheduling selecciona, para cada realización de canal, al subconjunto óptimo de $M$ usuarios que permite maximizar un determinado parámetro, como el sum-rate $\left(\sum_{m} R_{m}\right)$, o conseguir unas determinados calidades de servicio (QoS, Quality of Service) por usuario dada una restricción en potencia total transmitida. 
Scheduling óptimo. El algoritmo de scheduling óptimo requiere realizar una búsqueda exhaustiva sobre todas las posibles combinaciones de usuarios. Esta opción, aunque sí es óptima en cuanto a maximizar el sum-rate, por ejemplo, implica un alto coste computacional para un número de usuarios $M$ elevado.

Recientemente, se ha demostrado que las técnicas lineales de beamforming también consiguen maximizar el sum-rate cuando el número de usuarios $M$ es elevado (ver por ejemplo [27] o [28]).

Greedy methods. Estos métodos comprenden un conjunto de soluciones subóptimas que permiten reducir la complejidad computacional de las soluciones óptimas de scheduling. Por ejemplo, en el algoritmo greedy que selecciona a los usuarios con el objetivo de maximizar el sum-rate, en primer lugar se selecciona al usuario cuyo canal presenta una mayor capacidad. Después, se elige al usuario que proporciona el mayor sumrate de entre los restantes. El algoritmo se repite iterativamente hasta seleccionar los $M$ usuarios. De esta forma, la complejidad se reduce frente a la búsqueda exhaustiva que proporciona la solución óptima.

A la hora de calcular el sum-rate, pueden utilizarse esquemas de transmisión lineales o no lineales, aunque esta última opción todavía es bastante reciente y no existen muchos resultados. Por otro lado, el algoritmo greedy puede seleccionar otros parámetros de optimización distintos al sum-rate.

\subsubsection{Técnicas MIMO multi-usuario: enlace UL}

En este apartado se repasan brevemente las técnicas y esquemas que se utilizan en el enlace UL de los sistemas MIMO multi-usuario, puesto que en esta Tesis el interés se centra en el enlace DL del sistema.

Siguiendo el modelo de sistema (2.23), cada usuario transmite la señal $u_{m}=\sqrt{q_{m}} s_{m}$, siendo $q_{m}$ la potencia con que el usuario $m$-ésimo transmite la señal $s_{m}$.

Técnicas lineales, $N_{m}=1$

Las técnicas lineales consisten, básicamente, en implementar un filtrado lineal en la estación base mediante una técnica de beamforming (2.23):

$$
\mathbf{z}=\mathbf{W}^{H} \mathbf{v}=\mathbf{W}^{H} \mathbf{H}^{H} \sqrt{\mathbf{Q}} \mathbf{s}+\mathbf{W}^{H} \mathbf{w},
$$


donde la matriz de beamforming $\mathbf{W}^{H}$ es una matriz compleja $\left[M \times N_{t}\right]$ y la matriz de canal incluye los canales entre el transmisor y todos los usuarios:

$$
\mathbf{H}^{H}=\left[\mathbf{h}_{1}^{H} \ldots \mathbf{h}_{M}^{H}\right]
$$

siendo $\mathbf{h}_{m}^{H}$ el canal $\left[N_{t} \times 1\right]$ del usuario $m$. La matriz $\mathbf{Q}=\operatorname{diag}\left\{\left[q_{1} \ldots q_{M}\right]\right\}$ es una matriz diagonal que incluye las potencias transmitidas por cada usuario del sistema.

Técnicas no lineales, $N_{m}=1$

El método óptimo de detección es el detector ML, donde el receptor compara todas las posibles combinaciones de símbolos que podrían haberse transmitido con lo que recibe. Si $\mathbf{s}=\left[s_{1} \ldots s_{M}\right]^{T}$ :

$$
\hat{\mathbf{s}}=\arg \min _{\mathbf{s} \in \mathcal{A}}\left\{\left\|\mathbf{v}-\mathbf{H}^{H} \sqrt{\mathbf{Q}} \mathbf{s}\right\|^{2}\right\},
$$

donde $\mathcal{A}$ es el alfabeto de símbolos de la modulación.

\section{Múltiples antenas por usuario}

Cuando los usuarios disponen de más de una antena, $N_{m}>1$, las técnicas MIMO multi-usuario se reducen a implementar un esquema de beamforming si los usuarios disponen de CSI o, en caso contrario, a utilizar técnicas de STC o de multiplexado espacial.

\subsubsection{Criterios de optimización: enlace UL}

En el enlace UL, los parámetros a optimizar para satisfacer un determinado criterio son la matriz de beamforming en recepción dadas unas potencias de transmisión $q_{m}$ o bien, las potencias de los usuarios, $q_{m}$, dada una matriz de beamforming en recepción.

Entre los posibles criterios de optimización se encuentran:

- Optimización de la SINR: se fija un umbral de calidad de servicio en términos de SINR para cada usuario, $\gamma_{m}$. La SINR medida en el transmisor a partir de la señal transmitida por cada usuario, debe superar el umbral correspondiente:

$$
\operatorname{SINR}_{m}^{U L} \geq \gamma_{m}, \forall m
$$


- Minimización de potencia: este criterio es una extensión del criterio anterior. Los usuarios, además de cumplir la restricción en SINR, deben transmitir con la mínima potencia:

$$
\text { mín } \sum_{m} q_{m}, \quad \text { s.t. } \operatorname{SINR}_{m}^{U L} \geq \gamma_{m}, \forall m .
$$

- Maximizar el sum-rate del sistema: se intenta maximizar la suma de las tasas de transmisión de datos de todos los usuarios del sistema, estableciendo unas restricciones de potencia individuales para cada usuario:

$$
\text { máx } \sum_{m} \log _{2}\left\{1+\operatorname{SINR}_{m}^{U L}\right\} .
$$

Este criterio de optimización se puede interpretar como un caso particular de un criterio más general que trata de maximizar la suma ponderada de las tasas de transmisión de datos:

$$
\text { máx } \sum_{m} \alpha_{m} \log _{2}\left\{1+\operatorname{SINR}_{m}^{U L}\right\},
$$

donde los parámetros $\alpha_{m}$ representan un peso que modela la calidad de servicio requerida de cada usuario.

- Maximizar la mínima tasa de transmisión de datos presente en el sistema: manteniendo las restricciones de potencia individuales para cada usuario, se intenta maximizar la menor tasa de transmisión de datos del sistema:

$$
\text { máx }\left\{\operatorname{mín}_{m} \log _{2}\left\{1+\operatorname{SINR}_{m}^{U L}\right\}\right\} \text {. }
$$

La mayor parte de estos criterios de optimización utilizan técnicas de beamforming en recepción, como el beamfomer ZF o el MMSE. El objetivo del beamformer ZF en recepción es extraer la señal de cada usuario, sin interferencia, con la mayor SNR posible:

$$
\mathbf{W}^{H}=\sqrt{\mathbf{Q}}^{-1}\left(\mathbf{H H}^{H}\right)^{-1} \mathbf{H} .
$$

Cuando el canal es deficiente en rango, el beamformer ZF amplifica la potencia de ruido.

El beamformer MMSE se plantea para evitar los problemas de amplificación de ruido. El objetivo es resolver el siguiente criterio de optimización:

$$
\mathbf{W}=\arg \min \left\{E\left[\left\|\mathbf{W}^{H} \mathbf{v}-\left[s_{1} s_{2} \ldots s_{M}\right]\right\|^{2}\right]\right\},
$$


con lo que se obtiene el siguiente beamformer:

$$
\mathbf{W}=\left(\mathbf{I}+\mathbf{H}^{H} \mathbf{Q H}\right)^{-1} \mathbf{H}^{H} \mathbf{A},
$$

donde $\mathbf{A}$ es una matriz que normaliza el módulo del beamformer de cada usuario a la unidad.

\subsubsection{Técnicas MIMO multi-usuario: enlace DL}

Gracias a la teoría de la información, se sabe que DPC es la técnica que maximiza el sum-rate para el enlace DL de los sistemas MIMO multiusuario. La técnica de DPC, óptima teóricamente, es un concepto de teoría de la información complejo de implementar en entornos prácticos.

Las técnicas que se comentan en este apartado requieren de CSIT. En [16] se presentan algunas técnicas de transmisión cuando se dispone de CSIT parcial, como por ejemplo opportunistic random beamforming [26], mientras que en [25] se estudia el comportamiento de algunas de estas técnicas en términos de capacidad teórica.

\section{Técnicas lineales, $N_{m}=1$}

Las técnicas lineales presentan una solución intermedia entre complejidad y resultados obtenidos: no suelen ser muy complejas computacionalmente, pero generalmente no consiguen alcanzar soluciones óptimas.

Cuando los usuarios disponen de una sola antena, y separando la asignación de potencia del beamformer, el transmisor o estación base transmite la señal:

$$
\mathbf{x}=\mathbf{W} \sqrt{\mathbf{P}} \mathbf{s}
$$

donde $\mathbf{W}$ es la matriz de beamforming $\left[N_{t} \times M\right]$ en transmisión, $\mathbf{P}=$ $\operatorname{diag}\left\{\left[p_{1} \ldots p_{M}\right]\right\}$ es la matriz de asignación de potencias en DL y $\mathbf{s}=$ $\left[s_{1} \ldots s_{M}\right]^{T}$ contiene los símbolos transmitidos a los usuarios del sistema. La señal recibida por todos los usuarios viene dada por un vector columna $[M \times 1](2.26):$

$$
\mathbf{y}=\mathbf{H W} \sqrt{\mathbf{P}} \mathbf{s}+\mathbf{n} .
$$

Al igual que en el enlace UL, las técnicas lineales más sencillas en transmisión son el beamformer ZF (también conocido como channel inversion) o el MMSE (o regularized channel inversion) [29]. 
Para el criterio ZF, el objetivo es mandar señales libres de interferencia, pero cumpliendo una restricción de potencia en transmisión $P_{\max }$ :

$$
\mathbf{W}=\frac{1}{\sqrt{\lambda}} \mathbf{H}^{H}\left(\mathbf{H H}^{H}\right)^{-1} \sqrt{\mathbf{P}}^{-1},
$$

donde $\lambda$ se elige de forma que la potencia media transmitida sea $P_{\max }$,

$$
\lambda=\frac{1}{P_{\max }} \operatorname{Tr}\left[\left(\mathbf{H H}^{H}\right)^{-1}\right] .
$$

Cuando el canal es deficiente en rango, al menos uno de los valores singulares de $\left(\mathbf{H H}^{H}\right)^{-1}$ será muy grande, con lo que también lo será $\lambda$ y la SINR recibida en los usuarios será baja. La técnica de ZF en recepción (2.36), amplificaba la potencia de ruido cuando el canal era deficiente en rango. Sin embargo, cuando se aplica en transmisión, el efecto es el de atenuar la potencia de la señal recibida en los usuarios.

Como ocurre en recepción, cuando el canal es deficiente en rango, el hecho de introducir un factor de regularización en la inversa mejora las prestaciones del beamformer. Si el ruido es blanco, regularizar la inversa del canal equivale a plantear un criterio MMSE dada una restricción de potencia máxima $P_{\max }$ :

$$
\mathbf{W}=\arg \operatorname{mín}\left\{E\left[\|\mathbf{s}-\mathbf{y}\|^{2}\right]\right\}=\arg \operatorname{mín}\left\{E\left[\|\mathbf{s}-\mathbf{H W} \sqrt{\mathbf{P}} \mathbf{s}\|^{2}\right]\right\} \text {. }
$$

La solución es la siguiente:

$$
\mathbf{W}=\mathbf{H}^{H}\left(\alpha \mathbf{I}+\mathbf{H H}^{H}\right)^{-1} \sqrt{\mathbf{P}}^{-1},
$$

donde la constante $\alpha$ se elige de forma que $\operatorname{Tr}\left[\mathbf{W P} \mathbf{W}^{H}\right]=P_{\max }$.

Cuando el conjunto de usuarios seleccionados no son lo suficientemente separables espacialmente, estas soluciones pueden obtener resultados bastante alejados de las soluciones óptimas. Este efecto se mitiga cuando el número de usuarios en el sistema MIMO multi-usuario es elevado y por tanto es más probable encontrar usuarios casi-ortogonales con buena SNR [27]. La desventaja de estas técnicas es que no son fácilmente aplicables a sistemas multi-usuario con múltiples antenas en recepción.

Técnicas lineales, $N_{m}>1$

A partir del modelo (2.26), si $\mathbf{s}_{m}$ representa el vector $m$-ésimo de símbolos transmitidos, $\mathbf{W}_{m} \mathbf{s}_{m}$ es el vector de señal transmitido al usuario $m$, 
donde $\mathbf{W}_{m}$ es la matriz de precoding diseñada para el mismo usuario. Asumiendo que el algoritmo de scheduling selecciona un subconjunto de $M$ usuarios, el modelo (2.26) se modifica según:

$$
\mathbf{y}_{m}=\mathbf{H}_{m} \mathbf{W}_{m} \mathbf{s}_{m}+\mathbf{H}_{m} \sum_{\substack{i=1 \\ i \neq m}}^{M} \mathbf{W}_{i} \mathbf{s}_{i}+\mathbf{n}_{m} .
$$

Cada usuario dispone de $N_{m}$ antenas y puede decodificar $S_{m} \leq N_{m}$ conjuntos de datos, que constituyen su señal deseada. El objetivo del precoding lineal es diseñar las matrices $\mathbf{W}_{m}$ a partir del canal, de forma que un determinado parámetro se maximice para cada conjunto de datos. Hay que destacar que un beamformer en transmisión se corresponde con un caso particular de los precoders lineales [30].

Una extensión del criterio ZF es la llamada Block Diagonalization (BD) [31], que asume $N_{m}=S_{m}>1$ y $\sum_{m} N_{m}=N_{t}$. La idea es diseñar las matrices $\mathbf{W}_{m}$ de forma que $\mathbf{H}_{i} \mathbf{W}_{m}=0, \forall i \neq m$, eliminando la interferencia en (2.44), con lo que la señal recibida por el usuario $m$ se reduce a:

$$
\mathbf{y}_{m}=\mathbf{H}_{m} \mathbf{W}_{m} \mathbf{s}_{m}+\mathbf{n}_{m} .
$$

La técnica BD parte del llamado canal compuesto, que para un usuario $m$ se define como:

$$
\tilde{\mathbf{H}}_{m}=\left[\begin{array}{llllll}
\mathbf{H}_{1}^{H} & \cdots & \mathbf{H}_{m-1}^{H} & \mathbf{H}_{m+1}^{H} & \cdots & \mathbf{H}_{M}^{H}
\end{array}\right]^{H} .
$$

La descomposición SVD de este canal compuesto puede expresarse como:

$$
\tilde{\mathbf{H}}_{m}=\tilde{\mathbf{U}}_{m} \tilde{\mathbf{D}}_{m}\left[\begin{array}{ll}
\tilde{\mathbf{V}}_{m}^{(1)} & \tilde{\mathbf{V}}_{m}^{(0)}
\end{array}\right]^{H},
$$

donde $\tilde{\mathbf{U}}_{m}$ y $\tilde{\mathbf{D}}_{m}$ son la matriz de vectores singulares por la izquierda y la matriz de valores singulares, respectivamente, y $\tilde{\mathbf{V}}_{m}^{(1)}$ y $\tilde{\mathbf{V}}_{m}^{(0)}$ son las matrices de vectores singulares por la derecha relacionadas con los valores singulares no nulos y valores singulares nulos, respectivamente. Cualquier matriz $\mathbf{W}_{m}$ que sea una combinación lineal de las columnas de $\tilde{\mathbf{V}}_{m}^{(0)}$, satisface la restricción $\mathbf{H}_{i} \mathbf{W}_{m}=0, \forall i \neq m$. Si la matriz compuesta $\tilde{\mathbf{H}}_{m}$ es de rango completo, el transmisor necesita tener tantas antenas como sea la suma de las antenas de todos los usuarios del sistema para poder cancelar la interferencia [31].

La desventaja de la técnica de BD es que necesita cumplir $N_{m}=S_{m}$. Para resolverlo, puede incluirse cierto procesado en los receptores, como 
por ejemplo una matriz lineal $\mathbf{V}_{m}$ para cada usuario, con lo que la señal recibida queda:

$$
\mathbf{y}_{m}=\mathbf{V}_{m}^{H} \mathbf{H}_{m} \mathbf{W}_{m} \mathbf{s}_{m}+\mathbf{V}_{m}^{H} \mathbf{H}_{m} \sum_{\substack{i=1 \\ i \neq m}}^{M} \mathbf{W}_{i} \mathbf{s}_{i}+\mathbf{V}_{m}^{H} \mathbf{n}_{m} .
$$

Las matrices $\mathbf{W}_{m}$ y $\mathbf{V}_{m}$ para cada usuario deben cumplir:

$$
\mathbf{V}_{m}^{H} \mathbf{H}_{m} \sum_{\substack{i=1 \\ i \neq m}}^{M} \mathbf{W}_{i}=0, \forall m .
$$

Este problema de diseño suele resolverse mediante métodos iterativos.

\section{Técnicas no lineales}

Las técnicas lineales proporcionan buenos resultados pero pueden quedar lejos de la solución óptima DPC cuando el número de usuarios $M$ es pequeño. Dos de las técnicas no lineales más habituales son la vectorperturbation y una extensión espacial del precoding Tomlinson-Harashima.

La técnica de vector-perturbation [32] introduce una perturbación en el vector de señal transmitida para evitar los problemas que aparecen con los esquemas ZF en transmisión cuando el canal es deficiente en rango. En este caso, el factor $\lambda$ en (2.41) es elevado y el vector de datos se alinea con el vector singular que se corresponde con el mayor valor singular de $\left(\mathbf{H H}^{H}\right)^{-1}$. La idea es perturbar el vector de datos transmitidos de forma que se alinee con los vectores singulares correspondientes a los valores singulares más pequeños de $\left(\mathbf{H H}^{H}\right)^{-1}$. Además, los receptores pueden seguir decodificando el vector de símbolos sin conocer la perturbación introducida en transmisión.

Suponiendo que los usuarios disponen de una sola antena, la matriz de canal que incluye todos los canales de propagación del sistema sería una matriz $\mathbf{H}$ de dimensiones $\left[M \times N_{t}\right]$. El objetivo es encontrar un vector perturbación, $\mathbf{p}^{\prime}=\tau(\mathbf{a}+j \mathbf{b})$, que minimice la potencia transmitida. Este vector se obtiene resolviendo:

$$
\mathbf{p}=\arg \operatorname{mí}_{\mathbf{p}^{\prime}}\left\{\left\|\mathbf{G}\left(\mathbf{s}+\mathbf{p}^{\prime}\right)\right\|^{2}\right\},
$$

donde $\mathbf{G}$ es una matriz de transmisión, por ejemplo, ZF o MMSE, que cumple una restricción en potencia $\operatorname{Tr}\left[\mathbf{G G}^{H}\right] \leq P_{\max }$, s es la señal trasmitida y $\mathbf{p}^{\prime}$ depende de la modulación utilizada. 


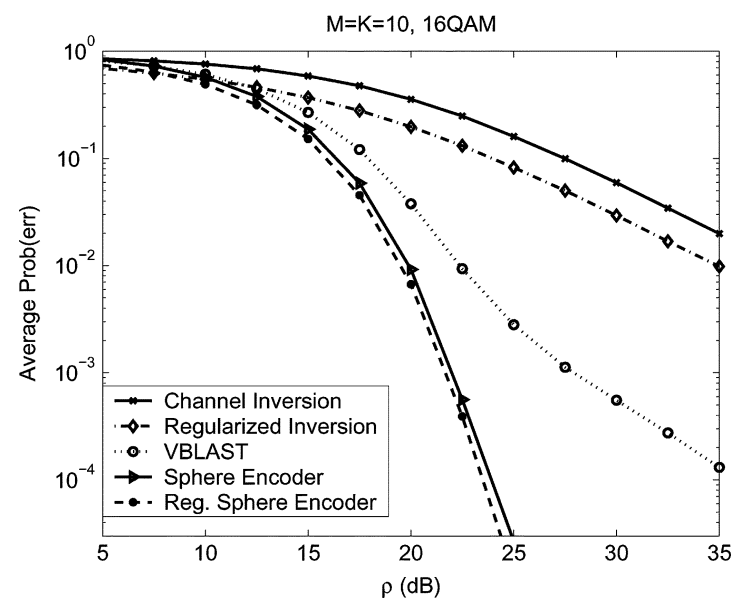

Figura 2.8: Probabilidad de error de símbolo para distintas técnicas de transmisión DL en función de $P_{\max }$ (con permiso de los autores [32]).

Por otra parte, cabe destacar que cuando se implementa la técnica de DPC en el enlace DL de los sistemas MIMO multi-usuario, se combina con un esquema lineal de beamforming, similar al ZF. Este esquema se basa en realizar una descomposición LQ de la matriz de canal, donde la matriz $\mathbf{L}$ es una matriz triangular inferior, y la matriz $\mathbf{Q}$ es una matriz unitaria, $\mathbf{Q Q}^{\dagger}=\mathbf{I}$. La matriz de beamforming (2.39) se elige de forma que $\mathbf{W}=\mathbf{Q}^{\dagger}$, y los usuarios reciben:

$$
\mathbf{y}=\mathbf{H x}+\mathbf{n}=\mathbf{L Q} \mathbf{x}+\mathbf{n}=\mathbf{L Q} \mathbf{Q}^{\dagger} \sqrt{\mathbf{P}} \mathbf{d}+\mathbf{n}=\mathbf{L} \sqrt{\mathbf{P}} \mathbf{d}+\mathbf{n},
$$

donde $\mathbf{d}=\left[d_{1} \ldots d_{M}\right]^{T}$ es el vector de símbolos codificado con DPC.

La técnica de vector perturbation no requiere realizar una descomposición LQ del canal y admite una implementación más eficiente, denominada por los autores sphere encoder, puesto que utiliza un algoritmo similar al algoritmo de sphere decoder pero en transmisión [32]. La figura 2.8 (con permiso de los autores [32]) muestra la probabilidad de error de símbolo para distintas técnicas de transmisión DL en función de $P_{\max }$, para $N_{t}=10$ y $N_{m}=10$. La curva reg. sphere encoder combina la sphere encoder con el beamformer MMSE o regularized channel inversion en DL. 


\subsubsection{Criterios de optimización: enlace DL}

Al igual que en el apartado de criterios de optimización en el enlace UL, se pueden plantear distintos problemas de optimización en el enlace DL. Suponiendo un sistema MIMO multi-usuario donde un transmisor, estación base o access point, se comunica con $M$ usuarios, los dos problemas clásicos de optimización serían:

- Maximizar el sum-rate del sistema dada una restricción en potencia total transmitida $P_{\max }$ :

$$
\operatorname{máx} \sum_{m} \log _{2}\left\{1+\operatorname{SINR}_{m}^{D L}\right\},
$$

donde $\operatorname{SINR}_{m}^{D L}$ es la SINR medida en el usuario $m$.

- Problema de minimización de potencia. En este caso, el objetivo es minimizar la potencia total transmitida en el sistema, asegurando que los usuarios cumplen una determinada restricción de QoS, generalmente medida en términos de SINR:

$$
\text { mín } \sum_{m} p_{m}, \quad \text { s.t. } \quad \operatorname{SINR}_{m}^{D L} \geq \gamma_{m}, \forall m \text {. }
$$

El problema de minimización de potencia se presenta habitualmente como un caso particular del llamado problema de balanceado de SINR o problema max-min. El problema de balanceado de SINR pretende mejorar el comportamiento del sistema desde un punto de vista de fairness; el objetivo es maximizar la mínima SINR normalizada en DL $\left(\mathrm{SINR}_{m}^{D L} / \gamma_{m}\right)$ dada una restricción en potencia total transmitida:

$$
\operatorname{máx}\left\{\operatorname{mín}_{m} \frac{\operatorname{SINR}_{m}^{D L}}{\gamma_{m}}\right\}, \quad \text { s.t. } \quad \sum_{m} p_{m} \leq P_{\max } .
$$

En el punto óptimo del problema, las SINR recibidas por los usuarios del sistema están balanceadas:

$$
\frac{\operatorname{SINR}_{1}^{D L}}{\gamma_{1}}=\ldots=\frac{\operatorname{SINR}_{M}^{D L}}{\gamma_{M}}
$$

La literatura asociada a estos problemas de optimización es muy extensa y comprende también propuestas que modifican ligeramente los problemas de optimización originales. 
En este apartado se repasan brevemente las principales referencias de los problemas de minimización de potencia y de maximización del sumrate, prestando más atención a las referencias que abordan el problema de minimización de potencia. En general, estas propuestas asumen que el parámetro que determina la QoS es la SINR en DL que perciben los usuarios, promediada sobre una determinada ventana de tiempo.

Una idea fundamental que aparece tanto en las soluciones del problema de minimización de potencia como en las de maximización del sum-rate, es la de aprovechar la posible dualidad entre el enlace UL y el DL de un mismo sistema. La técnica de la dualidad consiste en resolver el problema que se ha planteado en DL a través de un problema UL dual, siempre que el problema a resolver admita esta posibilidad. La clave está en el hecho de que un mismo problema es siempre mucho más sencillo de resolver en el enlace UL que en el DL.

Existen distintos tipos de dualidades. La más conocida, aparece en los escenarios en los que algún esquema de beamforming se utiliza en transmisión [33]. Es bien conocido que las técnicas de beamforming son mucho más sencillas de implementar en UL que en DL; mientras que en UL el beamformer procesa todas las señales recibidas para poder obtener la señal de un usuario concreto con la mejor calidad posible, en DL, la señal transmitida desde la estación base o access point afecta al usuario deseado y a todos los usuarios pertenecientes a la misma celda y a las adyacentes.

En [33] se demuestra que dada una restricción en la potencia total transmitida en el sistema, el conjunto de SINR que pueden alcanzar los usuarios en DL es el mismo que se obtiene al resolver un problema UL dual. El problema UL dual se plantearía con las mismas matrices de canal y la misma restricción en la potencia total transmitida, sólo que ahora se aplicaría a la suma de las potencias transmitidas por los usuarios.

Otro ejemplo importante de dualidad es la que aparece en el contexto de las regiones de capacidad de los canales MAC y BC para un sistema MIMO multi-usuario. Como se ha visto anteriormente, existe una dualidad entre ambas regiones de capacidad cuando se implementa la técnica de DPC. Esta dualidad permite obtener la región de capacidad $\mathrm{BC}$ resolviendo una región de capacidad MAC equivalente, donde la restricción en potencia se aplica a la suma de las potencias transmitidas por los usuarios y no a cada potencia individual.

Finalmente, otra dualidad entre el enlace UL y el DL aparece en el contexto del problema de minimizar el MSE (Mean Square Error) en DL 
entre los símbolos transmitidos y los estimados. En [34], se considera que los problemas UL y DL comparten la misma región MSE bajo una misma restricción de potencia total transmitida.

En general, es mucho más sencillo resolver un problema DL en términos de su UL dual. El inconveniente, como se verá en los sistemas MIMO multiusuario multi-celda, aparece con la restricción de potencia total transmitida.

\section{Algoritmos que maximizan el sum-rate}

En el análisis de la región de capacidad BC de un sistema MIMO multiusuario, se ha visto como, desde el punto de vista de teoría de la información, la técnica de DPC es óptima a la hora de caracterizar la región de capacidad del enlace DL. Sin embargo, todavía sería necesario determinar cuáles son las matrices de correlación $\boldsymbol{\Sigma}_{m}$ más adecuadas, es decir, cuál es la estrategia de transmisión óptima que se corresponde con la sum-capacity.

Por otra parte, aplicando la dualidad entre los problemas MAC y BC, es posible resolver el problema $\mathrm{BC}$ a partir de un problema $\mathrm{MAC}$ donde los usuarios están sujetos a una restricción en la potencia total transmitida y no a restricciones individuales. Aplicando esta propiedad, [24] propone algoritmos iterativos para caracterizar las matrices óptimas de correlación, muy similares al algoritmo de waterfilling propuesto originalmente para un canal MAC con restricciones individuales de potencia.

Actualmente, se sigue investigando para encontrar esquemas eficientes y prácticos que presenten un buen compromiso entre complejidad y prestaciones. Dentro de esta línea de investigación, se encuentran las soluciones que aplican esquemas lineales de beamforming, como [30,35,36].

\section{Algoritmos que minimizan la potencia total transmitida}

Habitualmente, el problema de minimización de potencia se plantea en un escenario donde el transmisor implementa un algoritmo de control de potencia y beamforming óptimo (JPCOB, Joint Power Control and Optimal Beamforming). Por tanto, el objetivo es encontrar la matriz óptima de beamforming y la asignación óptima de potencia, que minimicen la potencia total transmitida en el sistema, permitiendo al mismo tiempo que los usuarios alcancen las QoS requeridas.

Generalmente, las soluciones algorítmicas clásicas asumen que se dispone de CSI perfecta, y que se trata de un sistema MISO multi-usuario, o lo que es lo mismo, que el transmisor o estación base dispone de un array de 
antenas y que trata de dar servicio a $M$ usuarios que disponen de una única antena. Al mismo tiempo, suele asumirse que el sistema MISO multiusuario se ubica en única celda aislada o en un entorno multi-celda donde no se permite ningún tipo de coordinación entre celdas.

Bajo estas suposiciones, las referencias clásicas para tratar el problema de minimización de potencia en DL son los algoritmos propuestos por Rashid-Farrokhi et al. [33], Bengtsson y Ottersten [37], y Schubert y Boche [38]. En general, la forma más sencilla de abordar el diseño de un algoritmo JPCOB para un sistema MIMO multi-usuario multi-celda coordinado, es extender las referencias clásicas planteadas para sistemas MIMO multiusuario (y tal vez multi-celda) convencionales. Por este motivo, las referencias clásicas $[33,37,38]$ se desarrollan con más detalle en el Capítulo 3. Otras extensiones con variaciones interesantes se pueden encontrar en [30,39-42].

Como ya se ha comentado, el problema de minimización de potencia puede interpretarse como un caso particular del problema de balanceado de SINR o problema max-min. Algunos autores, plantean tanto las relaciones entre los dos problemas como soluciones algorítmicas válidas para ambos $[30,38,42]$.

La importancia de la dualidad entre las regiones de SINR del problema UL y del DL, recae en que es bien conocido que el problema de minimización de potencia en UL es mucho más sencillo de resolver [43]. Esta dualidad se planteó por primera vez en [33] y [40] para un sistema MISO multi-usuario donde los usuarios disponían de una única antena en recepción. Ambos trabajos demuestran, con matices ligeramente distintos, que el mínimo del problema de minimización de potencia en DL es el mismo que el que se obtiene si se plantea el problema UL dual con las mismas restricciones de SINR. De hecho, los beamformers óptimos en transmisión coinciden, teniendo en cuenta un factor de escala, con los beamformers óptimos en recepción del problema dual en UL.

En [33], Rashid-Farrokhi et al. plantean un algoritmo iterativo que alcanza el óptimo global del problema de minimización de potencia. Esta propuesta utiliza la dualidad entre el problema en DL y el mismo problema planteado en un UL virtual para obtener los beamformers en transmisión a partir de la formulación del UL virtual, mucho más sencilla. La principal desventaja de esta propuesta es que no estudia la feasibility del problema, es decir, si existe una solución con las condiciones iniciales planteadas (restricciones de QoS y $P_{\max }$ ). 
Desde un punto de vista distinto, Schubert y Boche resuelven tanto el problema de minimización de potencia como el de optimización maxmin [38]. Proponen un algoritmo más rápido y estable que en un primer paso comprueba la feasibility del problema. Schubert y Boche también utilizan la dualidad UL-DL, pero resuelven el problema max-min y el de minimización de potencia reescribiéndolos como problemas de valores propios. Posteriormente, los mismos autores añaden la técnica de DPC a la solución anterior [44].

Por último, Bengtsson y Ottersten formulan el problema de minimización de potencia como un problema de optimización semidefinida (SDP, Semidefinite Program) [37]. Su formulación centralizada permite encontrar un óptimo global y detectar un escenario no feasible, pero conlleva un alto coste computacional. Como alternativa, [41] plantea un algoritmo iterativo en dos pasos para resolver la versión distribuida de [37]. Recientemente, Hammarwall, Bengtsson y Ottersten extienden los algoritmos propuestos por Schubert y Boche en [38], añadiendo restricciones adicionales en el diseño del beamformer. Estas restricciones adicionales permiten considerar parámetros distintos de la SINR como restricciones de QoS, así como aspectos de diversidad o restricciones de tipo físico en la optimización [42].

Wiesel et al. plantean, desde un escenario distinto, una aproximación muy interesante a los problemas de minimización de potencia y max-min: consideran un sistema MIMO general donde el receptor permanece fijo [30]. De esta forma, se incluyen las configuraciones MISO multi-usuario y la single-user MIMO. En este contexto, analizan el problema de diseñar precoders lineales, entendiendo el precoder como una transformación lineal de los símbolos transmitidos (como caso particular, comprende el problema de beamforming en transmisión). Además, un punto a favor de esta propuesta es que los precoders obtenidos son independientes del rango del canal, es decir, sus prestaciones no se degradan cuando el canal es deficiente en rango (por ejemplo, cuando hay más usuarios que antenas en el transmisor).

En primer lugar, [30] plantea el problema de minimización de potencia utilizando herramientas de optimización cónica. En concreto, los autores formulan el problema como un SOCP (Second-order Cone Program) y como un SDP. Obviamente, el problema podría resolverse directamente utilizando paquetes software estándar de optimización. Sin embargo, los autores proponen una solución alternativa cuya complejidad computacional es menor y que además no necesita recurrir a los paquetes software. 
La idea es reescribir el problema de optimización de potencia y derivar las condiciones KKT (Karush-Kuhn-Tucker) de los programas cónicos. Aunque no reescriben el problema de forma convexa, los autores demuestran que para ese caso concreto las condiciones KKT son necesarias y suficientes para demostrar que la solución es óptima. A partir de las variables duales de Lagrange, proponen una expresión sencilla para el precoder óptimo. Por último, proponen dos métodos para obtener las variables duales de Lagrange, una iteración de punto fijo y un programa dual SDP con mucho menor coste que la formulación SOCP y SDP original.

Con respecto al problema max-min, plantean el problema mediante un GEVP (Generalized Eigenvalue Problem). Al igual que para la minimización de potencia, el problema GEVP puede resolverse directamente con paquetes de optimización software. Aprovechando las relaciones que existen entre el problema max-min y el de minimización de potencia, Wiesel et al. proponen la misma expresión para el precoder óptimo y presentan de nuevo un algoritmo de punto fijo que permite obtener las variables duales de Lagrange.

Yu y Lan, consideran un sistema de una única celda donde se aplican restricciones de potencia por antena en el transmisor, en lugar de la restricción en potencia habitual sobre todo el array [39]. En este escenario, los autores estudian tanto el problema de minimización de potencia en un contexto MISO multi-usuario, como el problema de maximizar el sum-rate cuando los usuarios disponen de múltiples antenas. La idea fundamental de [39] es proponer un nuevo punto de vista de la dualidad UL y DL: demuestran que la dualidad en SINR y la dualidad en la región de capacidad se pueden incluir en un concepto de dualidad más general que aparece al plantear el problema dual de Lagrange del problema original (en el apéndice B se incluye la derivación del problema dual de Lagrange a partir de la formulación del problema de minimización de potencia).

Mediante esta dualidad de Lagrange, Yu y Lan transforman el problema de optimización del transmisor en DL en un problema UL dual, añadiendo un término de ruido indeterminado. Para el problema de minimización de potencia, los autores plantean un método iterativo que actualiza las matrices de correlación de señal y ruido duales en cada iteración y un método de punto interior que las actualiza en un mismo paso. 


\subsubsection{Tendencias}

Actualmente, el estudio de los sistemas MIMO multi-usuario se centra en aspectos fundamentalmente prácticos, como por ejemplo, el diseño de técnicas que permiten reducir la cantidad de información que el usuario necesita realimentar a la estación base en los sistemas en los que la CSIT se obtiene mediante un canal de realimentación en el enlace UL (técnicas de limited feedback), aspectos de interacción entre usuarios (usuarios que compiten o colaboran entre sí) y cuestiones que relacionan a la capa de nivel físico con las capas superiores (técnicas de scheduling y métodos de optimización cross-layer).

Teóricamente, los sistemas MIMO multi-usuario pueden proporcionar las capacidades que necesitan los futuros sistemas de comunicaciones inalámbricos. Sin embargo, el desarrollo de aplicaciones comerciales con este tipo de sistemas está todavía en su fase inicial. El problema recae, especialmente, en cómo disponer de CSI en transmisión. 


\subsection{Sistemas MIMO multi-usuario multi-celda}

Como se vio en los sistemas single-user MIMO y MIMO multi-usuario, los sistemas MIMO mejoran sus prestaciones cuando los elementos de la matriz de canal permanecen incorrelados. Por tanto, el siguiente paso en la evolución de los sistemas MIMO ha sido plantear escenarios que puedan proporcionar fuentes adicionales de diversidad a estos sistemas.

Es en este punto donde surge la última de las configuraciones MIMO, la configuración MIMO multi-usuario multi-celda, que implica considerar la interacción entre varios sistemas single-user o MIMO multi-usuario. Al igual que los sistemas MIMO multi-usuario, esta configuración también puede interpretarse como si las antenas de un único sistema MIMO se dividieran en subgrupos y se distribuyeran espacialmente.

Desde un punto de vista teórico, al considerar la interacción de varios sistemas MIMO, pueden darse dos situaciones distintas. En la primera, los sistemas MIMO se interfieren entre ellos, dando lugar a un sistema MIMO Interferente como muestra la figura 2.9. En la segunda situación, denominada sistema MIMO Cooperativo, se permite que los transmisores o receptores, o ambos, cooperen entre ellos, tal y como muestra la figura 2.10 .

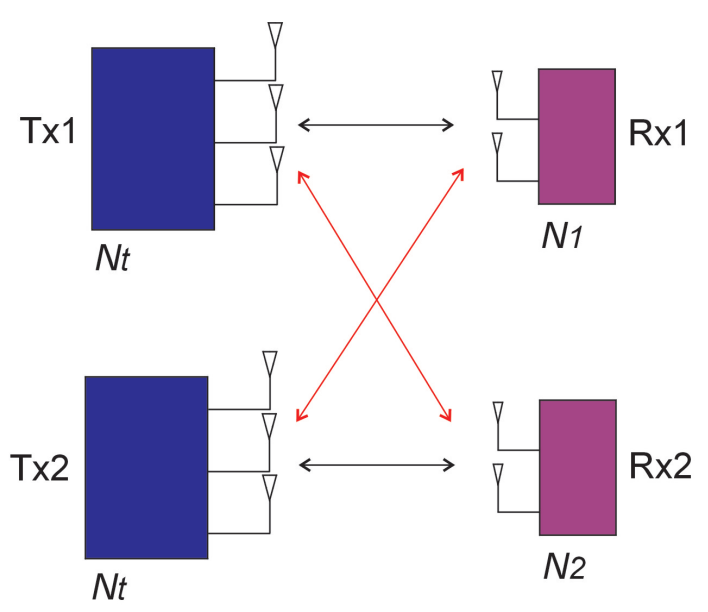

Figura 2.9: Sistema MIMO Interferente. 


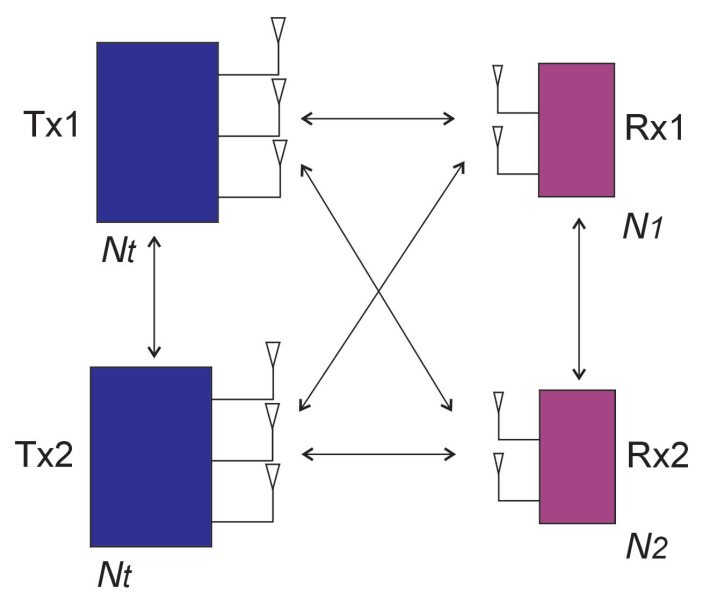

Figura 2.10: Sistema MIMO Cooperativo.

\subsubsection{Aspectos teóricos}

Desde el punto de vista de la teoría de la información, sobre las configuraciones anteriores se pueden definir distintos tipos de canales MIMO, que son físicamente idénticos, pero que difieren en las restricciones que se aplican en los mensajes a transmitir.

Con el fin de simplificar la notación, se asume que los transmisores disponen del mismo número de antenas transmisoras, $N_{t}$. En un sistema con dos transmisores, $K=2$, y dos receptores, $M=2$, donde $N_{m}$ es el número de antenas receptoras de cada usuario, $\mathbf{y}_{m}$ es la $\left[N_{m} \times 1\right]$ señal recibida en el usuario $m$, y puede expresarse con las siguientes ecuaciones:

$$
\begin{aligned}
& \mathbf{y}_{1}=\mathbf{H}_{11} \mathbf{x}_{1}+\mathbf{H}_{12} \mathbf{x}_{2}+\mathbf{n}_{1}, \\
& \mathbf{y}_{2}=\mathbf{H}_{21} \mathbf{x}_{1}+\mathbf{H}_{22} \mathbf{x}_{2}+\mathbf{n}_{2} .
\end{aligned}
$$

$\mathbf{H}_{m k}$ es la matriz de canal $\left[N_{m} \times N_{t}\right]$ para el enlace entre el transmisor $k \mathrm{y}$ el receptor $m, \mathbf{x}_{k}$ son las señales $\left[N_{t} \times 1\right]$ transmitidas por cada transmisor, y $\mathbf{n}_{m}$ es el vector de ruido AWGN añadido en recepción.

Las señales a transmitir en DL, $\mathbf{x}_{1}$ y $\mathbf{x}_{2}$ podrían expresarse como:

$$
\begin{aligned}
& \mathbf{x}_{1}=M_{11} \mathbf{v}_{11}+M_{21} \mathbf{v}_{21}, \\
& \mathbf{x}_{2}=M_{12} \mathbf{v}_{12}+M_{22} \mathbf{v}_{22},
\end{aligned}
$$

donde $M_{m k}$ son los datos a transmitir a cada usuario desde cada transmisor, y los vectores $\mathbf{v}_{m k}$ representan una transformación lineal de los datos. En 
general, puede haber hasta 4 mensajes $M_{m k}$ en el sistema, independientes y distintos, $M_{11}, M_{12}, M_{21}$, y $M_{22}$.

La importancia de los sistemas MIMO reside en la posibilidad de aprovechar la dimensión espacial, junto con el tiempo y la frecuencia. Sin embargo, en los sistemas MIMO multi-usuario, el hecho de distribuir espacialmente las antenas en el lado del receptor impide procesar conjuntamente las muestras recibidas, de forma que se modifican los grados de libertad espaciales disponibles.

Por este motivo, [45] y [46] estudian los grados de libertad espaciales que existen en un sistema MIMO como el determinado por las ecuaciones (2.51)-(2.54). En su análisis, distinguen distintos tipos de canales MIMO, que sólo se diferencian en las restricciones asociadas a los mensajes $M_{m k}$.

El canal MIMO X es el canal más general, donde se transmiten todos los mensajes, es decir, la señal que transmite en DL cada transmisor contiene señales útiles para ambos receptores. El canal MIMO Interferente coincide con el representado en la figura 2.9 y anula los mensajes $M_{12}=M_{21}=0$, es decir, el TX 1 no transmite al segundo usuario y el TX 2 no transmite al primer usuario. Por último, el canal MIMO Z es un caso especial del canal MIMO X, donde $M_{21}=0$ y $M_{11}$ está disponible en el segundo usuario, de forma que éste ayuda a eliminar la interferencia recibida por el primer usuario.

En [45], el canal MIMO X se identifica como una combinación del canal $\mathrm{MAC}$, el canal BC y el canal Interferente. Las estrategias óptimas para MAC y BC son conocidas (successive decoding y DPC, respectivamente). Aunque éste no es el caso del canal Interferente, se sabe que el esquema ZF consigue la mayor ganancia de multiplexado. De esta forma, los autores en [45] aprovechan las componentes MAC, BC e Interferente del canal para definir un esquema específico para el enlace DL del canal MIMO X, el esquema MMK, que combina successive decoding, DPC y ZF.

\subsubsection{Aspectos prácticos}

Los conceptos teóricos de sistema MIMO Interferente o Cooperativo encuentran su equivalente práctico en las redes ad hoc inalámbricas y en los llamados sistemas MIMO Multi-usuario Multi-celda o sistemas M3.

Una red ad hoc inalámbrica se compone de un gran número de nodos o usuarios, que pueden moverse y que se comunican entre sí mediante enlaces inalámbricos sin el apoyo de una infraestructura planeada (las redes inalámbricas tradicionales, como por ejemplo las redes celulares, necesitan 
una determinada infraestructura para intercambiar la información entre usuarios). De esta forma, dos nodos pueden comunicarse entre sí directamente. Sin embargo, los canales inalámbricos suelen ser adversos, lo que implica que si dos usuarios en la red ad hoc se comunican mediante un único salto entre nodos, la potencia de transmisión necesaria es bastante elevada, aumentando al mismo tiempo la interferencia en el sistema. Aquí aparece la posibilidad de llegar a destino utilizando nodos intermedios que van a cooperar con el nodo fuente.

El estudio de esquemas cooperativos en redes ad hoc es todavía una línea abierta de investigación, que tomó relevancia a partir del trabajo de Sendonaris et al. [47]. Una opción para mejorar las condiciones del canal es utilizar múltiples antenas en transmisión y recepción, aprovechando entonces las ventajas de los sistemas MIMO. El problema es que esta solución es inviable, especialmente en redes que requieren nodos pequeños y sencillos como las redes de sensores.

Una posible solución para conseguir diversidad espacial sin necesitar varias antenas es la llamada cooperative diversity: se trata de agrupar varios nodos de una única antena para formar un array virtual de mayor tamaño, que puede funcionar en transmisión o recepción. Estas agrupaciones de nodos se forman mediante negociaciones entre nodos vecinos, sin necesidad de implementar un control centralizado. Ni los nodos que actúan como transmisores ni los que actúan como receptores disponen de los datos que dispone el otro nodo, para conocerlos tienen que intercambiarlos a través del canal inalámbrico.

El relay channel es un canal de 3 nodos donde un nodo intermedio ayuda a un nodo fuente a alcanzar al nodo destino. En la cooperative diversity, los nodos también utilizan relaying para intercambiar los mensajes. Sin embargo, el hecho de tener múltiples fuentes introduce interferencia en el sistema, con lo que la cooperative diversity es fundamentalmente diferente al relay channel.

La figura 2.11 muestra las configuraciones más sencillas con cooperative diversity en un sistema con dos nodos transmisores y dos nodos receptores de una red ad hoc inalámbrica [48]. Cuando cooperan los dos nodos transmisores, primero intercambian los mensajes entre sí y después actúan como si fueran un único canal BC con dos antenas en transmisión. Por el contrario, cuando cooperan los nodos receptores, también intercambian la información recibida y se comportan como un receptor con dos antenas. En general, mediante cooperación en transmisión o en recepción, los nodos se 


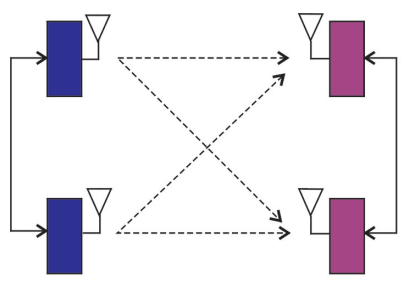

Canal MIMO

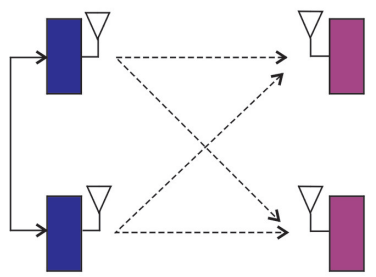

Canal BC

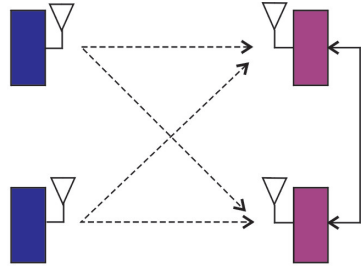

Canal MAC

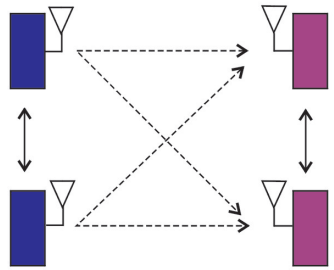

Canal Cooperativo

Figura 2.11: Configuraciones básicas en un sistema ad hoc con cooperative diversity.

comportan como un enlace MIMO punto a punto, consiguiendo las ganancias asociadas a éste sin necesidad de implementar múltiples antenas en los dispositivos.

En [48], Høst-Madsen caracteriza los límites de la región de capacidad de una red ad hoc inalámbrica con cooperative diversity.

La idea de cooperación surge en las redes ad hoc inalámbricas para mitigar los efectos adversos de los canales inalámbricos. Sin embargo, los sistemas celulares siguen siendo la opción más común para proporcionar servicio a usuarios en movimiento y también constituyen un escenario propicio para aplicar los conceptos de cooperación. Como sistema celular, se entiende un sistema inalámbrico donde los usuarios se conectan a algún tipo de infraestructura, ya sea una estación base (BS) o access point (AP).

Dentro del escenario celular, la cooperación puede ser basada en usuarios o basada en infraestructura. La cooperación basada en usuarios es básicamente el esquema convencional de relaying entre terminales con una única antena, que añade ganancia por diversidad. Dentro de la cooperación basada en infraestructura, existen varias aproximaciones, como la basada en la gestión de recursos y la basada en procesado de señal.

La cooperación basada en infraestructura presenta un gran potencial en el enlace DL de los sistemas MIMO multi-usuario multi-celda o sistemas 
M3 limitados por las interferencias, donde las BSs o APs transmiten las señales a los usuarios de su propia celda y un usuario ve como interferentes las señales procedentes de las celdas vecinas. Esta situación se presenta en el primer sistema de la figura 2.12, donde las contribuciones de señal representadas en rojo son interferencias radiadas al resto de los usuarios. En la aproximación basada en la gestión de recursos, las BSs o APs cooperan implementando un esquema conjunto de asignación de recursos [49]. En estos esquemas, los usuarios se siguen comunicando con su BS asignada pero reciben menos interferencias de las celdas adyacentes.

Si las BSs o APs se conectan entre sí e intercambian información mediante un enlace de alta capacidad (por ejemplo, mediante fibra óptica), es posible tener una forma más avanzada de cooperación, la basada en procesado de señal. En este caso, se dice que las BSs cooperan en datos y las antenas de cada BS se interpretan como las antenas distribuidas de un único array MIMO a gran escala, manteniendo las restricciones en potencia individuales por BS.

Básicamente, las antenas de las distintas BSs transmiten coordinadamente a múltiples usuarios, por ejemplo mediante un beamformer multibase. ${ }^{1}$ De esta forma, cada usuario recibe señal útil desde todas las BSs, y la interferencia de las celdas adyacentes pasa a ser señal deseada, como muestra el segundo sistema de la figura 2.12. En teoría, puesto que las BSs cooperan en datos, todas las BSs disponen de una copia perfecta de la señal a transmitir a cada usuario. Esta configuración cooperativa es lo que se denomina en esta Tesis sistema M3 coordinado o sistema CM3 (Coordinated Multi-cell Multi-user MIMO).

\footnotetext{
${ }^{1}$ Si tenemos un sistema M3 con $K$ BSs equipadas con $N_{t}$ antenas cada una, un beamformer multi-base es un beamformer que se ha diseñado globalmente, teniendo en cuenta las $K N_{t}$ antenas disponibles en transmisión, y no localmente en cada BS.
} 

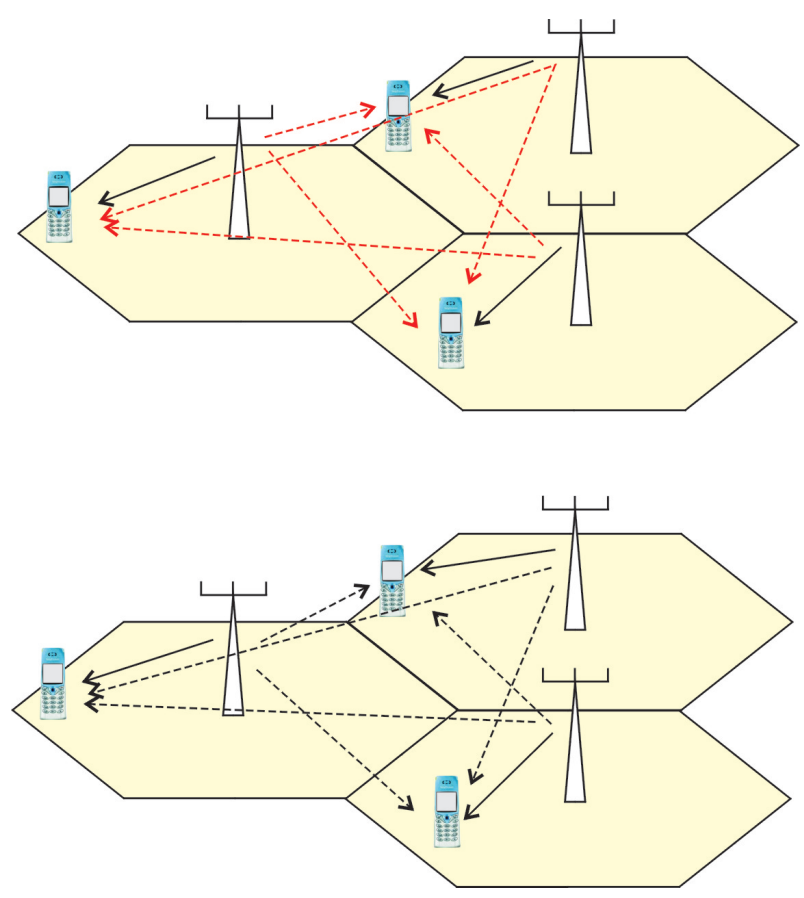

Figura 2.12: Sistema celular convencional y sistema CM3. 


\subsection{Sistemas MIMO multi-usuario multi-celda coordinados}

El estudio de esta configuración cooperativa basada en infraestructura y procesado de señal se encuentra todavía en su fase inicial. De hecho, todavía no ha recibido una denominación común por parte de los grupos de investigación que trabajan en ella. Lo que aquí denominamos sistema MIMO multi-usuario multi-celda coordinado (CM3), recibe en la literatura los nombres de joint transmission [50], network coordination [51], distributed antenna systems [52] o cooperative spatial multiplexing [53], y puede también interpretarse como un sistema MIMO multi-usuario espacialmente distribuido. Hay que destacar que las propuestas [50-53] presentan ligeras diferencias entre sí a la hora de definir un sistema CM3.

El término distributed antenna systems, también se utiliza para denominar aquellos sistemas no cooperativos que distribuyen espacialmente varias antenas para dar cobertura en el interior de grandes edificios o para extender la cobertura en túneles o entornos de difícil acceso [54]. En estos casos las unidades remotas son antenas de bajo coste que actúan a modo de repetidor o como extensión de la BS central. El procesado de señal lo realiza la BS central, y los puertos remotos no cooperan en ningún caso.

Esta configuración mejora las prestaciones del sistema en el enlace UL, debido a la macro-diversidad que proporciona el hecho de distribuir las antenas espacialmente. Sin embargo, en el enlace DL, si las antenas se limitan a funcionar como repetidores de la BS central, las prestaciones del sistema pueden llegar a degradarse debido al aumento de interferencia que se produce. Estudios recientes demuestran que esta configuración, cuando las unidades remotas pueden transmitir distintas señales en DL, presenta una mejora de la capacidad, medida en número de usuarios que puede dar servicio el sistema $[55,56]$. La figura 2.13 muestra un ejemplo de un sistema típico de antenas distribuidas [57].

En esta Tesis, un sistema CM3 es un sistema donde varias BSs, unidas mediante un enlace de alta capacidad, transmiten coordinadamente a múltiples usuarios, mediante una forma de cooperación basada en procesado de señal. Al mismo tiempo, las BSs cooperan perfectamente en datos, con lo que cada BS dispone de una copia de la señal a transmitir a cada usuario. Las BSs no pueden cooperar en potencia, lo que significa que existe una restricción de potencia total transmitida en DL por cada BS. 


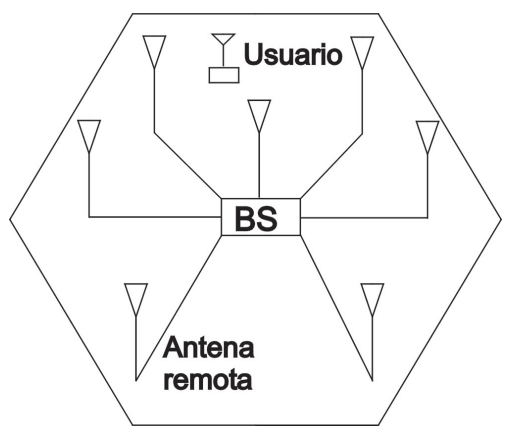

Figura 2.13: Sistema de antenas distribuidas.

Por otra parte, algunos trabajos sobre sistemas CM3 asumen que las BSs no están conectadas entre sí, sino que se conectan mediante un enlace de alta capacidad a un dispositivo central que es el que se encarga de procesar las señales y distribuirlas a las BSs, con lo que las BSs siguen transmitiendo coordinadamente a los usuarios del sistema.

Un sistema CM3 es fundamentalmente distinto del modelo de canal MIMO X presentado para los sistemas M3 (2.54): en el canal MIMO X, la señal $M_{m k}$ que recibe el usuario desde ambas BSs es distinta e independiente, mientras que en el sistema CM3, todas las BSs del sistema disponen de una copia de la señal a transmitir al usuario.

En la actualidad se pueden distinguir claramente dos líneas de investigación. Por una parte se está tratando de caracterizar desde un punto de vista de teoría de la información la región de capacidad de estos sistemas y por otra, se está desarrollando la algorítmica necesaria para tratar distintos problemas de optimización, como el problema de minimización de potencia y el de maximización del sum-rate.

\subsubsection{Capacidad del sistema}

El canal MAC y BC estudiados en el apartado de sistemas MIMO multiusuario son representaciones teóricas del enlace UL y DL de un sistema de comunicaciones de una única celda. El análisis de la capacidad de un sistema CM3 es un problema que todavía no se ha resuelto, pese a ser determinante para caracterizar cómo de eficiente u óptimo es un algoritmo o esquema aplicado a esta configuración. Los primeros estudios sobre la región de capacidad se obtuvieron extendiendo los resultados de los enlaces MAC y BC de un sistema MIMO multi-usuario al entorno CM3. 
En un sistema CM3 con $K$ BSs y $M$ usuarios, donde todas las BSs disponen del mismo número de antenas, $N_{t}$, y cada usuario dispone de $N_{m}$ antenas en recepción, el canal total DL de cada usuario se define como una matriz $\left[N_{m} \times K N_{t}\right]$ que agrupa los canales DL entre el usuario y todas las BSs:

$$
\mathbf{H}_{m}=\left[\mathbf{H}_{m 1} \cdots \mathbf{H}_{m K}\right],
$$

donde las matrices $\mathbf{H}_{m k}$ representan el canal DL $\left[N_{m} \times N_{t}\right]$ para el enlace entre la BS $k$ y el usuario $m$. De esta forma, el usuario $m$-ésimo recibe la señal $\left[N_{m} \times 1\right](2.26)$ :

$$
\mathbf{y}_{m}=\sum_{k=1}^{K} \mathbf{H}_{m k} \sum_{i=1}^{M} \mathbf{x}_{i k}+\mathbf{n}_{m},
$$

donde $\mathbf{x}_{i k}$ es la señal $\left[N_{t} \times 1\right]$ transmitida desde la BS $k$ para el usuario $i$.

En el enlace UL, la señal recibida por la BS $k$ se expresaría como (2.23):

$$
\mathbf{v}_{k}=\sum_{m=1}^{M} \mathbf{H}_{m k}^{H} \mathbf{u}_{m}+\mathbf{w}_{k},
$$

donde $\mathbf{u}_{m}$ es la señal $\left[N_{m} \times 1\right]$ transmitida por cada usuario y $\mathbf{w}_{k}$ es el vector de ruido $\left[N_{t} \times 1\right]$ añadido en la BS.

Suponiendo que existe una coordinación perfecta en datos entre las BSs del sistema, lo que significa que todas las BSs disponen de una copia perfecta de la señal a transmitir a todos los usuarios, se puede asumir que el conjunto de BSs equivale a un único array de antenas distribuido sobre una determinada área. En este caso, [58] propone que la región de capacidad MAC de un sistema CM3 con $K$ BSs y $M$ usuarios, coincide con la región de capacidad MAC de un sistema MIMO multiusuario, pero teniendo en cuenta los canales totales de cada usuario $\mathbf{H}_{m}$ tal y como se definen en (2.55). De esta forma la región de capacidad MAC definida en (2.25) para un sistema MIMO multi-usuario, pasaría a ser $C_{M A C}\left(P_{1}, \ldots, P_{M}, \mathbf{H}_{1}^{H}, \ldots, \mathbf{H}_{M}^{H}\right)$, siendo $P_{m}$ las restricciones de potencia individuales en cada usuario, $\operatorname{Tr}\left[E\left[\mathbf{u}_{m} \mathbf{u}_{m}^{H}\right]\right] \leq P_{m}, \forall m$.

Con respecto al canal $\mathrm{BC}$, puesto que las BSs cooperan perfectamente en datos, [59] implementa la técnica de DPC multi-base, es decir, utilizando las $K N_{t}$ antenas disponibles para transmitir las señales a cada usuario. En esta propuesta, las BSs cooperan en potencia y la restricción en potencia total transmitida se aplica sobre todo el sistema y no individualmente a cada BS, $\sum_{k=1}^{K} \sum_{m=1}^{M} \operatorname{Tr}\left[E\left[\mathbf{x}_{m k} \mathbf{x}_{m k}^{H}\right]\right] \leq P_{\text {max }}$. 
Volviendo a [58], los autores también estudian la capacidad del canal BC. Hay que tener en cuenta que el problema de la región de capacidad del canal BC podría resolverse de forma similar al canal MAC si las BSs pudieran cooperar tanto en datos como en potencia. De esta forma, podría implementarse la técnica de DPC como propone [59] y la región de capacidad DPC definida para sistemas MIMO multi-usuario (2.29), podría obtenerse también en el canal BC de un sistema CM3 [60].

Sin embargo, los sistemas reales no cooperan en potencia, en la práctica cada BS tiene su propia restricción de potencia, $\sum_{m=1}^{M} \operatorname{Tr}\left[E\left[\mathbf{x}_{m k} \mathbf{x}_{m k}^{H}\right]\right] \leq$ $P_{\max }, \forall k$. Cuando la restricción en potencia total transmitida aplica a cada BS de forma individual, no se sabe si la técnica de DPC es óptima y de hecho, ni siquiera la dualidad MAC-BC se mantiene.

Recientemente, aparecen trabajos sobre sistemas MIMO multi-usuario donde las restricciones de potencia total transmitida aplican a cada una de las antenas del array de la BS o a subgrupos de éstas [39,61]. Aunque los sistemas CM3 son esencialmente diferentes, este tipo de resultados caracteriza de una forma más general la región de capacidad de los sistemas CM3 con restricciones de potencia individuales en cada BS.

En [61], el autor intenta unificar las dos aproximaciones que derivaron la región de capacidad del canal $\mathrm{BC}$ de un sistema MIMO multi-usuario, la que utiliza la dualidad entre el MAC-BC [23] y la aproximación alternativa de [20], que descompone el canal BC en una serie de canales single-user MIMO, eliminando previamente la interferencia en el transmisor. En este contexto, el autor demuestra que la sum-capacity de un canal BC con restricciones de potencia por antena en el transmisor, es la misma que la sum-capacity de un canal MAC dual con una restricción de potencia total sobre todos los usuarios, donde la potencia de ruido es indeterminada.

Posteriormente, el mismo autor analiza en [39] el problema de diseñar un transmisor óptimo para el enlace DL de un sistema MIMO multi-usuario con múltiples antenas en transmisión y con restricciones de potencia individuales por antena en el transmisor. Asumiendo CSIT perfecta, plantea el diseño óptimo para obtener toda la región de capacidad cuando el usuario también dispone de múltiples antenas en recepción. El autor generaliza la dualidad MAC-BC para obtener toda la región de capacidad y no sólo la sum-capacity como en [61]: la región de capacidad del canal BC cuando se aplican un conjunto fijo de restricciones de potencia por antena, $\left(P_{1}, \ldots, P_{M}\right)$, coincide con la región de capacidad del canal MAC dual con una restricción en potencia total $\sum_{m} P_{m}$ y con un término de ruido inde- 
terminado. Este ruido se caracteriza mediante una matriz de correlación diagonal, $\sigma^{2} \hat{\mathbf{Q}}$, que satisface $\sum_{m=1}^{M} \hat{\mathbf{Q}}_{m m} P_{m} \leq \sum_{m} P_{m}$. Esta región de capacidad se mantiene para un número arbitrario de antenas transmisoras y receptoras.

\subsubsection{Soluciones algorítmicas en DL}

Esta Tesis se centra en el estudio de soluciones algorítmicas para el enlace DL de los sistemas CM3. Sin embargo, hay que destacar que también existe una línea de investigación bastante activa, que se centra en analizar las ventajas asociadas al enlace UL de estos sistemas (ver por ejemplo [62]).

En este apartado se presentan en primer lugar las cuatro aproximaciones que podríamos denominar clásicas, puesto que son las aproximaciones que sirven como punto de partida de la mayor parte de trabajos sobre sistemas CM3. A continuación, se comentan brevemente algunas de las propuestas más recientes para esta configuración de sistemas MIMO.

\section{Aproximación de Shamai y Zaidel}

Shamai y Zaidel fueron los primeros en proponer que las BSs cooperaran a nivel de procesado de señal, para mejorar las prestaciones en el enlace DL de un sistema limitado por las interferencias [59]. Los autores consideran un sistema CM3 síncrono, donde las BSs se organizan según un modelo lineal, y con único usuario activo en cada celda. Se supone que la cooperación entre BSs se realiza a través de un dispositivo centralizado, que dispone de CSIT perfecta con respecto a todos los canales del sistema y que decide las señales a transmitir desde cada BS. Tanto las BSs como los usuarios disponen de una única antena y las BSs pueden cooperar en potencia.

En este escenario, puesto que el dispositivo central conoce los términos de interferencia que recibe cada usuario, los autores diseñan un esquema de beamforming multi-base lineal, similar al ZF, obtenido a partir de la factorización LQ del canal, y lo combinan con la técnica de DPC. De esta forma, en un sistema CM3 SISO como el planteado por [59], un usuario $m$ recibiría la siguiente señal $(2.50)$ :

$$
y_{m}=l_{m m} d_{m}+\sum_{i=1}^{m-1} l_{m i} d_{i}+n_{m}, m=1, \ldots, M,
$$

donde al ruido AWGN se le suma un término de interferencia que es una combinación lineal de los símbolos transmitidos a los usuarios con un índice 
menor. Los autores muestran que este esquema simplificado de DPC es óptimo para relaciones altas de SINR.

\section{Aproximación de Jafar, Goldsmith y Foschini}

Jafar y Goldsmith parten de la idea de Shamai y Zaidel de aplicar la técnica de DPC en el enlace DL de un sistema CM3 para diseñar un esquema de transmisión óptimo desde el punto de vista de maximizar el sum-rate del sistema [63]. Las BSs cooperan en datos, pero mantienen restricciones individuales de potencia total transmitida. El diseño del esquema de transmisión es centralizado, pero no existe un dispositivo específico que se conecte a todas las BSs y realice el procesado requerido como en [59], sino que las BSs intercambian entre sí toda la información necesaria.

La dualidad MAC-BC no se mantiene cuando existen restricciones de potencia para cada BS. Jafar y Goldsmith proponen en [63] soluciones iterativas y subóptimas para maximizar el sum-rate de un sistema CM3 donde las BSs disponen de arrays de antenas y los usuarios cuentan con una única antena. Los autores proponen un algoritmo de control de potencia iterativo que decide en el problema UL dual qué potencias asignar a cada usuario, ignorando las restricciones de potencia por BS. Posteriormente, mediante las transformaciones UL-DL planteadas para sistemas MIMO multi-usuario, el algoritmo comprueba si alguna BS está superando su restricción en potencia total transmitida. Si esto ocurre, todas las matrices de canal asociadas a esa determinada BS, $\mathbf{H}_{m k}, \forall m$, se anulan en la siguiente iteración del algoritmo. En caso contrario, en la siguiente iteración el algoritmo realiza un pequeño incremento de la potencia asignada a cada usuario. El algoritmo finaliza cuando todas las BSs transmiten a la máxima potencia permitida.

Paralelamente, Jafar, Foschini y Goldsmith estudian la evolución con el tiempo de un sistema CM3, considerando distintas formas de admitir nuevos usuarios [58,64]. Entre las situaciones estudiadas, interesa destacar el problema en el que tratan de maximizar la tasa de transmisión de datos de un nuevo usuario en el sistema, sin que los usuarios ya presentes en el sistema se vean afectados. En este caso, los autores plantean un algoritmo de control de potencia que extiende el algoritmo clásico de waterfilling al entorno CM3, pero manteniendo unas restricciones de potencia por BS y por usuario $P_{m k}(2.56)$ :

$$
\operatorname{Tr}\left[E\left[\mathbf{x}_{m k} \mathbf{x}_{m k}^{H}\right]\right] \leq P_{m k}, \forall m, k .
$$


Básicamente, si el sistema CM3 cuenta con $K$ BSs, la idea es implementar un algoritmo de waterfilling de $K$ etapas. Si se utiliza la técnica de DPC, la máxima tasa de transmisión de datos que percibe un usuario $m$-ésimo sería [64]:

$$
R_{m}=\operatorname{máx}_{\mathbf{Q}_{m}} \log _{2}\left\{\operatorname{det}\left(\mathbf{I}+\left(\mathbf{I}+\sum_{j=1}^{m-1} \mathbf{H}_{j} \mathbf{Q}_{j} \mathbf{H}_{j}^{H}\right)^{-1} \mathbf{H}_{m} \mathbf{Q}_{m} \mathbf{H}_{m}^{H}\right)\right\},
$$

donde $\mathbf{H}_{m}$ es el canal total del usuario $m$ (2.55) y las matrices de correlación $\mathbf{Q}_{m}$ se obtienen al incluir en un único vector $\mathbf{x}_{m}$ las señales $\mathbf{x}_{m k}$ a transmitir al usuario desde todas las BSs.

Aunque no es necesario, la tasa de transmisión de datos del usuario $m$ puede descomponerse en $K$ componentes, cada una asociada a la matriz de correlación de una BS:

$$
\begin{aligned}
& R_{m}=R_{m 1}+R_{m 2}+\ldots+R_{m K} \\
& \mathbf{Q}_{m}=\mathbf{Q}_{m 1}+\mathbf{Q}_{m 2}+\ldots+\mathbf{Q}_{m K}
\end{aligned}
$$

Para obtener la matriz de correlación $\mathbf{Q}_{m}$ óptima, el algoritmo de $w a$ terfilling en $K$ etapas sigue los siguientes pasos:

1. El algoritmo empieza a asignar potencia a partir de la descomposición SVD del canal total efectivo:

$$
\left(\mathbf{I}+\sum_{j=1}^{m-1} \mathbf{H}_{j} \mathbf{Q}_{j} \mathbf{H}_{j}^{H}\right)^{-1 / 2} \mathbf{H}_{m}
$$

hasta que una BS, por ejemplo la primera, alcanza su restricción en potencia para el usuario $m, P_{m 1}$. En ese momento se obtiene la matriz de correlación óptima $\mathbf{Q}_{m 1}$. La tasa de transmisión de datos de esta componente sería:

$$
R_{m 1}=\log _{2}\left\{\operatorname{det}\left(\mathbf{I}+\left(\mathbf{I}+\sum_{j=1}^{m-1} \mathbf{H}_{j} \mathbf{Q}_{j} \mathbf{H}_{j}^{H}\right)^{-1} \mathbf{H}_{m} \mathbf{Q}_{m 1} \mathbf{H}_{m}^{H}\right)\right\} .
$$


2. Puesto que la primera BS ya no dispone de potencia para transmitir al usuario $m$, se redefine el canal total del usuario anulando la matriz de canal correspondiente a la primera BS:

$$
\hat{\mathbf{H}}_{m}=\left[\begin{array}{llll}
\mathbf{0} & \mathbf{H}_{m 2} & \ldots & \mathbf{H}_{m K}
\end{array}\right],
$$

y el algoritmo vuelve a asignar la potencia a partir de la descomposición SVD del nuevo canal total efectivo:

$$
\left(\mathbf{I}+\sum_{j=1}^{m-1} \mathbf{H}_{j} \mathbf{Q}_{j} \mathbf{H}_{j}^{H}+\mathbf{H}_{m} \mathbf{Q}_{m 1} \mathbf{H}_{m}^{H}\right)^{-1 / 2} \hat{\mathbf{H}}_{m} .
$$

El proceso se repite hasta que todas las BSs alcanzan la restricción en potencia de transmisión para el usuario $m$, con lo que se obtienen las matrices de correlación óptimas para ese usuario.

\section{Aproximación de Zhang et al.}

Zhang et al. presentan en [50,65] uno de los análisis más completos sobre sistemas CM3, modelados como un conjunto de BSs que se interconectan entre sí y no a un dispositivo central como en [59]. Los autores analizan el sistema CM3 desde un punto de vista teórico y desde un punto de vista práctico, y repasan algunas ventajas de la transmisión coordinada, como por ejemplo, la ganancia en potencia, la mejora en rango de la matriz de canal y la protección que proporciona la macro-diversidad frente a efectos adversos del canal de propagación como el shadowing. También comparan los resultados del sistema CM3 con los obtenidos por un sistema convencional donde se realiza una asignación de BS para cada usuario.

Para un sistema CM3 formado por $K \mathrm{BSs}$, con $N_{t}$ antenas cada una, y $M$ usuarios equipados con $N_{m}$ antenas cada uno, [50] extiende el esquema propuesto en [59] para un sistema que cumple $M=K$ (el esquema original se planteó con BSs y usuarios de una única antena).

A la hora de plantear esquemas de transmisión DL con restricciones de potencia total transmitida por BS, los trabajos [58] y [63] muestran que el hecho de aplicar la técnica de DPC con este tipo de restricciones conduce a soluciones iterativas bastante complejas. Por este motivo, Zhang et al. proponen soluciones subóptimas con respecto a las prestaciones de la técnica de DPC, pero mucho más atractivas desde un punto de vista práctico. En este punto interesa destacar un procedimiento que permite 
diseñar de forma sencilla un esquema lineal y multi-base de transmisión en DL con restricciones de potencia total transmitida por BS, siempre que $M=K$.

El procedimiento, en grandes líneas, es el siguiente: la matriz de beamforming multi-base lineal $\mathbf{W}$, se incluye en otra matriz,

$$
\mathbf{T}=\mathbf{W} \Omega,
$$

donde $\boldsymbol{\Omega}$ es una matriz diagonal que incluye la potencia asignada en DL a cada usuario desde cada BS:

$$
\boldsymbol{\Omega}=\operatorname{diag}\left\{\left[\mu_{1} \mathbf{I} \ldots \mu_{K} \mathbf{I}\right]\right\} .
$$

Al mismo tiempo se define otra matriz,

$$
\mathbf{Q}=\left[\begin{array}{cccc}
\left\|\mathbf{w}_{11}\right\|_{F}^{2} & \left\|\mathbf{w}_{12}\right\|_{F}^{2} & \cdots & \left\|\mathbf{w}_{1 K}\right\|_{F}^{2} \\
\left\|\mathbf{w}_{21}\right\|_{F}^{2} & \left\|\mathbf{w}_{22}\right\|_{F}^{2} & \cdots & \left\|\mathbf{w}_{2 K}\right\|_{F}^{2} \\
\vdots & \vdots & \vdots & \vdots \\
\left\|\mathbf{w}_{M 1}\right\|_{F}^{2} & \left\|\mathbf{w}_{M 2}\right\|_{F}^{2} & \cdots & \left\|\mathbf{w}_{M K}\right\|_{F}^{2}
\end{array}\right]
$$

donde cada vector $\mathbf{w}_{m k}$ se corresponde con el beamformer diseñado para el enlace entre el usuario $m$ y la BS $k$. Si $\mathbf{P}=\left[P_{\max }(1) \ldots P_{\max }(K)\right]$ incluye las restricciones de potencia por BS, $P_{\max }(k), \boldsymbol{\Omega}$ se obtiene resolviendo:

$$
\boldsymbol{\mu}=\left[\mu_{1}^{2} \ldots \mu_{K}^{2}\right]^{T}=\mathbf{Q}^{-1} \mathbf{P} .
$$

Puesto que $\boldsymbol{\mu}$ representa las potencias transmitidas en DL, la solución será feasible cuando todas las potencias obtenidas sean positivas. En caso contrario, se pueden obtener nuevas potencias con:

$$
\boldsymbol{\Omega}=\boldsymbol{\mu} \mathbf{I}, \boldsymbol{\mu}=\min _{\forall k} \frac{P_{\max }(k)}{\left\|\mathbf{W}_{k}\right\|_{F}^{2}},
$$

donde $\mathbf{W}_{k}$ representa a las filas de la matriz $\mathbf{W}$ que se corresponden con los beamformers de la BS $k$.

Independientemente de que la solución óptima en cuanto a maximizar el sum-rate requiera aplicar la técnica de DPC, este esquema lineal de transmisión se trata de una solución subóptima, puesto que la expresión (2.69) permite que todas las BS transmitan a su potencia máxima permitida, pero en la alternativa (2.70), sólo la BS que cumple con el valor mínimo transmite a máxima potencia, el resto transmite con una menor potencia de la permitida. 


\section{Aproximación de Foschini, Karakayali et al.}

Recientemente, Foschini, Karakayali et al. sientan las bases de los sistemas CM3. Puesto que la región de capacidad de estos sistemas es todavía desconocida, empiezan por caracterizar un límite de esta región desde un punto de vista de fairness. Para ello plantean el problema max-min con restricciones de potencia por BS, y definen la QoS mediante la tasa de transmisión de datos de cada usuario $[51,66,67]$.

Con el objetivo de resolver el problema max-min, aplican el esquema propuesto en [59] (DPC combinada con un beamformer multi-base similar al ZF obtenido a partir de la descomposición LQ del canal) para sistemas CM3 con una única antena tanto en las BSs como en los usuarios. Posteriormente, al igual que Zhang et al. para sistemas que cumplen $M=K$ [50], extienden el esquema para sistemas con múltiples antenas en transmisión y recepción.

La figura 2.14 (con permiso de los autores [51]) muestra una comparativa en eficiencia espectral de los esquemas de transmisión convencionales y coordinados evaluados en [51]. Los esquemas de transmisión coordinada (CCT, Coherent Coordination), superan claramente a los esquemas convencionales de asignación de BS (SBT, Single-Base Transmission), que en este caso son la transmisión a máxima potencia por parte de la BS asignada (FP-SBT) y un algoritmo de control de potencia más refinado que intenta igualar la tasa de transmisión de datos de todos los usuarios (ER-SBT). Los esquemas de transmisión coordinada incluyen la técnica de DPC con un beamformer multi-base ZF (ER-DPC-CCT) y el beamformer multi-base ZF sin codificación DPC (ER-ZF-CCT).

El procedimiento para extender el esquema que combina la técnica de DPC con el beamformer multi-base ZF a un sistema CM3 con $K$ BSs, cada una con $N_{t}$ antenas, y $M$ usuarios, equipados cada uno con $N_{m}$ antenas, es el siguiente [51].

Como se ha visto anteriormente, la señal $\left[N_{m} \times 1\right]$ recibida por el usuario $m$ puede expresarse como (2.56):

$$
\mathbf{y}_{m}=\mathbf{H}_{m} \mathbf{x}+\mathbf{n}_{m},
$$

donde $\mathbf{H}_{m}$ es el canal total DL del usuario, de dimensiones $\left[N_{m} \times K N_{t}\right]$, x es la señal transmitida coordinadamente por las $K$ BSs y $\mathbf{n}_{m}$ es el vector de ruido añadido en recepción.

Puesto que los usuarios disponen de múltiples antenas, es posible transmitir simultáneamente hasta $N_{m}$ subconjuntos de datos independientes 


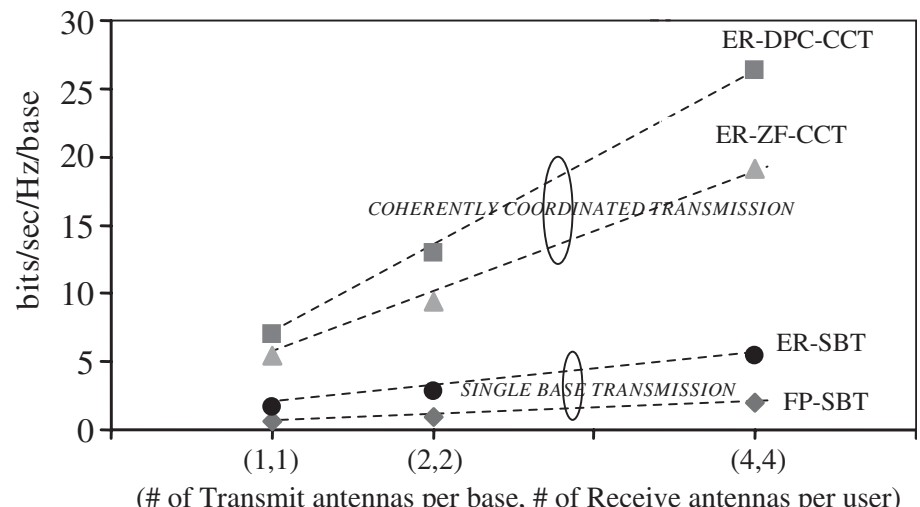

Figura 2.14: Eficiencia espectral de distintos esquemas convencionales y coordinados de transmisión, variando el número de antenas transmisoras y receptoras (con permiso de los autores [51]).

para cada usuario. Al mismo tiempo, dado que las BSs transmiten coordinadamente, la señal $\left[K N_{t} \times 1\right]$ transmitida en DL está formada por los datos a transmitir a los $M$ usuarios de la celda:

$$
\mathbf{x}=\sum_{j=1}^{N_{m}} b_{1 j} \mathbf{w}_{1 j}+\cdots+\sum_{j=1}^{N_{m}} b_{M j} \mathbf{w}_{M j},
$$

donde el vector $\mathbf{w}_{m j}$, de dimensiones $\left[K N_{t} \times 1\right]$, representa el beamformer multi-base diseñado para cada usuario $m$, mientras que $b_{m j}$ son los datos a transmitir a cada usuario.

En el esquema que combina la técnica de DPC con el beamformer multibase, es el beamformer el que elimina la interferencia que no puede eliminar la técnica de DPC. Para el usuario $m$, si el orden de los usuarios viene dado por $[\pi(1) \ldots \pi(M)]$, los autores proponen diseñar los beamformers de los usuarios $\pi(i)$ tales que $i>m$, de forma que sean ortogonales al canal del usuario $\pi(m)$ :

$$
\mathbf{H}_{\pi(m)} \mathbf{w}_{\pi(i) j}=0,\left\|\mathbf{w}_{\pi(i) j}\right\|^{2}=1, \forall i>m, j=1, \ldots, N_{m} .
$$


El primer paso es descomponer el canal de los usuarios $i>m$ como la suma de dos vectores:

$$
\left[\mathbf{H}_{\pi(i)}\right]_{(l,:)}=\mathbf{q}_{\pi(i) l}+\mathbf{q}_{\pi(i) l}^{\prime}
$$

donde $\left[\mathbf{H}_{\pi(i)}\right]_{(l,:)}$ es la fila $l$-ésima del canal total del usuario $\pi(i)$, que se corresponde con el canal $\left[1 \times K N_{t}\right]$ visto desde la antena $l$-ésima del usuario.

El vector $\mathbf{q}_{\pi(i) l}^{\prime}$ representa la parte del canal $\left[\mathbf{H}_{\pi(i)}\right]_{(l,:)}$ que recae en el subespacio formado por los canales de los usuarios $\pi(m), i>m$. La misma descomposición se puede aplicar sobre el canal global, de forma que se obtiene:

$$
\mathbf{H}_{\pi(i)}=\mathbf{Q}_{\pi(i)}+\mathbf{Q}_{\pi(i)}^{\prime}
$$

Los beamformers se diseñan de forma que la señal del usuario $\pi(i)$ queda confinada en el subespacio generado por las filas de la matriz $\mathbf{Q}_{\pi(i)}$. Para encontrar las bases del subespacio, se realiza una descomposición SVD de $\mathbf{Q}_{\pi(i)}$ :

$$
\mathbf{Q}_{\pi(i)}=\mathbf{U}_{\pi(i)} \mathbf{S}_{\pi(i)} \mathbf{V}_{\pi(i)}^{\dagger}
$$

Las primeras $N_{m}$ columnas de $\mathbf{V}_{\pi(i)}$ son los beamformers $\mathrm{ZF} \mathbf{w}_{\pi(i) j}, j=$ $1, \ldots, N_{m}$, del usuario $\pi(i)$.

Finalmente, la señal recibida por el usuario $\pi(m)$ se escribe como:

$$
\begin{aligned}
\mathbf{y}_{\pi(m)} & =\mathbf{H}_{\pi(m)} \mathbf{x}+\mathbf{n}_{\pi(m)}= \\
& =\mathbf{H}_{\pi(m)}\left(\sum_{j=1}^{N_{m}} b_{1 j} \mathbf{w}_{1 j}+\ldots+\sum_{j=1}^{N_{m}} b_{M j} \mathbf{w}_{M j}\right)+\mathbf{n}_{\pi(m)}= \\
& =\mathbf{H}_{\pi(m)}\left(\sum_{j=1}^{N_{m}} b_{\pi(m) j} \mathbf{w}_{\pi(m) j}\right)+ \\
& +\mathbf{H}_{\pi(m)}\left(\sum_{i>m} \sum_{j=1}^{N_{m}} b_{\pi(i) j} \mathbf{w}_{\pi(i) j}\right)+\mathbf{n}_{\pi(m)}= \\
& =\mathbf{H}_{\pi(m)}\left(\sum_{j=1}^{N_{m}} b_{\pi(m) j} \mathbf{w}_{\pi(m) j}\right)+\mathbf{n}_{\pi(m)}= \\
& =\left(\mathbf{Q}_{\pi(m)}+\mathbf{Q}_{\pi(m)}^{\prime}\right)\left(\sum_{j=1}^{N_{m}} b_{\pi(m) j} \mathbf{w}_{\pi(m) j}\right)+\mathbf{n}_{\pi(m)}=
\end{aligned}
$$




$$
\begin{aligned}
& =\mathbf{Q}_{\pi(m)}\left(\sum_{j=1}^{N_{m}} b_{\pi(m) j} \mathbf{w}_{\pi(m) j}\right)+\mathbf{n}_{\pi(m)}= \\
& =\mathbf{U}_{\pi(m)} \mathbf{S}_{\pi(m)} \mathbf{V}_{\pi(m)}^{\dagger}\left(\sum_{j=1}^{N_{m}} b_{\pi(m) j} \mathbf{w}_{\pi(m) j}\right)+\mathbf{n}_{\pi(m)}= \\
& =\mathbf{U}_{\pi(m)}\left[\begin{array}{c}
\lambda_{\pi(m) 1}^{1 / 2} b_{\pi(m) 1} \\
\lambda_{\pi(m) 2}^{1 / 2} b_{\pi(m) 2} \\
\vdots \\
\lambda_{\pi(m) N_{m}}^{1 / 2} b_{\pi(m) N_{m}}
\end{array}\right]+\mathbf{n}_{\pi(m)} .
\end{aligned}
$$

En la expresión anterior hay que tener en cuenta que (2.75) proviene del hecho de que el usuario con índice $\pi(m)$ no sufre interferencia por parte de los usuarios $\pi(i)$ con menores índices $(i<m)$ debido a la técnica de DPC. La interferencia que DPC no puede eliminar, (2.76), la eliminan los vectores diseñados conforme a (2.73). La expresión (2.77) se corresponde con la señal recibida por el usuario tras el efecto de DPC y el beamformer. Por último, hay que tener en cuenta que el vector $\mathbf{w}_{\pi(m) j}$ pertenece a la base del subespacio generado por las filas de $\mathbf{Q}_{\pi(m)}$, por lo que es ortogonal al espacio generado por las filas de $\mathbf{Q}_{\pi(m)}^{\prime}(2.78)$.

El usuario $\pi(m)$ recupera su señal realizando un filtrado adaptado con $\mathbf{U}_{\pi(m)}^{\dagger}$ :

$$
\tilde{\mathbf{y}}_{\pi(m)}=\mathbf{U}_{\pi(m)}^{\dagger} \mathbf{y}_{\pi(m)}=\left[\begin{array}{c}
\lambda_{\pi(m) 1}^{1 / 2} b_{\pi(m) 1} \\
\lambda_{\pi(m) 2}^{1 / 2} b_{\pi(m) 2} \\
\vdots \\
\lambda_{\pi(m) N_{m}}^{1 / 2} b_{\pi(m) N_{m}}
\end{array}\right]+\tilde{\mathbf{n}}_{\pi(m)}
$$

De esta forma, la tasa de transmisión de datos que se obtiene a partir de los canales paralelos de (2.79) sería:

$$
\sum_{j=1}^{N_{m}} \log _{2}\left\{1+\lambda_{\pi(m) j} E\left[\left|b_{\pi(m) j}\right|^{2}\right]\right\}
$$


Finalmente, el problema max-min se plantea como el problema de maximizar una tasa de transmisión de datos $R_{0}$ común a todos los usuarios del sistema (fairness):

$$
\begin{aligned}
\text { máx } & R_{0} \\
\text { s.t. } & \sum_{j=1}^{N_{m}} \log _{2}\left\{1+\lambda_{\pi(m) j} E\left[\left|b_{\pi(m) j}\right|^{2}\right]\right\} \geq R_{0}, m=1, \ldots, M,
\end{aligned}
$$

cumpliendo la restricción en potencia por BS. Los autores resuelven el problema de optimización anterior utilizando técnicas de optimización convexa.

\section{Comparativa entre las aproximaciones clásicas}

En este punto, interesa destacar y comparar las principales diferencias entre las cuatro aproximaciones clásicas.

En primer lugar, hay que remarcar que todas las aproximaciones se centran en el problema de maximizar el sum-rate del sistema CM3, con el objetivo de obtener una primera caracterización de la región de capacidad de este tipo de sistemas.

Desde este punto de vista, y teniendo en cuenta los resultados disponibles para sistemas MIMO multi-usuario, Shamai y Zaidel proponen un primer esquema que combina la técnica de DPC con un esquema de beamforming multi-base, similar al ZF, que se obtiene a partir de la factorización LQ del canal. Sin embargo, estos autores consideran un modelo de sistema CM3 simplificado, donde las BSs se ubican en línea y pueden cooperar en potencia. Además, sólo existe un usuario activo por celda y tanto las BSs como los usuarios disponen de una única antena.

El resto de aproximaciones ya presentan un modelo de sistema CM3 más realista, donde las BSs no pueden cooperar en potencia y están sujetas a restricciones de potencia individuales, y donde tanto las BSs como los usuarios disponen de múltiples antenas. Sin embargo, Jafar, Foschini y Goldsmith no especifican de qué forma se distribuyen las BSs del sistema, mientras que Zhang et al. y Foschini, Karakayali et al., sí que concretan que las BSs siguen la distribución habitual de los sistemas de comunicaciones celulares, es decir, se ubican en el centro de celdas hexagonales.

Con respecto a las soluciones algorítmicas presentadas, tanto Zhang et al. como Foschini, Karakayali et al., extienden el algoritmo de Shamai y Zaidel para sistemas CM3 que disponen de múltiples antenas en las BSs 
y en los usuarios. Foschini, Karakayali et al. se centran en aplicar este algoritmo al problema max-min, puesto que las restricciones de potencia por BS dificultan la resolución del problema de maximización del sum-rate.

Por otro lado, Zhang et al. también proporcionan un método subóptimo para diseñar esquemas de beamforming multi-base, respetando las restricciones de potencia transmitida por BS.

Desde un punto de vista distinto, Jafar, Foschini y Goldsmith tratan el problema de maximizar el sum-rate del sistema CM3 derivando una primera aproximación de la extensión del algoritmo de waterfilling a sistemas CM3. La principal dificultad de esta extensión radica en las restricciones de potencia transmitida por BS, por lo que estos autores modifican este parámetro, planteando una restricción de potencia transmitida por BS y por usuario.

En esta Tesis se plantea el problema de minimización de potencia en el enlace downlink de un sistema CM3. Tras revisar las características de las aproximaciones clásicas, se observa que ninguna de ellas aborda este problema de optimización. De hecho, la única referencia en la que puede encontrarse una primera aproximación a este problema es [68], donde el sistema CM3 se simplifica a un sistema sin coordinación antes de plantear el problema de minimización de potencia.

Desde este punto de vista, puede afirmarse que el trabajo presentado en esta Tesis representa una aportación significativa al estudio de los sistemas CM3.

\section{Aproximaciones recientes}

Dado que la técnica de DPC es un esquema complejo de implementar en sistemas reales, y más todavía en el caso de los sistemas CM3, recientemente aparecen una serie de trabajos que analizan las prestaciones en sum-rate de distintos esquemas lineales de menor complejidad, basados en el diseño de beamformers multi-base [69-72].

En [69] se propone un algoritmo de beamforming multi-base para mitigar la interferencia entre celdas, cuando las BSs, organizadas en un array lineal, disponen de canales de comunicación entre ellas libres de interferencias. En esta propuesta, no se necesita ningún tipo de dispositivo que realice un procesado centralizado de la información, sino que los beamformers multi-base se diseñan de forma distribuida, mediante el intercambio de información entre BSs adyacentes.

En los trabajos de [70-72], las BSs se organizan circularmente, y se evalúan las prestaciones en sum-rate de distintos esquemas de beamforming 
multi-base con diferentes suposiciones sobre el modelo del canal y distribución de usuarios en el sistema.

En esta misma línea, [52] resume desde un punto de vista teórico las principales características de los sistemas CM3 y deriva las expresiones del sum-rate de distintos esquemas tanto para el enlace UL como el enlace DL del sistema. Este trabajo presenta un modelo de sistema CM3 donde los APs distribuidos se conectan mediante enlaces de alta capacidad a una unidad central, que es la que realiza el procesado conjunto.

Otra línea interesante es la propuesta por $[53,73]$, donde se evalúan las prestaciones de un sistema CM3 cuando las BSs disponen de distintos grados de CSIT. Los autores introducen el concepto de hybrid channel knowledge, que representa la situación en la que una BS dispone de CSIT perfecta con respecto a los usuarios que se encuentran dentro de su área de cobertura, pero donde sólo dispone de información estadística de los canales entre el resto de BSs y usuarios. Esta aproximación permitiría reducir la carga de señalización asociada a los sistemas CM3.

Por último, como se ha visto anteriormente, los resultados presentados en [36] y [39] para sistemas MIMO multi-usuario con restricciones de potencia por antena en el array de la BS, también pueden extrapolarse a los sistemas CM3 con restricciones de potencia por BS. Las propuestas de [39] se comentan en el apartado 2.3.6 de criterios de optimización en el enlace DL de un sistema MIMO multi-usuario. Con respecto al trabajo de Boccardi [36], destacar que plantea el diseño de un beamformer ZF con restricciones de potencia por antena con el objetivo de maximizar el sumrate ponderado del sistema, y demuestra que cuando el número de usuarios en la celda crece, el beamformer ZF consigue los mismos resultados que la técnica de DPC con una única restricción de potencia sobre todo el array.

\subsubsection{Criterios de diseño}

Los sistemas CM3 presentan una serie de características que hacen que estos sistemas sean fundamentalmente distintos del resto de configuraciones MIMO. Por ejemplo, si se compara un sistema single-user MIMO con un sistema CM3, se observa que este último presenta una macro-diversidad asociada por el hecho de distribuir espacialmente las antenas, frente a la micro-diversidad que conllevan los sistemas con un único array de antenas en transmisión.

A la hora de modelar los canales, hay que tener en cuenta que en un sistema single-user MIMO los canales de propagación entre antenas consecu- 
tivas presentan condiciones de propagación similares, mientras que en los sistemas CM3 los canales que experimenta un usuario con respecto a todas las BSs pueden presentar condiciones de propagación totalmente distintas.

Al mismo tiempo, en los sistemas single-user MIMO la restricción en potencia total transmitida se aplica a todo el array, mientras que en los sistemas CM3 sin cooperación en potencia estas restricciones se aplican individualmente a cada BS.

Generalmente, los trabajos presentes en la literatura de los sistemas CM3 asumen que las BSs cooperan perfectamente en datos mediante un enlace de capacidad ilimitada y sin errores, y que disponen de CSIT perfecta. Algunos autores empiezan a considerar restricciones en la cantidad de información que las BSs pueden intercambiar, con lo que las prestaciones del sistema pueden empeorar [74].

Otro punto bastante importante en el contexto de los sistemas CM3, es el del sincronismo. La mayor parte de las referencias anteriores, asumen que el usuario recibe en el mismo instante de tiempo tanto la señal deseada como las interferencias procedentes de todas las BSs del sistema. Esta suposición es muy poco realista, puesto que la diferencia de caminos de propagación entre un usuario y todas las BSs, provoca que la señal que llega al usuario sea de naturaleza asíncrona.

Los únicos autores que consideran el problema del sincronismo son Zhang et al. $[50,65]$. En sus trabajos, asumen que las BSs, que disponen de CSIT perfecta, pueden compensar las señales con sus respectivos retardos DL antes de transmitir, de forma que al transmitir en instantes de tiempo distintos, el usuario recibe síncronamente el término de señal deseada procedente de las distintas BSs.

Con respecto al modelo del sistema $\mathrm{CM} 3$, muchas referencias consideran un modelo donde las BSs están situadas en un array lineal [59,69], en un modelo lineal de Wyner $^{2}$ [52] (ver figura 2.15) y en un modelo circular de Wyner ${ }^{3}$ [70-72] (ver figura 2.16). Únicamente Zhang et al. consideran un sistema más realista donde el sistema coordinado lo forman tres celdas coordinadas hexagonales, como muestra la figura $2.17[50,65]$.

\footnotetext{
${ }^{2}$ Un conjunto de BSs de una única antena se distribuyen en forma de array lineal y los usuarios ubicados en una celda sólo reciben la señal de la BS de su celda y de las dos celdas adyacentes.

${ }^{3}$ Las celdas se organizan en círculo, y la primera y última celda son adyacentes, de forma que el sistema es perfectamente simétrico.
} 


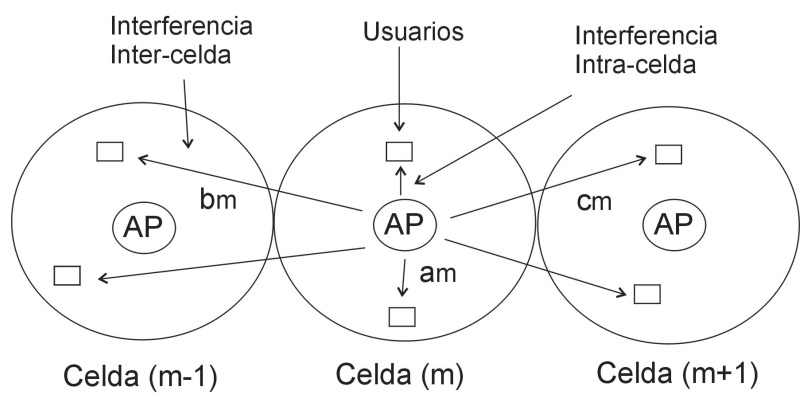

Figura 2.15: Modelo lineal de Wyner.

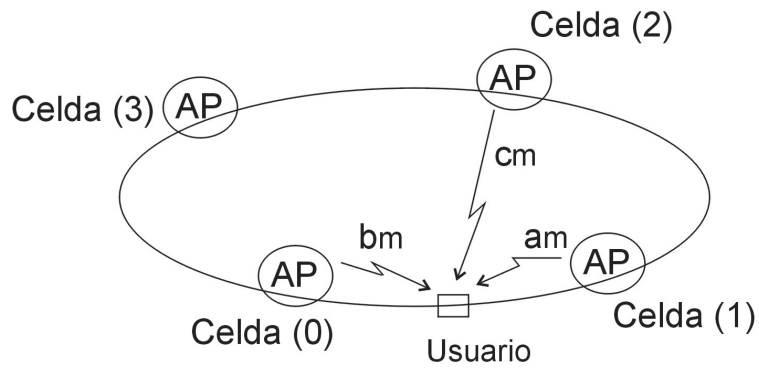

Figura 2.16: Modelo circular de Wyner.

Las matrices de canal de los sistemas que siguen modelos de Wyner para ubicar las BSs se caracterizan por presentar una estructura de bandas diagonal, como por ejemplo:

$$
\mathbf{H}=\left[\begin{array}{cccccc}
\mathbf{a}_{0} & \alpha \mathbf{c}_{0} & \mathbf{0} & \ldots & \mathbf{0} & \mathbf{0} \\
\alpha \mathbf{b}_{1} & \mathbf{a}_{1} & \alpha \mathbf{c}_{1} & \mathbf{0} & \ldots & \mathbf{0} \\
\mathbf{0} & \alpha \mathbf{b}_{2} & \mathbf{a}_{2} & \alpha \mathbf{c}_{2} & \ddots & \vdots \\
\vdots & \mathbf{0} & \alpha \mathbf{b}_{3} & \ddots & \ddots & \mathbf{0} \\
\mathbf{0} & \vdots & \ddots & \ddots & \mathbf{a}_{K-2} & \alpha \mathbf{c}_{K-2} \\
\mathbf{0} & \mathbf{0} & \cdots & \mathbf{0} & \alpha \mathbf{b}_{K-1} & \mathbf{a}_{K-1}
\end{array}\right]
$$

donde $\mathbf{a}_{m}, \mathbf{b}_{m}$ y $\mathbf{c}_{m}$ representan los canales que ven los usuarios ubicados en la celda $m$-ésima, desde la celda $m$, la $m-1$ y la $m+1$ celdas, respectivamente. 


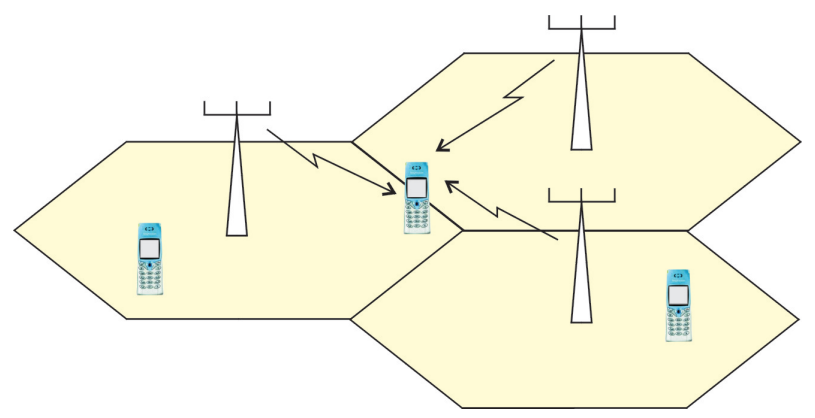

Figura 2.17: Modelo celular realista de un sistema CM3.

Por otra parte, muchas referencias también consideran que hay un único usuario activo en la celda, normalmente cercano al borde de la misma, ya sea mediante un algoritmo de scheduling [70] o suponiendo algún tipo de división en tiempo o frecuencia [59,67,69,70,72].

Otro aspecto en el que difieren los modelos de sistemas CM3 es en el hecho de considerar la existencia o no de un dispositivo que realiza de forma centralizada el procesado de señal asociado con esta forma de cooperación. Este es el caso, por ejemplo de $[52,59]$, mientras que el resto de referencias asumen que no existe este dispositivo.

Por último, los sistemas CM3 también pueden clasificarse en función del número de antenas en las BSs y en los usuarios. De esta forma tenemos sistemas CM3 SISO, como en [59], sistemas CM3 MISO como en [58] o sistemas CM3 MIMO [64].

\subsubsection{Tendencias}

Al igual que en los sistemas MIMO multi-usuario, el principal problema a la hora de desarrollar soluciones comerciales para los sistemas CM3 va a ser la cuestión de cómo disponer de CSI en transmisión. Además, este aspecto se complica en los sistemas CM3, donde las BSs tienen que intercambiar entre ellas esta CSI, y donde la complejidad es creciente con el número de BSs y de usuarios del sistema.

En este punto empieza el interés por esquemas de transmisión DL para sistemas CM3 que permiten implementaciones distribuidas, como las que ya existen para los sistemas M3 que cooperan a nivel de gestión de recursos. Sin embargo, esta línea de investigación es muy reciente, puesto que otros aspectos de los sistemas CM3 más fundamentales, como el estudio teórico 


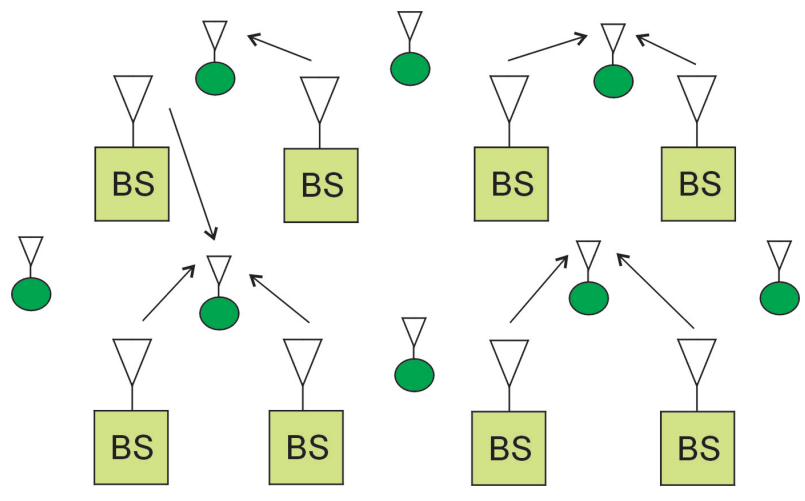

Figura 2.18: Sistema CM3 modelado como un grafo.

de la región de capacidad, siguen también abiertos.

En [75], Skjevling y Gesbert proponen una solución mixta, que combina cooperación en gestión de recursos y en procesado de señal. El objetivo es maximizar el sum-rate del sistema, que se interpreta como un grafo.

El esquema de transmisión DL propuesto, combina un esquema de beamforming multi-base distribuido con varias técnicas de scheduling, que establecen qué BSs sirven a cada usuario. Aunque cada BS sólo puede servir a un usuario, que se determina según la técnica de scheduling aplicada, es posible que un usuario reciba coordinadamente su señal desde más de una BS, como muestra la figura 2.18 [75].

El diseño del beamformer multi-base es distribuido porque asume hybrid channel knowledge, donde cada BS sólo necesita conocer información local de sus canales e información estadística de los canales entre el resto de usuarios y BSs. Por otra parte, las técnicas de scheduling estudiadas también comprenden soluciones coordinadas distribuidas.

En general, la tendencia actual es ubicar los sistemas CM3 en entornos reales, donde, por ejemplo, cada usuario va a recibir de forma asíncrona tanto la señal deseada como las interferentes desde las diferentes BSs [76]. Por otro lado, la dificultad asociada al hecho de disponer de CSIT perfecta en todas las BSs del sistema, conduce a una nueva línea de estudio de los sistemas CM3 que supone que sólo determinadas BSs del sistema se van a coordinar entre sí [77]. 



\section{Parte II}

\section{Solución}

Virtual uplink-based

para requerimiento de QoS en el enlace downlink de un sistema MIMO multi-usuario multi-celda coordinado 



\section{Capítulo 3}

\section{Algoritmos clásicos para sistemas MIMO multi-usuario multi-celda no coordinados}

La interferencia multi-usuario disminuye significativamente las capacidades teóricas que prometen los sistemas single-user MIMO. Este efecto es todavía más acusado en los sistemas MIMO multi-usuario multi-celda coordinados, que podrían definirse como limitados por interferencias. En estos sistemas, cualquier actuación dirigida a disminuir el nivel de interferencia que perciben los usuarios, conlleva una mejora de la capacidad del sistema, entendida con parámetros como por ejemplo el número de usuarios admitidos o el sum-rate del sistema (suma de las tasas de transmisión de datos de todos los usuarios).

En esta segunda Parte, la Tesis se centra en el enlace downlink (DL) de un sistema MIMO multi-usuario multi-celda coordinado (CM3). El objetivo es estudiar cómo mejorar el nivel de interferencia que reciben los usuarios de estos sistemas. Para este fin, se plantea el problema de minimización de potencia, donde se intenta minimizar la potencia total transmitida en el sistema CM3, pero asegurando en los usuarios una determinada calidad de servicio (QoS). En este caso, las restricciones de QoS se establecen mediante valores mínimos o umbrales de SINR. Al mismo tiempo, se imponen restricciones de potencia transmitida a cada estación base del sistema. 
Generalmente, el problema de minimización de potencia se transforma en el problema de diseñar un algoritmo de control de potencia y beamforming óptimo (JPCOB, Joint Power Control and Optimal Beamforming). En este Capítulo se repasan las aproximaciones clásicas a este problema, que son las presentadas por Schubert y Boche [38], Bengtsson y Ottersten [37], y Rashid-Farrokhi et al. [33], para sistemas de una única celda o sistemas multi-celda no coordinados. Estas tres aproximaciones clásicas, representan en realidad las tres formas básicas de abordar el diseño de un algoritmo JPCOB desde un planteamiento de minimización de la potencia total transmitida.

El algoritmo propuesto por Rashid-Farrokhi et al. va a servir como punto de partida de los algoritmos JPCOB propuestos en esta Tesis para el enlace DL de los sistemas CM3. Por este motivo, este algoritmo se presenta con más detalle que el resto de aproximaciones. Para completar aspectos como la convergencia del resto de referencias básicas, hay que recurrir a los trabajos [37,38].

El modelo de sistema es el siguiente: el sistema está formado por $K$ estaciones base (BSs), equipadas con arrays de $N_{t}$ antenas cada una, y $M$ usuarios activos que disponen de una única antena en recepción. El subíndice $m k$ representa el enlace DL entre la BS $k$-ésima y el usuario $m$-ésimo, de forma que los vectores $\mathbf{h}_{m k}(t)$ y $\mathbf{w}_{m k}$ representan, respectivamente, el canal de propagación y el beamformer para el enlace $m k$ usuario-BS del sistema. La matriz de correlación de canal se define mediante $\mathbf{R}_{m k}=E\left[\mathbf{h}_{m k}(t) \mathbf{h}_{m k}^{H}(t)\right]$. Por último, $s_{m}(t)$ es la señal transmitida en DL al usuario $m$ con una potencia $p_{m k}$.

\subsection{Aproximación de Schubert y Boche}

Schubert y Boche [38] consideran el enlace DL de un sistema formado por una única celda, donde la potencia de transmisión de la BS está limitada a $P_{\max }$. En este escenario, cada uno de los usuarios del sistema tiene que alcanzar un umbral de QoS que se traduce en un valor mínimo de SINR, $\left(\gamma_{1}, \ldots, \gamma_{M}\right)$. Todos los usuarios alcanzan su umbral simultáneamente si y solo si el valor mínimo de la SINR recibida en cada usuario está por encima del umbral:

$$
\min _{1 \leq m \leq M} \frac{\operatorname{SINR}_{m}^{D L}}{\gamma_{m}} \geq 1
$$


donde $\operatorname{SINR}_{m}^{D L}$ representa la SINR medida en el usuario $m$, que depende de los beamformers y potencias de transmisión de la BS.

Schubert y Boche resuelven el problema de minimización de potencia desde dos puntos de vista. Desde un punto de vista más general, una condición necesaria y suficiente para que el problema tenga solución se consigue maximizando el mínimo de las relaciones $\left(\operatorname{SINR}_{m}^{D L} / \gamma_{m}\right)$ sobre todos los posibles beamformers y potencias de transmisión:

$$
\operatorname{máx}\left\{\operatorname{mín}_{1 \leq m \leq M} \frac{\operatorname{SINR}_{m}^{D L}}{\gamma_{m}}\right\},
$$

con la restricción de potencia total $P_{\max }$. Este criterio de optimización es el que se denota en el apartado 2.3.6 de los sistemas MIMO multi-usuario como problema de balanceado de SINR o problema max-min.

Por otro lado, si el conjunto de valores de SINR umbral es feasible (es decir, el problema max-min tiene solución dada la restricción en potencia $P_{\max }$ y el vector de potencias que se obtiene para el enlace DL es estrictamente positivo), el problema de minimización de potencia puede plantearse de la forma habitual: minimizar la potencia total transmitida en el sistema manteniendo:

$$
\operatorname{SINR}_{m}^{D L} \geq \gamma_{m}, m=1, \ldots, M
$$

Este segundo planteamiento es un criterio eficiente en potencia, puesto que disminuye la interferencia radiada a las celdas vecinas. Sin embargo, a la hora de analizar soluciones algorítmicas para este segundo caso, hay que tener en cuenta que el conjunto de $\gamma_{m}$ establecidas como umbrales puede que no sea feasible con la restricción de potencia $P_{\text {max }}$. Por tanto, hay que incluir un primer paso que compruebe que las $\gamma_{m}$ requeridas en el problema son feasible.

En el sistema analizado por Schubert y Boche, la BS dispone de un array de $N_{t}$ antenas. La matriz $\mathbf{W}=\left[\mathbf{w}_{1} \ldots \mathbf{w}_{M}\right]$ recoge los $M$ beamformers de módulo unidad, $\left\|\mathbf{w}_{m}\right\|_{2}=1$, uno por usuario. Esta normalización implica que las potencias de transmisión DL sólo dependen de la potencia de las señales transmitidas a cada usuario, $p_{m}=E\left[\left|s_{m}(t)\right|^{2}\right]$.

Las potencias de transmisión DL y UL también se agrupan en los vectores $\mathbf{p}$ y $\mathbf{q}$, respectivamente, de forma que la potencia total de transmisión se obtiene, para cada enlace, sumando los elementos del vector correspondiente. Además, la restricción en potencia total transmitida $P_{\max }$, aplica tanto al enlace UL como al DL del sistema. 
El canal de propagación es un canal flat fading, donde $\mathbf{h}_{m}(t)$ representa el canal complejo entre el usuario $m$ y la BS. Las matrices de correlación de canal en DL se obtienen mediante $\mathbf{R}_{m}=E\left[\mathbf{h}_{m}(t) \mathbf{h}_{m}^{H}(t)\right], 1 \leq m \leq M$. Se asume que existe reciprocidad entre los canales UL y DL, es decir, ambos canales se pueden representar con la misma matriz de correlación.

En la aproximación de Schubert y Boche, la interferencia multi-usuario se modela mediante una matriz no negativa $\boldsymbol{\Psi}$, que depende de la matriz de beamforming $\mathbf{W}$ :

$$
[\mathbf{\Psi}(\mathbf{W})]_{(m, i)}=\left\{\begin{array}{cc}
\mathbf{w}_{i}^{H} \mathbf{R}_{m} \mathbf{w}_{i}, & i \neq m \\
0, & i=m
\end{array}\right.
$$

A partir del modelo de sistema considerado, la SINR recibida en UL y en DL puede expresarse, respectivamente, como:

$$
\begin{aligned}
& \operatorname{SINR}_{m}^{U L}\left(\mathbf{w}_{m}, \mathbf{q}\right)=\frac{q_{m} \mathbf{w}_{m}^{H} \mathbf{R}_{m} \mathbf{w}_{m}}{\mathbf{w}_{m}^{H}\left(\sum_{i=1, i \neq m}^{M} q_{i} \mathbf{R}_{i}+\sigma_{m}^{2} \mathbf{I}\right) \mathbf{w}_{m}}, \forall m, \\
& \operatorname{SINR}_{m}^{D L}(\mathbf{W}, \mathbf{p})=\frac{p_{m} \mathbf{w}_{m}^{H} \mathbf{R}_{m} \mathbf{w}_{m}}{\sum_{i=1, i \neq m}^{M} p_{i} \mathbf{w}_{i}^{H} \mathbf{R}_{m} \mathbf{w}_{i}+\sigma_{m}^{2}}, \forall m .
\end{aligned}
$$

Observando las dos expresiones anteriores, se puede comprobar que el hecho de que el beamformer diseñado en transmisión para un usuario afecte al resto de usuarios, dificulta una optimización directa de la SINR en DL.

\subsubsection{Problema JPCOB en DL, planteamiento max-min}

Schubert y Boche proponen un algoritmo JPCOB iterativo para resolver el problema de balanceado de SINR o problema max-min. Este algoritmo iterativo consta de dos pasos, un primer paso de optimización de los beamformers y un segundo paso donde se obtiene la asignación óptima de potencias en DL. Para demostrar que el algoritmo alcanza un óptimo global, primero demuestran que la solución obtenida en cada uno de los pasos es óptima, cuando la otra variable se mantiene fija.

Cuando la matriz de beamforming es fija, $\tilde{\mathbf{W}}=\left[\tilde{\mathbf{w}}_{1} \ldots \tilde{\mathbf{w}}_{M}\right]$, donde $\left\|\tilde{\mathbf{w}}_{m}\right\|_{2}=1$, la asignación óptima de potencias en DL es conocida [78]. En este caso, el problema de optimización (3.2) se reduce a maximizar la 


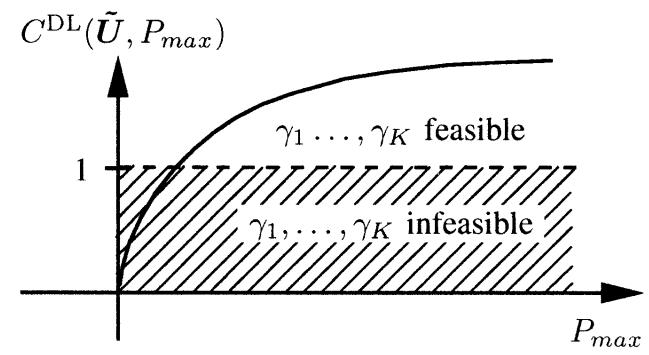

Figura 3.1: La función $C^{D L}\left(\tilde{\mathbf{W}}, P_{\max }\right)$ es estrictamente monótona creciente con la potencia total de transmisión $P_{\max }$ (con permiso de los autores [38]).

mínima SINR sobre todo el conjunto posible de asignación de potencias:

$$
\begin{aligned}
& C^{D L}\left(\tilde{\mathbf{W}}, P_{\text {max }}\right)=\operatorname{máx}_{\mathbf{p}}\left\{\operatorname{mín}_{1 \leq m \leq M} \frac{\operatorname{SINR}_{m}^{D L}(\tilde{\mathbf{W}}, \mathbf{p})}{\gamma_{m}}\right\}, \\
& \text { s.t. } \sum_{m=1}^{M} p_{m} \leq P_{\text {max }} .
\end{aligned}
$$

Como muestra la figura 3.1 (con permiso de los autores [38]), la función $C^{D L}\left(\tilde{\mathbf{W}}, P_{\max }\right)$ es estrictamente monótona creciente con la potencia total de transmisión $P_{\max }$, mientras que las SINR umbrales son feasible si y solo si $C^{D L}\left(\tilde{\mathbf{W}}, P_{\max }\right) \geq 1$.

La asignación óptima de potencias del problema (3.7) se caracteriza por forzar a la BS a transmitir utilizando toda la potencia disponible: si $\tilde{\mathbf{p}}$ es un máximo global entonces:

$$
\begin{aligned}
C^{D L}\left(\tilde{\mathbf{W}}, P_{\text {max }}\right) & =\frac{\operatorname{SINR}_{m}^{D L}(\tilde{\mathbf{W}}, \tilde{\mathbf{p}})}{\gamma_{m}}, 1 \leq m \leq M, \\
P_{\text {max }} & =\sum_{m=1}^{M} \tilde{p}_{m} .
\end{aligned}
$$

En este punto, las SINR en DL de todos los usuarios están balanceadas y son las máximas posibles:

$$
C^{D L}\left(\tilde{\mathbf{W}}, P_{\text {max }}\right)=\frac{\operatorname{SINR}_{1}^{D L}}{\gamma_{1}}=\ldots=\frac{\operatorname{SINR}_{M}^{D L}}{\gamma_{M}}
$$


Las $M$ primeras ecuaciones definidas por (3.8) pueden escribirse en forma matricial como:

$$
\tilde{\mathbf{p}} \frac{1}{C^{D L}\left(\tilde{\mathbf{W}}, P_{\max }\right)}=\mathbf{D} \Psi(\tilde{\mathbf{W}}) \tilde{\mathbf{p}}+\mathbf{D} \boldsymbol{\sigma}
$$

donde se ha tenido en cuenta (3.4) y se han definido el vector $\boldsymbol{\sigma}=\left[\sigma_{1}^{2} \ldots \sigma_{M}^{2}\right]^{T}$ y la matriz diagonal $\mathbf{D}=\operatorname{diag}\left\{\left[\left(\gamma_{1} /\left(\tilde{\mathbf{w}}_{1}^{H} \mathbf{R}_{1} \tilde{\mathbf{w}}_{1}\right)\right) \ldots\left(\gamma_{M} /\left(\tilde{\mathbf{w}}_{M}^{H} \mathbf{R}_{M} \tilde{\mathbf{w}}_{M}\right)\right)\right]\right\}$.

Multiplicando ambos lados por $\mathbf{1}^{T}=\left[\begin{array}{lll}1 & 1 & \ldots\end{array}\right]$, y teniendo en cuenta que $P_{\max }=\sum_{m} \tilde{p}_{m}$, queda:

$$
\frac{1}{C^{D L}\left(\tilde{\mathbf{W}}, P_{\max }\right)}=\frac{1}{P_{\max }} \mathbf{1}^{T} \mathbf{D} \mathbf{\Psi}(\tilde{\mathbf{W}}) \tilde{\mathbf{p}}+\frac{1}{P_{\max }} \mathbf{1}^{T} \mathbf{D} \boldsymbol{\sigma} .
$$

Finalmente, definiendo un vector extendido de potencia, $\tilde{\mathbf{p}}_{e x t}=\left[\begin{array}{c}\tilde{\mathbf{p}} \\ 1\end{array}\right], \mathrm{y}$ una matriz extendida de interferencia:

$$
\mathbf{\Upsilon}\left(\mathbf{W}, P_{\max }\right)=\left[\begin{array}{cc}
\mathbf{D} \boldsymbol{\Psi}(\mathbf{W}) & \mathbf{D} \boldsymbol{\sigma} \\
\frac{1}{P_{\max }} \mathbf{1}^{T} \mathbf{D} \mathbf{\Psi}(\mathbf{W}) & \frac{1}{P_{\max }} \mathbf{1}^{T} \mathbf{D} \boldsymbol{\sigma}
\end{array}\right],
$$

se observa que las ecuaciones (3.9) y (3.10) forman un problema de valores propios:

$$
\mathbf{\Upsilon}\left(\tilde{\mathbf{W}}, P_{\max }\right) \tilde{\mathbf{p}}_{e x t}=\frac{1}{C^{D L}\left(\tilde{\mathbf{W}}, P_{\max }\right)} \tilde{\mathbf{p}}_{e x t},\left[\tilde{\mathbf{p}}_{e x t}\right]_{M+1}=1 .
$$

$C^{D L}\left(\tilde{\mathbf{W}}, P_{\max }\right)$ es, por tanto, un valor propio recíproco de la matriz extendida de interferencia no negativa $\boldsymbol{\Upsilon}$. Sin embargo, no todos los valores propios tienen sentido físico, sólo aquellos en los que se cumple $\tilde{\mathbf{p}}_{\text {ext }}>0 \mathrm{y}$ $C^{D L}\left(\tilde{\mathbf{W}}, P_{\max }\right)>0$.

Por el Teorema de Perron-Frobenius, sabemos que el mayor valor propio de la matriz extendida de interferencia, $\lambda_{\max }(\mathbf{\Upsilon})$, y el vector propio asociado a éste, son siempre no negativos (en el apéndice A se introduce brevemente el Teorema de Perron-Frobenius y su relación con el problema de minimización de potencia). Además, en [78], se demuestra que para una matriz $\Upsilon$ no negativa ${ }^{1}$ con la estructura de (3.11), el mayor valor propio y el vector propio asociado son estrictamente positivos, ningún otro valor propio cumple el requisito de ser positivo, como consecuencia del crecimiento

\footnotetext{
${ }^{1}$ Si las matrices de correlación de canal $\mathbf{R}_{m}$ son definidas positivas, los elementos de $\Upsilon$ son estrictamente positivos.
} 
monótono de la función $C^{D L}\left(\tilde{\mathbf{W}}, P_{\max }\right)$. Por tanto, la solución al problema (3.7) es [78]:

$$
C^{D L}\left(\tilde{\mathbf{W}}, P_{\max }\right)=\frac{1}{\lambda_{\max }\left(\mathbf{\Upsilon}\left(\tilde{\mathbf{W}}, P_{\max }\right)\right)},
$$

y el vector de potencias extendidas $\tilde{\mathbf{p}}_{\text {ext }}$ se corresponde con el vector propio dominante de $\mathbf{\Upsilon}\left(\tilde{\mathbf{W}}, P_{\max }\right)$. De esta forma, el vector de potencias óptimas transmitidas $\tilde{\mathbf{p}}$ está formado por las $M$ primeras componentes de $\tilde{\mathbf{p}}_{\text {ext }}$, que puede escalarse de forma que la última componente $(M+1)$ sea igual a la unidad.

El problema (3.7) también puede plantearse en el enlace UL, con la misma restricción en potencia total transmitida $P_{\max }$, los mismos umbrales de SINR, $\left(\gamma_{1}, \ldots, \gamma_{M}\right)$, y la misma matriz fija de beamforming que en el caso DL. De forma similar al caso DL, se puede demostrar que el valor óptimo viene dado por [78]:

$$
C^{U L}\left(\tilde{\mathbf{W}}, P_{\max }\right)=\frac{1}{\lambda_{\max }\left(\boldsymbol{\Lambda}\left(\tilde{\mathbf{W}}, P_{\max }\right)\right)},
$$

donde $\boldsymbol{\Lambda}$ es la matriz UL extendida de interferencia. Del mismo modo, el vector de potencias óptimas $\tilde{\mathbf{q}}$ se construye con las $M$ primeras componentes del vector propio dominante de $\boldsymbol{\Lambda}\left(\tilde{\mathbf{W}}, P_{\max }\right)$, escaladas de forma que la componente $(M+1)$ sea igual a la unidad:

$$
\boldsymbol{\Lambda}\left(\tilde{\mathbf{W}}, P_{\max }\right) \tilde{\mathbf{q}}_{e x t}=\lambda_{\max }\left(\boldsymbol{\Lambda}\left(\tilde{\mathbf{W}}, P_{\max }\right)\right) \tilde{\mathbf{q}}_{e x t}
$$

Una vez que se conoce la asignación óptima de potencias para un conjunto de beamformers fijos, se puede plantear el problema general de diseñar un algoritmo JPCOB:

$$
\begin{aligned}
& C_{\text {opt }}^{D L}\left(P_{\text {max }}\right)=\operatorname{máx}_{\mathbf{W}, \mathbf{p}}\left\{\operatorname{mín}_{1 \leq m \leq M} \frac{\operatorname{SINR}_{m}^{D L}(\mathbf{W}, \mathbf{p})}{\gamma_{m}}\right\}, \\
& \text { s.t. } \quad \sum_{m=1}^{M} p_{m} \leq P_{\text {max }}, \\
& \quad\left\|\mathbf{w}_{m}\right\|_{2}=1,1 \leq m \leq M .
\end{aligned}
$$

El objetivo es, por tanto, maximizar la mínima SINR normalizada, dada por la relación $\left(\mathrm{SINR}_{m}^{D L} / \gamma_{m}\right)$, sobre todo el conjunto posible de potencias de 
transmisión $\mathbf{p}$ y beamformers $\mathbf{W}$, manteniendo la potencia total transmitida por debajo del máximo $P_{\max }$ y el módulo de los beamformers en transmisión igual a la unidad.

De todo el conjunto de beamformers $\mathcal{M}$ que cumplen (3.16), el subconjunto de beamformers óptimo, $\mathbf{W}^{\text {opt }} \in \mathcal{M}$, es aquel que balancea las relaciones de SINR normalizadas $\left(\operatorname{SINR}_{m}^{D L} / \gamma_{m}\right)$ a un nivel común máximo $C_{\text {opt }}^{D L}\left(P_{\max }\right)$. Este óptimo se obtiene al maximizar $C^{D L}\left(\mathbf{W}, P_{\max }\right)$ sobre todos los posibles beamformers de módulo unidad. Puesto que $C^{D L}\left(\mathbf{W}, P_{\max }\right)$ se relaciona con el mayor valor propio de la matriz extendida de interferencia $\mathbf{\Upsilon}\left(\mathbf{W}, P_{\max }\right)$, el valor óptimo de los beamformers se obtiene minimizando el mayor valor propio de la matriz extendida de interferencia:

$$
C_{o p t}^{D L}\left(P_{\max }\right)=\frac{1}{\min _{\mathbf{W}} \lambda_{\max }\left(\mathbf{\Upsilon}\left(\mathbf{W}, P_{\max }\right)\right)} .
$$

El mismo problema puede formularse para el enlace UL a partir de la matriz $\boldsymbol{\Lambda}\left(\mathbf{W}, P_{\max }\right)$.

\section{Dualidad uplink-downlink}

El siguiente paso es comparar los niveles balanceados obtenidos a partir de una determinada matriz de beamforming $\tilde{\mathbf{W}}$ en el enlace DL y UL, $C^{D L}\left(\tilde{\mathbf{W}}, P_{\max }\right)$ y $C^{U L}\left(\tilde{\mathbf{W}}, P_{\max }\right)$, respectivamente.

En trabajos anteriores, Schubert y Boche demuestran que si $P_{\max } \rightarrow \infty$, los dos enlaces tienen solución en las mismas regiones de SINR [79]. En este caso, interesa saber si el mismo resultado se mantiene cuando se tiene una restricción en potencia $P_{\max }$ y potencias de ruido individuales en los terminales. Para el caso concreto en que todas las potencias de ruido en recepción sean iguales, se cumple lo siguiente:

$$
C^{U L}\left(\tilde{\mathbf{W}}, P_{\max }\right)=C^{D L}\left(\tilde{\mathbf{W}}, P_{\max }\right) .
$$

Una consecuencia inmediata de la relación anterior es que si se tiene un conjunto de SINR umbrales, $\left(\gamma_{1}, \ldots, \gamma_{M}\right)$, feasible en el enlace UL, las mismas SINR umbrales son feasible en el enlace DL y viceversa. Más aún, se obtienen con los mismos beamformers. La lectura positiva de este resultado es que la solución al problema de beamforming en DL (3.16), puede obtenerse a partir de una formulación UL más sencilla.

Si la potencia de ruido en los usuarios no es la misma, $\sigma_{i}^{2} \neq \sigma_{m}^{2}, i \neq m$, la relación anterior no tiene por qué cumplirse, pero todavía es posible utilizar 
la idea de la dualidad para resolver el problema DL. Volviendo a la expresión de la SINR en DL para un usuario $m$ (3.6), se observa que la expresión se mantiene si se aplica un mismo escalado sobre $\mathbf{R}_{m}$ y $\sigma_{m}^{2}$. Por tanto, pueden utilizarse las matrices de correlación escaladas $\tilde{\mathbf{R}}_{m}=\mathbf{R}_{m} / \sigma_{m}^{2}$ de forma que $\sigma_{m}^{2}=1,1 \leq m \leq M$. Aunque este escalado no afecta al problema de optimización en DL, sí que cambia la expresión de la SINR en UL:

$$
\operatorname{SINR}_{m}^{U L}\left(\mathbf{w}_{m}, \mathbf{q}\right)=\frac{q_{m} \mathbf{w}_{m}^{H} \tilde{\mathbf{R}}_{m} \mathbf{w}_{m}}{\sum_{i=1, i \neq m}^{M} q_{i} \mathbf{w}_{m}^{H} \tilde{\mathbf{R}}_{i} \mathbf{w}_{m}+1}, \forall m .
$$

La expresión anterior coincide con la expresión real del enlace UL (3.5), si se cumple $\sigma_{1}^{2}=\ldots=\sigma_{M}^{2}$, es decir, si todos los usuarios tienen la misma potencia de ruido. En caso contrario, la expresión (3.19) se corresponde con la SINR recibida en un enlace uplink virtual. A partir de (3.18) se deriva que los beamformers que balancean de forma óptima las SINR virtuales de (3.19) también son óptimos con respecto al problema original (3.16). Por tanto, la solución al problema DL (3.16) se obtiene resolviendo el problema UL virtual:

$$
C_{\text {opt }}^{D L}\left(P_{\max }\right)=\frac{1}{\operatorname{mín}_{\mathbf{W}} \lambda_{\max }\left(\boldsymbol{\Lambda}\left(\mathbf{W}, P_{\max }\right)\right)} .
$$

Si $\mathbf{W}^{\text {opt }}$ es una solución óptima de (3.20), $\mathbf{W}^{\text {opt }} \in \mathcal{M}$. Los vectores óptimos de potencia en los dos enlaces se obtienen calculando los vectores propios derechos dominantes de las matrices $\boldsymbol{\Lambda}\left(\mathbf{W}^{\text {opt }}, P_{\max }\right)$ y $\mathbf{\Upsilon}\left(\mathbf{W}^{\text {opt }}, P_{\max }\right)$.

Sin embargo, obtener los beamformers óptimos a partir de (3.20) no es inmediato, puesto que la función de coste a minimizar no es lineal con la matriz de beamforming $\mathbf{W}$ y una búsqueda exhaustiva aumentaría excesivamente el coste computacional de la solución. Por este motivo, Schubert y Boche aprovechan la estructura de la matriz extendida de interferencia $\boldsymbol{\Lambda}$, para plantear una formulación equivalente de (3.20), que es fundamental para llegar al algoritmo definitivo:

$$
C_{\text {opt }}^{D L}\left(P_{\max }\right)^{-1}=\min _{\mathbf{W}}\left\{\operatorname{mín}_{\mathbf{q}_{e x t}>0} \hat{\lambda}\left(\mathbf{W}, \mathbf{q}_{\text {ext }}\right)\right\},
$$

donde $\mathbf{q}_{\text {ext }}$ es el vector extendido de potencias UL, y $\hat{\lambda}\left(\mathbf{W}, \mathbf{q}_{\text {ext }}\right)$ es la función de coste:

$$
\hat{\lambda}\left(\mathbf{W}, \mathbf{q}_{e x t}\right)=\operatorname{máx}_{\mathbf{x}>0} \frac{\mathbf{x}^{T} \boldsymbol{\Lambda}\left(\mathbf{W}, P_{\max }\right) \mathbf{q}_{e x t}}{\mathbf{x}^{T} \mathbf{q}_{e x t}},
$$


donde $\mathbf{x}$ es una variable sin significado físico.

Operando sobre (3.15), los autores demuestran que para una matriz de beamforming fija $\tilde{\mathbf{W}}$, el vector de potencias $\mathbf{q}_{\text {ext }}$ obtenido al resolver el problema de valores propios (3.15) en UL, es el que minimiza la función de coste $\hat{\lambda}\left(\tilde{\mathbf{W}}, \mathbf{q}_{\text {ext }}\right)$. Una vez que la asignación óptima de potencias está definida, interesa saber cómo obtener los beamformers $\hat{\mathbf{W}}$ óptimos para una potencia $\mathbf{q}_{\text {ext }}$ dada:

$$
\hat{\mathbf{W}}=\arg \min _{\mathbf{W}}\left\{\hat{\lambda}\left(\mathbf{W}, \mathbf{q}_{e x t}\right)\right\} .
$$

Schubert y Boche demuestran que dado un vector de potencias $\mathbf{q}_{\text {ext }}$, el mínimo de la función de coste $\hat{\lambda}\left(\mathbf{W}, \mathbf{q}_{\text {ext }}\right)$ puede conseguirse maximizando de forma independiente las distintas SINR virtuales en UL. Por tanto, el problema de optimización conjunta se transforma en $M$ problemas independientes:

$$
\begin{aligned}
\hat{\mathbf{w}}_{m}= & \arg \operatorname{máx}_{\mathbf{w}_{m}}\left\{\frac{\mathbf{w}_{m}^{H} \tilde{\mathbf{R}}_{m} \mathbf{w}_{m}}{\mathbf{w}_{m}^{H} \mathbf{Q}_{m}\left(\mathbf{q}_{e x t}\right) \mathbf{w}_{m}}\right\}, \\
\text { s.t. } & \left\|\mathbf{w}_{m}\right\|_{2}=1, \forall m, \\
\text { donde } & \mathbf{Q}_{m}\left(\mathbf{q}_{e x t}\right)=\sum_{i=1, i \neq m}^{M}\left[\mathbf{q}_{e x t}\right]_{i} \tilde{\mathbf{R}}_{i}+\mathbf{I}, \forall m .
\end{aligned}
$$

Las matrices $\mathbf{Q}_{m}$ son no singulares y simétricas, por lo que (3.24) puede resolverse con los vectores propios dominantes de los conjuntos $\left(\mathbf{R}_{m}, \mathbf{Q}_{m}\left(\mathbf{q}_{e x t}\right)\right)$, $1 \leq m \leq M$.

\section{Solución algorítmica JPCOB}

Schubert y Boche demuestran que la solución óptima del problema DL (3.16) se obtiene resolviendo el problema UL equivalente (3.20). A partir de este hecho, plantean un algoritmo iterativo compuesto por dos pasos: en un primer paso, se obtienen los beamformers que maximizan la SINR virtual recibida en la BS para cada usuario (3.24), mientras que en segundo paso, se obtiene la asignación óptima de potencias (3.15), también en el enlace UL. Una vez que el algoritmo ya ha convergido, obtienen la asignación óptima de potencias en DL (3.12), aprovechando que tanto el enlace UL como el DL alcanzan la misma región de SINR (3.18). 
En general, las estrategias de optimización alternada suelen tener propiedades de convergencia pobres, aunque los mismos Schubert y Boche demuestran en su trabajo que ese no es el caso del algoritmo que ellos proponen.

La siguiente tabla resume el algoritmo iterativo propuesto para resolver el problema (3.16):

1. Inicialización: $n_{i t}=0, \mathbf{q}(0)=[0 \ldots 0]^{T}$.

\section{Repetir:}

$$
\begin{aligned}
& \tilde{\mathbf{R}}_{m}=\mathbf{R}_{m} / \sigma_{m}^{2}, \forall m . \\
& \sigma_{m}^{2}=1, \forall m .
\end{aligned}
$$

Hasta que:

$$
\begin{aligned}
& n_{i t}=n_{i t}+1, \\
& \mathbf{w}_{m}\left(n_{i t}\right)=\mathbf{v}_{\max }\left(\tilde{\mathbf{R}}_{m}, \mathbf{Q}_{m}\left(\mathbf{q}\left(n_{i t}-1\right)\right)\right), \forall m, \\
& \mathbf{w}_{m}\left(n_{i t}\right)=\mathbf{w}_{m}\left(n_{i t}\right) /\left\|\mathbf{w}_{m}\left(n_{i t}\right)\right\|_{2}, \forall m, \\
& \text { resolver } \\
& \boldsymbol{\Lambda}\left(\mathbf{W}\left(n_{i t}\right), P_{\max }\right)\left[\begin{array}{c}
\mathbf{q}\left(n_{i t}\right) \\
1
\end{array}\right]=\lambda_{\max }\left(n_{i t}\right)\left[\begin{array}{c}
\mathbf{q}\left(n_{i t}\right) \\
1
\end{array}\right], \\
& C\left(n_{i t}\right)=1 / \lambda_{\max }\left(n_{i t}\right),
\end{aligned}
$$

3. Control

$$
\lambda_{\max }\left(n_{i t}-1\right)-\lambda_{\max }\left(n_{i t}\right)<\epsilon .
$$

de potencia:

$$
\mathbf{\Upsilon}\left(\mathbf{W}\left(n_{i t}\right), P_{\max }\right)\left[\begin{array}{c}
\mathbf{p}^{o p t} \\
1
\end{array}\right]=\lambda_{\max }\left(n_{i t}\right)\left[\begin{array}{c}
\mathbf{p}^{o p t} \\
1
\end{array}\right]
$$

En la tabla anterior, $n_{i t}$ representa el número de la iteración del algoritmo, mientras que el vector propio dominante del conjunto de matrices $(\mathbf{A}, \mathbf{B})$ se denota con $\mathbf{v}_{\max }(\mathbf{A}, \mathbf{B}), \lambda_{\max }\left(n_{i t}\right):=\lambda_{\max }\left(\boldsymbol{\Lambda}\left(\mathbf{W}\left(n_{i t}\right), P_{\max }\right)\right)$ y $C\left(n_{i t}\right):=C^{D L}\left(\mathbf{W}\left(n_{i t}\right), P_{\max }\right)$.

\subsubsection{Problema JPCOB en DL, planteamiento de minimización de potencia}

Schubert y Boche presentan el problema de minimización de potencia como un caso particular del problema de balanceado de SINR o problema max-min. Una vez se tiene un conjunto de beamformers, potencias de transmisión y umbrales de SINR tales que el problema es feasible, $\left(C_{o p t}^{D L}>1\right)$, 
es posible plantear el criterio de minimización de potencia:

$$
\begin{aligned}
P_{\text {min }}^{\text {opt }} & =\min _{\mathbf{W}, \mathbf{p}} \sum_{m=1}^{M} p_{m}, \quad \text { s.t. } \quad \operatorname{SINR}_{m}^{D L}(\mathbf{W}, \mathbf{p}) \geq \gamma_{m}, \\
\left\|\mathbf{w}_{m}\right\|_{2} & =1,1 \leq m \leq M .
\end{aligned}
$$

Esta formulación y la del problema max-min (3.16) se relacionan de la siguiente forma: ambos problemas obtienen la misma solución si en el problema max-min se fija $P_{\max }=P_{\text {min }}^{\text {opt }}$. Puesto que el problema max-min es, en teoría, siempre feasible, algunos autores proponen resolver el problema maxmin en un primer paso para poder plantear el problema de minimización de potencia sobre un escenario feasible [42].

El algoritmo propuesto para minimizar la potencia total transmitida en el sistema, es básicamente el mismo que el propuesto para el problema maxmin, sólo se modifica el paso de actualización de potencias en UL. La idea es que una vez el problema max-min es feasible en UL, $C^{U L}\left(\mathbf{W}\left(n_{i t}\right), P_{\max }\right) \geq$ 1 , dados unos umbrales de SINR $\gamma_{m}$, el valor mínimo de potencia total transmitida se obtiene cuando $\operatorname{SINR}_{m}^{U L}=\gamma_{m}, \forall m$. Esta afirmación se debe a lo siguiente: la expresión de la SINR en UL para un usuario $m$ es monótonamente creciente con $q_{m}$ y monótonamente decreciente con $q_{i}$, si $m \neq i(3.5)$. Si el problema es feasible, la potencia mínima transmitida en el sistema se obtiene cuando $\operatorname{SINR}_{m}^{U L}=\gamma_{m}, \forall m$. En caso contrario, sería posible disminuir el valor de alguna $q_{m}$ sin incumplir los umbrales de SINR, con lo que la potencia total transmitida no sería la mínima posible.

Para una matriz de beamforming dada, las condiciones $\operatorname{SINR}_{m}^{U L}=$ $\gamma_{m}, \forall m$, pueden escribirse en forma matricial, de forma que la asignación óptima de potencia en UL se obtendría resolviendo:

$$
\mathbf{q}\left(n_{i t}\right)=\left(\mathbf{I}-\mathbf{D} \Psi^{T}\left(\mathbf{W}\left(n_{i t}\right)\right)\right)^{-1} \mathbf{D} \mathbf{1},
$$

que proviene de escribir las $M$ condiciones $\operatorname{SINR}_{m}^{U L}=\gamma_{m}$ en forma matricial.

La siguiente tabla resume el algoritmo propuesto para resolver (3.25). Las operaciones incluidas en el primer paso se encargan de obtener un escenario feasible, mientras que el segundo paso es el que se encarga de actualizar las potencias UL. Tras la convergencia, que se define como el punto en que todas las SINR normalizadas están balanceadas en el enlace UL, el algoritmo obtiene la asignación óptima de potencias DL, aprovechando el hecho de que los beamformers óptimos para el enlace UL, también lo son para el DL: 
1. Inicialización: $\quad n_{i t}=0, \mathbf{q}(0)=[0 \ldots 0]^{T}, C(0)=0$.

$\tilde{\mathbf{R}}_{m}=\mathbf{R}_{m} / \sigma_{m}^{2}, \forall m$.

2. Repetir:

$$
\sigma_{m}^{2}=1, \forall m \text {. }
$$

Si $C\left(n_{i t}\right)<1$

$$
\begin{aligned}
& n_{i t}=n_{i t}+1 \\
& \mathbf{w}_{m}\left(n_{i t}\right)=\mathbf{v}_{\max }\left(\tilde{\mathbf{R}}_{m}, \mathbf{Q}_{m}\left(\mathbf{q}\left(n_{i t}-1\right)\right)\right), \forall m, \\
& \mathbf{w}_{m}\left(n_{i t}\right)=\mathbf{w}_{m}\left(n_{i t}\right) /\left\|\mathbf{w}_{m}\left(n_{i t}\right)\right\|_{2}, \forall m,
\end{aligned}
$$

(primer paso)

si no

\section{resolver}

$$
\begin{aligned}
& \boldsymbol{\Lambda}\left(\mathbf{W}\left(n_{i t}\right), P_{\max }\right)\left[\begin{array}{c}
\mathbf{q}\left(n_{i t}\right) \\
1
\end{array}\right]=\lambda_{\max }\left(n_{i t}\right)\left[\begin{array}{c}
\mathbf{q}\left(n_{i t}\right) \\
1
\end{array}\right], \\
& C\left(n_{i t}\right)=1 / \lambda_{\max }\left(n_{i t}\right),
\end{aligned}
$$

(segundo paso)

Hasta que:

$$
\begin{aligned}
& \mathbf{q}\left(n_{i t}\right)=\left(\mathbf{I}-\mathbf{D} \Psi^{T}\left(\mathbf{W}\left(n_{i t}\right)\right)\right)^{-1} \mathbf{D} \mathbf{1}, \\
& P_{\text {sum }}^{n_{i t}}=\sum_{m} q_{m}\left(n_{i t}\right) .
\end{aligned}
$$

\section{Control}

$$
\operatorname{máx}_{1 \leq m \leq M} \frac{\gamma_{m}}{\operatorname{SINR}_{m}^{U L}\left(n_{i t}\right)}-\operatorname{mín}_{1 \leq m \leq M} \frac{\gamma_{m}}{\operatorname{SINR}_{m}^{U L}\left(n_{i t}\right)}<\epsilon .
$$

de potencia:

$$
\mathbf{p}^{o p t}=\left(\mathbf{I}-\mathbf{D} \Psi\left(\mathbf{W}\left(n_{i t}\right)\right)\right)^{-1} \mathbf{D} \mathbf{1}
$$

\subsubsection{Caracterización del óptimo}

En este apartado se repasa brevemente la forma de caracterizar el óptimo de la solución obtenida por los algoritmos de Schubert y Boche. Esta caracterización es necesaria para, posteriormente, relacionar entre sí las tres aproximaciones clásicas presentadas en este Capítulo.

Para una matriz de beamforming fija $\tilde{\mathbf{W}}$, si no existe ninguna restricción de potencia y $\lambda_{\max }\left(\mathbf{D} \Psi^{T}(\tilde{\mathbf{W}})\right)<1$, por el Teorema de Perron-Frobenius (apéndice A, apartado A.1), existe una asignación de potencias UL estrictamente positivas [80]:

$$
\tilde{\mathbf{q}}=\boldsymbol{\sigma}^{2}\left(\mathbf{D}^{-1}-\Psi^{T}(\tilde{\mathbf{W}})\right)^{-1} \mathbf{1}
$$


tales que:

$$
\operatorname{SINR}_{m}^{U L}\left(\tilde{\mathbf{w}}_{m}, \tilde{\mathbf{q}}\right)=\gamma_{m}, \forall m .
$$

De la misma forma, se puede demostrar que el conjunto de SINR umbrales en DL, $\left(\gamma_{1}, \ldots, \gamma_{M}\right)$, es feasible si y solo si $\lambda_{\max }(\mathbf{D} \Psi(\tilde{\mathbf{W}}))<1$, con lo que la asignación óptima de potencias en DL se obtiene resolviendo:

$$
\tilde{\mathbf{p}}=\boldsymbol{\sigma}^{2}\left(\mathbf{D}^{-1}-\mathbf{\Psi}(\tilde{\mathbf{W}})\right)^{-1} \mathbf{1}
$$

En [79], se demuestra que $\lambda_{\max }\left(\mathbf{D} \Psi^{T}(\tilde{\mathbf{W}})\right)=\lambda_{\max }(\mathbf{D} \boldsymbol{\Psi}(\tilde{\mathbf{W}}))$. Por tanto, cuando no existen restricciones de potencia, el enlace UL y el DL alcanzan la misma región de SINR. Esta relación es la llamada dualidad débil entre el problema UL y el DL.

El siguiente paso es asumir que existe una restricción en potencia $P_{\max }$ y que se tiene un conjunto arbitrario de valores umbrales de SINR en UL, $\left(\gamma_{1}, \ldots, \gamma_{M}\right)$. Para cumplir estas restricciones de SINR, la asignación óptima de potencia en UL viene dada por un vector $\tilde{\mathbf{q}}>0$, de forma que la potencia total transmitida en UL sería la suma de los elementos de este vector, $\sum_{m} \tilde{q}_{m}$. A partir de la llamada dualidad débil, la misma región de SINR es feasible en el enlace DL. Si $\tilde{\mathbf{p}}>0$ es la asignación óptima de potencias en DL, y $\sum_{m} \tilde{p}_{m}$ es la potencia total transmitida en DL, se cumple:

$$
\begin{aligned}
\sum_{m=1}^{M} \tilde{p}_{m} & =\mathbf{1}^{T} \tilde{\mathbf{p}}=\boldsymbol{\sigma}^{2} \mathbf{1}^{T}\left(\mathbf{D}^{-1}-\mathbf{\Psi}(\tilde{\mathbf{W}})\right)^{-1} \mathbf{1} \\
& =\boldsymbol{\sigma}^{2} \mathbf{1}^{T}\left(\mathbf{D}^{-1}-\mathbf{\Psi}^{T}(\tilde{\mathbf{W}})\right)^{-1} \mathbf{1}=\mathbf{1}^{T} \tilde{\mathbf{q}}=\sum_{m=1}^{M} \tilde{q}_{m} .
\end{aligned}
$$

Por tanto, se necesita la misma potencia total transmitida en ambos enlaces para alcanzar los mismos umbrales de SINR (3.18). Esto es lo que se conoce como dualidad fuerte entre el problema UL y el DL [80]: sólo si se cumple la relación (3.29), es posible resolver el problema DL a través del planteamiento UL dual. En [80], Schubert y Boche generalizan las relaciones de dualidad entre ambos problemas para potencias de ruido arbitrarias. 


\subsection{Aproximación de Bengtsson y Ottersten}

Bengtsson y Ottersten [37] consideran un sistema multi-celda no coordinado, donde $K$ BSs, equipadas con arrays de $N_{t}$ antenas cada una, dan servicio a $M$ usuarios activos en el sistema (en este punto, interesa recordar que la aproximación de Schubert y Boche contempla un sistema formado por una única BS). La señal que recibe el usuario $m$ puede expresarse como:

$$
r_{m}(t)=\sum_{k=1}^{K} \mathbf{h}_{m k}^{H} \mathbf{x}_{k}(t)+n_{m}(t),
$$

donde $\mathbf{x}_{k}(t)$ es la señal transmitida por el array de antenas de la BS $k$ y $n_{m}(t)$ representa el término de ruido aditivo, que se supone blanco con potencia $\sigma_{m}^{2}=E\left[\left|n_{m}(t)\right|^{2}\right]$.

La variable $\mathcal{K}(m)$ designa cuál de las BSs sirve al usuario $m$, mientras que el conjunto $I(k)=\{m ; \mathcal{K}(m)=k\}$ representa a los usuarios a los que da servicio la BS $k$. Un usuario es servido por una única BS, de forma que la señal transmitida por la BS $k$ se expresa:

$$
\mathbf{x}_{k}(t)=\sum_{m \in I(k)} \mathbf{w}_{m} s_{m}(t)
$$

donde $\mathbf{w}_{m}$ es el beamformer y $s_{m}(t)$ es la señal a transmitir al usuario $m$. Se asume que las distintas señales $s_{m}(t)$ son señales de potencia unidad, $E\left[\left|s_{m}(t)\right|^{2}\right]=1$, incorreladas entre sí.

\subsubsection{Problema JPCOB en DL, planteamiento de minimización de potencia}

Bengtsson y Ottersten plantean el criterio clásico de minimización de potencia. A partir de las ecuaciones (3.30) y (3.31), la señal recibida en el usuario $m$ puede descomponerse en:

$$
r_{m}(t)=\mathbf{h}_{m \mathcal{K}(m)}^{H} \mathbf{w}_{m} s_{m}(t)+\sum_{\substack{i=1 \\ i \neq m}}^{M} \mathbf{h}_{m \mathcal{K}(i)}^{H} \mathbf{w}_{i} s_{i}(t)+n_{m}(t),
$$

donde el primer sumando se corresponde con la señal deseada, el segundo sumando representa las interferencias multi-usuario recibidas en el usuario $m$ y el tercer término representa el ruido en recepción. 
Por tanto, la SINR en el usuario $i$ puede calcularse como:

$$
\operatorname{SINR}_{m}^{D L}=\frac{\mathbf{w}_{m}^{H} \mathbf{R}_{m \mathcal{K}(m)} \mathbf{w}_{m}}{\sum_{i=1, i \neq m}^{M} \mathbf{w}_{i}^{H} \mathbf{R}_{m \mathcal{K}(i)} \mathbf{w}_{i}+\sigma_{m}^{2}} .
$$

Teniendo en cuenta que la potencia empleada en la BS $\mathcal{K}(m)$ para transmitir la señal $s_{m}(t)$ es $E\left[\left\|\mathbf{w}_{m} s_{m}(t)\right\|^{2}\right]=\mathbf{w}_{m}^{H} \mathbf{w}_{m}$, el problema de minimización de potencia puede escribirse como:

$$
\begin{aligned}
\min & \sum_{m=1}^{M} \mathbf{w}_{m}^{H} \mathbf{w}_{m}, \\
\text { s.t. } & \frac{\mathbf{w}_{m}^{H} \mathbf{R}_{m \mathcal{K}_{m}} \mathbf{w}_{m}}{\sum_{i=1, i \neq m}^{M} \mathbf{w}_{i}^{H} \mathbf{R}_{m \mathcal{K}(i)} \mathbf{w}_{i}+\sigma_{m}^{2}} \geq \gamma_{m}, m=1, \ldots, M .
\end{aligned}
$$

$\mathrm{O}$, de forma equivalente,

$$
\begin{array}{ll}
\operatorname{mín} & \sum_{m=1}^{M} \mathbf{w}_{m}^{H} \mathbf{w}_{m}, \\
\text { s.t. } & \mathbf{w}_{m}^{H} \mathbf{R}_{m \mathcal{K}_{m}} \mathbf{w}_{m}-\gamma_{m} \sum_{i=1, i \neq m}^{M} \mathbf{w}_{i}^{H} \mathbf{R}_{m \mathcal{K}(i)} \mathbf{w}_{i} \geq \gamma_{m} \sigma_{m}^{2}, m=1, \ldots, M .
\end{array}
$$

Observando el planteamiento del problema de minimización de potencia, se aprecia que todos los beamformers del sistema están incluidos en las restricciones. Esto implica que la solución del problema debe calcularse de forma centralizada. Además, es necesario conocer todos los enlaces entre cualquier BS y cualquier usuario, complicando la implementación de esta estrategia en un escenario real.

En principio, el problema de minimización de potencia planteado en (3.34) es un problema de optimización cuadrático con restricciones cuadráticas no convexas. Por tanto, el problema no es convexo, pero la formulación (3.34) permite transformarlo [30] o relajarlo, como hacen Bengtsson y Ottersten, para obtener un problema de optimización convexa.

La estrategia de Bengtsson y Ottersten consiste en obtener una relajación SDP (Semidefinite Program) de la formulación original (3.34). Para reescribir el problema, asumen que los beamformers varían con el tiempo con una distribución determinada. Con esta suposición, introducen las matrices de correlación de los beamformers, $\mathbf{W}_{m}=E\left[\mathbf{w}_{m}(t) \mathbf{w}_{m}^{H}(t)\right]$, que son 
matrices semidefinidas positivas ${ }^{2}$. Con la introducción de estas matrices de correlación, se añaden grados de libertad adicionales en la búsqueda del óptimo. Aunque posteriormente demuestran que las matrices óptimas $\mathbf{W}_{m}$ son en realidad de rango uno, lo que significa que la solución óptima es utilizar beamformers fijos, la ventaja de esta estrategia es que resolver el problema con las matrices $\mathbf{W}_{m}$ es en algunos aspectos más sencillo que resolverlo directamente sobre los vectores $\mathbf{w}_{m}$.

Aplicando la relación $\operatorname{Tr}[\mathbf{A B}]=\operatorname{Tr}[\mathbf{B A}]$, se deduce que $\mathbf{w}^{H} \mathbf{R} \mathbf{w}=$ $\operatorname{Tr}\left[\mathbf{R} \mathbf{w w}^{H}\right]=\operatorname{Tr}[\mathbf{R W}]$. Como consecuencia, el problema original DL (3.34) se puede reescribir como:

$$
\begin{array}{ll}
\operatorname{mín} & \sum_{m=1}^{M} \operatorname{Tr}\left[\mathbf{W}_{m}\right], \\
\text { s.t. } & \operatorname{Tr}\left[\mathbf{R}_{m \mathcal{K}(m)} \mathbf{W}_{m}\right]-\gamma_{m} \sum_{\substack{i=1 \\
i \neq m}}^{M} \operatorname{Tr}\left[\mathbf{R}_{m \mathcal{K}(i)} \mathbf{W}_{i}\right] \geq \gamma_{m} \sigma_{m}^{2}, \forall m, \\
& \mathbf{W}_{m}=\mathbf{W}_{m}^{H}, \\
& \mathbf{W}_{m} \succeq 0 .
\end{array}
$$

Las restricciones (3.37) y (3.38) se añaden para asegurar que las matrices de correlación $\mathbf{W}$ sean hermíticas, y semidefinidas positivas, respectivamente. La notación $\mathbf{W} \succeq 0$ indica que $\mathbf{W}$ es semidefinida positiva.

A partir de la formulación (3.35)-(3.38), se tiene una función de coste que es lineal con $\mathbf{W}$, al igual que las restricciones (3.36) y (3.37). La restricción semidefinida (3.38) es no lineal e incluso no diferenciable, pero es convexa. Por tanto, el problema es convexo, lo que significa que no existen mínimos locales y que el conjunto de soluciones óptimas son convexas (generalmente, un único punto). Los problemas que cuentan con una función de coste lineal, restricciones lineales y restricciones semidefinidas se denominan problemas semidefinidos o SDP. Para resolver este tipo de problemas, se dispone de métodos como los de punto de interior y de herramientas software estándar.

En realidad, la formulación (3.35)-(3.38) se trata de una relajación SDP del problema original (3.34), ya que relaja la restricción de que los beamformers óptimos sean de rango uno, como requiere el problema original. La formulación (3.35)-(3.38) no garantiza a priori que las matrices de correlación óptimas $\mathbf{W}$ sean de rango uno; es probable que se encuentre una

\footnotetext{
${ }^{2} \mathbf{x}^{T} \mathbf{W} \mathbf{x} \geq 0, \forall \mathbf{x}$.
} 
solución con menor coste que en el problema (3.34) pero con matrices $\mathbf{W}_{m}$ de mayor rango. Este sería un resultado típico de este tipo de técnicas de relajación, ya que suelen utilizarse para obtener límites inferiores de los problemas de optimización cuadrática.

Si al problema (3.35)-(3.38) se le añade la restricción de que el rango de las matrices de correlación cumpla rango $\left[\mathbf{W}_{m}\right]=1$, el problema de optimización (3.35)-(3.38) se correspondería exactamente con el problema (3.34).

Bengtsson y Ottersten demuestran que la relajación semidefinida (3.35)(3.38) siempre tiene al menos una solución óptima donde todas las matrices $\mathbf{W}_{m}$ son de rango uno. Esto es así porque la formulación (3.35)-(3.38) no es una relajación estricta del problema original, sino más bien una formulación equivalente. En el supuesto de que alguna matriz de correlación $\mathbf{W}_{m}$ fuera de mayor rango, los autores proponen un método para refinar la solución y ajustar el rango de las matrices.

\section{Solución algorítmica JPCOB}

Resumiendo, el algoritmo para obtener los beamformers óptimos $\mathbf{w}_{i}$ es el siguiente:

\section{Resolver:}

mediante paquetes software

de optimización semidefinida.

2. Encontrar $\mathbf{w}_{m}$ tal que: $\mathbf{w}_{m} \mathbf{w}_{m}^{H}=\mathbf{W}_{m}, \forall m$.

3. Si alguna $\mathbf{W}_{m}$ no es de rango uno, recalcular $\mathbf{w}_{m}$ :

Obtener los $\mathbf{u}_{m} \in \operatorname{span}\left[\mathbf{W}_{m}\right]$ Formar $\boldsymbol{\eta}=\left[\sigma_{1}^{2} \gamma_{1} \ldots \sigma_{M}^{2} \gamma_{M}\right]^{T}$, y la matriz $\mathbf{F}$ tal que:

$[\mathbf{F}]_{(m, i)}=\left\{\begin{array}{cc}\mathbf{u}_{m}^{H} \mathbf{R}_{m} \mathbf{u}_{m}, & i=m, \\ -\gamma_{m} \mathbf{u}_{i}^{H} \mathbf{R}_{m \mathcal{K}(i)} \mathbf{u}_{i}, & i \neq m,\end{array}\right.$

Calcular:

$\mathbf{p}=\mathbf{F}^{-1} \boldsymbol{\eta}$, $\mathbf{w}_{m}=\sqrt{[\mathbf{p}]_{m}} \mathbf{u}_{m}$.

En la tabla anterior, $\mathbf{u}_{m} \in \operatorname{span}\left[\mathbf{W}_{m}\right]$ implica considerar cualquier vector $\mathbf{u}_{m}$ incluido en el espacio vectorial formado por todas las posibles combinaciones lineales del conjunto $\mathbf{W}_{m}$. 
Una ventaja de este algoritmo es la gran flexibilidad que proporciona a la hora de formular el problema y sobre todo, la facilidad con la que se pueden añadir restricciones adicionales al planteamiento original del problema [42]. Por otro lado, las técnicas desarrolladas para problemas SDP pueden manejar un escenario no feasible. Sin embargo, el hecho de optimizar sobre matrices $\mathbf{W}_{m}$ con mayores grados de libertad que las del problema original, aumenta considerablemente el coste computacional de esta solución. 


\subsection{Aproximación de Rashid-Farrokhi et al.}

La aproximación de Rashid-Farrokhi et al. [33] se basa, al igual que la aproximación de Schubert y Boche, en un algoritmo iterativo que resuelve el diseño del algoritmo JPCOB a través de la dualidad entre el problema UL y el DL. Sin embargo, el algoritmo de Rashid-Farrokhi et al. presenta unas características particulares que hacen que sea elegido como punto de partida para desarrollar un algoritmo JPCOB válido para sistemas CM3.

Rashid-Farrokhi et al., como Bengtsson y Ottersten, sólo plantean el problema de minimización de potencia.

\subsubsection{Problema JPCOB en UL, planteamiento de minimización de potencia}

Aunque esta Tesis se centra en el enlace DL del sistema, en esta aproximación, la solución del problema de minimización de potencia en el enlace UL juega un papel fundamental.

El sistema está formado por un conjunto de $M$ enlaces co-canal, donde cada enlace $m$ está formado por un usuario y la BS que le da servicio. Con este modelo, se tienen en cuenta tanto un sistema de una única celda como un sistema de varias celdas.

El canal de propagación entre una BS y un usuario está formado por $L$ contribuciones multi-camino. Si se realiza un muestro de la señal a periodo de símbolo, y $N$ representa la duración de la respuesta impulsional, la señal recibida en la BS del enlace $i$ puede escribirse como:

$$
\mathbf{x}_{i}(n)=\sum_{m=1}^{M} \mathbf{H}_{m i} \sqrt{p_{m}} \mathbf{s}_{m}(n)+\mathbf{n}_{i}(n),
$$

donde la matriz de canal $\mathbf{H}_{m i}$, de dimensiones $\left[N_{t} \times N\right]$, contiene las respuestas discretas del canal:

$$
\begin{aligned}
\mathbf{H}_{m i} & =\left[\mathbf{h}_{m i}(0) \ldots \mathbf{h}_{m i}(N-1)\right], \\
\mathbf{h}_{m i} & =\left[h_{m i}^{1}(n) \ldots h_{m i}^{N_{t}}(n)\right]^{T},
\end{aligned}
$$

el vector $\mathbf{s}_{m}(n)$ contiene $N$ muestras de la señal transmitida por el usuario $m$ con una potencia $p_{m}$, mientras que $\mathbf{n}_{i}(n)$ es el vector de ruido a la salida del filtro adaptado de la BS del enlace $i$.

La figura 3.2 muestra el diagrama de bloques de un sistema con beamformers en recepción. La señal en la BS para el usuario $i$ tras el paso por 


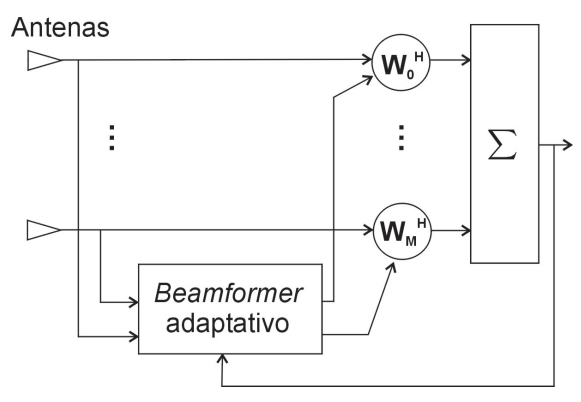

Figura 3.2: Diagrama de bloques de un sistema con beamformers en recepción.

el beamformer $\mathbf{w}_{i}$, viene dada por $\mathbf{e}_{i}(n)=\mathbf{w}_{i}^{H} \mathbf{x}_{i}(n)$. La potencia media de esta señal quedaría:

$$
\mathcal{E}_{i}=E\left[\mathbf{w}_{i}^{H} \mathbf{x}_{i}(n) \mathbf{x}_{i}^{H}(n) \mathbf{w}_{i}\right]=\mathbf{w}_{i}^{H} \boldsymbol{\Phi}_{i} \mathbf{w}_{i} .
$$

Suponiendo que las señales $s_{m}(t)$ transmitidas por los usuarios son de media nula e incorreladas entre sí, y que el ruido es espacial y temporalmente blanco, la matriz de correlación de la señal recibida en el enlace UL del usuario $i$-ésimo puede descomponerse en una matriz de correlación de señal deseada y en una matriz de correlación de interferencias más ruido, respectivamente:

$$
\begin{aligned}
\boldsymbol{\Phi}_{i} & =\boldsymbol{\Phi}_{i}^{S}+\boldsymbol{\Phi}_{i}^{I}, \\
\boldsymbol{\Phi}_{i}^{S} & =p_{i} \mathbf{h}_{i i}(0) \mathbf{h}_{i i}^{H}(0), \\
\boldsymbol{\Phi}_{i}^{I} & =\sum_{n \neq 0} p_{i} \mathbf{h}_{i i}(n) \mathbf{h}_{i i}^{H}(n)+\sum_{m \neq i} p_{m} \mathbf{H}_{m i} \mathbf{H}_{m i}^{H}+N_{i} \mathbf{I} .
\end{aligned}
$$

Finalmente, la expresión de la SINR a la salida del beamformer en recepción para el enlace $i$-ésimo es la siguiente:

$$
\operatorname{SINR}_{i}^{U L}=\frac{\mathbf{w}_{i}^{H} \boldsymbol{\Phi}_{i}^{S} \mathbf{w}_{i}}{\mathbf{w}_{i}^{H} \boldsymbol{\Phi}_{i}^{I} \mathbf{w}_{i}}=\frac{p_{i} \mathbf{w}_{i}^{H} \mathbf{G}_{i i}^{S} \mathbf{w}_{i}}{\sum_{m=1}^{M} p_{m} \mathbf{w}_{i}^{H} \mathbf{G}_{m i}^{I} \mathbf{w}_{i}+N_{i} \mathbf{w}_{i}^{H} \mathbf{w}_{i}}
$$

donde las matrices $\mathbf{G}_{i i}^{S}$ y $\mathbf{G}_{m i}^{I}$ son las matrices de correlación de canal de señal y de interferencias, respectivamente:

$$
\begin{aligned}
\mathbf{G}_{i i}^{S} & =\mathbf{h}_{i i}(0) \mathbf{h}_{i i}^{H}(0), \\
\mathbf{G}_{m i}^{I} & =\left\{\begin{array}{cc}
\mathbf{G}_{m i}=\mathbf{H}_{m i} \mathbf{H}_{m i}^{H}, & m \neq i \\
\mathbf{G}_{m i}-\mathbf{G}_{m i}^{S}, & m=i .
\end{array}\right.
\end{aligned}
$$


El problema de minimización de potencia en el enlace UL consiste en encontrar los beamformers en recepción, $\mathbf{A}=\left[\mathbf{w}_{1} \ldots \mathbf{w}_{M}\right]$, y las potencias de transmisión de los usuarios, $\mathbf{p}=\left[p_{1} \ldots p_{M}\right]^{T}$, que minimizan la potencia total transmitida en el sistema, asegurando al mismo tiempo que la SINR en UL para cada usuario supera el umbral requerido $\gamma_{i}$ :

$$
\begin{aligned}
\min _{\mathbf{A}, \mathbf{p}} & \sum_{i=1}^{M} p_{i} \\
\text { s.t. } & \operatorname{SINR}_{i}^{U L} \geq \gamma_{i}, i=1, \ldots, M .
\end{aligned}
$$

Tal y como plantean Schubert y Boche, es posible estudiar el problema de optimización de cada variable (beamformers o potencia transmitida por los usuarios) cuando la otra variable permanece fija.

El objetivo del problema de beamforming en recepción cuando las potencias transmitidas por los usuarios permanecen fijas, es diseñar los pesos de los beamformers de forma que se maximice la SINR de cada usuario a la salida del beamformer de la BS.

Por otro lado, como se ha visto en la aproximación de Schubert y Boche, cuando los beamformers en recepción permanecen fijos, la mínima potencia transmitida en UL se consigue cuando:

$$
\operatorname{SINR}_{i}^{U L}=\gamma_{i}, i=1, \ldots, M .
$$

El vector óptimo de potencias transmitidas en UL se calcula a partir de la expresión (3.43) mediante esquemas iterativos de control de potencia [43]:

$$
\begin{aligned}
p_{i}\left(n_{i t}+1\right) & =\gamma_{i} p_{i}\left(n_{i t}\right) \frac{\mathbf{w}_{i}^{H} \mathbf{\Phi}_{i}^{I} \mathbf{w}_{i}}{\mathbf{w}_{i}^{H} \mathbf{\Phi}_{i}^{S} \mathbf{w}_{i}} \\
& =\gamma_{i} \frac{\sum_{m=1}^{M} p_{m}\left(n_{i t}\right) \mathbf{w}_{i}^{H} \mathbf{G}_{m i}^{I} \mathbf{w}_{i}+N_{i} \mathbf{w}_{i}^{H} \mathbf{w}_{i}}{\mathbf{w}_{i}^{H} \mathbf{G}_{i i}^{S} \mathbf{w}_{i}}, i=1, \ldots, M,
\end{aligned}
$$

donde $n_{i t}$ representa el número de la iteración y $p_{i}\left(n_{i t}\right)$ es la potencia transmitida en cada iteración por el usuario $i$. En [43] se demuestra, utilizando las propiedades de convergencia de la iteración de Jacobi, que la iteración anterior converge a la asignación óptima de potencia transmitida en UL para cualquier vector $\mathbf{p}$ arbitrario de potencia inicial (en el apéndice A se introduce brevemente la iteración de Jacobi y su relación con el problema 
de minimización de potencia). Al mismo tiempo, hay que destacar que la expresión (3.45) se corresponde con un control de potencia distribuido, puesto que, como puede observarse, para actualizar la potencia transmitida por el usuario $i$ sólo se necesitan los canales o las potencias locales de la BS correspondiente al enlace $i$.

Volviendo al problema general de minimización de potencia en el enlace UL, (3.44), dado un conjunto de beamformers en recepción, se dice que este problema es feasible si existe un vector de potencias UL, y una matriz de beamforming $\mathbf{A}$, tales que los umbrales de SINR se superan en cada enlace, es decir:

$$
\operatorname{SINR}_{i}^{U L} \geq \gamma_{i}, i=1, \ldots, M .
$$

La relación anterior, teniendo en cuenta (3.43), puede expresarse de forma matricial como:

$$
[\mathbf{I}-\mathbf{D F}] \mathbf{p} \geq \mathbf{u}
$$

donde

$$
\begin{aligned}
{[\mathbf{F}]_{(i, m)} } & =\mathbf{w}_{i}^{H} \mathbf{G}_{m i}^{I} \mathbf{w}_{i} \\
\mathbf{D} & =\operatorname{diag}\left\{\left[\frac{\gamma_{1}}{\mathbf{w}_{1}^{H} \mathbf{G}_{11}^{S} \mathbf{w}_{1}} \ldots \frac{\gamma_{M}}{\mathbf{w}_{M}^{H} \mathbf{G}_{M M}^{S} \mathbf{w}_{M}}\right]\right\} \\
{[\mathbf{u}]_{i} } & =\frac{\gamma_{i} N_{i} \mathbf{w}_{i}^{H} \mathbf{w}_{i}}{\mathbf{w}_{i}^{H} \mathbf{G}_{i i}^{S} \mathbf{w}_{i}} .
\end{aligned}
$$

A partir del Teorema de Perron-Frobenius (apéndice A, apartado A.1), si existe un conjunto de beamformers $\mathbf{A}$, tales que $\rho(\mathbf{D F})<1$, donde $\rho(\cdot)$ representa el radio espectral ${ }^{3}$ de la matriz $[\mathbf{D F}]$, la matriz $[\mathbf{I}-\mathbf{D F}]$ es invertible y la potencia que minimiza la función objetivo se obtiene mediante $\mathbf{p}=[\mathbf{I}-\mathbf{D F}]^{-1} \mathbf{u}$. En este caso, el vector óptimo de potencias también puede obtenerse mediante la expresión iterativa (3.45).

En [43], Rashid-Farrokhi et al. estudian el problema JPCOB en UL de (3.44). Los autores demuestran que si el problema es feasible, existe un único conjunto de beamformers y de asignación de potencias en UL, entre todos los posibles, que minimizan la potencia total transmitida en UL. Este análisis es el que permite desarrollar una solución JPCOB para el problema

\footnotetext{
${ }^{3}$ Básicamente, el radio espectral de una matriz $\mathbf{A}$, representa el valor máximo de los valores absolutos de los valores propios de $\mathbf{A}$. Hay que tener en cuenta que la aproximación de Schubert y Boche, cuando se refiere al mayor valor propio de las matrices extendidas de interferencia, en realidad se está refiriendo al radio espectral de las mismas.
} 
de minimización de potencia en el enlace DL, a través del concepto del uplink virtual.

Los pasos del algoritmo JPCOB en UL para cada iteración $n_{i t}$ serían:

1. Beamformers en recepción: para cada usuario, se obtienen maximizando la SINR recibida en la BS del enlace correspondiente.

$$
\begin{aligned}
\mathbf{w}_{i}\left(n_{i t}\right) & =\arg \operatorname{máx}_{\mathbf{w}_{i}}\left\{\frac{\mathbf{w}_{i}^{H} \mathbf{\Phi}_{i}^{S} \mathbf{w}_{i}}{\mathbf{w}_{i}^{H} \mathbf{\Phi}_{i}^{I} \mathbf{w}_{i}}\right\} \\
& =\arg \operatorname{máx}_{\mathbf{w}_{i}}\left\{\frac{p_{i}\left(n_{i t}\right) \mathbf{w}_{i}^{H} \mathbf{G}_{i i}^{S} \mathbf{w}_{i}}{\sum_{m=1}^{M} p_{m}\left(n_{i t}\right) \mathbf{w}_{i}^{H} \mathbf{G}_{m i}^{I} \mathbf{w}_{i}+N_{i} \mathbf{w}_{i}^{H} \mathbf{w}_{i}}\right\} .
\end{aligned}
$$

2. Potencias UL: se actualizan mediante el control iterativo y distribuido de potencia, pero considerando en cada iteración los beamformers actualizados.

$$
\begin{aligned}
p_{i}\left(n_{i t}+1\right) & =\gamma_{i} p_{i}\left(n_{i t}\right) \frac{\mathbf{w}_{i}^{H}\left(n_{i t}\right) \mathbf{\Phi}_{i}^{I} \mathbf{w}_{i}\left(n_{i t}\right)}{\mathbf{w}_{i}^{H}\left(n_{i t}\right) \mathbf{\Phi}_{i}^{S} \mathbf{w}_{i}\left(n_{i t}\right)} \\
& =\gamma_{i} \frac{\sum_{m=1}^{M} p_{m}\left(n_{i t}\right) \mathbf{w}_{i}^{H}\left(n_{i t}\right) \mathbf{G}_{m i}^{I} \mathbf{w}_{i}\left(n_{i t}\right)+N_{i} \mathbf{w}_{i}^{H}\left(n_{i t}\right) \mathbf{w}_{i}\left(n_{i t}\right)}{\mathbf{w}_{i}^{H}\left(n_{i t}\right) \mathbf{G}_{i i}^{S} \mathbf{w}_{i}\left(n_{i t}\right)} .
\end{aligned}
$$

\subsubsection{Problema JPCOB en DL, planteamiento de minimización de potencia}

La figura 3.3 muestra el diagrama de bloques de un sistema con beamformers en transmisión. En este problema, sólo las BSs están equipadas con un array de antenas.

En el enlace DL, la señal recibida por cada usuario es la superposición de las señales transmitidas por las distintas BSs. Si se realiza un muestreo de la señal a periodo de símbolo, y $N$ representa la duración de la respuesta impulsional, la señal recibida por el usuario $i$ es la siguiente:

$$
\tilde{z}_{i}(n)=\sum_{b=1}^{M} \mathbf{w}_{b}^{H} \mathbf{H}_{i b} \sqrt{\tilde{p}_{b}} \tilde{\mathbf{s}}_{b}(n)+\tilde{n}_{i}(n)
$$

donde el vector $\tilde{\mathbf{s}}_{b}(n)$ contiene $N$ muestras de la señal transmitida desde la BS $b$ y $\tilde{n}_{i}(n)$ es el ruido en recepción. 


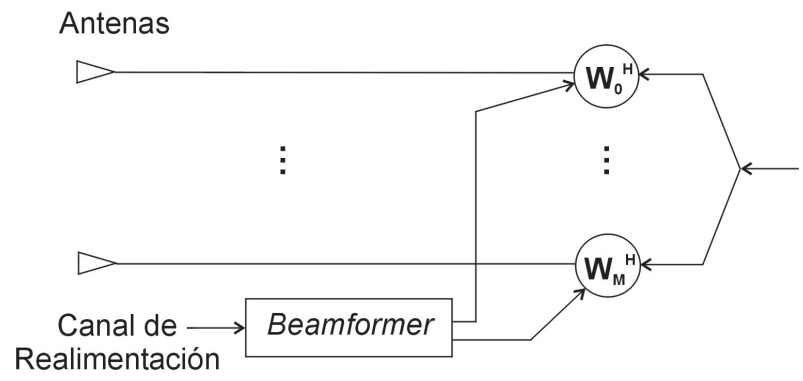

Figura 3.3: Diagrama de bloques de un sistema con beamformers en transmisión.

Si la señal transmitida por la BS del enlace $b$ se expresa como:

$$
\tilde{\mathbf{x}}_{i b}(n)=\mathbf{H}_{i b} \sqrt{\tilde{p}_{b}} \tilde{\mathbf{s}}(n),
$$

la señal recibida en el usuario $i$ quedaría:

$$
\tilde{z}_{i}(n)=\sum_{b=1}^{M} \mathbf{w}_{b}^{H} \tilde{\mathbf{x}}_{i b}(n)+\tilde{n}_{i}(n) .
$$

A partir de la expresión anterior, la SINR en DL en el usuario $i$ se calcula como:

$$
\operatorname{SINR}_{i}^{D L}=\frac{\tilde{p}_{i} \mathbf{w}_{i}^{H} \mathbf{G}_{i i}^{S} \mathbf{w}_{i}}{\sum_{b=1}^{M} \tilde{p}_{b} \mathbf{w}_{b}^{H} \mathbf{G}_{i b}^{I} \mathbf{w}_{b}+\sigma_{i}^{2}},
$$

donde las matrices de correlación de canal $\mathbf{G}_{i i}^{S}$ y $\mathbf{G}_{i b}^{I}$ se definen como en el problema UL y $\sigma_{i}^{2}$ es la potencia de ruido térmico en el usuario.

Contrariamente a lo que sucede al plantear el problema JPCOB en UL, en el problema JPCOB en DL no existe una solución que minimice la potencia transmitida por cada BS de forma independiente. Por tanto, el problema de minimización de potencia se plantea sobre todo el sistema, es decir, sobre los $M$ enlaces co-canal. Rashid-Farrokhi et al. no normalizan los beamformers a la unidad, por lo que la potencia transmitida en DL en cada enlace del sistema se puede separar en dos componentes, la potencia propiamente dicha, $\tilde{p}_{i}$, y la contribución de cada beamformer, $\left\|\mathbf{w}_{i}\right\|^{2}$.

El problema de minimización de potencia en DL consiste, por tanto, en obtener el conjunto de beamformers, A, y el vector de asignación de potencias DL, $\tilde{\mathbf{p}}$, tales que la potencia transmitida en todo el sistema sea la mínima posible, pero asegurando que la SINR recibida en DL supere en 
cada usuario el umbral $\gamma_{i}$ establecido:

$$
\operatorname{mín}_{\mathbf{A}, \tilde{\mathbf{p}}} \sum_{i=1}^{M} \tilde{p}_{i}\left\|\mathbf{w}_{i}\right\|^{2} \text {, s.t. } \operatorname{SINR}_{i}^{D L} \geq \gamma_{i}, i=1, \ldots, M \text {. }
$$

Como ya se ha visto en las aproximaciones anteriores, la mínima potencia transmitida en el sistema se consigue cuando la SINR en cada usuario es igual al valor umbral de QoS:

$$
\operatorname{SINR}_{i}^{D L}=\gamma_{i}, i=1, \ldots, M
$$

que en forma matricial se expresaría como:

$$
\tilde{\mathbf{p}}=\mathbf{D F}^{T} \tilde{\mathbf{p}}+\tilde{\mathbf{u}}
$$

donde

$$
\begin{aligned}
{[\mathbf{F}]_{(i, m)} } & =\mathbf{w}_{i}^{H} \mathbf{G}_{m i}^{I} \mathbf{w}_{i} \\
\mathbf{D} & =\operatorname{diag}\left\{\left[\frac{\gamma_{1}}{\mathbf{w}_{1}^{H} \mathbf{G}_{11}^{S} \mathbf{w}_{1}} \ldots \frac{\gamma_{M}}{\mathbf{w}_{M}^{H} \mathbf{G}_{M M}^{S} \mathbf{w}_{M}}\right]\right\}, \\
{[\tilde{\mathbf{u}}]_{i} } & =\frac{\gamma_{i} \sigma_{i}^{2}}{\mathbf{w}_{i}^{H} \mathbf{G}_{i i}^{S} \mathbf{w}_{i}} .
\end{aligned}
$$

Rashid-Farrokhi et al. presentan un algoritmo iterativo para obtener la solución óptima, si existe al menos una solución posible, del problema de minimización de potencia. Este algoritmo funciona a partir de la construcción de un uplink virtual.

En el enlace UL virtual del sistema, las matrices de canal son idénticas a las matrices de canal del enlace DL. En un primer paso, se obtienen los beamformers en recepción que maximizan la SINR recibida por las BSs en el UL virtual. Estos mismos beamformers se utilizan después en transmisión. En un segundo paso, se actualizan las potencias UL virtuales, que a priori no tienen significado físico, y las potencias de transmisión DL. La base del algoritmo es el análisis que se realiza del problema UL real en [43].

Los pasos del algoritmo JPCOB en DL para cada iteración $n_{i t}$ serían: 
1. Beamformers en transmisión: para cada usuario, se obtienen maximizando la SINR recibida en la BS, a partir del problema UL virtual.

$$
\begin{aligned}
\mathbf{w}_{i}\left(n_{i t}\right) & =\arg \operatorname{máx}_{\mathbf{w}_{i}}\left\{\frac{\mathbf{w}_{i}^{H} \mathbf{\Phi}_{i}^{S} \mathbf{w}_{i}}{\mathbf{w}_{i}^{H} \mathbf{\Phi}_{i}^{I} \mathbf{w}_{i}}\right\} \\
& =\arg \operatorname{máx}_{\mathbf{w}_{i}}\left\{\frac{p_{i}\left(n_{i t}\right) \mathbf{w}_{i}^{H} \mathbf{G}_{i i}^{S} \mathbf{w}_{i}}{\sum_{m=1}^{M} p_{m}\left(n_{i t}\right) \mathbf{w}_{i}^{H} \mathbf{G}_{m i}^{I} \mathbf{w}_{i}+\mathbf{w}_{i}^{H} \mathbf{w}_{i}}\right\} .
\end{aligned}
$$

Comparando la expresión anterior con la expresión de la SINR recibida en la BS en el enlace UL real, (3.43), es importante destacar que la expresión correspondiente al problema UL virtual mantiene el término de potencia de ruido igual a la unidad, $N_{i}=1$.

2. Potencias UL virtuales: se actualizan mediante el control iterativo y distribuido de potencia, pero considerando en cada iteración los beamformers actualizados.

$$
\mathbf{p}\left(n_{i t}+1\right)=\mathbf{D}\left(n_{i t}\right) \mathbf{F}\left(n_{i t}\right) \mathbf{p}\left(n_{i t}\right)+\mathbf{u}\left(n_{i t}\right)
$$

\section{Potencias DL:}

$$
\tilde{\mathbf{p}}\left(n_{i t}+1\right)=\mathbf{D}\left(n_{i t}\right) \mathbf{F}^{T}\left(n_{i t}\right) \tilde{\mathbf{p}}\left(n_{i t}\right)+\tilde{\mathbf{u}}\left(n_{i t}\right) .
$$

El beamformer $\mathbf{w}_{i}\left(n_{i t}\right)$ es el beamformer óptimo para la asignación de potencia de la iteración $n_{i t} . \mathbf{F}\left(n_{i t}\right), \mathbf{D}\left(n_{i t}\right)$ y $\tilde{\mathbf{u}}\left(n_{i t}\right)$ se definen como en las ecuaciones (3.53) pero sustituyendo $\mathbf{w}_{i}$ por $\mathbf{w}_{i}\left(n_{i t}\right) \mathbf{u}\left(n_{i t}\right)$ sería similar a la expresión correspondiente al problema UL real, (3.46), pero considerando $N_{i}=1$ :

$$
\left[\mathbf{u}\left(n_{i t}\right)\right]_{i}=\frac{\gamma_{i}\left\|\mathbf{w}_{i}\left(n_{i t}\right)\right\|^{2}}{\mathbf{w}_{i}^{H}\left(n_{i t}\right) \mathbf{G}_{i i}^{S} \mathbf{w}_{i}\left(n_{i t}\right)}
$$

\subsubsection{Convergencia del algoritmo JPCOB en DL}

Para demostrar que el algoritmo converge a una solución feasible en DL, se utiliza el hecho de que la asignación de potencias y los beamformers en el problema UL convergen a unos valores constantes y óptimos. Además, la matriz de ganancia UL, $\left[\mathbf{D}\left(n_{i t}\right) \mathbf{F}\left(n_{i t}\right)\right]$, converge a una matriz constante con valores propios menores que la unidad. Sabiendo que los valores propios de la matriz de ganancia en UL y de la matriz de ganancia en DL, 
$\left[\mathbf{D}\left(n_{i t}\right) \mathbf{F}^{T}\left(n_{i t}\right)\right]$, son los mismos, se concluye que la iteración DL converge si converge la iteración UL virtual.

El primer paso es definir un sistema asintóticamente constante: un sistema lineal

$$
\mathbf{x}(n+1)=\mathbf{C}(n) \mathbf{x}(n)+\mathbf{d}(n),
$$

es asintóticamente constante si

$$
\mathbf{C}(n)=\mathbf{C}+\mathbf{E}(n),
$$

y

$$
\lim _{n \rightarrow \infty}\|\mathbf{E}(n)\|=0 .
$$

Además, un sistema asintóticamente constante es estable si todos los valores propios de la matriz $\mathbf{C}$ son menores que la unidad.

El algoritmo JPCOB en DL propuesto en (3.54)-(3.56) es un sistema asintóticamente estable. Los dos primeros pasos del algoritmo son similares a los dos pasos del algoritmo planteado para el problema UL real. En la referencia [43] se demuestra que los dos primeros pasos del algoritmo sobre el problema UL real convergen a un vector de potencia fijo p. Por tanto, los beamformers también convergen a un valor fijo dado por:

$$
\hat{\mathbf{w}}_{i}=\arg \operatorname{máx}_{\mathbf{w}_{i}}\left\{\frac{p_{i} \mathbf{w}_{i}^{H} \mathbf{G}_{i i}^{S} \mathbf{w}_{i}}{\sum_{m=1}^{M} p_{m} \mathbf{w}_{i}^{H} \mathbf{G}_{m i}^{I} \mathbf{w}_{i}+\mathbf{w}_{i}^{H} \mathbf{w}_{i}}\right\} .
$$

Como consecuencia, la matriz de ganancia del UL virtual, $\left[\mathbf{D}\left(n_{i t}\right) \mathbf{F}\left(n_{i t}\right)\right]$, y la matriz de ganancia DL, $\left[\mathbf{D}\left(n_{i t}\right) \mathbf{F}^{T}\left(n_{i t}\right)\right]$, convergen a matrices constantes. Desde el punto de vista del problema UL virtual, la matriz de ganancia $\left[\mathbf{D}\left(n_{i t}\right) \mathbf{F}\left(n_{i t}\right)\right]$ converge a una matriz cuyos valores propios son menores que la unidad. De hecho, los valores propios del problema UL virtual y del problema DL son iguales:

$$
\begin{aligned}
|\mathbf{D F}-\lambda \mathbf{I}| & =|\mathbf{D}|\left|\mathbf{F}-\lambda \mathbf{D}^{-1}\right|=|\mathbf{D}|\left|\left(\mathbf{F}-\lambda \mathbf{D}^{-1}\right)^{T}\right| \\
& =\left|\mathbf{D} \| \mathbf{F}^{T}-\lambda \mathbf{D}^{-1}\right|=\left|\mathbf{D F} \mathbf{F}^{T}-\lambda \mathbf{I}\right|=0,
\end{aligned}
$$

con lo que la matriz de ganancia DL es una matriz asintóticamente constante con valores propios menores que la unidad. Por tanto, la iteración DL converge, y el algoritmo converge hacia una solución feasible.

Al mismo tiempo, se puede concluir que el radio espectral de las matrices $[\mathbf{D F}]$ y $\left[\mathbf{D F}^{T}\right]$ es el mismo, con lo que $\rho(\mathbf{D F})<1$ y $\rho\left(\mathbf{D F}^{T}\right)<1$ y la 
feasibility del problema DL y del UL virtual es la misma. Por feasibility, en la aproximación de Rashid-Farrokhi et al. se denomina a la situación en la que el problema de minimización de potencia tiene solución dado un conjunto de valores de SINR umbrales $\left(\gamma_{1}, \ldots, \gamma_{M}\right)$, y el vector óptimo de potencias es estrictamente positivo.

\subsubsection{Caracterización del óptimo}

A continuación, se puede demostrar que el algoritmo propuesto en (3.54)(3.56) también minimiza la potencia total transmitida en el enlace DL del sistema:

$$
[\hat{\mathbf{A}}, \hat{\mathbf{p}}]=\arg \min _{\mathbf{A}, \tilde{\mathbf{p}}}\left\{\sum_{i=1}^{M} \tilde{p}_{i}\left\|\mathbf{w}_{i}\right\|^{2}\right\},
$$

donde $\hat{\mathbf{A}}$ es el conjunto de beamformers óptimos $\hat{\mathbf{A}}=\left[\hat{\mathbf{w}}_{1} \ldots \hat{\mathbf{w}}_{M}\right]$.

La potencia total transmitida en el enlace DL puede expresarse como $\mathbf{1}_{w}^{T} \tilde{\mathbf{p}}$, donde:

$$
\mathbf{1}_{w}=\left[\left\|\mathbf{w}_{1}\right\|^{2} \ldots\left\|\mathbf{w}_{M}\right\|^{2}\right]^{T} .
$$

A partir del Teorema de Perron-Frobenius (apéndice A, apartado A.1), si existe un conjunto de beamformers $\hat{\mathbf{A}}$, tales que $\rho(\mathbf{D F})<1$, la iteración UL virtual converge a:

$$
\mathbf{p}=(\mathbf{I}-\mathbf{D F})^{-1} \mathbf{u}=(\mathbf{I}-\mathbf{D F})^{-1} \mathbf{D} \mathbf{1}_{w}
$$

donde $\mathbf{u}=\mathbf{D} \mathbf{1}_{w}$.

Definiendo $\boldsymbol{\sigma}^{2}=\left[\sigma_{1}^{2} \ldots \sigma_{M}^{2}\right]^{T}$, donde $\sigma_{i}^{2}$ es la potencia de ruido térmico en el usuario $i$, se obtiene la relación $\tilde{\mathbf{u}}=\mathbf{D} \boldsymbol{\sigma}^{2}$ (3.53). Si se construye una suma ponderada de las potencias transmitidas en el enlace UL virtual, $\mathbf{p}^{T} \boldsymbol{\sigma}^{2}$, y se desarrolla:

$$
\begin{aligned}
\mathbf{p}^{T} \boldsymbol{\sigma}^{2} & =\mathbf{1}_{w}^{T} \mathbf{D}\left(\mathbf{I}-\mathbf{D F}^{T}\right)^{-1} \boldsymbol{\sigma}^{2} \\
& =\mathbf{1}_{w}^{T}\left(\mathbf{I}-\mathbf{D} \mathbf{F}^{T}\right)^{-1} \mathbf{D} \boldsymbol{\sigma}^{2}=\mathbf{1}_{w}^{T} \tilde{\mathbf{p}},
\end{aligned}
$$

se puede afirmar que esta suma ponderada es igual a la potencia total transmitida en DL. Puesto que en el vector óptimo de potencias UL cada elemento es mínimo con respecto a todos los vectores posibles [43], una suma ponderada del mismo también será mínima. Se concluye pues, que en el óptimo, la suma de la potencia transmitida por todas las BSs del sistema es mínima. 


\subsection{Comparativa entre las distintas aproximaciones}

En este Capítulo se han repasado las tres aproximaciones clásicas propuestas para resolver el problema de minimización de potencia en sistemas MIMO multi-usuario multi-celda no coordinados $[33,37,38]$. Como se ha comentado al principio del Capítulo, estas tres aproximaciones representan las formas básicas de enfrentarse al diseño de un algoritmo JPCOB enfocado a minimizar la potencia total transmitida en un sistema convencional sin coordinación.

A primera vista, se puede afirmar que estas tres aproximaciones básicas se reducen a dos ideas fundamentales. La primera es la de aprovechar la dualidad entre el problema UL y DL para resolver el problema DL a partir de una formulación mucho más sencilla, como hacen Schubert y Boche o Rashid-Farrokhi et al.. La segunda, implica resolver directamente el problema DL reescribiendo el problema en forma convexa para poder utilizar técnicas estándar de optimización convexa, como proponen Bengtsson y Ottersten. Sin embargo, si se estudia con más detenimiento la parte donde Bengtsson y Ottersten demuestran que la relajación SDP no es estrictamente una relajación, sino una formulación equivalente del problema original, se observa que la demostración teórica utiliza en un punto, además de la formulación dual de Lagrange de la relajación SDP, la equivalencia de la formulación original (3.34) con una formulación que, al menos algebraicamente, es equivalente a plantear el problema en un enlace UL virtual ( [37], páginas 25-30).

\section{Dualidad UL-DL}

A la hora de resolver el problema de minimización de potencia en sistemas convencionales, tanto Schubert y Boche, como Rashid-Farrokhi et al., proponen algoritmos iterativos de optimización alternada entre beamformers y potencias, que utilizan la técnica de la dualidad UL-DL para simplificar el diseño de los beamformers en transmisión.

Schubert y Boche definen el problema de minimización de potencia como un caso concreto del problema max-min o de balanceado de SINR en DL. Realmente, la actualización de potencias UL que proponen en el segundo paso de su algoritmo, cuando el escenario ya es feasible (3.26), es equivalente a la que plantean Rashid-Farrokhi et al. para actualizar las 
potencias UL virtuales (3.55). Sin embargo, Schubert y Boche actualizan estas potencias en un único paso, mientras que Rashid-Farrokhi et al. utilizan una expresión distribuida que necesita actualizarse de forma iterativa, y por tanto más lentamente. Hay que destacar que la iteración de potencias en el UL virtual es necesaria, puesto que el algoritmo de Rashid-Farrokhi et al. no comprueba que el problema sea feasible antes de empezar a ejecutarse y podría no converger si las potencias se actualizaran en un único paso.

Cuando se compara la aproximación de Schubert y Boche con la de Rashid-Farrokhi et al., se observa que en la expresión de la SINR recibida en la BS en el enlace UL, Schubert y Boche introducen un escalado por la potencia de ruido que afecta a las matrices de correlación (3.19), y es a esta expresión escalada a lo que denominan UL virtual. A partir de la expresión (3.54) del cálculo de los beamformers en el enlace UL virtual, se aprecia que Rashid-Farrokhi et al. no consideran este escalado. El hecho de considerar o no este escalado para constituir el enlace UL virtual, repercute en el valor de las potencias UL que se obtienen con el algoritmo y en la caracterización del óptimo de la solución.

El Teorema de Perron-Frobenius (apéndice A, apartado A.1) es la base con la que ambas aproximaciones caracterizan el óptimo del problema. Más aún, lo que Schubert y Boche denominan dualidad débil y fuerte, también se cumple para la solución de Rashid-Farrokhi et al.. Sin embargo, el hecho de que Rashid-Farrokhi et al. no apliquen el escalado por la potencia de ruido sobre la expresión de la SINR recibida en el enlace UL virtual, provoca que, en lo que sería su condición de dualidad fuerte (3.57), se necesite una suma ponderada de las potencias UL virtuales por las potencias de ruido (comparar con (3.29)). Este hecho también provoca que aparentemente, los valores obtenidos para las potencias UL virtuales en (3.55), no tengan significado físico.

Por otro lado, hay que destacar que muchos autores, cuando referencian el algoritmo propuesto por Rashid-Farrokhi et al., siempre destacan que este algoritmo se propuso de forma independiente en el trabajo de Visotsky y Madhow [40]. Esta afirmación no es correcta, puesto que Visotsky y Madhow se basan en el algoritmo de Rashid-Farrokhi et al.. Además, Visotsky y Madhow aseguran que el algoritmo JPCOB, tal y como lo plantearon Rashid-Farrokhi et al., no consigue una solución óptima a menos que se aplique el escalado por la potencia de ruido para constituir el UL virtual. Posteriormente, los mismos Bengtsson y Ottersten, recurriendo a 
herramientas de optimización convexa, desmienten que este escalado sea necesario, y afirman que el algoritmo propuesto por Rashid-Farrokhi et al. alcanza la solución óptima del problema de minimización de potencia [81].

Como puede verse, existe mucha confusión con respecto a si es necesario o no escalar la expresión de la SINR en UL por la potencia de ruido para que el algoritmo JPCOB obtenga una solución óptima. De hecho, según cuál sea la referencia elegida, si el trabajo de Rashid-Farrokhi et al. o el de Visotsky y Madhow, los autores aplican o no este escalado.

Sin embargo, como apuntan Yu y Lan en [39], la dualidad UL-DL tal como se presenta en los trabajos de Schubert y Boche y Rashid-Farrokhi et al., realmente se engloba dentro de una dualidad más general obtenida a partir de plantear el problema dual de Lagrange del problema de minimización de potencia. En las referencias más recientes, como [30,42], ya se observa claramente cómo, al plantear el problema de minimización de potencia en el enlace UL virtual, no se aplica el escalado por la potencia de ruido y se utilizan unas potencias UL normalizadas, que se corresponden con una normalización por la potencia de ruido de las potencias UL virtuales del algoritmo de Rashid-Farrokhi et al.

En el apéndice B, se incluye la derivación del problema dual de Lagrange a partir de la formulación original del problema de minimización de potencia.

\section{Optimización convexa}

A primera vista la formulación básica del problema de minimización de potencia no es convexa (3.34). Sin embargo, aunque el problema no es aparentemente convexo, es posible relajarlo, como hacen Bengtsson y Ottersten, para obtener un problema SDP (Semidefinite Program), o bien transformarlo en un problema SOCP (Second-order Cone Program), como proponen Wiesel et al. [30]. De esta forma, es posible aplicar las herramientas estándar de optimización convexa a un problema que aparentemente no lo es.

Por otro lado, el algoritmo de Rashid-Farrokhi et al. converge más rápidamente que el propuesto por Bengtsson y Ottersten. Sin embargo, si el problema original está cerca de no ser feasible, como es el caso de situaciones de alta carga del sistema, la convergencia del algoritmo de RashidFarrokhi et al. se retarda, aumentando el número de iteraciones necesarias para converger. La formulación SDP, por el contrario, necesita el mismo número de iteraciones para converger independientemente del escenario y 
de las condiciones del sistema. Puesto que en cada iteración aumenta la complejidad, este método es más lento en situaciones normales, pero es significativamente más rápido para factores de carga del sistema elevados. 



\section{Capítulo 4}

\section{Algoritmo JPCOB-VUL para un sistema MIMO multi-usuario multi-celda coordinado}

El objetivo general de la Tesis es estudiar mecanismos que mejoren el nivel de interferencia que perciben los usuarios de un sistema Multinodo B. El primer paso, que coincide con el primer objetivo de este Capítulo, es plantear un modelo de sistema válido y realista para la arquitectura Multinodo B. Como se verá, el modelo de sistema elegido coincide con el de un sistema MIMO multi-usuario multi-celda coordinado (CM3).

A partir de este modelo de sistema, se desarrolla analíticamente el problema de minimización de potencia y se propone un primer algoritmo JPCOB que parte de la solución propuesta por Rashid-Farrokhi et al. [33]. Este primer algoritmo JPCOB, utiliza la dualidad entre el problema DL y su equivalente UL virtual para simplificar el diseño de los beamformers en transmisión. Por este motivo, se le denomina algoritmo JPCOB-VUL o Virtual uplink-based Joint Power Control and Optimal Beamforming [82-86].

Tras presentar el algoritmo JPCOB-VUL, se analizan aspectos como la convergencia o la caracterización del óptimo del algoritmo. Además, se plantea una formulación alternativa para conseguir una implementación eficiente o de bajo coste del algoritmo. 


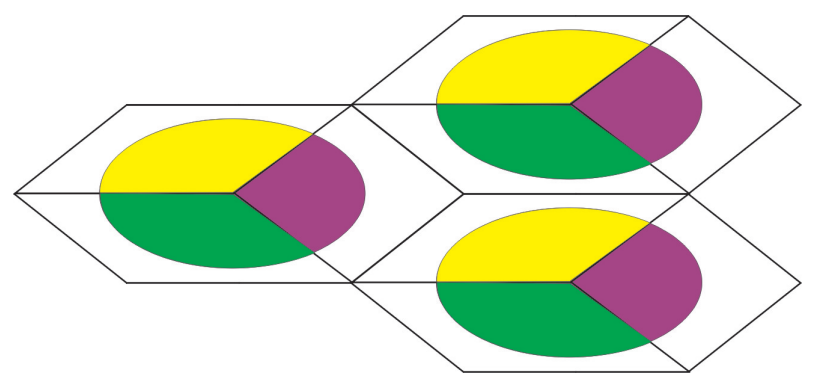

Figura 4.1: Arquitectura UMTS convencional.

Finalmente, se estudian los aspectos prácticos de la implementación del algoritmo JPCOB-VUL en sistemas CM3 y se realizan simulaciones para caracterizar los parámetros básicos que influyen en el comportamiento del algoritmo.

\subsection{Modelo del sistema MIMO multi-usuario multi-celda coordinado}

En un sistema UMTS convencional, las estaciones base (BSs) se ubican en el centro de cada celda. La figura 4.1 muestra un sistema UMTS convencional de tres celdas, donde el plan de frecuencias incluye una sectorización de cada celda en tres sectores.

Un posible modelo de sistema de la arquitectura Multinodo B, consistiría en distribuir espacialmente varias antenas por la celda, y unirlas mediante un enlace de alta capacidad, como fibra óptica, a la BS central. Aunque el concepto es diferente, de esta forma se obtendría un modelo de sistema muy similar al de los sistemas de antenas distribuidas presentados en el apartado de sistemas MIMO multi-usuario multi-celda coordinados (ver figura 2.13).

En esta Tesis, el modelo de sistema elegido para caracterizar la arquitectura Multinodo B coincide con las especificaciones de los sistemas MIMO multi-usuario multi-celda coordinados. Por tanto, a partir de este punto, la Tesis se centra en el estudio del enlace DL de un sistema W-CDMA CM3.

El modelo de sistema es el siguiente: se considera un sistema W-CDMA CM3 de $K$ celdas, con BSs ubicadas en el centro de cada celda. Cada BS dispone de un array de $N_{t}$ antenas, de forma que en el sistema hay un total de $K \cdot N_{t}$ antenas. Las $K$ BSs están unidas mediante un enlace 


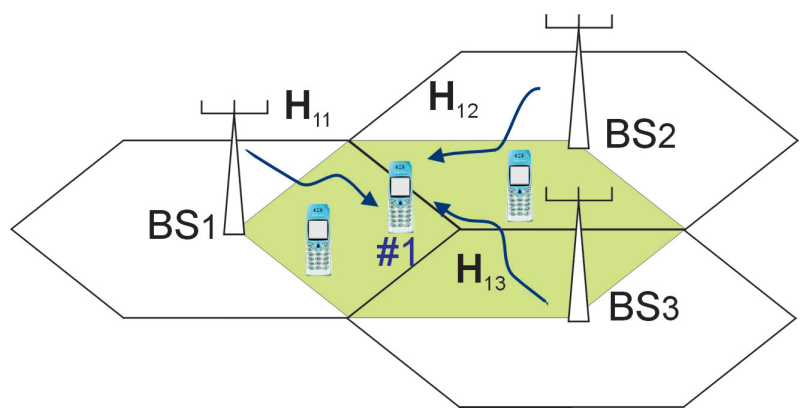

Figura 4.2: Sistema MIMO multi-usuario multi-celda coordinado.

de alta capacidad, que facilita una cooperación perfecta en datos entre ellas, es decir, todas las BSs disponen de todas las señales a transmitir a todos los usuarios, y pueden transmitir coordinadamente a cada usuario, por ejemplo, mediante un esquema de beamforming multi-base o mediante un algoritmo de control de potencia centralizado. Sin embargo, las BSs no pueden cooperar en potencia y cada BS está sujeta a una restricción individual en la potencia total transmitida, $P_{\max }$.

La figura 4.2 muestra un sistema CM3 de $K=3$ celdas. En realidad, los $M$ usuarios del sistema podrían ubicarse en cualquier punto del área de las celdas (ver figura 2.12). Sin embargo, estudios previos de los sistemas CM3, como [50], muestran que la ganancia por coordinación entre BSs es más significativa cuando el usuario se encuentra a una distancia similar con respecto a las $K$ BSs. Otro factor a considerar, es que en esta Tesis interesa evaluar soluciones JPCOB en entornos donde la potencia de interferencia sea muy importante.

Por estos motivos, a lo largo de la Tesis se evalúa únicamente el área compartida por las $K$ celdas del sistema CM3, que se corresponde con la zona sombreada de la figura 4.2, puesto que en esta zona los usuarios se ubican más o menos a la misma distancia de todas las BSs, y al mismo tiempo, la potencia de interferencia que percibe cada usuario debido a la transmisión coordinada es mucho mayor. De hecho, cada vez que se indique que existen $M$ usuarios activos en el sistema CM3, si no se especifica lo contrario, en realidad se está indicando que existen $M$ usuarios distribuidos sobre la zona sombreada de la figura 4.2.

En este primer análisis de un sistema CM3 se considera un modelo de sistema simplificado, para poder evaluar parámetros básicos como por ejemplo, la configuración óptima de un sistema CM3 en términos de número 
de BSs o de número de antenas por BS. Por este motivo, en este Capítulo se considera que los usuarios activos en el sistema CM3 son usuarios co-canal, lo que en un sistema CDMA significa que comparten el mismo código de canalización.

En este punto hay que tener en cuenta una diferencia fundamental entre los procesos de soft handover y los sistemas CM3. En el enlace DL de los procesos de soft handover, cada BS viene identificada por un código de scrambling, pero además, el usuario consume un código de canalización en cada BS [87].

Por el contrario, en los sistemas CM3, la zona compartida por las BSs se comporta a todos los efectos como una única celda, de forma que las $K$ BSs utilizan el mismo código de scrambling, y un usuario consume el mismo código de canalización en todas las BSs.

A lo largo de este Capítulo, se supone que las BSs del sistema CM3 disponen de conocimiento perfecto del estado del canal por el que van a transmitir (CSIT perfecta), aunque esta suposición sea en principio poco realista. En sistemas FDD, las transmisiones UL y DL se realizan sobre distintas frecuencias, de forma que el enlace UL y el DL se ven afectados por distintas atenuaciones producidas por el desvanecimiento rápido o fast fading, mientras que la atenuación por desvanecimiento lento o shadowing, y las pérdidas de propagación son similares en ambos enlaces. Una manera de obtener una CSIT perfecta sería suponiendo que existe algún tipo de canal de realimentación entre cada usuario y cada BS, que permitiría estimar el canal DL. Por otro lado, puesto que las BSs cooperan perfectamente en datos, cualquier BS puede disponer de cualquier canal de propagación del sistema.

Por último, el sufijo $m k$ representa, según sea el caso, el enlace UL o DL entre el usuario $m$ y la BS $k$.

\subsubsection{Modelo de canal}

En W-CDMA, es habitual que los canales sean frequency selective. Además, en un sistema CM3 hay que tener en cuenta que cada usuario ve $K$ canales de propagación, uno por cada BS del sistema, y que cada uno de estos canales podría presentar un Power Delay Profile $(\mathrm{PDP})^{1}$ diferente.

En un sistema CM3 con propagación multi-camino, existen $K \cdot M$ canales

\footnotetext{
${ }^{1}$ En el Power Delay Profile o perfil de retardo, se define la potencia y retardo relativo de cada contribución o tap respecto a la primera.
} 
de propagación $\mathbf{H}_{m k}$, uno por cada par BS-usuario del sistema, donde cada canal $\mathbf{H}_{m k}$ se modela mediante una matriz de dimensiones $\left[L \times N_{t}\right]$. Si $T_{c}$ es el periodo de chip, y $L_{m k}^{\prime} T_{c}$ representa el valor máximo del delay spread entre todos los canales de propagación del sistema, $L$ se define de forma que $L=L_{m k}^{\prime}$. Una suposición bastante habitual es considerar que la duración de un símbolo transmitido es mucho mayor que el máximo delay spread, $L T_{c}$, de forma que la interferencia entre símbolos o ISI (Inter-symbol Interference) se supone nula.

En el caso particular en el que los canales entre cada usuario y cada BS son canales flat fading, formados por una única contribución multi-camino, cada usuario recibe su señal deseada a través de $K$ contribuciones multicamino independientes, asociada cada una de ellas a una de las BSs del sistema.

La respuesta al impulso discreta del canal para la contribución multicamino $l$-ésima entre la BS $k$ y el usuario $m$ viene dada por:

$$
\mathbf{h}_{m k,(l)}=\left[\mathbf{H}_{m k}\right]_{(l,:)}=\sqrt{\beta_{s} \beta_{m k} \alpha_{m k}(l)} \mathbf{a}_{m k}(\theta(l)) g_{m k},
$$

donde $\beta_{s}$ representa el fading debido al shadowing, $\beta_{m k}$ representa las pérdidas de propagación o path loss, $\alpha_{m k}(l)$ es el fading a pequeña escala de la contribución l-ésima, $\mathbf{a}_{m k}(\theta(l))$ es la respuesta del array de antenas de la BS a la señal del usuario incidente en la dirección $\theta(l)$ y $g_{m k}$ incluye los efectos del transmisor y receptor y de la forma del pulso de transmisión.

\subsubsection{Enlace DL del sistema}

En este apartado, se presenta el modelo de señal del enlace DL del sistema CM3 desde el punto de vista de cada uno de los usuarios del sistema. Este modelo de señal se corresponde con una aproximación simplificada del sistema CM3, donde los $M$ usuarios son usuarios co-canal y los canales de propagación entre cada BS y cada usuario son canales flat fading constituidos por una única contribución multi-camino. Además, se supone que en recepción el usuario es capaz de gestionar de alguna forma el asincronismo de las señales que recibe desde cada una de las BSs. En los Capítulos 5 y 6 , se tratará con más detalle el proceso que tiene lugar en el receptor de cada usuario para afrontar la naturaleza asíncrona de las señales procedentes de las distintas BSs.

En W-CDMA, las señales a transmitir a cada usuario se canalizan mediante códigos ortogonales, que comparten el mismo periodo de chip $T_{c}$. 
Puesto que los $M$ usuarios del sistema CM3 son usuarios co-canal, todos utilizan el mismo código de canalización $c_{m}(t)$ :

$$
c_{m}(t)=\sum_{n=0}^{N-1} c_{m}[n] p\left(t-n T_{c}\right)
$$

donde $c_{m}[n]$ es el código de canalización, $p(t)$ es la forma del pulso banda base y $N$ es el factor de spreading. En principio, las BSs del sistema CM3 comparten el mismo código de scrambling, por lo que éste se ignora en el modelo de señal.

Suponiendo que el sistema utiliza una modulación QPSK, la señal que se transmite a cada usuario se expresaría como:

$$
u_{m}(t)=\sum_{d=-\infty}^{\infty} a_{m}[d] c_{m}(t-d T)
$$

donde $a_{m}[d] \in\left[ \pm \frac{\sqrt{2}}{2} \pm j \frac{\sqrt{2}}{2}\right]$ es la señal de información a transmitir al usuario $m, T=N T_{c}$ es el periodo de símbolo y $E\left[\left|u_{m}(t)\right|^{2}\right]=1$. Las señales $a_{m}[d]$ son a su vez señales de potencia unidad, incorreladas entre sí.

En cada BS, se diseñan beamformers específicos para transmitir la señal CDMA $u_{m}(t)$ al usuario $m$ con una potencia $p_{m k}$. Por tanto, la señal $\left[N_{t} \times 1\right]$ transmitida por cada BS se expresa como:

$$
\mathbf{x}_{k}(t)=\sum_{m=1}^{M} \mathbf{w}_{m k} \sqrt{p_{m k}} u_{m}(t), k=1, \ldots, K
$$

En la expresión anterior, hay que destacar que no es necesario que se realice una asignación previa del usuario $m$ a la BS $k$, puesto que todas las BSs transmiten la misma señal $u_{m}(t)$ al usuario $m$. Por otro lado, la potencia total transmitida al usuario $m$ desde una BS $k$ se compone de dos contribuciones, la asociada al beamformer en transmisión, $\left\|\mathbf{w}_{m k}\right\|^{2}$, y la asignada por el mecanismo de control de potencia, $p_{m k}$, tal y como ocurre en la referencia de Rashid-Farrokhi et al. (apartado 3.3.2).

En el sistema CM3, cada BS transmite a los $M$ usuarios del sistema. Por tanto, aunque se considere un escenario flat fading, la señal que recibe un usuario $m$ es realmente una señal multi-camino, donde cada contribución se modela con un vector $\mathbf{h}_{m k}$, de dimensiones $\left[1 \times N_{t}\right]$, y un retardo de propagación asociado $\tau_{m k}$ distinto. Si la variable $L$ representa el máximo 
delay spread que experimenta $\mathbf{x}_{k}$, para todo $k$, y si el periodo de símbolo es mucho mayor que el máximo delay spread, $L T_{c}<T$, no existe interferencia entre símbolos, pero sí interferencia entre chips. Tras el paso por el canal, la señal recibida en el usuario $m$ se expresaría como:

$$
y_{m}(t)=\sum_{k=1}^{K} \mathbf{h}_{m k} \mathbf{x}_{k}\left(t-\tau_{m k}\right)+n_{m}^{0}(t),
$$

donde $n_{m}^{0}(t)$ representa el ruido térmico en recepción.

En este punto, se supone que el receptor del usuario puede gestionar de alguna forma el hecho de que las señales procedentes de las distintas BSs del sistema puedan llegar de forma asíncrona, ya que, en general, $\tau_{m 1} \neq$ $\ldots \neq \tau_{m K}$. Por tanto, la señal disponible a la salida del receptor del usuario $m$ es:

$$
\begin{aligned}
y_{m}(t) & =\sum_{k=1}^{K} \mathbf{h}_{m k} \mathbf{w}_{m k} \sqrt{p_{m k}} a_{m}(t) \\
& +\sum_{k=1}^{K} \sum_{\substack{i=1 \\
i \neq m}}^{M} \mathbf{h}_{m k} \mathbf{w}_{i k} \sqrt{p_{i k}} a_{i}(t) \\
& +n_{m}(t)
\end{aligned}
$$

que se corresponde con la expresión que se obtendría si los $K$ canales de propagación llegaran de forma síncrona al usuario, $\tau_{m 1}=\ldots=\tau_{m K}$. El primer sumando representa la señal de interés, que incluye la transmisión de todas las BSs del sistema, el segundo sumando se corresponde con el término de interferencia co-canal, y por el último, el tercer sumando representa el ruido térmico y una contribución de interferencia de acceso múltiple o MAI (Multiple Access Interference).

El término de MAI se debe a que el modelo de señal considera $M$ usuarios co-canal distribuidos en el sistema CM3, pero además, añade la interferencia MAI que introduce un número indeterminado de usuarios no co-canal también presentes en el sistema. Esta interferencia MAI se interpreta como un pedestal de ruido en recepción.

\subsubsection{Modelo matricial del sistema}

En este apartado, se presenta el modelo de señal desarrollado en el apartado anterior desde un punto de vista matricial y discreto. La señal 


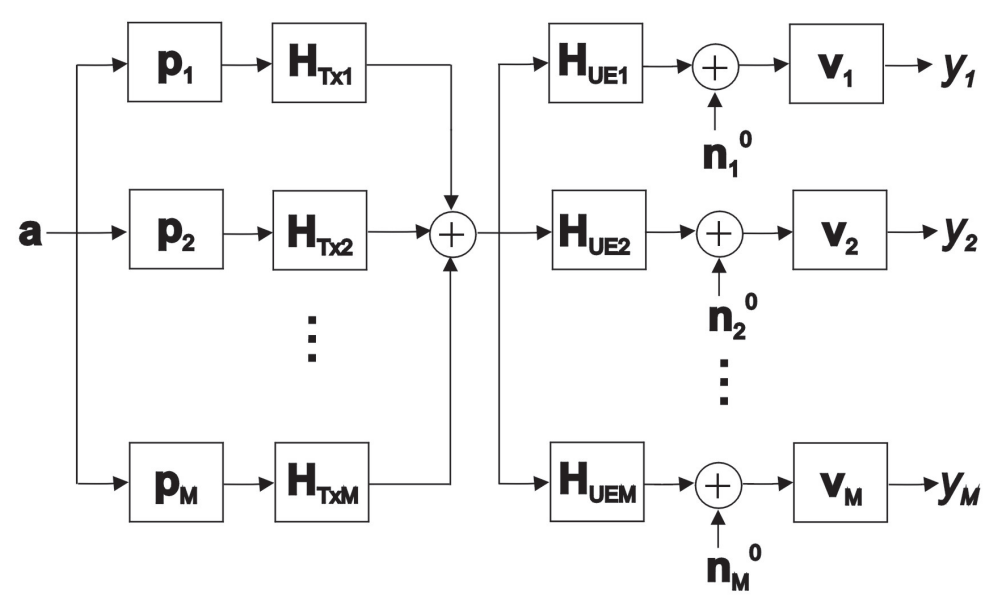

Figura 4.3: Diagrama de bloques de un sistema MIMO multi-usuario multi-celda coordinado.

(4.5) puede reescribirse en forma multi-usuario como:

$$
\underbrace{\left[\begin{array}{c}
y_{1} \\
y_{2} \\
\vdots \\
y_{M}
\end{array}\right]}_{\mathbf{y}}=\mathbf{V}^{H} \mathbf{H}_{\mathrm{Ch}} \mathbf{H}_{\mathrm{Tx}} \mathbf{P} \underbrace{\left[\begin{array}{c}
a_{1} \\
a_{2} \\
\vdots \\
a_{M}
\end{array}\right]}_{\mathbf{a}}+\mathbf{n},
$$

donde $\mathbf{P}$ es una matriz diagonal a bloques, de dimensiones $[M K \times M]$, que incluye todas las potencias transmitidas en el enlace DL del sistema, $\mathbf{H}_{\mathrm{Tx}}$ es una matriz $\left[K N_{t} \times M K\right]$ que agrupa a todos los beamformers en transmisión del sistema, $\mathbf{H}_{\mathrm{Ch}}$ es una matriz $\left[M L \times K N_{t}\right]$ que contiene todos los canales de propagación del sistema, $\mathbf{V}$ es una matriz $[M L \times M]$ diagonal a bloques, formada por unos y ceros, y $\mathbf{n}$ es la concatenación del término de ruido térmico y de MAI de los $M$ usuarios del sistema. La figura 4.3 muestra el diagrama de bloques del modelo de señal matricial y discreto del sistema CM3. 
Las matrices $\mathbf{P}, \mathbf{H}_{\mathrm{Tx}}, \mathbf{H}_{\mathrm{Ch}}$ y $\mathbf{V}$ presentan una estructura interna que permite relacionar cada subbloque con la transmisión a un usuario:

$$
\begin{aligned}
\mathbf{P} & =\operatorname{diag}\left\{\left[\mathbf{p}_{1} \ldots \mathbf{p}_{M}\right]\right\}, \\
\mathbf{H}_{\mathrm{Tx}} & =\left[\mathbf{H}_{\mathrm{Tx} 1} \ldots \mathbf{H}_{\mathrm{Tx} M}\right], \\
\mathbf{H}_{\mathrm{Ch}} & =\left[\mathbf{H}_{\mathrm{UE} 1}^{T} \ldots \mathbf{H}_{\mathrm{UEM}}^{T}\right]^{T}, \\
\mathbf{V}^{H} & =\operatorname{diag}\left\{\left[\mathbf{v}_{1}^{H} \ldots \mathbf{v}_{M}^{H}\right]\right\},
\end{aligned}
$$

donde $\mathbf{H}_{\mathrm{Tx} m}$ es una matriz diagonal a bloques $\left[K N_{t} \times K\right], \mathbf{H}_{\mathrm{UEm}}$ es la matriz $\left[L \times K N_{t}\right]$ que contiene todos los canales de propagación que recibe el usuario $m$ y $\mathbf{p}_{m}$ es un vector columna $[K \times 1]$ que contiene todas las potencias transmitidas en DL al usuario $m$. Por último, $\mathbf{v}_{m}^{H}$ es el vector que caracterizaría el funcionamiento del receptor, y que en este modelo simplificado, es un vector todo unos de dimensiones $[1 \times L]$.

La transmisión coordinada en DL a un usuario $m$ se representa mediante la matriz $\mathbf{H}_{\mathrm{Tx} m}$ y el vector $\mathbf{p}_{m}$. Las matrices $\mathbf{H}_{\mathrm{Tx} m}$ contienen los beamformers diseñados en las $K$ BSs para transmitir al usuario $m$ :

$$
\mathbf{H}_{\mathrm{Tx} m}=\operatorname{diag}\left\{[\underbrace{\mathbf{w}_{m 1}}_{\mathrm{BS}_{1}} \cdots \underbrace{\mathbf{w}_{m K}}_{\mathrm{BS}_{K}}]\right\},
$$

donde $\mathbf{w}_{m k}$ es el beamformer en transmisión, $\left[N_{t} \times 1\right]$, para el enlace $m k$ usuario-BS. Por su parte, el vector $\mathbf{p}_{m}$ contiene la raíz cuadrada de las potencias de transmisión DL para el usuario $m$ :

$$
\mathbf{p}_{m}=\left[\sqrt{p_{m 1}} \cdots \sqrt{p_{m K}}\right]^{T} .
$$

En recepción, la señal recibida por el usuario $m$ es la suma ponderada (matrices $\mathbf{H}_{\mathrm{Tx}}$ y $\mathbf{P}$ ) del vector de símbolos transmitidos sobre el canal $\mathbf{H}_{\mathrm{UEm}}$ (4.1):

$$
\mathbf{H}_{\mathrm{UEm}}=\left[\mathbf{H}_{m 1} \ldots \mathbf{H}_{m K}\right]=\left[\begin{array}{ccc}
\mathbf{h}_{m 1,(1)} & \cdots & \mathbf{h}_{m K,(1)} \\
\mathbf{h}_{m 1,(2)} & \cdots & \mathbf{h}_{m K,(2)} \\
\vdots & \cdots & \vdots \\
\mathbf{h}_{m 1,(L)} & \cdots & \mathbf{h}_{m K,(L)}
\end{array}\right] .
$$

Para un usuario $m$, cada una de las matrices $\mathbf{H}_{m k}, k=1, \ldots, K$, de dimensiones $\left[L \times N_{t}\right]$, se define a partir del PDP del canal $m k$ entre el usuario $m$ y la BS $k$ correspondiente. 
En el caso particular de canales flat fading, cada usuario del sistema únicamente recibe $K$ contribuciones multi-camino independientes, de forma que en la matriz $\mathbf{H}_{\mathrm{UEm}}$, la mayoría de los elementos serían nulos. En este caso, lo más adecuado es simplificar la expresión de la matriz $\mathbf{H}_{\mathrm{UEm}}$ e incluir únicamente los elementos $\mathbf{h}_{m k,(l)}, k=1, \ldots, K$, no nulos, que se corresponden con las contribuciones multi-camino asociadas con los retardos de propagación $\left(\tau_{m 1}, \ldots, \tau_{m K}\right)$. En el caso general en el que las $K$ contribuciones multi-camino llegan a cada usuario del sistema en instantes de tiempo separados, la matriz $\mathbf{H}_{\mathrm{UEm}}$ (4.13) se reescribe como:

$$
\mathbf{H}_{\mathrm{UEm}}=\operatorname{diag}\left\{\left[\mathbf{h}_{m 1} \ldots \mathbf{h}_{m K}\right]\right\},
$$

donde $\mathbf{h}_{m k}=\mathbf{h}_{m k,(1)}$, puesto que sólo existe una contribución multi-camino por cada canal. Además, las dimensiones de las matrices en (4.6) se modifican de forma que $L=K$.

Por último, la señal a la salida del usuario $m$ puede expresarse en forma matricial como (4.5):

$$
y_{m}=\mathbf{v}_{m}^{H} \mathbf{H}_{\mathrm{UE} m} \mathbf{H}_{\mathrm{Tx}} \mathbf{P a}+n_{m} .
$$

\subsection{Algoritmo JPCOB-VUL}

A la hora de diseñar un algoritmo JPCOB para minimizar la potencia total transmitida en un sistema CM3, el primer paso es plantearse si alguno de los algoritmos clásicos propuestos para sistemas convencionales MIMO multi-usuario admitiría una extensión coordinada sin aumentar con ello excesivamente la complejidad del algoritmo.

Hay que tener en cuenta que aunque las BSs coordinadas, cuando cooperan perfectamente en datos, se pueden interpretar como una única BS donde las $K \cdot N_{t}$ antenas se distribuyen espacialmente en grupos de $N_{t}$ antenas, las restricciones de potencia individuales $P_{\max }$ aplicadas a cada BS, impiden que las dos situaciones sean totalmente equivalentes.

De entre los algoritmos clásicos presentados en el Capítulo anterior, el algoritmo de Rashid-Farrokhi et al., se presenta, a priori, como la aproximación más sencilla de modificar para obtener una solución algorítmica válida para sistemas CM3. Este algoritmo se formula originalmente para sistemas TDD y trabaja con usuarios co-canal, puesto que ésta es una de las interferencias más importantes en los sistemas de comunicaciones móviles de segunda generación. 
En este Capítulo, el contexto es diferente. El sistema es un sistema W-CDMA CM3, y la interferencia que van a experimentar los usuarios se debe a dos factores, a la transmisión coordinada por parte de las BSs, y al hecho de que los $M$ usuarios considerados por el algoritmo comparten el mismo código de canalización. Existe otro término de interferencia, de menor importancia, que se debe a la presencia de más usuarios en el sistema, asociados con distintos códigos de canalización.

En esta segunda Parte, se presenta la primera solución JPCOB propuesta en la Tesis para minimizar la potencia total transmitida en un sistema W-CDMA CM3. Este algoritmo, denominado JPCOB-VUL o Virtual uplink-based Joint Power Control and Optimal Beamforming [82-86], es una extensión de la propuesta de Rashid-Farrokhi et al. [33].

El algoritmo JPCOB-VUL permite que las BSs transmitan de forma coordinada a cada usuario, de forma que no es necesario que se realice una asignación previa de usuarios a BSs y se evitan posibles handovers internos en la zona compartida por las BSs del sistema CM3. Este algoritmo, al igual que el de referencia de Rashid-Farrokhi et al., es un algoritmo iterativo que utiliza la dualidad entre el problema DL y su equivalente UL virtual para simplificar el diseño de los beamformers en transmisión. La iteración puede dividirse en dos pasos: el cálculo de los beamformers en transmisión mediante una iteración en el problema UL virtual y un segundo paso de actualización de las potencias de transmisión en DL.

Siguiendo la referencia, el algoritmo JPCOB-VUL obtiene un beamfor$m e r \mathbf{w}_{m k}$ y una potencia de transmisión $p_{m k}$ para cada enlace BS-usuario del sistema. Al mismo tiempo, la potencia transmitida en DL para cada usuario consta de dos contribuciones, una obtenida a partir del control de potencia y otra introducida por el módulo de cada beamformer. De esta forma, el problema de minimización de potencia en el sistema W-CDMA CM3 se formula de la forma habitual: el objetivo es minimizar la potencia total transmitida en DL (es decir, la suma de la potencia transmitida por todas las BSs), pero asegurando que se alcancen en los $M$ usuarios del sistema unas QoS determinadas, que se representan en este caso mediante unos valores umbrales de SINR en cada usuario:

$$
\begin{aligned}
\operatorname{mín} & \sum_{k=1}^{K} \sum_{m=1}^{M} p_{m k}\left\|\mathbf{w}_{m k}\right\|^{2} \\
\text { s.t. } & \operatorname{SINR}_{m}^{D L} \geq \gamma_{m}, m=1, \ldots, M .
\end{aligned}
$$




\subsubsection{Beamforming en transmisión}

A partir de la expresión de la señal recibida por el usuario $m$ del sistema (4.5), la SINR se calcula como:

$$
\operatorname{SINR}_{m}^{D L}=\frac{\sum_{k=1}^{K} p_{m k} \mathbf{w}_{m k}^{H} \mathbf{H}_{m k} \mathbf{w}_{m k}}{\sum_{k=1}^{K} \sum_{i=1, i \neq m}^{M} p_{i k} \mathbf{w}_{i k}^{H} \mathbf{H}_{m k} \mathbf{w}_{i k}+\sigma_{m}^{2}}
$$

donde $\mathbf{H}_{m k}=E\left[\mathbf{h}_{m k}^{H} \mathbf{h}_{m k}\right]$ es el estadístico de segundo orden del canal DL $m k$, mientras que $\sigma_{m}^{2}$ representa la potencia de ruido térmico y MAI en el usuario. En la expresión anterior, se asume que los canales $\mathbf{h}_{m k}$ y $\mathbf{h}_{m k^{\prime}}$ entre dos BSs distintas, $k \neq k^{\prime}$, y el usuario $m$, están incorrelados gracias a la separación espacial entre las distintas BSs. Del mismo modo, las señales $a_{i}(t)$ y $a_{m}(t)$ también están incorreladas entre sí.

La solución del problema de beamforming óptimo, definido como aquel que maximiza la SINR (4.17) en el usuario, implica una búsqueda exhaustiva sobre todos los beamformers $\mathbf{w}_{m k}$ que no es inmediata. Sin embargo, puesto que el algoritmo JPCOB-VUL toma como punto de partida el algoritmo propuesto por Rashid-Farrokhi et al., se sabe que es posible simplificar este diseño aplicando la dualidad entre el problema DL y su equivalente UL virtual. Por tanto, el beamformer diseñado por la BS $k$ para transmitir al usuario $m, \mathbf{w}_{m k}$, se obtiene maximizando la SINR virtual recibida en la BS $k$ desde el usuario $m$, no la expresión DL (4.17):

$$
\mathrm{SINR}_{m k}^{V U}=\frac{p_{m k}^{u} \mathbf{w}_{m k}^{H} \mathbf{H}_{m k} \mathbf{w}_{m k}}{\mathbf{w}_{m k}^{H}\left(\sum_{i=1, i \neq m}^{M} p_{i k}^{u} \mathbf{H}_{i k}+\mathbf{I}\right) \mathbf{w}_{m k}}
$$

donde $p_{i k}^{u}$ son las potencias de transmisión UL virtuales. Al igual que en el algoritmo de Rashid-Farrokhi et al., las matrices de correlación de canal que se consideran en la expresión anterior se corresponden con las matrices del enlace DL, mientras que el término de potencia de ruido en recepción se mantiene igual a la unidad (3.54).

Aunque el algoritmo JPCOB-VUL es una extensión coordinada de la referencia de Rashid-Farrokhi et al., los beamformers en transmisión se calculan localmente en cada BS. Por tanto, el diseño de los beamformers en el algoritmo JPCOB-VUL es una solución distribuida y desde el punto de vista de coordinación, subóptima. 


\subsubsection{Control de potencia}

El control de potencia, se encarga de actualizar las potencias UL virtuales, $p_{m k}^{u}$, y las potencias DL, $p_{m k}$, para cada enlace $m k$ usuario-BS del sistema.

A partir de las soluciones JPCOB clásicas presentadas en el Capítulo 3, se sabe que la potencia mínima transmitida en el enlace DL del sistema se consigue cuando todos los usuarios alcanzan con igualdad los valores de SINR establecidos como QoS, es decir, cuando se cumple la siguiente relación:

$$
\operatorname{SINR}_{m}^{D L}=\gamma_{m}, m=1, \ldots, M,
$$

donde la SINR en DL se corresponde con la formulada en (4.17).

La expresión de la SINR en DL (4.17) considera todas las potencias de transmisión $p_{m k}$ para todos los enlaces BS-usuario del sistema. Además, se observa que para calcular la SINR en DL para un determinado usuario $m$, se necesitan conocer los canales de propagación entre las $K$ BSs y este usuario. Esto indica que el control de potencia en el enlace DL requiere una implementación centralizada, al contrario que el diseño de los beamformers.

Utilizando un sencillo cambio de notación en los subíndices $m k$ asociados a las potencias de transmisión, a los beamformers y a las matrices de canal, es posible expresar de forma sencilla y unificada la actualización de las potencias en el enlace DL. Tomando como punto de partida la primera BS, $k=1$, sus $M$ potencias de transmisión $p_{i 1}$ se renombran según $p_{i 1} \rightarrow p_{j}, j=1, \ldots, M$. De la misma manera, las $M$ potencias de la segunda BS se renombran como $p_{i 2} \rightarrow p_{j}$, con $j=M+1, \ldots, 2 M$. Generalizando, dada una BS $k$ y un usuario $m$, los nuevos índices quedarían:

$$
\left.\begin{array}{rl}
p_{m k} & \rightarrow p_{j} \\
p_{m k}^{u} & \rightarrow p_{j}^{u} \\
\mathbf{H}_{m k} & \rightarrow \mathbf{H}_{j} \\
\mathbf{w}_{m k} & \rightarrow \mathbf{w}_{j}
\end{array}\right\} j=(k-1) M+m
$$

con $k=1, \ldots, K$, y $m=1, \ldots, M$.

Con la nueva notación, la igualdad (4.19), teniendo en cuenta la expresión de la SINR en el enlace DL (4.17), se expresa de forma simple para un usuario $m$ según:

$$
\sum_{j} p_{j} \mathbf{w}_{j}^{H} \mathbf{H}_{j} \mathbf{w}_{j}=\gamma_{m} \sum_{n} p_{n} \mathbf{w}_{n}^{H} \mathbf{H}_{j} \mathbf{w}_{n}+\gamma_{m} \sigma_{m}^{2},
$$


donde el índice $j$ indexa los términos de señal deseada, $j=(k-1) M+m$ con $k=1, \ldots, K$, mientras que el índice $n$ se utiliza para indexar los términos de interferencia, $n=(k-1) M+i$, para $k=1, \ldots, K, i=1, \ldots, M$, e $i \neq m$.

Si definimos el vector de potencias transmitidas en el enlace DL como un vector columna de dimensiones $[K M \times 1]$ :

$$
\mathbf{p}=[\underbrace{p_{1} \ldots p_{M}}_{\mathrm{BS}_{1}} \cdots \underbrace{p_{(K-1) M+1} \cdots p_{K M}}_{\mathrm{BS}_{K}}]^{T},
$$

la ecuación de la actualización de potencias (4.20) puede expresarse de forma multi-usuario y matricial según:

$$
\mathrm{Dp}=\mathbf{F} \mathbf{p}+\mathbf{u}
$$

En la expresión anterior, la matriz $\mathbf{D}$ es una matriz $[M \times K M]$, que contiene los términos de potencia media de señal deseada recibidos por cada usuario. Esta matriz presenta una estructura de $K$ matrices diagonales $\mathbf{D}_{k}$ de dimensiones $[M \times M]$, cuyos elementos no nulos son:

$$
\begin{aligned}
\mathbf{D} & =\left[\mathbf{D}_{1}\left|\mathbf{D}_{2}\right| \cdots \mid \mathbf{D}_{K}\right], \\
{\left[\mathbf{D}_{k}\right]_{(m, m)} } & =\mathbf{w}_{j}^{H} \mathbf{H}_{j} \mathbf{w}_{j} .
\end{aligned}
$$

El índice $j$ se relaciona con la BS $k$ y el usuario $m$ mediante la relación $j=(k-1) M+m$.

De la misma manera, la matriz $\mathbf{F},[M \times K M]$, contiene un valor escalado de la potencia media de interferencia co-canal. Esta matriz también presenta una estructura de $K$ matrices $\mathbf{F}_{k}$ de dimensiones $[M \times M]$ cuyos elementos cumplen:

$$
\begin{aligned}
\mathbf{F} & =\left[\mathbf{F}_{1}\left|\mathbf{F}_{2}\right| \cdots \mid \mathbf{F}_{K}\right], \\
{\left[\mathbf{F}_{k}\right]_{(m, i)} } & =\left\{\begin{array}{cc}
\gamma_{m} \mathbf{w}_{n}^{H} \mathbf{H}_{j} \mathbf{w}_{n}, & m \neq i \\
0, & m=i .
\end{array}\right.
\end{aligned}
$$

El índice $j$ se relaciona con la BS $k$ y el usuario $m$ mediante la relación $j=(k-1) M+m$, mientras que el índice $n$ se relaciona con la BS $k$ y el usuario $i$ a través de $n=(k-1) M+i$, siendo $i \neq m$.

Por último, el vector $\mathbf{u}$ incluye un valor escalado de la potencia de ruido y MAI percibida por los $M$ usuarios co-canal:

$$
\mathbf{u}=\left[\gamma_{1} \sigma_{1}^{2} \ldots \gamma_{M} \sigma_{M}^{2}\right]^{T}
$$


La expresión de actualización de las potencias, (4.22), es una expresión que se corresponde con una iteración de Jacobi (apéndice A, apartado A.2), de forma que las potencias óptimas de transmisión se obtienen iterando:

$$
\mathbf{p}\left(n_{i t}+1\right)=\mathbf{D}^{\dagger}\left(\mathbf{F} \mathbf{p}\left(n_{i t}\right)+\mathbf{u}\right),
$$

donde $n_{i t}$ es el número de la iteración correspondiente.

De nuevo, hay que tener en cuenta que el algoritmo JPCOB-VUL es una extensión de la propuesta de Rashid-Farrokhi et al.. El algoritmo JPCOBVUL no comprueba en un primer paso que el problema de minimización de potencia sea feasible para el conjunto de valores umbrales de SINR, $\left(\gamma_{1}, \ldots, \gamma_{M}\right)$, establecidos para cada usuario (por feasible se entiende la situación en la que el problema de minimización de potencia tiene solución y el vector de potencias que se obtiene para el enlace DL es estrictamente positivo). Por este motivo, necesita actualizar las potencias DL de forma iterativa y no en un único paso como ocurre en la aproximación de Schubert y Boche:

$$
\mathbf{p}=\left(\mathbf{I}-\mathbf{D}^{\dagger} \mathbf{F}\right)^{-1} \mathbf{D}^{\dagger} \mathbf{u} .
$$

Sin embargo, si el problema es feasible, las dos expresiones para obtener las potencias de transmisión en el enlace DL son equivalentes.

La actualización de las potencias UL virtuales está relacionada con el diseño de los beamformers en transmisión. Puesto que el algoritmo JPCOBVUL mantiene un diseño local de los beamformers, la actualización de estas potencias se realiza también de forma distribuida en cada BS, mediante un control de potencia clásico distribuido, como el propuesto en [88]. En el apartado 4.2.6 de caracterización del óptimo del algoritmo JPCOB-VUL, se explica por qué no es posible, en principio, seguir el ejemplo del algoritmo de referencia de Rashid-Farrokhi et al. y obtener una expresión similar a (4.22) que represente en forma matricial y multi-usuario la actualización de todas las potencias UL virtuales del sistema.

\subsubsection{Solución algorítmica}

El algoritmo JPCOB-VUL es un algoritmo iterativo. En cada iteración, se calculan los beamformers en transmisión en el enlace UL virtual y se actualizan las potencias DL y UL virtuales.

Los pasos del algoritmo JPCOB-VUL para cada iteración $n_{i t}$ serían:

1. Inicializar las potencias UL virtuales, $p_{j}^{u}(0)$, y las potencias DL, $p_{j}(0)$, para todos los enlaces del sistema, $j=1, \ldots, K M$, con valores positivos. 
2. Para $n_{i t}=0,1,2, \ldots$, hasta llegar a la convergencia:

2.1. Beamforming. Calcular los beamformers subóptimos en transmisión $\mathbf{w}_{j}$ para cada enlace $j=1, \ldots, K M$, maximizando para cada enlace la SINR recibida en el UL virtual:

$$
\mathbf{w}_{j}\left(n_{i t}\right)=\arg \operatorname{máx}_{\mathbf{w}_{j}}\left\{\frac{p_{j}^{u}\left(n_{i t}\right) \mathbf{w}_{j}^{H} \mathbf{H}_{j} \mathbf{w}_{j}}{\mathbf{w}_{j}^{H}\left(\sum_{n} p_{n}^{u}\left(n_{i t}\right) \mathbf{H}_{n}+\mathbf{I}\right) \mathbf{w}_{j}}\right\},
$$

donde $n$ toma $M-1$ valores relacionados con $j$ de la siguiente forma: si $j=(k-1) M+m$, entonces $n=(k-1) M+i$ con $i=1, \ldots, M$, e $i \neq m$. Esto es debido a que en el UL virtual, los usuarios interferentes del enlace $j$-ésimo son los restantes $M-1$ enlaces asociados a la misma BS $k$.

En la práctica, cada uno de los beamformers $\mathbf{w}_{j}$ se calcula mediante la expresión MMSE:

$$
\mathbf{w}_{j}\left(n_{i t}\right)=\sqrt{p_{j}^{u}\left(n_{i t}\right)} \boldsymbol{\Phi}_{j}^{-1}\left(n_{i t}\right) \mathbf{h}_{j},
$$

donde la matriz $\boldsymbol{\Phi}_{j}\left(n_{i t}\right)$ es la matriz de correlación de la señal recibida en el UL virtual del enlace $j$ :

$$
\mathbf{\Phi}_{j}\left(n_{i t}\right)=p_{j}^{u}\left(n_{i t}\right) \mathbf{H}_{j}+\sum_{n} p_{n}^{u}\left(n_{i t}\right) \mathbf{H}_{n}+\mathbf{I} .
$$

2.2. Control de potencia, enlace UL virtual. Las potencias UL virtuales, $p_{j}^{u}$, se actualizan para cada enlace, $j=1, \ldots, K M$, mediante un control de potencia clásico distribuido [88]:

$$
p_{j}^{u}\left(n_{i t}+1\right)=\frac{\gamma_{m}}{\operatorname{SINR}_{j}^{V U}\left(n_{i t}\right)} p_{j}^{u}\left(n_{i t}\right),
$$

donde $\operatorname{SINR}_{j}^{V U}\left(n_{i t}\right)$ es la SINR en el UL virtual dada en la ecuación (4.18), pero considerando las potencias UL virtuales y los beamformers de la iteración correspondiente:

$$
\operatorname{SINR}_{j}^{V U}\left(n_{i t}\right)=\frac{p_{j}^{u}\left(n_{i t}\right) \mathbf{w}_{j}^{H}\left(n_{i t}\right) \mathbf{H}_{j} \mathbf{w}_{j}\left(n_{i t}\right)}{\mathbf{w}_{j}^{H}\left(n_{i t}\right)\left(\sum_{n} p_{n}^{u}\left(n_{i t}\right) \mathbf{H}_{n}+\mathbf{I}\right) \mathbf{w}_{j}\left(n_{i t}\right)} .
$$

En la expresión anterior, los índices $j$ y $n$ cumplen $j=(k-1) M+m$, $n=(k-1) M+i$, siendo $i=1, \ldots M$, e $i \neq m$. 
Puesto que el algoritmo JPCOB-VUL mantiene la estructura distribuida de la iteración en el enlace UL virtual, la actualización de las potencias UL virtuales se realiza como en el algoritmo de RashidFarrokhi et al., pero de forma independiente en cada BS. De hecho, si se expresa (4.32) en forma matricial, y por separado en cada BS, se obtienen $K$ ecuaciones del tipo (3.55).

2.3. Control de potencia, enlace DL. El vector $\mathbf{p}$ de potencias DL se actualiza mediante:

$$
\mathbf{p}\left(n_{i t}+1\right)=\mathbf{D}^{\dagger}\left(n_{i t}\right)\left(\mathbf{F}\left(n_{i t}\right) \mathbf{p}\left(n_{i t}\right)+\mathbf{u}\right),
$$

donde las matrices $\mathbf{D}\left(n_{i t}\right)$ y $\mathbf{F}\left(n_{i t}\right)$ se obtienen considerando los beamformers en transmisión correspondientes a la iteración $n_{i t}, \mathrm{y}(\cdot)^{\dagger}$ representa la pseudo-inversa definida como $\mathbf{D}^{\dagger}=\left(\mathbf{D}^{T} \mathbf{D}\right)^{-1} \mathbf{D}^{T}$ (la matriz $\mathbf{D}$ es una matriz real).

La ecuación de actualización de potencias DL, (4.33), admite una formulación mediante la descomposición SVD de la matriz $\mathbf{D}\left(n_{i t}\right)$. Si esta descomposición se expresa como (suponiendo que esta matriz es de rango completo, es decir que todos sus valores singulares son no nulos) [89]:

$$
\mathbf{D}\left(n_{i t}\right)=\sum_{m=1}^{M} \lambda_{m} \mathbf{b}_{m} \mathbf{c}_{m}^{H},
$$

la ecuación de actualización de potencias DL podría expresarse como [82]:

$$
\mathbf{p}\left(n_{i t}+1\right)=\sum_{m=1}^{M} \frac{1}{\lambda_{m}} \mathbf{b}_{m}^{H}\left(\mathbf{F}\left(n_{i t}\right) \mathbf{p}\left(n_{i t}\right)+\mathbf{u}\right) \mathbf{c}_{m},
$$

donde $n_{i t}$ representa la iteración correspondiente del algoritmo.

En principio, la descomposición SVD tiene el mismo coste computacional que realizar la inversa de una matriz. Sin embargo, la matriz $\mathbf{D}$ es dispersa (sólo los elementos de la diagonal de cada submatriz $\mathbf{D}_{k}$ son no nulos), con lo que se minimiza el coste computacional de realizar la descomposición SVD de una matriz de dimensiones $[M \times K M]$. Además, en el siguiente apartado se demuestra cómo la estructura de la matriz $\mathbf{D}$, junto con su descomposición SVD, permite obtener una expresión computacionalmente eficiente para calcular la pseudo-inversa de $\mathbf{D}$. 


\subsubsection{Versión eficiente}

La estructura de la matriz $\mathbf{D}$, permite expresar la actualización de las potencias DL mediante la descomposición SVD (4.34). Un estudio más detallado de las propiedades de la matriz $\mathbf{D}$, formada por bloques de matrices diagonales, permite además reducir drásticamente el número de operaciones necesarias para realizar el cálculo de la pseudo-inversa en (4.33). De esta forma, es posible obtener una versión computacionalmente eficiente del algoritmo JPCOB-VUL [84].

La descomposición SVD de la matriz $\mathbf{D}$ se define ahora como:

$$
\mathbf{D}=\mathbf{B} \boldsymbol{\Lambda} \mathbf{C}^{T},
$$

donde $\mathbf{B}$ es una matriz unitaria $[M \times M]$ de vectores singulares izquierdos, $\boldsymbol{\Lambda}$ es una matriz $[M \times K M]$ que contiene los valores singulares y $\mathbf{C}$ es una matriz unitaria $[K M \times K M]$ que contiene los vectores singulares derechos.

Debido a la estructura de la matriz $\mathbf{D}$, el producto $\mathbf{D D}^{T}$ es una matriz $[M \times M]$ diagonal, por lo que la matriz de vectores singulares izquierdos $\mathbf{B}$ resulta ser la matriz identidad, como muestra la siguiente relación:

$$
\mathbf{D} \mathbf{D}^{T}=\mathbf{B} \boldsymbol{\Lambda} \mathbf{C}^{T} \mathbf{C} \boldsymbol{\Lambda} \mathbf{B}^{T}=\mathbf{B} \boldsymbol{\Lambda}^{2} \mathbf{B}^{T}=\boldsymbol{\Lambda}^{2} .
$$

Por tanto, teniendo en cuenta que $\mathbf{B}=\mathbf{I}$, la descomposición SVD de (4.35) se reescribe como:

$$
\mathbf{D}=\boldsymbol{\Lambda} \mathbf{C}^{T}
$$

En la expresión anterior, la matriz $\boldsymbol{\Lambda}$ es una matriz $[M \times K M]$ con elementos nulos excepto en el primer bloque $[M \times M]$, que es diagonal, es decir, $\boldsymbol{\Lambda}=[\tilde{\boldsymbol{\Lambda}} \mid \mathbf{0}]$, mientras que la matriz $\mathbf{C}$ es una matriz de dimensiones $[K M \times K M]$. La descomposición (4.37) puede escribirse finalmente como:

$$
\mathbf{D}=\boldsymbol{\Lambda} \mathbf{C}^{T}=\left[\tilde{\boldsymbol{\Lambda}}_{M \times M} \mid \mathbf{0}_{M \times(K-1) M}\right]\left[\tilde{\mathbf{C}}_{K M \times M} \mid \hat{\mathbf{C}}\right]^{T}=\tilde{\Lambda} \tilde{\mathbf{C}}^{T} .
$$

Por otro lado, teniendo en cuenta que la matriz $\mathbf{C}$ es unitaria, $\mathbf{C C}^{T}=$ I, la pseudo-inversa de la matriz $\mathbf{D}$ puede calcularse a partir de (4.37) mediante:

$$
\mathbf{D}^{\dagger}=\mathbf{C} \boldsymbol{\Lambda}^{\dagger} .
$$

Desarrollando esta expresión como en (4.38), se obtiene:

$$
\mathbf{D}^{\dagger}=\mathbf{C} \boldsymbol{\Lambda}^{\dagger}=\left[\tilde{\mathbf{C}}_{K M \times M} \mid \hat{\mathbf{C}}\right]\left[\frac{\tilde{\boldsymbol{\Lambda}}^{-1}}{\mathbf{0}}\right]=\tilde{\mathbf{C}} \tilde{\boldsymbol{\Lambda}}^{-1},
$$


donde la matriz $\tilde{\mathbf{\Lambda}}^{-1}$ se calcula invirtiendo cada uno de los elementos de la diagonal de $\tilde{\boldsymbol{\Lambda}}$.

Volviendo a la expresión (4.38), la matriz de vectores singulares puede expresarse como

$$
\tilde{\mathbf{C}}=\mathbf{D}^{T} \tilde{\boldsymbol{\Lambda}}^{-1}
$$

de forma que la ecuación (4.39) se resume en:

$$
\mathbf{D}^{\dagger}=\mathbf{D}^{T} \tilde{\mathbf{\Lambda}}^{-2} .
$$

Por último, sustituyendo (4.40) en (4.33), la actualización de potencias DL se realiza mediante:

$$
\mathbf{p}\left(n_{i t}+1\right)=\mathbf{D}^{T}\left(n_{i t}\right) \tilde{\mathbf{\Lambda}}^{-2}\left(n_{i t}\right)\left(\mathbf{F}\left(n_{i t}\right) \mathbf{p}\left(n_{i t}\right)+\mathbf{u}\right) .
$$

Aunque la matriz $\tilde{\boldsymbol{\Lambda}}$ es el primer bloque $[M \times M]$ de la matriz de valores singulares, esta matriz está directamente relacionada con la matriz diagonal $\mathbf{D D}^{T}$, tal y como se deduce de la relación (4.36):

$$
\mathbf{D D}^{T}=\boldsymbol{\Lambda}^{2}=\left[\tilde{\boldsymbol{\Lambda}}_{M \times M} \mid \mathbf{0}_{M \times(K-1) M}\right]\left[\tilde{\boldsymbol{\Lambda}}_{M \times M} \mid \mathbf{0}_{M \times(K-1) M}\right]^{T}=\tilde{\boldsymbol{\Lambda}}^{2},
$$

es decir, la matriz $\tilde{\Lambda}^{2}$ contiene los valores propios de la matriz diagonal $\mathbf{D D}^{T}$. Por tanto, para calcular la pseudo-inversa de $\mathbf{D}$, primero se obtiene $\tilde{\mathbf{\Lambda}}^{2}$ calculada como $\mathbf{D} \mathbf{D}^{T}$, y después se calcula $\tilde{\mathbf{\Lambda}}^{-2}$ invirtiendo cada uno de los elementos de la diagonal de $\tilde{\Lambda}^{2}$. De esta forma, para calcular la pseudoinversa de la matriz $\mathbf{D}$ no es necesaria ni la descomposición SVD ni ninguna inversión de matrices.

\subsubsection{Convergencia del algoritmo}

El algoritmo JPCOB-VUL es una extensión del algoritmo propuesto por Rashid-Farrokhi et al. [33]. Por tanto, la demostración de la convergencia del algoritmo JPCOB-VUL es similar a la del algoritmo de referencia, exceptuando el hecho de que ahora todas las BSs transmiten a todos los usuarios y en el problema de optimización hay que considerar los $K \cdot M$ enlaces del sistema.

El algoritmo JPCOB-VUL es una extensión subóptima del algoritmo de referencia, porque en un contexto de coordinación entre BSs mantiene un diseño local de los beamformers en transmisión. Sin embargo, el algoritmo JPCOB-VUL es óptimo desde el punto de vista local de cada BS, puesto 
que los beamformers se diseñan para maximizar la SINR recibida en el UL virtual de cada usuario.

En principio, los pasos de diseño de los beamformers y de actualización de las potencias UL virtuales, expresiones (4.29) y (4.32), respectivamente, coinciden con los del algoritmo de referencia de Rashid-Farrokhi et al.. Sin embargo, en el algoritmo JPCOB-VUL el diseño de los beamformers y de las potencias UL virtuales se realiza de forma distribuida para cada BS.

Por tanto, los beamformers en transmisión y las potencias UL virtuales convergen a valores estables, y en teoría óptimos, de forma independiente en cada BS, es decir, la convergencia de la iteración sobre el problema UL virtual se evalúa de forma independiente en cada BS.

Si se reescribe la iteración de las potencias UL virtuales en una BS $k$ determinada de forma similar a (3.55), la matriz de ganancia en el UL virtual para esa BS determinada, $\left[\mathbf{D}_{k} \mathbf{F}_{k}\right]$, converge a una matriz constante cuyo radio espectral cumple:

$$
\rho\left(\mathbf{D}_{k} \mathbf{F}_{k}\right)<1 .
$$

Esta condición asegura que la iteración de las potencias UL virtuales, que se trata en realidad de una iteración de Jacobi (apéndice A, apartado A.2), converge para cualquier valor inicial de las potencias UL virtuales.

La convergencia de la iteración de actualización de las potencias en DL se demuestra de una forma ligeramente distinta. Para que la iteración DL converja es necesario que la iteración de los beamformers y de las potencias en el UL virtual converja de forma independiente en cada BS. Una vez se tienen unos beamformers en trasmisión óptimos para cada enlace, si la matriz de ganancia DL, $\left[\mathbf{D}^{\dagger} \mathbf{F}\right]$, obtenida a partir de (4.23) y (4.25), converge a una matriz constante y cumple la condición:

$$
\rho\left(\mathbf{D}^{\dagger} \mathbf{F}\right)<1
$$

la iteración de actualización de las potencias DL (4.33), que de nuevo es una iteración de Jacobi, converge al óptimo a partir de cualquier valor inicial del vector de potencias DL. La relación anterior sobre el radio espectral de la matriz de ganancia DL es lo que se denomina en esta Tesis condición de feasibility teórica del algoritmo JPCOB-VUL o equivalentemente, del problema de minimización de potencia: si esta relación se cumple, el problema de minimización de potencia tiene solución y las potencias DL óptimas son valores positivos. 
Por el Teorema de Perron-Frobenius (apéndice A, apartado A.1), las condiciones planteadas sobre el radio espectral de las matrices de ganancia en el enlace DL y UL virtual requieren que estas matrices sean irreducibles. En principio, si los canales entre los usuarios y las BSs no siguen una estructura específica, estas matrices no serían ni periódicas ni reducibles. Además, las situaciones en las que todos los usuarios del sistema se interfieren mutuamente, aseguran que la matriz de ganancia DL sea irreducible [80].

\subsubsection{Caracterización del óptimo}

En este apartado, el objetivo es demostrar que la potencia total transmitida en el enlace DL, definida como la suma de las potencias transmitidas por cada BS, es la mínima posible.

Por otro lado, también interesa aclarar por qué el algoritmo JPCOBVUL, que en teoría es una extensión para sistemas CM3 del algoritmo de Rashid-Farrokhi et al., mantiene un diseño local de los beamformers en transmisión, en lugar de aprovechar la cooperación en datos de las BSs y diseñar un beamformer multi-base con pesos $\left[K N_{t} \times 1\right]$ para cada usuario.

\section{Diseño multi-base de los beamformers}

Una extensión del algoritmo de referencia de Rashid-Farrokhi et al., incluyendo un diseño multi-base de los beamformers en transmisión, tendría que calcular $M$ beamformers $\mathbf{w}_{m}$, que contendrían los pesos de cada beamformer en todas las BSs:

$$
\mathbf{w}_{m}=\left[\begin{array}{lll}
\mathbf{w}_{m 1}^{H} & \ldots & \mathbf{w}_{m K}^{H}
\end{array}\right]^{H}, m=1, \ldots, M .
$$

Estos beamformers multi-base se diseñarían a partir de un problema UL virtual coordinado, donde la SINR recibida en el UL virtual para el usuario $m$ se expresaría como:

$$
\operatorname{SINR}_{m}^{V U}=\frac{p_{m}^{u} \mathbf{w}_{m}^{H} \mathbf{H}_{m} \mathbf{w}_{m}}{\sum_{i \neq m} p_{i}^{u} \mathbf{w}_{m}^{H} \mathbf{H}_{i} \mathbf{w}_{m}+\mathbf{w}_{m}^{H} \mathbf{w}_{m}},
$$

donde las matrices de correlación de canal $\mathbf{H}_{m}$ se obtendrían a partir de los canales totales de cada usuario:

$$
\mathbf{H}_{m}=E\left[\mathbf{h}_{m}^{H} \mathbf{h}_{m}\right]
$$


definidos de forma similar a $\mathbf{w}_{m}$ :

$$
\mathbf{h}_{m}=\left[\begin{array}{lll}
\mathbf{h}_{m 1} & \ldots & \mathbf{h}_{m K}
\end{array}\right] .
$$

Este diseño multi-base de los beamformers asignaría una única potencia UL virtual para cada usuario en el sistema, independientemente de que el algoritmo de asignación de potencias en DL (4.33) asigne una potencia de transmisión para cada enlace $m k$ usuario-BS en el sistema.

La cuestión es cómo afectaría este hecho a la convergencia del algoritmo. En el algoritmo de referencia de Rashid-Farrokhi et al., se demuestra que la potencia total transmitida en DL es mínima, porque coincide con una suma ponderada de las potencias UL virtuales (3.57), que son las mínimas posibles. Con la introducción del diseño multi-base de los beamformers a partir del problema UL virtual coordinado, la relación (3.57) se transformaría en:

$$
\sum_{m=1}^{M} p_{m}^{u} \sigma_{m}^{2}=\sum_{m=1}^{M} p_{m}\left\|\mathbf{w}_{m}\right\|^{2} .
$$

La expresión anterior implica considerar una única potencia en DL para cada usuario $m$, o lo que es lo mismo, las BSs estarían cooperando en potencia, actuando como una única BS, y no se cumpliría la restricción en potencia transmitida por BS.

\section{Diseño local de los beamformers}

Este es el caso del algoritmo JPCOB-VUL. El hecho de diseñar los beamformers de forma local en cada BS permite tener, para un usuario $m$, distintas potencias en el problema UL virtual, $\left(p_{m 1}^{u}, \ldots, p_{m K}^{u}\right)$.

De esta forma se asegura que la iteración UL virtual, en el punto óptimo, cumpla la relación (3.57), pero particularizada para cada una de las BSs del sistema:

$$
\sum_{m=1}^{M} p_{m k}^{u} \sigma_{m}^{2}=\sum_{m=1}^{M} p_{m k}\left\|\mathbf{w}_{m k}\right\|^{2}, k=1, \ldots, K .
$$

Esta aproximación local permite asignar al usuario distintas potencias DL desde cada $\mathrm{BS},\left(p_{m 1} \neq \ldots \neq p_{m K}\right)$, con lo que se mantiene la restricción en potencia transmitida por BS. Resumiendo, el diseño local de los beamformers aumenta los grados de libertad con respecto a la solución con un diseño multi-base de los beamformers. 


\section{Algoritmo JPCOB-VUL semi-coordinado}

Sin embargo, es posible incluir un cierto grado de coordinación entre BSs en el problema UL virtual, obteniendo una versión semi-coordinada del algoritmo JPCOB-VUL. Esta modificación continúa siendo una alternativa subóptima desde el punto de vista de coordinación, puesto que mantiene un diseño local de los beamformers y solamente introduce la coordinación en el paso de actualización de las potencias UL virtuales [84].

Siguiendo un procedimiento similar al del enlace DL, se puede obtener una expresión matricial compacta para la actualización de las potencias UL virtuales. La idea es modificar la expresión de la SINR en el enlace UL virtual (4.18), incluyendo un sumatorio de todas las contribuciones en las BSs para un determinado usuario $m$. De esta forma, la ecuación de actualización de las potencias UL virtuales puede escribirse en forma matricial y multi-usuario como:

$$
\mathbf{D} \overline{\mathbf{p}}=\tilde{\mathbf{F}} \overline{\mathbf{p}}+\tilde{\mathbf{u}},
$$

donde $\overline{\mathbf{p}}$ se define como en (4.21) pero para las potencias UL virtuales. La matriz $\tilde{\mathbf{F}}$ de interferencias, de dimensiones $[M \times K M]$, sigue presentando una estructura de $K$ matrices $\tilde{\mathbf{F}}_{k}$ de dimensiones $[M \times M]$ :

$$
\begin{aligned}
\tilde{\mathbf{F}} & =\left[\tilde{\mathbf{F}}_{1}\left|\tilde{\mathbf{F}}_{2}\right| \cdots \mid \tilde{\mathbf{F}}_{K}\right], \\
{\left[\tilde{\mathbf{F}}_{k}\right]_{(m, i)} } & =\left\{\begin{array}{cc}
\gamma_{m} \mathbf{w}_{j}^{H} \mathbf{H}_{n} \mathbf{w}_{j}, & m \neq i, \\
0, & m=i,
\end{array}\right.
\end{aligned}
$$

donde los índices $j$ y $n$ se relacionan con los índices $k$ y $m$ como en (4.20). Sin embargo, a partir de (4.18) se observa que el vector $\tilde{\mathbf{u}}$ no es único para el usuario $m$, depende de los índices $k$ y $m$. Por tanto, para obtener una expresión única, en el vector $\tilde{\mathbf{u}}$ sólo se considera, para cada usuario, el valor máximo del módulo de sus beamformers (el caso peor desde el punto de vista de potencia transmitida):

$$
\tilde{\mathbf{u}}=\left[\gamma_{1}\left\|\mathbf{w}_{1}\right\|^{2} \ldots \gamma_{M}\left\|\mathbf{w}_{M}\right\|^{2}\right]^{T}
$$

donde el vector $\mathbf{w}_{m}, m=1, \ldots, M$, se corresponde con el beamformer de mayor módulo del usuario $m$ de entre todas las BSs:

$$
\mathbf{w}_{m}=\operatorname{máx}_{j}\left\|\mathbf{w}_{j}\right\|,
$$


con $j=(k-1) M+m, k=1, \ldots, K$. Aprovechando la similitud entre la notación de los problemas (4.45) y (4.22), la solución iterativa de (4.45) se expresa como:

$$
\overline{\mathbf{p}}\left(n_{i t}+1\right)=\mathbf{D}^{T} \tilde{\boldsymbol{\Lambda}}^{-2}\left(\tilde{\mathbf{F}} \overline{\mathbf{p}}\left(n_{i t}\right)+\tilde{\mathbf{u}}\right) .
$$

Los pasos del algoritmo JPCOB-VUL semi-coordinado para cada iteración $n_{i t}$ serían:

1. Inicializar las potencias UL virtuales, $p_{j}^{u}(0)$, y las potencias $\mathrm{DL}, p_{j}(0)$ para todos los enlaces del sistema, $j=1, \ldots, K M$, con valores positivos.

2. Para $n_{i t}=0,1,2, \ldots$, hasta llegar a la convergencia:

2.1. Beamforming. Calcular los beamformers subóptimos en transmisión $\mathbf{w}_{j}$ para cada enlace $j=1, \ldots, K M$, maximizando para cada enlace la SINR recibida en el UL virtual, con las potencias UL virtuales correspondientes a la iteración $n_{i t}$.

En la práctica, cada uno de los beamformers $\mathbf{w}_{j}$ se calcula mediante la expresión MMSE:

$$
\mathbf{w}_{j}\left(n_{i t}\right)=\sqrt{p_{j}^{u}\left(n_{i t}\right)}\left(p_{j}^{u}\left(n_{i t}\right) \mathbf{H}_{j}+\sum_{n} p_{n}^{u}\left(n_{i t}\right) \mathbf{H}_{n}+\mathbf{I}\right)^{-1} \mathbf{h}_{j},
$$

donde $n$ representa a los enlaces interferentes del enlace $j$-ésimo, que se corresponden con los $M-1$ enlaces restantes asociados a la misma BS $k$ : si $j=(k-1) M+m$, entonces $n=(k-1) M+i$, con $i=1, \ldots, M$, e $i \neq m$.

2.2. Control de potencia, enlace UL virtual. Las potencias UL virtuales $\overline{\mathbf{p}}$ se actualizan mediante la expresión (4.48),

$$
\overline{\mathbf{p}}\left(n_{i t}+1\right)=\mathbf{D}^{T}\left(n_{i t}\right) \tilde{\boldsymbol{\Lambda}}^{-2}\left(n_{i t}\right)\left(\tilde{\mathbf{F}}\left(n_{i t}\right) \overline{\mathbf{p}}\left(n_{i t}\right)+\tilde{\mathbf{u}}\left(n_{i t}\right)\right),
$$

donde las matrices $\mathbf{D}\left(n_{i t}\right), \tilde{\mathbf{\Lambda}}^{-2}\left(n_{i t}\right), \tilde{\mathbf{F}}\left(n_{i t}\right)$ y el vector $\tilde{\mathbf{u}}\left(n_{i t}\right)$ se obtienen a partir de las expresiones (4.23), (4.42), (4.46) y (4.47), respectivamente, pero considerando los beamformers en transmisión correspondientes a la iteración $n_{i t}$.

2.3. Control de potencia, enlace DL. El vector $\mathbf{p}$ de potencias DL se actualiza mediante (4.41):

$$
\mathbf{p}\left(n_{i t}+1\right)=\mathbf{D}^{T}\left(n_{i t}\right) \tilde{\boldsymbol{\Lambda}}^{-2}\left(n_{i t}\right)\left(\mathbf{F}\left(n_{i t}\right) \mathbf{p}\left(n_{i t}\right)+\mathbf{u}\right),
$$


donde las matrices $\mathbf{D}\left(n_{i t}\right), \tilde{\boldsymbol{\Lambda}}^{-2}\left(n_{i t}\right)$ y $\mathbf{F}\left(n_{i t}\right)$ se obtienen a partir de las expresiones (4.23), (4.42) y (4.25), respectivamente, pero considerando los beamformers en transmisión correspondientes a la iteración $n_{i t}$. El vector $\mathbf{u}$ se calcula mediante la expresión (4.27).

Por último, hay que destacar que las modificaciones introducidas en el algoritmo JPCOB-VUL para plantear esta versión semi-coordinada no afectan a las propiedades de convergencia del algoritmo, que siguen siendo las presentadas en el apartado 4.2.5.

\subsection{Aspectos prácticos del algoritmo JPCOB-VUL}

Antes de analizar mediante simulaciones la potencia total transmitida en el sistema, parece lógico pensar que los sistemas CM3 transmiten más potencia que los sistemas de comunicaciones convencionales sin coordinación como UMTS, donde se contempla una asignación de usuarios a BSs.

Recientemente, en [68] se propone el problema de minimización de potencia en un sistema CM3. Los autores de [68], demuestran que para un sistema CM3 sin restricciones de potencia de ningún tipo, la mínima potencia total transmitida en el sistema se consigue cuando sólo una BS transmite a cada usuario, es decir, cuando no existe coordinación entre las BSs. En este caso, es necesario implementar algún tipo de esquema de asignación de usuarios a BSs.

Una de las ventajas de los sistemas CM3, es que no necesitan una asignación previa de usuarios a BSs, porque todas las BSs transmiten a todos los usuarios. En este punto, la complejidad del sistema se reduce con respecto a otros algoritmos JPCOB propuestos para sistemas no coordinados [90,91], que incluyen en el problema de optimización la asignación de usuarios a BSs. Además, la transmisión coordinada permite eliminar posibles handovers internos en la zona compartida por las BSs del sistema.

Por otra parte, la transmisión coordinada puede ser perjudicial en algunos casos, por ejemplo, cuando alguno de los canales de propagación entre un usuario y las $K$ BSs del sistema presenta unas pérdidas de propagación elevadas o un desvanecimiento pronunciado del canal. En este caso concreto, siguiendo el comportamiento del algoritmo, la BS correspondiente aumentaría la potencia $p_{j}$ asociada a ese enlace para cumplir con la SINR 
requerida en el usuario, aumentando al mismo tiempo la potencia de interferencia transmitida a los $M-1$ usuarios restantes. En la siguiente iteración del algoritmo JPCOB-VUL, el vector de pesos asociado a ese enlace podría conformarse erróneamente debido al bajo valor del estadístico $\mathbf{H}_{j}$ en (4.29). Esto conllevaría un aumento progresivo de la potencia UL virtual asociada a este enlace, $p_{j}^{u}$, para cumplir con (4.32), puesto que la SINR asociada con ese usuario en el problema UL virtual sería muy baja. Todo este proceso, acabaría afectando al diseño del resto de beamformers de la misma BS.

En este escenario, se añade al algoritmo JPCOB-VUL un esquema de selección de enlaces activos. Este esquema, a partir de un determinado parámetro, decide qué enlaces, del total de los $K \cdot M$ disponibles en el sistema CM3, se encuentran activos en cada momento.

En un primer momento, la introducción del esquema de selección de enlaces activos se debió a la necesidad de mejorar las prestaciones del algoritmo JPCOB-VUL, eliminando los enlaces que a priori, según un determinado parámetro, parecían empeorar las prestaciones del sistema. Sin embargo, a medida que avanza en la Tesis el análisis de las prestaciones de los algoritmos JPCOB, se observa que el esquema de selección de enlaces activos es una técnica eficiente para evaluar las prestaciones de los distintos algoritmos frente a diferentes grados de coordinación entre las BSs del sistema CM3.

Desde otro punto de vista, un esquema de selección de enlaces activos puede interpretarse como una técnica de scheduling multi-base centralizada. En [75], también se proponen técnicas de scheduling multi-base distribuidas para sistemas CM3. Sin embargo, el objetivo de la referencia [75] es maximizar el sum-rate del sistema CM3 y por este motivo, cada BS transmite a un único usuario a máxima potencia.

Hay que destacar que el objetivo del esquema de selección de enlaces activos no es, en ningún momento, realizar una asignación óptima de usuarios a BSs dentro de un sistema CM3. Hasta la fecha, ningún autor ha propuesto una solución para el problema conjunto de minimización de potencia y asignación óptima de usuarios a BSs en sistemas CM3. En [68] se realiza una primera aproximación a la solución. Sin embargo, en esta referencia, los autores utilizan el hecho de que según las condiciones de su sistema, la mínima potencia total transmitida se consigue cuando una única BS transmite a cada usuario, para realizar la asignación de usuarios a BSs mediante el algoritmo clásico de [43]. 
En esta Tesis, se proponen dos esquemas de selección de enlaces activos, que dan lugar a lo que se ha denominado como entorno Coordinado I y entorno Coordinado II del algoritmo JPCOB-VUL. Estos esquemas se presentan desde un punto de vista teórico, y se asume que se dispone de los canales de realimentación necesarios para llevarlos a la práctica.

Desde el punto de vista de programación del algoritmo JPCOB-VUL, los esquemas de selección de enlaces activos se implementan en la iteración de las potencias DL, (4.33), donde simplemente se anulan los beamformers en transmisión asociados a los enlaces inactivos del sistema CM3. Estos esquemas, no afectan a los pasos de la iteración UL del algoritmo por el siguiente motivo: la iteración UL comprende el diseño de los beamformers (4.29) y la actualización de las potencias UL virtuales (4.32), pero se resume en un único paso, que es el de obtener los beamformers en transmisión de cada usuario. Cuando se anula un enlace en el UL virtual, en realidad, lo que sucede es que en el diseño del beamformer en transmisión se está dejando de considerar una interferencia que sí que está presente en el enlace DL, por lo que el beamformer en transmisión se conforma erróneamente.

Los esquemas de selección de enlaces activos pueden interpretarse como una matriz de activación $\mathbf{H}_{a c t}$, de dimensiones $[M \times K]$, donde cada elemento de la matriz $\left[\mathbf{H}_{a c t}\right]_{(m, k)}$ se asocia con el enlace entre la BS k y el usuario $m$ y toma el valor 1 cuando el enlace está activo o 0 cuando el enlace no lo está. Por tanto, cuando el algoritmo llega a la actualización de potencias DL de (4.33), cada beamformer en transmisión $\mathbf{w}_{m k}$ se multiplica por el elemento correspondiente de la matriz de activación $\left[\mathbf{H}_{a c t}\right]_{(m, k)}$. Este punto de vista es el que se utiliza en [75] para plantear el sistema CM3 como un grafo.

\section{Algoritmo JPCOB-VUL: entorno Coordinado I}

En el entorno Coordinado I del algoritmo JPCOB-VUL, la selección de enlaces activos en el sistema CM3 se realiza de forma similar a los procesos de soft handover. La idea es que de las $K$ BSs del sistema CM3, sólo van a transmitir a cada usuario aquellas que se encuentren incluidas en su Active Set (AS) [87].

Para generar el AS de un determinado usuario, éste monitoriza continuamente la potencia que recibe de los canales piloto transmitidos por las $K$ BSs. Previamente, el sistema establece un parámetro, denominado ventana de AS o Active Set Window (ASW) y medido en $\mathrm{dB}$, que funciona como umbral de activación de los enlaces. 
Para formar un AS de BSs, el usuario compara las potencias de los $K$ canales piloto, uno por cada BS, y establece como valor de referencia la potencia máxima recibida. Teniendo en cuenta el valor del parámetro ASW, establece una ventana de activación de enlaces, fijando un umbral mínimo de activación al restar al valor de referencia el valor del ASW. El AS de BSs para ese usuario, estará constituido entonces por todas las BSs cuya potencia de canal piloto quede entre el valor de referencia y el umbral mínimo fijado por el parámetro ASW.

Por tanto, esta técnica depende de las condiciones de propagación de cada enlace del sistema, y a priori, va a eliminar los enlaces que presenten mayores pérdidas. La ventaja de esta técnica, es que el valor de la ventana de AS es un valor establecido a nivel de sistema. Esto podría aprovecharse para reconfigurar el sistema CM3 de forma dinámica (por ejemplo, modificando el valor de ASW, el algoritmo JPCOB-VUL puede adaptarse automáticamente a distintas cargas de tráfico y requerimientos de QoS).

Por último, hay destacar que esta técnica genera un AS de BSs por cada usuario del sistema, según sean las condiciones de los canales de propagación de cada usuario. Por tanto, para un mismo instante de tiempo, puede haber usuarios que sólo reciban su señal deseada desde una BS, y otros usuarios que estén recibiendo su señal deseada hasta de $K$ BSs de forma coordinada.

\section{Algoritmo JPCOB-VUL: entorno Coordinado II}

En este segundo entorno de coordinación del algoritmo JPCOB-VUL, el parámetro que determina qué enlaces se consideran activos, es el módulo de los beamformers de cada enlace. La idea es que transmitan a cada usuario sólo aquellas BSs cuyo beamformer, en módulo, supere un valor prefijado. De esta forma, se eliminan los enlaces que a priori, no contribuyen significativamente a la SINR recibida en el usuario.

La ventaja de este esquema de activación es que requiere una implementación puramente en transmisión, y por tanto, no se necesita que el usuario realimente a las BSs ningún tipo de información.

Por otro lado, la desventaja de este esquema es que el valor de activación de los enlaces, es decir, el valor mínimo del módulo de los beamformers, depende en gran medida de las condiciones del sistema, y por tanto, es un valor crítico y puede ser complejo de establecer. 


\subsection{Prestaciones del algoritmo JPCOB-VUL}

En este apartado, se evalúan las prestaciones del algoritmo JPCOBVUL en un sistema W-CDMA CM3 con $K$ BSs y un determinado número de usuarios, de los que $M$ son usuarios co-canal, distribuidos uniformemente sobre la zona sombreada de la figura 4.2.

El objetivo es evaluar el comportamiento de esta primera propuesta de algoritmo JPCOB en un entorno donde el nivel de potencia interferente es muy elevado, y donde en principio el algoritmo debería verse muy limitado, tanto por el diseño local de los beamformers como por las restricciones de potencia transmitida por cada BS.

El entorno elegido para programar el algoritmo y simular sus prestaciones es el programa MATLAB. En un primer paso, se simula el sistema W-CDMA CM3 de una forma realista, prestando especial atención a la generación de los canales de propagación conforme al estándar UMTS. Posteriormente, se implementan todos los bloques correspondientes al algoritmo JPCOB-VUL y se pasa a evaluar puntos concretos de su comportamiento.

En general, este Capítulo se centra en plantear un modelo de sistema y un algoritmo JPCOB válido para la arquitectura Multinodo B. Por este motivo, las simulaciones intentan reproducir lo más fielmente posible un sistema CM3 según los parámetros del estándar UMTS.

\subsubsection{Modelo de sistema}

Para evaluar y comparar las prestaciones del algoritmo JPCOB-VUL, se simulan dos modelos de sistema, representados en la figura 4.4. El primer modelo se corresponde con un sistema CM3 como el representado en la figura 4.2, aunque sólo se muestra la zona compartida entre las BSs. El segundo modelo de sistema, representa una celda sectorizada convencional. Estos dos tipos de sistema se caracterizan de forma que sean totalmente equivalentes. Por ejemplo, si el sistema CM3 dispone de $K=3$ BSs transmitiendo coordinadamente, la celda convencional se divide en 3 sectores, y en cada uno de ellos se ubica un array de antenas con el mismo número de antenas que las que dispone cada BS del sistema CM3. Al mismo tiempo, si cada BS del sistema CM3 está sujeta a una restricción de potencia $P_{\max }$, esta misma restricción se impone a cada uno de los arrays de antenas ubicados en la celda convencional. Para simplificar la notación, a los arrays de antenas de la celda convencional también se les denomina BSs. 


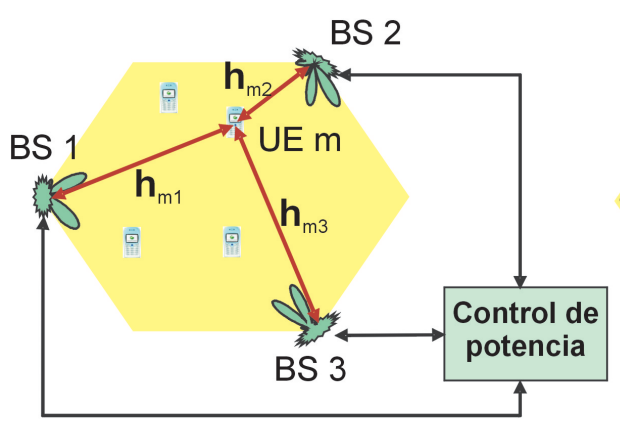

(a)

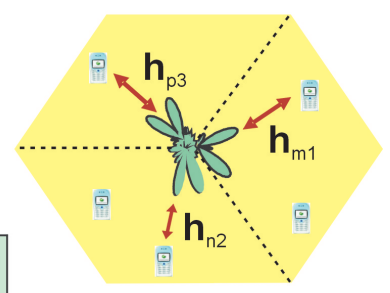

(b)

Figura 4.4: Ejemplo de (a) un sistema CM3 con $K=3$ BSs y (b) una celda sectorizada convencional sin coordinación con un array de $N_{t}$ antenas en cada sector.

Para generar el canal de propagación $\mathbf{h}_{m k}$, es necesario definir los parámetros celulares de distancia entre usuario-BS o las direcciones de llegada o DOAs (Direction of Arrival) de las contribuciones del canal.

Los parámetros comunes a los dos sistemas de la figura 4.4 son el radio de la celda $R$ y la altura del hexágono $h$. El radio de la celda se fija en 350 metros, para conseguir una distancia entre emplazamientos $2 h$ de 600 metros.

Los usuarios se ubican en el sistema mediante una rejilla rectangular. Esta rejilla tiene una capacidad máxima, sin repetir posiciones, de 51 usuarios en el caso de la configuración de sistema CM3, y de 50 usuarios para la configuración convencional.

La figura 4.5 representa la rejilla en el caso de tener un sistema CM3 de $K=3$ BSs. En este caso, no existe sectorización, puesto que las BSs transmiten coordinadamente a todos los usuarios y no se realiza ninguna asignación previa de usuarios a BSs.

Por otro lado, la figura 4.6 muestra el caso equivalente de tener tres BSs o arrays de antenas en la configuración central, una por sector. En esta configuración, la asignación de usuarios a BSs se realiza simplemente por posición, es decir, los usuarios que están ubicados en un sector, reciben su señal deseada desde la BS de ese sector. En el caso de tener usuarios cercanos al borde de un sector, la asignación de BS se realiza de forma aleatoria. 


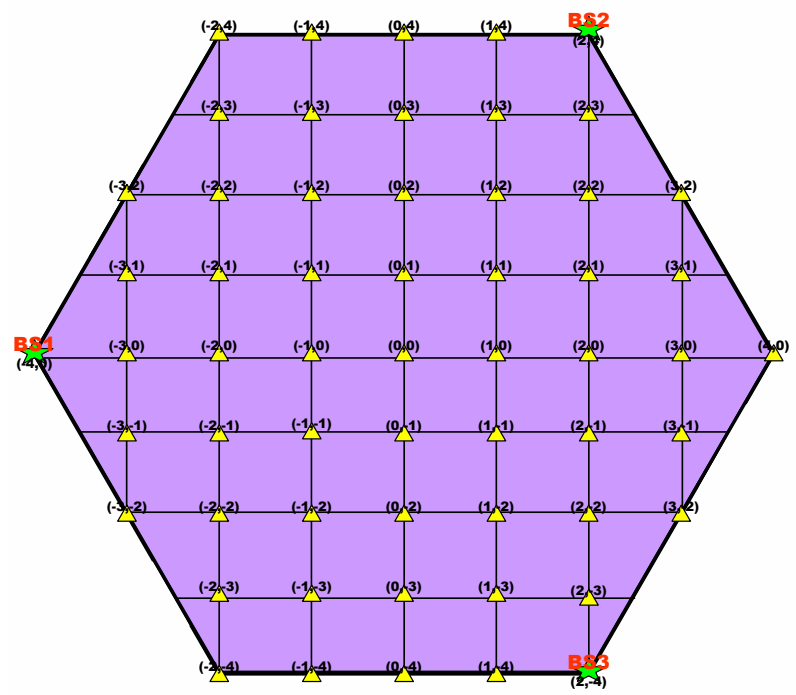

Figura 4.5: Sistema CM3, $K=3$ BSs.

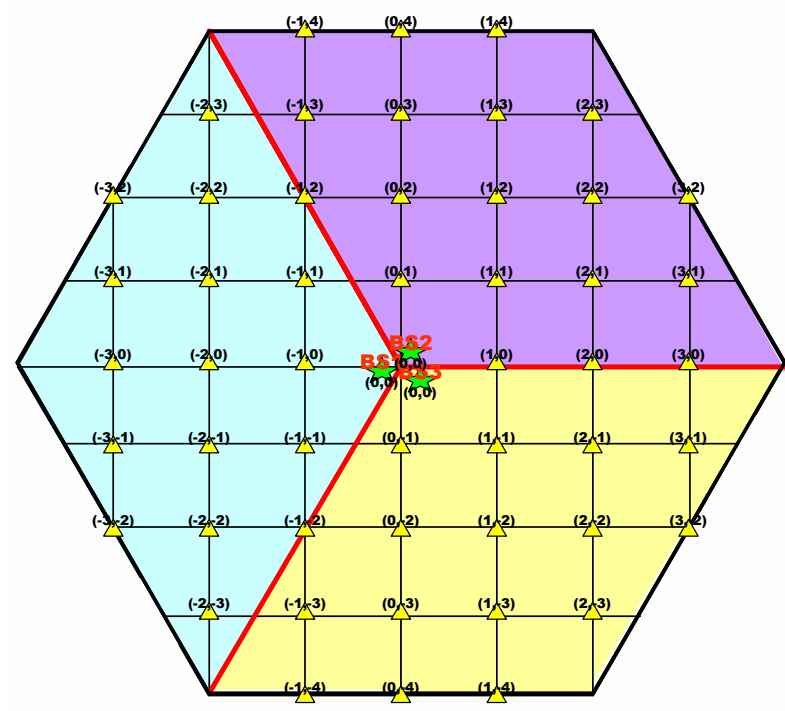

Figura 4.6: Celda sectorizada convencional, $K=3$ BSs centrales. 
Para definir la posición de cada usuario en el sistema, se establece un sistema de coordenadas rectangular cuyo origen se encuentra en el centro de la celda, de forma que cada posición sobre la rejilla queda definida por unas coordenadas $(x, y)$. La separación en los ejes $\mathbf{x}$ e $\mathbf{y}$ entre coordenadas es de $R / 4$ y $h / 4$, respectivamente.

Para añadir más flexibilidad a la rejilla rectangular, una vez escogida la posición del usuario sobre la rejilla, se le añade un desplazamiento aleatorio de $[ \pm R / 16, \pm h / 16]$ a la posición elegida. De esta forma, la distancia entre un usuario y una BS, ubicados en dos puntos de la celda $\left(x_{1}, y_{1}\right)$ y $\left(x_{2}, y_{2}\right)$, se calcula según:

$$
d=\sqrt{\left(x_{1}-x_{2}\right)^{2}+\left(y_{1}-y_{2}\right)^{2}} .
$$

En cuanto al procedimiento utilizado para ubicar a los usuarios sobre la rejilla, los programas pueden ubicarlos de forma aleatoria sobre todo el sistema o bien, de forma aleatoria dentro de un sector (en el caso del sistema CM3, existiría una división imaginaria en sectores, sólo útil para ubicar a los usuarios pero no para realizar una asignación de usuarios a BS).

Las DOA se modelan considerando solamente las DOA nominales de cada contribución multi-camino del canal [92]. Con el fin de acotar el ensanchamiento en ángulo o angle spread, se incluye un nuevo parámetro que indica en cuántos subsectores se divide el sector de $120^{\circ}$ asociado a una BS: los programas calculan los valores de la DOA aleatoriamente entre los que componen el subsector angular en el que se encuentra el usuario en cuestión. Por ejemplo, si cada sector se subdivide en tres subsectores de $40^{\circ}$, las DOA de un usuario ubicado en uno de ellos toma tantos valores como contribuciones de canal se considere, pero sobre un intervalo de 40 valores y no de 120 , como sería si se considerara todo el sector.

En las simulaciones, se considera una división en 4 subsectores, de forma que el ensanchamiento en ángulo se limita a $30^{\circ}$. Gráficamente, la figura 4.7 muestra la división en 4 subsectores de cada sector en el caso de la configuración central de tres BSs. La figura 4.8 muestra la división en subsectores de la configuración CM3 con tres BSs coordinadas; en este caso, hay que recalcar que esta división no implica realizar una asignación de usuarios a BSs, sino que únicamente se utiliza para el cálculo de las DOAs.

En el apéndice $\mathrm{C}$ se incluyen las figuras correspondientes a configuraciones de sistema CM3 y celdas sectorizadas convencionales equivalentes con $K=2$ BSs. 


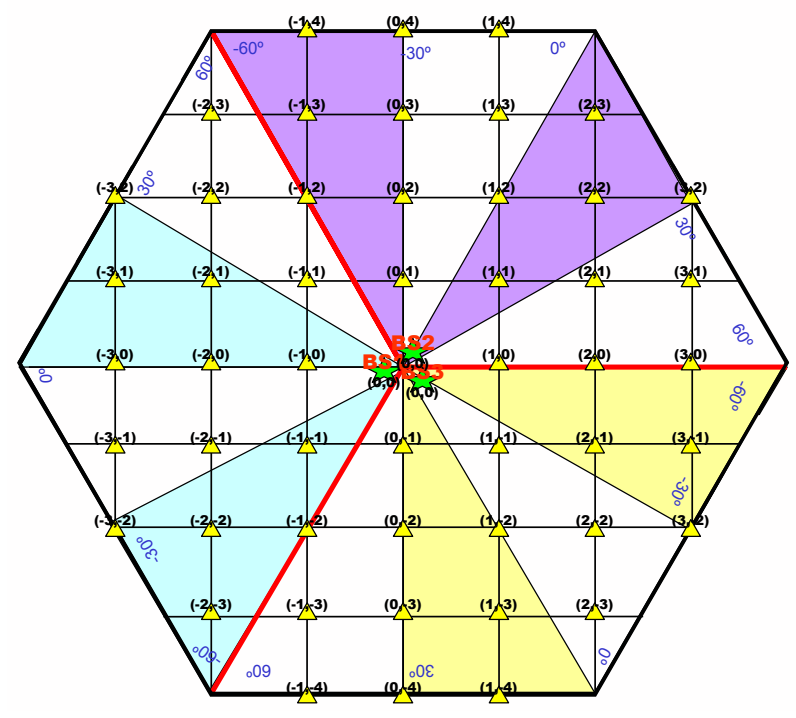

Figura 4.7: División en subsectores para el cálculo de las DOAs en una celda sectorizada convencional con $K=3$ BSs centrales.

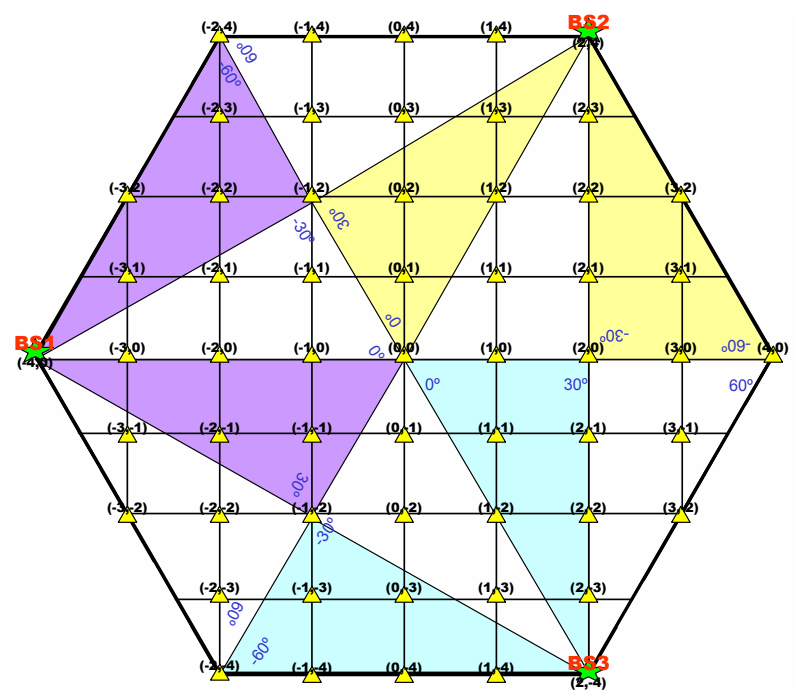

Figura 4.8: División en subsectores para el cálculo de las DOAs en un sistema CM3 con $K=3$ BSs. 


\subsubsection{Modelo de canal}

Uno de los puntos fundamentales de la simulación es la construcción del modelo de canal. En W-CDMA, los canales de propagación se componen de varios caminos de propagación o paths. El receptor de cada usuario se encarga de discretizar estos caminos en varias contribuciones de canal o taps, con retardos discretos que son múltiplos del periodo de chip $T_{c}$. Por tanto, lo que denominamos contribución multi-camino o tap, representa en realidad a un conjunto de caminos de propagación que se agrupan en el intervalo $\left[\tau, \tau+T_{c}\right]$.

En las simulaciones de este apartado, se considera un entorno flat fading donde cada canal $\mathbf{h}_{m k}$ está formado por una única contribución multicamino. Sin embargo, esta contribución se genera a partir de tres componentes con la misma potencia de fading (el retardo de todas las componentes se fija a cero). La DOA de cada componente se obtiene de forma aleatoria a partir de la ubicación del usuario en un subsector.

Volviendo a la ecuación que representa el modelo de canal (4.1),

$$
\mathbf{h}_{m k}=\sum_{l} \sqrt{\beta_{s} \beta_{m k} \alpha_{m k}(l)} \mathbf{a}_{m k}(\theta(l)) g_{m k},
$$

los parámetros necesarios para generar el vector de canal son la desviación estándar del shadowing del enlace, la distancia entre el usuario $m$ y la BS $k$, la frecuencia Doppler, y la potencia media de fading, el retardo y la DOA de cada componente.

Las DOAs y los parámetros relacionados con el PDP del canal, como la potencia media y los retardos de cada componente, se opta por dejarlos como parámetros opcionales, para poder construir modelos de canal conformes a alguna especificación; si no se introducen, el programa los calcula aleatoriamente entre todos los valores válidos posibles.

Las pérdidas de propagación del enlace se calculan a partir del modelo de rayo directo y rayo reflejado. De esta forma, el path loss $\beta_{m k}$ es proporcional a $r_{m k}^{-4}$, donde $r_{m k}$ es la distancia entre el usuario $m$ y la BS $k$. El shadowing $\beta_{s}$ se modela como una variable aleatoria log-normal, con una desviación estándar de $8 \mathrm{~dB}$.

Cada componente de la contribución de canal, $\alpha_{m k}(l)$, se genera como una variable aleatoria compleja Gaussiana, con parte real e imaginaria Gaussianas, de media nula e idéntica varianza (1/2), de forma que el módulo de la contribución presenta una distribución Rayleigh. Sin embargo, el valor 
de estos coeficientes se ajusta para cumplir con la potencia media de $f a$ ding establecida como parámetro de entrada en la simulación. La variación temporal de los coeficientes del canal se introduce modelando el espectro Doppler como un filtro IIR, a partir del modelo propuesto en [93, 94].

Aunque se simula un canal con una variación temporal lenta, las matrices de correlación $\mathbf{H}_{j}$ que se utilizan en la expresión de la SINR en UL y DL, y en el control de potencia, son un valor promediado sobre un determinado número de realizaciones del canal. De esta forma, antes de empezar la simulación del algoritmo JPCOB-VUL, lo que se hace es generar la respuesta del canal a lo largo de toda la longitud de la simulación, extrayendo después en cada iteración del algoritmo JPCOB-VUL el bloque de canal correspondiente.

Por último, se fija un número máximo de iteraciones para que el algoritmo JPCOB-VUL converja, o lo que es lo mismo, para que los $M$ usuarios co-canal alcancen la SINR umbral requerida, cumpliendo al mismo tiempo la restricción en potencia transmitida por BS, $P_{\max }$.

La tabla 4.1 resume los parámetros utilizados en la simulación. 


\begin{tabular}{|l|c|}
\hline \hline Parámetro & Valor \\
\hline \hline Frecuencia de la portadora & $2 \mathrm{GHz}$ \\
\hline Factor de spreading $N$ & 16 \\
\hline Tamaño máximo del active set & $10 \mathrm{dBm}$ \\
\hline Potencia de ruido AWGN y MAI & $20 \mathrm{~W}$ \\
\hline $\begin{array}{l}\text { Potencia máxima } P_{\text {max }} \\
\text { transmitida por BS }\end{array}$ & $600 \mathrm{~m}$ \\
\hline Distancia entre emplazamientos & $350 \mathrm{~m}$ \\
\hline Radio celular & $8 \mathrm{~dB}$ \\
\hline Desviación estándar del shadowing & $\beta_{m k} \propto r_{m k}^{-4}$ \\
\hline Pérdidas de propagación & 3 \\
\hline $\begin{array}{l}\text { Número de componentes } \\
\text { multi-camino por contribución }\end{array}$ & $0 \mathrm{~dB}$ \\
\hline $\begin{array}{l}\text { Potencia media de fading } \\
\text { de cada componente }\end{array}$ & 0 \\
\hline Retardo de cada componente & $4 \mathrm{~km} / \mathrm{h}$ \\
\hline Velocidad de los usuarios & 20 \\
\hline Máximo número de iteraciones del algoritmo $(I t)$ & $T_{s}=1 / 3,84 \cdot 10^{6}$ \\
\hline Periodo de muestreo & $n s i m b=3 \cdot N_{t}$ \\
\hline $\begin{array}{l}\text { Número de símbolos necesarios para estimar } \\
\text { la correlación de las matrices de canal }\end{array}$ & $N_{c o e f}=I t \cdot n s i m b$ \\
\hline Número de coeficientes del canal & \\
\hline
\end{tabular}

Cuadro 4.1: Parámetros de simulación. 


\subsubsection{Algoritmo JPCOB-VUL: entorno Coordinado I}

En este apartado se evalúan las prestaciones del algoritmo JPCOB-VUL de (4.29)-(4.33) en el entorno Coordinado I, donde la selección de enlaces activos se realiza configurando un active set (AS) de BSs para cada usuario del sistema.

\section{Comparativa con asignación óptima de usuarios a BSs}

Esta primera simulación se centra en una configuración de sistema CM3 como la de la figura 4.5, con $K=3 \mathrm{BSs}$, donde cada BS dispone de un array lineal de $N_{t}=4$ antenas, separadas entre sí media longitud de onda.

El objetivo es comparar el número medio de usuarios co-canal admitidos por el algoritmo JPCOB-VUL, con el admitido por un algoritmo de beamforming óptimo que también incluye una asignación óptima de usuarios a BSs [90].

El algoritmo propuesto por Bengtsson en [90], obtiene la solución óptima al problema conjunto de minimización de potencia y asignación de BS para un sistema convencional de una única celda. Esta solución óptima se obtiene a partir de una relajación del problema original, cercana a la idea de coordinación, donde se permite que todas las BSs transmitan a todos los usuarios. Además, este algoritmo también funciona a partir de la construcción del problema en el enlace UL virtual.

Por tanto, lo que se va a comparar en realidad es el número medio de usuarios co-canal que admite un sistema CM3 con coordinación o sin coordinación entre BSs. En el caso del algoritmo JPCOB-VUL, se obtiene el número medio de usuarios co-canal admitidos para distintos valores de ventana de AS, o lo que es lo mismo, para diferentes grados de coordinación entre las BSs.

La figura 4.19 muestra el diagrama de bloques de la simulación del algoritmo JPCOB-VUL. Para cada algoritmo simulado, el JPCOB-VUL y el de [90], el sistema empieza ubicando un único usuario en el sistema y sigue añadiendo usuarios co-canal mientras el algoritmo converja, es decir, mientras en cada uno de los usuarios se alcance la SINR umbral requerida en DL, sin superar el número máximo de iteraciones permitidas, $I t$, y la restricción en potencia de cada BS, $P_{\max }$.

La distribución de la posición inicial de los usuarios en el sistema es uniforme, asignando a cada uno una velocidad constante de $4 \mathrm{~km} / \mathrm{h}$, de forma que el canal varía lentamente con el tiempo. El resto de parámetros 
de simulación son los indicados en la tabla 4.1.

Ambos algoritmos se comparan sobre distintos valores de SINR umbral. De esta forma, una simulación viene definida por un valor de SINR umbral, común a todos los usuarios, y en el caso del algoritmo JPCOB-VUL, también con un valor de ventana de AS. Cada simulación se promedia sobre 200 realizaciones independientes:

- ASW: 0 dB (sin coordinación), 2 dB, 10 dB y 20 dB.

- SINR umbral: $5 \mathrm{~dB}, 8 \mathrm{~dB}, 11 \mathrm{~dB}$ y $14 \mathrm{~dB}$.

La configuración de $0 \mathrm{~dB}$ se corresponde con el caso en el que cada usuario sólo recibe señal deseada desde una única BS, es decir, no existe coordinación entre BSs. Sin embargo, la forma de seleccionar qué BS transmite a cada usuario depende del AS del usuario, que no trata de optimizar la asignación de usuarios a BSs. En principio, la configuración ASW $=0 \mathrm{~dB}$ del algoritmo JPCOB-VUL se corresponde exactamente con la propuesta de [90], exceptuando el hecho de que el algoritmo de [90] sí que realiza una asignación óptima de usuarios en base al criterio de minimización de potencia.

La figura 4.9 muestra el número medio de usuarios co-canal admitidos para los distintos valores de SINR y para los diferentes tamaños de ventana de coordinación [82]. En principio, el valor del tamaño de la ventana de AS lo establece el sistema, y es el mismo para todos los usuarios del sistema. En la figura se comparan los resultados del algoritmo JPCOB-VUL con los obtenidos por el algoritmo de beamforming óptimo y asignación óptima de BS de [90].

Estudiando los resultados del algoritmo JPCOB-VUL, parece que todos los valores de ASW consiguen resultados similares, siendo la configuración $\mathrm{ASW}=10 \mathrm{~dB}$ ligeramente mejor al resto. Este hecho contradice la idea de que cuantas más BSs estén activas en el sistema, mayor potencia de interferencia, por la que se esperaría un peor comportamiento de la configuración de $\mathrm{ASW}=10 \mathrm{~dB}$ frente a configuraciones de $\mathrm{ASW}=0 \mathrm{~dB}$ o $\mathrm{ASW}=2 \mathrm{~dB}$. La tabla 4.2 muestra el número medio de BSs que forman el AS para los distintos valores de ventana de AS simulados.

Por otro lado, el algoritmo de asignación óptima de BS supera de forma considerable los resultados obtenidos por el algoritmo JPCOB-VUL, como es de esperar, pero siempre a costa de una mayor complejidad computacional, puesto que este algoritmo realiza una búsqueda exhaustiva sobre el 


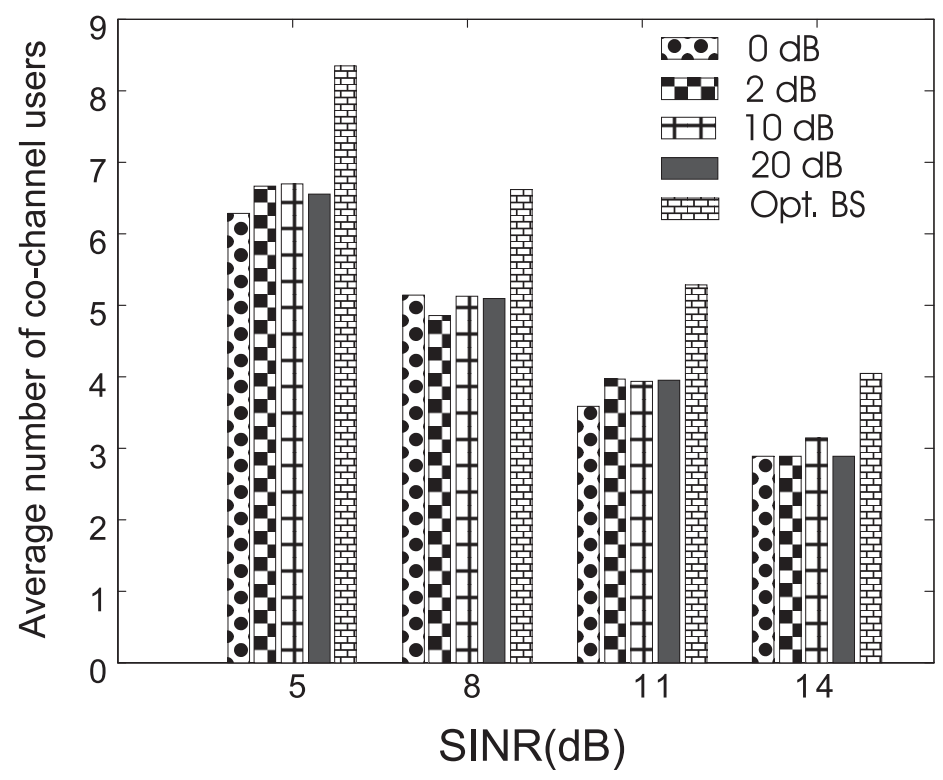

Figura 4.9: Número medio de usuarios co-canal admitidos por el algoritmo JPCOB-VUL para distintos valores de ventana de AS. Comparativa con los resultados obtenidos por el algoritmo de asignación óptima de usuarios a BS de [90].

\begin{tabular}{|c|c|}
\hline \hline Ventana (dB) & BSs en el AS \\
\hline \hline 0 & 1 \\
\hline 2 & 1,14 \\
\hline 10 & 1,7 \\
\hline 20 & 2,2 \\
\hline
\end{tabular}

Cuadro 4.2: Número medio de BSs en el AS para los valores de ventana simulados.

valor de la SINR en el UL virtual para obtener el óptimo del problema de asignación.

Por último, la diferencia entre los resultados del algoritmo JPCOB-VUL para $\mathrm{ASW}=0 \mathrm{~dB}$ y el algoritmo de asignación óptima de [90], confirma el hecho de que el esquema de selección de enlaces activos del entorno Coordinado I no realiza una selección óptima desde el punto de vista del criterio de minimización de potencia. 


\section{Estudio de la tasa de fallo}

Esta segunda simulación se centra en estudiar con más detalle el comportamiento del algoritmo JPCOB-VUL en el entorno Coordinado I. En este análisis, se simulan tres configuraciones distintas de sistema CM3, variando el número de BSs y de antenas disponibles en cada una de ellas. La tabla 4.3 resume el número de BSs, $K$, y el número de antenas por array, $N_{t}$, de cada configuración (en el apéndice C pueden consultarse las gráficas correspondientes a sistemas CM3 con $K=2 \mathrm{BSs}$ ), mientras que los parámetros de simulación siguen siendo los de la tabla 4.1.

\begin{tabular}{|c|c|c|}
\hline \hline Configuración & BSs & Antenas \\
\hline \hline 3BS-3ANT & 3 & 3 \\
\hline 2BS-4ANT & 2 & 4 \\
\hline 2BS-3ANT & 2 & 3 \\
\hline
\end{tabular}

Cuadro 4.3: Configuraciones de sistema CM3 simuladas.

El objetivo de esta simulación es evaluar cómo influyen los parámetros de SINR umbral y ventana de AS en el comportamiento del algoritmo JPCOB-VUL. En [95], se presenta un análisis similar de la influencia de la ventana de AS para un escenario de handover cooperativo, pero sin considerar el uso de beamformers en transmisión.

La figura 4.20 muestra el diagrama de bloques de la simulación del algoritmo JPCOB-VUL. En cada simulación, el sistema distribuye uniformemente $M=3$ usuarios co-canal, y el algoritmo JPCOB-VUL, para converger, tiene que conseguir que los usuarios alcancen la SINR umbral requerida dentro de un número máximo de iteraciones, $I t$, sin que la potencia transmitida por cada BS supere la restricción $P_{\max }$.

$\mathrm{Al}$ igual que en la primera simulación, una simulación se define mediante un valor de ventana de AS y un valor de SINR umbral, que es común a todos los usuarios del sistema. En este caso, se simulan 5 valores diferentes de ventana y cuatro valores de SINR umbral:

- ASW: $0 \mathrm{~dB}$ (sin coordinación), $5 \mathrm{~dB}, 10 \mathrm{~dB}, 15 \mathrm{~dB}$ y $20 \mathrm{~dB}$.

- SINR umbral: $6 \mathrm{~dB}, 8 \mathrm{~dB}, 10 \mathrm{~dB}$ y $12 \mathrm{~dB}$.

El parámetro que evalúa las prestaciones del algoritmo JPCOB-VUL para cada configuración del sistema CM3 es la tasa de fallo, definida como el número de realizaciones en las cuales el algoritmo no converge, sobre 
el número de realizaciones totales (3000). Esta tasa de fallo así definida es una estimación aproximada de la llamada probabilidad de outage; dada una determinada tasa de transmisión de datos, si $p$ es el porcentaje de outage de la misma, esa tasa puede transmitirse un $(100-p) \%$ del tiempo. De esta forma, la probabilidad de outage se define como la probabilidad de que esa determinada tasa de transmisión de datos no pueda ser transmitida por el canal, y viene dada por $(p / 100)$.

En esta simulación, esta probabilidad de outage se traduce en la probabilidad de que el algoritmo JPCOB-VUL, dado una valor de SINR umbral común a todos los usuarios, una restricción en potencia transmitida por BS y número máximo de iteraciones, no pueda dar servicio a $M=3$ usuarios co-canal.

Las 3000 realizaciones de cada simulación se realizaron en 15 grupos de 200 realizaciones cada uno, para disminuir la desviación estándar de los resultados. Las figuras 4.10 y 4.11 representan la tasa de fallo del algoritmo JPCOB-VUL en función de la ventana de AS para los distintos valores de SINR umbral [83].

A partir de las figuras, se observa que como tendencia general, la tasa de fallo disminuye para todas las configuraciones de sistema y parámetros de simulación al disminuir la SINR umbral requerida, como es lógico esperar.

Tomando como referencia el número total de antenas en el sistema, $K \cdot N_{t}$, se observa que para un número similar de antenas en el sistema (2BS-4ANT, 3BS-3ANT), la diversidad espacial que proporciona el hecho de distribuir 3 BSs mejora significativamente la tasa de fallo para todos los umbrales de SINR, aumentando por tanto la capacidad del sistema, frente al caso de considerar un sistema con 2 BSs.

Por el contrario, añadir una antena a cada BS en la configuración de 2 BSs (pasar de 2BS-3ANT a 2BS-4ANT) no representa ninguna mejora para un valor elevado de SINR umbral (figura 4.10), mientras que sí puede ser útil para servicios con un requerimiento de baja calidad de servicio (figura 4.11).

Respecto a la influencia del tamaño de ventana de AS, se puede observar que las configuraciones de 2 y 3 BSs se comportan de forma diferente. Las configuraciones de $2 \mathrm{BSs}$, en general, mejoran su comportamiento a medida que se permite una mayor coordinación entre ellas. Por el contrario, en la configuración de $3 \mathrm{BSs}$, parece que la mejor elección de ventana de AS queda entre los valores $\mathrm{ASW}=5 \mathrm{~dB}$ y $\mathrm{ASW}=15 \mathrm{~dB}$, aunque la mejora de la tasa de fallo entre transmisión coordinada y no coordinada $(\mathrm{ASW}=0 \mathrm{~dB})$ 

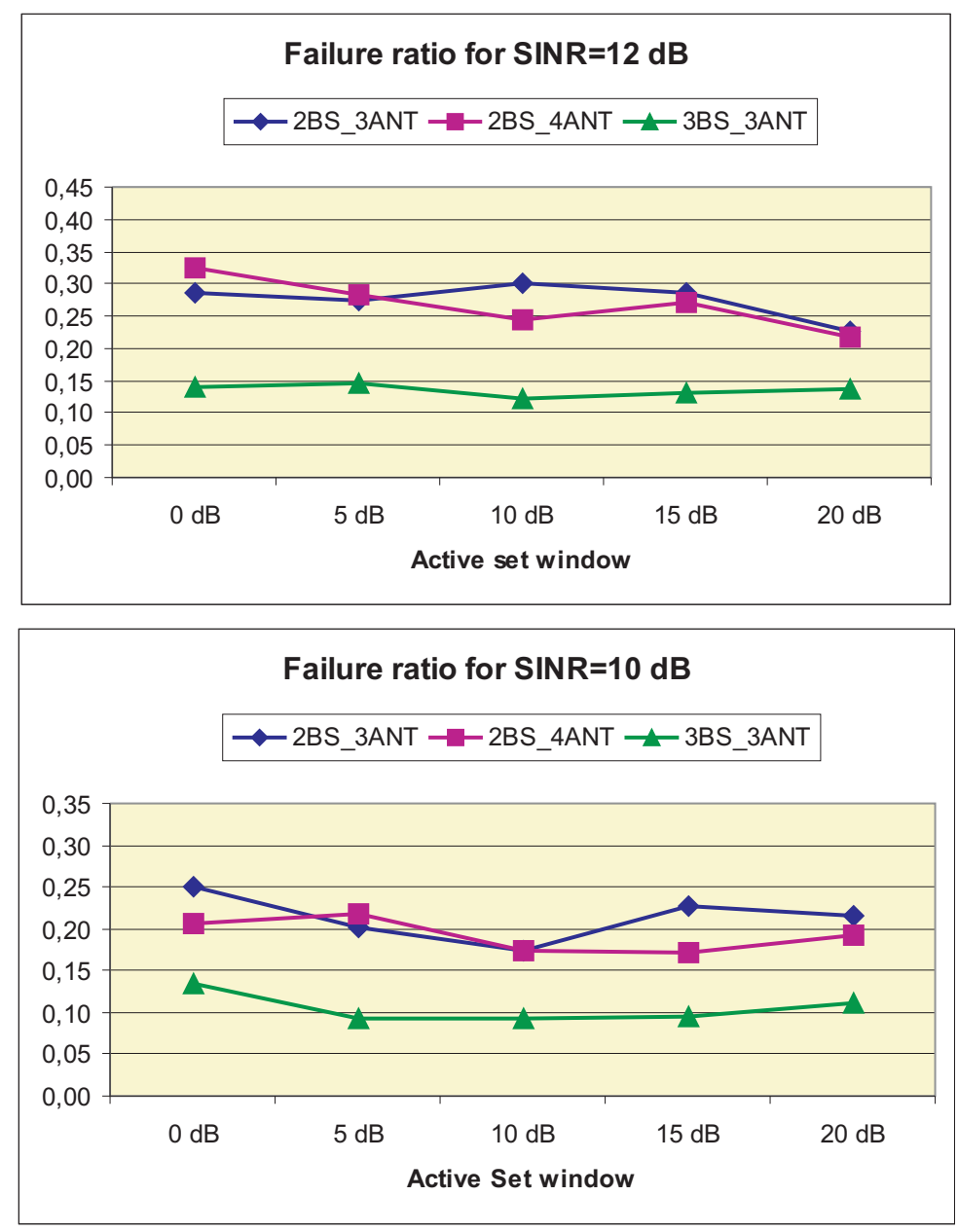

Figura 4.10: Tasa de fallo para una SINR umbral de 12 y $10 \mathrm{~dB}$.

no es muy pronunciada. Esta conclusión coincide con la presentada en [95], donde se establece que tener 3 BSs en el active set sólo aumenta en un $4 \%$ la capacidad de la red.

La figura 4.12 muestra la distribución de la potencia total transmitida en DL para todas las configuraciones de sistema de la tabla 4.3 y tamaños de ventana de AS, cuando la SINR umbral se fija en 12 y 6 dB [83]. Se puede observar una pequeña variación de las curvas sobre la media, pero ninguna de ellas presenta un comportamiento particularmente diferente. 

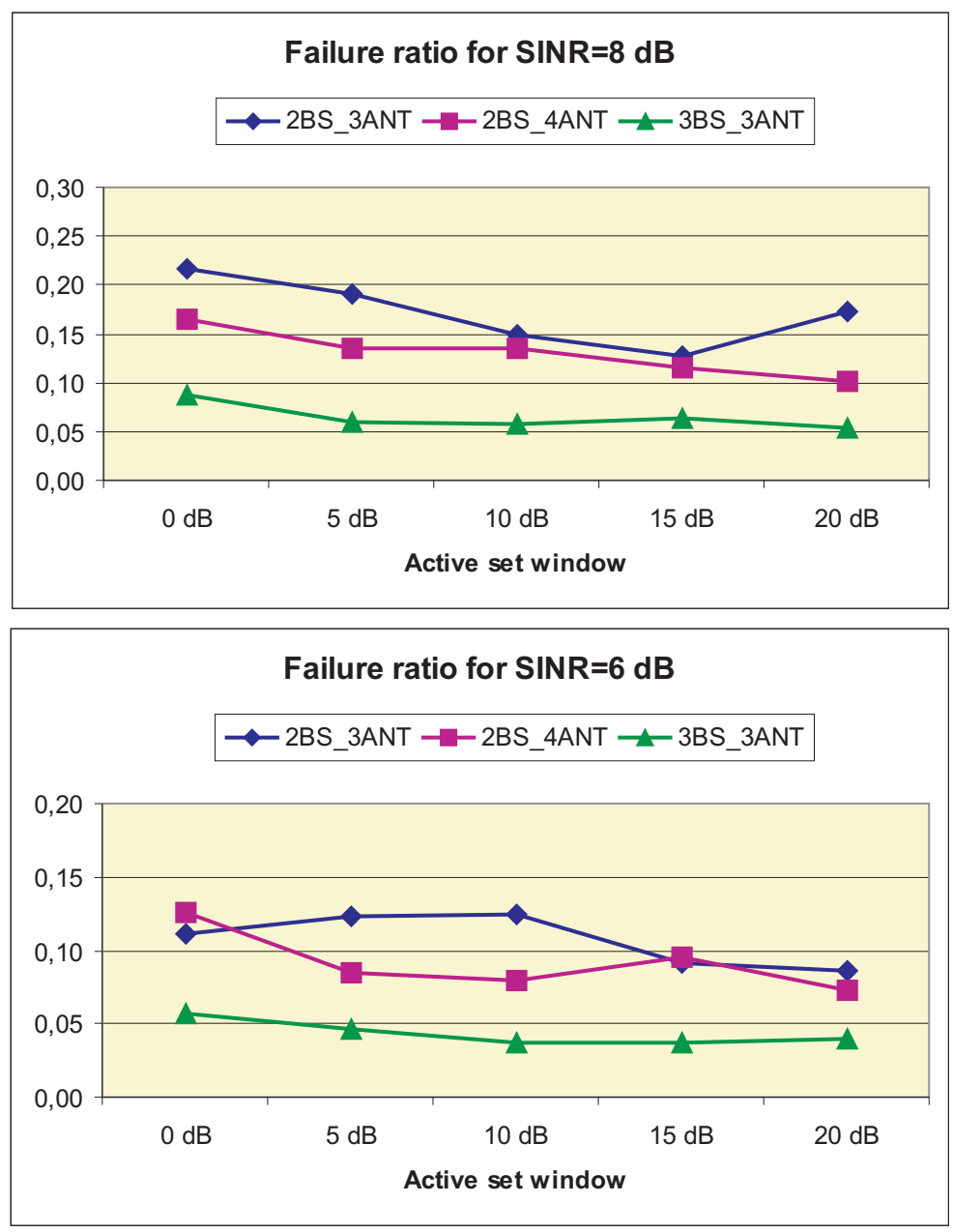

Figura 4.11: Tasa de fallo para una SINR umbral de 8 y 6 dB.

Estos resultados parecen indicar que el hecho de permitir coordinación entre BSs no está aumentando significativamente la potencia total transmitida en el sistema, de forma que el control de potencia centralizado del algoritmo JPCOB-VUL está asignando eficientemente la potencia disponible de las BSs incluidas en el active set de cada usuario.

Por último, la figura 4.13 muestra la composición en porcentaje de enlaces activos del active set de los usuarios en función del valor de ventana de AS para las configuraciones de $K=2$ y $K=3$ BSs [83]. Esta composición 

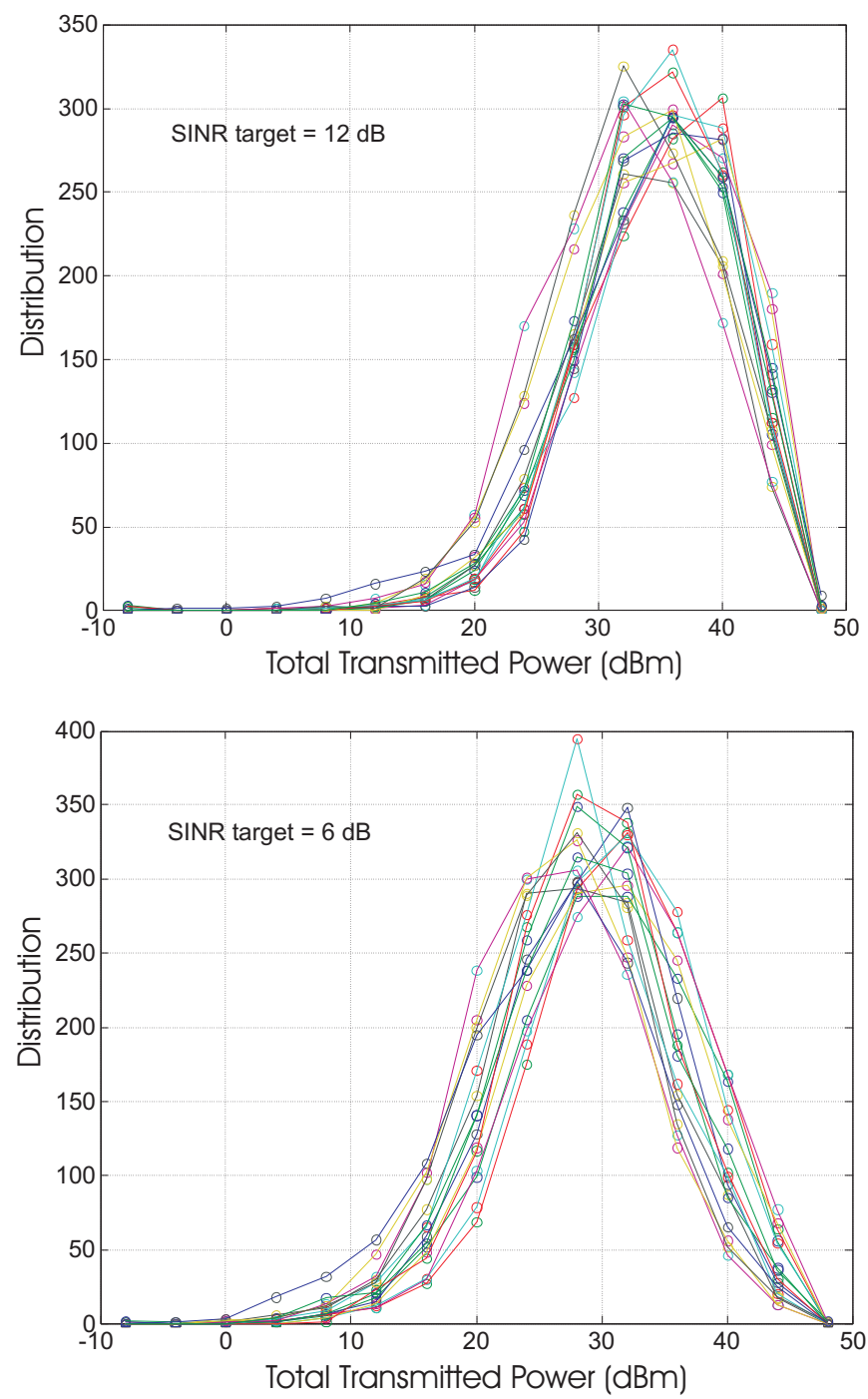

Figura 4.12: Distribución de la potencia total transmitida en DL para todas las configuraciones de sistema y tamaños de ventana de AS para la SINR umbral de 12 y $6 \mathrm{~dB}$.

no varía al cambiar el valor de la SINR umbral, ni el número de antenas de la BS, puesto que la coordinación en el sentido de enlaces activos depende 

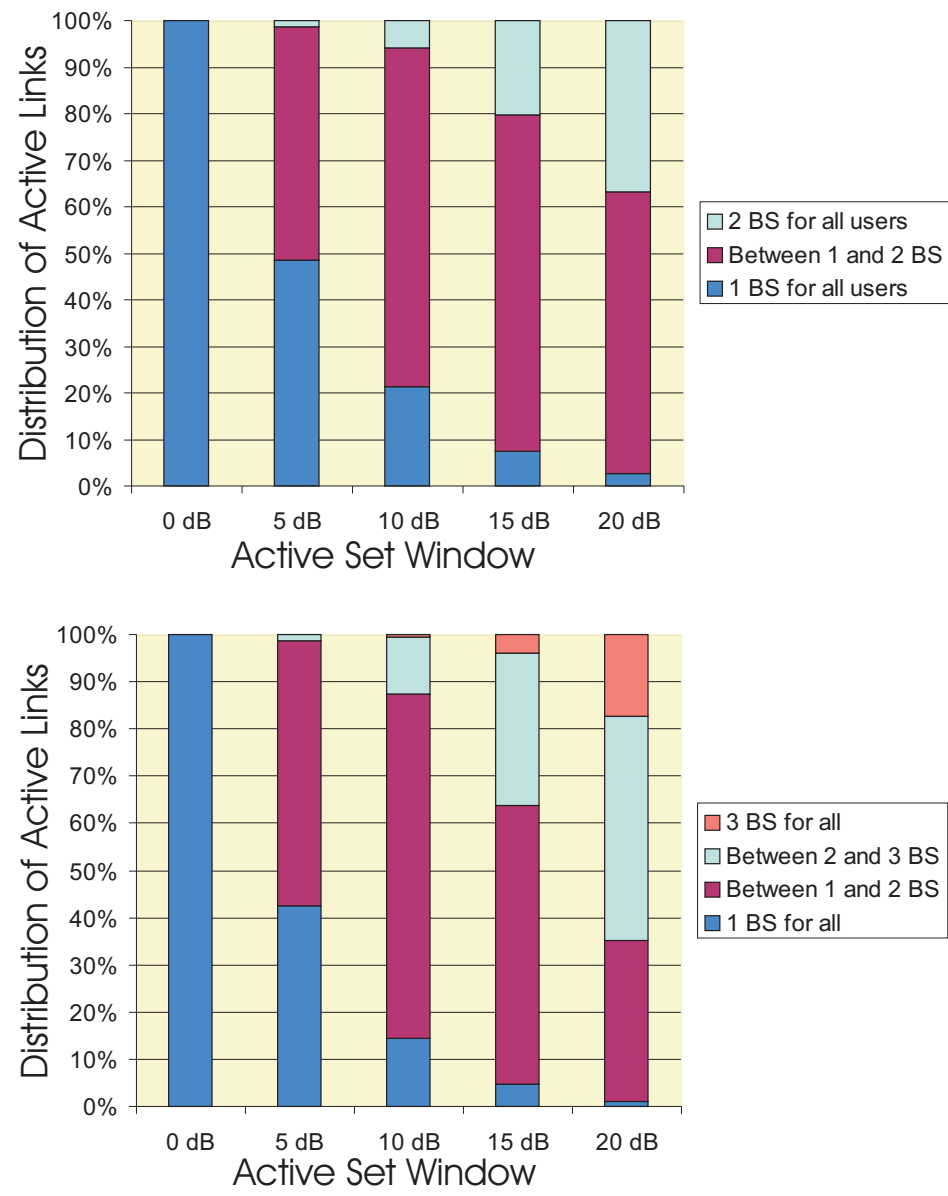

Figura 4.13: Porcentaje de enlaces activos para el conjunto de todas las simulaciones y configuraciones de sistema de $K=2$ y $K=3$ BSs.

del tamaño de ventana de AS y no de la SINR umbral requerida. 


\section{Estudio de la tasa de fallo, comparativa con una configuración de celda convencional}

En esta tercera simulación, se extiende el análisis de la tasa de fallo de la simulación anterior y se realiza una comparativa entre dos propuestas diferentes. Por una parte, se simula el algoritmo JPCOB-VUL sobre una configuración de sistema CM3, y por otra, se simula el algoritmo de referencia de Rashid-Farrokhi et al. [33], sobre una configuración de celda sectorizada convencional, donde el algoritmo se ejecuta de forma independiente en cada uno de los sectores de la celda (ver figura 4.4). La tabla 4.4 resume el número de BSs, $K$, y el número de antenas por array, $N_{t}$, de cada configuración, mientras que los parámetros de simulación siguen siendo los de la tabla 4.1.

\begin{tabular}{|c|c|c|c|}
\hline \hline Configuración & BSs & Antenas & Configuración \\
\hline \hline 3BS-3ANT & 3 & 3 & CM3 \\
\hline 2BS-4ANT & 2 & 4 & CM3 \\
\hline 2BS-3ANT & 2 & 3 & CM3 \\
\hline 3BS-3ANT-C & 3 & 3 & central \\
\hline 2BS-4ANT-C & 2 & 4 & central \\
\hline
\end{tabular}

Cuadro 4.4: Configuraciones simuladas.

En este punto, interesa recordar que las dos configuraciones de sistema son equivalentes, las BSs están sujetas a la misma restricción en potencia $P_{\max }$, la única diferencia es que en la configuración de sistema CM3, las BSs transmiten coordinadamente hacia el área compartida, y en la configuración de celda convencional, las BSs se ubican en el centro del área y transmiten de forma independiente a cada sector de la celda.

De nuevo, el objetivo de esta simulación es observar cómo varían las prestaciones del algoritmo JPCOB-VUL en función de los parámetros de SINR umbral y ventana de AS. Sin embargo, ahora además se comparan los resultados de dos configuraciones de sistema totalmente distintas, una configuración CM3 que permite coordinación entre BSs y una configuración convencional equivalente sin coordinación.

En cada simulación, el algoritmo JPCOB-VUL y el JPCOB de referencia [33], intentan dar servicio a $M=3$ usuarios co-canal, cada uno sobre su configuración de sistema correspondiente. Los usuarios se distribuyen uniformemente sobre cada configuración de sistema y se asume una velocidad constante para cada uno. Se considera que cada algoritmo converge cuando 
todos los usuarios del sistema alcanzan la SINR umbral requerida, dentro de un máximo de $I t$ iteraciones del algoritmo y sin que las BSs superen la restricción en potencia total transmitida $P_{\max }$. La figura 4.20 muestra el diagrama de bloques de la simulación del algoritmo JPCOB-VUL.

Como en las simulaciones anteriores, una simulación se define mediante un valor de ventana de AS y un valor de SINR umbral, común a todos los usuarios del sistema. Para cada simulación, se realizan 3000 realizaciones independientes:

- Ventana de AS: 0 dB (sin coordinación), 5 dB, 10 dB, 15 dB y 20 dB.

- SINR umbral: $6 \mathrm{~dB}, 8 \mathrm{~dB}, 10 \mathrm{~dB}$ y $12 \mathrm{~dB}$.

El parámetro que evalúa las prestaciones de los dos algoritmos sigue siendo la tasa de fallo, definida como el número de realizaciones en las cuales un algoritmo no converge, sobre el número de realizaciones totales. Las figuras 4.14 y 4.15 muestran la tasa de fallo para las distintas configuraciones de sistema de la tabla 4.4 y valores de ventana de AS y SINR umbral $[85,86]$.

Como en la simulación anterior, a partir de las figuras se observa que la tendencia general de la tasa de fallo es a disminuir, para todas las configuraciones de sistema y parámetros de simulación, a medida que disminuye la SINR umbral requerida. Sin embargo, a la hora de interpretar los resultados hay que tener en cuenta que, aunque las dos configuraciones de sistema, la central y la CM3, son físicamente equivalentes (en términos de antenas o potencia disponible por $\mathrm{BS}$ ), la potencia de interferencia que recibe un usuario en cada sistema es diferente.

En principio, el algoritmo de referencia de Rashid-Farrokhi et al. se ejecuta de forma independiente en cada uno de los sectores de la configuración central de sistema, es decir, que en cada sector, un usuario únicamente recibe potencia de interferencia si existe más de un usuario asignado a la misma BS. Por el contrario, en el sistema CM3, aunque se esté considerando una ventana de AS de $0 \mathrm{~dB}$, donde sólo una BS transmite a cada usuario, debido a la distribución espacial de las BSs, un usuario recibe la potencia de interferencia correspondiente al resto de usuarios del sistema. Además, los beamformers que el algoritmo JPCOB-VUL diseña en cada BS tienen que considerar a los $M$ usuarios del sistema, mientras que en la configuración central, los beamformers se diseñan para cada sector y por tanto, sólo tienen que considerar a los usuarios asignados a ese sector. 

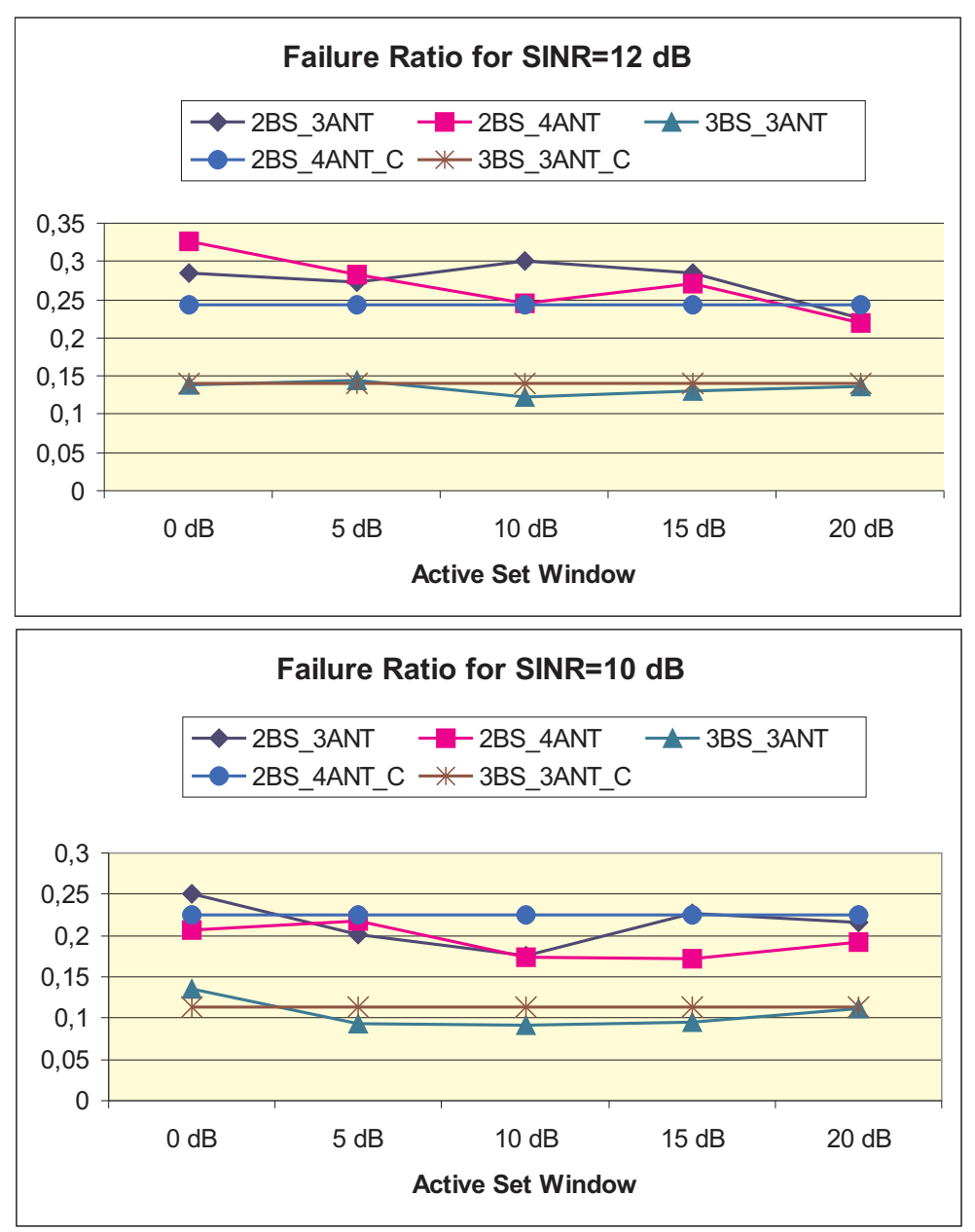

Figura 4.14: Tasa de fallo para una SINR umbral de 12 y $10 \mathrm{~dB}$.

Teniendo en cuenta estas diferencias, se observa que, para configuraciones de sistema con $K=2 \mathrm{BSs}$, y para requerimientos de baja y media SINR umbral (figura 4.15), el algoritmo JPCOB-VUL mejora la tasa de fallo con respecto al algoritmo de referencia para un mismo número de antenas por BS (2BS-4ANT, 2BS-4ANT-C); sin embargo, la restricción en potencia de las BSs impide que para valores elevados de SINR umbral se repita este comportamiento (figura 4.14). En este caso, el hecho de que cada BS de la configuración CM3 disponga de un array de antenas tal que $N_{t}>M$, pro- 

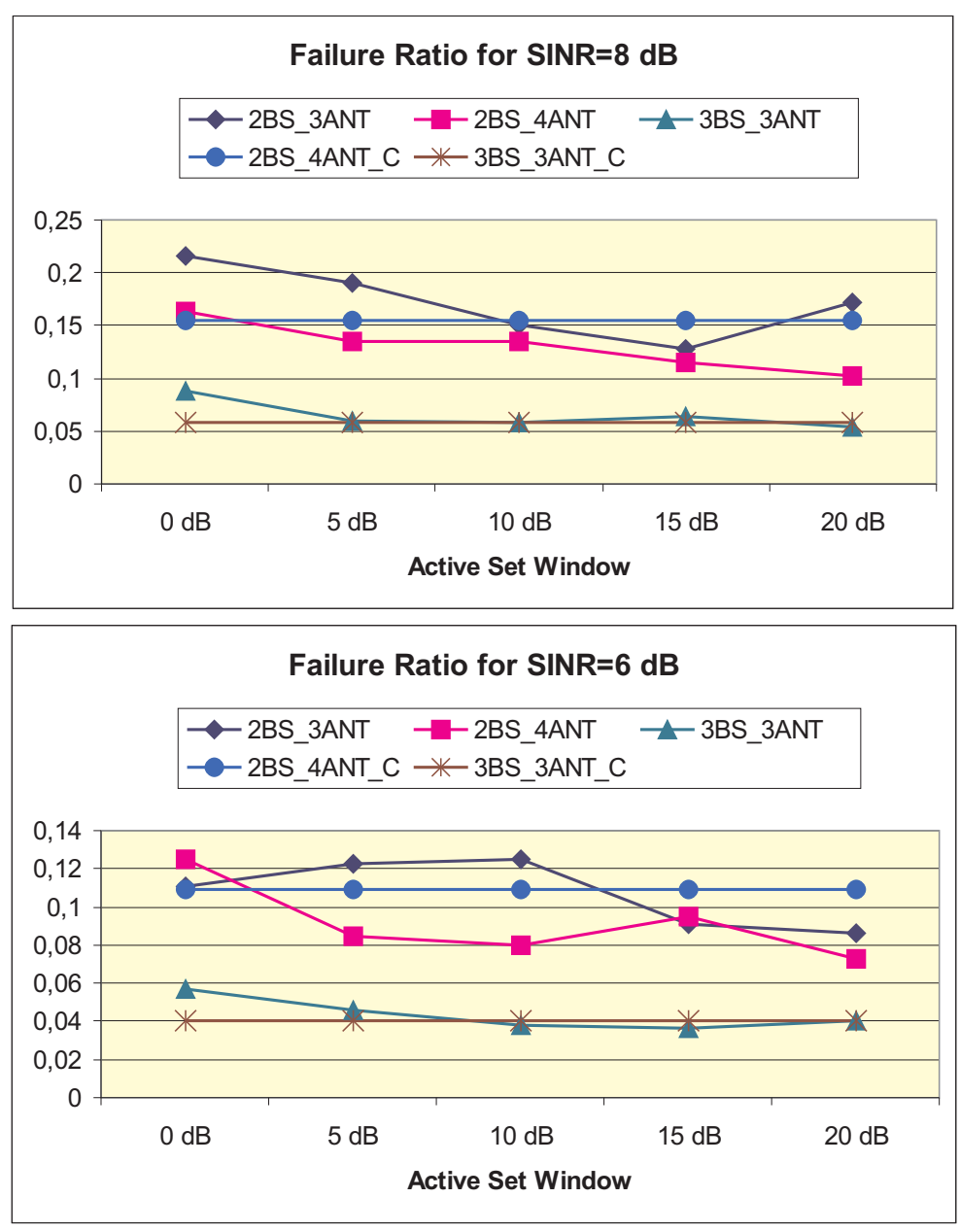

Figura 4.15: Tasa de fallo para una SINR umbral de 8 y 6 dB.

porciona los suficientes grados de libertad para mitigar la mayor potencia de interferencia que reciben los usuarios de la configuración CM3.

Por el contrario, para las configuraciones de sistema con $K=3 \mathrm{BSs}$ (3BS-3ANT, 3BS-3ANT-C), la configuración CM3 dispone de $N_{t}=M$ antenas en cada BS. Esto, junto con la mayor potencia de interferencia presente en esta configuración de sistema, provoca que las diferencias entre la tasa de fallo para ambas configuraciones no sean muy significativas. Aún así, las figuras muestran como el hecho de permitir cooperación entre las 
BSs de la configuración CM3, consigue mejorar ligeramente la tasa de fallo con respecto a los resultados de la configuración central.

La figura 4.16 muestra la distribución de la potencia total transmitida en DL para la configuración central y CM3 cuando se dispone de $K=3$ BSs, equipadas con un array de $N_{t}=3$ antenas cada una, para valores de SINR umbral de 12 y $6 \mathrm{~dB}$. Con respecto a los resultados del algoritmo JPCOBVUL sobre la configuración de sistema CM3, esta figura confirma de nuevo los resultados de la simulación anterior (figura 4.12), puesto que se observa una ligera variación de las curvas correspondientes a distintas ventanas de AS, pero en principio parece que el hecho de permitir que varias BSs transmitan coordinadamente $(\mathrm{ASW}>0 \mathrm{~dB}$ ) no aumenta significativamente la potencia total transmitida en la configuración de sistema CM3.

Comparando ahora los resultados de la configuración CM3 y central, se puede apreciar que la potencia total transmitida en la configuración central es ligeramente menor para el valor de SINR umbral de $6 \mathrm{~dB}$, mientras que esta diferencia disminuye para la SINR umbral de $12 \mathrm{~dB}$. De hecho, si se compara numéricamente la potencia total transmitida por el algoritmo JPCOB-VUL con ASW $=0 \mathrm{~dB}$ con la potencia total transmitida en la configuración central, se observa que la configuración central proporciona una reducción de entre el 4 y el 14\% (según sea el valor de la SINR umbral) en la potencia total transmitida $[85,86]$. Esto es beneficioso para esta única celda, pero hay que destacar que se trata de una potencia radiada hacia el exterior de la celda, mientras que en la configuración CM3 la potencia se radia hacia el área interior, transmitiendo más potencia para combatir el aumento de interferencia que esto supone, pero disminuyendo al mismo tiempo la potencia interferente radiada hacia otras celdas. 

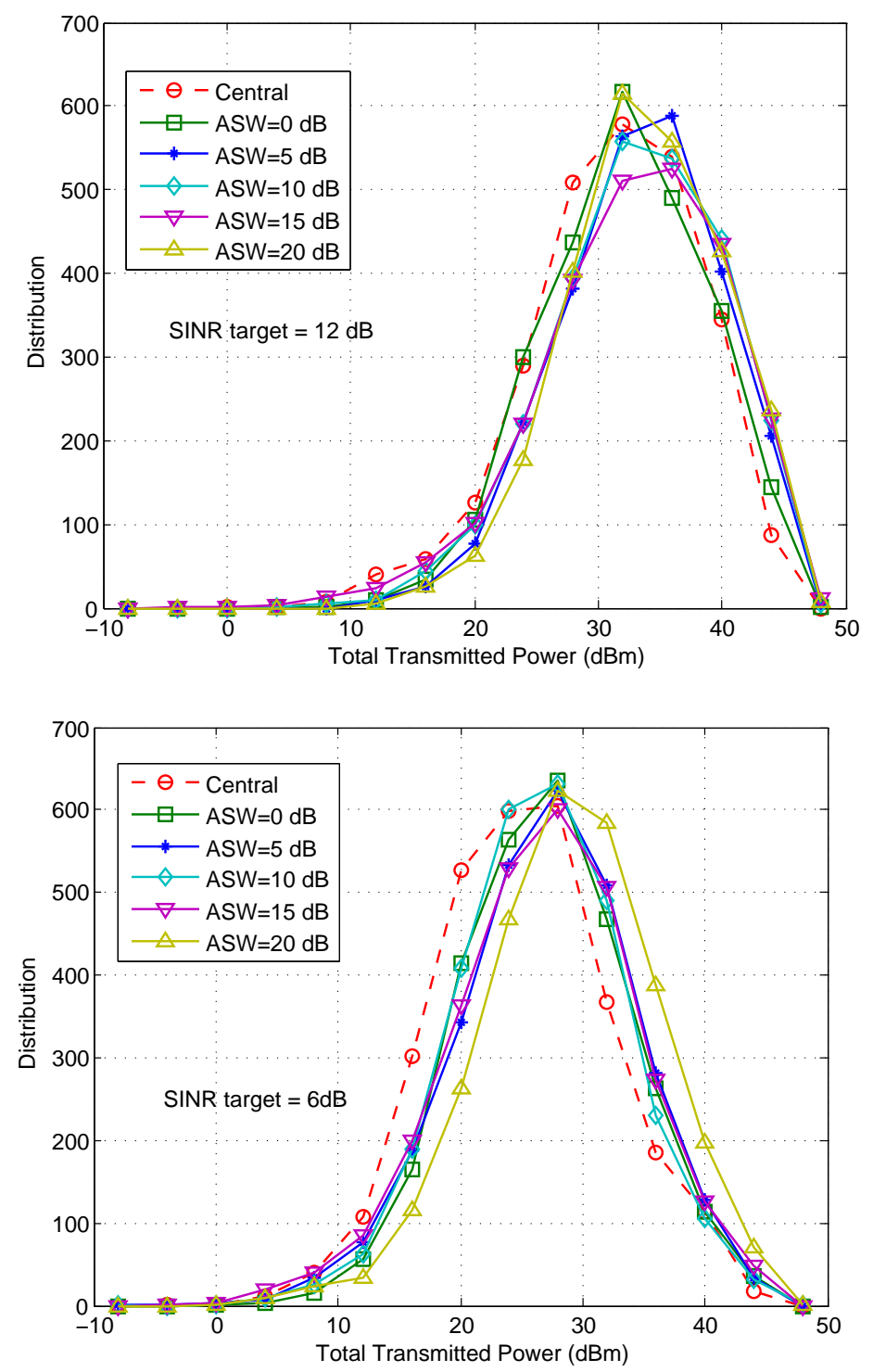

Figura 4.16: Distribución de la potencia total transmitida en DL para las configuraciones de sistema central y CM3 con $K=3 \mathrm{BSs}, N_{t}=3$. Resultados para la SINR umbral de 12 y 6 dB. 


\subsubsection{Algoritmo JPCOB-VUL: entorno Coordinado II}

En este apartado se evalúan las prestaciones del algoritmo JPCOBVUL en el entorno Coordinado II, donde la selección de enlaces activos en el sistema CM3 se realiza en función del módulo de los beamformers en transmisión. La versión elegida del algoritmo JPCOB-VUL es la solución semi-coordinada de (4.49)-(4.51). Además, se incluye el cálculo eficiente de la pseudo-inversa de la matriz D mediante (4.40).

\section{Comparativa con otros algoritmos JPCOB}

En esta primera simulación, el objetivo es comparar, sobre la misma configuración de sistema $\mathrm{CM} 3$, las prestaciones de la versión semi-coordinada y eficiente del algoritmo JPCOB-VUL, con dos algoritmos JPCOB que no permiten coordinación entre BSs. Uno de ellos es el algoritmo JPCOB de referencia de Rashid-Farrokhi et al. [33], donde la asignación de usuarios a BSs se realiza a partir de la EbNo (energía media de bit respecto a densidad espectral de ruido más interferencias) evaluada en el usuario, lo que equivale a asignar los usuarios con la BS cuyo canal piloto se recibe con mayor potencia, mientras que el otro algoritmo, propuesto por Bengtsson, incluye una asignación óptima de usuarios a BSs desde el punto de vista del criterio de minimización de potencia [90].

El sistema CM3 sobre el que se comparan los algoritmos JPCOB está formado por $K=3 \mathrm{BSs}$, donde cada BS dispone de un array lineal de $N_{t}=4$ antenas. Los parámetros de simulación son los de la tabla 4.1 y el valor que se establece como mínimo para considerar que un enlace está activo es de $\left\|\mathbf{w}_{m k}\right\|^{2}=5 \cdot 10^{-3}$, lo que equivale a considerar inactivos aquellos enlaces cuyo beamformer en transmisión aporte una contribución en potencia inferior a $6 \mathrm{dBm}$.

La figura 4.19 muestra el diagrama de bloques de la simulación del algoritmo JPCOB-VUL. Para cada algoritmo, el sistema empieza ubicando un único usuario en el sistema y sigue añadiendo usuarios mientras el algoritmo converja, es decir, mientras en cada uno de los usuarios se alcance la SINR umbral requerida en DL, sin superar el máximo de iteraciones permitidas, $I t$, y la restricción en potencia transmitida en cada BS, $P_{\max }$. La distribución de la posición inicial de cada usuario es uniforme y cada usuario posee una velocidad constante. 
El objetivo es comparar el número medio de usuarios co-canal admitidos por los tres algoritmos en función del valor de la SINR umbral, común a todos los usuarios, que en este caso se establece en los valores $5,8,11 \mathrm{y}$ 14 dB. Cada simulación se promedia sobre 200 realizaciones independientes.

La figura 4.17 muestra el número medio de usuarios co-canal admitidos y la potencia media total transmitida en el sistema en función de distintos umbrales de SINR para los tres algoritmos JPCOB [84]. La barra No Opt. BS assig. se corresponde con los resultados del algoritmo de RashidFarrokhi et al. [33], la de Coop. BS Tx. con los del algoritmo JPCOB-VUL semi-coordinado y la de Opt. BS assig. representa los resultados del algoritmo propuesto por Bengtsson [90].

Respecto al número de usuarios co-canal admitidos, en la gráfica se observa que el algoritmo JPCOB-VUL semi-coordinado consigue unos resultados intermedios para valores de SINR umbral medios y bajos. Para un umbral alto de SINR, la limitación en potencia de las BSs impide que el algoritmo admita a un mayor número de usuarios.

Si se analiza la potencia media total transmitida en el enlace DL del sistema, se observa que los resultados son similares para los tres algoritmos, lo que permite concluir, de forma similar a las anteriores simulaciones, que aunque la transmisión coordinada implica que un mayor número de BSs estén transmitiendo simultáneamente en el sistema, el control de potencia del algoritmo JPCOB-VUL consigue asignar de forma eficiente la potencia disponible de las BSs que transmiten a cada usuario, y no se transmite más potencia que en las transmisiones no coordinadas.

La figura 4.18 muestra el número medio de iteraciones que necesita cada algoritmo para converger en función de la SINR umbral [84]. A partir de la figura, se observa que el algoritmo JPCOB-VUL converge de forma más rápida que el algoritmo sin asignación óptima de [33], y mejora ligeramente la convergencia del algoritmo que incluye la asignación óptima de usuarios a BSs [90]. 

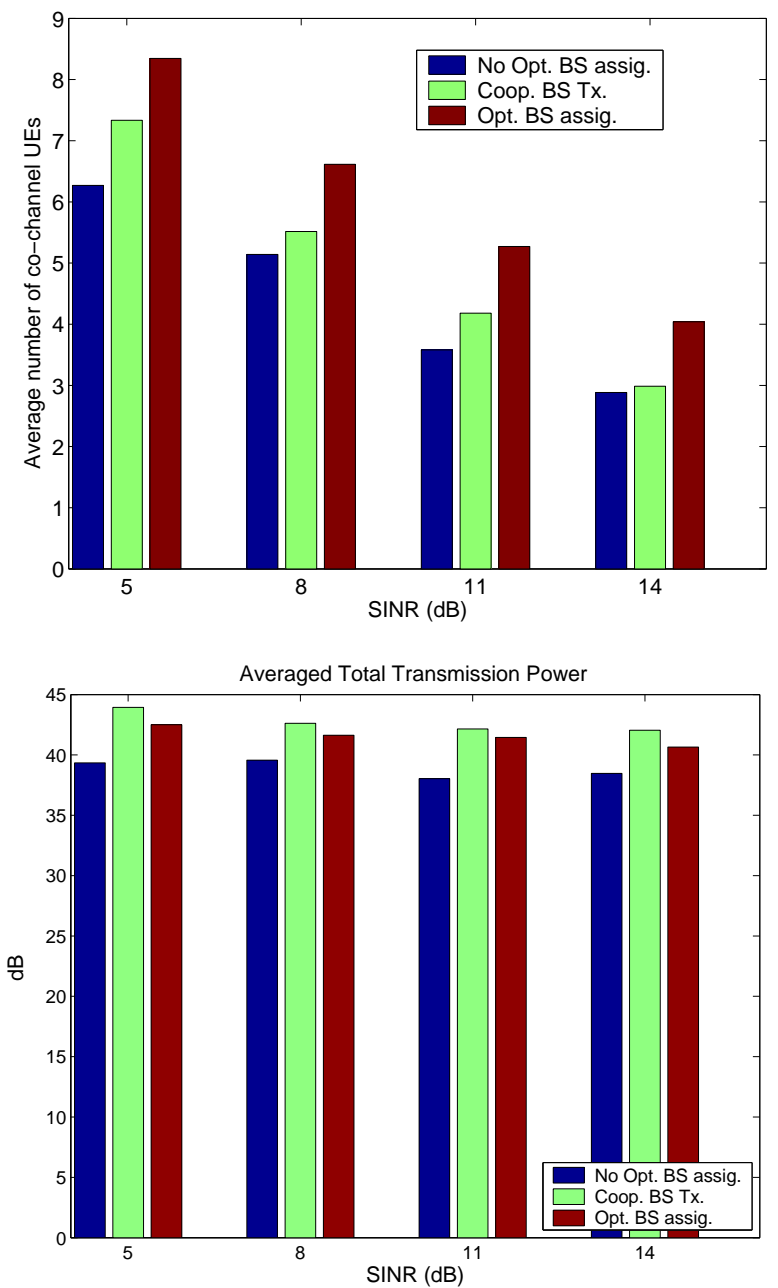

Figura 4.17: Número medio de usuarios co-canal admitidos y potencia media total transmitida en el sistema por los tres algoritmos JPCOB para distintos valores de SINR umbral. 


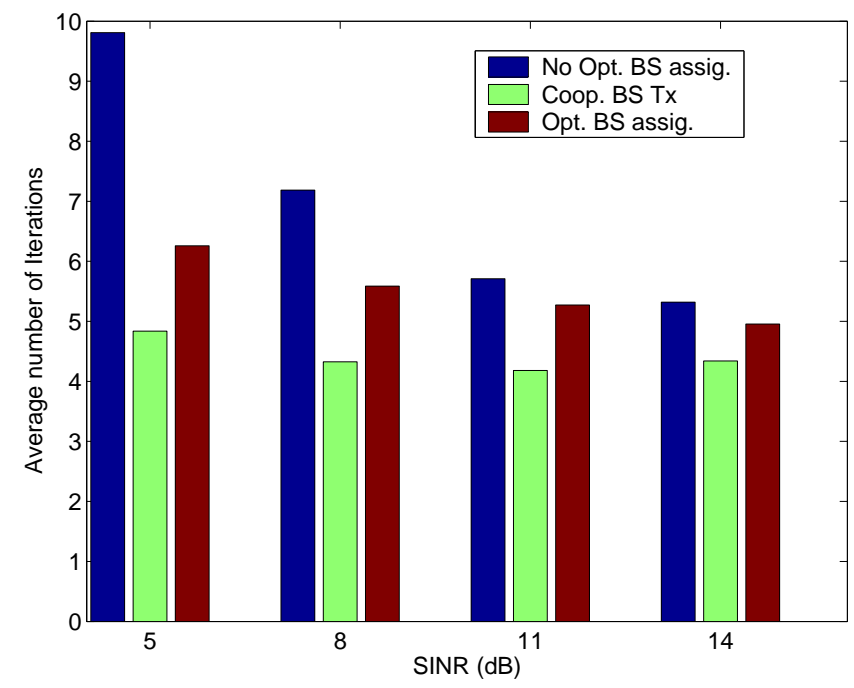

Figura 4.18: Número medio de iteraciones necesarias para converger para distintos valores de SINR umbral. 


\section{Estudio de la complejidad computacional}

Los resultados de la simulación anterior demuestran que la versión semicoordinada del algoritmo JPCOB-VUL presenta un comportamiento intermedio en términos de capacidad, sin aumentar de forma significativa la potencia total transmitida en el sistema. Además, es el algoritmo que más rápidamente converge.

En esta segunda simulación, el objetivo es comparar la complejidad computacional de los algoritmos que obtuvieron mejores resultados en la simulación anterior, en este caso, el algoritmo JPCOB-VUL semi-coordinado con cálculo eficiente de la pseudo-inversa de la matriz D y el algoritmo JPCOB propuesto por Bengtsson que incluye asignación óptima de usuarios a BSs [90]. El parámetro elegido para comparar la complejidad de los tres algoritmos es el número de operaciones MAC (Multiply and Accumulate) que necesita cada uno de ellos.

La tabla 4.5 resume el número de operaciones MAC que utilizan en cada paso el algoritmo de asignación óptima de usuarios a BSs de [90] y el algoritmo JPCOB-VUL semi-coordinado y eficiente, si el sistema CM3 se compone de $M$ usuarios y $K$ BSs, cada una equipada con un array de $N_{t}$ antenas [84].

\begin{tabular}{|l|c|c|}
\hline \hline & $\begin{array}{c}\text { Asignación óptima } \\
\text { de BS }[90]\end{array}$ & $\begin{array}{c}\text { JPCOB-VUL } \\
(4.49)-(4.51)\end{array}$ \\
\hline \hline Beamformers & $\left(N_{t}+2 N_{t}^{2}\right) K M$ & $\left(N_{t}+2 N_{t}^{2}\right) K M$ \\
\hline Potencias UL virtuales & $2 M$ & $(1+2 K+2 K M) M$ \\
\hline Potencias DL & $M N_{t}+M^{2}+M^{3}$ & $2 K\left(M^{2}\right)$ \\
\hline
\end{tabular}

Cuadro 4.5: Número de operaciones MAC.

Los dos algoritmos necesitan un número similar de operaciones para el cálculo de los beamformers. Sin embargo, en la actualización de las potencias UL virtuales, el algoritmo JPCOB-VUL semi-coordinado necesita del orden de $K M$ operaciones más que el algoritmo de asignación óptima propuesto por Bengtsson. Este coste adicional se debe al cálculo eficiente de la pseudo-inversa de la matriz $\mathbf{D}$ en el enlace UL virtual (4.50), mientras que el algoritmo propuesto por Bengtsson realiza una actualización de las potencias UL virtuales mediante el control de potencia clásico distribuido de [88], similar a la expresión (4.32). 
Sin embargo, hay que destacar que el paso de actualización de las potencias DL de la versión semi-coordinada del algoritmo JPCOB-VUL (4.51), utiliza exactamente la misma pseudo-inversa de la matriz $\mathbf{D}$ que el paso de actualización de las potencias UL virtuales, por lo que el coste computacional de la actualización de las potencias DL se simplifica notablemente. De hecho, teniendo en cuenta que un sistema de comunicaciones lo habitual es que el número de BSs $K$ permanezca constante, y aumente el número de usuarios $M$, la actualización de potencias DL del algoritmo de Bengtsson aumentaría con $M^{3}$, mientras que la del algoritmo JPCOB-VUL semicoordinado lo haría con $M^{2}$, compensando en cierta forma el coste adicional que se introduce en la actualización de las potencias UL virtuales.

Por otro lado, la capacidad de reconfiguración que ofrece el algoritmo JPCOB-VUL y la gestión de las situaciones de handover ya se contabilizan en las operaciones, mientras que en el algoritmo de asignación óptima de Bengtsson, habría que añadir a los valores mostrados en la tabla, el coste de gestionar los posibles handovers internos dentro del área compartida por las BSs del sistema CM3. 


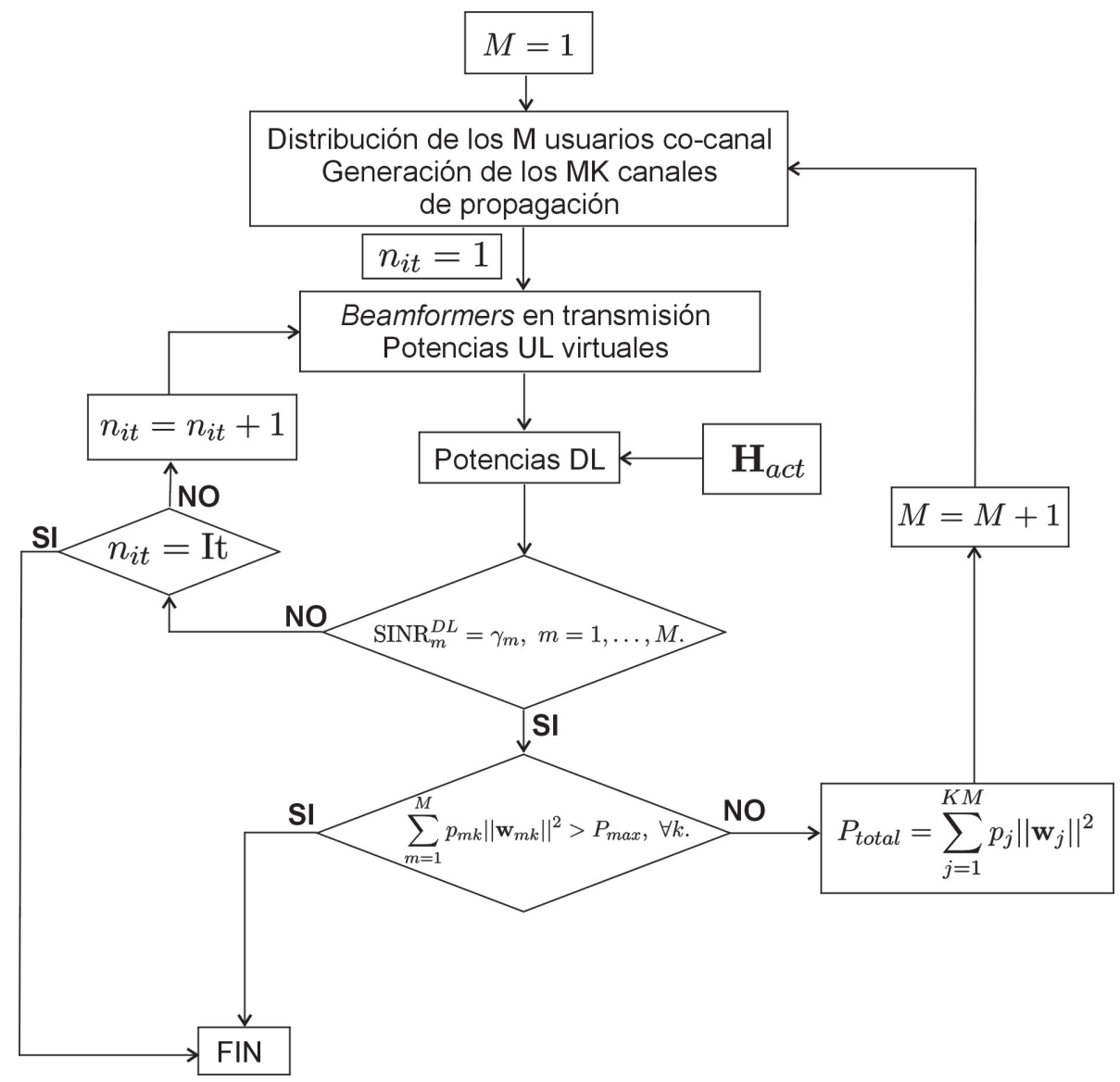

Figura 4.19: Diagrama de bloques de la simulación que evalúa el número medio de usuarios co-canal admitidos por el algoritmo JPCOB-VUL. 


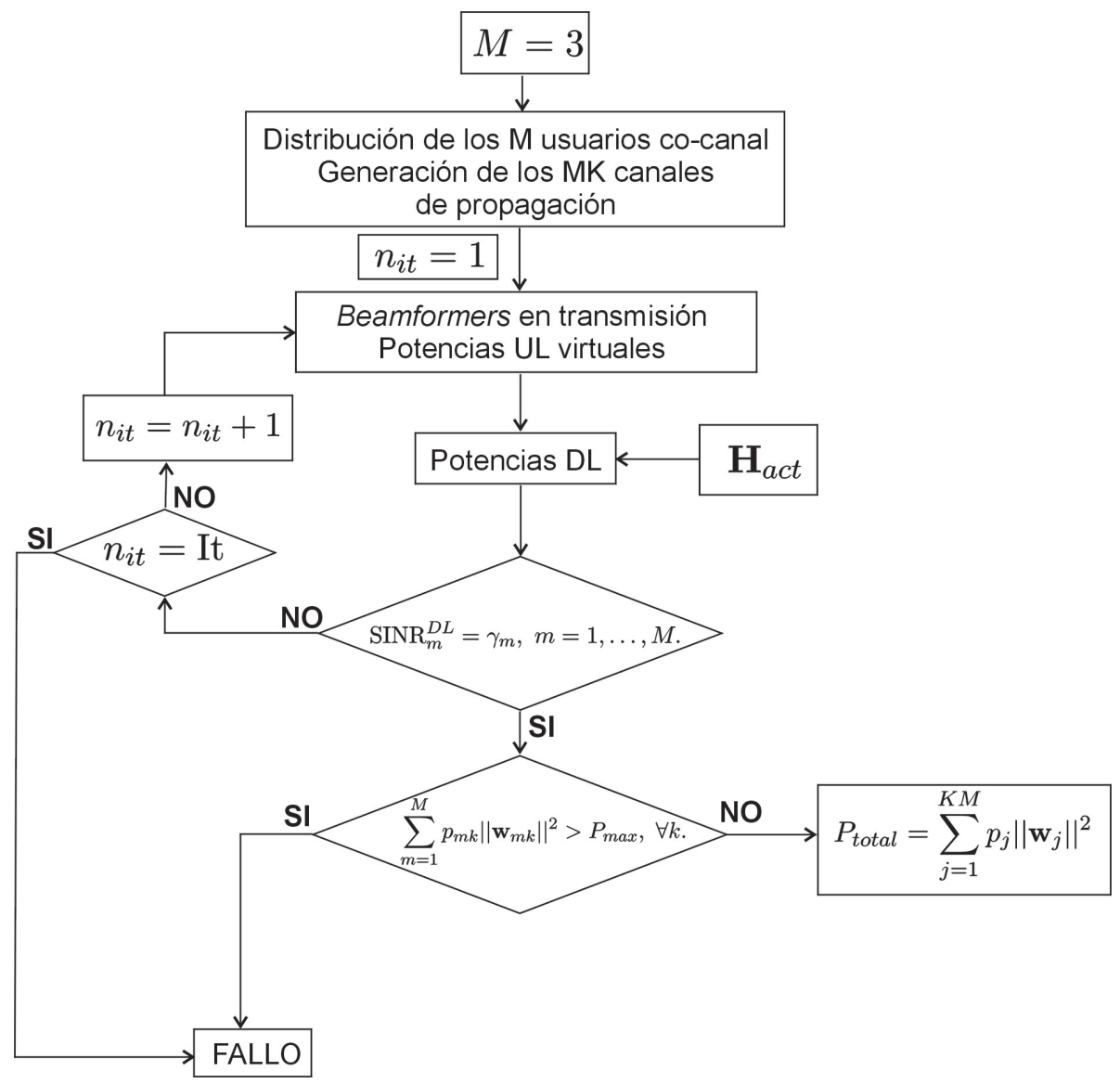

Figura 4.20: Diagrama de bloques de la simulación que evalúa la tasa de fallo del algoritmo JPCOB-VUL. 



\section{Capítulo 5}

\section{Algoritmo JPCOB-VUL: aplicaciones prácticas}

Hasta este punto del desarrollo de la Tesis, las prestaciones del sistema W-CDMA MIMO multi-usuario multi-celda coordinado (CM3) se han analizado considerando que los usuarios son usuarios co-canal. Además, en el modelo de sistema presentado en el Capítulo anterior, se simplificaba la situación en recepción. Recordando la expresión de la señal recibida por un usuario $m,(4.4)$, simplemente se ignoraban las cuestiones de sincronismo y se suponía que el usuario podía, de alguna forma, extraer perfectamente tanto los $K$ términos de señal deseada como los términos de interferencia co-canal.

En un sistema CM3 real, los $M$ usuarios se asocian con distintos códigos de canalización. La zona compartida por las estaciones base (figura 4.2) se comporta a todos los efectos como una única celda, de forma que las $K$ estaciones base (BSs) utilizan el mismo código de scrambling y un usuario consume un único código de canalización en todas las BSs.

Los códigos de canalización utilizados en UMTS son los códigos OVSF (Orthogonal Variable Spreading Factor) [96]. Estos códigos son ortogonales entre sí, y por tanto anulan perfectamente la interferencia entre usuarios, siempre y cuando estén perfectamente sincronizados. Sin embargo, en un sistema CM3, debido a la separación espacial de las BSs, las señales transmitidas por las distintas BSs llegan al usuario en diferentes instantes de tiempo. Aunque los canales entre un usuario y las $K$ BSs se modelen como canales flat fading, el usuario está recibiendo las $K$ señales en instantes de tiempo diferentes, y los códigos OVSF pierden parte de su ortogonalidad. Si 
además los canales son frequency selective, como es habitual en W-CDMA, la propagación multi-camino contribuirá a reducir aún más la ortogonalidad de los códigos OVSF, aumentando considerablemente la interferencia entre los usuarios.

Por tanto, una de las fuentes de interferencia más importante en los sistemas W-CDMA CM3 se corresponde con la interferencia de acceso múltiple o MAI (Multiple Access Interference). Habitualmente, este tipo de interferencia se asume Gaussiana y se modela mediante un factor de ortogonalidad.

El estudio de los sistemas CM3 se encuentra todavía en su fase inicial. En general, los estudios previos sobre sistemas CM3, como los presentados en la primera Parte de la Tesis, asumen que las señales procedentes de las $K$ BSs llegan perfectamente sincronizadas a cada usuario. Únicamente la referencia [50] presenta un modelo más realista, al considerar que las BSs, al disponer de CSIT perfecta, conocen los retardos de propagación con respecto al usuario, y por tanto pueden transmitir en instantes distintos de forma que las señales de las $K$ BSs lleguen síncronamente al usuario. Sin embargo, los términos de interferencia siguen llegando de forma asíncrona, no es posible compensar en transmisión tanto los términos de señal deseada como los interferentes.

Recientemente, en [76] se propone un modelo matemático para definir el asincronismo de las señales en recepción, y se demuestra la degradación que sufren las técnicas y algoritmos propuestos para sistemas CM3 que no tienen en cuenta este asincronismo en su proceso de diseño.

En este punto de la Tesis, se avanza un paso más en la caracterización del sistema W-CDMA CM3. En concreto, se abandona el concepto de usuarios co-canal y se consideran $M$ usuarios, cada uno asociado con un código de canalización distinto. En este contexto, se introduce en el modelo del sistema CM3 el asincronismo de las señales que llegan a cada usuario y se obtiene un modelo ajustado de las interferencias presentes en sistemas W-CDMA CM3. Además, los parámetros de simulación se modifican para modelar de la forma más realista posible, el peor caso posible de condiciones de propagación en un sistema CM3.

En este Capítulo, se presentan dos aplicaciones prácticas del algoritmo JPCOB-VUL propuesto para sistemas CM3. La primera aplicación plantea esquemas de asignación de códigos de canalización para sistemas CM3 [9799]. Estos esquemas se proponen a partir del nuevo modelo de los términos de interferencia presentes en un sistema W-CDMA CM3, y su propósito 
es mejorar el nivel de interferencia que perciben los usuarios, con el fin de aumentar la capacidad del sistema.

La segunda aplicación, vuelve a considerar usuarios co-canal, pero mantiene los nuevos parámetros de simulación. El objetivo es modificar el algoritmo JPCOB-VUL para afrontar las situaciones en las que las BSs del sistema disponen de CSIT perfecta con respecto a sus canales locales, pero donde sin embargo, existe algún tipo de restricción en la cantidad de información que pueden intercambiar a través del enlace de alta capacidad y sólo disponen de información estadística del resto de canales del sistema [100]. Esta situación se modela mediante una forma de conocimiento parcial del canal denominada hybrid channel knowledge [53,73].

\subsection{Modelado de las interferencias en un sistema W-CDMA MIMO multi-usuario multi-celda coordinado}

En este apartado, se presenta en primer lugar el modelo analítico de un sistema W-CDMA CM3, para el caso general de canales frequency selective para cada enlace usuario-BS del sistema. A partir de este modelo, se repasa brevemente la forma habitual de caracterizar el término de correlación entre códigos en los sistemas MIMO multi-usuario convencionales, para pasar después a modelar el valor de esta correlación en los sistemas CM3. Este modelo de los términos de correlación se utiliza para obtener una caracterización precisa de los términos de potencia de señal deseada y potencia de interferencia presentes en el sistema W-CDMA CM3.

\subsubsection{Modelo de sistema}

Consideramos un sistema W-CDMA CM3 como el de la figura 4.2, donde las $K$ BSs disponen de un array lineal de $N_{t}$ antenas. Al mismo tiempo, se distribuyen $M$ usuarios sobre la zona compartida por las BSs (área sombreada de la figura), cada uno identificado por una secuencia del tipo $\left[c_{m}[1], \ldots, c_{m}[N]\right]$, donde $N$ es el factor de spreading.

De esta forma, el código de canalización de cada usuario $m$ puede escribirse como:

$$
c_{m}(t)=\sum_{n=0}^{N-1} c_{m}[n] p\left(t-n T_{c}\right)
$$


donde $c_{m}[n]$ es el código de canalización, $p(t)$ es la forma del pulso banda base, $N$ es el factor de spreading y $T_{c}$ es el periodo de chip. En principio, se considera que las $K$ BSs del sistema CM3 comparten el mismo código de scrambling, de forma que éste se ignora en el desarrollo analítico del modelo de sistema.

Suponiendo que el sistema utiliza una modulación QPSK, la señal que se transmite a cada usuario se expresaría como:

$$
u_{m}(t)=\sum_{d=-\infty}^{\infty} a_{m}[d] c_{m}(t-d T)
$$

donde $a_{m}[d] \in\left[ \pm \frac{\sqrt{2}}{2} \pm j \frac{\sqrt{2}}{2}\right]$ es la señal de información a transmitir al usuario $m, T=N T_{c}$ es el periodo de símbolo y $E\left[\left|u_{m}(t)\right|^{2}\right]=1$. Las señales $a_{m}[d]$ son a su vez señales de potencia unidad, incorreladas entre sí.

En un entorno frequency selective, los canales de propagación entre cada BS y cada usuario del sistema estarán formados por varias contribuciones multi-camino, de forma que el canal se puede representar como una línea de retardos:

$$
\mathbf{h}_{m k}(t)=\sum_{l_{k}=1}^{L_{m k}} \mathbf{h}_{m k,\left(l_{k}\right)} \delta\left(t-\tau_{l_{k}}\right),
$$

donde $\mathbf{h}_{m k,\left(l_{k}\right)}$ es el vector $\left[1 \times N_{t}\right]$ que representa la contribución $l_{k}$-ésima para el enlace $m k$ usuario-BS y $\tau_{l_{k}}$ es el retardo de propagación asociado a esta contribución multi-camino.

La expresión (5.3) se corresponde con el canal de propagación que recibe un usuario $m$ desde la BS $k$, donde, suponiendo que los retardos de propagación $\tau_{l_{k}}$ son múltiplos enteros del periodo de chip, $\tau_{l_{k}}=l_{k} T_{c}$, el valor máximo del delay spread se corresponde con $L_{m k} T_{c}$.

En general, los canales de propagación entre un usuario y las $K$ BSs del sistema CM3 presentan distintas características y pueden caracterizarse mediante diferentes perfiles de retardo o Power Delay Profile (PDP). En este modelo de sistema, se supone que los canales de propagación entre un usuario y las $K$ BSs van a ser diferentes y por tanto pueden tener un número distinto de contribuciones multi-camino $L_{m k}$. Desde el punto de vista de cada usuario del sistema, es posible definir una especie de PDP compuesto, donde se establecerían las características de las $\sum_{k} L_{m k}$ contribuciones multi-camino que recibe cada usuario. 
Para construir el PDP desde el punto de vista del usuario, es necesario establecer los instantes de llegada de las distintas contribuciones multicamino. Si $L_{m k}^{\prime} T_{c}$ representa el valor máximo del delay spread entre los $K \cdot M$ canales de propagación del sistema, se define el parámetro $L$ de forma que $L=L_{m k}^{\prime}$. De esta forma, a una frecuencia de muestreo igual a la frecuencia de chip, todas las contribuciones multi-camino recibidas por cada uno de los usuarios del sistema se corresponden con un instante de llegada múltiplo del periodo de chip, $\tau_{l} \in\left[T_{c}, L T_{c}\right]$, y la expresión (5.3) se modifica según:

$$
\mathbf{h}_{m k}(t)=\sum_{l=1}^{L} \mathbf{h}_{m k,(l)} \delta\left(t-\tau_{l}\right),
$$

donde determinadas componentes $\mathbf{h}_{m k,(l)}$ serán nulas según sean las características de los $K$ canales de propagación que conforman el PDP visto por el usuario.

Bajo las suposiciones anteriores y teniendo en cuenta que se trata de un sistema CM3, la BS $k$ transmite la señal:

$$
\mathbf{x}_{k}(t)=\sum_{i=1}^{M} \mathbf{w}_{i k} \sqrt{p_{i k}} u_{i}(t),
$$

donde $\mathbf{w}_{i k}$ representa el beamformer en DL de la BS $k$ diseñado para transmitir la señal CDMA $u_{i}(t)$ al usuario $i$ con una potencia $p_{i k}$.

Tras el paso por el canal, la señal recibida en el usuario $m$, procedente de la BS $k$, quedaría:

$$
y_{m k}(t)=\sum_{l=1}^{L} \mathbf{h}_{m k,(l)} \mathbf{x}_{k}\left(t-\tau_{l}\right)+n_{m}^{0}(t) .
$$

Desarrollando (5.6), la señal recibida en el usuario $m$ se expresa según:

$$
y_{m}(t)=\sum_{k=1}^{K} \sum_{l=1}^{L} \mathbf{h}_{m k,(l)} \sum_{i=1}^{M} \mathbf{w}_{i k} \sqrt{p_{i k}} u_{i}\left(t-\tau_{l}\right)+n_{m}^{0}(t) .
$$

Para simplificar el modelo de sistema, se supone que no existe interferencia entre símbolos (ISI, Inter-symbol Interference), y que sólo se tiene interferencia entre fingers (IFI, Inter-finger Interference), para lo cual el valor máximo del delay spread no debe superar el periodo de símbolo, es decir:

$$
L T_{c}<T \text {. }
$$


En recepción, cada usuario dispone de un banco de $Q=K$ correladores que se encarga del despreading de la señal. En general, un correlador sincroniza la contribución multi-camino de mayor ganancia del canal de propagación entre el usuario y una de las BSs del sistema. De esta forma, el correlador $q$ del usuario $m$ se sincroniza con la contribución multi-camino $l_{q}$, que resulta ser la contribución de mayor ganancia del canal de la BS $k=q$ que recibe el usuario. La señal del usuario $m$ a la salida del correlador $q$-ésimo sería:

$$
\begin{aligned}
y_{m,(q)}[d] & =\frac{1}{T_{c}} \int_{(d-1) T}^{d T} y_{m}\left(t+\tau_{q}\right) c_{m}^{*}(t) d t \\
& =\sum_{k=1}^{K} \sum_{l=1}^{L} \mathbf{h}_{m k,(l)} \sum_{i=1}^{M} \mathbf{w}_{i k} \sqrt{p_{i k}} \rho_{m i}^{q, l} a_{i}[d]+n_{m,(q)}[d], \\
q & =1,2, \ldots Q,
\end{aligned}
$$

donde los términos de correlación entre códigos se corresponden con:

$$
\rho_{m i}^{q, l}=\frac{1}{T_{c}} \int_{0}^{T} c_{i}\left(t-\left(\tau_{l}-\tau_{q}\right)\right) c_{m}^{*}(t) d t
$$

y el término de ruido se caracteriza por:

$$
n_{m,(q)}[d]=\frac{1}{T_{c}} \int_{d T}^{(d+1) T} n_{m}^{0}\left(t+\tau_{q}\right) c_{m}^{*}(t-d T) d t .
$$

Hay que destacar que debido a la discretización del canal asumida previamente, los retardos $\tau_{l}$ y $\tau_{q}$ son múltiplos del periodo de chip $T_{c}$.

En este Capítulo, se presenta un modelo de sistema un poco más evolucionado para definir el proceso que tiene lugar en recepción. En teoría, a la salida del banco de correladores del usuario $m$ existe un ecualizador caracterizado por unos coeficientes $\mathbf{v}_{m}$. En este modelo, se mantienen los coeficientes del ecualizador de cada usuario igual a la unidad, por lo que en el usuario $m$, la señal tras el ecualizador quedaría:

$$
y_{m}[d]=\sum_{q=1}^{Q}\left(\sum_{k=1}^{K} \sum_{l=1}^{L} \mathbf{h}_{m k,(l)} \sum_{i=1}^{M} \mathbf{w}_{i k} \sqrt{p_{i k}} \rho_{m i}^{q, l} a_{i}[d]+n_{m,(q)}[d]\right) .
$$

Esta expresión se corresponde con el caso más general del sistema. 
Por otro lado, la señal a la salida del ecualizador del usuario $m$ puede descomponerse en los términos de señal deseada o SOI (Signal Of Interest), interferencia entre fingers o IFI, interferencia de acceso múltiple o MAI y un término de ruido:

$$
y_{m}[d]=\mathrm{SOI}_{m}[d]+\operatorname{IFI}_{m}[d]+\operatorname{MAI}_{m}[d]+\mathrm{N}_{m}[d] .
$$

Estos términos son fácilmente identificables a partir de la expresión (5.10):

$$
\begin{aligned}
\operatorname{SOI}_{m}[d] & =\sum_{q=1}^{Q} \sum_{k=1}^{K} \mathbf{h}_{m k,\left(l_{q}\right)} \mathbf{w}_{m k} \sqrt{p_{m k}} \rho_{m m}^{q, l_{q}} a_{m}[d], \\
\operatorname{IFI}_{m}[d] & =\sum_{q=1}^{Q} \sum_{k=1}^{K} \sum_{\substack{l=1 \\
l \neq l_{q}}}^{L} \mathbf{h}_{m k,(l)} \mathbf{w}_{m k} \sqrt{p_{m k}} \rho_{m m}^{q, l} a_{m}[d], \\
\operatorname{MAI}_{m}[d] & =\sum_{q=1}^{Q} \sum_{k=1}^{K} \sum_{l=1}^{L} \mathbf{h}_{m k,(l)} \sum_{\substack{i=1 \\
i \neq m}}^{M} \mathbf{w}_{i k} \sqrt{p_{i k}} \rho_{m i}^{q, l} a_{i}[d], \\
\mathrm{N}_{m}[d] & =\sum_{q=1}^{Q} n_{m,(q)}[d],
\end{aligned}
$$

donde $l_{q}$ se corresponde con la contribución multi-camino sincronizada por el correlador $q$-ésimo, de forma que en la expresión de la SOI, $\rho_{m m}^{q, l_{q}}=1$, puesto que $\tau_{q}=\tau_{l_{q}}$. Observando la expresión de la IFI y de la MAI, se aprecia que los términos de correlación entre códigos en cada uno de los correladores, $\rho_{m m}^{q, l}$ o $\rho_{m i}^{q, l}$, dependen de un desplazamiento relativo entre ellos $\left(\tau_{l}-\tau_{q}\right)(5.9)$.

El objetivo de este apartado es caracterizar estos términos de correlación para obtener después, a partir de las expresiones (5.11)-(5.13), un modelo ajustado de las potencias de señal deseada e interferencia presentes en los sistemas W-CDMA CM3. Como se verá a continuación, los modelos estadísticos que se utilizan habitualmente para modelar los términos de correlación entre códigos no se corresponden exactamente con las características que presentan los sistemas CM3. 


\subsubsection{Modelo tradicional de correlación entre códigos}

En UMTS, el proceso de spreading consta de dos pasos. En un primer paso, se ensancha la señal del usuario mediante un código de canalización, que es un código de la familia de los códigos OVSF, y en un segundo paso, la señal ensanchada se concatena con un código de scrambling, que suele ser una secuencia de Gold [101].

Los códigos OVSF son códigos ortogonales entre sí siempre y cuando estén perfectamente sincronizados. Sin embargo, estos códigos presentan unas propiedades de auto-correlación y correlación cruzada muy variables cuando se considera un desplazamiento temporal entre ellos [102]. Por este motivo, los códigos de canalización OVSF se concatenan con los códigos de scrambling, que presentan una correlación cruzada muy baja para cualquier desplazamiento temporal, excepto para situaciones de superposición.

En el enlace UL de UMTS, las señales transmitidas por los usuarios llegan de forma asíncrona a la BS. En esta situación, los códigos OVSF pierden ortogonalidad, y es el código de scrambling el que permite distinguir a los distintos usuarios. Por el contrario, en el enlace DL de UMTS, la BS transmite de forma síncrona a todos los usuarios, y los códigos OVSF mantienen su ortogonalidad para los usuarios de una misma celda. Las señales de las distintas BSs no están sincronizadas entre sí, de forma que es el código de scrambling el que permite distinguir usuarios de celdas diferentes.

En general, todos los trabajos de investigación que se centran en la tecnología W-CDMA, tienen que caracterizar de alguna forma la pérdida de ortogonalidad que introduce la propagación multi-camino. Desde el punto de vista analítico, una de las formas más habituales de caracterizar el comportamiento de los códigos es mediante un modelo estadístico de sus propiedades de auto-correlación y correlación cruzada. El problema es que estos estadísticos varían entre autores según sean las suposiciones iniciales planteadas.

Por otra parte, otra de las formas de caracterizar la pérdida de ortogonalidad de los códigos es introduciendo un factor de ortogonalidad. Básicamente, el factor de ortogonalidad representa la fracción instantánea de potencia recibida en DL que se transforma en interferencia entre usuarios debido a la propagación multi-camino.

En este apartado se repasan brevemente algunas referencias de ambos planteamientos para justificar la necesidad de desarrollar un modelo estadístico propio para caracterizar la pérdida de ortogonalidad, en este caso 
de los códigos de canalización, en los sistemas W-CDMA CM3.

\section{Caracterización de Fong et al.}

En [103] se presenta uno de los estudios fundamentales de lo que se conoce como scrambling de una secuencia de canalización.

Los autores derivan los estadísticos de la auto-correlación y correlación cruzada para una secuencia obtenida a partir de la concatenación (operación módulo 2) de secuencias ortogonales de Walsh-Hadamard con una secuencia real pseudo-aleatoria, para mejorar las propiedades de correlación cruzada que presentan las versiones desplazadas temporalmente de las secuencias de Walsh-Hadamard. En esta referencia también se introduce la idea de definir conjuntos de secuencias de Walsh para minimizar la interferencia entre usuarios cuando se tienen distintos tipos de tráfico. Este hecho se plantea después en UMTS mediante la utilización de los códigos OVSF.

En el estudio de la correlación entre distintos códigos, los autores evalúan la varianza del término interferente, puesto que la media que obtienen es nula. En el caso de una transmisión DL asíncrona, cuando el retardo entre secuencias es mayor que el periodo de chip, $\tau>T_{c}$, la varianza del término interferente es:

$$
\frac{2}{3} G T_{c}^{2}
$$

donde $G$ es la ganancia de procesado ${ }^{1}$.

En la misma situación, $\tau>T_{c}$, la varianza de la auto-correlación es la misma que la de la correlación cruzada, lo que significa una mejora sustancial con respecto a los códigos de Walsh-Hadamard, que presentan una varianza de la auto-correlación mucho mayor.

Estos mismos estadísticos se utilizan en [104] en un sistema con códigos aleatorios, puesto que los autores defienden la idea de que los códigos de Walsh en presencia de propagación multi-camino presentan unas propiedades de correlación similares a las de los códigos aleatorios.

\section{Caracterización de Soon et al.}

En [105], los autores realizan un estudio con secuencias i.i.d. binarias y aleatorias, donde el pulso es perfectamente rectangular, y caracterizan la

\footnotetext{
${ }^{1}$ La ganancia de procesado es la relación entre el ancho de banda de la señal ensanchada y el de la señal original. En el caso de modulaciones binarias, la ganancia de procesado coincide con el factor de spreading.
} 
correlación cruzada entre códigos como una variable de media nula y una varianza de:

$$
\frac{2}{3} G
$$

donde $G$ es de nuevo la ganancia de procesado. La referencia para justificar este resultado es la Tesis de A. F. Naguib [106] sobre antenas adaptativas en entornos CDMA.

\section{Caraterización de Hara et al.}

En [107] se estudia el comportamiento de un receptor Rake en un sistema W-CDMA de una única celda donde la BS implementa una técnica de beamforming. Los autores estudian las propiedades de una secuencia formada por un código de Walsh y un código de scrambling.

En este caso, la novedad radica en la definición de un coeficiente de correlación generalizado:

$$
\xi(a, b, \nabla p)=\frac{1}{G} \sum_{p=q G+a}^{q G+b} c_{k}^{*}\left(p T_{c}\right) c_{i}\left((p+\nabla p) T_{c}\right)
$$

que depende del símbolo $q$ y que permite derivar una matriz de correlación de la interferencia a la salida de los filtros de despreading, antes de tener en cuenta el peso del Rake. Los elementos de esta matriz de correlación de interferencias son no nulos y presentan una cierta correlación cuando $r_{1}-l_{1}=r_{2}-l_{2}$ : el finger $r_{1}$ desensancha el camino $\left(r_{1}+\triangle l\right)$ y el finger $r_{2}$ desensancha el camino $\left(r_{2}+\triangle l\right)$, por lo que en la señal desensanchada de los dos fingers aparecen los mismos términos de correlación.

\section{Caracterización de Schubert y Boche}

En [108], se define el término de correlación cruzada como:

$$
\phi_{i q}^{k l}[r]=\sum_{n=-\infty}^{\infty} b_{k}[n] \frac{1}{T_{c}} \int_{0}^{T_{b}} c_{i}^{*}(t) c_{k}\left(t-n T_{b}-l T_{c}+r T_{b}+q T_{c}\right) d t
$$

donde $i$ es el usuario receptor, $k$ es el código transmitido, $l$ representa la contribución multi-camino l-ésima del canal, $q$ es el finger del Rake y $r$ es el símbolo que se está detectando. 
Para caracterizar este término de correlación, los autores consideran que cuando el finger $q$ sintoniza la contribución multi-camino $l$-ésima, $q=l$, los códigos son perfectamente ortogonales, de forma que:

$$
\phi_{i q}^{k l}[r]=\left\{\begin{array}{cc}
b_{i}[r] G, & i=k, q=l, \\
0, & i \neq k, q=l .
\end{array}\right.
$$

Cuando $q \neq l$, es decir, cuando se considera una contribución multicamino distinta a la que está sintonizando el finger del Rake, el término de correlación es difícil de caracterizar, por lo que los autores consideran que es una variable aleatoria independiente e idénticamente distribuida, según la referencia [109], que se caracterizaría por:

$$
E\left[\left|\phi_{i q}^{k l}[r]\right|^{2}\right]=G, l \neq q,
$$

donde $G$ es la ganancia de procesado.

En [109] se considera un sistema CDMA donde los códigos son secuencias binarias y pseudo-aleatorias. Estas secuencias tendrían una función de auto-correlación $r_{i, i}(\tau)$ estrecha:

$$
\left|r_{i, i}(\tau)\right|=\left|\int_{t_{0}}^{t_{0}+T_{b}} c_{i}(t) c_{i}(t-\tau) d t\right|=\left\{\begin{array}{cc}
N, & \tau=0, \\
\approx \sqrt{N}, & |\tau \geq 0|,
\end{array}\right.
$$

donde $N$ es el factor de spreading, y una correlación cruzada baja:

$$
\left|r_{i, j}(\tau)\right|=\left|\int_{t_{0}}^{t_{0}+T_{b}} c_{i}(t) c_{j}(t-\tau) d t\right| \approx \sqrt{N}, i \neq j, \nabla \tau .
$$

Schubert y Boche puntualizan, sin embargo, que las propiedades de correlación que se especifican en [109] no suelen cumplirse al mismo tiempo, es decir, no suele tenerse una auto-correlación estrecha y una correlación cruzada baja, sino que una buena auto-correlación implica una correlación cruzada alta, como se justifica en la referencia [102]. De esta forma, Schubert y Boche contemplan en su planteamiento la posibilidad de que las propiedades de correlación de las secuencias no sean necesariamente ideales, por lo que la interferencia entre fingers del Rake y la MAI deben incluirse siempre en cualquier análisis que se realice de un sistema W-CDMA.

\section{Caracterización del factor de ortogonalidad}

El factor de ortogonalidad es un parámetro que mide la pérdida de ortogonalidad que introduce la propagación multi-camino en los códigos de spreading. 
Aunque el valor de este parámetro varía con el tiempo, tradicionalmente, en las simulaciones del enlace DL de un sistema W-CDMA se utiliza su valor promediado en el tiempo, puesto que no existe una expresión analítica cerrada para representar el valor medio del factor de ortogonalidad sobre todas las posibles distribuciones del multi-camino.

En [110], se estudia una expresión analítica para el factor de ortogonalidad instantáneo, y mediante simulaciones se demuestra que su valor promediado aumenta con la dispersión del canal. Este hecho se analiza posteriormente en [111], donde se define el parámetro de Factor de Dispersión, similar a la desviación estándar normalizada del canal, que caracteriza el perfil del canal. Los autores demuestran que este parámetro está inversamente relacionado con el valor promediado en el tiempo del factor de ortogonalidad.

Los estudios $[110,111]$ demuestran que el factor de ortogonalidad necesita una caracterización más precisa que la habitual, especialmente porque se trata de un parámetro que afecta directamente a la capacidad de los sistemas W-CDMA. Recientemente, en [112] se caracteriza el comportamiento del factor de ortogonalidad para distintos PDP mediante simulaciones, reforzando la idea de que utilizar un mismo factor de ortogonalidad para todos los usuarios de un mismo sistema no es una caracterización lo suficientemente precisa de este parámetro.

\subsubsection{Estudio de la correlación entre códigos}

En un sistema W-CDMA CM3, la señal de un usuario a la salida del banco de correladores y tras el ecualizador (5.10), puede separarse en el término de señal deseada o SOI (5.11), el término de interferencia entre fingers o IFI (5.12), el término de interferencia de acceso múltiple o MAI (5.13) y el término de ruido (5.14). Estos términos son los que definen la potencia de señal deseada y las potencias de interferencia, y como puede observarse, dependen de la auto-correlación y de la correlación cruzada de los códigos de canalización OVSF, $\rho_{m m}^{q, l}$ y $\rho_{m i}^{q, l}$, respectivamente.

En este punto, habría sido posible utilizar cualquiera de los estadísticos anteriores para transformar los valores instantáneos $\rho_{m m}^{q, l}$ y $\rho_{m i}^{q, l}$ en una realización de una variable aleatoria. También se podría haber considerado un factor de ortogonalidad fijo para todo el sistema W-CDMA CM3. Sin embargo, los estudios de [110-112] ya demuestran que es poco realista considerar un único factor de ortogonalidad para todos los usuarios de un sistema convencional. Tampoco existe ningún estudio que justifique que la 


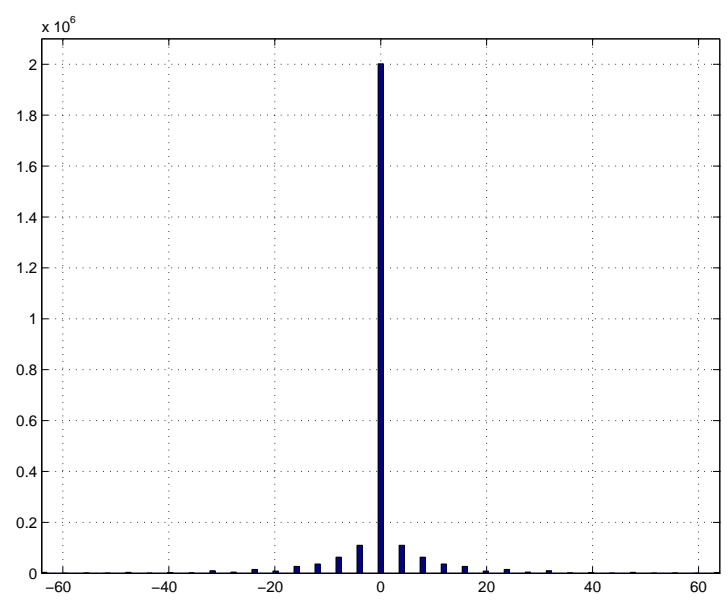

Figura 5.1: Histograma de correlaciones entre códigos OVSF.

MAI en el caso de sistemas W-CDMA CM3 puede modelarse como una interferencia Gaussiana. Pero es que además, en este modelo de sistema CM3, las BSs comparten el mismo código de scrambling y cada usuario se canaliza con un mismo código OVSF desde cada una de ellas.

Por todos estos motivos, se lleva a cabo una caracterización propia de las propiedades de correlación de los códigos OVSF en un sistema CM3. En un primer paso, se estudian las propiedades de correlación de los códigos OVSF con un factor de spreading de $N=64$ mediante una herramienta de simulación que genera dos códigos OVSF de $100 \cdot N$ chips y que calcula la correlación entre ambos para desplazamientos entre 0 y $N-1$ chips. Esta correlación se mide para un total de $10 N^{2}$ realizaciones, considerando aleatoriamente en cada una de ellas dos de los 64 códigos posibles y obteniendo para cada realización un vector de $N-1$ elementos (tantos como posibles desplazamientos).

Al final, se dispone de $10(N-1) N^{2}$ valores de correlación entre códigos. La figura 5.1 muestra el histograma de estos valores de correlación, mientras que la figura 5.2 presenta una ampliación de la figura 5.1. En ambas figuras, se presentan los valores de correlación sin normalizar, lo que significa que la correlación puede tomar cualquier valor entre $-N$ y $N$ (correlación total).

En un segundo paso, se intenta modelar los histogramas mediante alguna función distribución de probabilidad estándar, pero el punto en el que la correlación vale 0 distorsiona cualquier función distribución de probabili- 


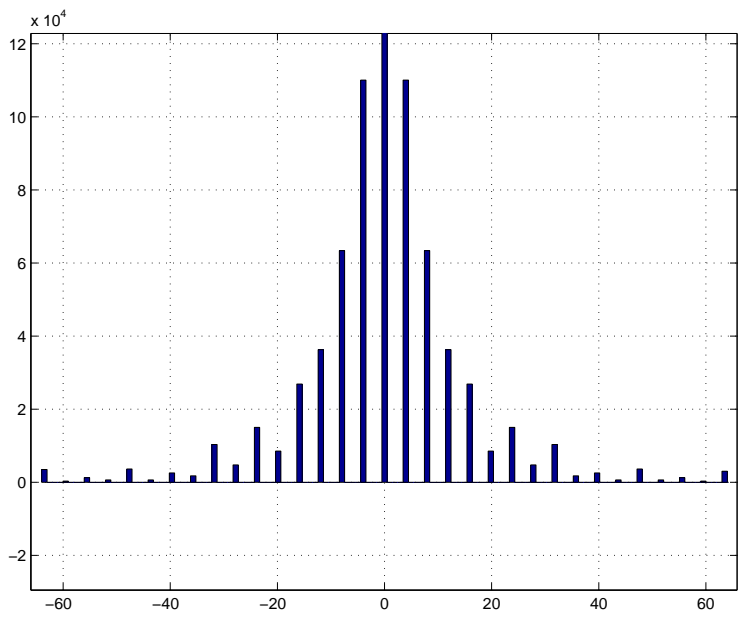

Figura 5.2: Detalle del histograma de correlaciones entre códigos OVSF.

dad conocida. Lo que también queda claro es que esta correlación no puede simplemente modelarse como una distribución Gaussiana de media nula, y que en ocasiones no despreciables puede llegar a tomar valores muy altos.

Para continuar con el análisis, se decide cambiar de estrategia. En cualquier sistema, las BSs conocen en todo momento qué códigos OVSF se están utilizando, de forma que se podría obtener una cierta estimación de la correlación concreta que existe entre los códigos activos, evaluados dos a dos. Este valor de correlación depende del desplazamiento entre los códigos, al que no es posible asignarle una distribución determinada, puesto que está relacionado con la distribución espacial de las BSs y con el PDP de cada enlace. Sin embargo, en el siguiente apartado, a partir de un modelo exhaustivo de la señal a la salida del ecualizador de cada usuario, se consigue un modelo estadístico adecuado de los términos de correlación entre códigos en sistemas W-CDMA CM3.

\subsubsection{Modelo de las interferencias}

Tradicionalmente, la interferencia de acceso múltiple o MAI se modela o bien mediante el factor de ortogonalidad o bien como un término de interferencia Gaussiana. Sin embargo, no existe ningún resultado que asegure que el factor de ortogonalidad o la suposición de interferencia Gaussiana son parámetros válidos para modelar las interferencias presentes en un sistema 


\section{W-CDMA CM3.}

En este apartado, el análisis anterior de los términos de correlación se amplía y se sitúa en el contexto de un sistema W-CDMA CM3, donde hay que considerar que el desplazamiento entre los códigos está relacionado con las características de propagación del canal, y que por tanto, va a depender de ciertas probabilidades que se extraen del PDP de cada canal.

Para caracterizar correctamente los términos de correlación entre códigos, es conveniente tener en cuenta la relación que existe entre el PDP compuesto que se puede obtener desde el punto de vista del usuario, (5.4), y el PDP de cada canal de propagación (5.3):

$$
\tau_{l}=\tau_{m k}+\tau_{l_{k}}
$$

donde el retardo $\tau_{l_{k}}$ está relacionado con el PDP del canal entre el usuario $m$ y la BS $k$. El retardo de propagación $\tau_{m k}$ se debe a la distribución espacial de las BSs en el sistema, es decir, en el caso de que los $K$ canales de propagación que recibe un usuario $m$ se caracterizaran mediante un mismo PDP, existiría un retardo $\tau_{m k}$ adicional y posiblemente distinto para cada enlace usuario$\mathrm{BS}$, que provocaría que las contribuciones multi-camino de cada uno de los canales llegaran al usuario en instantes de tiempo distintos, como muestra la figura 5.3. Por tanto, el retardo $\tau_{m k}$ se añade para considerar el caso general en el que los canales procedentes de las $K$ BSs llegan al usuario de forma asíncrona. Este retardo $\tau_{m k}$ define de esta forma el instante de llegada de la primera contribución multi-camino del canal entre el usuario $m$ y la BS $k$.

El término de correlación $\rho_{m i}^{q, l}$ (5.9) entre los códigos $m$ e $i$, puede expresarse entonces como:

$$
\rho_{m i}^{q, l}\left(\tau_{m k}\right)=\frac{1}{T_{c}} \int_{0}^{T} c_{i}\left(t-\left(\tau_{l_{k}}-\tau_{q}\right)-\tau_{m k}\right) c_{m}^{*}(t) d t,
$$

donde $\tau_{l_{k}}, \tau_{q}$ y $\tau_{m k}$ son múltiplos enteros del periodo de chip $T_{c}$.

En el sistema W-CDMA CM3 planteado en este Capítulo, se desprecia la interferencia entre símbolos o ISI, de forma que las interferencias presentes en el sistema se reducen a la interferencia de acceso múltiple o MAI y a la interferencia entre fingers o IFI. A continuación, teniendo en cuenta la nueva expresión del término de correlación entre códigos, (5.15), y retomando la expresión de la señal a la salida del ecualizador de cada usuario, (5.10), se obtiene un modelo estadístico de los términos de correlación entre códigos, que permite plantear un modelo realista de la potencia de señal 

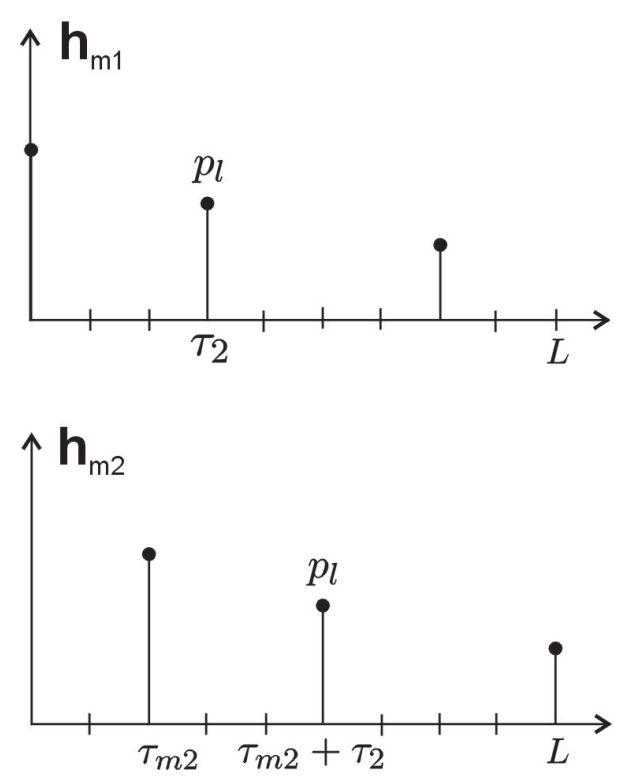

Figura 5.3: Ejemplo de desplazamiento de los perfiles de retardo para los canales de propagación entre el usuario $m$ y las BSs $k=1$ y $k=2$.

deseada, potencia de interferencia entre fingers y potencia de interferencia de acceso múltiple presentes en un sistema W-CDMA CM3.

\section{Señal deseada, SOI}

El término de señal deseada se expresa mediante (5.11):

$$
\mathrm{SOI}_{m}[d]=\sum_{q=1}^{Q} \sum_{k=1}^{K} \mathbf{h}_{m k,\left(l_{q}\right)} \mathbf{w}_{m k} \sqrt{p_{m k}} a_{m}[d]
$$

puesto que $\rho_{m m}^{q, l_{q}}=1$, donde $l_{q}$ representa la contribución multi-camino sincronizada por el correlador q-ésimo, que se corresponde con la contribución multi-camino de mayor ganancia del canal entre el usuario $m$ y la BS $k=q$.

Definiendo un vector de canal $\left[1 \times N_{t} Q\right]$ :

$$
\dot{\mathbf{h}}_{m k}=\left[\mathbf{h}_{m k,\left(l_{q}=1\right)} \ldots \mathbf{h}_{m k,\left(l_{q}=Q\right)}\right]
$$

y un vector de beamformers:

$$
\dot{\mathbf{w}}_{m k}=\left[\mathbf{1}_{[Q \times 1]} \otimes \mathbf{w}_{m k}\right],
$$


de dimensiones $\left[N_{t} Q \times 1\right]$, el término de señal deseada se puede expresar de forma compacta según:

$$
\mathrm{SOI}_{m}[d]=\sum_{k=1}^{K} \dot{\mathbf{h}}_{m k} \dot{\mathbf{w}}_{m k} \sqrt{p_{m k}} a_{m}[d],
$$

de forma que la potencia media de señal deseada se calcularía como:

$$
P_{\mathrm{SOI}}=E\left[\left|\mathrm{SOI}_{m}[d]\right|^{2}\right]=\sum_{k=1}^{K} p_{m k} \dot{\mathbf{w}}_{m k}^{H} \dot{\mathbf{H}}_{m k} \dot{\mathbf{w}}_{m k}
$$

donde $\dot{\mathbf{H}}_{m k}=E\left[\dot{\mathbf{h}}_{m k}^{H} \dot{\mathbf{h}}_{m k}\right]$ es el estadístico de segundo orden del canal. Para calcular este estadístico, se asume que las contribuciones multi-camino provenientes de distintas BSs, así como las que pertenecen a un mismo canal, están incorreladas entre sí:

$$
\begin{aligned}
& E\left[\mathbf{h}_{m k,(q)}^{H} \mathbf{h}_{m k,(l)}\right]=\left\{\begin{array}{cl}
0, & q \neq l, \\
\mathbf{H}_{m k,(q)}, & q=l,
\end{array}\right. \\
& E\left[\mathbf{h}_{m k,(q)}^{H} \mathbf{h}_{m k^{\prime},(l)}\right]=\left\{\begin{array}{cc}
0, & q \neq l, k \neq k^{\prime}, \\
\mathbf{H}_{m k,(q)}, & q=l, k=k^{\prime} .
\end{array}\right.
\end{aligned}
$$

\section{Interferencia entre fingers, IFI}

El término de interferencia entre fingers se expresa mediante (5.12):

$$
\operatorname{IFI}_{m}[d]=\sum_{q=1}^{Q} \sum_{k=1}^{K} \sum_{\substack{l=1 \\ l \neq l_{q}}}^{L} \mathbf{h}_{m k,(l)} \mathbf{w}_{m k} \sqrt{p_{m k}} \rho_{m m}^{q, l}\left(\tau_{m k}\right) a_{m}[d],
$$

donde

$$
\rho_{m m}^{q, l}\left(\tau_{m k}\right)=\frac{1}{T_{c}} \int_{0}^{T} c_{m}\left(t-\left(\tau_{l_{k}}-\tau_{q}\right)-\tau_{m k}\right) c_{m}^{*}(t) d t .
$$

Este término de auto-correlación depende del retardo de propagación de la contribución multi-camino l-ésima del PDP compuesto visto por el móvil y del correlador $q$-ésimo. El objetivo es modelar este término como una variable aleatoria independiente del desplazamiento entre códigos.

El retardo de propagación $\tau_{m k}$ está relacionado con la distribución espacial de las BSs a lo largo del sistema CM3. Si la distribución de las BSs 
es uniforme, puede suponerse que el retardo $\tau_{m k}$ presenta una distribución uniforme entre 1 y $n_{\max }$ periodos de chip. Por el contrario, las probabilidades asociadas a los retardos $\tau_{l_{k}}$ y $\tau_{q}$ se obtienen a partir del PDP del canal $m k$ correspondiente.

El modelo de sistema W-CDMA CM3 contempla el caso general en el que todas las contribuciones multi-camino de cada uno de los $K$ canales de propagación llegan al usuario en instantes de tiempo arbitrarios, incluidos en el intervalo $\left[T_{c}, L T_{c}\right]$. Sin pérdida de generalidad, y desde el punto de vista del PDP compuesto visto por el usuario, se asume que la señal de una de las BSs, $k^{\prime}$, llegará antes que el resto, con lo cual, $\tau_{m k^{\prime}}=0$, como muestra la figura 5.3.

Si $p_{l}$ representa la probabilidad asociada a la contribución multi-camino l-ésima, la media y la varianza del término de auto-correlación que aparece en la componente de IFI se definen como:

$$
\begin{aligned}
& \left.m_{I F I}^{0}(m)\right\rfloor_{\tau_{m k}=0}=\sum_{q=1}^{Q} \sum_{\substack{l=1 \\
l \neq q}}^{L} p_{q} p_{l} \rho_{m m}^{q, l}(0), \\
& \left.m_{I F I}(m)\right\rfloor_{\tau_{m k} \neq 0}=\frac{1}{n_{\max }} \sum_{n=1}^{n_{\max }} \sum_{q=1}^{Q} \sum_{\substack{l=1 \\
l \neq q}}^{L} p_{q} p_{l} \rho_{m m}^{q, l}\left(n T_{c}\right),
\end{aligned}
$$

y

$$
\begin{aligned}
\left.\left(\sigma_{I F I}^{0}(m)\right)^{2}\right\rfloor_{\tau_{m k}=0} & =\sum_{q=1}^{Q} \sum_{\substack{l=1 \\
l \neq q}}^{L} p_{q} p_{l}\left(\rho_{m m}^{q, l}(0)-m_{I F I}^{0}(m)\right)^{2}, \\
\left.\sigma_{I F I}^{2}(m)\right\rfloor_{\tau_{m k} \neq 0} & =\frac{1}{n_{\max }} \sum_{n=1}^{n_{\max }} \sum_{\substack{q=1 \\
q}}^{Q} \sum_{\substack{l=1 \\
l \neq q}}^{L} p_{q} p_{l}\left(\rho_{m m}^{q, l}\left(n T_{c}\right)-m_{I F I}(m)\right)^{2},
\end{aligned}
$$

donde $p_{q}$ y $p_{l}$ son las probabilidades asociadas al correlador y a la contribución multi-camino l-ésima, respectivamente. Estas probabilidades se obtienen haciendo corresponder a la potencia media de cada contribución multi-camino de la expresión (5.4) una probabilidad normalizada $p_{l}$ (ver figura 5.3), de forma que:

$$
p_{l}=\frac{\left\|\mathbf{h}_{m k,(l)}\right\|^{2}}{\sum_{l}\left\|\mathbf{h}_{m k,(l)}\right\|^{2}} .
$$


Una vez definido el término de auto-correlación $\rho_{m m}^{q, l}$ mediante su media y varianza, es posible obtener, como en el caso del término de señal deseada, una expresión compacta del término de interferencia entre fingers. Definiendo el vector de canal $\left[1 \times N_{t}(L-1)\right]$ :

$$
\ddot{\mathbf{h}}_{m k, l_{q}}=\left[\mathbf{h}_{m k,(l=1)} \ldots \mathbf{h}_{m k,\left(l=l_{q}-1\right)} \mathbf{h}_{m k,\left(l=l_{q}+1\right)} \ldots \mathbf{h}_{m k,(l=L)}\right],
$$

y el vector de beamformers:

$$
\ddot{\mathbf{w}}_{m k}=\left[\mathbf{1}_{[(L-1) \times 1]} \otimes \mathbf{w}_{m k}\right],
$$

de dimensiones $\left[N_{t}(L-1) \times 1\right]$, el término de interferencia entre fingers se expresaría según:

$$
\operatorname{IFI}_{m}[d]=\sum_{k=1}^{K} \rho_{I F I}(m) \sum_{q=1}^{Q} \ddot{\mathbf{h}}_{m k, l_{q}} \ddot{\mathbf{w}}_{m k} \sqrt{p_{m k}} a_{m}[d],
$$

donde $\rho_{I F I}(m)$ es una realización de la variable aleatoria que caracteriza el término de auto-correlación. Teniendo en cuenta el valor de $\tau_{m k}$, la potencia media de interferencia entre fingers se calcularía mediante:

$$
\begin{aligned}
P_{\mathrm{IFI}}=E\left[\left|\operatorname{IFI}_{m}[d]\right|^{2}\right] & =E\left[\left(\rho_{I F I}^{0}(m)\right)^{2}\right] p_{m k^{\prime}} \ddot{\mathbf{w}}_{m k^{\prime}}^{H} \ddot{\mathbf{H}}_{m k^{\prime}} \ddot{\mathbf{w}}_{m k^{\prime}} \\
& +\sum_{\substack{k=1 \\
k \neq k^{\prime}}}^{K} E\left[\rho_{I F I}^{2}(m)\right] p_{m k} \ddot{\mathbf{w}}_{m k}^{H} \ddot{\mathbf{H}}_{m k} \ddot{\mathbf{w}}_{m k},
\end{aligned}
$$

donde $\ddot{\mathbf{H}}_{m k}=\sum_{q=1}^{Q} \ddot{\mathbf{H}}_{m k}^{q}$, y $\ddot{\mathbf{H}}_{m k}^{q}=E\left[\ddot{\mathbf{h}}_{m k, l_{q}}^{H} \ddot{\mathbf{h}}_{m k, l_{q}}\right]$. El término de correlación $\rho_{I F I}^{0}(m)$ es una realización concreta de la variable aleatoria cuyo valor cuadrático medio es $E\left[\left(\rho_{I F I}^{0}(m)\right)^{2}\right]=\left(\left(m_{I F I}^{0}(m)\right)^{2}+\left(\sigma_{I F I}^{0}(m)\right)^{2}\right)$, mientras que $\rho_{I F I}(m)$ es una realización de la variable aleatoria definida por el valor cuadrático medio $E\left[\rho_{I F I}^{2}(m)\right]=\left(m_{I F I}^{2}(m)+\sigma_{I F I}^{2}(m)\right)$.

\section{Interferencia de acceso múltiple, MAI}

El término de interferencia de acceso múltiple se expresa mediante (5.13):

$$
\operatorname{MAI}_{m}[d]=\sum_{q=1}^{Q} \sum_{k=1}^{K} \sum_{l=1}^{L} \mathbf{h}_{m k,(l)} \sum_{\substack{i=1 \\ i \neq m}}^{M} \mathbf{w}_{i k} \sqrt{p_{i k}} \rho_{m i}^{q, l}\left(\tau_{m k}\right) a_{i}[d],
$$


donde

$$
\rho_{m i}^{q, l}\left(\tau_{m k}\right)=\frac{1}{T_{c}} \int_{0}^{T} c_{i}\left(t-\left(\tau_{l_{k}}-\tau_{q}\right)-\tau_{m k}\right) c_{m}^{*}(t) d t .
$$

Este término de correlación cruzada se modela como el término de autocorrelación de la componente de IFI, pero considerando distintos códigos $m$ e $i$ y permitiendo $l=l_{q}$ :

$$
\begin{aligned}
& \left.m_{M A I}^{0}(m i)\right\rfloor_{\tau_{m k}=0}=\sum_{q=1}^{Q} \sum_{l=1}^{L} p_{q} p_{l} \rho_{m i}^{q, l}(0), \\
& \left.m_{M A I}(m i)\right\rfloor_{\tau_{m k} \neq 0}=\frac{1}{n_{\max }} \sum_{n=1}^{n_{\max }} \sum_{q=1}^{Q} \sum_{l=1}^{L} p_{q} p_{l} \rho_{m i}^{q, l}\left(n T_{c}\right),
\end{aligned}
$$

$\mathrm{y}$

$$
\begin{aligned}
\left.\left(\sigma_{M A I}^{0}(m i)\right)^{2}\right\rfloor_{\tau_{m k}=0} & =\sum_{q=1}^{Q} \sum_{l=1}^{L} p_{q} p_{l}\left(\rho_{m i}^{q, l}(0)-m_{M A I}^{0}(m i)\right)^{2} \\
\left.\sigma_{M A I}^{2}(m i)\right\rfloor_{\tau_{m k} \neq 0} & =\frac{1}{n_{\max }} \sum_{n=1}^{n_{\max }} \sum_{q=1}^{Q} \sum_{l=1}^{L} p_{q} p_{l}\left(\rho_{m i}^{q, l}\left(n T_{c}\right)-m_{M A I}(m i)\right)^{2} .
\end{aligned}
$$

Una vez que el término de correlación cruzada $\rho_{m i}^{q, l}$ se modela como una variable aleatoria independiente del desplazamiento entre códigos y del correlador $q$, desaparece la dependencia del término de interferencia de acceso múltiple (5.13) con el correlador $q$ del usuario. Para obtener una expresión compacta del término de interferencia de acceso múltiple, se define un vector de canal $\left[1 \times N_{t} L\right]$ :

$$
\tilde{\mathbf{h}}_{m k}=\left[\mathbf{h}_{m k,(l=1)} \ldots \mathbf{h}_{m k,(l=L)}\right]
$$

y un vector de beamformers:

$$
\tilde{\mathbf{w}}_{i k}=\left[\mathbf{1}_{[L \times 1]} \otimes \mathbf{w}_{i k}\right],
$$

de dimensiones $\left[N_{t} L \times 1\right]$, de forma que el término de interferencia de acceso múltiple se expresa según:

$$
\operatorname{MAI}_{m}[d]=\sum_{k=1}^{K} \sum_{\substack{i=1 \\ i \neq m}}^{M} \rho_{M A I}(m i) \tilde{\mathbf{h}}_{m k} \tilde{\mathbf{w}}_{i k} \sqrt{p_{i k}} a_{i}[d] .
$$


Por último, y considerando la distinción en función del valor de $\tau_{m k}$, la potencia media de interferencia de acceso múltiple sería:

$$
\begin{aligned}
P_{\mathrm{MAI}}=E\left[\left|\mathrm{MAI}_{m}[d]\right|^{2}\right] & =\sum_{\substack{i=1 \\
i \neq m}}^{M} E\left[\left(\rho_{M A I}^{0}(m i)\right)^{2}\right] p_{i k^{\prime}} \tilde{\mathbf{w}}_{i k^{\prime}}^{H} \tilde{\mathbf{H}}_{m k^{\prime}} \tilde{\mathbf{w}}_{i k^{\prime}} \\
& +\sum_{\substack{k=1 \\
k \neq k^{\prime}}}^{K} \sum_{\substack{i \neq 1 \\
i \neq m}}^{M} E\left[\rho_{M A I}^{2}(m i)\right] p_{i k} \tilde{\mathbf{w}}_{i k}^{H} \tilde{\mathbf{H}}_{m k} \tilde{\mathbf{w}}_{i k},
\end{aligned}
$$

donde $\tilde{\mathbf{H}}_{m k}=E\left[\tilde{\mathbf{h}}_{m k}^{H} \tilde{\mathbf{h}}_{m k}\right]$ es el estadístico de segundo orden del canal. De nuevo, $\rho_{M A I}^{0}(m i)$ es una realización de la variable aleatoria definida por el valor cuadrático medio $E\left[\left(\rho_{M A I}^{0}(m i)\right)^{2}\right]=\left(\left(m_{M A I}^{0}(m i)\right)^{2}+\left(\sigma_{M A I}^{0}(m i)\right)^{2}\right)$, mientras que $\rho_{M A I}(m i)$ es una realización de la definida por el valor cuadrático medio $E\left[\rho_{M A I}^{2}(m i)\right]=\left(m_{M A I}^{2}(m i)+\sigma_{M A I}^{2}(m i)\right)$. 


\subsection{Esquemas de asignación de códigos OVSF}

A partir de la expresión de la potencia media de señal deseada (5.16), potencia media de interferencia entre fingers (5.21) y potencia media de interferencia de acceso múltiple (5.26), se observa que el valor cuadrático medio del término de correlación entre códigos (expresado como la suma del cuadrado de la media y de la varianza), influye directamente en el nivel de las interferencias presentes en los sistemas W-CDMA CM3.

Una posible aplicación de la caracterización de las interferencias presentes en el sistema W-CDMA CM3, es la de actuar sobre el valor cuadrático medio de los distintos términos de correlación para tratar de mejorar el nivel de interferencia que experimentan los usuarios del sistema.

La opción elegida en esta Tesis, es la de desarrollar distintos esquemas multi-base de asignación de códigos OVSF para el sistema W-CDMA $\mathrm{CM} 3$, de forma que se reduzca la interferencia que perciben los usuarios y aumente con ello la capacidad del sistema. En este contexto, el término multi-base se refiere al hecho de que la señal de cada usuario se transmite mediante el mismo código OVSF desde todas las BSs del sistema, por tanto, la asignación del código se realiza sobre las $K$ BSs del sistema.

Tradicionalmente, los códigos OVSF se asignan de forma que se mantenga la ortogonalidad entre ellos. Los códigos OVSF se generan en forma de árbol: a partir de un código $[x]$, se generan dos códigos de longitud doble, $[x x]$ y $[x-x]$, que se corresponden con un mismo factor de spreading. Estos códigos son ortogonales entre sí, y al mismo tiempo, son ortogonales con respecto a los códigos de otros niveles del árbol, siempre y cuando uno de los códigos no sea el código ascendente del otro (es decir, no se haya generado un código a partir del otro). Concretamente, un usuario puede canalizarse mediante un determinado código si y solo si ningún otro código del camino entre ese código y la raíz del árbol, o en los niveles que se derivan de ese código, ya está asignado.

La literatura asociada a los esquemas de asignación de códigos OVSF para UMTS es muy amplia, y algunos ejemplos interesantes pueden encontrarse en [113-116].

En este apartado, se presentan distintos esquemas multi-base de asignación de códigos OVSF, cuando todos los usuarios del sistema comparten el mismo requerimiento de QoS, expresado en forma de un valor umbral de SINR. De esta forma, el problema se reduce a asignar eficientemente los códigos OVSF que pertenecen a un mismo nivel o factor de spreading 
del árbol de códigos. Por tanto, los esquemas de asignación de códigos propuestos son esquemas sencillos, que no contemplan aspectos como el bloqueo de códigos ${ }^{2}$.

En un primer paso, se estudia el problema para un sistema con canales flat fading [97,98], para pasar después a profundizar en el estudio del mejor esquema de asignación de códigos OVSF en un entorno con canales frequency selective [99].

La principal diferencia de esta propuesta con los trabajos de referencia como [113], es que en estos últimos se suele definir un nuevo tipo de códigos que minimiza la interferencia en el sistema actuando sobre ciertos parámetros como por ejemplo, la firma espacio-temporal de cada usuario. La propuesta de esta Tesis, sin embargo, busca minimizar la interferencia en el sistema CM3 utilizando los códigos que ya están definidos en el estándar UMTS.

\subsubsection{Entorno flat fading}

En un entorno flat fading, la expresión del canal de propagación entre un usuario $m$ y una BS $k$ se reduce a $(5.3)$ :

$$
\mathbf{h}_{m k}(t)=\mathbf{h}_{m k} \delta\left(t-\tau_{m k}\right)
$$

donde $\mathbf{h}_{m k}$ es un vector $\left[1 \times N_{t}\right]$ y $\tau_{m k}$ es el retardo de propagación que modela, desde el punto de vista del usuario, el comportamiento asíncrono de las contribuciones multi-camino $\mathbf{h}_{m k}, \forall k$, en recepción. De esta forma, aunque se trata de un entorno flat fading, el usuario percibe un canal formado por $K$ contribuciones multi-camino independientes, una por BS.

En un sistema W-CDMA CM3 con canales de propagación flat fading, la interferencia dominante es la interferencia de acceso múltiple o MAI. Por este motivo, en el análisis de los esquemas de asignación de códigos en el entorno flat fading se desprecia la contribución de la interferencia entre fingers o IFI. A continuación, se revisan las modificaciones que necesita el algoritmo JPCOB-VUL en el entorno Coordinado I con canales flat fading cuando se considera el nuevo modelo de correlación entre códigos.

\footnotetext{
${ }^{2}$ Una situación de bloqueo de códigos se define como aquella en la que un nuevo usuario podría aceptarse en base al análisis de las interferencias y la capacidad del sistema, pero donde se rechaza debido a las asignaciones previas de códigos.
} 


\section{Modificación del algoritmo JPCOB-VUL en el entorno Coordinado I}

El algoritmo JPCOB-VUL definido en (4.29)-(4.33) necesita unas pequeñas modificaciones para incluir los términos de correlación entre códigos asociados a la presencia en el sistema CM3 de usuarios no co-canal [97, 98].

La expresión de la SINR recibida en el enlace UL virtual entre el usuario $m$ y la BS $k$ se modifica como (4.18):

$$
\operatorname{SINR}_{m k}^{V U}=\frac{p_{m k}^{u} \mathbf{w}_{m k}^{H} \mathbf{H}_{m k} \mathbf{w}_{m k}}{\mathbf{w}_{m k}^{H}\left(\sum_{i=1, i \neq m}^{M} p_{i k}^{u} \rho_{m i}^{2} \mathbf{H}_{i k}+\mathbf{I}\right) \mathbf{w}_{m k}},
$$

donde $\rho_{m i}$ es una realización concreta de la variable aleatoria que define la correlación cruzada entre los códigos $m$ e $i$ y $\mathbf{H}_{m k}=E\left[\mathbf{h}_{m k}^{H} \mathbf{h}_{m k}\right]$.

De la misma forma, la SINR recibida en DL por un usuario $m$ se reescribe como (4.17):

$$
\operatorname{SINR}_{m}^{D L}=\frac{\sum_{k=1}^{K} p_{m k} \mathbf{w}_{m k}^{H} \mathbf{H}_{m k} \mathbf{w}_{m k}}{\sum_{k=1}^{K} \sum_{i=1, i \neq m}^{M} p_{i k} \rho_{m i}^{2} \mathbf{w}_{i k}^{H} \mathbf{H}_{m k} \mathbf{w}_{i k}+\sigma_{m}^{2}},
$$

donde de nuevo los coeficientes de correlación son realizaciones concretas de las distintas variables aleatorias.

Siguiendo los pasos (4.19)-(4.21), la ecuación de la actualización de potencias puede expresarse de forma multi-usuario y matricial según:

$$
\mathbf{D p}=\mathbf{F} \mathbf{p}+\mathbf{u},
$$

donde las matrices $\mathbf{D}$ y $\mathbf{F}$ se modifican de la siguiente forma (4.23)-(4.26):

$$
\begin{aligned}
\mathbf{D} & =\left[\mathbf{D}_{1}\left|\mathbf{D}_{2}\right| \cdots \mid \mathbf{D}_{K}\right], \\
{\left[\mathbf{D}_{k}\right]_{(m, m)} } & =\mathbf{w}_{j}^{H} \mathbf{H}_{j} \mathbf{w}_{j}, \\
\mathbf{F} & =\left[\begin{array}{c}
\left.\mathbf{F}_{1}\left|\mathbf{F}_{2}\right| \cdots \mid \mathbf{F}_{K}\right], \\
{\left[\mathbf{F}_{k}\right]_{(m, i)}}
\end{array}=\left\{\begin{array}{cc}
\gamma_{m} \rho_{m i}^{2} \mathbf{w}_{n}^{H} \mathbf{H}_{j} \mathbf{w}_{n}, & m \neq i, \\
0, & m=i .
\end{array}\right.\right.
\end{aligned}
$$

EL vector $\mathbf{u}$ se mantiene como en el algoritmo JPCOB-VUL original (4.27).

Los pasos de esta modificación del algoritmo JPCOB-VUL, para cada iteración $n_{i t}$ serían:

1. Inicializar las potencias UL virtuales, $p_{j}^{u}(0)$, y las potencias DL, $p_{j}(0)$, para todos los enlaces del sistema, $j=1, \ldots, K M$, con valores positivos. 
2. Para $n_{i t}=0,1,2, \ldots$, hasta llegar a la convergencia:

2.1. Beamforming. Calcular los beamformers subóptimos en transmisión $\mathbf{w}_{j}$ para cada enlace, $j=1, \ldots, K M$, maximizando para cada enlace la SINR recibida en el UL virtual:

$$
\mathbf{w}_{j}\left(n_{i t}\right)=\arg \operatorname{máx}_{\mathbf{w}_{j}}\left\{\frac{p_{j}^{u}\left(n_{i t}\right) \mathbf{w}_{j}^{H} \mathbf{H}_{j} \mathbf{w}_{j}}{\mathbf{w}_{j}^{H}\left(\sum_{n} p_{n}^{u}\left(n_{i t}\right) \rho_{m i}^{2} \mathbf{H}_{n}+\mathbf{I}\right) \mathbf{w}_{j}}\right\},
$$

donde $n$ toma $M-1$ valores relacionados con $j$ de la siguiente forma: si $j=(k-1) M+m$, entonces $n=(k-1) M+i$ con $i=1, \ldots, M$, e $i \neq m$. Esto es debido a que en el UL virtual, los usuarios interferentes del enlace $j$-ésimo son los restantes $M-1$ enlaces asociados a la misma BS $k$.

En la práctica, cada uno de los beamformers $\mathbf{w}_{j}$ se calcula mediante la expresión MMSE:

$$
\mathbf{w}_{j}\left(n_{i t}\right)=\sqrt{p_{j}^{u}\left(n_{i t}\right)} \boldsymbol{\Phi}_{j}^{-1}\left(n_{i t}\right) \mathbf{h}_{j},
$$

donde la matriz $\boldsymbol{\Phi}_{j}\left(n_{i t}\right)$ es la matriz de correlación de la señal recibida en el UL virtual del enlace $j$ (4.31). El procedimiento para obtener esta matriz de correlación también se modifica para incluir los términos de correlación entre códigos. Estos términos son necesarios para ponderar correctamente la potencia de interferencia en la BS, que a su vez repercute directamente en la conformación del beamformer.

2.2. Control de potencia, enlace UL virtual. Las potencias UL virtuales, $p_{j}^{u}$, se actualizan para cada enlace, $j=1, \ldots, K M$, mediante un control de potencia clásico distribuido [88]:

$$
p_{j}^{u}\left(n_{i t}+1\right)=\frac{\gamma_{m}}{\operatorname{SINR}_{j}^{V U}\left(n_{i t}\right)} p_{j}^{u}\left(n_{i t}\right),
$$

donde $\operatorname{SINR}_{j}^{V U}\left(n_{i t}\right)$ es la SINR en el UL virtual dada en la ecuación (5.28), pero considerando las potencias UL virtuales y los beamformers de la iteración correspondiente.

2.3. Control de potencia, enlace DL. El vector $\mathbf{p}$ de potencias DL se actualiza mediante:

$$
\mathbf{p}\left(n_{i t}+1\right)=\mathbf{D}^{\dagger}\left(n_{i t}\right)\left(\mathbf{F}\left(n_{i t}\right) \mathbf{p}\left(n_{i t}\right)+\mathbf{u}\right),
$$


donde las matrices $\mathbf{D}\left(n_{i t}\right)$ y $\mathbf{F}\left(n_{i t}\right)$ se obtienen a partir de las expresiones (5.31) y (5.33), respectivamente, pero considerando los beamformers en transmisión correspondientes a la iteración $n_{i t}$.

En principio, la inclusión de los términos de correlación entre códigos no afecta a las propiedades de convergencia de esta modificación del algoritmo JPCOB-VUL, que siguen siendo las presentadas en el apartado 4.2.5.

\section{Parámetros de la simulación}

En este Capítulo, las simulaciones ya no tratan de caracterizar parámetros básicos de los sistemas CM3, sino que se plantean para evaluar las aplicaciones prácticas del algoritmo JPCOB-VUL sobre una configuración de sistema CM3.

El diagrama de bloques de la figura 4.19 sigue siendo válido para resumir los pasos de esta simulación del algoritmo JPCOB-VUL. En cada simulación, se distribuyen de forma uniforme $M$ usuarios iniciales sobre el sistema CM3. Después, se ejecuta el algoritmo JPCOB-VUL de (5.35)(5.38), considerando el esquema de selección de enlaces activos del entorno Coordinado I.

El algoritmo dispone de $I t$ iteraciones para converger, es decir, para que todos los usuarios alcancen la SINR umbral, manteniendo la potencia total transmitida por cada BS por debajo de la restricción $P_{\max }$. Si el algoritmo converge, se añade un nuevo usuario al sistema, $M=M+1$, manteniendo la ubicación de los usuarios anteriores, y se vuelve a ejecutar el algoritmo. La simulación se interrumpe cuando el algoritmo no es capaz de admitir nuevos usuarios, ya sea porque algún usuario no alcanza la SINR umbral, porque el algoritmo necesita más de $I t$ iteraciones o porque alguna BS supera la restricción en potencia $P_{\max }$. Por cuestiones prácticas, el máximo número de usuarios que puede admitir el algoritmo se fija para mantener un factor de carga del sistema del $80 \%$.

Una simulación viene definida por un valor umbral de SINR común a todos los usuarios del sistema y por un valor de tamaño de ventana de active set o ASW. Los resultados de las simulaciones se evalúan mediante la probabilidad de admisión que presenta el algoritmo JPCOB-VUL para un determinado número de usuarios activos.

En las simulaciones de este Capítulo, la probabilidad de admisión de usuarios del algoritmo se representa mediante una modificación de la función de distribución estimada o CDF (Cumulative Distribution Function). 
De esta forma, para un número determinado de usuarios activos, un valor de probabilidad de admisión representa el número de realizaciones, sobre el total, en las que el algoritmo admite al menos ese número de usuarios activos, es decir, que un número igual o menor de usuarios alcanza el valor umbral de SINR, sin que el algoritmo supere las restricciones en número de iteraciones y en potencia total transmitida por BS.

La tabla 5.1 resume los parámetros utilizados en la simulación. Con respecto a estos parámetros, es importante destacar que las simulaciones presentadas en este Capítulo modifican el modelo de propagación con respecto al Capítulo anterior, al obtener las pérdidas de propagación mediante el modelo del COST 273, particularizado para el escenario MORANS [117]. Este modelo de propagación se corresponde con un escenario que introduce unas pérdidas de propagación considerables y que podría considerarse como el caso peor con respecto a condiciones de propagación. Por el contrario, el shadowing se sigue modelando a partir de su desviación estándar, sin tener en cuenta otras características como la correlación o distancia de decorrelación. El resto de parámetros se mantienen igual que en las simulaciones del Capítulo anterior.

Como se ha visto en el apartado 5.1.4, el parámetro $n_{\max }$ se utiliza para definir la distribución uniforme con la que se caracteriza al retardo $\tau_{m k}$ asociado con la distribución espacial de las BSs. En el entorno flat fading, el desplazamiento entre códigos del término de correlación cruzada depende del valor de $\tau_{m k}$, que en este caso se modela mediante una distribución uniforme entre $\left[T_{c}, n_{\max } T_{c}\right]$.

Teniendo en cuenta esta distribución uniforme, es posible evaluar dos a dos los $N$ posibles códigos OVSF, obteniendo para cada par de códigos un vector de $n_{\max }$ valores de correlación (tantos como desplazamientos posibles). Tomando estos valores como realizaciones de una determinada variable aleatoria, se puede realizar una estima de la media y la desviación estándar que presentan los $n_{\max }$ valores de correlación de cada par de códigos OVSF.

A partir de estos valores, en las simulaciones del entorno flat fading se decide modelar los términos de correlación cruzada como variables aleatorias Gaussianas, de media nula y con la desviación estándar promedio obtenida a partir de las simulaciones. Estos valores de desviación estándar se utilizan posteriormente para definir distintos esquemas de asignación de códigos OVSF. 


\begin{tabular}{|c|c|}
\hline Parámetro & Valor \\
\hline Frecuencia de la portadora & $2 \mathrm{GHz}$ \\
\hline Factor de carga & $80 \%$ \\
\hline EbNo umbral & $4,4 \mathrm{~dB}$ \\
\hline Factor de spreading $N$ & 64 \\
\hline$n_{\max }$ & $N / 4$ \\
\hline Tamaño máximo del active set. & $3 \mathrm{BSs}$ \\
\hline Altura del usuario & $1,5 \mathrm{~m}$ \\
\hline Altura de la BS & $17 \mathrm{~m}$ \\
\hline Altura de los edificios & $12 \mathrm{~m}$ \\
\hline Potencia de ruido & $-108,2 \mathrm{dBm}$ \\
\hline Figura de ruido & $7 \mathrm{~dB}$ \\
\hline Ganancia de la antena omnidireccional & $11 \mathrm{dBi}$ \\
\hline $\begin{array}{l}\text { Potencia máxima } P_{\max } \\
\text { transmitida por BS }\end{array}$ & $20 \mathrm{~W}$ \\
\hline Distancia entre emplazamientos & $1 \mathrm{~km}$ \\
\hline Radio celular & $577 \mathrm{~m}$ \\
\hline Desviación estándar del shadowing & $8 \mathrm{~dB}$ \\
\hline Pérdidas de propagación (COST 273) & $\beta_{m k}(d B)=143+39,6 \log (\mathrm{R}(\mathrm{km}))$ \\
\hline $\begin{array}{l}\text { Número de componentes } \\
\text { multi-camino por contribución }\end{array}$ & 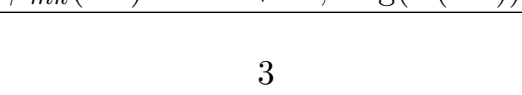 \\
\hline $\begin{array}{l}\text { Potencia media de fading } \\
\text { de cada componente }\end{array}$ & $0 \mathrm{~dB}$ \\
\hline Retardo de cada componente & 0 \\
\hline Velocidad de los usuarios & $4 \mathrm{~km} / \mathrm{h}$ \\
\hline $\begin{array}{l}\text { Máximo número de iteraciones } \\
\text { del algoritmo }(I t)\end{array}$ & 15 \\
\hline Periodo de muestreo & $T_{s}=1 / 3,84 \cdot 10^{6}$ \\
\hline $\begin{array}{l}\text { Número de símbolos necesarios } \\
\text { para estimar la correlación } \\
\text { de las matrices de canal }\end{array}$ & $n \operatorname{simb}=3 \cdot N_{t}$ \\
\hline Número de coeficientes del canal & $N_{\text {coef }}=I t \cdot n \operatorname{simb}$ \\
\hline
\end{tabular}

Cuadro 5.1: Parámetros de simulación. 


\section{Estudio de la probabilidad de admisión de usuarios}

El objetivo de esta primera simulación en el entorno flat fading es comparar la probabilidad de admisión del algoritmo JPCOB-VUL de (5.35)(5.38) en el entorno Coordinado I, con la probabilidad de admisión del algoritmo JPCOB de referencia de [33], (3.54)-(3.56), que también se modifica para tener en cuenta los términos de correlación entre códigos.

Además, estos algoritmos se ejecutan sobre distintas configuraciones de sistema (ver figura 4.4): el algoritmo JPCOB-VUL se ejecuta sobre una configuración CM3 donde las BSs transmiten coordinadamente a todos los usuarios, mientras que el algoritmo JPCOB de referencia se ejecuta de forma independiente en cada uno de los sectores de la configuración central. Hay que recordar que las dos configuraciones de sistema son totalmente equivalentes en cuando a número total de antenas y restricción de potencia transmitida por BS, $P_{\max }$.

Cada simulación empieza con $M=15$ usuarios, que se distribuyen uniformemente sobre la celda y a los que se les asigna una velocidad de $4 \mathrm{~km} / \mathrm{h}$. Una simulación puede añadir hasta un total de $M=50$ usuarios, que representa un factor de carga del $80 \%$ para un factor de spreading de $N=64$. Teniendo en cuenta que la EbN0 umbral es de $4,4 \mathrm{~dB}$, y que el sistema utiliza una modulación QPSK, el valor umbral de SINR antes del despreading se fija en $-16,6 \mathrm{~dB}$.

Las simulaciones consideran configuraciones de sistema con $K=2$ y $K=3 \mathrm{BSs}$, equipadas con arrays lineales de $N_{t}=3$ o $N_{t}=4$ antenas. Asimismo, en el caso del algoritmo JPCOB-VUL, se simulan distintos valores de ventana de AS: $0 \mathrm{~dB}$ (sin coordinación), $2 \mathrm{~dB}$ y $5 \mathrm{~dB}$, evaluando 500 realizaciones independientes para cada configuración de BSs y antenas y valor de ventana de AS.

Las figuras 5.4 y 5.5 representan la función de distribución estimada o CDF de la probabilidad de admisión de ambos algoritmos y configuraciones de sistema, para las combinaciones de número de BSs y antenas por BS que obtienen resultados más significativos [98].

Como puede apreciarse en las figuras, en general, el algoritmo JPCOBVUL sobre la configuración CM3 mejora la probabilidad de admisión para todos los tamaños de ventana de AS frente al algoritmo JPCOB de referencia de [33] sobre la configuración central.

Interesa destacar que para el algoritmo JPCOB-VUL, un tamaño de ventana de AS de $2 \mathrm{~dB}$ aumenta la probabilidad de admisión frente a una configuración no coordinada $(\mathrm{ASW}=0 \mathrm{~dB})$. Sin embargo, un tamaño ma- 


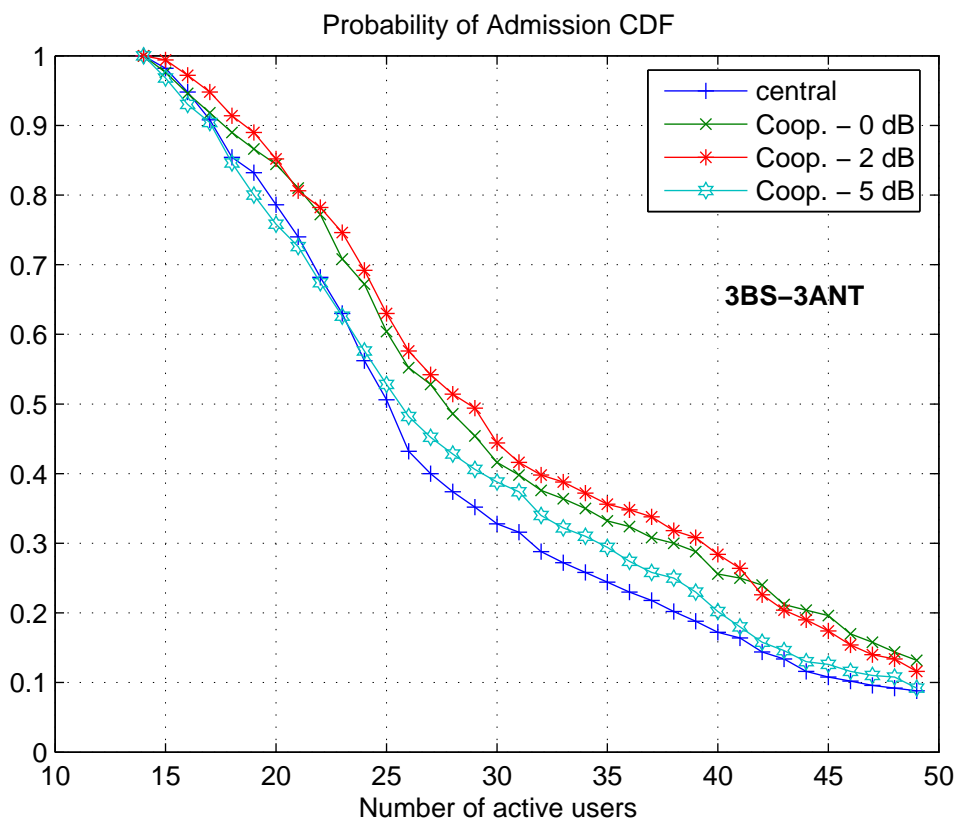

Figura 5.4: Función de distribución estimada de la probabilidad de admisión, $K=3, N_{t}=3$. La configuración CM3 se denota por Coop., indicando asimismo el tamaño de la ventana de AS (0, 2 ó 5 dB).

yor de ventana, $\mathrm{ASW}=5 \mathrm{~dB}$, aunque mejora las prestaciones del algoritmo JPCOB de referencia sobre una configuración central, consigue una probabilidad menor de admisión que la ventana de $2 \mathrm{~dB}$, debido al aumento del nivel de interferencias que supone considerar una mayor coordinación entre las BSs del sistema. 


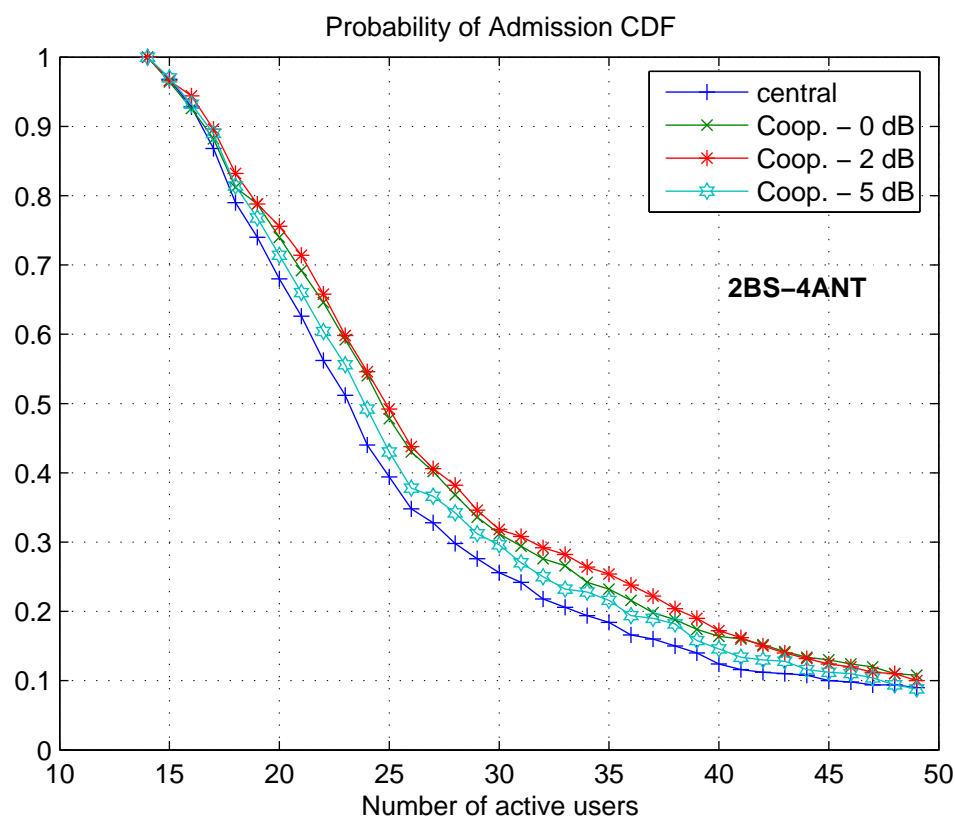

Figura 5.5: Función de distribución estimada de la probabilidad de admisión, $K=2, N_{t}=4$. La configuración CM3 se denota por Coop., indicando asimismo el tamaño de la ventana de $\mathrm{AS}(0,2$ ó $5 \mathrm{~dB})$. 


\section{Esquemas de asignación de códigos OVSF a nuevos usuarios}

En esta segunda simulación en el entorno flat fading, se proponen cuatro estrategias que permiten obtener cuatro órdenes distintos de asignación de códigos OVSF. Estos órdenes de asignación se utilizan para asignar el código de canalización a cada uno de los nuevos usuarios que se van añadiendo al sistema a lo largo de una simulación [97].

El objetivo de estos esquemas de asignación de códigos es mejorar el nivel de interferencia que perciben los usuarios, de forma que el sistema admita el mayor número posible de usuarios. Por este motivo, los esquemas de asignación de códigos se plantean a partir de una matriz de desviación estándar, $\mathbf{R}_{s t d}$, de dimensiones $[N \times N]$, donde el elemento $\left[\mathbf{R}_{s t d}\right]_{(i, j)}$ es la desviación estándar del término de correlación cruzada entre los códigos $i$ y $j$, ya que en cierta manera, este valor de desviación estándar caracteriza cuánto de interferente puede llegar a ser un código con respecto a otro.

El primer esquema o esquema Natural, es simplemente el orden natural de los códigos OVSF. Puesto que todos los usuarios comparten un mismo requerimiento de QoS, los códigos OVSF que se van a asignar pertenecen al mismo nivel del árbol OVSF, en concreto al nivel de $N=64$. De esta forma, el esquema Natural proporciona un orden de asignación sucesivo, empezando en el código 1 y acabando en el $N$.

El segundo esquema o esquema MT (Minimum Total), proporciona un orden de asignación en el cual se asignan los códigos en función de la interferencia que introducen en el resto. Para obtener este orden, se suman las filas de la matriz $\mathbf{R}_{s t d}$, obteniendo así la desviación estándar total que introduce cada uno de los códigos:

$$
\sum_{i=1}^{N}\left[\mathbf{R}_{s t d}\right]_{(i, j)}, j=1, \ldots, N .
$$

Esta desviación estándar total puede interpretarse como cuánto de interferente es un código con respecto al resto de códigos. Los valores de desviación estándar total se ordenan de menor a mayor, obteniendo así el orden de asignación del esquema MT (ver figura 5.6).

El tercer esquema o esquema mMT (modified Minimum Total), sigue los pasos del esquema MT, pero se obtiene a partir de una matriz $\mathbf{R}_{s t d}$ modificada, donde los elementos de la diagonal principal son nulos.

El cuarto y último esquema, el esquema MP o Minimum Partial, obtiene un orden de asignación que, cuando llega un nuevo usuario, asigna el código 


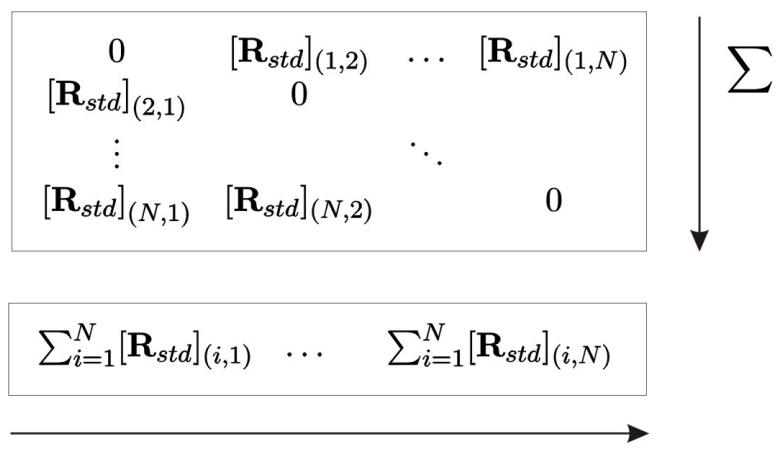

Figura 5.6: Esquema MT o Minimum Total.

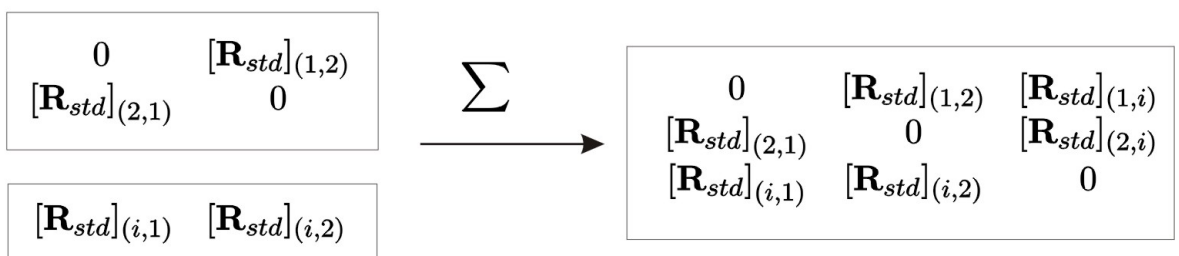

Figura 5.7: Esquema MP o Minimum Partial.

que menor desviación estándar total introduce con respecto a los códigos que ya están activos en la celda. La figura 5.7 muestra un ejemplo práctico del funcionamiento de este esquema. En este ejemplo, el esquema empieza asignando los códigos 1 y 2. En el momento en que tiene que asignar un tercer código, realiza una búsqueda entre el resto de códigos disponibles, y selecciona aquel código que, sumando sus valores de desviación estándar con respecto a los códigos que ya están activos en la celda, obtiene un menor valor de desviación estándar total. Este proceso se repite hasta ordenar los $N$ códigos disponibles.

Los valores de desviación estándar de los distintos términos de correlación son valores fijos y conocidos antes de la simulación, puesto que no dependen de una realización concreta del canal, sino de la distribución del retardo $\tau_{m k}$. Por tanto, los cuatro esquemas de asignación de códigos proporcionan un orden de asignación fijo y conocido antes de cualquier simulación, independientemente del número de BSs o usuarios del sistema. 


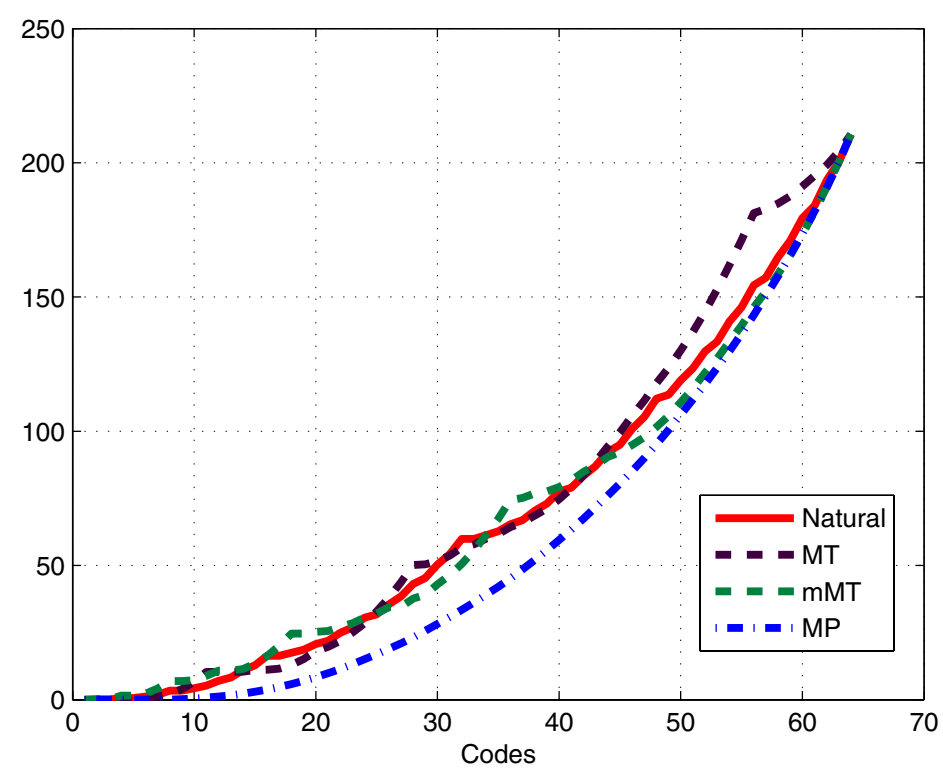

Figura 5.8: Suma acumulada de la desviación estándar para los cuatro órdenes de asignación de códigos.

La figura 5.8 muestra la suma acumulada de la desviación estándar de los términos de correlación de los cuatro órdenes que proporcionan los esquemas Natural, MT, mMT y MP [97]. La gráfica se interpreta de la siguiente manera: para un esquema determinado, un valor del eje $x$, por ejemplo el punto codes $=30$, indica que se consideran activos los 30 primeros códigos del orden, y el valor en el eje $y$ indica cuál es la suma acumulada de la desviación estándar para esos 30 códigos. En cierta manera, esta gráfica parece indicar que el esquema MP es el que presenta una menor desviación estándar acumulada y, por tanto, es el que introduce una menor potencia de interferencia de acceso múltiple en el sistema.

En esta segunda simulación del entorno flat fading, se vuelve a comparar el algoritmo JPCOB-VUL sobre una configuración CM3, con el algoritmo JPCOB de referencia de [33] sobre una configuración central equivalente (ver figura 4.4). Sin embargo, sólo se considera una configuración de sistema con $K=3 \mathrm{BSs}$, cada una equipada con un array lineal de $N_{t}=3$ antenas.

En principio, los esquemas de asignación de códigos se proponen para mejorar la interferencia que perciben los usuarios de los sistemas CM3, 


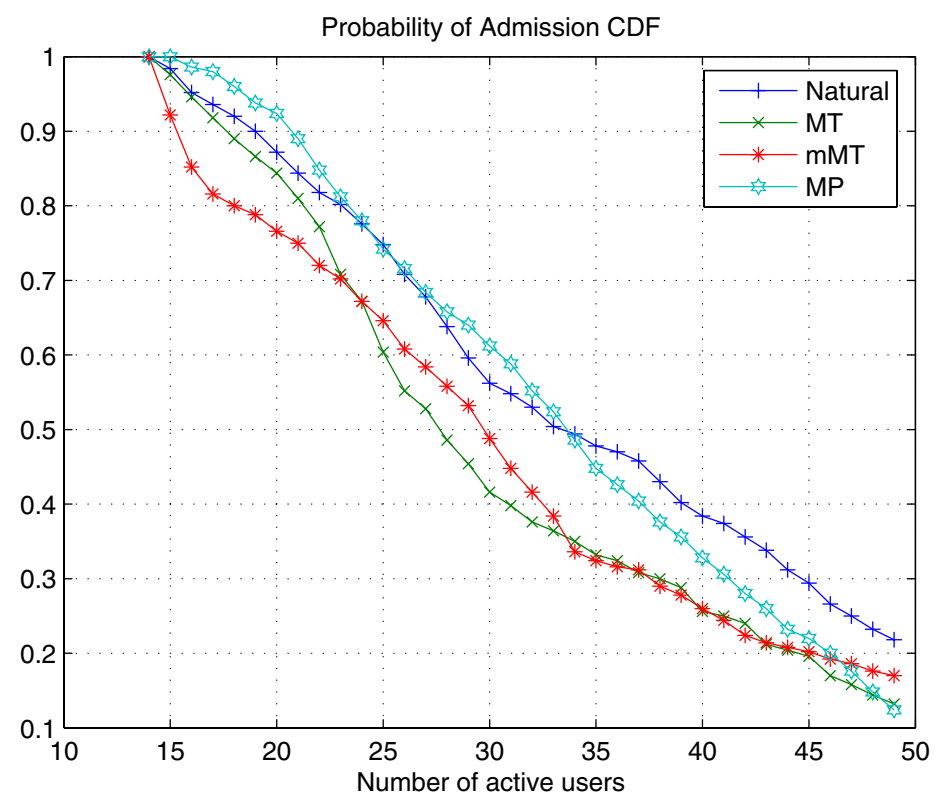

Figura 5.9: Función de distribución estimada de la probabilidad de admisión del algoritmo JPCOB-VUL, para $K=3, N_{t}=3 \mathrm{y} \mathrm{ASW}=0 \mathrm{~dB}$.

cuando las señales de las distintas BSs llegan al usuario siguiendo una distribución uniforme entre $\left[T_{c}, n_{\max } T_{c}\right]$. Podría parecer entonces que no tiene sentido aplicar los órdenes así obtenidos sobre una configuración central de sistema, con BSs ubicadas en el centro y donde el algoritmo JPCOB de referencia se ejecuta de forma independiente en cada sector, puesto que en realidad cada usuario sólo recibe señal de una única BS. Sin embargo, como se verá en los resultados de la simulación, incluso en esta configuración, el hecho de realizar una asignación de códigos, que no es óptima para esta configuración, también mejora la capacidad del sistema.

La simulación sigue la misma estructura que la simulación anterior, sólo que ahora el objetivo es evaluar las prestaciones de los cuatro órdenes de asignación de códigos OVSF propuestos. Para cada simulación, definida por un orden de asignación de códigos y una ventana de AS para el caso coordinado, se promedian 500 realizaciones independientes.

Las figuras 5.9 y 5.10 presentan la función de distribución estimada o CDF de la probabilidad de admisión del algoritmo JPCOB-VUL sobre la 


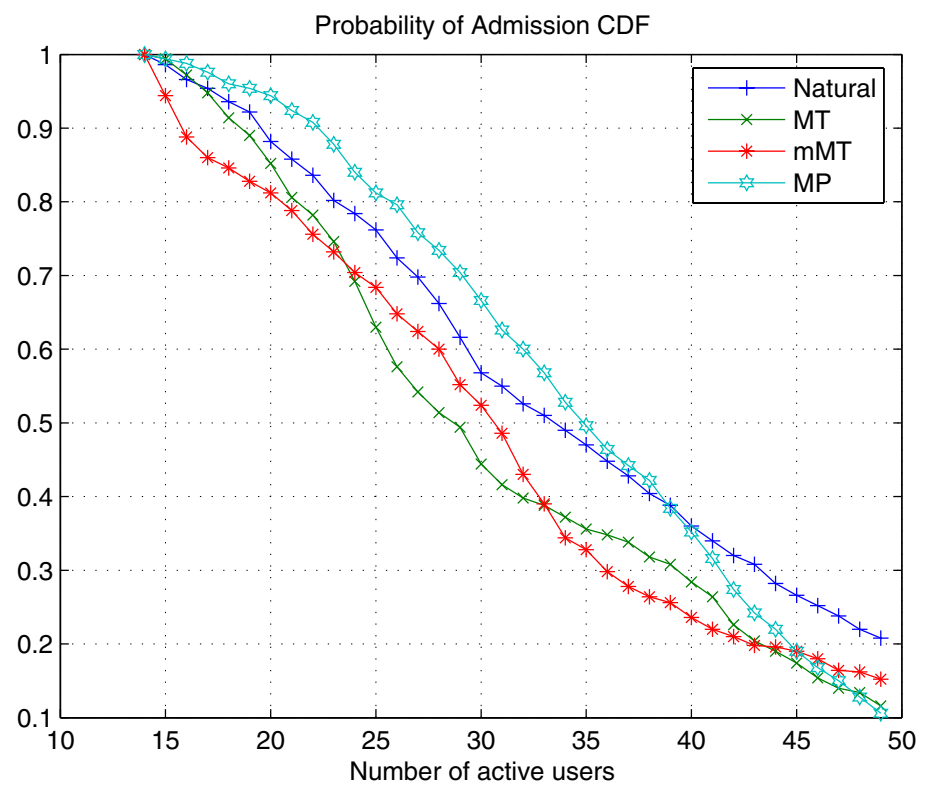

Figura 5.10: Función de distribución estimada de la probabilidad de admisión del algoritmo JPCOB-VUL, para $K=3, N_{t}=3$ y $\mathrm{ASW}=2 \mathrm{~dB}$.

configuración CM3 para los cuatro órdenes de asignación de códigos, para una configuración sin coordinación ( $\mathrm{ASW}=0 \mathrm{~dB}$ ) y una configuración coordinada (ASW $=2 \mathrm{~dB}$ ), respectivamente [97].

Las figuras 5.9 y 5.10 muestran que en el caso concreto del algoritmo JPCOB-VUL, los órdenes de asignación Natural y MP aumentan considerablemente la probabilidad de admisión del algoritmo con respecto a los órdenes MT y mMT. Si nos centramos en el efecto de la ventana de AS, y comparamos ambas figuras, el hecho de permitir una ligera coordinación entre BSs, $\mathrm{ASW}=2 \mathrm{~dB}$, también mejora la probabilidad de admisión del algoritmo. Por ejemplo, para un factor de carga del 48\% (30 usuarios activos), el orden MP para un tamaño de ventana de $2 \mathrm{~dB}$ (figura 5.10) consigue un incremento de casi un $8 \%$ en la probabilidad de admisión con respecto al caso no coordinado de $0 \mathrm{~dB}$ (figura 5.9).

Por otro lado, también interesa destacar en la figura 5.9 que el orden Natural mejora la probabilidad de admisión del orden MP para más de 35 usuarios activos. Este punto se mueve hasta los 40 usuarios activos cuando se permite coordinación entre BSs (figura 5.10). 


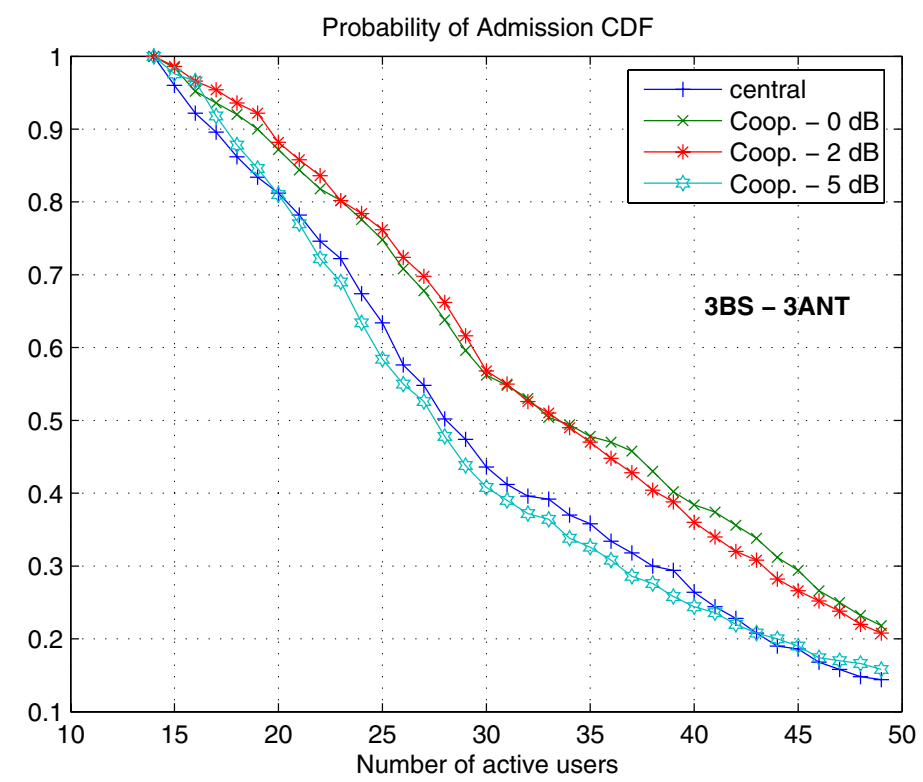

Figura 5.11: Función de distribución estimada de la probabilidad de admisión del orden Natural, para $K=3, N_{t}=3$. La configuración CM3 se denota por Coop., indicando asimismo el tamaño de la ventana de $\mathrm{AS}(0,2$ ó $5 \mathrm{~dB})$.

Las figuras 5.11 y 5.12 muestran la función de distribución estimada o CDF de la probabilidad de admisión del algoritmo JPCOB-VUL sobre la configuración de sistema CM3, y la probabilidad de admisión del algoritmo JPCOB de referencia sobre una configuración central de sistema, para los órdenes de asignación Natural y MP, respectivamente [97].

Las figuras 5.11 y 5.12 justifican de nuevo los resultados obtenidos en simulaciones anteriores, donde el algoritmo JPCOB-VUL sobre una configuración CM3 mejora las prestaciones del algoritmo JPCOB de referencia sobre una configuración central. Comparando ambas figuras, se observa que el comportamiento de los resultados asociados al orden MP es más estable que el que presentan los resultados asociados al orden Natural. Además, la figura 5.11 muestra que permitir una ventana de AS de $5 \mathrm{~dB}$ en el orden Natural disminuye considerablemente la probabilidad de admisión, mientras que el orden MP suaviza este efecto (figura 5.12).

Por último, hay que destacar que el orden MP consigue un incremento notable de la probabilidad de admisión con respecto al orden Natural para 


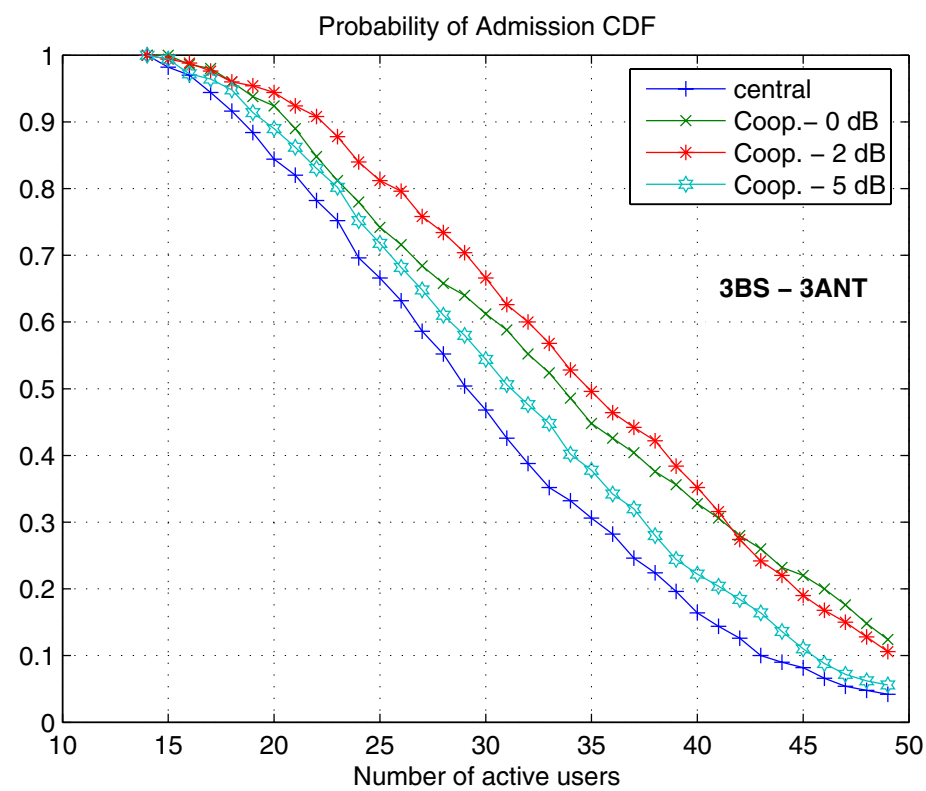

Figura 5.12: Función de distribución estimada de la probabilidad de admisión del orden MP, para $K=3, N_{t}=3$. La configuración CM3 se denota por Coop., indicando asimismo el tamaño de la ventana de $\mathrm{AS}(0,2$ ó $5 \mathrm{~dB})$.

ambos algoritmos cuando la carga del sistema es inferior al $55 \%$ (menos de 35 usuarios activos).

Volviendo a la figura 5.8, los resultados presentados en estas dos simulaciones en el entorno flat fading demuestran que las prestaciones de los distintos órdenes de asignación se relacionan de alguna manera con la suma acumulada de la desviación estándar mostrada en esta figura.

Para terminar, es interesante recordar que en los sistemas CM3, un usuario consume un único código de canalización en todas las BSs del sistema, a diferencia de las situaciones de soft handover de los sistemas de comunicaciones convencionales, donde el usuario consume un código de canalización por cada una de las BSs involucradas. Por tanto, desde el punto de vista de gestión de recursos, los sistemas CM3 presentan una opción más eficiente, siempre y cuando se actúe de alguna manera sobre el aumento de interferencia que supone utilizar un mismo código de canalización en todas las BSs del sistema. 


\subsubsection{Entorno frequency selective fading}

En un sistema CM3 con canales de propagación frequency selective, la potencia media de señal deseada viene dada por (5.16), la potencia media de interferencia entre fingers se obtiene mediante (5.21) y la potencia media de interferencia de acceso múltiple se expresa como (5.26). En estas expresiones ya se incluye el término de correlación entre códigos como una realización concreta de una variable aleatoria. En concreto, este término de correlación se representa en función de su valor cuadrático medio, o suma del cuadrado de la media y de la varianza.

Por tanto, el hecho de considerar usuarios no co-canal, y de introducir en recepción el banco de correladores y el ecualizador, también obligan a modificar el algoritmo JPCOB-VUL original de (4.29)-(4.33). De nuevo, el esquema de selección de enlaces activos elegido es el del entorno Coordinado I, donde los enlaces activos se eligen a partir de la construcción de un active set de BSs para cada usuario del sistema.

\section{Modificación del algoritmo JPCOB-VUL en el entorno Coordinado I}

La primera modificación afecta al cálculo de los beamformers en el problema UL virtual. Los trabajos de $[92,108,118]$ demuestran que en un sistema MIMO multi-usuario convencional, la dualidad entre el problema DL y el problema UL virtual no se mantiene en un entorno W-CDMA con canales frequency selective si se tienen en cuenta los coeficientes del ecualizador. Esto se debe a que, en el algoritmo de referencia de [33], la matriz de ganancia en UL, [DF], y la matriz de ganancia en DL, $\left[\mathbf{D F}^{T}\right]$, no presentan los mismos valores propios debido a que $\mathbf{F} \neq \mathbf{F}^{T}$.

Tradicionalmente, la solución a este problema pasa por realizar algunas aproximaciones sobre el término de IFI, como [92,118], o bien en plantear una matriz de correlación de canal subóptima, como en [108].

En este Capítulo, todavía no se aborda el diseño de los coeficientes del ecualizador, por lo que éstos no intervienen en el problema de minimización de potencia, y en principio no existiría ningún problema en plantear el problema de la forma habitual. De hecho, en la referencia original de RashidFarrokhi et al. [33], se consideran canales con varias contribuciones multicamino y por tanto, existe tanto interferencia entre símbolos como interferencia de acceso múltiple.

Sin embargo, el algoritmo JPCOB-VUL mantiene un diseño local de los 
beamformers. Además, se considera un beamformer de banda estrecha. Es poco realista suponer que en un entorno frequency selective, el beamformer que se obtiene de esta forma va a poder apuntar de forma efectiva al usuario deseado.

En esta modificación del algoritmo JPCOB-VUL, lo que se hace es diseñar el beamformer de forma que elimine las contribuciones multi-camino de mayor potencia interferente, es decir, el beamformer se diseña teniendo en cuenta únicamente las contribuciones de mayor ganancia de cada canal:

$$
\mathbf{h}_{m k}^{0}=\operatorname{máx}_{l}\left\|\mathbf{h}_{m k,(l)}\right\|^{2}, \forall m, k .
$$

La expresión de la SINR recibida en el enlace UL virtual entre el usuario $m$ y la BS $k$ se modifica como (4.18):

$$
\operatorname{SINR}_{m k}^{V U}=\frac{p_{m k}^{u} \mathbf{w}_{m k}^{H} \mathbf{H}_{m k}^{0} \mathbf{w}_{m k}}{\mathbf{w}_{m k}^{H}\left(\sum_{i=1, i \neq m}^{M}\left(\rho_{M A I}^{0}(m i)\right)^{2} p_{i k}^{u} \mathbf{H}_{i k}^{0}+\mathbf{I}\right) \mathbf{w}_{m k}},
$$

donde $\mathbf{H}_{m k}^{0}=E\left[\left(\mathbf{h}_{m k}^{0}\right)^{H} \mathbf{h}_{m k}^{0}\right]$ y el término de correlación cruzada se modela según la variable aleatoria definida por $\left(\left(m_{M A I}^{0}(m i)\right)^{2}+\left(\sigma_{M A I}^{0}(m i)\right)^{2}\right)$.

A partir de las expresiones de los términos de potencia media de señal deseada (5.16), potencia media de interferencia entre fingers (5.21) y potencia media de interferencia de acceso múltiple (5.26), la SINR recibida en el usuario $m$ se expresaría (4.17):

$$
\operatorname{SINR}_{m}^{D L}=\frac{P_{\mathrm{SOI}}}{P_{\mathrm{IFI}}+P_{\mathrm{MAI}}+\sigma_{m}^{2}}
$$

donde $\sigma_{m}^{2}$ es la potencia de ruido en recepción.

Siguiendo los pasos (4.19)-(4.21), la ecuación de la actualización de potencias puede expresarse de forma multi-usuario y matricial según:

$$
\mathbf{D p}=\mathbf{F p}+\mathbf{u},
$$

donde las matrices $\mathbf{D}$ y $\mathbf{F}$ se modifican de la siguiente forma (4.23)-(4.26):

$$
\begin{aligned}
& \mathbf{D}=\left[\begin{array}{l|l|l|l}
\mathbf{D}_{1} & \mathbf{D}_{2} & \cdots & \mathbf{D}_{K}
\end{array}\right], \\
& {\left[\mathbf{D}_{k}\right]_{(m, m)}=\dot{\mathbf{w}}_{j}^{H} \dot{\mathbf{H}}_{j} \dot{\mathbf{w}}_{j},} \\
& \mathbf{F}=\left[\mathbf{F}_{1}\left|\mathbf{F}_{2}\right| \cdots \mid \mathbf{F}_{K}\right], \\
& {\left[\mathbf{F}_{k}\right]_{(m, i)}=\left\{\begin{array}{cc}
\gamma_{m} \rho_{M A I}^{2}(m i) \tilde{\mathbf{w}}_{n}^{H} \tilde{\mathbf{H}}_{j} \tilde{\mathbf{w}}_{n}, & m \neq i, \\
\gamma_{m} \rho_{I F I}^{2}(m) \ddot{\mathbf{w}}_{j}^{H} \ddot{\mathbf{H}}_{j} \ddot{\mathbf{w}}_{j}, & m=i .
\end{array}\right.}
\end{aligned}
$$


EL vector $\mathbf{u}$ se mantiene como en el algoritmo JPCOB-VUL original (4.27).

Hay que tener en cuenta que los términos de correlación que se han presentado simplemente como $\rho_{I F I}^{2}(m)$ y $\rho_{M A I}^{2}(m i)$ provienen de variables aleatorias que presentan distintas distribuciones en función del valor del retardo $\tau_{m k}$ (ver expresiones $(5.21)$ y $(5.26)$ ).

Los pasos de esta segunda modificación del algoritmo JPCOB-VUL, para cada iteración $n_{i t}$ serían:

1. Inicializar las potencias $\mathrm{UL}$ virtuales, $p_{j}^{u}(0)$, y las potencias $\mathrm{DL}, p_{j}(0)$, para todos los enlaces del sistema, $j=1, \ldots, K M$, con valores positivos.

2. Para $n_{i t}=0,1,2, \ldots$, hasta llegar a la convergencia:

2.1. Beamforming. Calcular los beamformers subóptimos en transmisión $\mathbf{w}_{j}$ para cada enlace, $j=1, \ldots, K M$, maximizando para cada enlace la SINR recibida en el UL virtual (5.39):

$$
\mathbf{w}_{j}\left(n_{i t}\right)=\arg \operatorname{máx}_{\mathbf{w}_{j}}\left\{\frac{p_{j}^{u}\left(n_{i t}\right) \mathbf{w}_{j}^{H} \mathbf{H}_{j}^{0} \mathbf{w}_{j}}{\mathbf{w}_{j}^{H}\left(\sum_{n}\left(\rho_{M A I}^{0}(m i)\right)^{2} p_{n}^{u}\left(n_{i t}\right) \mathbf{H}_{n}^{0}+\mathbf{I}\right) \mathbf{w}_{j}}\right\},
$$

donde $n$ toma $M-1$ valores relacionados con $j$ de la siguiente forma: si $j=(k-1) M+m$, entonces $n=(k-1) M+i$ con $i=1, \ldots, M$, e $i \neq m$. En la práctica, cada uno de los beamformers $\mathbf{w}_{j}\left(n_{i t}\right)$ se calcula mediante la expresión MMSE (5.36), incluyendo únicamente en la matriz de correlación $\boldsymbol{\Phi}_{j}\left(n_{i t}\right)$ las matrices de correlación de los canales de mayor ganancia, $\mathbf{H}_{j}^{0}$.

2.2. Control de potencia, enlace UL virtual. Las potencias UL virtuales, $p_{j}^{u}$, se actualizan para cada enlace, $j=1, \ldots, K M$, mediante un control de potencia clásico distribuido [88]:

$$
p_{j}^{u}\left(n_{i t}+1\right)=\frac{\gamma_{m}}{\operatorname{SINR}_{j}^{V U}\left(n_{i t}\right)} p_{j}^{u}\left(n_{i t}\right) .
$$

donde $\operatorname{SINR}_{j}^{V U}\left(n_{i t}\right)$ es la SINR en el UL virtual dada en la ecuación (5.39), pero considerando las potencias UL virtuales y los beamformers de la iteración correspondiente.

2.3. Control de potencia, enlace DL. El vector $\mathbf{p}$ de potencias DL se actualiza mediante:

$$
\mathbf{p}\left(n_{i t}+1\right)=\mathbf{D}^{\dagger}\left(n_{i t}\right)\left(\mathbf{F}\left(n_{i t}\right) \mathbf{p}\left(n_{i t}\right)+\mathbf{u}\right),
$$


donde las matrices $\mathbf{D}\left(n_{i t}\right)$ y $\mathbf{F}\left(n_{i t}\right)$ se obtienen a partir de las expresiones (5.42) y (5.44), respectivamente, pero considerando los beamformers en transmisión correspondientes a la iteración $n_{i t}$.

De nuevo, la inclusión de los términos de correlación entre códigos y el nuevo diseño de los beamformers no afectan a las propiedades de convergencia de esta modificación del algoritmo JPCOB-VUL, que siguen siendo las presentadas en el apartado 4.2.5.

\section{Esquemas de asignación de códigos OVSF a nuevos usuarios}

En este apartado, el análisis de los esquemas de asignación de códigos se centra en el esquema MP o Minimum Partial, que para entornos flat fading presentaba las mejores prestaciones para factores de carga del sistema por debajo del $55 \%$.

En un escenario con canales flat fading, la única interferencia presente tanto en el sistema CM3 como en la configuración central es la interferencia de acceso múltiple. Además, todos los términos de correlación se pueden obtener a partir de un mismo modelo de variable aleatoria, en concreto a partir de una variable Gaussiana de media nula y desviación estándar obtenida a partir de simulaciones.

Cuando los canales son frequency selective, las dos configuraciones de sistema, la configuración CM3 y la central, presentan características distintas con respecto a los términos de interferencia. Además, para el caso CM3, la composición de la interferencia varía según el número de BSs incluidas en el AS de cada usuario.

Por otro lado, como se ha visto en las expresiones de potencia media de SOI, potencia media de IFI y potencia media de MAI, (5.16)-(5.21)-(5.26), respectivamente, un mismo término de correlación se modela con variables aleatorias diferentes según sea el valor del retardo $\tau_{m k}$.

Todo esto motiva la introducción de ciertos cambios en el esquema MP de asignación de códigos, puesto que hay que tener en cuenta más variables a la hora de establecer el orden óptimo de asignación.

En primer lugar nos centramos en la configuración central de sistema, donde las BSs o arrays de antenas se ubican en la zona central y cada BS transmite a los usuarios ubicados en su sector. En este caso, aunque un usuario sólo recibe señal desde una BS, el canal de propagación está formado por varias contribuciones multi-camino y habría que considerar tanto 
los términos de auto-correlación como los de correlación cruzada asociados al retardo $\tau_{m k}=0$, modelados según $\left(\left(m_{I F I}^{0}(m)\right)^{2}+\left(\sigma_{I F I}^{0}(m)\right)^{2}\right)$ y $\left(\left(m_{M A I}^{0}(m i)\right)^{2}+\left(\sigma_{M A I}^{0}(m i)\right)^{2}\right)$, respectivamente.

En el sistema CM3, en principio habría que considerar todos los términos de correlación posibles, los asociados con $\tau_{m k}=0$, que se modelan mediante las variables $\left(\left(m_{I F I}^{0}(m)\right)^{2}+\left(\sigma_{I F I}^{0}(m)\right)^{2}\right)$ y $\left(\left(m_{M A I}^{0}(m i)\right)^{2}+\right.$ $\left.\left(\sigma_{M A I}^{0}(m i)\right)^{2}\right)$, y los que se obtienen cuando este retardo no es nulo, modelados mediante $\left(m_{I F I}^{2}(m)+\sigma_{I F I}^{2}(m)\right)$ y $\left(m_{M A I}^{2}(m i)+\sigma_{M A I}^{2}(m i)\right)$.

En el enlace UL virtual de ambas configuraciones de sistema, se sigue considerando únicamente la contribución multi-camino de mayor ganancia del canal, de forma que para calcular la SINR en el UL virtual y diseñar los beamformers, sólo se consideran los términos de correlación cruzada modelados mediante $\left(\left(m_{M A I}^{0}(m i)\right)^{2}+\left(\sigma_{M A I}^{0}(m i)\right)^{2}\right)$.

El esquema MP en el entorno flat fading operaba sobre una matriz de desviaciones estándar $\mathbf{R}_{s t d}$, y obtenía un único orden de asignación para la configuración CM3 y la configuración central de sistema. Sin embargo, en el entorno frequency selective, las interferencias presentes en el sistema varían según sea la configuración del sistema, de forma que ahora el esquema MP va a obtener un orden de asignación de códigos distinto para cada configuración de sistema, CM3 y central.

El esquema MP de asignación de códigos OVSF en el entorno frequency selective construye en primer lugar una matriz $\boldsymbol{\Xi}$ de valores cuadráticos medios de correlación de códigos para cada configuración de sistema.

Para la configuración central, la construcción de esta matriz es bastante inmediata, puesto que los únicos valores cuadráticos medios a considerar son los asociados al retardo $\tau_{m k}=0$, es decir, $\left(\left(m_{I F I}^{0}(m)\right)^{2}+\left(\sigma_{I F I}^{0}(m)\right)^{2}\right)$ $\mathrm{y}\left(\left(m_{M A I}^{0}(m i)\right)^{2}+\left(\sigma_{M A I}^{0}(m i)\right)^{2}\right)$.

En el caso de la configuración CM3, en principio, la presencia de los valores cuadráticos medios depende que cuántas BSs estén activas en el AS de un usuario. Por ejemplo, si a un usuario sólo le transmite una BS, el término modelado por $\left(m_{I F I}^{2}(m)+\sigma_{I F I}^{2}(m)\right)$ se anula porque sólo le llega su señal deseada en $\tau_{m k}=0$, mientras que si el mismo usuario dispone de $K$ BSs activas en su AS, uno de los términos de correlación se modela según $\left(\left(m_{I F I}^{0}(m)\right)^{2}+\left(\sigma_{I F I}^{0}(m)\right)^{2}\right)$, mientras que los $K-1$ términos restantes se modelan como $\left(m_{I F I}^{2}(m)+\sigma_{I F I}^{2}(m)\right)$. 


\begin{tabular}{|c|c|c|}
\hline \hline Configuración & IFI & MAI \\
\hline \hline Central & $\left(\left(m_{I F I}^{0}(m)\right)^{2}+\left(\sigma_{I F I}^{0}(m)\right)^{2}\right)$ & $\left(\left(m_{M A I}^{0}(m i)\right)^{2}+\left(\sigma_{M A I}^{0}(m i)\right)^{2}\right)$ \\
\hline $\mathrm{CM} 3-\mathrm{AS}=1$ & $\left(\left(m_{I F I}^{0}(m)\right)^{2}+\left(\sigma_{I F I}^{0}(m)\right)^{2}\right)$ & $\left(\left(m_{M A I}^{0}(m i)\right)^{2}+\left(\sigma_{M A I}^{0}(m i)\right)^{2}\right)$ \\
& & $2 \cdot\left(m_{M A I}^{2}(m i)+\sigma_{M A I}^{2}(m i)\right)$ \\
\hline $\mathrm{CM} 3-\mathrm{AS}=2$ & $\left(\left(m_{I F I}^{0}(m)\right)^{2}+\left(\sigma_{I F I}^{0}(m)\right)^{2}\right)$ & $\left(\left(m_{M A I}^{0}(m i)\right)^{2}+\left(\sigma_{M A I}^{0}(m i)\right)^{2}\right)$ \\
& $\left(m_{I F I}^{2}(m)+\sigma_{I F I}^{2}(m)\right)$ & $2 \cdot\left(m_{M A I}^{2}(m i)+\sigma_{M A I}^{2}(m i)\right)$ \\
\hline $\mathrm{CM} 3-\mathrm{AS}=3$ & $\left(\left(m_{I F I}^{0}(m)\right)^{2}+\left(\sigma_{I F I}^{0}(m)\right)^{2}\right)$ & $\left(\left(m_{M A I}^{0}(m i)\right)^{2}+\left(\sigma_{M A I}^{0}(m i)\right)^{2}\right)$ \\
& $2 \cdot\left(m_{I F I}^{2}(m)+\sigma_{I F I}^{2}(m)\right)$ & $2 \cdot\left(m_{M A I}^{2}(m i)+\sigma_{M A I}^{2}(m i)\right)$ \\
\hline
\end{tabular}

Cuadro 5.2: Ponderación de los valores cuadráticos medios de correlación de códigos en función de las características del sistema.

La tabla 5.2 presenta un resumen de la ponderación de cada uno de los valores cuadráticos medios que intervienen en la señal recibida por un usuario, según sean las características del sistema, donde AS indica el número de BSs que se incluyen en el active set del usuario.

El esquema de asignación de códigos MP o Minimum Partial sigue los siguientes pasos:

1. Construye la matriz $\boldsymbol{\Xi}$ de dimensiones $[N \times N]$ definida mediante:

$$
\boldsymbol{\Xi}=\left(\begin{array}{cccc}
\xi_{11}^{2} & \xi_{12}^{2} & \cdots & \xi_{1 N}^{2} \\
\xi_{21}^{2} & \xi_{22}^{2} & \cdots & \xi_{2 N}^{2} \\
\vdots & \vdots & \ddots & \vdots \\
\xi_{N 1}^{2} & \xi_{N 2}^{2} & \cdots & \xi_{N N}^{2}
\end{array}\right)
$$

donde cada término $\xi_{i j}^{2}$ es el valor cuadrático medio resultante de la suma de todos los valores cuadráticos medios asociados a la pareja de códigos $(i, j)$. Para el caso de la configuración central de sistema sería:

$$
\xi_{i j}^{2}=\left(\left(m_{I F I}^{0}(i)\right)^{2}+\left(\sigma_{I F I}^{0}(i)\right)^{2}\right)+\left(\left(m_{M A I}^{0}(i j)\right)^{2}+\left(\sigma_{M A I}^{0}(i j)\right)^{2}\right),
$$

mientras que para la configuración de sistema CM3 con 3 BSs en el active set se obtendría mediante:

$$
\begin{aligned}
\xi_{i j}^{2} & =\left(\left(m_{I F I}^{0}(i)\right)^{2}+\left(\sigma_{I F I}^{0}(i)\right)^{2}\right)+\left(\left(m_{M A I}^{0}(i j)\right)^{2}+\left(\sigma_{M A I}^{0}(i j)\right)^{2}\right) \\
& +2 \cdot\left(m_{I F I}^{2}(i)+\sigma_{I F I}^{2}(i)\right)+2 \cdot\left(m_{M A I}^{2}(i j)+\sigma_{M A I}^{2}(i j)\right) .
\end{aligned}
$$


2. Suponemos la situación en la que el código \#1 es el único asignado (este código se utiliza habitualmente en canales de control), y llega un nuevo usuario al sistema. En este caso el esquema MP busca entre el resto de códigos disponibles aquel código $j$ tal que la suma de valores cuadráticos $\xi_{1 j}^{2}+\xi_{j j}^{2}$ sea mínima.

3. Una vez que en el sistema hay más de dos códigos activos, y llega un nuevo usuario, el siguiente código $j$ en asignarse es aquel que minimiza la suma de los valores cuadráticos medios con respecto a los códigos ya activos en la celda:

$$
j=\arg \min \left\{\xi_{j j}^{2}+\sum_{i \text { activos }} \xi_{j i}^{2}\right\} .
$$

En un escenario real, los términos $\xi_{j j}^{2}$ debidos a la interferencia entre fingers serían prácticamente despreciables frente a los producidos por la interferencia de acceso múltiple, $\xi_{j i}^{2}$. Sin embargo, en las simulaciones de este Capítulo no se desprecia ninguna contribución, puesto que el principal interés es caracterizar lo más fielmente posible la potencia de interferencia que recibe un usuario en un sistema W-CDMA CM3.

Por otro lado, destacar de nuevo que el esquema de asignación de códigos se llama Minimum Partial porque a la hora de asignar un nuevo código, sólo tiene en cuenta la interferencia introducida hacia el conjunto de códigos activos en el sistema.

De la misma forma que en los esquemas propuestos para el caso de canales flat fading, el esquema MP en un entorno frequency selective puede proporcionar un orden de asignación óptimo y fijo, puesto que se obtiene a partir de una serie de parámetros que sólo varían si se produce alguna variación en el PDP de alguno de los canales del sistema.

\section{Estudio de la probabilidad de admisión de usuarios}

Esta tercera simulación continúa con el estudio de la probabilidad de admisión del esquema MP, ahora para canales frequency selective [99].

El objetivo es de nuevo comparar la probabilidad de admisión del algoritmo JPCOB-VUL modificado de (5.46)-(5.48) en el entorno Coordinado I, con la probabilidad de admisión del algoritmo JPCOB de referencia de [33], (3.54)-(3.56), que también se modifica para tener en cuenta el nuevo modelo de los términos de correlación entre códigos. Cada uno de estos algoritmos 


\begin{tabular}{|c|c|c|c|}
\hline \hline Tap & Retardo (nseg.) & Potencia media (dB) & Espectro Doppler \\
\hline \hline 1 & 0 & 0 & clásico \\
\hline 2 & 200 & $-0,9$ & clásico \\
\hline 3 & 800 & $-4,9$ & clásico \\
\hline 4 & 1200 & $-8,0$ & clásico \\
\hline 5 & 2300 & $-7,8$ & clásico \\
\hline 6 & 3700 & $-23,9$ & clásico \\
\hline
\end{tabular}

Cuadro 5.3: Outdoor to Indoor and Pedestrian Test Environment Tapped-DelayLine Parameters.

se ejecuta sobre la configuración de sistema para la que se propusieron: un sistema CM3 para el caso del algoritmo JPCOB-VUL y un sistema central equivalente en el caso del algoritmo JPCOB de referencia (ver figura 4.4).

En esta simulación, se varían algunos parámetros para acortar el tiempo de simulación. En primer lugar, el factor de spreading se fija en $N=32$, y la EbN0 se establece en 12,1 dB, lo que se corresponde con un valor umbral de SINR antes del despreading, suponiendo modulación QPSK, de -5,6 dB para cada usuario.

Para cada algoritmo y configuración de sistema, una simulación empieza con $M=8$ usuarios, que se distribuyen uniformemente por el sistema y a los que se les asigna una velocidad de $4 \mathrm{~km} / \mathrm{h}$, y continúa añadiendo usuarios mientras el algoritmo converja (mientras necesite menos de It iteraciones para converger, cada BS no supere su restricción de potencia máxima transmitida y todos los usuarios alcancen la SINR umbral).

En general, los parámetros de simulación siguen siendo los de la tabla 5.1. Para la configuración de sistema CM3, se simulan sólo los valores de ventana de $\mathrm{AS}$ de $0 \mathrm{~dB}$ (sin coordinación), $2 \mathrm{~dB}$ y $5 \mathrm{~dB}$, aunque en los resultados de las simulaciones en el entorno flat fading la ventana de AS de $2 \mathrm{~dB}$ mejora significativamente las prestaciones de la ventana de AS de 5 dB. Para cada algoritmo y cada configuración de sistema, CM3 o central, se evalúan 500 realizaciones independientes.

Con respecto a la caracterización de las variables aleatorias que modelan los términos de auto-correlación y correlación cruzada entre los códigos, se asume que todos los canales del sistema presentan un PDP del tipo Outdoorto-Indoor and Pedestrian Channel B. La tabla 5.3 resume las características de este canal [117]. 


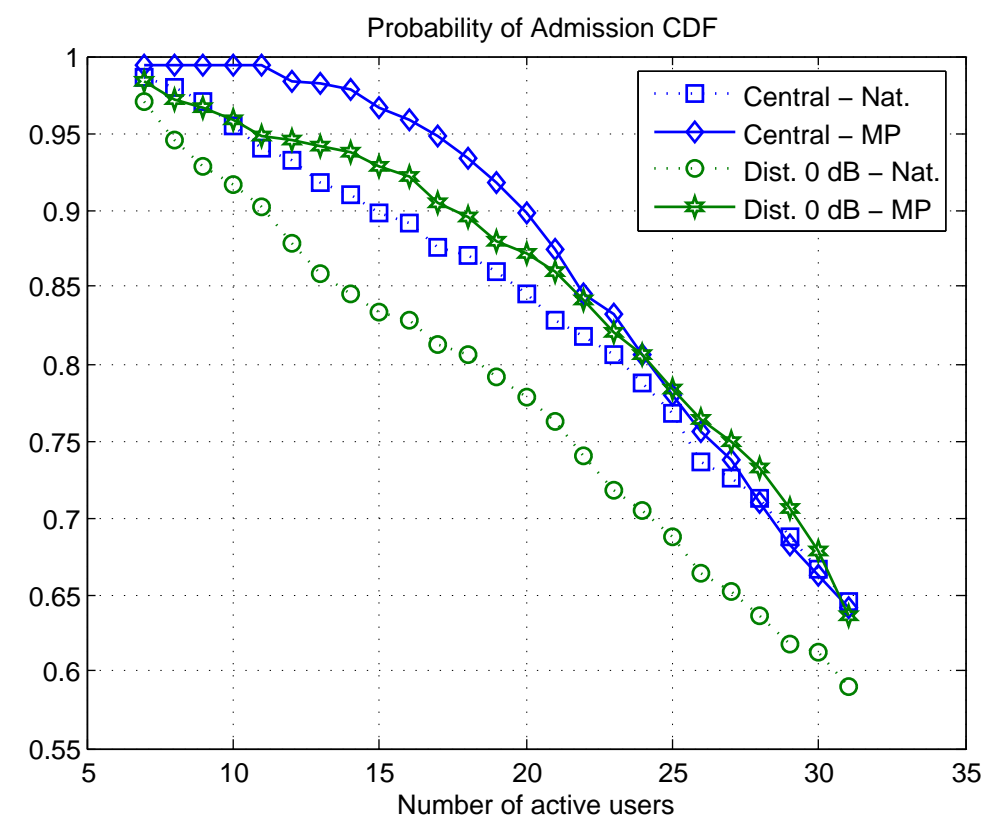

Figura 5.13: Función de distribución estimada de la probabilidad de admisión del orden Natural y MP, para configuraciones de sistema CM3 y centrales con $K=3$ y $N_{t}=3$. La configuración CM3 o Dist., se presenta para ASW $=0 \mathrm{~dB}$.

El valor de la media y varianza que caracteriza a cada una de las variables aleatorias se obtiene mediante simulación, comparando los códigos dos a dos, y extrayendo las probabilidades de cada contribución a partir del PDP del canal.

Por otro lado, es necesario definir un mecanismo que determine, en la configuración de sistema CM3, qué BS es la que va a asociarse con el retardo $\tau_{m k}=0$. En las simulaciones, para un usuario $m$, se asocia con el retardo $\tau_{m k}=0$ aquella BS cuya potencia de canal piloto se establece como valor de referencia para construir el active set de BSs del usuario.

La figura 5.13 compara la función de distribución estimada o CDF de la probabilidad de admisión del orden Natural (los códigos se asignan de forma sucesiva, empezando en el 1 y acabando en el $N$ ) y del orden MP, para el algoritmo JPCOB-VUL sobre la configuración CM3 y el algoritmo JPCOB de referencia sobre la configuración central. Ambas configuraciones de sistema disponen de $K=3 \mathrm{BSs}$, equipada cada una de ellas con arrays 
lineales de $N_{t}=3$ antenas. Los resultados de la configuración CM3 se presentan para el caso de $\mathrm{ASW}=0 \mathrm{~dB}$, donde sólo una BS transmite a cada usuario, por lo que ambas configuraciones sólo se diferencian en la ubicación espacial de las BSs en el sistema.

Desde el punto de vista de los esquemas de asignación de códigos, y para la configuración de sistema CM3, el esquema MP aumenta considerablemente la probabilidad de admisión con respecto al esquema Natural para cualquier factor de carga del sistema. De hecho, el esquema MP consigue un incremento constante del $10 \%$ en la probabilidad de admisión con respecto al esquema Natural. En el caso de la configuración central de BSs, esta mejora en la probabilidad de admisión disminuye a medida que aumenta el número de usuarios admitidos.

Si ahora nos centramos en un mismo esquema de asignación de códigos, y comparamos la probabilidad de admisión de las dos configuraciones de sistema, CM3 y central, se observa que la configuración central de BSs aumenta la probabilidad de admisión con respecto a la configuración CM3. Esta mejora es más acusada en el caso del esquema Natural de asignación de códigos, mientras que la diferencia se reduce para el esquema MP, donde la configuración CM3 llega incluso a mejorar la probabilidad de admisión de la configuración central para factores elevados de carga del sistema (más de 25 usuarios activos).

El hecho de que la configuración central de BSs aumente la probabilidad de admisión con respecto a la configuración de sistema CM3 se puede explicar recordando el estudio de la tasa de fallo del Capítulo 4, donde se comenta que aunque las dos configuraciones de sistema, la central y la CM3, son físicamente equivalentes, la potencia de interferencia que recibe un usuario en cada sistema es diferente. En la configuración central de sistema, puesto que el algoritmo de referencia de Rashid-Farrokhi et al. se ejecuta de forma independiente en cada uno de de los sectores, un usuario únicamente recibe potencia de interferencia de los usuarios asignados a su mismo sector. Por el contrario, en la configuración CM3, aunque se considere una ventana de AS de $0 \mathrm{~dB}$, donde sólo una BS transmite a cada usuario, un usuario recibe la potencia de interferencia correspondiente al resto de usuarios del sistema.

Las figuras 5.14 y 5.15 presentan la función de distribución estimada o CDF de la probabilidad de admisión de los esquemas Natural y MP para una configuración de sistema CM3 con $K=3$ BSs, cuando cada BS dispone de un array lineal de $N_{t}=2$ o $N_{t}=3$ antenas, respectivamente. En las 


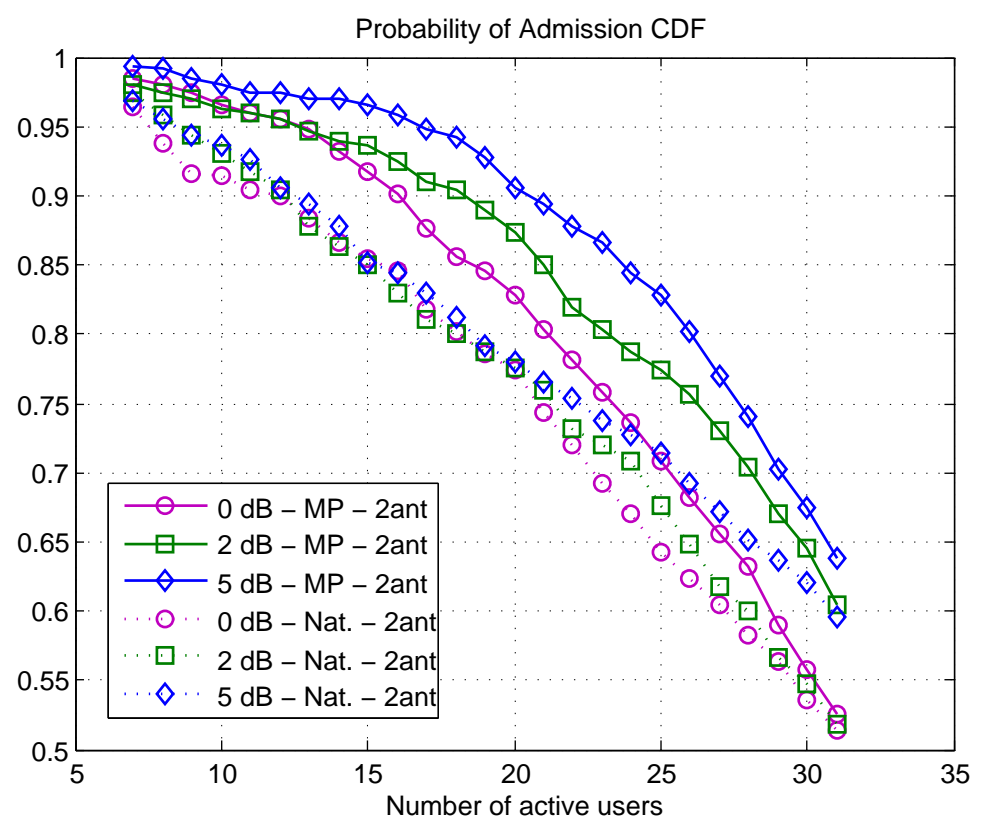

Figura 5.14: Función de distribución estimada de la probabilidad de admisión del orden Natural y MP, para una configuración de sistema CM3 con $K=3$ y $N_{t}=2$, indicando asimismo el tamaño de la ventana de $\operatorname{AS}(0,2$ ó $5 \mathrm{~dB})$.

figuras se presentan los resultados obtenidos por las distintas ventanas de AS simuladas, $\mathrm{ASW}=0, \mathrm{ASW}=2 \mathrm{y} \mathrm{ASW}=5 \mathrm{~dB}$.

En general, los resultados de ambas figuras siguen la pauta de los presentados en la figura 5.13 con respecto a los esquemas de asignación de códigos: el esquema MP aumenta la probabilidad de admisión con respecto al esquema Natural para cualquier número de antenas en la BS y para los tamaños de ventana de AS simulados. Sin embargo, hay que destacar que para factores de carga de sistema superiores a los 25 usuarios (figura 5.14), el esquema Natural con $\mathrm{ASW}=5 \mathrm{~dB}$ mejora la probabilidad de admisión del esquema MP con $\mathrm{ASW}=0 \mathrm{~dB}$. Este punto se desplaza hasta los 30 usuarios cuando las BSs disponen de arrays de $N_{t}=3$ antenas (figura 5.15).

Desde el punto de vista de un mismo esquema de asignación de códigos, la conclusión más sorprendente de estas figuras es el hecho de que un pequeño grado de coordinación entre las BSs, como es el caso de las ventanas de $\mathrm{AS}$ de $2 \mathrm{~dB}$ y $5 \mathrm{~dB}$, permite mejorar significativamente las prestaciones del sistema en términos de probabilidad de admisión. Esta mejora es to- 


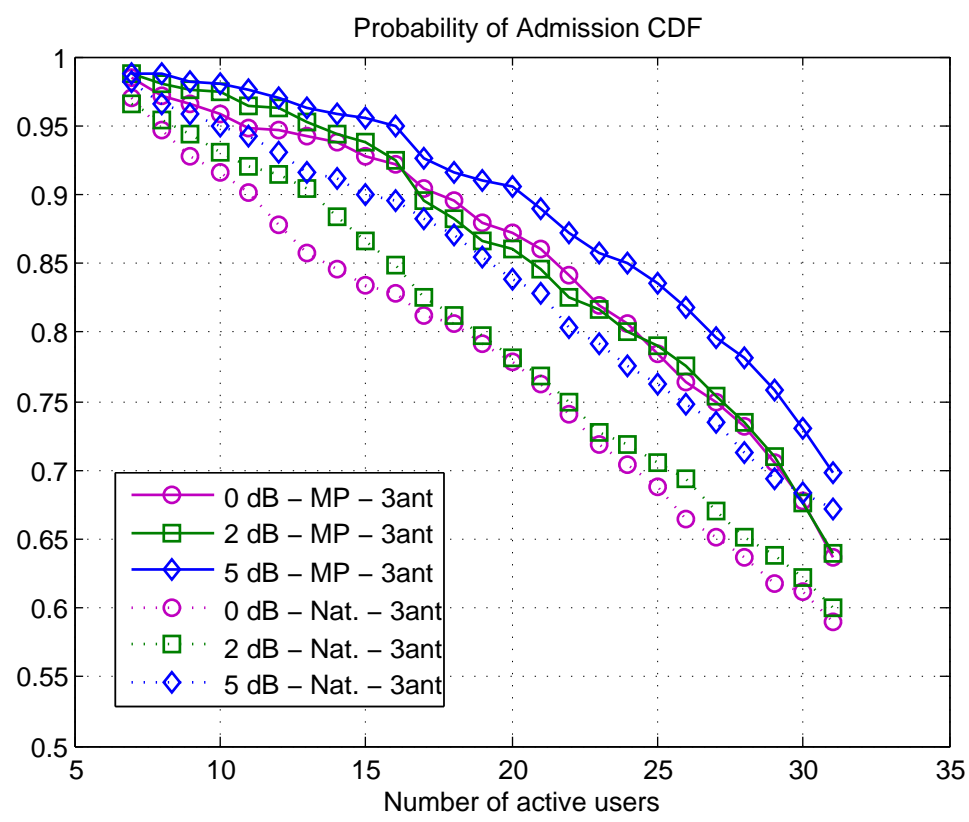

Figura 5.15: Función de distribución estimada de la probabilidad de admisión del orden Natural y MP, para una configuración de sistema CM3 con $K=3$ y $N_{t}=3$, indicando asimismo el tamaño de la ventana de AS (0, 2 ó $\left.5 \mathrm{~dB}\right)$.

davía más acusada cuando además de permitir cierta coordinación entre las BSs del sistema, se implementa un esquema de asignación de códigos OVSF como el MP, diseñado para reducir la potencia de interferencia que perciben los usuarios del sistema CM3. Por ejemplo, en la configuración de sistema con $N_{t}=2$, figura 5.14, la combinación del esquema MP con la ventana de AS de $5 \mathrm{~dB}$ consigue una mejora de la probabilidad de admisión del orden del $15 \%$ con respecto a la ventana de $0 \mathrm{~dB}$, mientras que esta mejora aumenta hasta un $20 \%$ si se compara con la curva del esquema Natural sin coordinación, ASW $=0 \mathrm{~dB}$.

Por último, comparando las figuras 5.14 y 5.15 se observa que aumentar el número de antenas disponibles en cada BS, es decir, pasar de $N_{t}=2$ a $N_{t}=3$, conlleva una mejora de la probabilidad de admisión general para factores de carga del sistema superiores a los 20 usuarios activos. 


\subsection{Control robusto de potencia para escenarios con hybrid channel knowledge}

Hasta el momento, las simulaciones que se presentan para analizar las prestaciones del algoritmo JPCOB-VUL asumen que las BSs del sistema CM3 disponen de CSIT perfecta y que cooperan perfectamente en datos, por lo que cualquier BS del sistema tiene acceso al canal de propagación de cualquier enlace usuario-BS del sistema.

En principio, el algoritmo JPCOB-VUL realiza un diseño distribuido o local de los beamformers en transmisión para cada usuario, pero requiere implementar el control de potencia de forma centralizada, puesto que necesita conocer los canales de propagación de todos los enlaces del sistema.

En [119], se realiza un primer estudio para proponer soluciones robustas de beamforming para el algoritmo JPCOB-VUL, cuando las BSs no disponen de CSIT perfecta. Sin embargo, teniendo en cuenta la estructura del algoritmo JPCOB-VUL, el control de potencia es el paso del algoritmo que, con toda probabilidad, sufriría una mayor degradación en un escenario CM3 real, debido a la dificultad de disponer de forma centralizada de todos los canales de propagación del sistema.

En este apartado, se considera un sistema W-CDMA CM3 en el que las BSs disponen de CSIT perfecta, pero en el que existe algún tipo de restricción en la cantidad de información que las BSs pueden intercambiar a través del enlace de alta capacidad, o lo que es lo mismo, no es posible disponer de forma centralizada y perfecta de todos los canales de propagación del sistema. Esta situación se puede modelar mediante una forma de conocimiento parcial del canal denominada hybrid channel knowledge [53,73].

El concepto de hybrid channel knowledge se plantea para modelar los casos en los que en un sistema CM3, cada BS dispone de CSIT perfecta con respecto a los usuarios que se encuentran dentro de su área de cobertura, pero sólo dispone de información estadística de los canales de propagación del resto de enlaces del sistema. Este modelo de CSI parcial se ajusta perfectamente a un sistema CM3 donde existe un determinado límite en la cantidad de información que pueden intercambiar las BSs.

En esta segunda aplicación práctica del algoritmo JPCOB-VUL, se utiliza el concepto de hybrid channel knowledge para modelar aquellas situaciones en las que no es posible disponer de forma centralizada de todos los canales de propagación del sistema. Al mismo tiempo, se modifica el paso de actualización de potencias DL del algoritmo JPCOB-VUL para incluir 
un control de potencia robusto y adaptado a este conocimiento parcial del canal, siguiendo el modelo propuesto en [120].

De esta forma, tenemos un sistema CM3 donde las BSs disponen de hybrid channel knowledge para ejecutar el algoritmo JPCOB-VUL. En las simulaciones de este Capítulo, se simplifica el entorno de simulación para poder evaluar de forma eficiente las prestaciones de esta modificación robusta del algoritmo JPCOB-VUL. Concretamente, se generan canales de propagación flat fading para cada enlace del sistema y se vuelven a considerar usuarios co-canal.

\section{Modificación del algoritmo JPCOB-VUL en el entorno Coordinado I}

La primera modificación afecta al diseño de los beamformers. Los beamformers de cada usuario se siguen calculando mediante la dualidad UL-DL, sin embargo, se abandona la expresión MMSE (4.30) y se plantea un diseño ZF (zero forcing), siguiendo la propuesta de [119]:

$$
\mathbf{w}_{m k}=\left(\sqrt{\mathbf{P}_{k}^{u}} \mathbf{H}_{k}^{H}\right)^{\dagger} \mathbf{e}_{m},
$$

donde $\sqrt{\mathbf{P}_{k}^{u}}$ es una matriz diagonal $[M \times M]$ que incluye la raíz cuadrada de las potencias UL virtuales recibidas en la BS $k$,

$$
\sqrt{\mathbf{P}_{k}^{u}}=\operatorname{diag}\left\{\left[\sqrt{p_{1 k}^{u}} \cdots \sqrt{p_{M k}^{u}}\right]\right\},
$$

la matriz de canales $\mathbf{H}_{k}$, de dimensiones $\left[N_{t} \times M\right]$, incluye los canales flat fading entre la BS $k$ y todos los usuarios del sistema:

$$
\mathbf{H}_{k}=\left[\mathbf{h}_{1 k}^{H} \ldots \mathbf{h}_{M k}^{H}\right],
$$

y $\mathbf{e}_{m}$ es la columna $m$-ésima de la matriz identidad $[M \times M]$.

La actualización de las potencias UL virtuales permanece como en el algoritmo JPCOB-VUL original, puesto que las BSs disponen de CSIT local perfecta.

El paso de actualización de potencias DL se modifica para conseguir un mecanismo de control de potencia robusto frente a situaciones de CSI parcial. La expresión habitual de la SINR recibida en el usuario $m$ :

$$
\operatorname{SINR}_{m}^{D L}=\frac{\sum_{k=1}^{K} p_{m k} \mathbf{w}_{m k}^{H} \mathbf{H}_{m k} \mathbf{w}_{m k}}{\sum_{k=1}^{K} \sum_{i=1, i \neq m}^{M} p_{i k} \mathbf{w}_{i k}^{H} \mathbf{H}_{m k} \mathbf{w}_{i k}+\sigma_{m}^{2}},
$$


depende de la matriz de correlación de canal entre el usuario $m$ y la BS $k, \mathbf{H}_{m k}=E\left[\mathbf{h}_{m k}^{H} \mathbf{h}_{m k}\right]$. Si existe algún tipo de error en la estima del canal, esta matriz de correlación de canal puede expresarse como:

$$
\mathbf{H}_{m k}=\tilde{\mathbf{H}}_{m k}+\mathbf{E}_{m k},
$$

donde $\tilde{\mathbf{H}}_{m k}$ es la matriz de correlación de canal estimada, $\mathbf{H}_{m k}$ es la matriz de correlación de canal real y $\mathbf{E}_{m k}$ es una matriz de error desconocida, cuya norma de Frobenius está acotada por una constante que sí que es conocida [120]:

$$
\left\|\mathbf{E}_{m k}\right\|_{F} \leq \epsilon \cdot\left\|\tilde{\mathbf{H}}_{m k}\right\|_{F}=\epsilon_{m k} .
$$

El modelo de error (5.54) permite modificar las restricciones de SINR del criterio de minimización de potencia (4.16) para conseguir un control de potencia robusto frente a los errores en la estima del canal:

$$
\frac{\sum_{k=1}^{K} p_{m k} \mathbf{w}_{m k}^{H}\left(\tilde{\mathbf{H}}_{m k}+\mathbf{E}_{m k}\right) \mathbf{w}_{m k}}{\sum_{k=1}^{K} \sum_{i=1, i \neq m}^{M} p_{i k} \mathbf{w}_{i k}^{H}\left(\tilde{\mathbf{H}}_{m k}+\mathbf{E}_{m k}\right) \mathbf{w}_{i k}+\sigma_{m}^{2}} \geq \gamma_{m}, m=1, \ldots, M,
$$

para todas las posibles matrices de error tales que $\left\|\mathbf{E}_{m k}\right\|_{F} \leq \epsilon_{m k}$, y donde $\gamma_{m}$ es la SINR umbral requerida para el usuario $m$.

La expresión (5.55) se corresponde con la SINR que recibiría el usuario en el caso peor. Si se introduce una cota inferior:

$$
\frac{\sum_{k=1}^{K} p_{m k} \operatorname{mín}_{m k}^{H}\left(\tilde{\mathbf{H}}_{m k}+\mathbf{E}_{m k}\right) \mathbf{w}_{m k}}{\sum_{k=1}^{K} \sum_{i=1, i \neq m}^{M} p_{i k} \operatorname{máx} \mathbf{w}_{i k}^{H}\left(\tilde{\mathbf{H}}_{m k}+\mathbf{E}_{m k}\right) \mathbf{w}_{i k}+\sigma_{m}^{2}}, m=1, \ldots, M,
$$

y se aplica el lema 1 de [120]:

Para cualquier vector $\mathbf{w}$, matriz $\mathbf{H}$ y un escalar positivo $\delta$,

$$
\begin{aligned}
\text { mín } \quad & \mathbf{w}^{H}(\mathbf{H}+\mathbf{E}) \mathbf{w}=\mathbf{w}^{H}(\mathbf{H}-\delta \mathbf{I}) \mathbf{w} \\
\operatorname{máx} & \mathbf{w}^{H}(\mathbf{H}+\mathbf{E}) \mathbf{w}=\mathbf{w}^{H}(\mathbf{H}+\delta \mathbf{I}) \mathbf{w} \\
\|E\|_{F} \leq & \delta,
\end{aligned}
$$

se obtiene la expresión de la SINR recibida en los usuarios que permite derivar el control robusto de potencia:

$$
\frac{\sum_{k=1}^{K} p_{m k} \mathbf{w}_{m k}^{H}\left(\tilde{\mathbf{H}}_{m k}-\epsilon_{m k} \mathbf{I}\right) \mathbf{w}_{m k}}{\sum_{k=1}^{K} \sum_{i=1, i \neq m}^{M} p_{i k} \mathbf{w}_{i k}^{H}\left(\tilde{\mathbf{H}}_{m k}+\epsilon_{m k} \mathbf{I}\right) \mathbf{w}_{i k}+\sigma_{m}^{2}}=\gamma_{m}, m=1, \ldots, M,
$$


donde $\mathbf{I}$ es una matriz identidad de dimensiones $\left[N_{t} \times N_{t}\right]$.

Siguiendo los pasos (4.19)-(4.21) a partir de la expresión (5.57), la ecuación de la actualización de potencias puede expresarse de forma multiusuario y matricial según:

$$
\mathrm{Dp}=\mathbf{F p}+\mathbf{u}
$$

donde las matrices D y $\mathbf{F}$ se modifican de la siguiente forma (4.23)-(4.26):

$$
\begin{aligned}
& \mathbf{D}=\left[\begin{array}{l|l|l|l}
\mathbf{D}_{1} & \mathbf{D}_{2}|\cdots| \mathbf{D}_{K}
\end{array}\right], \\
& {\left[\mathbf{D}_{k}\right]_{(m, m)}=\mathbf{w}_{j}^{H}\left(\tilde{\mathbf{H}}_{j}-\epsilon_{j} \mathbf{I}\right) \mathbf{w}_{j} \text {, }} \\
& \mathbf{F}=\left[\begin{array}{l|l|l|l}
\mathbf{F}_{1} & \mathbf{F}_{2}|\cdots| \mathbf{F}_{K}
\end{array}\right], \\
& {\left[\mathbf{F}_{k}\right]_{(m, i)}=\left\{\begin{array}{cc}
\gamma_{m} \mathbf{w}_{n}^{H}\left(\tilde{\mathbf{H}}_{j}+\epsilon_{j} \mathbf{I}\right) \mathbf{w}_{n}, & m \neq i, \\
0, & m=i .
\end{array}\right.}
\end{aligned}
$$

EL vector u se mantiene como en el algoritmo JPCOB-VUL original (4.27).

Los pasos de esta versión robusta del algoritmo JPCOB-VUL, para cada iteración $n_{i t}$ serían:

1. Inicializar las potencias UL virtuales, $p_{j}^{u}(0)$, y las potencias DL, $p_{j}(0)$, para todos los enlaces del sistema, $j=1, \ldots, K M$, con valores positivos.

2. Para $n_{i t}=0,1,2, \ldots$, hasta llegar a la convergencia:

2.1. Beamforming. Calcular los beamformers subóptimos en transmisión $\mathbf{w}_{j}$ para cada enlace, $j=1, \ldots, K M$, mediante la expresión del criterio ZF (5.51):

$$
\mathbf{w}_{j}\left(n_{i t}\right)=\left(\sqrt{\mathbf{P}_{k}^{u}\left(n_{i t}\right)} \tilde{\mathbf{H}}_{k}^{H}\right)^{\dagger} \mathbf{e}_{m}
$$

donde $j=(k-1) M+m$ y la matriz de canales $\tilde{\mathbf{H}}_{k}$ se construye a partir de los canales estimados $\tilde{\mathbf{h}}_{m k}$.

2.2. Control de potencia, enlace UL virtual. Las potencias UL virtuales, $p_{j}^{u}$, se actualizan para cada enlace, $j=1, \ldots, K M$, mediante un control de potencia clásico distribuido [88]:

$$
p_{j}^{u}\left(n_{i t}+1\right)=\frac{\gamma_{m}}{\operatorname{SINR}_{j}^{V U}\left(n_{i t}\right)} p_{j}^{u}\left(n_{i t}\right),
$$


donde $\operatorname{SINR}_{j}^{V U}\left(n_{i t}\right)$ es la SINR en el UL virtual dada en la ecuación (4.18), pero considerando las potencias UL virtuales y los beamformers de la iteración $n_{i t}$, así como la matriz de correlación de canal estimada del enlace correspondiente:

$$
\operatorname{SINR}_{j}^{V U}\left(n_{i t}\right)=\frac{p_{j}^{u}\left(n_{i t}\right) \mathbf{w}_{j}^{H}\left(n_{i t}\right) \tilde{\mathbf{H}}_{j} \mathbf{w}_{j}\left(n_{i t}\right)}{\mathbf{w}_{j}^{H}\left(n_{i t}\right)\left(\sum_{n} p_{n}^{u}\left(n_{i t}\right) \tilde{\mathbf{H}}_{n}+\mathbf{I}\right) \mathbf{w}_{j}\left(n_{i t}\right)} .
$$

En la expresión anterior, los índices $j$ y $n$ cumplen $j=(k-1) M+m$, $n=(k-1) M+i$, siendo $i=1, \ldots M$, e $i \neq m$.

2.3. Control de potencia, enlace DL. El vector $\mathbf{p}$ de potencias DL se actualiza mediante:

$$
\mathbf{p}\left(n_{i t}+1\right)=\mathbf{D}^{\dagger}\left(n_{i t}\right)\left(\mathbf{F}\left(n_{i t}\right) \mathbf{p}\left(n_{i t}\right)+\mathbf{u}\right),
$$

donde las matrices $\mathbf{D}\left(n_{i t}\right)$ y $\mathbf{F}\left(n_{i t}\right)$ se obtienen a partir de las expresiones (5.59) y (5.61), respectivamente, pero considerando los beamformers en transmisión correspondientes a la iteración $n_{i t}$.

El hecho de modificar el diseño de los beamformers y el paso de actualización de las potencias DL para incluir un mecanismo robusto frente a situaciones de CSI parcial, no influye en la convergencia de esta versión robusta del algoritmo JPCOB-VUL, que mantiene las propiedades de convergencia del algoritmo JPCOB-VUL original.

\section{Hybrid channel knowledge}

A la hora de incorporar el concepto de hybrid channel knowledge a la versión robusta del algoritmo JPCOB-VUL, la opción elegida es suponer que los $K$ canales de propagación que relacionan a un usuario con las BSs del sistema CM3 se modelan mediante distintos grados de precisión, es decir, que cada uno de los canales del usuario $m$ presenta una matriz de error distinta:

$$
\mathbf{E}_{m}=\left[\mathbf{E}_{m 1} \ldots \mathbf{E}_{m K}\right]
$$

En las simulaciones, para cada usuario $m$ del sistema, se evalúa la norma de Frobenius de las matrices de correlación de canal estimadas, $\left\|\tilde{\mathbf{H}}_{m k}\right\|_{F}, \quad k=1, \ldots, K$, y se asocia con una estima perfecta del canal al enlace $m k$ que presenta la mayor norma. Los $K-1$ canales restantes se asocian con una matriz de error desconocida generada a partir del modelo (5.53)-(5.54). 


\section{Prestaciones del control robusto de potencia}

El objetivo de las simulaciones es evaluar las prestaciones de la versión robusta del algoritmo JPCOB-VUL en un sistema CM3 donde las BSs disponen de una forma de conocimiento parcial del canal denominada hybrid channel knowledge.

Una simulación consiste en la evaluación de la versión robusta del algoritmo JPCOB-VUL de (5.63)-(5.65), y de una versión no robusta, sobre una misma realización del canal. La versión no robusta del algoritmo JPCOBVUL mantiene el diseño de los beamformers de la expresión (5.63) y la actualización de las potencias UL de (5.64). Sin embargo, el paso de actualización de potencias DL se mantiene como en el algoritmo JPCOB-VUL original, (4.33), pero considerando las matrices de correlación de canal estimadas $\tilde{\mathbf{H}}_{j}$. Tras la convergencia de la versión robusta y no robusta del algoritmo JPCOB-VUL, se calcula la SINR real que están alcanzando los usuarios del sistema en ambos casos. Esta SINR se calcula de la forma habitual, (5.52), pero considerando las matrices de correlación de canal reales, $\mathbf{H}_{j}$, y las potencias DL obtenidas por la versión robusta en un caso, y la versión no robusta en el otro.

En las simulaciones, la versión robusta y no robusta del algoritmo JPCOB-VUL se evalúan sobre una misma realización del canal, variando además de forma progresiva el grado de coordinación entre BSs o parámetro ASW y el valor de $\epsilon$ del modelo de error (5.54). Cada una de estas versiones del algoritmo converge cuando los $M$ usuarios del sistema alcanzan la SINR umbral requerida, sin superar el máximo de $I t$ iteraciones ni la restricción en potencia total transmitida por BS, $P_{\max }$. Para evaluar la convergencia del algoritmo, la versión robusta utiliza la expresión (5.57) de la SINR en DL, mientras que la versión no robusta recurre a la expresión habitual de la SINR, (5.52), pero considerando las matrices de correlación de canal estimadas. En total, se evalúan 1000 realizaciones independientes de cada simulación.

El escenario de simulación es el siguiente. Se considera un sistema CM3 con $K=3 \mathrm{BSs}$, donde cada una de ellas dispone de un array lineal de $N_{t}=3$ antenas. En cada simulación, se distribuyen uniformemente $M=9$ usuarios co-canal a los que se les asigna una velocidad de $4 \mathrm{~km} / \mathrm{h}$. El factor de spreading se fija en $N=32$, y el valor umbral de SINR se establece en -12 dB. Los canales de propagación son canales flat fading. El resto de parámetros de simulación son los presentados en la tabla 5.1. 


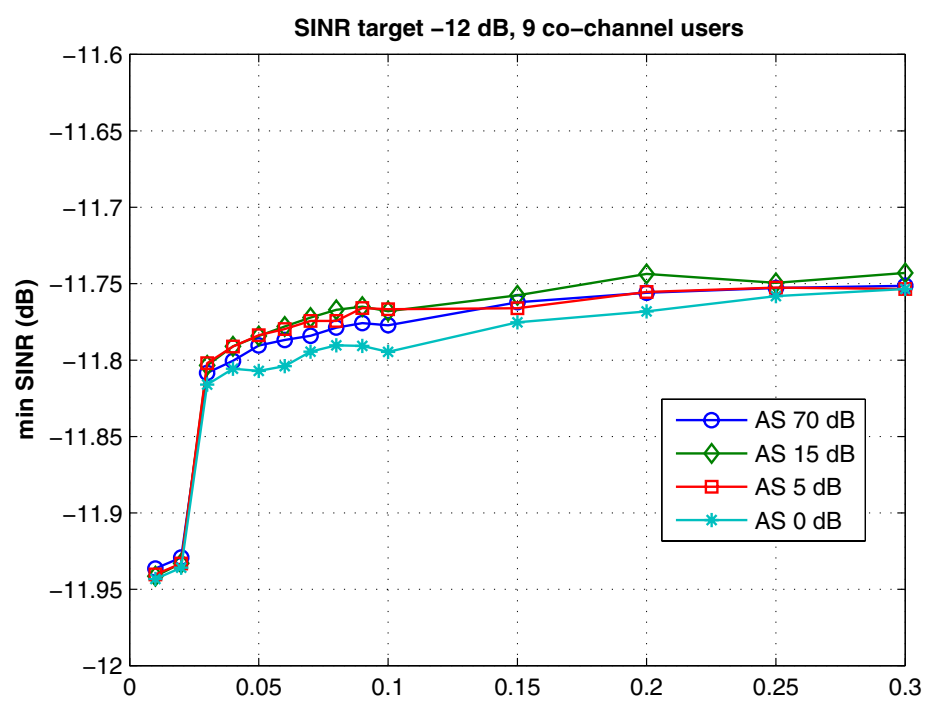

Figura 5.16: Valor medio de la mínima SINR real obtenido por el control robusto de potencia, en función del valor de $\epsilon$.

De nuevo, el esquema de selección de enlaces activos simulado es el del entorno Coordinado I. Concretamente, se simulan cuatro valores de ventana de $\mathrm{AS}, 0 \mathrm{~dB}, 5 \mathrm{~dB}, 15 \mathrm{~dB}$ y $70 \mathrm{~dB}$, que proporcionan los grados de coordinación entre BSs más significativos: sin coordinación (0 dB), coordinación entre una y dos BSs ( $5 \mathrm{~dB})$, coordinación entre dos o tres BSs (15 dB), o coordinación total (70 dB).

La figura 5.16 presenta el valor medio de la mínima SINR real que alcanzan los usuarios cuando se varía el valor de la constante $\epsilon$ y el tamaño de ventana de $\mathrm{AS}$ en el control robusto de potencia, mientras que la figura 5.17 representa los resultados obtenidos en los mismos casos por el control no robusto de potencia [100].

Se puede observar en la figura 5.16 que el control robusto de potencia consigue que el valor medio de la mínima SINR alcanzada en el sistema supere la SINR umbral requerida, independientemente del valor de $\epsilon$. Hay que destacar que la curva de $\mathrm{ASW}=0 \mathrm{~dB}$, cuando sólo una BS transmite a cada usuario, se corresponde con los resultados presentados en el algoritmo de control robusto de potencia de referencia [120].

Por otro lado, el control no robusto de potencia (figura 5.17) falla en la mayoría de los casos y el valor medio de la mínima SINR alcanzada en 


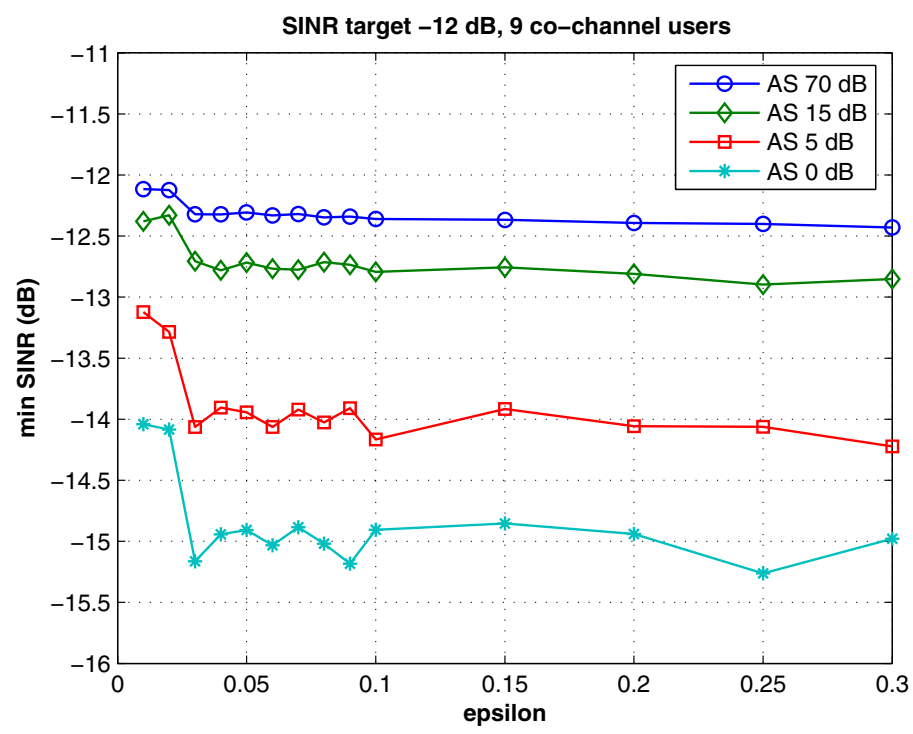

Figura 5.17: Valor medio de la mínima SINR real obtenido por el control no robusto de potencia, en función del valor de $\epsilon$.

el sistema no cumple el valor de SINR umbral requerido. Sin embargo, en la configuración con coordinación total, $\mathrm{ASW}=70 \mathrm{~dB}$, incluso ejecutando un control no robusto de potencia, el valor medio de la mínima SINR se ajusta bastante al valor de SINR umbral requerida. Este resultado confirma la idea de que un sistema MIMO multi-usuario multi-celda coordinado, es un sistema intrínsecamente robusto, por todos los motivos expuestos en el Capítulo 2 (rango de los canales, ganancia por diversidad espacial...).

La figura 5.18 compara la potencia media total transmitida en el sistema CM3 para los casos de control robusto y no robusto de potencia, cuando existe coordinación total entre BSs, ASW = $70 \mathrm{~dB}$ [100]. Como se puede observar, el control robusto de potencia aumenta ligeramente la potencia total transmitida en el sistema, pero las prestaciones que conlleva este mecanismo compensan el aumento de interferencias que supone transmitir a una mayor potencia.

El algoritmo JPCOB-VUL, incluida la versión robusta presentada en este apartado, no comprueba la feasibility teórica del problema antes de empezar a ejecutarse (4.43). La figura 5.19 muestra una estimación de la probabilidad de feasibility práctica del control robusto de potencia para los 


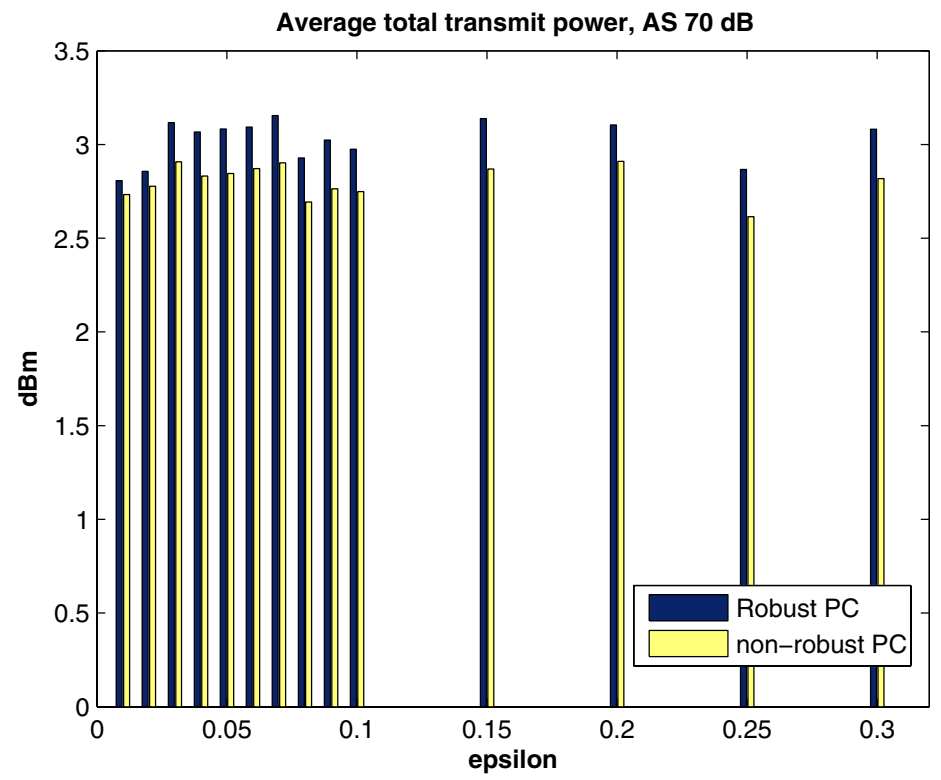

Figura 5.18: Potencia media total transmitida en el sistema CM3 para el control robusto y no robusto de potencia, cuando se permite coordinación total entre BSs, $\mathrm{ASW}=70 \mathrm{~dB}$.

distintos grados de coordinación entre BSs y valores de $\epsilon$ [100]. Esta probabilidad se calcula dividiendo, sobre el total, el número de realizaciones en las que el algoritmo converge, es decir, todos los usuarios alcanzan el valor de SINR umbral, sin que se supere la restricción en potencia transmitida de cada BS y el número máximo de iteraciones permitidas del algoritmo.

En esta figura se observa como la probabilidad de feasibility práctica del control robusto de potencia para coordinación total entre BSs, ASW = $70 \mathrm{~dB}$, es casi uno independientemente del error que se introduzca en la estima de las matrices de correlación de canal. Sin embargo, la probabilidad de feasibility práctica del resto de configuraciones está muy influenciada por el error en esta estima. Por tanto, aunque estas configuraciones alcanzan en la figura 5.16 la SINR umbral requerida, hay que tener en cuenta que su probabilidad de feasibility práctica es inaceptable según sea el valor de $\epsilon$. 


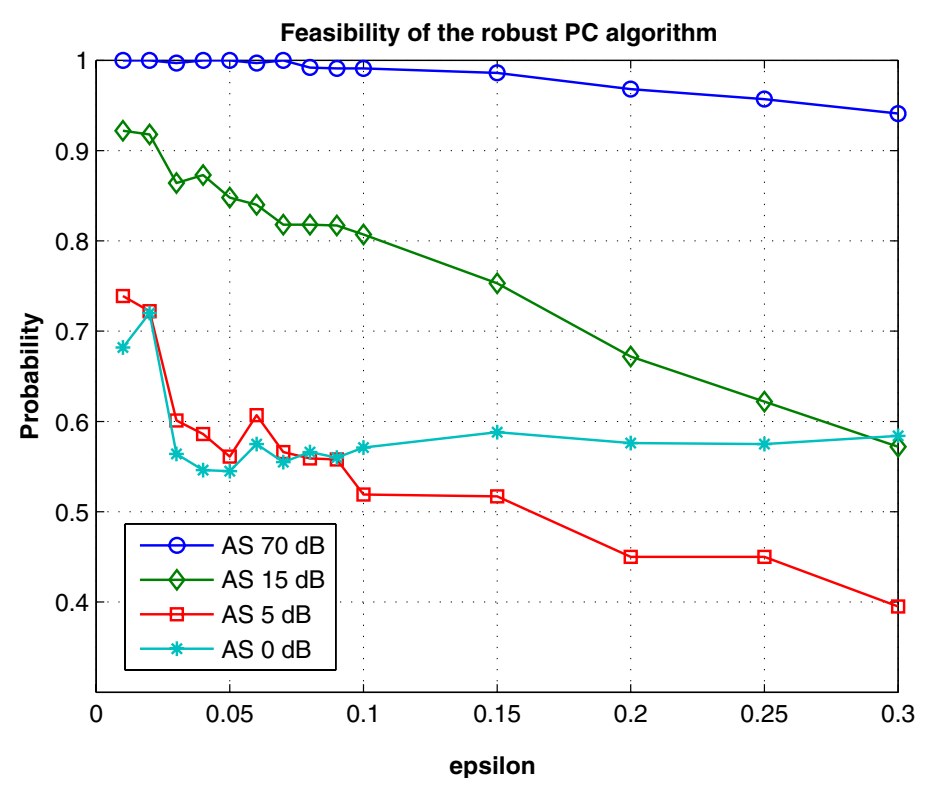

Figura 5.19: Probabilidad de feasibility práctica del control robusto de potencia para distintos valores de ventana de AS.

Por último, comparando las figuras 5.18 y 5.19, se aprecia que el ligero aumento en potencia transmitida que presenta en general el control robusto de potencia es perfectamente asumible, especialmente en el caso de coordinación total entre BSs, puesto que este aumento conlleva una probabilidad de feasibility práctica casi total del algoritmo. 


\section{Parte III}

\section{Soluciones}

\section{Downlink-based}

para requerimiento de QoS en el enlace downlink de un sistema MIMO multi-usuario multi-celda coordinado 



\section{Capítulo 6}

\section{Algoritmos JPCOB-DL y JPCOB-SDL para un sistema MIMO multi-usuario multi-celda coordinado}

La segunda Parte de la Tesis empieza definiendo una configuración de sistema adecuada para la arquitectura Multinodo B, que coincide con la de un sistema MIMO multi-usuario multi-celda coordinado (CM3). Sobre esta configuración CM3, se desarrolla analíticamente un modelo de sistema que sirve como marco de referencia para proponer un primer algoritmo JPCOB, el JPCOB-VUL o Virtual uplink-based Joint Power Control and Optimal Beamforming, modificando una de las tres referencias clásicas propuestas para resolver el problema de minimización de potencia en sistemas sin coordinación.

En general, las simulaciones que se recogen en la segunda Parte de la Tesis tratan de evaluar las prestaciones y los límites del algoritmo JPCOBVUL en un entorno W-CDMA CM3. Además, este entorno de simulación se modela siguiendo lo más fielmente posible los parámetros del estándar UMTS.

En la práctica, el principal problema al que se enfrenta un sistema CM3 es a disponer de CSIT perfecta en todas las estaciones base (BSs) del sistema. Además, las prestaciones de cualquier algoritmo propuesto para este tipo de sistemas van a depender del tipo de información de canal que puedan 
disponer las BSs. Desde este punto de vista, el algoritmo JPCOB-VUL se presenta como una buena opción, por el hecho de diseñar los beamformers de forma distribuida, y por incluir un mecanismo de control de potencia sencillo y fácilmente adaptable a situaciones de conocimiento parcial del canal.

En esta tercera Parte, la Tesis se reorienta hacia el análisis y desarrollo de algoritmos alternativos al JPCOB-VUL para resolver el problema de minimización de potencia en un sistema W-CDMA CM3. Por una parte, se avanza en el planteamiento analítico del modelo de sistema, proponiendo un modelo matricial compacto que reproduce exactamente las características de un sistema W-CDMA CM3, y donde además, se caracteriza completamente el proceso que realiza el receptor de cada usuario [121]. Este modelo matricial se apoya en el desarrollo propuesto por Wiesel et al. para el caso general de diseño de precoders en transmisión [30]. Por otro lado, se vuelve a un entorno de simulación básico y estándar, con el objetivo de independizar en la medida de lo posible el comportamiento de los algoritmos del entorno de simulación.

En este escenario, se propone un algoritmo Downlink-based que ya no depende de la dualidad entre el problema DL y un problema UL virtual equivalente, el algoritmo JPCOB-DL o Downlink-based Joint Power Control and Optimal Beamforming [121]. Este algoritmo comparte la misma estructura iterativa en dos pasos que el algoritmo JPCOB-VUL, pero se diferencia en el diseño del beamformer en transmisión. En concreto, el algoritmo JPCOB-DL implementa un beamformer multi-base en transmisión para cada usuario, donde el diseño del beamformer de cada usuario se realiza de forma centralizada teniendo en cuenta las $K \cdot N_{t}$ antenas disponibles en el sistema CM3.

En principio, el hecho de que tanto el diseño de los beamformers como el control de potencia requieran de una implementación centralizada, disminuye las posibilidades de conseguir una implementación de este algoritmo en un sistema CM3 real. Por este motivo, se propone una versión simplifica$d a$ del algoritmo JPCOB-DL, el JPCOB-SDL o Simplified Downlink-based Joint Power Control and Optimal Beamforming, para aquellos casos en los que existe algún tipo de restricción en la cantidad de información que el usuario puede realimentar a la BS. Desde este punto de vista, es posible considerar al algoritmo JPCOB-SDL como una técnica de limited feedback, puesto que estas técnicas no sólo se utilizan para realimentar información de canal, sino que en general se proponen para reducir la cantidad total de 
información que un usuario necesita realimentar a la BS.

\subsection{Modelo matricial del sistema}

En este apartado, se amplía el modelo matricial del sistema W-CDMA CM3 para canales flat fading, representado por el diagrama de bloques de la figura 4.3 o por la expresión (4.6), al caso de un sistema W-CDMA CM3 con canales frequency selective. En este caso, se recupera la expresión de la señal a la salida del usuario, (5.10), y se completa para caracterizar el proceso completo que tiene lugar en el receptor de cada usuario.

Por tanto, se considera un sistema W-CDMA CM3 formado por $K$ BSs, cada una equipada con un array lineal de $N_{t}$ antenas, donde los canales de propagación entre cada usuario y cada BS del sistema son canales frequency selective. En el sistema, además, se distribuyen uniformemente $M$ usuarios sobre el área compartida por las $K$ BSs (zona sombreada de la figura 4.2).

En un sistema CM3, la diferencia de caminos de propagación entre un usuario y las $K$ BSs del sistema provoca que la señal que recibe el usuario sea de naturaleza asíncrona. Por este motivo, en recepción, cada usuario dispone de un banco de $Q=K$ correladores, cada uno de ellos sincronizado con la contribución multi-camino de mayor ganancia del canal de propagación entre el usuario y una de las BSs del sistema. Tras el banco de correladores, un ecualizador se encarga de combinar, siguiendo un determinado criterio, las señales obtenidas a la salida de cada correlador.

La figura 6.1 muestra el diagrama de bloques del sistema W-CDMA CM3. El vector a, de dimensiones $[M \times 1]$, incluye los símbolos que se van a modular y a transmitir a cada usuario del sistema. Estos símbolos, de potencia unidad, están incorrelados entre sí. Siguiendo el desarrollo de [30], y suponiendo que el proceso que tiene lugar en el receptor de cada usuario es lineal, la señal a la salida del receptor de cada usuario, $y_{m}$, puede expresarse de forma compacta y multi-usuario como (ver figura 6.1):

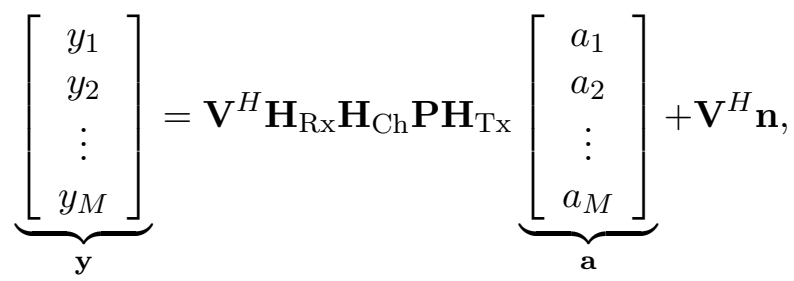

donde la matriz $\mathbf{H}_{\mathrm{Tx}}$, de dimensiones $\left[M K N_{t} \times M\right]$, es una matriz diagonal a bloques que agrupa los beamformers en transmisión de todo el sistema, $\mathbf{P}$ 


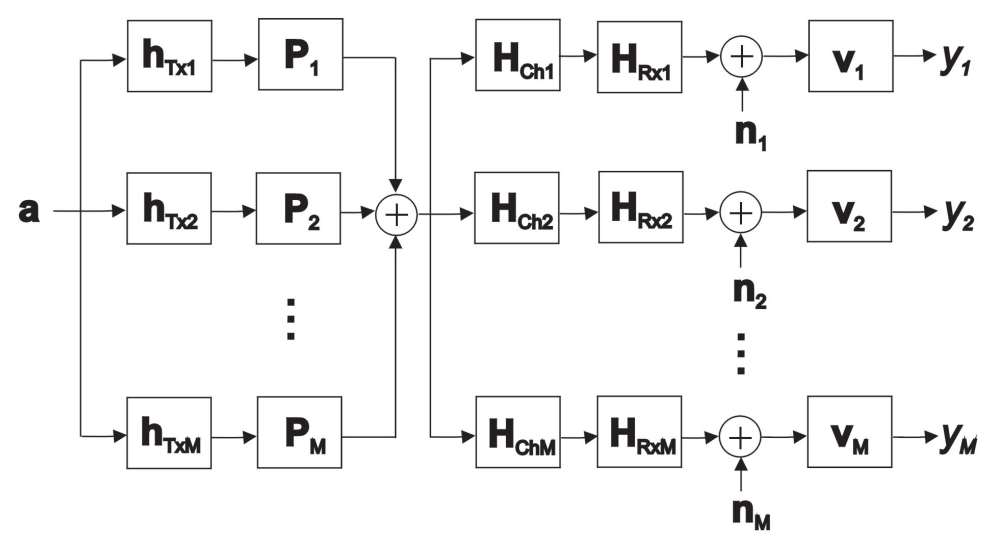

Figura 6.1: Diagrama de bloques de un sistema MIMO multi-usuario multi-celda coordinado.

es también una matriz diagonal a bloques, $\left[M K N_{t} \times M K N_{t}\right]$, que incluye todas las potencias transmitidas en el enlace DL del sistema y $\mathbf{H}_{\mathrm{Ch}}$ es una matriz $\left[M^{2} L \times M K N_{t}\right]$ que contiene los canales frequency selective entre todos los usuarios y BSs del sistema. En recepción, la matriz $\mathbf{H}_{\mathrm{Rx}}$ es una matriz $\left[M Q \times M^{2} L\right]$ que caracteriza el proceso que realiza el banco de $Q$ correladores de cada usuario. Tras los correladores, el vector $\mathbf{n},[M Q \times 1]$, es el resultado de concatenar las contribuciones de ruido térmico a la salida del banco de correladores de cada usuario, $\mathbf{n}_{m}$. Por último, la matriz $\mathbf{V}$, $[M Q \times M]$, incluye los pesos del ecualizador presente a la salida del banco de correladores de cada usuario.

Las matrices $\mathbf{H}_{\mathrm{Tx}}, \mathbf{P}, \mathbf{H}_{\mathrm{Ch}}, \mathbf{H}_{\mathrm{Rx}}$ y $\mathbf{V}$ pueden descomponerse en $M$ bloques independientes que se relacionan directamente con la transmisión a cada usuario. Siguiendo la figura 6.1:

$$
\begin{aligned}
& \mathbf{H}_{\mathrm{Tx}}=\operatorname{diag}\left\{\left[\mathbf{h}_{\mathrm{Tx} 1} \mathbf{h}_{\mathrm{Tx} 2} \ldots \mathbf{h}_{\mathrm{Tx} M}\right]\right\}, \\
& \mathbf{P}=\operatorname{diag}\left\{\left[\begin{array}{llll}
\mathbf{P}_{1} & \mathbf{P}_{2} & \ldots & \mathbf{P}_{M}
\end{array}\right]\right\}, \\
& \mathbf{H}_{\mathrm{Ch}}=\left[\begin{array}{llll}
\mathbf{H}_{\mathrm{Ch} 1}^{T} & \mathbf{H}_{\mathrm{Ch} 2}^{T} \ldots \mathbf{H}_{\mathrm{Ch} M}^{T}
\end{array}\right]^{T},
\end{aligned}
$$

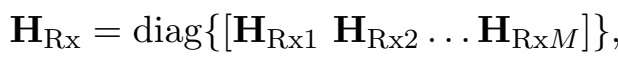

$$
\begin{aligned}
& \mathbf{V}=\operatorname{diag}\left\{\left[\begin{array}{llll}
\mathbf{v}_{1} & \mathbf{v}_{2} & \ldots & \mathbf{v}_{M}
\end{array}\right]\right\},
\end{aligned}
$$

donde $\mathbf{P}_{m}, \mathbf{H}_{\mathrm{Chm}}$ y $\mathbf{H}_{\mathrm{Rxm}}$ son, respectivamente, matrices de dimensiones $\left[K N_{t} \times K N_{t}\right],\left[M L \times M K N_{t}\right]$ y $[Q \times M L]$, mientras que $\mathbf{h}_{\mathrm{Tx} m}$ y $\mathbf{v}_{m}$ son vectores $\left[K N_{t} \times 1\right]$ y $[Q \times 1]$. Hay que destacar que la matriz $\mathbf{H}_{\mathrm{Ch}}$ se forma 
simplemente concatenando los bloques $\mathbf{H}_{\mathrm{Ch} m}$.

La transmisión coordinada en DL a un usuario $m$ se representa mediante el vector $\mathbf{h}_{\mathrm{Tx} m}$ y la matriz $\mathbf{P}_{m}$. El vector $\mathbf{h}_{\mathrm{Tx} m}$ contiene los beamformers en transmisión diseñados para el usuario $m$ desde todas las BSs del sistema:

$$
\mathbf{h}_{\mathrm{Tx} m}=\operatorname{vec}\left\{[\underbrace{\mathbf{w}_{m 1}}_{\mathrm{BS}_{1}} \cdots \underbrace{\mathbf{w}_{m K}}_{\mathrm{BS}_{K}}]\right\},
$$

donde $\mathbf{w}_{m k}$ es el beamformer en transmisión, $\left[N_{t} \times 1\right]$, para el enlace entre la BS $k$ y el usuario $m$. Por su parte, la matriz $\mathbf{P}_{m}$ es una matriz diagonal, que agrupa a la raíz cuadrada de las potencias de transmisión DL asignadas desde todas las BSs para transmitir a un usuario $m$ :

$$
\mathbf{P}_{m}=\operatorname{diag}\left\{\left[\left[\begin{array}{lll}
\sqrt{p_{m 1}} & \cdots & \sqrt{p_{m K}}
\end{array}\right]^{T} \otimes \mathbf{1}_{N_{t}}\right]\right\} .
$$

En recepción, la señal recibida por el usuario $m$ es la suma ponderada (matrices $\mathbf{H}_{\mathrm{Tx}}$ y $\mathbf{P}$ ) del vector de símbolos transmitidos sobre la matriz de canal $\mathbf{H}_{\mathrm{Ch} m}$ :

$$
\mathbf{H}_{\mathrm{Ch} m}=\mathbf{I}_{M} \otimes \mathbf{H}_{\mathrm{UEm}},
$$

donde $\mathbf{H}_{\mathrm{UEm}}$ es una matriz $\left[L \times K N_{t}\right]$ que contiene los canales de propagación entre las $K$ BSs y el usuario $m$ :

$$
\mathbf{H}_{\mathrm{UE} m}=\left[\mathbf{H}_{m 1} \ldots \mathbf{H}_{m K}\right] .
$$

Las matrices de canal $\mathbf{H}_{m k}$, de dimensiones $\left[L \times N_{t}\right]$, representan de forma discreta el canal de propagación para el enlace $m k$ entre la BS $k$ y el usuario $m$ del sistema. En este modelo de sistema, se contempla de nuevo la discretización del canal presentada en el Capítulo 5, de forma que el parámetro $L$ se define a partir del valor máximo del delay spread entre los $K \cdot M$ canales de propagación del sistema. Este modelo discreto se obtiene tras plantear un power delay profile o PDP compuesto, definido desde el punto de vista de los usuarios del sistema. De esta forma, a una frecuencia de muestreo igual a la frecuencia de chip, cada una de las contribuciones de los $K$ canales de propagación $\mathbf{H}_{m k}$ que recibe un usuario se puede asociar a un instante de llegada incluido en el intervalo $\left[T_{c}, L T_{c}\right]$ (5.3)-(5.4). Con este modelo discreto del canal, se tiene en cuenta cualquier posible combinación de canales $\mathbf{H}_{\mathrm{UEm}}$ en recepción, asíncronos o solapados en el tiempo. Por otro lado, siguiendo el modelo de canal presentado en el Capítulo 5, se supone que el valor máximo del delay spread no supera el periodo de símbolo, $\left[L T_{c}<T\right]$, y por tanto, no existe interferencia entre símbolos. 
El receptor de cada usuario está formado por la concatenación de un banco de correladores y un ecualizador, que se diseña siguiendo un determinado criterio. La matriz $\mathbf{H}_{\mathrm{Rxm}}$ caracteriza el proceso que tiene lugar en el banco de correladores de cada usuario. Esta matriz, incluye los términos de auto-correlación y correlación cruzada de los códigos OVSF utilizados para canalizar a los $M$ usuarios del sistema en el enlace DL:

$$
\mathbf{H}_{\mathrm{Rx} m}=\left[\mathbf{H}_{\mathrm{Rx} m 1} \ldots \mathbf{H}_{\mathrm{Rx} m M}\right],
$$

donde el elemento $(q, l)$ de cada una de las matrices $\mathbf{H}_{\mathrm{Rxmi}}, i=1, \ldots, M$, de dimensiones $[Q \times L]$, se define como:

$$
\left[\mathbf{H}_{\mathrm{Rx} m i}\right]_{(q, l)}=\frac{1}{N} \sum_{n=0}^{N-1} c_{m}^{*}(n) c_{i}(n-(q-l)) .
$$

En este caso, $N$ representa el mayor factor de spreading de entre los $M$ usuarios activos en el sistema CM3.

Por último, la señal a la salida del usuario $m$ puede expresarse en forma matricial como:

$$
y_{m}=\mathbf{v}_{m}^{H} \mathbf{H}_{\mathrm{Rx} m} \mathbf{H}_{\mathrm{Ch} m} \mathbf{P} \mathbf{H}_{\mathrm{Tx}} \mathbf{a}+\mathbf{v}_{m}^{H} \mathbf{n}_{m},
$$

donde el vector $\mathbf{v}_{m}^{H}$ se define como el complejo conjugado de los pesos del ecualizador del receptor del usuario $m$.

Este modelo matricial de sistema, al igual que el propuesto en [30], es un modelo compacto y general que facilita cualquier modificación para contemplar casos concretos del sistema W-CDMA CM3, como por ejemplo, el caso de usuarios co-canal o de un sistema CM3 sin coordinación [100].

\subsection{Algoritmos Downlink-based}

Los sistemas W-CDMA CM3 necesitan implementar técnicas o mecanismos que les permitan reducir el nivel de interferencias que perciben los usuarios, con el fin de mejorar la capacidad del sistema. En esta Tesis, se ha optado por plantear algoritmos JPCOB diseñados a partir de plantear el problema de minimización de potencia en el enlace DL del sistema CM3. En este problema de optimización, el objetivo es minimizar la potencia total transmitida en el sistema, entendida como la suma de la potencia que transmite cada BS, asegurando que los usuarios cumplen un determinado 
requisito de QoS, expresado en este caso como un valor mínimo de la SINR medida en el usuario. A partir del modelo de sistema (6.1), el problema de minimización de potencia se plantea como:

$$
\begin{aligned}
\underset{\mathbf{P}, \mathbf{H}_{\mathrm{Tx}}}{\min _{m=1}} & \sum_{m}^{M}\left\|\mathbf{P}_{m} \mathbf{h}_{\mathrm{Tx} m}\right\|^{2} \\
\text { s.t. } & \operatorname{SINR}_{m}^{D L}\left(\mathbf{P}, \mathbf{H}_{\mathrm{Tx}}\right) \geq \gamma_{m}, m=1, \ldots, M,
\end{aligned}
$$

donde $\operatorname{SINR}_{m}^{D L}$ es la SINR medida en el usuario $m$ del sistema y $\gamma_{m}$ representa el valor umbral de SINR que se establece como requerimiento de QoS para ese usuario concreto.

El algoritmo JPCOB-VUL presentado en la segunda Parte de la Tesis, se basa en la dualidad entre el problema DL y un problema UL virtual equivalente para obtener los beamformers en transmisión para cada usuario, localmente en cada BS. Aunque este algoritmo se presenta como una opción muy interesante al constituir una solución intermedia entre solución distribuida y centralizada, las prestaciones de los beamformers son claramente insuficientes para situaciones en las que el número de usuarios en el sistema es mayor que el número de antenas en el array de cada una de las BSs, $M>N_{t}$. Desafortunadamente, esta situación es la habitual en cualquier sistema de comunicaciones.

Por otra parte, está demostrado que en sistemas CM3 con CSIT perfecta en todas las BSs, el hecho de aprovechar todas las antenas disponibles en el sistema para diseñar beamformers en transmisión para cada usuario de forma multi-base, mejora sustancialmente las prestaciones del sistema en términos de capacidad, entendida, por ejemplo, en número de usuarios admitidos (en este caso, el beamformer podría manejar sin problemas hasta $M=K \cdot N_{t}$ usuarios en el sistema) [69,70,72].

Por tanto, en esta tercera Parte de la Tesis, se propone un algoritmo alternativo al algoritmo JPCOB-VUL, el algoritmo JPCOB-DL o Downlinkbased Joint Power Control and Optimal Beamforming. Este algoritmo se clasifica como downlink-based porque no necesita de la dualidad UL-DL para obtener los beamformers en transmisión.

El algoritmo JPCOB-DL comparte la estructura del algoritmo JPCOBVUL, es decir, es un algoritmo iterativo que consta de dos pasos, por una parte, se obtienen los beamformers en transmisión para cada usuario, y en un segundo paso se actualizan las potencias transmitidas en el enlace DL a cada usuario. Este algoritmo elimina la iteración en el problema UL virtual, 
pero mantiene exactamente el mismo mecanismo de control de potencia. La diferencia fundamental entre ambos algoritmos se encuentra entonces en el diseño de los beamformers en transmisión.

El algoritmo JPCOB-VUL obtiene los beamformers en transmisión para cada usuario, maximizando en cada BS el valor de la SINR recibida en el enlace UL virtual de ese usuario. En la práctica, cada uno de los beamformers se calcula mediante un criterio MMSE. En el algoritmo JPCOB-DL, el diseño del beamformer en transmisión de cada usuario se realiza considerando las antenas de todas las BSs del sistema, es decir, se trata de un diseño multi-base. En esta Tesis, el algoritmo JPCOB-DL implementa una extensión multi-base de la solución MMSE propuesta por Choi y Perreau en [122], donde la interferencia entre los usuarios de un sistema W-CDMA se minimiza utilizando únicamente procesado en la dimensión espacial.

De esta forma, el diseño de los beamformers que realiza el algoritmo JPCOB-DL es un diseño óptimo, puesto que minimiza espacialmente la interferencia entre los usuarios, y además es óptimo desde el punto de vista de coordinación, puesto que utiliza las $K \cdot N_{t}$ antenas disponibles en el sistema CM3.

La principal desventaja del algoritmo JPCOB-DL es que necesita una implementación centralizada, tanto del diseño de los beamformers, como del control de potencia. Sin embargo, como se ha visto en la segunda Parte de la Tesis, el mecanismo de control de potencia que comparten el algoritmo JPCOB-VUL y el algoritmo JPCOB-DL es un mecanismo que permite implementar de forma sencilla un control robusto de potencia, adaptado a situaciones de conocimiento parcial del canal. Por este motivo, en este Capítulo se presenta también un segundo algoritmo, el algoritmo JPCOBSDL o Simplified Downlink-based Joint Power Control and Optimal Beamforming, que modifica el paso del diseño del beamformer en transmisión del algoritmo JPCOB-DL, pensando especialmente en las situaciones en las que existe algún tipo de restricción en la cantidad de información que un usuario puede realimentar a una BS. Esta propuesta alternativa, que se puede considerar como una técnica de limited feedback o incluso como una solución robusta de beamforming, asume que las BSs siguen intercambiando información entre ellas a través del enlace de alta capacidad.

\subsubsection{Beamforming en transmisión: JPCOB-DL}

El algoritmo JPCOB-DL diseña un beamformer multi-base en transmisión para cada usuario, a partir de la propuesta de la referencia [122]. En 
esta referencia, Choi y Perreau proponen un beamformer MMSE en transmisión para un sistema W-CDMA de una única celda, donde la interferencia entre los usuarios se reduce únicamente mediante procesado espacial. Además, la potencia total transmitida se ajusta de forma que todos los usuarios del sistema reciben la misma potencia de señal deseada.

En el caso del algoritmo JPCOB-DL, la potencia transmitida a cada usuario desde una BS está formada por dos contribuciones, una contribución asociada al beamformer y otra que depende del mecanismo de control de potencia. Además, la suma de las potencias transmitidas por cada BS está sujeta a una restricción en potencia total transmitida:

$$
\sum_{m=1}^{M} p_{m k}\left\|\mathbf{w}_{m k}\right\|^{2} \leq P_{\max }, \forall k .
$$

En recepción, cada usuario del sistema dispone de un banco de correladores y de un ecualizador, para hacer frente al asincronismo de las señales que recibe desde las $K$ BSs. En este caso, se supone que en el banco de correladores de un usuario, donde $Q=K$, el correlador $q$-ésimo se sincroniza con la contribución multi-camino $l_{q}$, que resulta ser la contribución de mayor ganancia del canal de la BS $k=q$ que transmite al usuario. De esta forma, tras el banco de correladores, se dispone de un ecualizador diseñado según un criterio MRC (Maximum Ratio Combining), de forma que los pesos del ecualizador para el usuario $m, \mathbf{v}_{m}$, se diseñarían según:

$$
\left[\mathbf{v}_{m}\right]_{q}=\left[\mathbf{H}_{m k_{q}}\right]_{\left(l_{q},:\right)} \mathbf{w}_{m k_{q}}
$$

donde $k_{q}$ es la BS cuyo canal sintoniza el correlador $q$-ésimo y $\left[\mathbf{H}_{m k_{q}}\right]_{\left(l_{q},:\right)}$ es la contribución multi-camino sincronizada por ese correlador.

La extensión multi-base del beamformer MMSE de [122] se obtiene planteando el siguiente criterio (6.1):

$$
\mathbf{H}_{\mathrm{Tx}}^{\mathrm{MMSE}}=\arg \operatorname{mín}_{\mathbf{H}_{\mathrm{Tx}}}\left\{E\left[\|\mathbf{a}-\mathbf{y}\|^{2}\right]\right\} .
$$

Definiendo una matriz $\mathbf{G}$, de dimensiones $[M \times M]$, como:

$$
\mathbf{G}=\mathbf{I}_{M}-\mathbf{H H}_{\mathrm{Tx}}
$$

donde $\mathbf{H}=\mathbf{V}^{H} \mathbf{H}_{\mathrm{Rx}} \mathbf{H}_{\mathrm{Ch}} \mathbf{P}$, la expresión del criterio MMSE se reescribe de la siguiente forma:

$$
\mathbf{H}_{\mathrm{Tx}}^{\mathrm{MMSE}}=\arg \operatorname{mín}_{\mathbf{H}_{\mathrm{Tx}}}\left\{E\left[\|\mathbf{G a}\|^{2}\right]\right\}
$$


En la expresión anterior, el vector de ruido recibido por cada usuario, $\mathbf{V}^{H} \mathbf{n}$, no se introduce en la formulación del criterio MMSE puesto que no influye en el diseño de los beamformers óptimos en transmisión.

Por otra parte, la siguiente relación se cumple para una determinada realización del canal [89]:

$$
E\left[\|\mathbf{G a}\|^{2}\right]=E\left[\operatorname{Tr}\left[\mathbf{G a a}{ }^{H} \mathbf{G}^{H}\right]\right]=\operatorname{Tr}\left[\mathbf{G G}{ }^{H}\right]=\|\mathbf{G}\|_{F}^{2},
$$

puesto que cada símbolo $a_{m}$ es de potencia unidad.

Aplicando la relación (6.19) al criterio MMSE de (6.18), se obtiene:

$$
\mathbf{H}_{\mathrm{Tx}}^{\mathrm{MMSE}}=\arg \min _{\mathbf{H}_{\mathrm{Tx}}}\left\{\left\|\mathbf{I}_{M}-\mathbf{H} \mathbf{H}_{\mathrm{Tx}}\right\|_{F}^{2}\right\} .
$$

El operador vec $\{\cdot\}$ es útil para transformar expresiones en función de matrices en una expresión vectorial equivalente. Aplicando este operador a la norma matricial de (6.20) y teniendo en cuenta que:

$$
\operatorname{vec}\{\mathbf{A B C}\}=\left(\mathbf{C}^{T} \otimes \mathbf{A}\right) \operatorname{vec}\{\mathbf{B}\}
$$

donde $\otimes$ es el operador de Kronecker, la expresión (6.20) se transforma en:

$$
\mathbf{H}_{\mathrm{Tx}}^{\mathrm{MMSE}}=\arg \operatorname{mín}_{\mathbf{H}_{\mathrm{Tx}}}\left\{\left\|\operatorname{vec}\left\{\mathbf{I}_{M}\right\}-\left(\mathbf{I}_{M} \otimes \mathbf{H}\right) \operatorname{vec}\left\{\mathbf{H}_{\mathrm{Tx}}\right\}\right\|_{2}^{2}\right\},
$$

donde la relación (6.21) se aplica sobre $\operatorname{vec}\left\{\mathbf{H H}_{\mathrm{Tx}} \mathbf{I}_{M}\right\}$.

Puesto que el resultado del producto $\left(\mathbf{I}_{M} \otimes \mathbf{H}\right)$ es una matriz $\left[M^{2} \times\right.$ $\left.M^{2} K N_{t}\right]$ diagonal a bloques, y teniendo en cuenta que $\operatorname{vec}\left\{\mathbf{H}_{\mathrm{Tx}}\right\}$ puede expresarse como un vector columna $\left[M^{2} K N_{t} \times 1\right]$ [122]:

$$
\operatorname{vec}\left\{\mathbf{H}_{\mathrm{Tx}}\right\}=\left[\begin{array}{lll}
\left(\mathbf{Q}_{1} \mathbf{h}_{\mathrm{Tx} 1}\right)^{T} & \ldots & \left(\mathbf{Q}_{M} \mathbf{h}_{\mathrm{Tx} M}\right)^{T}
\end{array}\right]^{T},
$$

donde $\mathbf{Q}_{m}$ es una matriz de dimensiones $\left[M K N_{t} \times K N_{t}\right]$ :

$$
\mathbf{Q}_{m}=\left[\mathbf{e}_{m} \otimes \mathbf{I}_{K N_{t}}\right]
$$

siendo $\mathbf{e}_{m}$ la columna $m$-ésima de la matriz identidad $\mathbf{I}_{M}$, el criterio MMSE puede subdividirse en $M$ problemas independientes, cada uno asociado al diseño del beamformer multi-base de un usuario:

$$
\left\|\operatorname{vec}\left\{\mathbf{I}_{M}\right\}-\left(\mathbf{I}_{M} \otimes \mathbf{H}\right) \operatorname{vec}\left\{\mathbf{H}_{\mathrm{Tx}}\right\}\right\|^{2}=\sum_{m=1}^{M}\left\|\mathbf{e}_{m}-\mathbf{H} \mathbf{Q}_{m} \mathbf{h}_{\mathrm{Tx} m}\right\|^{2} .
$$


Por último, la expresión del beamformer multi-base óptimo para cada usuario se obtiene a través de la solución least square:

$$
\mathbf{h}_{\mathrm{Tx} m}^{\mathrm{MMSE}}=\left(\mathbf{H} \mathbf{Q}_{m}\right)^{\dagger} \mathbf{e}_{m}, m=1, \ldots, M
$$

En esta expresión, el producto $\mathbf{H Q}_{m}$ selecciona $K N_{t}$ columnas de la matriz $\mathbf{H}$, concretamente aquellas columnas que se corresponden con los índices $\left[(m-1) K N_{t}+1, m K N_{t}\right]$.

\subsubsection{Beamforming en transmisión: JPCOB-SDL}

Si se analiza con detenimiento la expresión del beamformer multi-base que se obtiene en el algoritmo JPCOB-DL, (6.25), se observa que en la matriz $\mathbf{H}$ se incluyen las matrices asociadas con el funcionamiento del receptor del usuario, $\mathbf{V}^{H}$ y $\mathbf{H}_{\mathrm{Rx}}$.

El algoritmo JPCOB-SDL se plantea para las situaciones en las que existe algún tipo de restricción en la cantidad de información que los usuarios pueden realimentar a la BS, pero donde las BSs siguen intercambiando datos entre ellas a través del enlace de alta capacidad.

En el algoritmo JPCOB-SDL, este tipo de situaciones se traducen en que las BSs del sistema no pueden disponer de información sobre qué tipo de ecualizador se está utilizando en recepción para combinar las señales a la salida del banco de correladores del usuario. En este caso, el criterio MMSE se plantea justo a la salida del banco de correladores, de tal manera que en cada uno de los $Q$ correladores se obtenga el mínimo error cuadrático medio entre su salida y el símbolo enviado:

$$
\mathbf{H}_{\mathrm{Tx}}^{\mathrm{MMSE}}=\arg \operatorname{mín}_{\mathbf{H}_{\mathrm{Tx}}}\left\{E\left[\left\|\mathbf{B a}-\mathbf{y}^{S}\right\|^{2}\right]\right\}
$$

donde la matriz $\mathbf{B}$, de dimensiones $[M Q \times M]$, se expresa como:

$$
\mathbf{B}=\left[\mathbf{I}_{M} \otimes \mathbf{1}_{Q}\right]
$$

e $\mathbf{y}^{S}$ se define como un vector $[M Q \times 1]$ :

$$
\mathbf{y}^{S}=\mathbf{H}^{S} \mathbf{H}_{\mathrm{Tx}} \mathbf{a}=\mathbf{H}_{\mathrm{Rx}} \mathbf{H}_{\mathrm{Ch}} \mathbf{P} \mathbf{H}_{\mathrm{Tx}} \mathbf{a} .
$$

El criterio MMSE, tal y como se plantea en la expresión (6.26), fuerza el diseño del beamformer en transmisión para que a la salida de cada correlador del usuario ya se obtenga la potencia de señal deseada necesaria para 
que el usuario alcance el valor mínimo de SINR, independientemente del procesado que realice después el ecualizador. Esta solución, no es una opción eficiente en cuanto a la gestión de la potencia disponible en el sistema, pero proporciona un diseño robusto del beamformer que puede ser útil en determinadas situaciones.

Siguiendo las expresiones (6.17)-(6.18), y aplicando la relación (6.19), el criterio MMSE se reescribe como:

$$
\mathbf{H}_{\mathrm{Tx}}^{\mathrm{MMSE}}=\arg \min _{\mathbf{H}_{\mathrm{Tx}}}\left\{\left\|\mathbf{B}-\mathbf{H}^{S} \mathbf{H}_{\mathrm{Tx}}\right\|_{F}^{2}\right\} .
$$

Aplicando de nuevo el operador vec $\{\cdot\}$ y teniendo en cuenta (6.21) y (6.23), el criterio MMSE se divide de nuevo en $M$ problemas independientes, relacionados con el diseño de los beamformers multi-base de cada uno de los usuarios:

$$
\left\|\operatorname{vec}\{\mathbf{B}\}-\left(\mathbf{I}_{M} \otimes \mathbf{H}^{S}\right) \operatorname{vec}\left\{\mathbf{H}_{\mathrm{Tx}}\right\}\right\|^{2}=\sum_{m=1}^{M}\left\|\left(\mathbf{e}_{m} \otimes \mathbf{1}_{Q}\right)-\mathbf{H}^{S} \mathbf{Q}_{m} \mathbf{h}_{\mathrm{Tx} m}\right\|^{2},
$$

donde $\mathbf{e}_{m}$ es la columna $m$-ésima de la matriz identidad $\mathbf{I}_{M}$.

La solución del beamformer multi-base óptimo para cada usuario se obtiene a través de la solución least square de cada uno de los $M$ problemas independientes:

$$
\mathbf{h}_{\mathrm{T} \times m}^{\mathrm{MMSE}}=\left(\mathbf{H}^{S} \mathbf{Q}_{m}\right)^{\dagger}\left(\mathbf{e}_{m} \otimes \mathbf{1}_{Q}\right), m=1, \ldots, M .
$$

De nuevo, el producto $\mathbf{H}^{S} \mathbf{Q}_{m}$ selecciona $K N_{t}$ columnas de la matriz $\mathbf{H}^{S}$, concretamente las asociadas con los índices $\left[(m-1) K N_{t}+1, m K N_{t}\right]$.

\subsubsection{Control de potencia}

El control de potencia de los algoritmos JPCOB-DL y JPCOB-SDL coincide con el control de potencia del algoritmo JPCOB-VUL. Sin embargo, en este Capítulo ya se considera el proceso completo que tiene lugar en el receptor de cada usuario, por lo que es necesario volver a definir las matrices $\mathbf{D}$ y $\mathbf{F}$, y el vector $\mathbf{u}$ involucrados en la actualización de las potencias DL. Además, el modelo de sistema matricial presentado en este Capítulo permite también obtener una expresión compacta de la actualización de las potencias DL.

El primer paso es reordenar el modelo matricial de sistema (6.1) según:

$$
\mathbf{y}=\mathbf{V}^{H} \mathbf{H}_{\mathrm{Rx}} \mathbf{H}_{\mathrm{Ch}} \mathbf{H}_{\mathrm{Tx}}^{D} \mathbf{P}^{D} \mathbf{a}+\mathbf{V}^{H} \mathbf{n},
$$


de forma que la matriz de beamforming $\mathbf{H}_{\mathrm{Tx}}^{D}$ es una matriz diagonal a bloques de dimensiones $\left[M K N_{t} \times M K\right]$, donde cada subbloque $\mathbf{H}_{\mathrm{Tx} m}^{D}$ es a su vez una matriz diagonal a bloques $\left[K N_{t} \times K\right]$ :

$$
\begin{aligned}
\mathbf{H}_{\mathrm{Tx}}^{D} & =\operatorname{diag}\left\{\left[\mathbf{H}_{\mathrm{Tx} 1}^{D} \ldots \mathbf{H}_{\mathrm{Tx} M}^{D}\right]\right\}, \\
\mathbf{H}_{\mathrm{Tx} m}^{D} & =\operatorname{diag}\left\{\left[\mathbf{w}_{m 1} \ldots \mathbf{w}_{m K}\right]\right\} .
\end{aligned}
$$

De la misma manera, la matriz de potencias DL, $\mathbf{P}^{D}$, es una matriz $[M K \times$ $M]$ diagonal a bloques, donde cada subbloque $\mathbf{p}_{m}^{D}$ es un vector columna $[K \times 1]$ que incluye la raíz cuadrada de las potencias de transmisión DL asignadas desde todas las BSs para transmitir a un usuario $m$ :

$$
\begin{aligned}
\mathbf{P}^{D} & =\operatorname{diag}\left\{\left[\mathbf{p}_{1}^{D} \ldots \mathbf{p}_{M}^{D}\right]\right\}, \\
\mathbf{p}_{m}^{D} & =\left[\sqrt{p_{m 1}} \cdots \sqrt{p_{m K}}\right]^{T} .
\end{aligned}
$$

A partir del modelo de sistema de la expresión (6.31), la señal a la salida del usuario $m$ puede expresarse en forma matricial como:

$$
y_{m}=\mathbf{v}_{m}^{H} \mathbf{H}_{\mathrm{Rx} m} \mathbf{H}_{\mathrm{Ch} m} \mathbf{H}_{\mathrm{Tx}}^{D} \mathbf{P}^{D} \mathbf{a}+\mathbf{v}_{m}^{H} \mathbf{n}_{m},
$$

donde $\mathbf{v}_{m}^{H}, \mathbf{H}_{\mathrm{Rx} m}$ y $\mathbf{H}_{\mathrm{Ch} m}$ se definen como en (6.13) y $\mathbf{H}_{\mathrm{Tx}}^{D} \mathbf{P}^{D}=\mathbf{P H}_{\mathrm{Tx}}$.

Para obtener la expresión de la SINR recibida por un usuario del sistema, interesa identificar en la expresión (6.36) los términos de señal deseada o SOI (Signal Of Interest) (6.37), interferencia entre fingers o IFI (Inter-finger Interference) (6.38) e interferencia de acceso múltiple o MAI (Multiple Access Interference) (6.39):

$$
\begin{aligned}
\mathrm{SOI}_{m} & =\mathbf{v}_{m}^{H} \mathbf{H}_{\mathrm{Rx} m m}^{\mathrm{S}} \mathbf{H}_{\mathrm{UE} m} \mathbf{H}_{\mathrm{Tx} m}^{D} \mathbf{p}_{m}^{D} a_{m} \\
\mathrm{IFI}_{m} & =\mathbf{v}_{m}^{H} \mathbf{H}_{\mathrm{Rx} m m}^{\mathrm{I}} \mathbf{H}_{\mathrm{UE} m} \mathbf{H}_{\mathrm{Tx} m}^{D} \mathbf{p}_{m}^{D} a_{m} \\
\mathrm{MAI}_{m} & =\mathbf{v}_{m}^{H} \sum_{\substack{i=1 \\
i \neq m}}^{M} \mathbf{H}_{\mathrm{Rx} m i} \mathbf{H}_{\mathrm{UE} m} \mathbf{H}_{\mathrm{Tx} i}^{D} \mathbf{p}_{i}^{D} a_{i} .
\end{aligned}
$$

La matriz $\mathbf{H}_{\mathrm{Rx} m m}^{\mathrm{S}}$ es una matriz de dimensiones $[Q \times L]$ que contiene los términos de auto-correlación entre códigos asociados a las contribuciones multi-camino sincronizadas por cada uno de los correladores, es decir, contiene únicamente $Q$ términos no nulos e iguales a la unidad en las posiciones 
$\left(q, l_{q}\right)$ (5.11), mientras que $\mathbf{H}_{\mathrm{Rxmm}}^{\mathrm{I}}$ incluye los términos de auto-correlación entre códigos que intervienen en los términos de IFI (5.12):

$$
\mathbf{H}_{\mathrm{Rx} m m}^{\mathrm{I}}=\mathbf{H}_{\mathrm{Rx} m m}-\mathbf{H}_{\mathrm{Rx} m m}^{\mathrm{S}} .
$$

La suma de las potencias transmitidas por todas las BSs es mínima cuando todos los usuarios alcanzan su valor mínimo de SINR con igualdad:

$$
\operatorname{SINR}_{m}^{D L}=\gamma_{m}, m=1, \ldots, M .
$$

Para el usuario $m$, y a partir de las expresiones de la SOI, IFI y MAI, (6.37)-(6.39), la SINR se calcula como:

$$
\operatorname{SINR}_{m}^{D L}=\frac{E\left[\left|\mathrm{SOI}_{m}\right|^{2}\right]}{E\left[\left|\mathrm{IFI}_{m}\right|^{2}\right]+E\left[\left|\mathrm{MAI}_{m}\right|^{2}\right]+\sigma_{n}^{2}} .
$$

En la expresión anterior, se asume que la potencia de ruido térmico, $\sigma_{n}^{2}=$ $E\left[\left|\mathbf{v}_{m}^{H} \mathbf{n}_{m}\right|^{2}\right]$, es la misma para todos los usuarios del sistema. Además, tanto los símbolos $a_{m}$ como las contribuciones multi-camino de los canales $\mathbf{H}_{m k}$ y $\mathbf{H}_{m k^{\prime}}$, donde $k \neq k^{\prime}$, están incorreladas entre sí.

Desarrollando (6.41), (6.42) puede escribirse para cada usuario como:

$$
E\left[\left|\mathrm{SOI}_{m}\right|^{2}\right]=\gamma_{m}\left(E\left[\left|\mathrm{IFI}_{m}\right|^{2}\right]+E\left[\left|\mathrm{MAI}_{m}\right|^{2}\right]+\sigma_{n}^{2}\right) .
$$

Esta expresión es el equivalente de la relación (4.20), y también puede expresarse de forma matricial y multi-usuario como:

$$
\mathbf{D p}=\mathbf{F} \mathbf{p}+\mathbf{u},
$$

donde $\mathbf{p}$ es un vector columna de dimensiones $[K M \times 1]$ :

$$
\begin{aligned}
\mathbf{p} & =\operatorname{vec}\left\{\left[\mathbf{p}_{1} \ldots \mathbf{p}_{M}\right]\right\}, \\
\mathbf{p}_{m} & =\left[p_{m 1} \ldots p_{m K}\right]^{T} .
\end{aligned}
$$

En la expresión (6.44), la matriz $\mathbf{D}$ es una matriz diagonal a bloques de dimensiones $[M \times K M]$, que contiene los términos de potencia media de señal deseada recibidos por cada usuario. Los vectores fila $\mathbf{d}_{m}$, de dimensiones $[1 \times K]$, se calculan como:

$$
\begin{aligned}
\mathbf{D} & =\operatorname{diag}\left\{\left[\mathbf{d}_{1} \ldots \mathbf{d}_{M}\right]\right\}, \\
{\left[\mathbf{d}_{m}\right]_{k} } & =E\left[\left|\mathbf{v}_{m}^{H} \mathbf{H}_{\mathrm{Rx} m m}^{\mathrm{S}}\left[\mathbf{H}_{\mathrm{UEm}} \mathbf{H}_{\mathrm{Tx} m}^{D}\right]_{(:, k)}\right|^{2}\right] .
\end{aligned}
$$


Hay que destacar que esta matriz $\mathbf{D}$ presenta una estructura diferente a la matriz D del algoritmo JPCOB-VUL, (4.23), puesto que los elementos del vector de potencias $\mathbf{p}$ se agrupan en el algoritmo JPCOB-DL por usuarios, $\mathbf{p}_{m}$, y no por $B S s$ como en el algoritmo JPCOB-VUL (4.21).

De la misma manera, la matriz $\mathbf{F},[M \times K M]$, contiene un valor escalado de la potencia media de IFI y MAI. Esta matriz presenta una estructura de $M$ matrices $\mathbf{F}_{m}$ de dimensiones $[M \times K]$, cuyos elementos representan la potencia de interferencia que introduce la transmisión a un usuario del sistema:

$$
\begin{aligned}
\mathbf{F} & =\left[\mathbf{F}_{1}\left|\mathbf{F}_{2}\right| \cdots \mid \mathbf{F}_{M}\right], \\
{\left[\mathbf{F}_{i}\right]_{(m, k)} } & =\left\{\begin{array}{cl}
\gamma_{m} E\left[\left|\mathbf{v}_{m}^{H} \mathbf{H}_{\mathrm{Rx} m m}^{\mathrm{I}}\left[\mathbf{H}_{\mathrm{UEm} m} \mathbf{H}_{\mathrm{Tx} m}^{D}\right]_{(:, k)}\right|^{2}\right], & m=i, \\
\gamma_{m} E\left[\left|\mathbf{v}_{m}^{H} \mathbf{H}_{\mathrm{Rx} m i}\left[\mathbf{H}_{\mathrm{UEm} m} \mathbf{H}_{\mathrm{Tx} i}^{D}\right]_{(:, k)}\right|^{2}\right], & m \neq i .
\end{array}\right.
\end{aligned}
$$

Por último, el vector $\mathbf{u}$ incluye un valor escalado de la potencia de ruido percibida por los $M$ usuarios del sistema:

$$
\mathbf{u}=\left[\gamma_{1} \sigma_{n}^{2} \ldots \gamma_{M} \sigma_{n}^{2}\right]^{T}
$$

La expresión de actualización de las potencias, (6.44), es una expresión que se corresponde con una iteración de Jacobi (apéndice A, apartado A.2), de forma que las potencias óptimas de transmisión en el enlace DL se obtienen iterando:

$$
\mathbf{p}\left(n_{i t}+1\right)=\mathbf{D}^{\dagger}\left(\mathbf{F} \mathbf{p}\left(n_{i t}\right)+\mathbf{u}\right)
$$

donde $n_{i t}$ es el número de la iteración correspondiente.

\subsubsection{Solución algorítmica}

Los algoritmos JPCOB-DL y JPCOB-SDL comparten la estructura y el mecanismo de control de potencia del algoritmo JPCOB-VUL, sin embargo, implementan un beamformer multi-base para cada usuario sin recurrir a la dualidad UL-DL. De hecho, ambos algoritmos únicamente se diferencian en el diseño de los beamformers en transmisión.

\section{Algoritmo JPCOB-DL}

Los pasos del algoritmo JPCOB-DL para cada iteración $n_{i t}$ serían:

1. Inicializar las potencias DL, $p_{m k}(0)$, para todos los enlaces del sistema, $m=1, \ldots, M$, y $k=1, \ldots, K$, con valores positivos. 
2. Para $n_{i t}=0,1,2, \ldots$, hasta llegar a la convergencia:

2.1. Beamforming. Los beamformers en transmisión para cada usuario se obtienen a partir de la extensión multi-base del beamformer MMSE propuesto en [122]:

$$
\mathbf{h}_{\mathrm{Tx} m}^{\mathrm{MMSE}}\left(n_{i t}\right)=\left(\mathbf{H}\left(n_{i t}\right) \mathbf{Q}_{m}\right)^{\dagger} \mathbf{e}_{m},
$$

donde en $\mathbf{H}\left(n_{i t}\right)=\mathbf{V}^{H} \mathbf{H}_{\mathrm{Rx}} \mathbf{H}_{\mathrm{Ch}} \mathbf{P}\left(n_{i t}\right)$, se tienen en cuenta las potencias DL actualizadas según la iteración $n_{i t}$ correspondiente y donde $\mathbf{Q}_{m}=\left[\mathbf{e}_{m} \otimes \mathbf{I}_{K N_{t}}\right]$, siendo $\mathbf{e}_{m}$ la columna $m$-ésima de la matriz iden$\operatorname{tidad} \mathbf{I}_{M}$.

2.2. Control de potencia, enlace DL. El vector $\mathbf{p}$ de potencias DL se actualiza mediante:

$$
\mathbf{p}\left(n_{i t}+1\right)=\mathbf{D}^{\dagger}\left(n_{i t}\right)\left(\mathbf{F}\left(n_{i t}\right) \mathbf{p}\left(n_{i t}\right)+\mathbf{u}\right),
$$

donde las matrices $\mathbf{D}\left(n_{i t}\right)$ y $\mathbf{F}\left(n_{i t}\right)$ se obtienen mediante las expresiones $(6.47)$ y (6.49), respectivamente, considerando los beamformers en transmisión correspondientes a la iteración $n_{i t}, \mathrm{y}(\cdot)^{\dagger}$ representa la pseudo-inversa definida como $\mathbf{D}^{\dagger}=\left(\mathbf{D}^{T} \mathbf{D}\right)^{-1} \mathbf{D}^{T}$ (la matriz $\mathbf{D}$ es una matriz real).

\section{Algoritmo JPCOB-SDL}

Los pasos del algoritmo JPCOB-SDL para cada iteración $n_{i t}$ serían:

1. Inicializar las potencias DL, $p_{m k}(0)$, para todos los enlaces del sistema, $m=1, \ldots, M, \mathrm{y} k=1, \ldots, K$, con valores positivos.

2. Para $n_{i t}=0,1,2, \ldots$, hasta llegar a la convergencia:

2.1. Beamforming. Los beamformers en transmisión para cada usuario se obtienen a partir de una modificación de la extensión multi-base del beamformer MMSE propuesto en [122]:

$$
\mathbf{h}_{\mathrm{Tx} m}^{\mathrm{MMSE}}\left(n_{i t}\right)=\left(\mathbf{H}^{S}\left(n_{i t}\right) \mathbf{Q}_{m}\right)^{\dagger}\left(\mathbf{e}_{m} \otimes \mathbf{1}_{Q}\right),
$$

donde en $\mathbf{H}^{S}\left(n_{i t}\right)=\mathbf{H}_{\mathrm{Rx}} \mathbf{H}_{\mathrm{Ch}} \mathbf{P}\left(n_{i t}\right)$, se tienen en cuenta las potencias DL actualizadas según la iteración $n_{i t}$ correspondiente y donde $\mathbf{Q}_{m}=$ $\left[\mathbf{e}_{m} \otimes \mathbf{I}_{K N_{t}}\right]$, siendo $\mathbf{e}_{m}$ la columna $m$-ésima de la matriz identidad $\mathbf{I}_{M}$. 
2.2. Control de potencia, enlace DL. El vector $\mathbf{p}$ de potencias DL se actualiza mediante:

$$
\mathbf{p}\left(n_{i t}+1\right)=\mathbf{D}^{\dagger}\left(n_{i t}\right)\left(\mathbf{F}\left(n_{i t}\right) \mathbf{p}\left(n_{i t}\right)+\mathbf{u}\right),
$$

donde las matrices $\mathbf{D}\left(n_{i t}\right)$ y $\mathbf{F}\left(n_{i t}\right)$ se obtienen mediante las expresiones (6.47) y (6.49), respectivamente, considerando los beamformers en transmisión correspondientes a la iteración $n_{i t}, \mathrm{y}(\cdot)^{\dagger}$ representa la pseudo-inversa definida como $\mathbf{D}^{\dagger}=\left(\mathbf{D}^{T} \mathbf{D}\right)^{-1} \mathbf{D}^{T}$ (la matriz $\mathbf{D}$ es una matriz real).

\subsubsection{Convergencia de los algoritmos}

Aunque los algoritmos JPCOB-DL y JPCOB-SDL no recurren a la dualidad UL-DL, la demostración de su convergencia es muy similar a la demostración de la convergencia del algoritmo JPCOB propuesto por RashidFarrokhi et al. en [33].

La base de la convergencia del algoritmo JPCOB-DL y del algoritmo JPCOB-SDL es que la matriz de ganancia DL, $\left[\mathbf{D}^{\dagger}\left(n_{i t}\right) \mathbf{F}\left(n_{i t}\right)\right]$, calculada para cada algoritmo siguiendo las expresiones de $\mathbf{D}$ y $\mathbf{F}$ correspondientes, converge a una matriz constante. Esto es debido a que la única realimentación que reciben los beamformers en transmisión en cada iteración es la de las potencias DL, y este hecho permite que los beamformers en transmisión también converjan a un valor constante para ambos algoritmos.

De esta forma, la expresión de actualización de las potencias DL, (6.52), se corresponde en ambos algoritmos con una iteración de Jacobi, que converge siempre y cuando el radio espectral de la matriz de ganancia DL de cada algoritmo sea menor que la unidad (apéndice A, apartado A.2):

$$
\rho\left(\mathbf{D}^{\dagger} \mathbf{F}\right)<1 \text {. }
$$

Desde el punto de vista teórico, el algoritmo JPCOB-DL, o el algoritmo JPCOB-SDL, es feasible, si, dado un conjunto de valores umbrales de SINR, $\left(\gamma_{1}, \ldots, \gamma_{M}\right)$, el problema de minimización de potencia (6.14) tiene solución, y las potencias DL óptimas son valores positivos. La relación (6.57) proporciona una definición equivalente de feasibility del problema: el problema de minimización de potencia (6.14) es feasible, si se cumple la relación (6.57) sobre el radio espectral de la matriz de ganancia DL. Por tanto, la condición (6.57) representa en realidad la condición de feasibility teórica de los algoritmos JPCOB-DL y JPCOB-SDL. 
De la misma forma que ocurría en el algoritmo JPCOB-VUL, el algoritmo JPCOB-DL y el JPCOB-SDL no comprueban si el problema es feasible para el conjunto de valores de SINR umbrales establecidos para cada usuario. En caso contrario, no sería necesario recurrir a una actualización iterativa de las potencias DL, sino que el cálculo de las potencias DL óptimas podría obtenerse en un único paso como:

$$
\mathbf{p}=\left(\mathbf{I}-\mathbf{D}^{\dagger} \mathbf{F}\right)^{-1} \mathbf{D}^{\dagger} \mathbf{u} .
$$

Como se verá en el siguiente apartado, un problema que teóricamente es feasible, puede verse seriamente afectado por las restricciones asociadas a una implementación práctica de los algoritmos en un sistema CM3 real.

\subsection{Prestaciones de los algoritmos Downlink-based}

En este apartado, el objetivo es comparar, sobre una misma configuración de sistema W-CDMA CM3, las prestaciones del algoritmo JPCOBDL y su versión simplificada, el JPCOB-SDL, con las prestaciones del algoritmo JPCOB-VUL presentado en la segunda Parte de la Tesis.

La configuración del sistema W-CDMA CM3 es la mostrada por la figura 4.2: $K=3$ BSs equipadas con arrays de $N_{t}=3$ antenas transmiten coordinadamente a $M$ usuarios, que se distribuyen uniformemente sobre el área sombreada compartida por las BSs. En recepción, cada usuario dispone de un banco de $Q=3$ correladores y de un ecualizador que implementa un criterio MRC en el caso del algoritmo JPCOB-DL, o un criterio no conocido en el algoritmo JPCOB-SDL.

Teóricamente, las BSs están conectadas entre sí mediante un enlace de alta capacidad, de forma que cooperan perfectamente en datos, ya sea intercambiando las señales a transmitir a cada usuario o bien intercambiando información de los canales (CSI). Sin embargo, las BSs no cooperan en potencia, sino que cada BS está sujeta a una restricción en la potencia total que puede transmitir en DL.

En este apartado se intenta disminuir la variabilidad del canal e independizar en la medida de lo posible el comportamiento de los algoritmos del entorno de simulación. Por este motivo, los usuarios del sistema vuelven a ser usuarios co-canal, y los canales para cada enlace entre usuario y BS del sistema se modelan como canales flat fading de una única contribución multi-camino. Además, la forma de generar estos canales se simplifica, implementando un modelo estándar de generación de canales para sistemas 


\begin{tabular}{|l|c|}
\hline \hline Parámetro & Valor \\
\hline \hline Frecuencia de la portadora & $2 \mathrm{GHz}$ \\
\hline Factor de spreading $N$ & 32 \\
\hline Tamaño máximo del active set. & $3 \mathrm{BSs}$ \\
\hline Potencia de ruido & $-108,2 \mathrm{dBm}$ \\
\hline Figura de ruido & $7 \mathrm{~dB}$ \\
\hline $\begin{array}{l}\text { Potencia máxima } P_{\text {max }} \\
\text { transmitida por BS }\end{array}$ & $20 \mathrm{~W}$ \\
\hline Distancia entre emplazamientos & $867 \mathrm{~m}$ \\
\hline Radio celular & $500 \mathrm{~m}$ \\
\hline Desviación estándar del shadowing & $8 \mathrm{~dB}$ \\
\hline Pérdidas de propagación & $\beta_{m k}=r_{m k}^{-2}$ \\
\hline Máximo número de iteraciones del algoritmo $(I t)$ & 30 \\
\hline
\end{tabular}

Cuadro 6.1: Parámetros de simulación.

MIMO multi-usuario. El vector de canal, $\left[1 \times N_{t}\right]$, entre un usuario $m$ y una $\mathrm{BS} k$ se genera mediante:

$$
\mathbf{h}_{m k}=\beta_{m k} \beta_{s} \mathbf{g} \mathbf{R}_{t}^{1 / 2}
$$

donde $\beta_{m k}=r_{m k}^{-2}$ representa el path loss o las pérdidas de propagación para una distancia $r_{m k}$ entre el usuario y la BS y $\beta_{s}$ es el shadowing del enlace, que se modela como una variable aleatoria log-normal, con una desviación estándar de $8 \mathrm{~dB}$. El vector $\mathbf{g}$, de dimensiones $\left[1 \times N_{t}\right]$, se modela mediante una distribución Rayleigh, de forma que sus elementos son variables i.i.d. complejas Gaussianas, con parte real e imaginaria independientes, de media nula e idéntica varianza $(1 / 2)$. Por último, la matriz $\mathbf{R}_{t}$ define la correlación entre las antenas de cada BS, que se establece en 0,5 en las simulaciones.

En este modelo de canal, la distancia $r_{m k}$ entre cada usuario y las BSs del sistema se sigue calculando mediante la rejilla de la figura 4.5. Sin embargo, la forma de generar el canal se simplifica, eliminando la parte de modelado de la DOA de cada contribución o el efecto Doppler. De la misma forma, en las simulaciones se utiliza el valor instantáneo de las matrices de correlación de canal. La tabla 6.1 resume los parámetros utilizados en las simulaciones.

Los algoritmos se comparan sobre distintos valores de SINR umbral (se considera un mismo valor de SINR umbral para todos los usuarios del sistema). Además, se introduce el esquema de selección de enlaces activos del entorno Coordinado I para comparar las prestaciones de los algoritmos 
frente a distintos grados de coordinación entre las BSs. De esta forma, una simulación viene definida por un valor de SINR umbral, común a todos los usuarios, y por un valor de ventana de active set (AS). Cada simulación se promedia sobre 3000 realizaciones independientes:

- ASW: $0 \mathrm{~dB}$ (sin coordinación), $5 \mathrm{~dB}, 10 \mathrm{~dB}, 20 \mathrm{~dB}$ y $90 \mathrm{~dB}$ (coordinación total).

- SINR umbral (tras el despreading): $3 \mathrm{~dB}, 7 \mathrm{~dB}$ y $12 \mathrm{~dB}$.

Aunque las simulaciones se llevan a cabo para cinco valores de ventana de AS, en las gráficas de este apartado sólo se representan los resultados más significativos, que generalmente se asocian a la ventana de $0 \mathrm{~dB}$, cuando no existe coordinación entre BSs y cada usuario recibe su señal desde una única BS, $90 \mathrm{~dB}$, que se corresponde para este modelo de sistema con una situación de coordinación total donde todos los usuarios son servidos por las $K$ BSs, y un tamaño de ventana que se corresponde con un grado intermedio de coordinación entre BSs, en este caso, $20 \mathrm{~dB}$.

En las simulaciones, el concepto de feasibility del problema cambia, puesto que hay que tener en cuenta las restricciones de tipo práctico impuestas a cada algoritmo. El problema de minimización de potencia es feasible, y por tanto el algoritmo simulado también lo es, si todos los usuarios del sistema alcanzan la SINR umbral requerida sin que las BSs superen la restricción en potencia máxima de transmisión en DL y sin que el algoritmo supere el número máximo de iteraciones permitidas. Por tanto, es posible encontrar una situación teóricamente feasible, en la que se cumple la relación sobre el radio espectral (6.57), y donde sin embargo el algoritmo no converge, con lo que el problema de minimización de potencia no es feasible, porque, por ejemplo, el algoritmo necesita más iteraciones de las establecidas como máximo para converger. En las simulaciones, los algoritmos disponen de un máximo de 30 iteraciones para converger.

Por otro lado, la condición de convergencia básica, $\operatorname{SINR}_{m}^{D L}=\gamma_{m}, \forall m$, afecta sensiblemente a las propiedades de convergencia los distintos algoritmos. En la literatura asociada a los algoritmos de control de potencia, como por ejemplo [88], se introduce una ligera tolerancia sobre la condición anterior, es decir:

$$
\operatorname{SINR}_{m}^{D L}=\gamma_{m} \pm \nabla \mathrm{SINR}, \forall m,
$$

donde $\nabla$ SINR es un valor que habitualmente oscila entre 0,5 y $1 \mathrm{~dB}$. Esto es debido a que los algoritmos JPCOB pueden invertir muchas iteraciones 
en ajustar exactamente la condición sobre la SINR, cuando si el problema es teóricamente feasible, necesitan típicamente de unas pocas iteraciones para converger.

En este apartado, se consideran las dos situaciones, un requerimiento estricto de SINR y un requerimiento de SINR con cierta tolerancia. En principio, el parámetro de interés para evaluar las prestaciones de los distintos algoritmos es la potencia total transmitida en el sistema. Sin embargo, el estudio de la probabilidad de feasibility práctica de los tres algoritmos, es decir, teniendo en cuenta las restricciones en potencia de las BSs y en el número de iteraciones, resulta también muy interesante para saber cómo evolucionarían estos algoritmos en un entorno real. En este punto, es interesante destacar que la probabilidad de feasibility práctica es una medida equivalente a la tasa de fallo evaluada en el Capítulo 4, sólo que en este Capítulo se enfoca hacia aspectos de convergencia del algoritmo.

\subsubsection{Requerimiento de QoS: estricto}

En los sistemas CM3 con beamformers multi-base, en principio, podría darse servicio a un total de $M=K \cdot N_{t}$ usuarios co-canal sin que las prestaciones del beamformer se vieran significativamente degradadas.

Como se ha comentado anteriormente, el algoritmo JPCOB-SDL diseña un beamformer multi-base en transmisión para las situaciones en las que existe algún tipo de restricción en la cantidad de información que realimenta el usuario a las BSs y que en este caso se interpreta como que las BSs no tienen ningún tipo de información de si existe un ecualizador en el receptor del usuario. Este algoritmo, fuerza el diseño del beamformer para que a la salida de cada uno de los correladores del receptor de un usuario, se disponga de $(1 / K)$-ésima parte de la potencia de señal deseada. Debido a este diseño, la relación entre el número de usuarios y antenas en el sistema se modifica de la siguiente manera:

$$
M Q \leq K N_{t}
$$

de forma que si $M Q>K N_{t}$, las prestaciones del beamformer multi-base diseñado por el algoritmo JPCOB-SDL se degradan rápidamente. En nuestro caso, $Q=K$, con lo que la condición en el número de usuarios se equipara con la de un diseño distribuido de los beamformers, como el que realiza el algoritmo JPCOB-VUL, donde $M \leq N_{t}$.

Puede concluirse pues, que la primera consecuencia de permitir un diseño robusto del beamfomer, o adaptado a situaciones de limited feed- 
back, es que la principal ventaja de los beamformers multi-base desaparece, es decir, no se puede admitir a un mayor número de usuarios que en los diseños distribuidos.

Por tanto, teniendo en cuenta la particularidad del algoritmo JPCOBSDL, y el diseño distribuido de los beamformers del algoritmo JPCOBVUL, se consideran $M=3$ usuarios co-canal en el sistema. Para comprobar el efecto de superar la condición en número de usuarios, se incluyen algunos resultados para $M=4$.

\section{Enlaces activos en el sistema}

Teóricamente, los algoritmos JPCOB-DL, JPCOB-SDL y JPCOB-VUL no deberían influir en el número de enlaces activos del sistema, puesto que es el esquema de selección de enlaces activos en el entorno Coordinado I el que se encarga de decidir qué enlaces se consideran activos mediante la construcción de un active set de BSs para cada usuario del sistema. Sin embargo, el análisis del número de enlaces activos en el sistema según el valor de la ventana de AS para los tres algoritmos bajo estudio, descubre una propiedad inherente a los beamformers diseñados por el algoritmo JPCOBDL.

La figura 6.2 presenta la función distribución estimada o CDF del número de enlaces entre usuarios y BSs activos en el sistema para un requerimiento de QoS común a todos los usuarios de $\mathrm{SINR}=7 \mathrm{~dB}$ y $M=3$ usuarios activos [121]. En este modelo de sistema, el número de enlaces activos oscila entre $3,(\mathrm{ASW}=0 \mathrm{~dB}$, sin coordinación, donde cada usuario es servido por una única BS y por tanto existen sólo $M$ enlaces activos en el sistema), y $9,(\mathrm{ASW}=90 \mathrm{~dB}$, coordinación total, donde cada usuario es servido por todas las BSs y en el sistema existen $K \cdot M$ enlaces activos). Por simplicidad, en la figura se representan los resultados del algoritmo JPCOB-DL y del algoritmo JPCOB-VUL para grados de coordinación entre BSs ASW $\geq 20 \mathrm{~dB}$ (los resultados del algoritmo JPCOB-SDL coinciden con los del algoritmo JPCOB-VUL).

La figura 6.2 se interpreta de la siguiente forma. Un valor de probabilidad, por ejemplo, 0,9, para un algoritmo (JPCOB-VUL) y un tamaño de ventana de AS determinado $(\mathrm{ASW}=20 \mathrm{~dB})$, indica que existe esa probabilidad concreta de que el número de enlaces activos en el sistema sea igual o menor que 7 .

En esta figura se observa que las curvas del algoritmo JPCOB-VUL y del algoritmo JPCOB-DL, que en teoría deberían coincidir, y que de hecho 


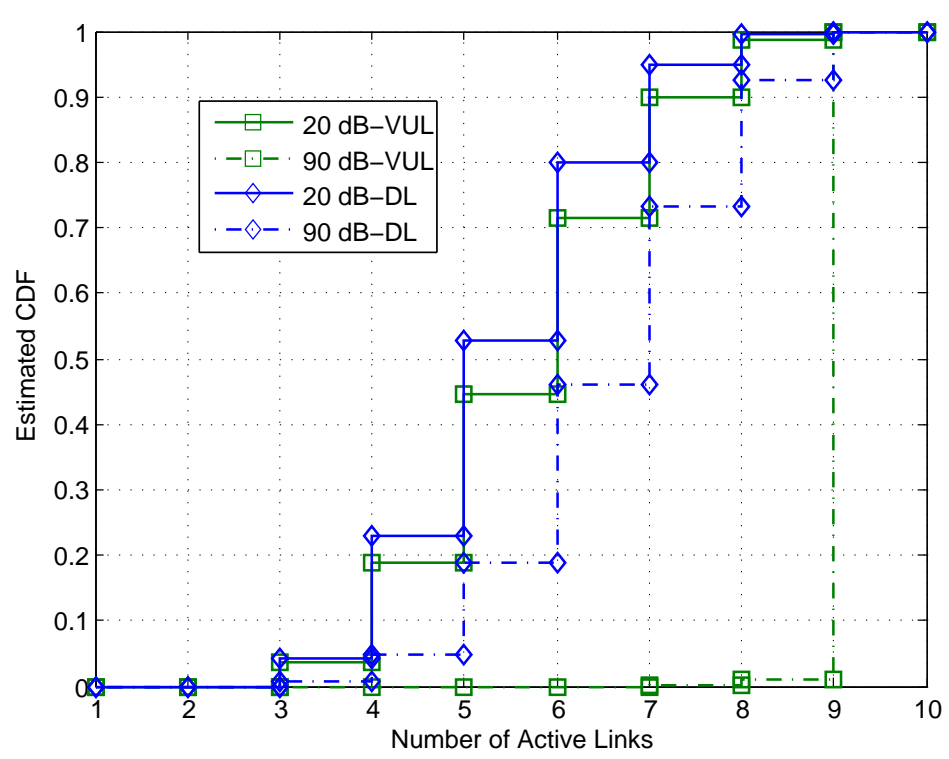

Figura 6.2: Función distribución estimada del número de enlaces activos en el sistema CM3 para un requerimiento de QoS de $\mathrm{SINR}=7 \mathrm{~dB}$ y $M=3$ usuarios co-canal.

coinciden para ASW $<20 \mathrm{~dB}$, se separan y que el algoritmo JPCOB-DL presenta un menor número de enlaces activos que el algoritmo JPCOB-VUL o el JPCOB-SDL. Por ejemplo, si nos centramos en la curva de ASW = $90 \mathrm{~dB}$ del algoritmo JPCOB-DL, existe una probabilidad del $45 \%$ de que existan 6 o menos enlaces activos en el sistema, mientras que el algoritmo JPCOB-VUL, para un mismo tamaño de ventana de AS, presenta una probabilidad prácticamente nula, es decir, siempre tiene más de 6 enlaces activos.

Analizando un poco más en detalle el funcionamiento del algoritmo JPCOB-DL, se observa que el paso de diseño de los beamformers, independientemente de las decisiones del esquema de selección de enlaces activos, anula los beamformers asociados a determinados enlaces que en teoría deberían estar activos. En las simulaciones, se ha considerado que un determinado enlace $m k$ entre una BS $k$ y un usuario $m$ está inactivo, cuando el beamformer en transmisión correspondiente a ese enlace presenta una contribución de potencia inferior a $\left\|\mathbf{w}_{m k}\right\|^{2}<10^{-6}$, lo que equivale a una potencia en transmisión inferior a $-30 \mathrm{dBm}$. 


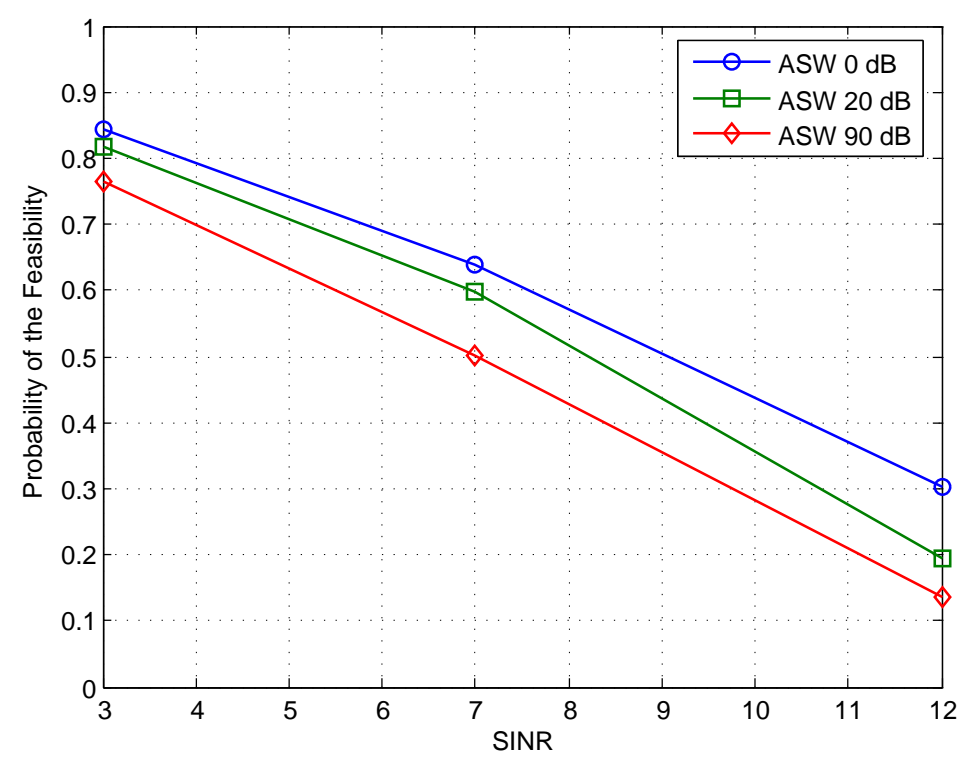

Figura 6.3: Probabilidad de feasibility práctica del algoritmo JPCOB-VUL para $M=3$ usuarios co-canal.

\section{Probabilidad de feasibility práctica de los distintos algoritmos}

En las figuras 6.3 y 6.4 se presentan los resultados de la probabilidad de feasibility práctica de los distintos algoritmos. Esta probabilidad se obtiene dividiendo el número de realizaciones en las que un algoritmo es feasible, es decir, los usuarios alcanzan el valor mínimo de SINR umbral sin que las BSs superen la restricción en potencia $P_{\max }$ y dentro de un número máximo de iteraciones del algoritmo, en este caso 30 iteraciones, sobre el número de realizaciones totales. Hay que recordar que esta feasibility práctica es una restricción añadida a la feasibility teórica, caracterizada por la condición sobre el radio espectral de la matriz de ganancia DL (6.57).

La figura 6.3 presenta la probabilidad de feasibility práctica del algoritmo JPCOB-VUL para distintos grados de coordinación entre BSs cuando se distribuyen uniformemente $M=3$ usuarios co-canal en el sistema. Para este número de usuarios, los algoritmos JPCOB-DL y JPCOB-SDL consiguen una probabilidad prácticamente igual a la unidad para todos los valores de ventana de AS. 


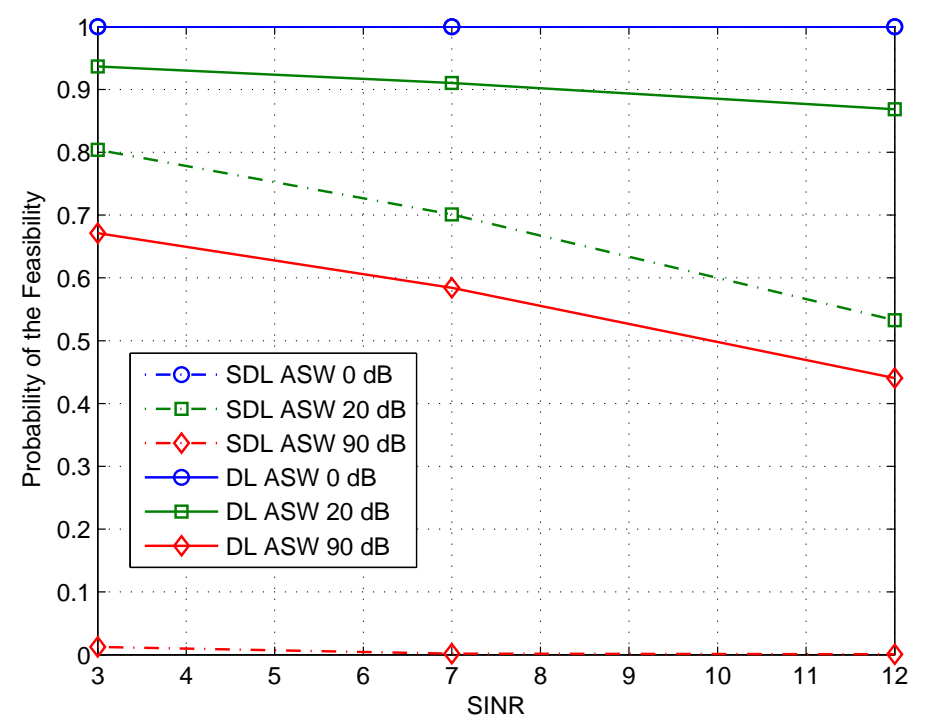

Figura 6.4: Probabilidad de feasibility práctica de los algoritmos JPCOB-DL y JPCOB-SDL para $M=4$ usuarios co-canal.

En teoría, para este número de usuarios, el diseño de los beamformers de los tres algoritmos es óptimo, ya que $M=N_{t}$. Sin embargo, la figura 6.3 muestra que la probabilidad de feasibility práctica del algoritmo JPCOB-VUL disminuye hasta un valor prácticamente no feasible a medida que aumenta el valor mínimo de SINR umbral requerida. Además, contrariamente a los resultados obtenidos en la segunda Parte de la Tesis, donde las prestaciones del algoritmo mejoraban al permitir cierto grado de coordinación entre las BSs, ahora la probabilidad de feasibility práctica disminuye a medida que se aumenta esta coordinación. Como se verá más adelante, este comportamiento de la probabilidad de feasibility práctica del algoritmo JPCOB-VUL se debe a que este algoritmo está muy limitado tanto por la restricción en la potencia que puede transmitir cada BS como por la restricción en el número máximo de iteraciones del algoritmo.

La figura 6.4 presenta la probabilidad de feasibility práctica cuando el número de usuarios co-canal en el sistema se incrementa a $M=4$, y por tanto, $M Q>K N_{t}$, manteniendo el requerimiento estricto en el valor mínimo de SINR. La figura 6.4 muestra únicamente los resultados de los algoritmos JPCOB-DL y JPCOB-SDL, puesto que el algoritmo JPCOB- 


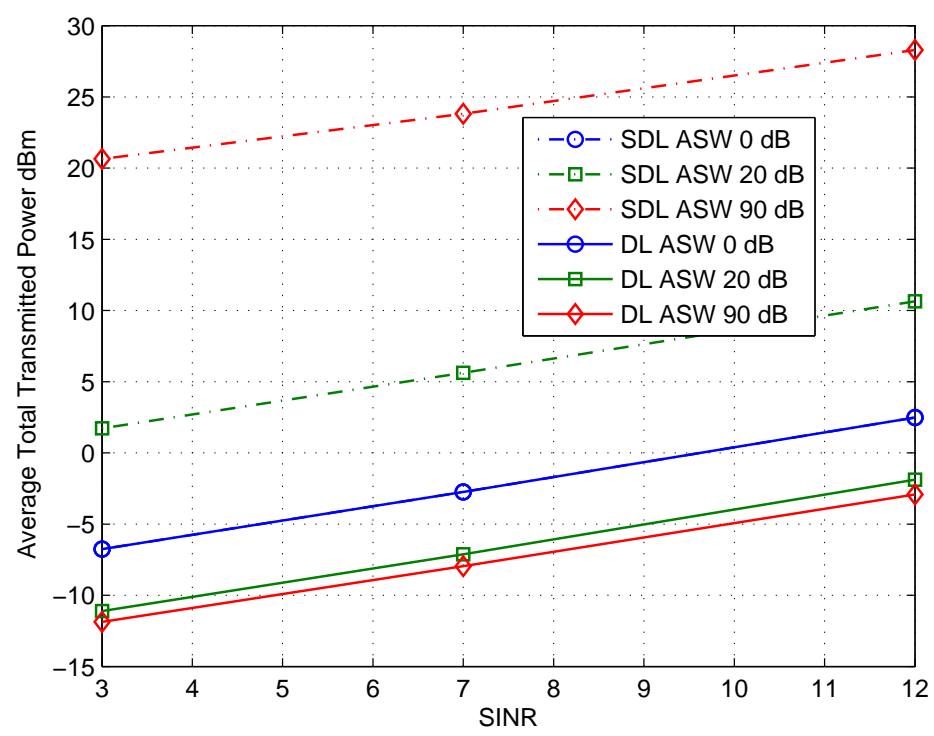

Figura 6.5: Potencia media total transmitida en el sistema por los algoritmos JPCOB-DL y JPCOB-SDL para $M=3$ usuarios co-canal.

VUL empeora los resultados de la figura 6.3.

Los algoritmos JPCOB-DL y JPCOB-SDL disminuyen su probabilidad de feasibility práctica a medida que aumenta el grado de coordinación entre BSs, aunque como se verá más adelante, el motivo es en este caso la limitación en el número de iteraciones del algoritmo. Además, la curva correspondiente a la configuración $\mathrm{ASW}=90 \mathrm{~dB}$ del algoritmo JPCOB-SDL es totalmente no feasible. En general, el algoritmo JPCOB-DL mejora las prestaciones del algoritmo JPCOB-SDL a costa de aumentar la cantidad de información que necesita realimentar el usuario a las BSs.

\section{Potencia media total transmitida en el sistema}

La figura 6.5 muestra la potencia media total transmitida en el sistema CM3 por los algoritmos JPCOB-DL y JPCOB-SDL para $M=3$ usuarios co-canal. Los resultados del algoritmo JPCOB-VUL no se incluyen en la gráfica puesto que la baja probabilidad de feasibility práctica de este algoritmo para valores umbrales de SINR medio-altos provoca que los resultados sean poco fiables. 
En general, ambos algoritmos aumentan la potencia media total transmitida en el sistema a medida que aumenta el valor de SINR umbral requerido a cada usuario.

El algoritmo JPCOB-SDL comparte el mecanismo de control de potencia del algoritmo JPCOB-DL, sin embargo, no aprovecha la presencia del ecualizador en el receptor del usuario y diseña un beamformer robusto para cada usuario, lo que provoca un aumento considerable de la potencia total transmitida con respecto al algoritmo JPCOB-DL.

Si nos centramos en la influencia del valor de ventana de AS, se observa que el algoritmo JPCOB-DL, contrariamente a los algoritmos JPCOB-VUL y JPCOB-SDL, disminuye la potencia total transmitida en el sistema a medida que se aumenta la coordinación entre BSs. Este comportamiento está relacionado con el hecho de que este algoritmo anula automáticamente determinados enlaces en función del beamformer obtenido para cada usuario del sistema.

Por último, hay que destacar que las curvas correspondientes a ASW = $0 \mathrm{~dB}$ coinciden para ambos algoritmos debido a que únicamente transmite una BS, y en este caso el diseño de los beamformers MMSE proporciona una solución similar.

\section{Número medio de iteraciones}

Con respecto al número medio de iteraciones que necesita cada algoritmo para converger, hay que destacar que para la configuración $M=3$, el algoritmo JPCOB-SDL necesita una media de únicamente 3 iteraciones para todos los valores de SINR umbral y valores de ventana de AS, mientras que el algoritmo JPCOB-VUL y el JPCOB-DL necesitan un número mayor de iteraciones para un mismo valor de SINR umbral a medida que se aumenta el grado de coordinación entre BSs. Sin embargo, el algoritmo JPCOB-DL necesita en media menos iteraciones para converger que el algoritmo JPCOB-VUL.

A la vista de estos resultados, se puede concluir que el algoritmo JPCOBSDL es un algoritmo más sencillo de implementar, pero que esta sencillez empeora sus prestaciones desde el punto de vista de potencia total transmitida en el sistema y probabilidad de feasibility práctica. Aún así, sirve como punto de referencia del comportamiento que podría presentar el algoritmo JPCOB-DL en un sistema CM3 con conocimiento parcial del canal. 


\subsubsection{Requerimiento de QoS: tolerante}

Los resultados que se han presentado hasta ahora, exigen que la condición básica de convergencia, $\operatorname{SINR}_{m}^{D L}=\gamma_{m}, \forall m$, se cumpla con una tolerancia estricta, $\nabla$ SINR $\pm 0,05 \mathrm{~dB}$. En la práctica, lo habitual es permitir una cierta tolerancia más amplia sobre la condición de SINR, con lo que en principio, los algoritmos mejorarían sus propiedades de convergencia.

A continuación se presenta una comparación de las prestaciones de los distintos algoritmos para una tolerancia de $\nabla \operatorname{SINR} \pm 0,5 \mathrm{~dB}$ sobre la condición básica de convergencia. El hecho de introducir esta tolerancia modifica el comportamiento de los algoritmos en cuanto a probabilidad de feasibility práctica, pero no modifica sustancialmente los resultados de potencia media total transmitida o número medio de iteraciones de cada algoritmo. Teniendo en cuenta además que el algoritmo JPCOB-SDL es un algoritmo de implementación relativamente sencilla, que proporciona una solución robusta de beamforming, pero que no representa una solución eficiente en cuanto a potencia total transmitida en el sistema, en las figuras de esta simulación que evalúan la potencia media total transmitida en el sistema, únicamente se incluyen los resultados obtenidos por los algoritmos JPCOBDL y JPCOB-VUL.

\section{Probabilidad de feasibility práctica de los distintos algoritmos}

La figura 6.6, siguiendo los resultados de la figura 6.3, muestra la probabilidad de feasibility práctica del algoritmo JPCOB-VUL para distintos grados de coordinación entre las BSs cuando se distribuyen uniformemente $M=3$ usuarios co-canal en el sistema, pero permitiendo ahora una cierta tolerancia en la condición básica de convergencia [121]. Para este número de usuarios, la probabilidad de feasibility práctica de los algoritmos JPCOBDL y JPCOB-SDL es igual a la unidad para todos los valores de ventana de AS.

Como se puede observar en la figura, la probabilidad de feasibility práctica del algoritmo JPCOB-VUL aumenta con respecto a los resultados presentados en la figura 6.3, y lo que es más importante, estos nuevos resultados ya siguen la tendencia de los resultados obtenidos en la segunda Parte de la Tesis, donde el hecho de permitir una cierto grado de coordinación entre las BSs del sistema mejoraba las prestaciones del algoritmo JPCOB-VUL para valores medio-bajos de SINR umbral. 


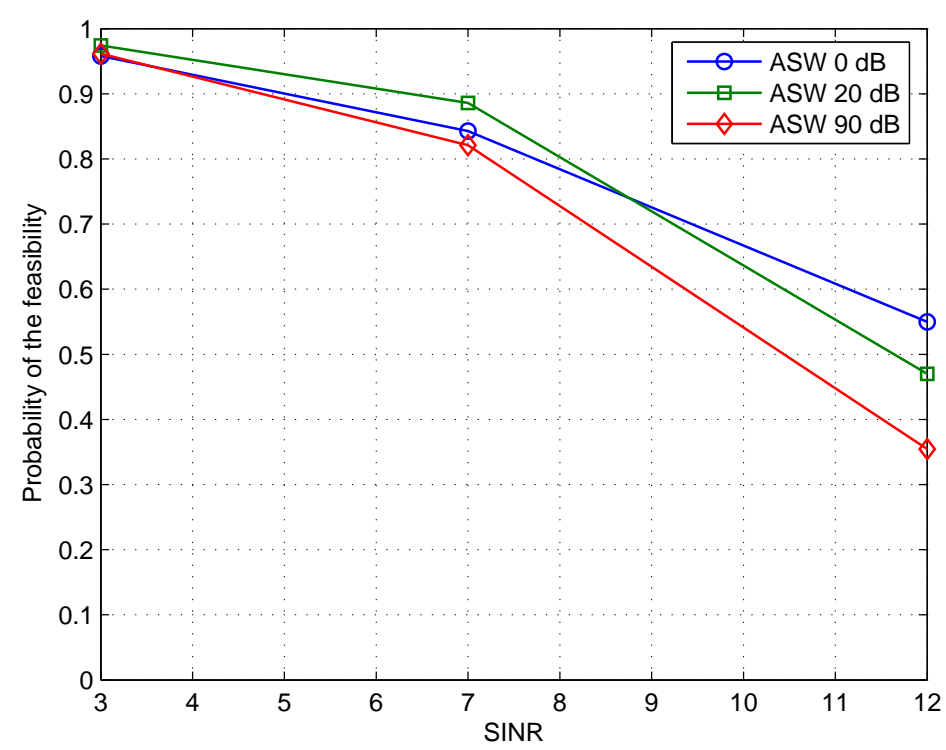

Figura 6.6: Probabilidad de feasibility práctica del algoritmo JPCOB-VUL para $M=3$ usuarios co-canal.

Por otro lado, la figura 6.7 presenta la probabilidad de feasibility práctica de los algoritmos JPCOB-DL, JPCOB-SDL y JPCOB-VUL para $M=4$ usuarios co-canal [121]. Claramente, el hecho de considerar más usuarios en el sistema que antenas dispone cada BS, $N_{t}<M$, afecta al diseño distribuido de los beamformers del algoritmo JPCOB-VUL, lo que unido a la restricción en potencia de cada BS, impide que el mecanismo de control de potencia del algoritmo mitigue la interferencia que el diseño de los beamformers no puede gestionar.

Comparando las figuras 6.4 y 6.7 , se observa un aumento general en la probabilidad de feasibility práctica de los algoritmos JPCOB-DL y JPCOBSDL. Si nos centramos en el algoritmo JPCOB-DL, el hecho de permitir una cierta tolerancia en la condición básica de convergencia permite que las configuraciones de ASW $<90 \mathrm{~dB}$ presenten una probabilidad prácticamente igual a la unidad para todos los valores de SINR umbral, mientras que la curva de $\mathrm{ASW}=90 \mathrm{~dB}$ experimenta un incremento de un $35 \%$ para un valor de SINR umbral de $12 \mathrm{~dB}$. Con respecto al algoritmo JPCOB-SDL, las configuraciones de ASW $<90 \mathrm{~dB}$ siguen la tendencia de los resultados del JPCOB-DL y consiguen valores de probabilidad de feasibility práctica 


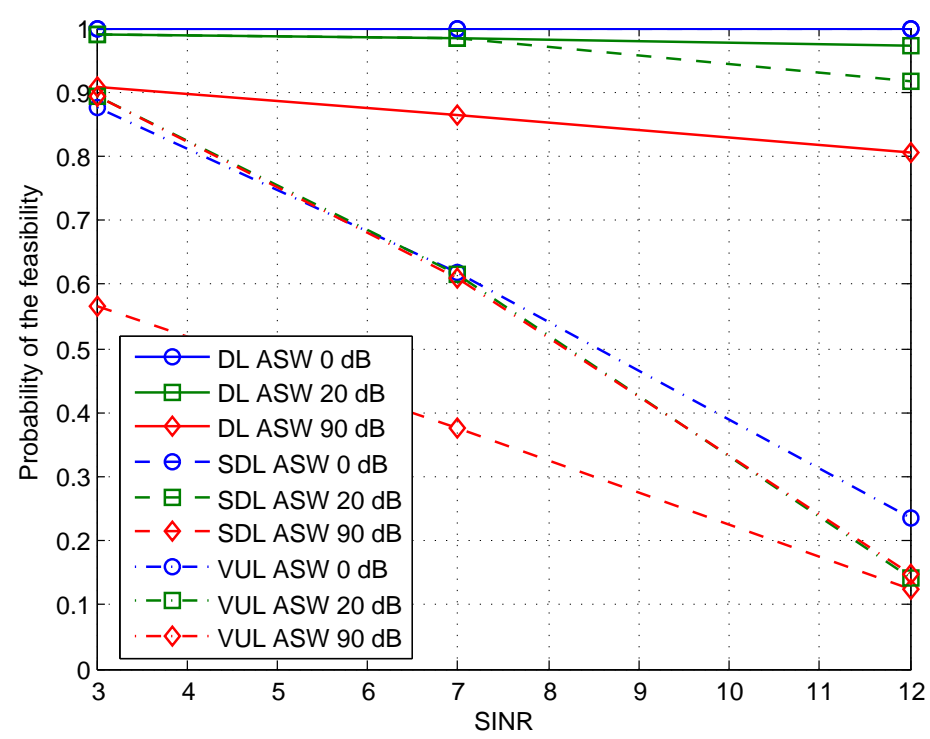

Figura 6.7: Probabilidad de feasibility práctica de los algoritmos JPCOB-DL, JPCOB-SDL y JPCOB-VUL para $M=4$ usuarios co-canal.

cercanos a la unidad para todos los valores de SINR umbral. Además, la curva de $\mathrm{ASW}=90 \mathrm{~dB}$ consigue una mejora de un $55 \%$ para el valor de SINR umbral de $3 \mathrm{~dB}$, aunque esta mejora disminuye al aumentar el valor de SINR umbral, llegando hasta un incremento del $10 \%$ para el valor de SINR umbral de $12 \mathrm{~dB}$.

\section{Potencia media total transmitida en el sistema}

La figura 6.8 muestra la potencia media total transmitida en el sistema CM3 por los algoritmos JPCOB-DL y JPCOB-VUL para $M=4$ usuarios co-canal en el sistema [121]. De nuevo, hay que tener en cuenta que la probabilidad de feasibility práctica del algoritmo JPCOB-VUL es muy baja para valores altos de SINR umbral, lo que influye en la fiabilidad de los resultados en potencia total transmitida.

En general, ambos algoritmos siguen la tendencia de la figura previa 6.5 a aumentar la potencia media total transmitida en el sistema a medida que aumenta el valor de SINR umbral. De nuevo, el algoritmo JPCOBDL disminuye la potencia total transmitida en el sistema a medida que se incrementa la coordinación entre BSs. Por su parte, el algoritmo JPCOB- 


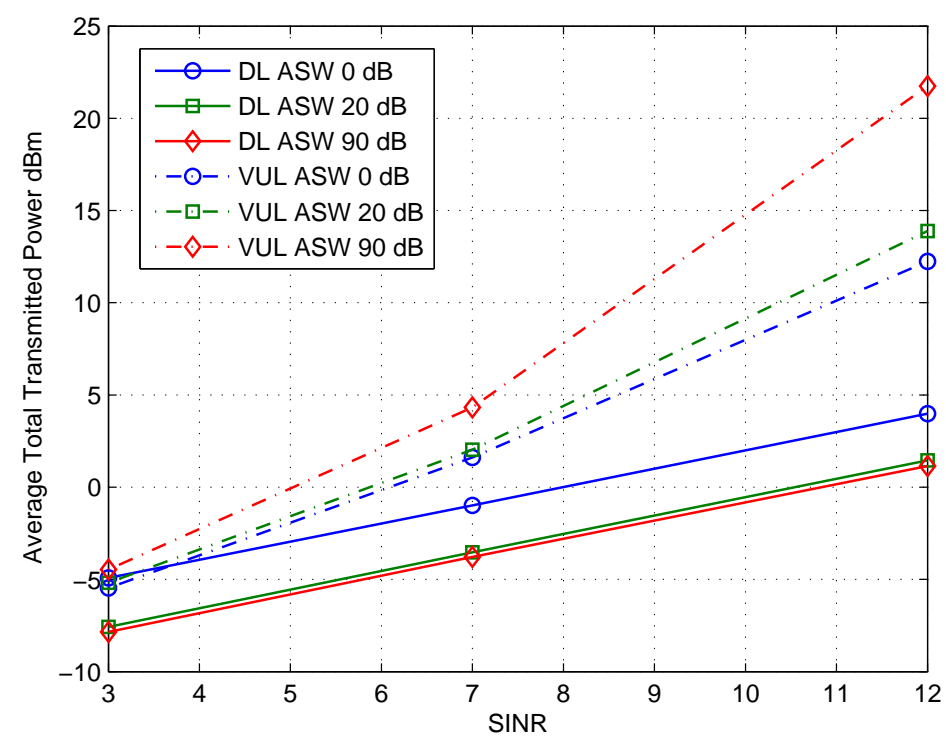

Figura 6.8: Potencia media total transmitida en el sistema por los algoritmos JPCOB-DL y JPCOB-VUL para $M=4$ usuarios co-canal.

VUL presenta el comportamiento opuesto, es decir, aumenta la potencia total transmitida a medida que se aumenta el grado de coordinación entre BSs (en realidad, tal y como ocurría en las simulaciones del Capítulo 4, las configuraciones de sistema tales que $\mathrm{ASW}<90 \mathrm{~dB}$, no aumentan significativamente la potencia total transmitida en el sistema, como se puede observar en esta figura al comparar las curvas ASW $=0 \mathrm{~dB}$ y ASW $=20 \mathrm{~dB}$ del algoritmo JPCOB-VUL). Además, en las curvas correspondientes a la configuración de $\mathrm{ASW}=0 \mathrm{~dB}$ es donde se aprecia que el diseño de los beamformers del algoritmo JPCOB-DL, por el hecho de ser multi-base y centralizado, es más eficiente en potencia que la solución distribuida del algoritmo JPCOB-VUL. 


\subsubsection{Influencia de las restricciones adicionales en la convergencia y estabilidad de los algoritmos}

En los resultados que se han presentado en este Capítulo, se puede observar como la feasibility práctica del algoritmo, o del problema de minimización de potencia, es una restricción añadida a la feasibility teórica dada por la relación (6.57).

Se ha comentado brevemente que tanto la probabilidad de feasibility teórica del algoritmo JPCOB-VUL como la del algoritmo JPCOB-DL se ve influenciada por las restricciones adicionales de potencia transmitida por BS y número máximo de iteraciones del algoritmo. Las figuras 6.3, 6.4, 6.6 y 6.7 , muestran la probabilidad de feasibility práctica de ambos algoritmos para un requerimiento estricto o con una tolerancia de $\pm 0,5 \mathrm{~dB}$ en la condición de convergencia sobre la SINR. Sin embargo, estas figuras no precisan de qué manera influyen las restricciones adicionales de potencia máxima transmitida por BS, $P_{\max }$, y número máximo de iteraciones del algoritmo, It, es decir, qué porcentaje de la probabilidad de feasibility práctica que falta para llegar a la probabilidad de feasibility total, se corresponde con un fallo del algoritmo por superar la restricción $P_{\max }$ o It.

Las gráficas 6.9 y 6.10 muestran la distribución de la probabilidad de feasibility práctica de los resultados de los algoritmos JPCOB-VUL y JPCOBDL presentados en las figuras 6.6 y 6.7 , respectivamente. Los resultados representados con $P_{\max }$ representan el número de realizaciones sobre el total en las que el algoritmo JPCOB-VUL no converge porque alguna BS supera la restricción en potencia $P_{\max }$, mientras que los resultados asociados a $I T_{\max }$ se corresponden con el número de realizaciones sobre el total en las que el algoritmo necesita más de 30 iteraciones para converger.

En las figuras de 6.9 se observa como la distribución de la probabilidad de feasibility práctica del algoritmo JPCOB-VUL varía en función del requerimiento de SINR umbral. Para un valor de SINR bajo o medio, y para valores de ventana de $\mathrm{AS}$ inferiores a $20 \mathrm{~dB}$, existe la misma probabilidad de no convergencia asociada a la restricción en potencia de las BSs que a la restricción en el número máximo de iteraciones del algoritmo. Sin embargo, en estos casos, a medida que aumenta la coordinación entre BSs, $\mathrm{ASW}>20 \mathrm{~dB}$, es la restricción en número máximo de iteraciones la que domina la probabilidad de no convergencia del algoritmo.

Por el contrario, si nos centramos en la figura correspondiente a un valor de SINR umbral de $12 \mathrm{~dB}$, se observa que la convergencia o probabilidad de feasibility práctica del algoritmo se encuentra muy limitada por la 


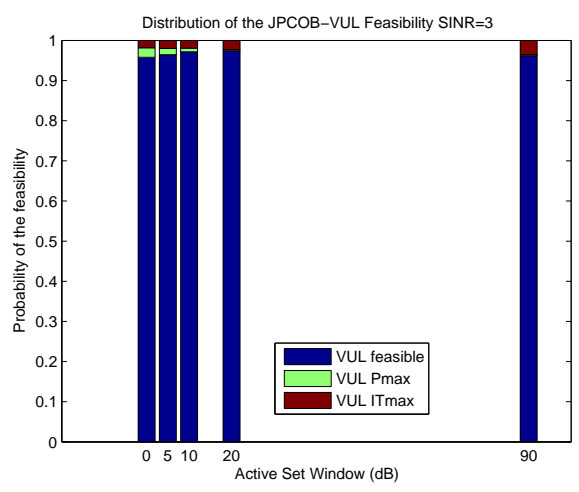

(a) $\mathrm{SINR}=3 \mathrm{~dB}$.

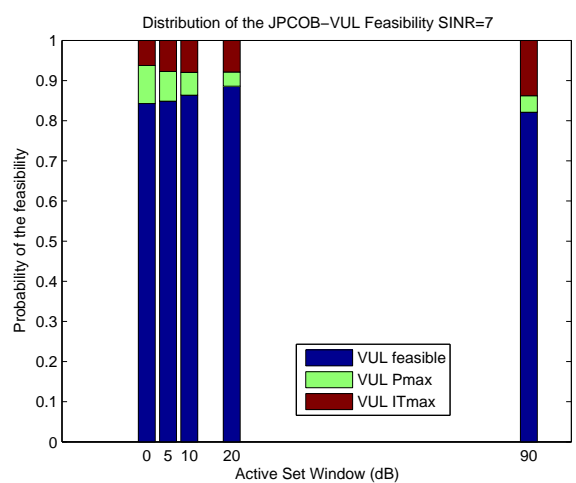

(b) $\mathrm{SINR}=7 \mathrm{~dB}$.

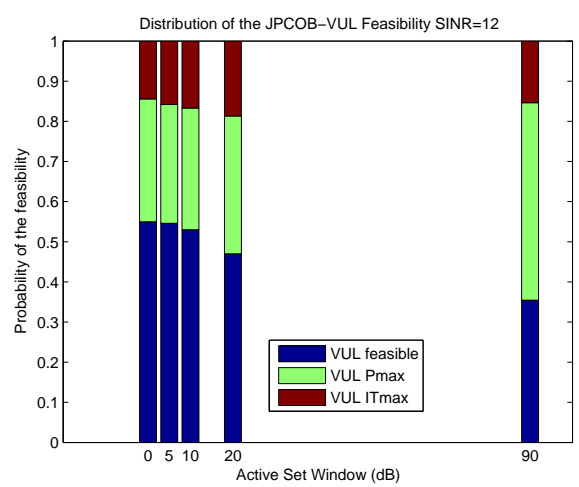

(c) $\mathrm{SINR}=12 \mathrm{~dB}$.

Figura 6.9: Distribución de la probabilidad de feasibility práctica del algoritmo JPCOB-VUL para $M=3$ usuarios co-canal (figura 6.6).

restricción en potencia de cada BS del sistema.

El algoritmo JPCOB-DL presenta una distribución de la probabilidad de feasibility práctica distinta a la del algoritmo JPCOB-VUL. En las figuras de 6.10 se observa como el hecho de diseñar un beamformer multi-base para cada usuario permite gestionar de manera más eficiente la potencia disponible de cada BS, de forma que la principal causa de no convergencia del algoritmo JPCOB-DL se debe a la restricción en el número máximo de iteraciones del algoritmo. 


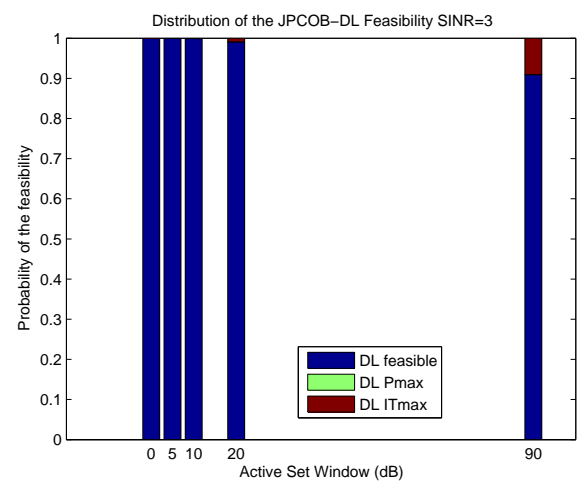

(a) $\operatorname{SINR}=3 \mathrm{~dB}$.

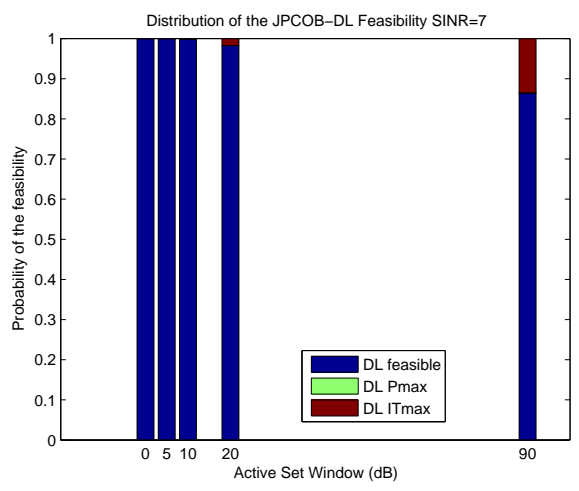

(b) $\mathrm{SINR}=7 \mathrm{~dB}$.

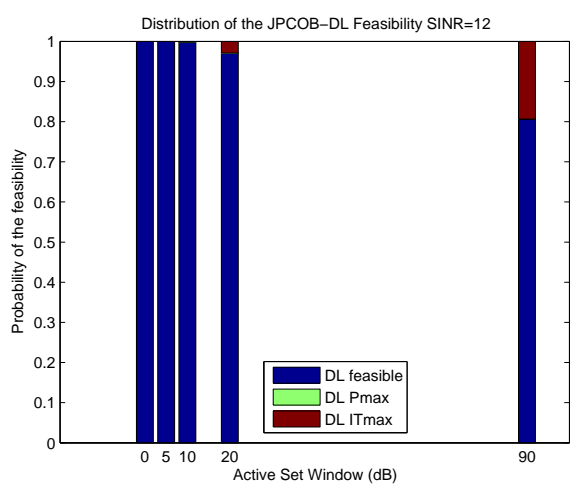

(c) $\mathrm{SINR}=12 \mathrm{~dB}$.

Figura 6.10: Distribución de la probabilidad de feasibility práctica del algoritmo JPCOB-DL para $M=4$ usuarios co-canal (figura 6.7).

Por otro lado, en situaciones donde el sistema está mal condicionado, por ejemplo, en sistemas donde $M Q \geq K N_{t}$ para el caso concreto del algoritmo JPCOB-SDL, estas restricciones $P_{\max }$ e $I t$ pueden provocar cierta inestabilidad en la convergencia de los algoritmos downlink-based. Se ha comprobado mediante simulaciones que para estos casos de sistemas mal condicionados, la solución de los beamformers obtenida por cualquiera de los algoritmos downlink-based entre en algunas ocasiones en una dinámica oscilatoria. 
En esta dinámica, el paso de diseño de los beamformers multi-base de los algoritmos JPCOB-SDL y JPCOB-DL se queda oscilando entre dos puntos entre una iteración y la siguiente, que se corresponden con una solución de beamforming distinta. De esta forma, las matrices de ganancia DL no convergen a matrices constantes y por tanto el mecanismo de control de potencia no puede converger. Esto no implica necesariamente que la condición de feasibility teórica sobre el radio espectral de la matriz de ganancia DL (6.57) no se esté cumpliendo, sino que las restricciones adicionales $P_{\max }$ e $I t$, junto con la situación adversa en número de usuarios en el sistema, provocan que la matriz de ganancia DL oscile entre una iteración y la siguiente entre dos valores fijos, asociados con dos soluciones distintas de beamforming.

Esta oscilación se ha analizado mediante simulaciones y se han podido caracterizar en grandes líneas las características principales de los beamformers asociados a cada punto de la oscilación.

En primer lugar, se obtiene un beamformer que se caracteriza por asegurar fairness entre todos los usuarios del sistema, ya que se obtiene en la primera iteración del algoritmo cuando todas las potencia DL que se consideran para diseñar por primera vez el beamformer de cada usuario se corresponden con las potencias de inicialización del algoritmo, y son, por tanto, iguales para todos los usuarios.

El segundo beamformer se obtiene en la siguiente iteración del algoritmo, a partir de las potencias DL que se obtienen en el paso de actualización de potencias al considerar el beamformer fairness de la primera iteración. En principio, este segundo beamformer es el que realmente se ajusta al diseño propuesto para los algoritmos JPCOB-DL y JPCOB-SDL. El problema es que en la tercera iteración de los algoritmos, este beamformer, en lugar de seguir ajustándose hasta converger a un beamformer fijo, cambia bruscamente al beamformer fairness de la primera iteración y ya no es posible salir de la dinámica oscilatoria entre ambos beamformers.

En grandes líneas, es posible afirmar que ambos beamformers se corresponden con dos filosofías distintas de beamformer MMSE; por una parte el beamformer fairness trata de minimizar la interferencia recibida por los usuarios, comportándose de forma similar a un beamformer ZF del canal, mientras que el beamformer que se obtiene en el segundo punto de la oscilación trata de maximizar la señal deseada recibida por el usuario. 
Si en una simulación de cualquiera de los dos algoritmos downlink-based se toma uno de los dos beamformers, se mantiene constante y se itera únicamente el control de potencia, el algoritmo converge siempre y cuando la matriz de ganancia DL resultante cumpla la restricción sobre el radio espectral (6.57). Si se analiza el radio espectral de las matrices de ganancia DL obtenidas de esta forma, se observa que, en general, el radio espectral asociado al beamformer fairness, que es el que proporciona mínima interferencia, es mucho menor que el asociado al beamformer del segundo punto de la oscilación, con lo que el mecanismo de control de potencia converge más rápidamente, y en principio, proporciona una menor potencia total transmitida en el sistema. Sin embargo, todo parece indicar que el beamformer al que debería converger el algoritmo es el que se obtiene a partir del segundo punto de la oscilación. 


\section{Capítulo 7}

\section{Algoritmo JPCOB-DL: aplicaciones prácticas}

Esta tercera Parte de la Tesis se centra en la propuesta y análisis de algoritmos JPCOB alternativos al algoritmo JPCOB-VUL para resolver el problema de minimización de potencia en sistemas W-CDMA MIMO multi-usuario multi-celda coordinados (CM3). En el Capítulo anterior se proponen dos algoritmos JPCOB downlink-based, es decir, que no recurren a la dualidad entre el problema de minimización de potencia en DL y un problema equivalente planteado en el UL virtual para simplificar el cálculo de los beamformers en transmisión para cada usuario del sistema CM3.

El algoritmo JPCOB-DL y su versión robusta o simplificada, el JPCOBSDL, mejoran significativamente las prestaciones del algoritmo JPCOBVUL. Sin embargo, cuando el sistema CM3 está mal condicionado, por ejemplo, cuando existen más usuarios activos que antenas disponibles en el sistema, $M>K N_{t}$, las propiedades de convergencia de estos algoritmos se degradan rápidamente, principalmente debido a la influencia de las restricciones de carácter práctico (número máximo de iteraciones y potencia disponible en la estación base) que se añaden en la simulación de cada algoritmo.

Por otro lado, la condición de feasibility teórica del algoritmo JPCOBDL y por tanto, del problema de minimización de potencia, viene dada por la relación (6.57) sobre el radio espectral de la matriz de ganancia DL del algoritmo, $\left[\mathbf{D}^{\dagger} \mathbf{F}\right]$. Esto es debido a que si la matriz de ganancia DL del algoritmo JPCOB-DL converge a una matriz constante, las propiedades de convergencia del algoritmo coinciden con las de la iteración de las potencias 
DL, que se trata en realidad de una iteración de Jacobi [89].

En aquellos algoritmos JPCOB en los que el mecanismo de control de potencia se puede expresar en forma de una iteración de Jacobi, el valor del radio espectral de la matriz de ganancia DL caracteriza aspectos fundamentales como por ejemplo la potencia total transmitida en el sistema, la velocidad de convergencia del algoritmo o la capacidad del sistema medida en número de usuarios. En general, cuanto menor sea el valor del radio espectral de la matriz de ganancia DL, menor será la potencia total transmitida en el sistema y mayor será tanto la velocidad de convergencia como la probabilidad de admisión del algoritmo [123,124].

En los problemas de control de potencia, o en los algoritmos JPCOB que admiten una formulación del control de potencia en forma de iteración de Jacobi, es bastante habitual actuar sobre el valor del radio espectral de la matriz de ganancia DL para mejorar las prestaciones del sistema [123-125]. Por ejemplo, las referencias [123] y [125] evalúan el gradiente del radio espectral de la matriz de ganancia DL para modificar su valor y ajustarlo según diferentes criterios de optimización. Por su parte, Schubert y Boche estudian en [124] las propiedades de convergencia de un mecanismo de control de potencia expresado como una iteración de Jacobi y proporcionan una interpretación geométrica de la feasibility teórica del problema de minimización de potencia en el enlace UL de un sistema de una única celda con dos usuarios.

En los sistemas W-CDMA CM3, y teniendo en cuenta las expresiones de las matrices D y F, (6.47) y (6.49), respectivamente, se observa que el valor del radio espectral de la matriz de ganancia DL depende de distintos parámetros que se relacionan tanto con aspectos de transmisión como de recepción del sistema.

En este Capítulo, se proponen dos esquemas iterativos para mejorar la probabilidad de feasibility teórica del algoritmo JPCOB-DL. Esta probabilidad se define a partir de la relación (6.57) como la probabilidad de que el valor del radio espectral de la matriz de ganancia DL del algoritmo JPCOB-DL sea inferior a la unidad. Los esquemas se formulan aprovechando la estructura de las matrices $\mathbf{D}$ y $\mathbf{F}$, por lo que se denominan esquema $\boldsymbol{D}$-based y esquema $\boldsymbol{F}$-based.

Básicamente, ambos esquemas modifican el valor del radio espectral de la matriz de ganancia DL disminuyendo de forma gradual el grado de coordinación entre las estaciones base (BSs) del sistema, por lo que en realidad se comportan como esquemas de selección de enlaces activos. Sin embargo, 
la base de estos esquemas es diferente a la de los esquemas presentados en la segunda Parte de la Tesis, puesto que en este caso se anulan de forma iterativa aquellos enlaces que según el esquema $\boldsymbol{D}$-based o el $\boldsymbol{F}$-based van a contribuir a reducir el valor del radio espectral de la matriz de ganancia DL.

Hay que destacar que el objetivo de estos esquemas no es resolver un problema de optimización conjunto de potencia, beamforming y selección de enlaces activos, sino que una vez planteado el problema de minimización de potencia en el enlace DL de un sistema CM3, si se produce una situación teóricamente no feasible, se ejecuta uno de estos esquemas para obtener una situación teóricamente feasible, es decir, que cumpla la condición de feasibility teórica (6.57).

Las simulaciones de este Capítulo demuestran que la tendencia de los esquemas $\boldsymbol{D}$-based y $\boldsymbol{F}$-based es a anular los $K$ enlaces correspondientes a un mismo usuario del sistema, con lo que en realidad estos esquemas podrían interpretarse como técnicas de scheduling o de control de admisión. De todas formas, el objetivo no es conseguir un control de admisión óptimo desde el punto de vista del problema de minimización de potencia, sino que ésta es una propiedad asociada a los esquemas propuestos en este Capítulo. La literatura asociada a la combinación del problema de control de potencia y control de admisión o scheduling es muy extensa. Algunas referencias interesantes pueden encontrarse en [126-128].

\subsection{Problema de minimización de potencia}

Siguiendo el modelo de sistema presentado en el Capítulo anterior, se considera un sistema W-CDMA CM3 formado por $K$ BSs, cada una equipada con un array lineal de $N_{t}$ antenas. En el sistema, se distribuyen uniformemente $M$ usuarios sobre el área compartida por las $K$ BSs (zona sombreada de la figura 4.2). En recepción, cada usuario dispone de un banco de $Q$ correladores y de un ecualizador que implementa un criterio MRC.

En las simulaciones de este Capítulo, interesa provocar aquellas situaciones en las que no se cumple la condición de feasibility teórica (6.57), para poder evaluar de forma eficiente los dos esquemas propuestos para mejorar la probabilidad de feasibility teórica del algoritmo JPCOB-DL. El problema es que estas situaciones suelen darse en los sistemas CM3 mal condicionados, $M>K N_{t}$, donde el control de potencia no tiene bastantes grados de libertad para suplir la degradación de las prestaciones de los 
beamformers multi-base y donde el paso de diseño de los beamformers del algoritmo JPCOB-DL puede entrar en una dinámica oscilatoria.

Por este motivo, el algoritmo JPCOB-DL se simplifica y se reduce únicamente al paso de actualización de las potencias DL, manteniendo un diseño de los beamformers multi-base fijo, y por tanto, subóptimo, en todas las iteraciones del algoritmo. De esta forma, el algoritmo JPCOB-DL se reduce a un mecanismo de control de potencia sujeto a las condiciones teóricas de convergencia de la iteración de Jacobi.

Por otro lado, se considera un entorno de propagación flat fading, donde el canal de propagación para cada enlace usuario-BS del sistema está formado por una única contribución multi-camino. En este caso concreto, y recordando el modelo de sistema presentado en el Capítulo 4, (4.14), la estructura de las matrices de canal $\mathbf{H}_{\mathrm{UEm}}$ en (6.1) se modifica de la siguiente manera:

$$
\mathbf{H}_{\mathrm{UEm}}=\operatorname{diag}\left\{\left[\mathbf{h}_{m 1} \ldots \mathbf{h}_{m K}\right]\right\},
$$

donde $\mathbf{h}_{m k}$ es el vector $\left[1 \times N_{t}\right]$ que caracteriza el canal de propagación del enlace entre la BS $k$ y el usuario $m$. La expresión anterior asume que las contribuciones de canal procedentes de cada BS del sistema llegan al usuario en distintos instantes de tiempo. Además, las dimensiones de las matrices en (6.1) se modifican de forma que $L=K$.

Teniendo en cuenta las modificaciones anteriores, el problema de minimización de potencia (6.14) se formula ahora como:

$$
\begin{aligned}
\operatorname{mín}_{\mathbf{P}} & \sum_{m=1}^{M}\left\|\mathbf{P}_{m} \mathbf{h}_{\mathrm{Tx} m}\right\|^{2} \\
\text { s.t. } & \operatorname{SINR}_{m}^{D L}\left(\mathbf{P}, \mathbf{H}_{\mathrm{Tx}}\right) \geq \gamma_{m}, m=1, \ldots, M,
\end{aligned}
$$

donde $\operatorname{SINR}_{m}^{D L}$ es la SINR medida en el usuario $m$ del sistema y $\gamma_{m}$ representa el valor umbral de SINR que se establece como requerimiento de QoS para ese usuario concreto.

\subsubsection{Beamforming en transmisión}

Con el fin de forzar situaciones teóricamente no feasible, el conjunto de beamformers multi-base en transmisión para los $M$ usuarios del sistema CM3 se calcula mediante un criterio ZF (Zero Forcing) [66]:

$$
\mathbf{H}_{\mathrm{Tx}}^{\mathrm{ZF}}=\mathbf{H}_{\mathrm{UE}}^{H}\left(\mathbf{H}_{\mathrm{UE}} \mathbf{H}_{\mathrm{UE}}^{H}\right)^{-1},
$$


donde $\mathbf{H}_{\mathrm{UE}}$ es una matriz de dimensiones $\left[M \times K N_{t}\right]$ cuyas filas se corresponden con los vectores $\mathbf{h}_{m}=\left[\mathbf{h}_{m 1} \ldots \mathbf{h}_{m K}\right]$, de dimensiones $\left[1 \times K N_{t}\right]$. El beamformer multi-base del usuario $m$, de dimensiones $\left[K N_{t} \times 1\right]$, se corresponde con la columna $m$-ésima de la matriz $\mathbf{H}_{\mathrm{Tx}}^{\mathrm{ZF}}$.

Si se compara la expresión anterior con la expresión de un beamformer ZF en transmisión para un sistema MIMO multi-usuario (2.41), se observa que en (7.3) no se incluye ningún tipo de restricción de potencia. En las simulaciones, la restricción en potencia de cada BS se evalúa sobre la contribución total de potencia, que incluye la potencia asignada por el beamformer, y la asignada por el mecanismo de control de potencia, $\sum_{m=1}^{M} p_{m k}\left\|\mathbf{w}_{m k}\right\|^{2}, \forall k$.

\subsubsection{Control de potencia}

Los esquemas $\boldsymbol{D}$-based y $\boldsymbol{F}$-based aprovechan la estructura de las matrices $\mathbf{D}$ y $\mathbf{F}$ involucradas en la condición de feasibility teórica de (6.57). En realidad, el esquema $\boldsymbol{D}$-based se basa en la versión eficiente de cálculo de la pseudo-inversa de la matriz $\mathbf{D}$ propuesta en la segunda Parte de la Tesis (4.40). Por este motivo, es necesario reorganizar la estructura de las matrices y vectores que forman parte de la actualización de potencias DL, siguiendo el modelo de (4.21):

$$
\mathbf{p}=[\underbrace{p_{11} \ldots p_{M 1}}_{\mathrm{BS}_{1}} \cdots \underbrace{p_{1 K} \ldots p_{M K}}_{\mathrm{BS}_{K}}]^{T} .
$$

En esta expresión, los elementos del vector de potencias DL se agrupan de nuevo por BSs y no por usuarios, como ocurría en (6.45). En realidad, la forma de organizar los elementos del vector $\mathbf{p}$ no afecta a la solución del control de potencia, pero sí que influye a la hora de aprovechar la estructura de las matrices presentes en la iteración de actualización de las potencias DL.

La mínima potencia total transmitida en el sistema se consigue cuando $\operatorname{SINR}_{m}^{D L}=\gamma_{m}, \forall m$, o de forma matricial:

$$
\mathbf{D p}=\mathbf{F p}+\mathbf{u} .
$$

Las matrices $\mathbf{D}$ y $\mathbf{F}$ de la expresión anterior, además de reorganizar sus elementos con respecto a las expresiones (6.47) y (6.49), tienen en cuenta que todos los canales de propagación del sistema son canales flat fading. 
Siguiendo la estructura de (4.23), la matriz $\mathbf{D}$ es una matriz $[M \times K M]$, que contiene los términos de potencia media de señal deseada recibidos por cada usuario. Esta matriz presenta una estructura de $K$ matrices diagonales $\mathbf{D}_{k}$ de dimensiones $[M \times M]$, cuyos elementos no nulos son:

$$
\begin{aligned}
\mathbf{D} & =\left[\mathbf{D}_{1}\left|\mathbf{D}_{2}\right| \cdots \mid \mathbf{D}_{K}\right], \\
{\left[\mathbf{D}_{k}\right]_{(m, m)} } & =E\left[\left|\mathbf{v}_{m}^{H} \mathbf{H}_{\mathrm{Rx} m m}^{\mathrm{S}}\left[\mathbf{H}_{\mathrm{UEm}} \mathbf{H}_{\mathrm{Tx} m}^{D}\right]_{(:, k)}\right|^{2}\right],
\end{aligned}
$$

donde la matriz de canales $\mathbf{H}_{\mathrm{UEm}}$ presenta la estructura (7.1) y $\mathbf{H}_{\mathrm{Tx} m}^{D}$ es a su vez una matriz diagonal a bloques de dimensiones $\left[K N_{t} \times K\right]$ :

$$
\mathbf{H}_{\mathrm{Tx} m}^{D}=\operatorname{diag}\left\{\left[\mathbf{w}_{m 1} \ldots \mathbf{w}_{m K}\right]\right\} .
$$

El beamformer en transmisión $\mathbf{w}_{m k}$ se extrae de la columna $m$-ésima de la matriz $\mathbf{H}_{\mathrm{Tx}}^{\mathrm{ZF}}$ (7.3), concretamente, se corresponde con los elementos asociados con la BS $k$, determinados por los índices $(k-1) N_{t}+1$ a $k N_{t}$.

La matriz $\mathbf{F},[M \times K M]$, contiene un valor escalado de la potencia media de IFI (Inter-finger Interference) y MAI (Multiple Access Interference). Esta matriz también presenta una estructura de $K$ matrices $\mathbf{F}_{k}$, de dimensiones $[M \times M]$, cuyos elementos cumplen:

$$
\begin{aligned}
\mathbf{F} & =\left[\mathbf{F}_{1}\left|\mathbf{F}_{2}\right| \cdots \mid \mathbf{F}_{K}\right], \\
{\left[\mathbf{F}_{k}\right]_{(m, i)} } & =\left\{\begin{array}{cc}
\gamma_{m} E\left[\left|\mathbf{v}_{m}^{H} \mathbf{H}_{\mathrm{Rx} m m}^{\mathrm{I}}\left[\mathbf{H}_{\mathrm{UEm} m} \mathbf{H}_{\mathrm{Tx} m}^{D}\right]_{(:, k)}\right|^{2}\right], & m=i, \\
\gamma_{m} E\left[\left|\mathbf{v}_{m}^{H} \mathbf{H}_{\mathrm{Rx} m i}\left[\mathbf{H}_{\mathrm{UEm} m} \mathbf{H}_{\mathrm{Tx} i}^{D}\right]_{(:, k)}\right|^{2}\right], & m \neq i .
\end{array}\right.
\end{aligned}
$$

Por último, el vector $\mathbf{u}$ incluye un valor escalado de la potencia de ruido percibida por los $M$ usuarios del sistema:

$$
\mathbf{u}=\left[\gamma_{1} \sigma_{1}^{2} \ldots \gamma_{M} \sigma_{M}^{2}\right]^{T}
$$

La expresión de actualización de las potencias, (7.5), es una expresión que se corresponde con una iteración de Jacobi (apéndice A, apartado A.2), de forma que las potencias óptimas de transmisión se obtienen iterando:

$$
\mathbf{p}\left(n_{i t}+1\right)=\mathbf{D}^{\dagger}\left(\mathbf{F} \mathbf{p}\left(n_{i t}\right)+\mathbf{u}\right)
$$

donde $n_{i t}$ es el número de la iteración correspondiente. 


\subsubsection{Solución algorítmica}

Los pasos de esta versión simplificada del algoritmo JPCOB-DL para cada iteración $n_{i t}$ serían:

1. En $n_{i t}=0$ :

1.1. Inicializar las potencias $\mathrm{DL}, p_{m k}(0)$, para todos los enlaces del sistema, $m=1, \ldots, M$, y $k=1, \ldots, K$, con valores positivos.

1.2. Obtener los beamformers en transmisión para cada usuario a partir de la expresión (7.3).

1.3. Calcular los pesos del ecualizador MRC de cada usuario del sistema, donde ahora cada correlador, $Q=K$, se sincroniza con la contribución de una de las BSs:

$$
\left[\mathbf{v}_{m}\right]_{q}=\mathbf{h}_{m k_{q}} \mathbf{w}_{m k_{q}},
$$

donde $k_{q}$ es la BS cuyo canal sintoniza el correlador $q$-ésimo.

1.4. Calcular las matrices $\mathbf{D}, \mathbf{F}$ y el vector $\mathbf{u}$ a partir de las expresiones (7.6), (7.9) y (7.11), respectivamente.

2. Esquemas de selección de enlaces activos. Se evalúa la condición de feasibility teórica sobre la matriz de ganancia DL:

$$
\rho\left(\mathbf{D}^{\dagger} \mathbf{F}\right)<1 .
$$

Si esta condición se cumple, se pasa a actualizar las potencias DL. En caso contrario, se ejecuta uno de los dos esquemas de selección de enlaces activos hasta conseguir una situación teóricamente feasible.

3. Si el problema es teóricamente feasible, el vector $\mathbf{p}$ de potencias DL se actualiza iterativamente mediante:

$$
\mathbf{p}\left(n_{i t}+1\right)=\mathbf{D}^{\dagger}\left(\mathbf{F} \mathbf{p}\left(n_{i t}\right)+\mathbf{u}\right),
$$

o, alternativamente,

$$
\mathbf{p}=\left(\mathbf{I}-\mathbf{D}^{\dagger} \mathbf{F}\right)^{-1} \mathbf{D}^{\dagger} \mathbf{u} .
$$

El esquema $\boldsymbol{D}$-based se basa en la expresión eficiente de cálculo de la pseudo-inversa de $\mathbf{D}$ propuesto en (4.40):

$$
\mathbf{D}^{\dagger}=\mathbf{D}^{T} \tilde{\mathbf{\Lambda}}^{-2},
$$


donde $\tilde{\boldsymbol{\Lambda}}$ es una matriz diagonal $[M \times M]$ que contiene los $M$ valores singulares resultantes de realizar la descomposición SVD de la matriz D. Recordando la relación (4.42):

$$
\mathrm{DD}^{T}=\tilde{\Lambda}^{2}
$$

para calcular la pseudo-inversa de $\mathbf{D}$, en primer lugar se obtiene la matriz $\tilde{\boldsymbol{\Lambda}}^{2}$ mediante la relación anterior. Puesto que $\tilde{\boldsymbol{\Lambda}}^{2}$ se trata de una matriz diagonal, la inversa $\tilde{\boldsymbol{\Lambda}}^{-2}$ se calcula invirtiendo cada uno de los elementos de la diagonal principal, y por último, se realiza el producto por $\mathbf{D}^{T}$. De esta forma, para obtener la pseudo-inversa de la matriz $\mathbf{D}$, no es necesario invertir ninguna matriz ni realizar la descomposición SVD de la matriz $\mathbf{D}$.

\subsection{Esquemas de selección de enlaces activos}

Los esquemas $\boldsymbol{D}$-based y $\boldsymbol{F}$-based, son esquemas iterativos que se proponen para mejorar la probabilidad de feasibility teórica del algoritmo JPCOB-DL, definida como la probabilidad de que el radio espectral de la matriz de ganancia DL sea inferior a la unidad (7.14).

La idea fundamental de estos esquemas es modificar el valor del radio espectral de la matriz de ganancia DL de aquellos casos teóricamente no feasible, disminuyendo gradualmente el grado de coordinación de las BSs del sistema, o lo que es lo mismo, cancelando de forma iterativa los enlaces usuario-BS que según los esquemas propuestos van a contribuir a obtener una situación teóricamente feasible.

En principio, estos esquemas no pueden eliminar usuarios del sistema, es decir, que los esquemas terminan de iterar en el momento en que sólo un enlace permanece activo para cada usuario del sistema.

\subsubsection{Esquema $F$-based}

La matriz $\mathbf{F}$ incluye un valor escalado de la potencia media de interferencia de IFI y MAI. Por tanto, el objetivo del esquema $\boldsymbol{F}$-based es cancelar aquellos enlaces usuario-BS que introducen la mayor potencia de interferencia en el sistema CM3. Como ocurría en los esquemas de selección de enlaces activos de la segunda Parte de la Tesis, cuando se dice que se cancela un enlace, lo que realmente se está haciendo es anular el beamformer en transmisión correspondiente a ese usuario y a esa BS. 
La siguiente relación entre el radio espectral y la norma infinito de una matriz (el valor máximo de la suma de las filas de una matriz), $\|\cdot\|_{\infty}$ :

$$
\rho(\mathbf{A}) \leq\|\mathbf{A}\|_{\infty}=\operatorname{máx}_{i} \sum_{j}[\mathbf{A}]_{(i, j)},
$$

parece indicar que, si se anulan los enlaces $m k$ relacionados con la fila de la matriz de ganancia DL $\left[\mathbf{D}^{\dagger} \mathbf{F}\right]$ cuyos elementos, sumados, proporcionan un valor máximo, se puede reducir también el valor del radio espectral. Observando las matrices (7.6) y (7.9), se deduce que la fila $j$-ésima de la matriz $\left[\mathbf{D}^{\dagger} \mathbf{F}\right], j=1, \ldots, K M$, está directamente relacionada con el beamformer en transmisión $\mathbf{w}_{j}$, donde $j=(k-1) M+m$, si $m$ es el usuario y $k$ es la BS.

Por tanto, para una situación teóricamente no feasible, el esquema $\boldsymbol{F}$ based realiza las siguientes operaciones:

1. Para $n_{i t}=1,2, \ldots$, hasta llegar a una situación teóricamente feasible, $\rho\left(\mathbf{D}^{\dagger} \mathbf{F}\right)<1$ :

1.1. Se calcula el vector columna $\mathbf{s}$, de dimensiones $[K M \times 1]$, donde cada elemento del vector contiene la suma de la fila correspondiente de la matriz $\left[\mathbf{D}^{\dagger} \mathbf{F}\right]$ :

$$
[\mathbf{s}]_{j}=\sum_{i=1}^{K M}\left[\mathbf{D}^{\dagger} \mathbf{F}\right]_{(j, i)} .
$$

Los elementos $[\mathbf{s}]_{j}, j=1, \ldots, K M$, se ordenan de forma descendente, de forma que el primer elemento representa el valor máximo de la suma de las filas de la matriz de ganancia DL.

1.2. Se anula el beamformer en transmisión $\mathbf{w}_{j}, j=(k-1) M+m$, relacionado con el valor máximo de la suma de las filas de $\left[\mathbf{D}^{\dagger} \mathbf{F}\right]$ (el primer elemento del vector $\mathbf{s}$ ).

1.3. Se obtiene la nueva matriz de ganancia DL, $\left[\mathbf{D}^{\dagger} \mathbf{F}\right]$, donde $\mathbf{D}^{\dagger}$ se calcula mediante la expresión eficiente (7.16) y la nueva matriz $\mathbf{F}$ se obtiene simplemente anulando la columna $j$-ésima (relacionada con el vector $\mathbf{w}_{j}$ ) de la matriz $\mathbf{F}$.

En teoría, la condición de feasibility teórica que sirve como criterio de parada del esquema $\boldsymbol{F}$-based podría sustituirse por la siguiente relación:

$$
\left\|\mathbf{D}^{\dagger} \mathbf{F}\right\|_{\infty}<1
$$


que no necesita evaluar el radio espectral de la matriz y es por tanto, computacionalmente más simple. Sin embargo, esta relación representa una condición de convergencia demasiado estricta, puesto que puede haber escenarios en los que:

$$
\rho\left(\mathbf{D}^{\dagger} \mathbf{F}\right)<1,\left\|\mathbf{D}^{\dagger} \mathbf{F}\right\|_{\infty}>1
$$

En estos casos, el criterio $\boldsymbol{F}$-based anula más enlaces que los estrictamente necesarios. Una conclusión similar se puede observar en la referencia [125].

\subsubsection{Esquema D-based}

El esquema $\boldsymbol{D}$-based afronta el problema desde un punto de vista distinto, este esquema trata de mejorar la probabilidad de feasibility teórica anulando aquellos enlaces del sistema CM3 asociados con una contribución de señal pobre.

Para determinar qué enlaces del sistema no están contribuyendo significativamente en términos de potencia de señal deseada, el esquema $\boldsymbol{D}$ based aprovecha la formulación eficiente de la pseudo-inversa de la matriz D (7.16):

$$
\mathbf{D}^{\dagger}=\left[\begin{array}{c}
\mathbf{D}_{1}^{-1} \\
\mathbf{D}_{2}^{-1} \\
\vdots \\
\mathbf{D}_{K}^{-1}
\end{array}\right]
$$

Las matrices $\mathbf{D}_{k}^{-1}$ son matrices diagonales. Además, los elementos de la diagonal de cada una de estas matrices son proporcionales a un determinado enlace $m k$ entre un usuario $m$ y una BS $k$ del sistema:

$$
\left[\mathbf{D}_{k}^{-1}\right]_{(m, m)} \propto \frac{\left(\alpha_{k} \mathbf{w}_{m k}\right)^{H}}{\sum_{k}\left\|\beta_{k} \mathbf{w}_{m k}\right\|^{2}},
$$

donde los factores $\alpha_{k}$ y $\beta_{k}$ dependen de la matriz de canal $\mathbf{H}_{\mathrm{UEm}}$ y de las matrices que caracterizan al receptor del usuario $m$ (7.6).

A partir de la expresión (7.21), el esquema $\boldsymbol{D}$-based anula aquellos enlaces que menos contribuyen en términos de potencia de señal deseada, cancelando los beamformers en transmisión relacionados con estos enlaces. 
Los pasos del esquema $\boldsymbol{D}$-based para una situación teóricamente no feasible son los siguientes:

1. In $n_{i t}=0$ : se calcula el vector columna d, de dimensiones $[K M \times 1]$, que incluye todos los elementos no nulos de la matriz $\mathbf{D}$ ordenados de forma ascendente, es decir, desde el punto de vista de potencia de señal deseada.

2. Para $n_{i t}=1,2, \ldots$, hasta llegar a una situación teóricamente feasible, $\rho\left(\mathbf{D}^{\dagger} \mathbf{F}\right)<1$ :

2.1. Se anula el beamformer en transmisión $\mathbf{w}_{j}, j=(k-1) M+m$, relacionado con el elemento $n_{i t}$ del vector $\mathbf{d}$.

2.2. Se obtiene la nueva matriz de ganancia DL, $\left[\mathbf{D}^{\dagger} \mathbf{F}\right]$, donde $\mathbf{D}^{\dagger}$ se calcula mediante la expresión eficiente (7.16) y la nueva matriz $\mathbf{F}$ se obtiene simplemente anulando la columna $j$-ésima (relacionada con el vector $\mathbf{w}_{j}$ ) de la matriz $\mathbf{F}$.

\subsubsection{Prestaciones de los esquemas $F$-based y $D$-based}

En este apartado, el objetivo es comparar, sobre una misma configuración de sistema W-CDMA CM3, las prestaciones de los esquemas $\boldsymbol{D}$ based y $\boldsymbol{F}$-based a la hora de mejorar la probabilidad de feasibility teórica del algoritmo JPCOB-DL.

La configuración del sistema W-CDMA CM3 es la mostrada por la figura 4.2: $K=3 \mathrm{BSs}$ equipadas con arrays de $N_{t}=3$ antenas transmiten coordinadamente a $M$ usuarios, que se distribuyen uniformemente sobre el área sombreada compartida por las BSs. En recepción, cada usuario dispone de un banco de $Q=3$ correladores y de un ecualizador que implementa un criterio MRC.

Teóricamente, las BSs están conectadas entre sí mediante un enlace de alta capacidad, de forma que cooperan perfectamente en datos, ya sea intercambiando las señales a transmitir a cada usuario o bien intercambiando información de los canales (CSI). Sin embargo, las BSs no cooperan en potencia, sino que cada BS está sujeta a una restricción en la potencia total que puede transmitir en DL.

En este caso, se permite coordinación total entre todas las BSs del sistema, es decir, que en teoría, cada usuario del sistema recibe su señal deseada desde las $K$ BSs. Para forzar situaciones teóricamente no feasible, se consideran $M=12$ usuarios co-canal en el sistema, de forma que $M>K N_{t}$. 


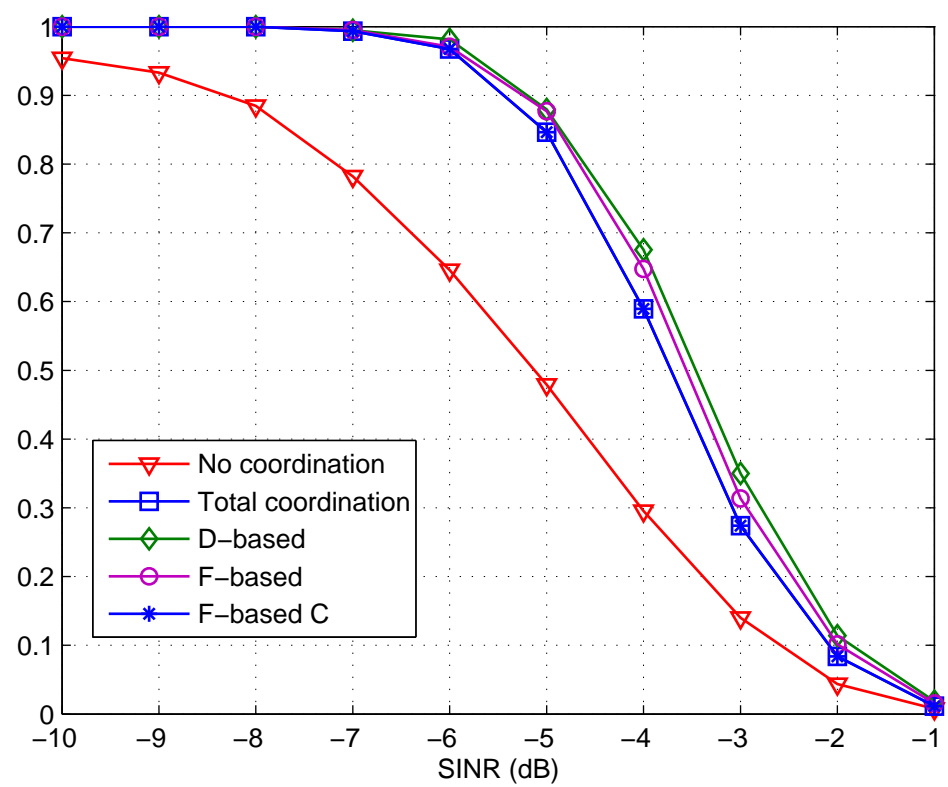

Figura 7.1: Probabilidad de feasibility práctica del algoritmo JPCOB-DL para los esquemas $\boldsymbol{D}$-based, $\boldsymbol{F}$-based y conservative $\boldsymbol{F}$-based.

En las figuras de este apartado se representa la probabilidad de feasibility práctica del algoritmo JPCOB-DL. En esta probabilidad, hay que tener en cuenta las restricciones de carácter práctico impuestas al algoritmo en cada simulación, es decir, la restricción en potencia transmitida de cada BS y la restricción en número máximo de iteraciones del control de potencia. Si una simulación se define mediante un valor de SINR umbral común a todos los usuarios y la implementación de un esquema determinado, la probabilidad de feasibility práctica se obtiene dividiendo el número de realizaciones, sobre el total, en las que el problema es teóricamente feasible, pero donde además se obtiene la asignación óptima de potencias sin superar la restricción en potencia transmitida de cada BS y el número máximo de iteraciones permitido. Los parámetros de simulación siguen siendo los del Capítulo anterior (ver tabla 6.1), evaluando 2000 realizaciones independientes para cada simulación.

La figura 7.1 presenta la probabilidad de feasibility práctica del algoritmo JPCOB-DL, para los esquemas $\boldsymbol{D}$-based, $\boldsymbol{F}$-based y conservative $\boldsymbol{F}$ based $(\boldsymbol{F}$-based $C)$ en función de distintos valores de SINR umbral, comunes 
a todos los usuarios del sistema. El esquema conservative $\boldsymbol{F}$-based utiliza la condición (7.18) como criterio de parada. La curva no coordination se corresponde con un escenario donde cada usuario recibe su señal deseada únicamente desde la BS cuya potencia de canal piloto recibida es mayor, mientras que la curva total coordination se corresponde con un escenario con coordinación total entre BSs.

La figura 7.1 muestra claramente como el hecho de permitir coordinación entre las BSs del sistema mejora las prestaciones del algoritmo JPCOB-DL, en este caso, la probabilidad de feasibility práctica. Si además de permitir coordinación entre BSs, se ejecuta uno de los dos esquemas propuestos en este Capítulo, el esquema $\boldsymbol{D}$-based o el esquema $\boldsymbol{F}$-based, esta probabilidad de feasibility práctica todavía puede mejorarse ligeramente. Sin embargo, la condición de parada del esquema conservative $\boldsymbol{F}$ based impide que este esquema pueda mejorar la probabilidad de feasibility práctica del algoritmo con respecto a la curva de coordinación total.

Aunque la figura 7.1 muestra resultados similares para los esquemas $\boldsymbol{D}$-based y $\boldsymbol{F}$-based, hay que tener en cuenta que su comportamiento es totalmente opuesto. Por una parte, el algoritmo $\boldsymbol{F}$-based anula aquellos enlaces que introducen una mayor potencia de interferencia en el sistema, introduciendo una perturbación significativa en las matrices $\mathbf{D}$ y $\mathbf{F}$ entre una iteración y la siguiente. Este proceso afecta a la continuidad del valor del radio espectral de la matriz de ganancia DL que obtiene esta solución entre una iteración y la siguiente. Por el contrario, el esquema $\boldsymbol{D}$-based cancela aquellos enlaces que menos contribuyen en el sistema en términos de potencia de señal deseada, introduciendo una pequeña perturbación en las matrices $\mathbf{D}$ y $\mathbf{F}$ entre iteraciones, asegurando por tanto la continuidad del valor del radio espectral de la matriz de ganancia DL.

La figura 7.2 ilustra, para una realización concreta del canal, la evolución del radio espectral de la matriz de ganancia DL de cada uno de los esquemas propuestos, en función del número de la iteración en la que se encuentre cada uno. Hay que destacar que la velocidad de convergencia del esquema $\boldsymbol{F}$-based es mayor que la de los esquemas $\boldsymbol{D}$-based y $\boldsymbol{F}$-based $C$. Sin embargo, el valor del radio espectral de la matriz de ganancia DL obtenido por el esquema $\boldsymbol{D}$-based es monótonamente decreciente con el número de la iteración, lo que en cierta manera asegura la estabilidad del esquema. Por último, el esquema $\boldsymbol{F}$-based $C$ continúa iterando, aunque el valor del radio espectral de la matriz de ganancia DL ya es menor que la unidad, hasta cumplir el criterio de parada (7.18). 


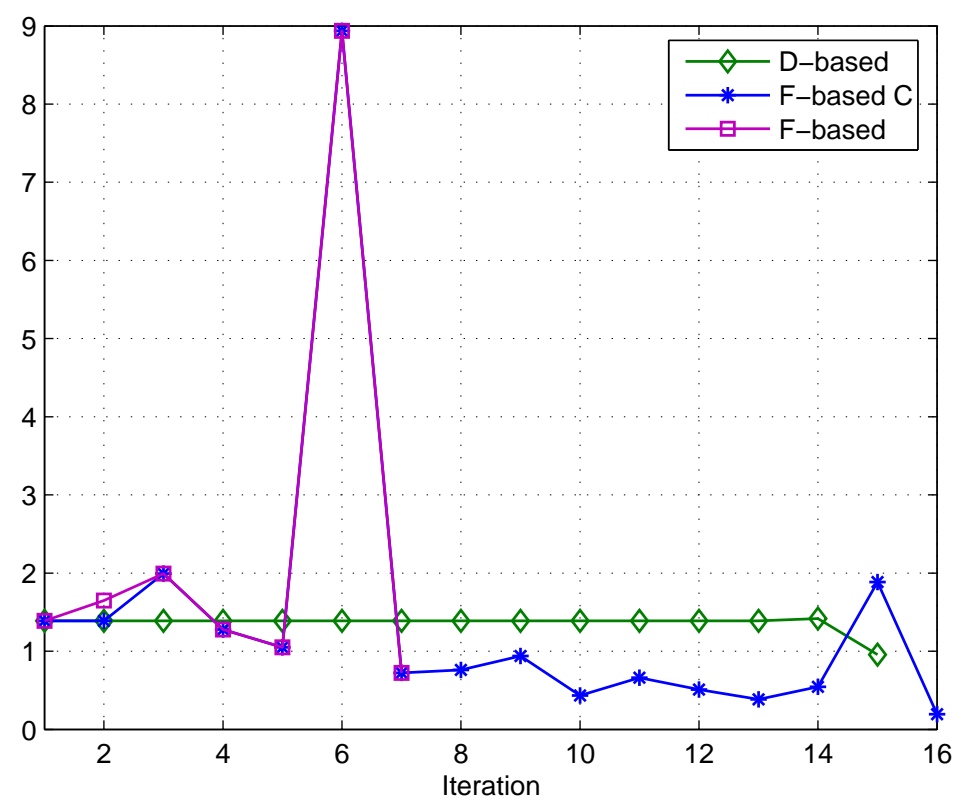

Figura 7.2: Evolución del radio espectral de la matriz de ganancia DL para los esquemas $\boldsymbol{D}$-based, $\boldsymbol{F}$-based y conservative $\boldsymbol{F}$-based.

En los esquemas propuestos, se exige que al menos uno de los enlaces de cada usuario permanezca activo, de forma que no se pueda eliminar usuarios activos del sistema. En las simulaciones, se observa que la tendencia natural de los esquemas $\boldsymbol{D}$-based y $\boldsymbol{F}$-based es a eliminar completamente los enlaces de un determinado usuario. Además, existen situaciones en las que tras ejecutar cualquiera de los dos esquemas, todos los usuarios del sistema permanecen con un único enlace activo y aún así, $\rho\left(\mathbf{D}^{\dagger} \mathbf{F}\right) \geq 1$. En estos casos, la única opción que permite obtener una situación teóricamente feasible es permitir a los esquemas $\boldsymbol{D}$-based y $\boldsymbol{F}$-based que continúen iterando, sabiendo que esto supone eliminar algún usuario del sistema.

La figura 7.3 muestra la probabilidad de feasibility práctica del algoritmo JPCOB-DL cuando a los esquemas $\boldsymbol{D}$-based y $\boldsymbol{F}$-based se les permite eliminar hasta dos usuarios del sistema (en este caso, $M$ sigue siendo mayor que $K N_{t}$ ). Como se observa en la figura, el hecho de eliminar únicamente un usuario del sistema, permite incrementar significativamente (hasta un $30 \%$ ) la probabilidad de feasibility práctica del algoritmo. Es en esta gráfica donde se detecta la utilidad de los esquemas $\boldsymbol{D}$-based y $\boldsymbol{F}$-based desde el 


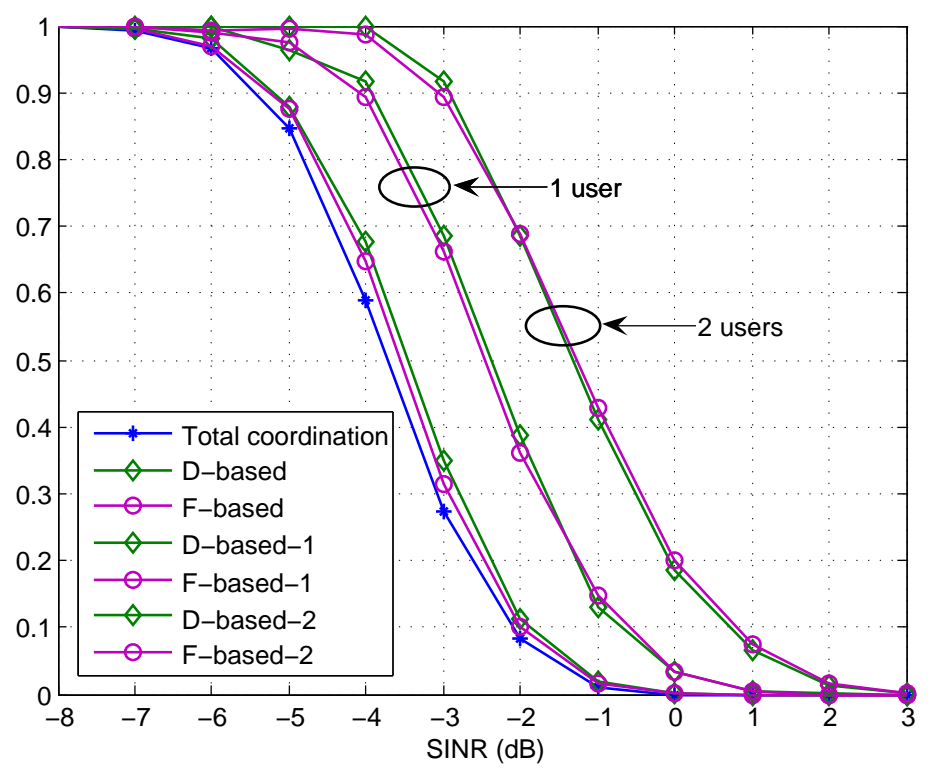

Figura 7.3: Probabilidad de feasibility práctica del algoritmo JPCOB-DL para los esquemas $\boldsymbol{D}$-based y $\boldsymbol{F}$-based. Posibilidad de eliminar hasta dos usuarios del sistema.

punto de vista de control de admisión o scheduling. Además, comparando estos esquemas con las propuestas de por ejemplo, [127], se observa que estos esquemas constituyen criterios computacionalmente sencillos para eliminar usuarios de un sistema CM3. 



\section{Capítulo 8}

\section{Conclusiones y líneas futuras}

\subsection{Conclusiones}

La Tesis se centra en el análisis de un sistema W-CDMA MIMO multiusuario multi-celda coordinado como posible modelo de sistema para la arquitectura Multinodo B de un sistema UMTS. Puesto que UMTS es un sistema limitado por interferencias, a lo largo de la Tesis se estudian soluciones algorítmicas JPCOB o Joint Power Control and Optimal Beamforming que resuelven el problema de minimización de potencia en el enlace downlink de una configuración de sistema W-CDMA MIMO multi-usuario multi-celda coordinado.

Las conclusiones se presentan agrupadas en tres bloques, que se corresponden con las tres partes principales en las que se estructura la Tesis.

\section{Primera Parte de la Tesis}

En la primera Parte de la Tesis se revisa la evolución de los sistemas MIMO, empezando con la configuración básica punto a punto y terminando con la configuración avanzada MIMO multi-usuario multi-celda. Para cada configuración MIMO, se presentan tanto los resultados proporcionados por la teoría de la información como las técnicas y algoritmos propuestos para ambos enlaces del sistema y desde el punto de vista de los problemas clásicos de optimización. En general, las técnicas en transmisión y los algoritmos que resuelven el problema de minimización de potencia se presentan con mayor detalle. 
Si se observa la evolución de las técnicas y algoritmos propuestos para cada configuración MIMO, se aprecia que en cada etapa, las técnicas y algoritmos asociados a una configuración concreta empiezan siendo una extensión de las soluciones propuestas para la configuración MIMO anterior, pero que se van perfeccionando progresivamente al incorporar las características propias de cada configuración. Esta metodología de trabajo permite obtener en un primer momento los límites superiores e inferiores de las prestaciones de cada configuración MIMO.

Con respecto al estado del arte de los sistemas MIMO multi-usuario multi-celda coordinados, hay que destacar que se trata de una configuración MIMO relativamente reciente y que ni siquiera existe un nombre común para designarla. Actualmente, las principales líneas de investigación se centran en obtener los primeros límites de la región de capacidad de estos sistemas, así como en proponer técnicas y algoritmos que aprovechen las características propias de esta configuración.

Si nos centramos en la parte de técnicas y algoritmos propuestos para esta configuración, se aprecia que la mayoría de las referencias simplifican el modelo de sistema, por ejemplo, ignorando la naturaleza asíncrona de las señales que llegan a cada usuario del sistema, o suponiendo que las estaciones base pueden cooperar en potencia, es decir, que la restricción en potencia se aplica a la potencia total transmitida en el sistema. Además, parece ser que la única referencia que plantea el problema de minimización de potencia sobre un sistema MIMO multi-usuario multi-celda coordinado es [68]. Sin embargo, esta referencia se centra en el problema de asignar usuarios a estaciones base, tras simplificar el modelo de sistema a un sistema sin coordinación.

Con respecto a esta primera Parte, se puede concluir que el trabajo presentado en esta Tesis cubre una necesidad que existía en el estudio de los sistemas MIMO multi-usuario multi-celda coordinados y aporta además, una nueva perspectiva al estado del arte de estos sistemas.

\section{Segunda Parte de la Tesis}

La segunda Parte de la Tesis plantea el problema de minimización de potencia en el enlace downlink de un sistema W-CDMA MIMO multi-usuario multi-celda coordinado, donde cada estación base está sujeta a una restricción en la potencia total transmitida. En primer lugar, se revisan las tres referencias clásicas que plantean algoritmos JPCOB para resolver el problema de minimización de potencia $[33,37,38]$. Entre estas referencias, que 
representan en realidad las tres formas básicas de abordar este problema, se elige el algoritmo propuesto por Rashid-Farrokhi et al. en [33] como punto de partida para proponer un primer algoritmo JPCOB para un sistema MIMO multi-usuario multi-celda coordinado.

El siguiente paso es obtener un modelo analítico del sistema que permita plantear el problema de minimización de potencia y derivar posteriormente una solución algorítmica JPCOB. En este primer modelo de sistema, se presenta una visión parcial del funcionamiento del sistema MIMO multiusuario multi-celda coordinado, puesto que se modela con detalle el proceso que tiene lugar en transmisión, pero se ignora el proceso que tiene lugar en el receptor del usuario [82-86]. En cierto modo, se está siguiendo la filosofía de los trabajos presentes en la literatura de sistemas MIMO multi-usuario multi-celda coordinados, donde se ignora la naturaleza asíncrona de las señales en recepción y se supone que el receptor del usuario puede separar perfectamente las señales procedentes de las distintas estaciones base, tanto la parte de señal deseada como la parte interferente.

El algoritmo JPCOB-VUL o Virtual uplink-based JPCOB [82-86], se obtiene modificando el algoritmo de referencia propuesto por Rashid-Farrokhi et al. en [33]. El algoritmo JPCOB-VUL es un algoritmo iterativo que utiliza la dualidad entre el problema en el enlace downlink y un equivalente uplink virtual para simplificar el diseño de los beamformers en transmisión. Este algoritmo mantiene un diseño local de los beamformers, es decir, obtiene los beamformers en transmisión para cada usuario por separado en cada estación base del sistema, mientras que implementa un mecanismo de control de potencia centralizado. Sin embargo, la estructura de las matrices que intervienen en el mecanismo de control de potencia permite obtener una versión computacionalmente eficiente del algoritmo JPCOB-VUL [84], disminuyendo de esta forma la complejidad asociada a realizar un control de potencia centralizado.

La simulación del algoritmo JPCOB-VUL sobre un sistema W-CDMA MIMO multi-usuario multi-celda coordinado conlleva el estudio de ciertos aspectos prácticos asociados a este algoritmo. En este punto, se proponen dos esquemas de selección de enlaces activos que determinan qué enlaces del sistema se consideran activos en base a un determinado parámetro [83,84]. Es importante destacar que el objetivo de estos esquemas no es determinar de forma óptima qué enlaces deben permanecer activos para minimizar la potencia total transmitida en el sistema, sino que la selección de enlaces activos se realiza conforme al valor de parámetros relacionados con 
el sistema. Estos esquemas, además, se revelan como una forma eficiente de evaluar las prestaciones del algoritmo JPCOB-VUL frente a distintos grados de coordinación entre las estaciones base del sistema.

En las simulaciones presentadas en el Capítulo 4, se analizan aspectos básicos del algoritmo JPCOB-VUL y del sistema MIMO multi-usuario multi-celda coordinado, como por ejemplo, la influencia en los resultados del número de estaciones base y de antenas por estación base disponibles en el sistema, o del grado de coordinación entre estaciones base. Estas simulaciones tratan de forzar situaciones donde la potencia de interferencia presente en el sistema sea muy elevada, para obtener de alguna manera el límite de las prestaciones del algoritmo JPCOB-VUL.

En grandes líneas, y teniendo en cuenta aspectos básicos de configuración del sistema, se puede concluir que las configuraciones de sistema con tres estaciones base mejoran los resultados de las configuraciones de sistema con dos estaciones base cuando el número total de antenas en el sistema es similar para las dos configuraciones, debido a la mayor diversidad espacial que proporciona la configuración con tres estaciones base [83]. Además, las simulaciones demuestran que cuando el algoritmo JPCOB-VUL se ejecuta sobre un sistema MIMO multi-usuario multi-celda coordinado, y se comparan sus prestaciones con las del algoritmo de referencia de [33], implementado sobre un sistema totalmente equivalente que ubica un mismo número de antenas en el centro de la zona bajo estudio y que no permite coordinación, la configuración de sistema MIMO multi-usuario multi-celda coordinado mejora los resultados en términos de probabilidad de fallo del algoritmo $[85,86]$.

Con respecto a la potencia total transmitida en el sistema, los resultados de las simulaciones permiten afirmar que la potencia total transmitida en el sistema no aumenta significativamente cuando se permite coordinación entre estaciones base. De hecho, comparando la potencia total transmitida por el sistema MIMO multi-usuario multi-celda coordinado con la potencia total transmitida por el sistema central equivalente, se aprecia que la potencia total transmitida en el sistema es ligeramente mayor en el caso del sistema MIMO multi-usuario multi-celda coordinado, pero hay que tener en cuenta que esta configuración es menos interferente con respecto al resto del sistema que la configuración central, puesto que se trata de una potencia de interferencia radiada hacia el interior de la zona bajo estudio $[85,86]$.

Si se compara el algoritmo JPCOB-VUL sobre un sistema MIMO multiusuario multi-celda coordinado con otros algoritmos JPCOB que no per- 
miten coordinación, se aprecia que el algoritmo JPCOB-VUL presenta un comportamiento intermedio, por ejemplo, en términos de capacidad, pero se observa que es un algoritmo más rápido en cuanto a número medio de iteraciones necesario para converger. Este análisis, junto con el análisis del coste computacional asociado a este algoritmo, permite concluir que el coste de implementar este algoritmo que permite coordinación entre estaciones base no conlleva necesariamente un aumento significativo de la complejidad con respecto a otras soluciones no coordinadas [84]. Por otro lado, el estudio comparativo del número medio de usuarios co-canal admitidos por el algoritmo JPCOB-VUL y por un algoritmo que no permite coordinación pero que realiza una asignación óptima de usuarios a estaciones base [82], confirma el hecho de que los esquemas de selección de enlaces activos propuestos en esta Parte de la Tesis no realizan una selección óptima desde el punto de vista del criterio de minimización de potencia.

En el Capítulo 5, se presenta el estudio de dos aspectos prácticos relacionados con el algoritmo JPCOB-VUL. A la hora de realizar las simulaciones del sistema W-CDMA MIMO multi-usuario multi-celda coordinado, se constató la necesidad de establecer un modelo que representara de forma adecuada las potencias de interferencia que intervenían en este tipo de sistemas. Por este motivo, en este Capítulo se lleva a cabo un modelado exhaustivo de las potencias de interferencia presentes en el sistema, y se plantea de nuevo el problema de minimización de potencia [97-99]. Sin embargo, también se actúa sobre los códigos de canalización asignados a cada usuario del sistema con el objetivo de reducir aún más la potencia de interferencia que perciben los usuarios, aumentando con ello la capacidad del sistema, medida en este caso en términos de probabilidad de admisión de usuarios en el sistema.

En este Capítulo, se considera un escenario donde los canales del sistema pueden ser flat o selective fading, y se proponen diversos esquemas de asignación de códigos de canalización [97-99]. Entre los esquemas propuestos, interesa destacar el esquema MP o Minimum Partial, que establece un orden de asignación de códigos de canalización que trata de minimizar la interferencia introducida por un nuevo código con respecto a los códigos que ya están activos en el sistema. Estos esquemas de asignación de códigos dependen de las características asociadas a los canales del sistema, de forma que obtienen un orden de asignación fijo mientras no varíe el power delay profile de algún canal. Con respecto a la influencia del grado de coordinación de las estaciones base del sistema en los resultados, resulta 
sorprendente comprobar que en términos de probabilidad de admisión de usuarios, un grado de coordinación relativamente pequeño permite mejorar significativamente las prestaciones del sistema. Este resultado es muy importante a la hora de considerar una posible implementación real del sistema MIMO multi-usuario multi-celda coordinado, puesto que cuanto menor sea el grado de coordinación entre las estaciones base del sistema, menor será la cantidad de información que se necesita disponer de forma centralizada.

El último aspecto práctico analizado con respecto al algoritmo JPCOBVUL se relaciona con el hecho de que el mecanismo de control de potencia requiere una implementación centralizada, lo que implica que las estaciones base del sistema deben de disponer de información de canal perfecta de sus canales locales y que además, necesitan intercambiar información a través del enlace de alta capacidad sin que esta información experimente ningún tipo de degradación. En este último análisis, se supone que las estaciones base van a poder estimar de forma perfecta los canales locales, pero que sin embargo, va a existir algún tipo de restricción en la cantidad de información que pueden intercambiar entre ellas [100]. Esta situación se modela mediante una forma de conocimiento parcial del canal denominada hybrid channel knowledge, donde las estaciones base disponen de información de los canales locales, pero donde sólo disponen de información estadística del resto de canales del sistema.

En este escenario, se propone una versión robusta del algoritmo JPCOBVUL [100], modificando el mecanismo de control de potencia para incorporar cierta robustez frente a errores en la estima de la matriz de correlación de canal. El concepto de hybrid channel knowledge se incorpora a las simulaciones asignando un error distinto a las matrices de correlación de canal entre cada usuario y todas las estaciones base del sistema.

Desde el punto de vista de gestión de recursos del sistema, la configuración de sistema MIMO multi-usuario multi-celda coordinado constituye una opción a tener en cuenta. Por una parte, el hecho de que todas las estaciones base del sistema transmitan coordinadamente a cada usuario permite evitar los procesos de handover entre celdas. Además, cada usuario consume un único código de canalización en todas las estaciones base. Sin embargo, la principal ventaja de esta configuración de sistema es la capacidad de reconfiguración que presenta, puesto que se puede variar el grado de coordinación entre las estaciones base del sistema en función de la carga del sistema o del requerimiento de calidad de servicio en los usuarios. 
La conclusión general de esta segunda Parte de la Tesis es que la estructura semi-distribuida del algoritmo JPCOB-VUL posibilita que este algoritmo se presente como una opción muy interesante dentro de la tendencia actual de proponer soluciones distribuidas para los sistemas MIMO multi-usuario multi-celda coordinados, debida a la dificultad de disponer de información de canal perfecta de todos los canales del sistema en un entorno real. Por otro lado, el mecanismo de control de potencia, además de permitir una formulación computacionalmente eficiente del algoritmo, presenta una estructura fácilmente adaptable a soluciones robustas frente a modelos de conocimiento parcial del canal.

\section{Tercera Parte de la Tesis}

En la tercera Parte de la Tesis, se avanza en el modelo analítico del sistema y se propone una formulación matricial compacta que caracteriza completamente el funcionamiento de un sistema W-CDMA MIMO multiusuario multi-celda coordinado. En este modelo de sistema ya se considera el proceso completo que tiene lugar en el receptor de cada usuario, teniendo en cuenta la naturaleza asíncrona de las señales que llegan al usuario [100, 121]. Además, este modelo de sistema constituye un modelo compacto y general que facilita cualquier modificación para contemplar casos concretos del sistema, como por ejemplo, el caso de usuarios co-canal o de un sistema MIMO multi-usuario multi-celda sin coordinación [100].

De nuevo, se plantea el problema de minimización de potencia en el enlace downlink de un sistema W-CDMA MIMO multi-usuario multi-celda coordinado. En este punto de la Tesis, se propone un algoritmo JPCOB que no recurre a la dualidad entre el problema downlink y un equivalente uplink virtual, por lo que se denomina algoritmo JPCOB-DL o Downlink-based JPCOB [121]. Este algoritmo comparte la estructura y el mecanismo de control de potencia del algoritmo JPCOB-VUL, pero diseña los beamformers en transmisión para cada usuario de forma multi-base, es decir, teniendo en cuenta todas las antenas disponibles en el sistema. Este diseño multi-base se basa en una extensión del beamformer MMSE propuesto en [122] para sistemas MIMO multi-usuario convencionales.

De esta forma, la propuesta alternativa al algoritmo JPCOB-VUL, el algoritmo JPCOB-DL, requiere una implementación centralizada tanto del paso de diseño de los beamformers en transmisión, como del mecanismo de control de potencia. En principio, esta solución, aunque óptima desde el punto de vista de sistemas coordinados, presentaría mayores dificultades 
a la hora de implementarse en un sistema W-CDMA MIMO multi-usuario multi-celda coordinado real. Por este motivo, se presenta una versión simplificada de este algoritmo, el algoritmo JPCOB-SDL o Simplified Downlinkbased JPCOB, para aquellos casos en los que existe algún tipo de restricción en la cantidad de información que un usuario puede realimentar a las estaciones base. En la Tesis, esta situación se traduce en que las estaciones base del sistema no disponen de ningún tipo de información del proceso que lleva a cabo el ecualizador del receptor de cada usuario, y por tanto, se plantea un diseño robusto del beamformer en transmisión. Esta solución JPCOBSDL también podría integrarse en la categoría de las llamadas técnicas de limited feedback.

En las simulaciones que se incluyen en Capítulo 6, el objetivo es comparar las prestaciones de los tres algoritmos JPCOB propuestos en la Tesis. A la hora de ajustar los parámetros de simulación, hay que tener en cuenta que el algoritmo JPCOB-VUL realiza un diseño local de los beamformers en transmisión de cada usuario, mientras que las características del beamformer diseñado por el algoritmo JPCOB-SDL requieren que se cumpla una determinada relación entre los parámetros de diseño del sistema. Teniendo en cuenta estos dos factores, para que el algoritmo JPCOB-VUL y el JPCOB-SDL encuentren un sistema bien condicionado, es necesario que el número de usuarios en el sistema sea menor que el número de antenas disponibles en cada estación base. Por tanto, la primera consecuencia de permitir un diseño robusto del beamformer, como hace el algoritmo JPCOB-SDL, es que la principal ventaja asociada a diseñar un beamformer multi-base desaparece, es decir, no se puede admitir a un mayor número de usuarios que en los diseños distribuidos.

En las simulaciones, se vuelve a considerar uno de los esquemas de selección de enlaces activos propuestos en la segunda Parte de la Tesis, con el fin de evaluar las prestaciones de los algoritmos frente a distintos grados de coordinación entre las estaciones base del sistema. En principio, el número medio de enlaces activos en el sistema no debería variar para los tres algoritmos, puesto que el esquema de selección de enlaces activos es independiente de la ejecución de cada algoritmo. Sin embargo, el análisis de este parámetro permite concluir que el diseño de los beamformers incluido en el algoritmo JPCOB-DL se encarga de anular, para grados significativos de coordinación entre estaciones base, determinados enlaces que el esquema de selección de enlaces activos determinó como activos con anterioridad [121]. 
Por otro lado, las propiedades de convergencia de los tres algoritmos dependen de cómo de estricto sea el requerimiento de calidad de servicio. De hecho, el comportamiento de los distintos algoritmos varía según se exija un requerimiento estricto de calidad de servicio o si se permite una cierta tolerancia sobre esta condición. Por este motivo, en las simulaciones del Capítulo 6 se distingue entre un requerimiento estricto y un requerimiento con un cierto margen de tolerancia en la condición de calidad de servicio. En estas simulaciones, se estudia la potencia media total transmitida en el sistema y la probabilidad de feasibility práctica de los tres algoritmos presentados en la Tesis. Esta probabilidad representa en cierto modo la probabilidad de convergencia de cada algoritmo, teniendo en cuenta las restricciones de carácter práctico que se incluyen en las simulaciones.

En general, y para ambos casos de requerimiento de calidad de servicio, el algoritmo JPCOB-DL es el que mejor probabilidad de feasibility práctica presenta, mientras que el algoritmo JPCOB-VUL disminuye progresivamente esta probabilidad hasta un valor no feasible, a medida que aumenta el grado de coordinación entre estaciones base o el valor del requerimiento de calidad de servicio [121]. El algoritmo JPCOB-SDL presenta un comportamiento intermedio, pero su convergencia se ve muy afectada en el caso de coordinación total entre estaciones base y un requerimiento estricto de calidad de servicio. Con respecto a la potencia media total transmitida, las simulaciones muestran un comportamiento sorprendente del algoritmo JPCOB-DL, puesto que este algoritmo, contrariamente a lo que ocurre en el caso de los algoritmos JPCOB-VUL y JPCOB-SDL, disminuye la potencia media total transmitida a medida que aumenta el grado de coordinación entre estaciones base [121]. Estas simulaciones también demuestran que el hecho de incluir un diseño robusto en el algoritmo JPCOB-SDL, provoca que este algoritmo no sea eficiente en cuanto a la potencia total transmitida en el sistema. Sin embargo, el análisis del número medio de iteraciones que necesitan los tres algoritmos para converger establece que el algoritmo JPCOB-SDL es el algoritmo que más rápidamente converge.

En la práctica, las propiedades de convergencia de los tres algoritmos propuestos en la Tesis se ven muy influenciadas por las restricciones de carácter práctico que se consideran en las simulaciones de cada algoritmo. De hecho, se puede concluir que el algoritmo JPCOB-VUL es un algoritmo fundamentalmente limitado por la restricción en la potencia total transmitida que se impone a cada estación base del sistema, puesto que el mecanismo de control de potencia es el que intenta compensar el hecho de 
que los beamformers en transmisión, debido a su diseño local, empiecen a degradarse en cuanto el número de usuarios en el sistema supera el número de antenas disponibles en cada estación base. Por el contrario, el algoritmo JPCOB-DL es un algoritmo fundamentalmente limitado por la restricción en el número máximo de iteraciones que se permite iterar a cada algoritmo.

En el Capítulo 7, se proponen dos esquemas para mejorar las propiedades de convergencia del algoritmo JPCOB-DL en el caso de sistemas MIMO multi-usuario multi-celda coordinados mal condicionados, donde, por ejemplo, el número de usuarios activos supera el número total de antenas disponibles en el sistema. Cada uno de estos esquemas aprovecha la estructura de una de las matrices que intervienen en el mecanismo de control de potencia, aunque comparten la idea de mejorar la probabilidad de feasibility teórica de este algoritmo reduciendo el grado de coordinación de las estaciones base del sistema. Aunque en principio estos esquemas se proponen y se simulan evitando eliminar usuarios activos, las simulaciones demuestran que ambos esquemas pueden utilizarse como criterios eficientes y computacionalmente sencillos para eliminar usuarios del sistema en los casos en los que el algoritmo JPCOB-DL no alcanza la convergencia.

Como conclusión de esta tercera Parte de la Tesis, hay que remarcar que el modelo de sistema presentado en el Capítulo 6 caracteriza completamente los procesos que tienen lugar tanto en transmisión como en recepción en un sistema W-CDMA MIMO multi-usuario multi-celda coordinado, y que este modelo de sistema no estaba disponible previamente en la literatura. Por otro lado, el algoritmo JPCOB-DL mejora significativamente las prestaciones del algoritmo JPCOB-VUL, además de presentar propiedades tan interesantes como una técnica inherente de selección de enlaces activos y de disminuir la potencia total transmitida en el sistema a medida que aumenta la coordinación entre las estaciones base del sistema. Finalmente, el algoritmo JPCOB-SDL es un algoritmo más sencillo de implementar en un sistema real, pero esta sencillez empeora sus prestaciones desde el punto de vista de potencia total transmitida y probabilidad de feasibility práctica. Aún así, sirve como punto de referencia del comportamiento que podría presentar el algoritmo JPCOB-DL bajo determinadas condiciones de conocimiento parcial de canal. 


\subsection{Líneas futuras}

La configuración de sistema MIMO multi-usuario multi-celda coordinado es una configuración avanzada y cooperativa de sistema MIMO, donde los transmisores están conectados entre sí mediante un enlace de alta capacidad que les permite transmitir de forma coordinada a todos los usuarios del sistema. Esta configuración MIMO es relativamente reciente, y de hecho, todavía no existe un nombre común para referirse a ella. En este sentido, todavía queda mucho trabajo pendiente, aunque probablemente lo más importante e inmediato sea proponer soluciones que tengan en cuenta las características propias de esta configuración de sistema y que no sean una mera extensión de las soluciones propuestas para los sistemas MIMO multi-usuario.

Por ejemplo, las restricciones en potencia total transmitida impuestas a cada estación base del sistema, dificultan la extensión de técnicas como el algoritmo de waterfilling a los sistemas MIMO multi-usuario multi-celda coordinados. De hecho, y aunque parece un problema trivial, no se dispone todavía de una expresión cerrada que permita implementar técnicas en transmisión multi-base y lineales, como un beamformer zero forcing, donde las estaciones base transmitan a potencia máxima. Como se ha visto en el Capítulo 2, por el momento sólo existe la solución subóptima planteada por Zhang et al. en [50,65], que obliga a alguna estación base a transmitir por debajo de su capacidad, y la solución presentada por Boccardi en [36] para sistemas MIMO multi-usuario convencionales donde la restricción en potencia se impone sobre cada antena del array de la estación base.

En la Tesis, se presentan posibles soluciones para modelar ciertas características propias de este tipo de sistemas MIMO, que hasta el momento no se habían considerado en la literatura. Por ejemplo, en la tercera Parte de la Tesis se propone un modelo de sistema donde se caracteriza el proceso que tiene lugar en el receptor de cada usuario para afrontar el asincronismo de las señales que llegan al usuario desde las distintas estaciones base. En cierta manera, es posible que el modelo aquí propuesto siga siendo demasiado ideal o dicho de otra forma, constituya el caso mejor que puede darse en recepción, puesto que este modelo supone que el receptor del usuario puede distinguir de alguna forma las señales que transmite cada estación base.

Del mismo modo, en la segunda Parte de la Tesis se propone un modelo exhaustivo de las interferencias presentes en el sistema, a partir de los términos de correlación entre los códigos de canalización de los usuarios. 
Algunos autores, establecen que este tipo de caracterización no es necesaria y que basta con considerar el modelo tradicional de correlación entre códigos. Sin embargo, los resultados de las simulaciones del Capítulo 5 indican que es posible esperar ciertos beneficios en la capacidad del sistema si se tienen en cuenta estas interferencias.

Por otro lado, a lo largo de la Tesis se asume que las estaciones base disponen de información de canal perfecta de todos los canales del sistema, aunque se presentan soluciones para dos casos concretos en los que esta suposición no se cumple, un primer caso en el que existe algún tipo de restricción en la cantidad de información que pueden intercambiar las estaciones base a través del enlace de alta capacidad, para el que se propone una versión robusta del algoritmo JPCOB-VUL, y un segundo caso en el que la restricción se aplica a la información que realimenta cada usuario a las estaciones base, donde se propone una version simplificada del algoritmo JPCOB-DL, el JPCOB-SDL.

A partir de la experiencia de los sistemas MIMO multi-usuario, parece claro que el principal inconveniente de un sistema MIMO multi-usuario multi-celda coordinado va a ser cómo disponer de la información de todos los canales de propagación del sistema. Las simulaciones de esta Tesis y el trabajo paralelo de otros grupos de investigación demuestran que la fuerza de esta configuración reside en la posibilidad de aprovechar la diversidad espacial que proporciona la transmisión coordinada utilizando todas las antenas disponibles en el sistema. Sin embargo, esto requiere una implementación centralizada, que en la práctica puede ser muy difícil de conseguir excepto para sistemas formados por un número no muy elevado de estaciones base y usuarios.

Por este motivo, la investigación sobre este tipo de configuraciones está derivando hacia la búsqueda de soluciones distribuidas, claramente subóptimas, pero más sencillas de implementar. Al mismo tiempo, otra tendencia reciente es coordinar únicamente un subgrupo de estaciones base dentro del sistema, para disminuir la carga asociada a esta configuración de sistema MIMO.

El objetivo de esta Tesis es, en grandes líneas, proponer soluciones algorítmicas que resuelvan el problema de minimización de potencia en el enlace downlink de un sistema W-CDMA MIMO multi-usuario multi-celda coordinado. El problema de minimización de potencia, junto con el de maximización del sum-rate, es un problema clásico de optimización para cualquier sistema de comunicación y como tal, se trata de un problema 
para el que la comunidad investigadora propone nuevas técnicas de forma continua.

Desde este punto de vista, sería posible conseguir una solución algorítmica que mejorara de alguna forma las prestaciones de los algoritmos propuestos en esta Tesis. Una opción muy interesante, sería desarrollar una extensión para sistemas MIMO multi-usuario multi-celda coordinados del trabajo presentado por Wiesel et al. en [30]. En este trabajo, estos autores proponen un algoritmo que no recurre a la dualidad entre el enlace uplink y el downlink, pero que aprovecha distintas técnicas de optimización convexa para presentar una solución que mejora la eficiencia del algoritmo iterativo de Rashid-Farrokhi et al., y por tanto la del algoritmo JPCOBVUL, puesto que optimiza los beamformers y la asignación de potencia en único paso, mientras que en la aproximación de Rashid-Farrokhi et al., estos parámetros se optimizan de forma independiente en cada iteración.

Por último, y pensando en un posible desarrollo comercial de este tipo de sistemas, habrá que esperar para saber qué pasa con las técnicas desarrolladas para los sistemas MIMO multi-usuario. Si estas técnicas se incorporan finalmente a los diversos estándares, los sistemas MIMO multiusuario multi-celda coordinados tendrán alguna oportunidad fuera del ámbito científico. 



\section{Apéndice A}

\section{Teorema de}

\section{Perron-Frobenius e}

Iteración de Jacobi

\section{A.1. Teorema de Perron-Frobenius}

El Teorema de Perron-Frobenius permite caracterizar de forma sencilla los valores y vectores propios de un determinado tipo de matrices con entradas no negativas. La importancia de este Teorema se debe a que los valores propios de este tipo de matrices aparecen en muchos problemas de ingeniería, como por ejemplo en las cadenas de Markov, en el problema de control de potencia en sistemas de comunicaciones inalámbricas, en modelos de crecimiento de la población o para explicar el funcionamiento del motor de búsqueda de los buscadores Web [129].

Oscar Perron formuló por primera vez el Teorema en 1907, y Frobenius lo generalizó en 1912. El Teorema de Perron se centra en matrices positivas, que son las matrices cuyos elementos son estrictamente positivos. Más generalmente, el Teorema de Perron se aplica a matrices primitivas: si $\mathbf{A}$ es una matriz no negativa cuyas entradas $[\mathbf{A}]_{(i, j)}$ son números no negativos, $\mathbf{A}$ es primitiva si para algún entero $m_{0}, \mathbf{A}^{m_{0}}$ es una matriz positiva, es decir $\left[\mathbf{A}^{m_{0}}\right]_{(i, j)}>0, \forall i, j$.

Para formular el Teorema de Perron es necesario definir el concepto de radio espectral de una matriz. El radio espectral de una matriz $\mathbf{A}$ representa 
el máximo de los valores absolutos de los valores propios de $\mathbf{A}$ :

$$
\rho(\mathbf{A})=\operatorname{máx}_{i}\left|\lambda_{i}(\mathbf{A})\right|,
$$

donde $\lambda_{i}(\mathbf{A})$ representa al $i$-ésimo valor propio de $\mathbf{A}$. Si $r_{i}$ y $c_{j}$ representan la suma de la fila $i$-ésima y de la columna $j$-ésima de $\mathbf{A}$ :

$$
r_{i}=\sum_{j}[\mathbf{A}]_{(i, j)} \quad c_{j}=\sum_{i}[\mathbf{A}]_{(i, j)}
$$

el radio espectral cumple las siguientes inecuaciones:

$$
\begin{aligned}
\min _{i} r_{i} & \leq \rho(\mathbf{A}) \leq \operatorname{máx}_{i} r_{i}, \\
\min _{j} c_{j} \leq \rho(\mathbf{A}) & \leq \operatorname{máx}_{j} c_{j} .
\end{aligned}
$$

En general, una matriz no tiene por qué tener un valor propio igual a su radio espectral, pero como muestra el Teorema de Perron, las matrices primitivas lo poseen.

En este apartado, se repasan brevemente las ideas básicas del Teorema de Perron-Frobenius. En la referencia [130], puede encontrarse un estudio más detallado del Teorema.

\section{A.1.1. Teorema de Perron}

Si $\mathbf{A}$ es una matriz $[n \times n]$ primitiva, y $\rho(\mathbf{A})$ es su radio espectral:

(a) $\rho(\mathbf{A})$ es real y positivo.

(b) La multiplicidad algebraica de $\rho(\mathbf{A})$ es igual a la unidad (el mayor valor propio de $\mathbf{A}$ es siempre positivo, simple e igual al radio espectral de A).

(c) Los vectores propios izquierdo y derecho de $\mathbf{A}$ correspondientes a $\rho(\mathbf{A})$ son estrictamente positivos (sus elementos son estrictamente positivos), es decir, existen unos vectores columna positivos $\mathbf{x}_{0}$ e $\mathbf{y}_{0}$ tales que:

$$
\begin{aligned}
\mathbf{A} \mathbf{x}_{0} & =\lambda_{0} \mathbf{x}_{0}, \\
\mathbf{y}_{0}^{T} \mathbf{A} & =\lambda_{0} \mathbf{y}_{0}^{T},
\end{aligned}
$$

donde $\lambda_{0}=\rho(\mathbf{A})$. En general, a los vectores $\mathbf{x}_{0}$ e $\mathbf{y}_{0}$ se los conoce como vectores propios de Perron de la matriz $\mathbf{A}$.

(d) Si $\lambda$ es otro valor propio de $\mathbf{A},|\lambda|<\rho(\mathbf{A})$. Concretamente, no existe ningún otro valor propio $\lambda$ tal que $|\lambda|=\rho(\mathbf{A})$. 


\section{A.1.2. Teorema de Perron-Frobenius}

Frobenius extiende el Teorema de Perron a matrices no negativas e irreducibles. Una matriz A es irreducible si no existe una matriz de permutación $\mathbf{S}$ tal que:

$$
\mathbf{S A S}^{T}=\left[\begin{array}{ll}
\mathbf{B} & \mathbf{0} \\
\mathbf{C} & \mathbf{D}
\end{array}\right]
$$

donde las matrices $\mathbf{B}$ y $\mathbf{D}$ son matrices cuadradas. En el caso de matrices reducibles, es posible encontrar una matriz de permutación que consigue reescribir $\mathbf{A}$ de forma que el bloque superior derecho sea un bloque todo ceros.

Si A es una matriz $[n \times n]$ no negativa e irreducible, y $\rho(\mathbf{A})$ es su radio espectral, las afirmaciones (a)-(c) del Teorema de Perron se mantienen. Sin embargo, la afirmación (d) se modifica, de forma que puede haber otro valor propio que iguale el radio espectral, sólo se puede afirmar que $|\lambda| \leq \rho(\mathbf{A})$.

\section{Aplicaciones del Teorema de Perron-Frobenius}

- Una condición necesaria y suficiente para obtener una solución x no negativa y no trivial $^{1}$ para la ecuación:

$$
(s \mathbf{I}-\mathbf{A}) \mathbf{x}=\mathbf{c},
$$

dado cualquier vector $\mathbf{c}$ no negativo y no trivial, es que:

$$
s>\rho(\mathbf{A}) .
$$

En ese caso, existe sólo una solución estrictamente positiva dada por:

$$
(s \mathbf{I}-\mathbf{A})^{-1} \mathbf{c} .
$$

- Si un vector y no negativo y no trivial cumple:

$$
\mathbf{A y} \leq s \mathbf{y}, s>0,
$$

entonces $\mathbf{y}>0, s \geq \rho(\mathbf{A})$. En este caso, $s=\rho(\mathbf{A})$ si y solo si $\mathbf{A y}=s \mathbf{y}$.

\footnotetext{
${ }^{1}$ Una solución trivial es aquella en la que los elementos de $\mathbf{x}$ son todos nulos.
} 


\section{A.1.3. Perron-Frobenius y el problema de minimización de potencia}

Una de las aplicaciones del Teorema de Perron-Frobenius aparece en el contexto del problema de minimización de potencia en un sistema de comunicaciones convencional. En este apartado, se especifica el papel que cumple el Teorema de Perron-Frobenius en el desarrollo de la solución de este problema [129].

Para simplificar la notación, se plantea el enlace UL de un sistema de comunicaciones de una única celda, donde el único mecanismo disponible para manejar la interferencia entre usuarios es el control de potencia, no se implementa ninguna técnica de beamforming en transmisión o recepción. En este caso concreto, el problema de minimización de potencia en el enlace UL se plantea como:

$$
\text { mín } \sum_{m=1}^{M} p_{m}, \text { s.t. } \operatorname{SINR}_{m}^{U L} \geq \gamma_{m}, m=1, \ldots, M,
$$

donde $\left(\gamma_{1}, \ldots, \gamma_{M}\right)$ son los umbrales de SINR establecidos como QoS.

La SINR recibida en la estación base (BS) para el usuario $m$ se obtiene mediante:

$$
\operatorname{SINR}_{m}^{U L}=\frac{p_{m}\left\|\mathbf{h}_{m}\right\|^{2}}{\sum_{i \neq m} p_{i}\left\|\mathbf{h}_{i}\right\|^{2}+\sigma^{2}},
$$

donde $\mathbf{h}_{m}$ representa el canal UL entre el usuario $m$ y la BS, y $\sigma^{2}$ es la potencia de ruido en la BS.

Como ya se ha comentado anteriormente, el hecho de que la SINR para un usuario sea monótonamente creciente con $p_{m}$ y monótonamente decreciente con $p_{i}$, implica que la potencia mínima transmitida se consigue cuando todos los umbrales de SINR se cumplen con igualdad, es decir:

$$
\operatorname{SINR}_{m}^{U L}=\gamma_{m}, \forall m .
$$

Reescribiendo la expresión anterior en forma matricial, la potencia mínima transmitida se consigue cuando:

$$
\left(\mathbf{I}_{M}-\mathbf{D F}\right) \mathbf{p}=\mathbf{D u},
$$

donde $\mathbf{I}_{M}$ es una matriz identidad $[M \times M]$, D es una matriz diagonal que incluye los términos de potencia de señal, $\mathbf{F}$ es la matriz que contiene los términos interferentes, $\mathbf{p}=\left[p_{1} \ldots p_{M}\right]^{T}$ es el vector que incluye las potencias 
transmitidas por los usuarios y $\mathbf{u}$ es un vector positivo que incluye el término de ruido:

$$
\begin{aligned}
& {[\mathbf{F}]_{(i, m)}=\left\{\begin{array}{cl}
\left\|\mathbf{h}_{i}\right\|^{2}, & i \neq m, \\
0, & i=m,
\end{array}\right.} \\
& \mathbf{D}=\operatorname{diag}\left\{\left[\frac{\gamma_{1}}{\left\|\mathbf{h}_{1}\right\|^{2}} \cdots \frac{\gamma_{M}}{\left\|\mathbf{h}_{M}\right\|^{2}}\right]\right\}, \\
& {[\mathbf{u}]_{m}=\gamma_{m} \sigma^{2} \text {. }}
\end{aligned}
$$

Si $\mathbf{A}=\mathbf{D F}$, y $\mathbf{b}=\mathbf{D u}$, la expresión (A.7) quedaría de la siguiente forma:

$$
(\mathbf{I}-\mathbf{A}) \mathbf{p}=\mathbf{b} .
$$

En principio, si los canales entre los usuarios y la BS no siguen una estructura específica, la matriz A no sería periódica ni reducible, sería una matriz primitiva no negativa con elementos nulos en la diagonal principal.

La condición necesaria y suficiente para que (A.8) tenga un vector solución no negativo de potencia para cualquier vector positivo $\mathbf{b}$, es que la inversa $(\mathbf{I}-\mathbf{A})^{-1}$ sea no negativa para $\mathbf{A} \geq 0$. Sin embargo, para cualquier $\mathbf{A} \geq 0$ :

$$
(\mathbf{I}-\mathbf{A})^{-1} \geq 0 \text { si y solo si } \quad \rho(\mathbf{A})=\left|\lambda_{\max }(\mathbf{A})\right|<1 .
$$

Por tanto, a partir de (A.9), la condición necesaria y suficiente para que (A.8) tenga un vector solución $\mathbf{p}$ positivo, es que el radio espectral de la matriz A sea estrictamente menor que la unidad.

\section{Condición suficiente}

Suponemos que $\rho(\mathbf{A})<1$. En este caso $\mathbf{A}^{k} \rightarrow 0$ y la serie $(\mathbf{I}-\mathbf{A})^{-1}=$ $\sum_{k=0}^{\infty} \mathbf{A}^{k} \geq 0$ converge. Entonces, la solución dada por:

$$
\mathbf{p}=(\mathbf{I}-\mathbf{A})^{-1} \mathbf{b}=\sum_{k=0}^{\infty} \mathbf{A}^{k} \mathbf{b}>0,
$$

es positiva para cualquier vector $\mathbf{b}$ positivo en (A.8). Por lo tanto, la condición (A.9) expresada en términos del radio espectral de la matriz $\mathbf{A}$ es suficiente.

La expresión (A.10) utiliza las propiedades de convergencia de las series de Neumann [130], en las que $(\mathbf{I}-\mathbf{A})^{-1}$ se descompone: 
Series de Neumann: para una matriz A cuadrada, $[n \times n]$, las siguiente afirmaciones son equivalentes:

- La serie de Neumann $\left[\mathbf{I}+\mathbf{A}+\mathbf{A}^{2}+\cdots\right]$ converge,

- $\rho(\mathbf{A})<1$,

- $\lim _{k \rightarrow \infty} \mathbf{A}^{k}=0$,

en cuyo caso, la inversa $(\mathbf{I}-\mathbf{A})^{-1}$ existe y puede expresarse como $\sum_{k=0}^{\infty} \mathbf{A}^{k}=$ $(\mathbf{I}-\mathbf{A})^{-1}$.

\section{Condición necesaria}

Para probar que la condición (A.9) también es necesaria, se supone que $\mathbf{A} \geq 0$ y que $(\mathbf{I}-\mathbf{A})^{-1} \geq 0$, como en (A.9). Si $\lambda$ y $\mathbf{x}$ representan cualquier pareja de valores y vectores propios, respectivamente, de la matriz A, entonces:

$$
\lambda \mathbf{x}=\mathbf{A} \mathbf{x} \Rightarrow|\lambda||\mathbf{x}|=|\mathbf{A} \mathbf{x}| \leq \mathbf{A}|\mathbf{x}|,
$$

puesto que $\mathbf{A} \geq 0$ ( $|\mathbf{x}|$ representa el valor absoluto elemento a elemento del vector), o bien:

$$
(\mathbf{I}-\mathbf{A})|\mathbf{x}| \leq(1-|\lambda|)|\mathbf{x}| .
$$

Por tanto,

$$
|\mathbf{x}| \leq(1-|\lambda|)(\mathbf{I}-\mathbf{A})^{-1}|\mathbf{x}|>0,
$$

puesto que $(\mathbf{I}-\mathbf{A})^{-1} \geq 0$.

Sin embargo, en la parte izquierda de la ecuación, $|\mathbf{x}| \geq 0$. Esto implica que en la parte derecha de la ecuación $|\lambda|<1$ para todo $|\lambda|$, con lo que se obtiene:

$$
\rho(\mathbf{A})<1 \quad \text { si } \quad(\mathbf{I}-\mathbf{A})^{-1} \geq 0,
$$

demostrando así la necesidad de la condición en términos del radio espectral en (A.9). 


\section{A.2. Iteración de Jacobi}

La iteración de Jacobi $[89,131]$ es una iteración de punto fijo que sirve para resolver un sistema de ecuaciones lineales del tipo:

$$
\mathbf{A x}=\mathbf{b}
$$

sin invertir explícitamente la matriz $\mathbf{A}$. La solución del sistema $\mathbf{A x}=\mathbf{b}$, también resuelve la expresión equivalente:

$$
\mathbf{x}=(\mathbf{I}-\mathbf{A}) \mathbf{x}+\mathbf{b},
$$

que se puede interpretar como una transformación afín [89]. El punto fijo del sistema de ecuaciones, si existe, es la solución:

$$
\mathbf{x}=\mathbf{A}^{-1} \mathbf{b} \text {. }
$$

La iteración de Jacobi, como la de Gauss-Seidel, forman parte de una serie de métodos iterativos que pueden expresarse de forma general como:

$$
\mathbf{x}\left(n_{i t}+1\right)=\mathbf{B} \mathbf{x}\left(n_{i t}\right)+\mathbf{c},
$$

donde la expresión de la matriz $\mathbf{B}$ y del vector $\mathbf{c}$ depende del tipo de método iterativo elegido.

Para demostrar la convergencia de la iteración (A.15), volvemos a recurrir al Teorema de Perron-Frobenius: para que la iteración (A.15) produzca una secuencia convergente a $(\mathbf{I}-\mathbf{B})^{-1} \mathbf{c}$ a partir de cualquier vector inicial $\mathbf{x}(0)$, es suficiente y necesario que el radio espectral de la matriz $\mathbf{B}$ sea menor que la unidad, $\rho(\mathbf{B})<1$. La demostración de la convergencia vuelve a recurrir a las series de Neumann y a las propiedades del radio espectral de una matriz [89].

En la iteración de Jacobi, la matriz A se descompone en la suma:

$$
\mathbf{A}=\mathbf{L}+\mathbf{D}+\mathbf{U},
$$

donde la matriz $\mathbf{D}$ es una matriz diagonal, y las matrices $\mathbf{L}$ y $\mathbf{U}$ son matrices triangulares inferiores y superiores, respectivamente. A partir de esta descomposición, la iteración de Jacobi puede expresarse como:

$$
\mathbf{x}\left(n_{i t}+1\right)=-\mathbf{D}^{-1}(\mathbf{L}+\mathbf{U}) \mathbf{x}\left(n_{i t}\right)+\mathbf{D}^{-1} \mathbf{b},
$$

de forma que comparando con (A.15), en la iteración de Jacobi se cumple $\mathbf{B}=-\mathbf{D}^{-1}(\mathbf{L}+\mathbf{U})$ y $\mathbf{c}=\mathbf{D}^{-1} \mathbf{b}$. 
Por otro lado, hay que destacar que la condición de convergencia sobre el radio espectral de la matriz $\mathbf{B}$, está directamente relacionada con cuánto de diagonal dominante sea la matriz $\mathbf{B}$, de hecho, cuánto más diagonal dominante sea esta matriz, más rápido converge la iteración [131].

\section{A.2.1. Jacobi y el problema de minimización de potencia}

La solución al problema de minimización de potencia (A.5), si existe $\rho(\mathbf{D F})<1$, puede calcularse de forma centralizada mediante (A.7):

$$
\mathbf{p}=(\mathbf{I}-\mathbf{D F})^{-1} \mathbf{D u},
$$

o alternativamente, de forma distribuida mediante la iteración de Jacobi:

$$
\mathbf{p}\left(n_{i t}+1\right)=\mathbf{D F} \mathbf{p}\left(n_{i t}\right)+\mathbf{D u},
$$

que converge para cualquier valor inicial de $\mathbf{p}$. 


\section{Apéndice B}

\section{Optimización convexa aplicada al problema de minimización de potencia}

Esta Tesis se centra en plantear el problema de minimización de potencia en el enlace DL de un sistema MIMO multi-usuario multi-celda coordinado. La mayor parte de los algoritmos planteados para resolver este problema de optimización en los sistemas MIMO multi-usuario sin coordinación, recurren a la dualidad entre el problema planteado en el enlace DL y un problema equivalente planteado en el enlace UL para simplificar el diseño de posibles soluciones algorítmicas.

Estudios recientes, como [39,61], demuestran que este concepto de dualidad se engloba dentro de una dualidad más general, que se observa el plantear el problema dual de Lagrange del problema de minimización de potencia. En este apéndice, se presenta un desarrollo simplificado de este planteamiento, que puede completarse con la referencia [132].

Suponemos un sistema de comunicaciones de una única celda, donde la BS dispone de un array de $N_{t}$ antenas y $\operatorname{los} M$ usuarios distribuidos por la celda sólo disponen de una antena en recepción. En este caso, para simplificar la notación, agrupamos el control de potencia y el diseño de los beamformers, de forma que la potencia transmitida en DL para cada usuario depende del módulo de los beamformers, $p_{i}=\left\|\mathbf{w}_{i}\right\|^{2}$.

La señal recibida por el usuario $i$-ésimo sería:

$$
y_{i}=\mathbf{h}_{i}^{H} \mathbf{x}+n_{i}, i=1, \ldots, M,
$$


donde el vector $\mathbf{h}_{i}^{H},\left[1 \times N_{t}\right]$, representa el canal entre la BS y el usuario $i$-ésimo, $n_{i}$ es el ruido AWGN añadido en recepción, y $\mathbf{x},\left[N_{t} \times 1\right]$, es la señal transmitida en DL por la BS, que sigue la forma:

$$
\mathbf{x}=\sum_{i=1}^{M} \mathbf{w}_{i} u_{i}
$$

El escalar $u_{i}$ representa la señal de información del usuario $i$, mientras que el vector $\mathbf{w}_{i}$ representa al beamformer en transmisión diseñado para transmitir esta señal de información. Si $E\left[\left|u_{i}\right|^{2}\right]=1$, la SINR recibida en el usuario $i$-ésimo se escribe como:

$$
\operatorname{SINR}_{i}^{D L}=\frac{\left|\mathbf{h}_{i}^{H} \mathbf{w}_{i}\right|^{2}}{\sum_{j=1, j \neq i}^{M}\left|\mathbf{h}_{i}^{H} \mathbf{w}_{j}\right|^{2}+\sigma^{2}} .
$$

En este escenario, el problema de minimización de potencia presentaría la forma habitual:

$$
\begin{array}{ll}
\min & \sum_{j=1}^{M}\left\|\mathbf{w}_{j}\right\|^{2} \\
\text { s.t. } & \frac{\left|\mathbf{h}_{i}^{H} \mathbf{w}_{i}\right|^{2}}{\sum_{j=1, j \neq i}^{M}\left|\mathbf{h}_{i}^{H} \mathbf{w}_{j}\right|^{2}+\sigma^{2}} \geq \gamma_{i}, \forall i,
\end{array}
$$

donde se asume que el conjunto de valores umbrales de $\operatorname{SINR},\left(\gamma_{1}, \ldots, \gamma_{M}\right)$, es feasible.

Como se ha visto en la aproximación de Bengtsson y Ottersten presentada en el Capítulo 3, las restricciones de SINR planteadas en (B.3) no son convexas. Sin embargo, este problema que aparentemente no es convexo, presenta muchas de las propiedades de los problemas convexos. De hecho se puede relajar [37] o transformar [30] en un problema de optimización convexo.

Técnicamente, para plantear el problema dual de Lagrange de (B.3), primero debería reescribirse en forma SOCP (Second-order Cone Program) [30], sin embargo, en este caso concreto, el problema dual de Lagrange de (B.3) y el obtenido a partir de la formulación SOCP coinciden. 
En primer lugar, se plantea el problema (B.3) mediante los operadores de Lagrange:

$$
\begin{aligned}
L\left(\mathbf{w}_{i}, \lambda_{i}\right) & =\sum_{j=1}^{M} \mathbf{w}_{j}^{H} \mathbf{w}_{j}-\sum_{i=1}^{M} \lambda_{i}\left\{\frac{1}{\gamma_{i}}\left|\mathbf{h}_{i}^{H} \mathbf{w}_{i}\right|^{2}-\sum_{j \neq i}\left|\mathbf{h}_{i}^{H} \mathbf{w}_{j}\right|^{2}-\sigma^{2}\right\} \\
& =\sum_{i=1}^{M} \lambda_{i} \sigma^{2}+\sum_{i=1}^{M} \mathbf{w}_{i}^{H}\left\{\mathbf{I}+\sum_{j \neq i} \lambda_{j} \mathbf{h}_{j} \mathbf{h}_{j}^{H}-\frac{\lambda_{i}}{\gamma_{i}} \mathbf{h}_{i} \mathbf{h}_{i}^{H}\right\} \mathbf{w}_{i} .
\end{aligned}
$$

La función objetivo dual de la expresión anterior sería:

$$
g\left(\lambda_{i}\right)=\operatorname{mín}_{\mathbf{w}_{i}} L\left(\mathbf{w}_{i}, \lambda_{i}\right)
$$

Si la matriz $\mathbf{I}+\sum_{j \neq i} \lambda_{j} \mathbf{h}_{j} \mathbf{h}_{j}^{H}-\left(\lambda_{i} / \gamma_{i}\right) \mathbf{h}_{i} \mathbf{h}_{i}^{H}$ no es semidefinida positiva, existe algún $\mathbf{w}_{i}$ tal que $g\left(\lambda_{i}\right)=-\infty$. Los parámetros $\lambda_{i}$ se eligen de forma que $g\left(\lambda_{i}\right)$ sea máxima, por lo que al plantear el problema dual de Lagrange, aparece una restricción semidefinida:

$$
\begin{aligned}
\operatorname{máx} & \sum_{i=1}^{M} \lambda_{i} \sigma^{2} \\
\text { s.t. } & \sum_{j=1}^{M} \lambda_{j} \mathbf{h}_{j} \mathbf{h}_{j}^{H}+\mathbf{I} \succeq\left(1+\frac{1}{\gamma_{i}}\right) \lambda_{i} \mathbf{h}_{i} \mathbf{h}_{i}^{H} .
\end{aligned}
$$

El aspecto interesante, es que se puede demostrar que la expresión anterior se corresponde con la expresión de un problema de minimización de potencia en el enlace UL, donde el parámetro $\lambda_{i}$ se corresponde con una potencia UL escalada, $\mathbf{h}_{j}$ es el canal UL y $\gamma_{i}$ es el valor umbral de SINR.

El problema de minimización de potencia en el enlace UL puede expresarse como:

$$
\begin{array}{ll}
\operatorname{mín} & \sum_{i=1}^{M} \rho_{i} \\
\text { s.t. } & \frac{\rho_{i}\left|\hat{\mathbf{w}}_{i}^{H} \mathbf{h}_{i}\right|^{2}}{\sum_{j \neq i} \rho_{j}\left|\hat{\mathbf{w}}_{i}^{H} \mathbf{h}_{j}\right|^{2}+\sigma^{2} \hat{\mathbf{w}}_{i}^{H} \hat{\mathbf{w}}_{i}} \geq \gamma_{i}, \forall i,
\end{array}
$$

donde el objetivo es encontrar los valores óptimos de las potencias UL, $\rho_{i}, \mathrm{y}$ los beamformers en recepción, $\hat{\mathbf{w}}_{i}$. En este caso, la expresión del beamformer 
óptimo en recepción es conocida y se corresponde con el criterio MMSE:

$$
\hat{\mathbf{w}}_{i}=\left(\sum_{j=1}^{M} \rho_{i} \mathbf{h}_{j} \mathbf{h}_{j}^{H}+\sigma^{2} \mathbf{I}\right)^{-1} \mathbf{h}_{i} .
$$

Sustituyendo la expresión del beamformer óptimo en (B.7), y tras reorganizar los términos, es posible demostrar que el problema de minimización de potencia en el enlace UL es equivalente a:

$$
\begin{aligned}
\operatorname{mín} & \sum_{i=1}^{M} \rho_{i} \\
\text { s.t. } & \sum_{j=1}^{M} \rho_{j} \mathbf{h}_{j} \mathbf{h}_{j}^{H}+\sigma^{2} \mathbf{I} \preceq\left(1+\frac{1}{\gamma_{i}}\right) \rho_{i} \mathbf{h}_{i} \mathbf{h}_{i}^{H} .
\end{aligned}
$$

Igualando $\rho_{i}=\lambda_{i} \sigma^{2}$, se observa que el problema (B.8) coincide con (B.6), exceptuando el hecho de que las funciones máx y mín están cambiadas, de la misma forma que las restricciones de SINR. Sin embargo, la mínima potencia transmitida se obtiene cuando las restricciones en SINR se cumplen con igualdad, y en este caso, tanto la función máx como la mín obtienen la misma solución.

Resumiendo, el desarrollo (B.4)-(B.8) demuestra que es posible identificar el problema dual de Lagrange del problema de minimización de potencia en el enlace DL, con un problema de minimización de potencia en el enlace UL. Además, los parámetros duales $\lambda_{i}$ se pueden interpretar como unas potencias UL, escaladas por la potencia del ruido $\left(\lambda_{i}=\rho_{i} / \sigma^{2}\right)$.

Por último, hay que destacar que la formulación dual de Lagrange del problema de minimización de potencia en el enlace DL, permite derivar soluciones algorítmicas eficientes para sistemas que utilizan técnicas lineales en transmisión, como han demostrado recientemente los trabajos de Wiesel et al. [30] y Hammarwall et al. [42]. 


\section{Apéndice $\mathrm{C}$}

\section{Configuraciones de celda}

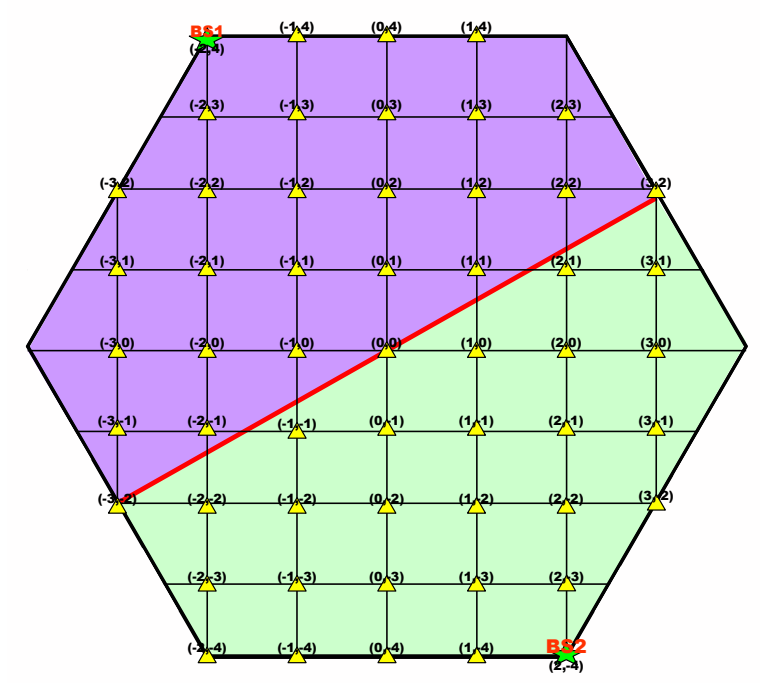

Figura C.1: Sistema CM3, $K=2$ BSs. 


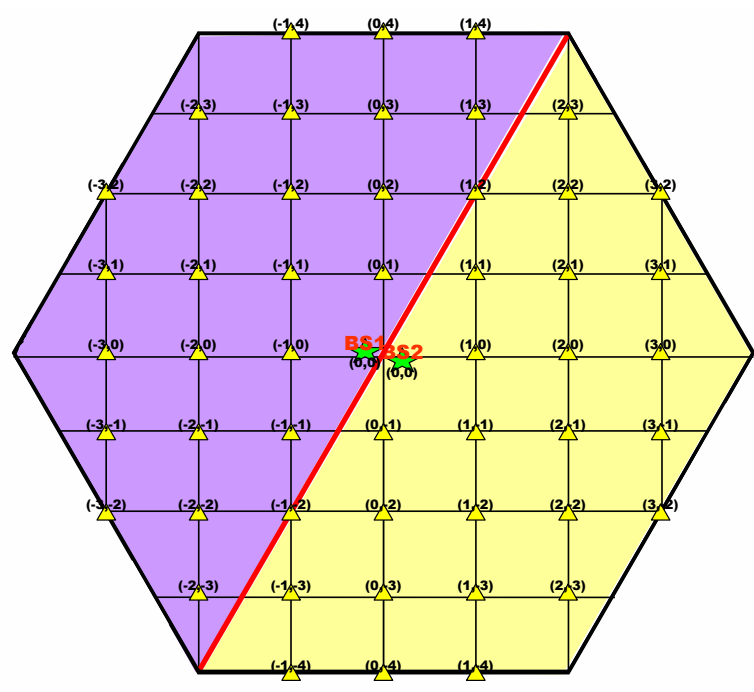

Figura C.2: Celda sectorizada convencional, $K=2$ BSs centrales.

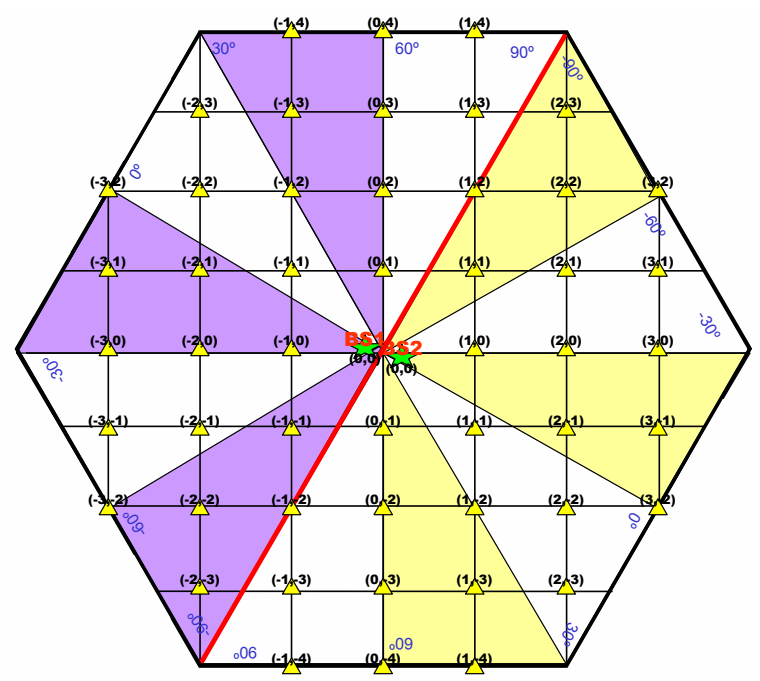

Figura C.3: División en subsectores para el cálculo de las DOAs en una celda sectorizada convencional con $K=2$ BSs centrales. 


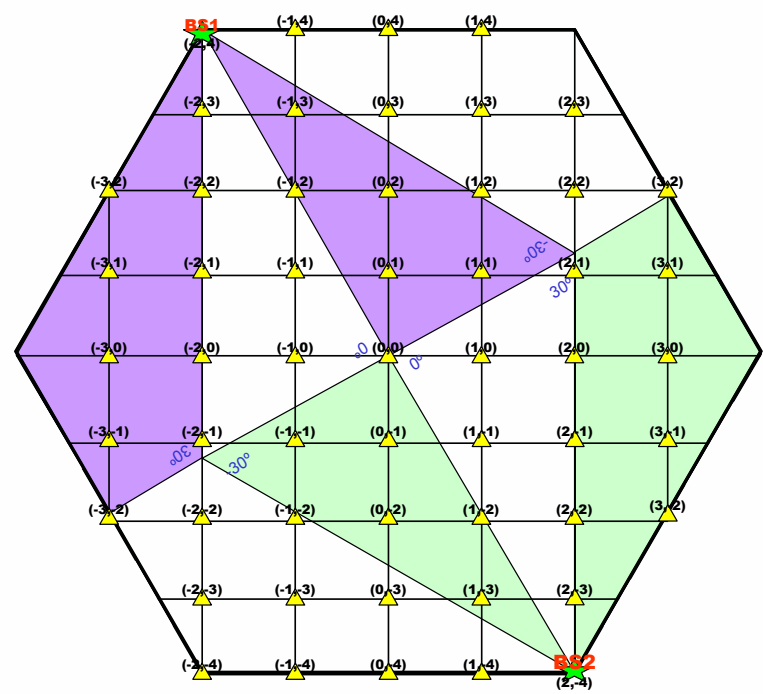

Figura C.4: División en subsectores para el cálculo de las DOAs en un sistema CM3 con $K=2$ BSs. 



\section{Bibliografía}

[1] H. Kaaranen. UMTS Networks: Architecture, Mobility and Services. John Wiley and sons, 2001.

[2] H. Holma and A. Toskala. WCDMA for UMTS - Radio Access for Third Generation Communications. John Wiley and sons, 2000.

[3] G.J. Foschini and M.J. Gans. On limits of wireless communications in a fading environment when using multiple antennas. Wireless Personal Communications, (no. 6):311-335, 1998.

[4] I.E. Telatar. Capacity of multi-antenna Gaussian channels. European Transactions on Telecommunications, vol. 10(no. 6):585-595, Noviembre 1999.

[5] D. Gesbert. Advances in multiuser MIMO systems (tutorial): Emerging topics in multiuser MIMO networks. Septiembre 2007.

[6] T.M. Cover. Elements of Information Theory. John Wiley and sons, 1991.

[7] J.C. Liberti and T.S. Rappaport. Smart Antennas for Wireless Communications. Prentice Hall, 1999.

[8] A. Goldsmith, S.A. Jafar, N. Jindal, and S. Vishwanath. Capacity limits of MIMO channels. IEEE Journal on Selected Areas in Communications, vol. 21(no. 5):684-702, Junio 2003.

[9] D. Gesbert, M. Shafi, D. Shiu, P.J. Smith, and A.Naguib. From theory to practice: an overview of MIMO space-time coded wireless systems. IEEE Journal on Selected Areas in Communications, vol. 21(no. 3):281-302, Abril 2003. 
[10] J. Winters. On the capacity of radio communication systems with diversity in a Rayleigh fading environment. IEEE Journal on Selected Areas in Communications, vol. 5(no. 5):871-878, Junio 1987.

[11] V. Tarokh, N. Seshadri, and A.R. Calderbank. Space-time codes for high data rate wireless communication: performance criterion and code construction. IEEE Transactions on Information Theory, vol. 44(no. 2):744-765, Marzo 1998.

[12] S.M. Alamouti. A simple transmit diversity technique for wireless communications. IEEE Journal on Selected Areas in Communications, vol. 16(no. 8):1451-1458, Octubre 1998.

[13] G.J. Foschini. Layered space-time architecture for wireless communication in a fading environment when using multi-element antennas. Technical report, Bell Labs Technical Journal, 1996.

[14] Zheng Lizhong and D.N.C. Tse. Diversity and multiplexing: a fundamental tradeoff in multiple-antenna channels. IEEE Transactions on Information Theory, vol. 49(no. 5):1073-1096, Mayo 2003.

[15] G. Caire and S. Shamai. On the achievable throughput of a multiantenna Gaussian broadcast channel. IEEE Transactions on Information Theory, vol. 49(no. 7):1691-1706, Julio 2003.

[16] D. Gesbert, M. Kountouris, R.W. Heath, C.-B Chae, and T. Salzer. Shifting the MIMO paradigm. IEEE Signal Processing Magazine, vol. 24(no. 5):36-46, Septiembre 2007.

[17] W. Yu, W. Rhee, S. Boyd, and J.M. Cioffi. Iterative water-filling for Gaussian vector multiple-access channels. IEEE Transactions on Information Theory, vol. 50(no. 1):145-152, Enero 2004.

[18] M. Costa. Writing on dirty paper. IEEE Transactions on Information Theory, vol. 29(no. 3):439-441, Mayo 1983.

[19] P. Viswanath and D.N.C. Tse. Sum capacity of the vector Gaussian broadcast channel and uplink-downlink duality. IEEE Transactions on Information Theory, vol. 49(no. 8):1912-1921, Agosto 2003.

[20] W. Yu and J.M. Cioffi. Sum capacity of Gaussian vector broadcast channels. IEEE Transactions on Information Theory, vol. 50(no. 9):1875-1892, Septiembre 2004. 
[21] S. Vishwanath, N. Jindal, and A. Goldsmith. Duality, achievable rates, and sum-rate capacity of Gaussian MIMO broadcast channels. IEEE Transactions on Information Theory, vol. 49(no. 10):26582668, Octubre 2003.

[22] H. Weingarten, Y. Steinberg, and S. Shamai. The capacity region of the Gaussian multiple-input multiple-output broadcast channel. IEEE Transactions on Information Theory, vol. 52(no. 9):3936-3964, Septiembre 2006.

[23] N. Jindal, S. Vishwanath, and A. Goldsmith. On the duality of Gaussian multiple-access and broadcast channels. IEEE Transactions on Information Theory, vol. 50(no. 5):768-783, Mayo 2004.

[24] N. Jindal, W. Rhee, S. Vishwanath, S.A. Jafar, and A. Goldsmith. Sum power iterative water-filling for multi-antenna Gaussian broadcast channels. IEEE Transactions on Information Theory, vol. 51(no. 4):1570-1580, Abril 2005.

[25] B. Hassibi and M. Sharif. Fundamental limits in MIMO broadcast channels. IEEE Journal on Selected Areas in Communications, vol. 25(no. 7):1333-1344, Septiembre 2007.

[26] M. Sharif and B. Hassibi. On the capacity of MIMO broadcast channels with partial side information. IEEE Transactions on Information Theory, vol. 51(no. 2):506-522, Febrero 2005.

[27] T. Yoo and A. Goldsmith. On the optimality of multiantenna broadcast scheduling using zero-forcing beamforming. IEEE Journal on Selected Areas in Communications, vol. 24(no. 3):528-541, Marzo 2006.

[28] M. Sharif and B. Hassibi. A comparison of time-sharing, DPC, and beamforming for MIMO broadcast channels with many users. IEEE Transactions on Communications, vol. 55(no. 1):11-15, Enero 2007.

[29] C.B. Peel, B.M. Hochwald, and A.L. Swindlehurst. A vectorperturbation technique for near-capacity multiantenna multiuser communication - part I: channel inversion and regularization. IEEE Transactions on Communications, vol. 53(no. 1):195-202, Enero 2005. 
[30] A. Wiesel, Y.C. Eldar, and S. Shamai. Linear precoding via conic optimization for fixed MIMO receivers. IEEE Transactions on Signal Processing, vol. 54(no. 1):161-176, Enero 2006.

[31] Q.H. Spencer, A.L. Swindlehurst, and M. Haardt. Zero-forcing methods for downlink spatial multiplexing in multiuser MIMO channels. IEEE Transactions on Signal Processing, vol. 52(no. 2):461-471, Febrero 2004.

[32] B.M. Hochwald, C.B. Peel, and A.L. Swindlehurst. A vectorperturbation technique for near-capacity multiantenna multiuser communication - part II: perturbation. IEEE Transactions on Communications, vol. 53(no. 3):537-544, Marzo 2005.

[33] F. Rashid-Farrokhi, K.J.R. Liu, and L. Tassiulas. Transmit beamforming and power control for cellular wireless systems. IEEE Journal on Selected Areas in Communications, vol. 16(no. 8):1437-1450, Octubre 1998.

[34] M. Schubert, S. Shi, E.A. Jorswieck, and H. Boche. Downlink sumMSE transceiver optimization for linear multi-user MIMO systems. In Asilomar Conference on Signals, Systems and Computers, 2005.

[35] M. Stojnic, H. Vikalo, and B. Hassibi. Rate maximization in multiantenna broadcast channels with linear processing. IEEE Transactions on Wireless Communications, vol. 5(no. 9):2338-2342, Septiembre 2006 .

[36] F. Boccardi, F. Tosato, and G. Caire. Precoding schemes for the MIMO-GBC. In International Zurich Seminar on Communications, 2006.

[37] M. Bengtsson and B. Ottersten. Optimum and suboptimum transmit beamforming, pages 18-1-18-33. CRC Press LLC, 2002.

[38] M. Schubert and H. Boche. Solution of the multiuser downlink beamforming problem with individual SINR constraints. IEEE Transactions on Vehicular Technology, vol. 53(no. 1):18-28, Enero 2004.

[39] Wei Yu and Tian Lan. Transmitter optimization for the multiantenna downlink with per-antenna power constraints. IEEE Transactions on Signal Processing, vol. 55(no. 6):2646-2660, Junio 2007. 
[40] E. Visotsky and U. Madhow. Optimum beamforming using transmit antenna arrays. In IEEE Vehicular Technology Conference, 1999.

[41] A. Ekbal and J.M. Cioffi. Distributed transmit beamforming in cellular networks - a convex optimization perspective. In IEEE International Conference on Communications, 2005.

[42] D. Hammarwall, M. Bengtsson, and B. Ottersten. On downlink beamforming with indefinite shaping constraints. IEEE Transactions on Signal Processing, vol. 54(no. 9):3566-3580, Septiembre 2006.

[43] F. Rashid-Farrokhi, L. Tassiulas, and K.J.R. Liu. Joint optimal power control and beamforming in wireless networks using antenna arrays. IEEE Transactions on Communications, vol. 46(no. 10):1313-1324, Octubre 1998.

[44] M. Schubert and H. Boche. Iterative multiuser uplink and dowlink beamforming under SINR constraints. IEEE Transactions on Signal Processing, vol. 53(no. 7):2324-2334, Julio 2005.

[45] M.A. Maddah-Ali, A.S. Motahari, and A.K. Khandani. Signaling over MIMO multi-base systems: combination of multi-access and broadcast schemes. In IEEE International Symposium on Information Theory, 2006.

[46] S.A. Jafar and M.J. Fakhereddin. Degrees of freedom for the MIMO interference channel. IEEE Transactions on Information Theory, vol. 53(no. 7):2637-2642, Julio 2007.

[47] A. Sendonaris, E. Erkip, and B. Aazhang. User cooperation diversity part I and II. IEEE Transactions on Communications, vol. 51(no. 11):1927-1948, Noviembre 2003.

[48] A. Høst Madsen. Capacity bounds for cooperative diversity. IEEE Transactions on Information Theory, vol. 52(no. 4):1522-1544, Abril 2006.

[49] D. Gesbert, S.G. Kiani, A. Gjendemsjø, and G.E. Øien. Adaptation, coordination, and distributed resource allocation in interference-limited wireless networks. Proceedings of the IEEE, vol. 95(no. 12):2393-2409, Diciembre 2007. 
[50] H. Zhang and H. Dai. Cochannel interference mitigation and cooperative processing in downlink multicell multiuser MIMO networks. EURASIP Journal on Wireless Communications and Networking, (no. 2):222-235, 2004.

[51] G.J. Foschini, K. Karakayali, and R.A. Valenzuela. Coordinating multiple antenna cellular networks to achieve enormous spectral efficiency. IEEE Procedings - Communications, vol. 153(no. 4):548-555, Agosto 2006.

[52] O. Somekh, O. Simeone, Y. Bar-Ness, A.M. Haimovich, U. Spagnolini, and S. Shamai. An information theoretic view of distributed antenna processing in cellular systems, pages 31-65. Auerbach publications, Junio 2007.

[53] H. Skjevling, D. Gesbert, and A. Hjørungnes. Receiver-enhanced cooperative spatial multiplexing with hybrid channel knowledge. In IEEE International Conference on Acoustics, Speech, and Signal Processing, 2006.

[54] A. Saleh, A. Rustako, and R. Roman. Distributed antennas for indoor radio communications. IEEE Transactions on Communications, vol. 35(no. 12):1245-1251, Diciembre 1987.

[55] Lin Dai, S. Zhou, and Yan Yao. Capacity analysis in CDMA distributed antenna systems. IEEE Transactions on Wireless Communications, vol. 4(no. 6):2613-2620, Noviembre 2005.

[56] W. Choi, J.G. Andrews, and C. Yi. The capacity of multicellular distributed antenna networks. In International Conference on Wireless Networks, Communications and Mobile Computing, 2005.

[57] Guan-Hong Chen, Chih-Min Yu, and Chia-Chi Huang. A simulation study of a distributed antenna-based CDMA system. In IEEE International Symposium on Personal, Indoor and Mobile Radio Communications, 1996.

[58] S.A. Jafar, G.J. Foschini, and A.J. Goldsmith. Phantomnet: exploring optimal multicellular multiple antenna systems. In IEEE Vehicular Technology Conference, 2002. 
[59] S. Shamai and B.M. Zaidel. Enhancing the cellular downlink capacity via co-processing at the transmitting end. In IEEE Vehicular Technology Conference, 2001.

[60] H. Huang and S. Venkatesan. Asymptotic downlink capacity of coordinated cellular networks. In Asilomar Conference on Signals, Systems and Computers, 2004.

[61] Wei Yu. Uplink-downlink duality via minimax duality. IEEE Transactions on Information Theory, vol. 52(no. 2):361-374, Febrero 2006.

[62] Y. Liang and A. Goldsmith. Symmetric rate capacity of cellular systems with cooperative base stations. In IEEE Global Telecommunications Conference, 2006.

[63] S.A. Jafar and A.J. Goldsmith. Transmitter optimization for multiple antenna cellular systems. In IEEE International Symposium on Information Theory, 2002.

[64] S.A. Jafar, G.J. Foschini, and A.J. Goldsmith. Phantomnet: exploring optimal multicellular multiple antenna systems. EURASIP Journal on Applied Signal Processing, (no. 5):591-604, 2004.

[65] H. Zhang, H. Dai, and Q. Zhou. Base station cooperation for multiuser MIMO: Joint transmission and BS selection. In Conference on Information Sciences and Systems, 2004.

[66] M.K. Karakayali, G.J. Foschini, and R.A. Valenzuela. Network coordination for spectrally efficient communications in cellular systems. IEEE Wireless Communications, vol. 13(no. 4):56-61, Agosto 2006.

[67] M.K. Karakayali, G.J. Foschini, R.A. Valenzuela, and R.D. Yates. On the maximum common rate achievable in a coordinated network. In IEEE International Conference on Communications, 2006.

[68] L. Smolyar, I. Bergel, and H. Messer. Joint downlink power allocation, beamforming weights and base assignment. In IEEE International Workshop on Signal Processing Advances in Wireless Communications, 2007.

[69] Boon Loong Ng, J.S. Evans, S.V. Hanly, and D. Aktas. Transmit beamforming with cooperative base stations. In IEEE International Symposium on Information Theory, 2005. 
[70] O. Somekh, O. Simeone, Y. Bar-Ness, and A.M. Haimovich. Distributed multi-cell zero-forcing beamforming in cellular downlink channels. In IEEE Global Telecommunications Conference, 2006.

[71] S. Jing, D.N.C. Tse, J. Hou, J.B. Soriaga, J.E. Smee, and R. Padovani. Multi-cell downlink capacity with coordinated processing. In Information Theory and Applications Workshop, 2007.

[72] S. Jing, D.N.C. Tse, J.B. Soriaga, J. Hou, J.E. Smee, and R. Padovani. Downlink macro-diversity in cellular networks. In IEEE International Symposium on Information Theory, 2007.

[73] D. Gesbert, A. Hjørungnes, and H. Skjevling. Cooperative spatial multiplexing with hybrid channel knowledge. In International Zurich Seminar on Communications, 2006.

[74] P. Marsch and G. Fettweis. A framework for optimizing the downlink performance of distributed antenna systems under a constrained backhaul. In European Wireless Conference, 2007.

[75] H. Skjevling, D. Gesbert, and A. Hjørungnes. A low complexity distributed multibase transmission scheme for improving the sum capacity of wireless networks. In IEEE International Workshop on Signal Processing Advances in Wireless Communications, 2007.

[76] H. Zhang, N.B. Mehta, A.F. Molisch, J. Zhang, and H. Dai. On the fundamentally asynchronous nature of interference in cooperative base station systems. In IEEE International Conference on Communications, 2007.

[77] F. Boccardi and H. Huang. Limited downlink network coordination in cellular networks. In IEEE International Symposium on Personal, Indoor and Mobile Radio Communications, 2007.

[78] Weidong Yang and Guanghan Xu. Optimal downlink power assignment for smart antenna systems. In IEEE International Conference on Acoustics, Speech, and Signal Processing, 1998.

[79] M. Schubert and H. Boche. A unifying theory for uplink and downlink multi-user beamforming. In International Zurich Seminar on Communications, 2002. 
[80] H. Boche and M. Schubert. A general duality theory for uplink and downlink beamforming. In IEEE Vehicular Technology Conference, 2002 .

[81] M. Bengtsson and B. Ottersten. Optimal downlink beamforming using semidefinite optimization. In Allerton Conference on Communication, Control, and Computing, 1999.

[82] G. Piñero, C. Botella, M. de Diego, A. González, and N. Cardona. Cooperative transmission for wireless networks using power control and beamforming. In European Signal Processing Conference, 2004.

[83] G. Piñero, C. Botella, A. González, M. de Diego, and N. Cardona. Downlink power control and beamforming for a cooperative wireless system. In IEEE International Symposium on Personal, Indoor and Mobile Radio Communications, 2004.

[84] G. Piñero, A. González, C. Botella, M. de Diego, and N. Cardona. An efficient algorithm for downlink cooperative transmission using joint power control and beamforming. In IEEE Sensor Array and Multichannel Signal Processing Workshop, 2004.

[85] C. Botella, G. Piñero, M. de Diego, A. González, and O. Lázaro. An efficient joint power control and beamforming algorithm for distributed base stations. In IEEE International Symposium on Wireless Communications Systems, 2004.

[86] C. Botella, G. Piñero, M. de Diego, and A. González. Algoritmos de procesado digital para una estructura Multi-nodo B. In Jornadas de Telecom $I+D, 2004$.

[87] 3GPP. TS 25.922, Radio resource management strategies.

[88] G.J. Foschini and Z. Miljanic. A simple distributed autonomous power control algorithm and its convergence. IEEE Transactions on Vehicular Technology, vol. 42(no. 4):641-646, Noviembre 1993.

[89] T.K. Moon and W.C. Stirling. Mathematical Methods and Algorithms for Signal Processing. Prentice Hall, 2000.

[90] M. Bengtsson. Jointly optimal downlink beamforming and base station assignment. In IEEE International Conference on Acoustics, Speech, and Signal Processing, 2001. 
[91] G. Piñero, C. Botella, O. Lázaro, A. González, and M. de Diego. New criteria for dynamic mobile assignment using joint power control and optimal beamforming. In 6th Baiona Workshop on Signal Processing in Communications, 2003.

[92] Ying-Chang Liang and F. Chin. Two suboptimal algorithms for downlink beamforming in FDD DS-CDMA mobile radio. IEEE Journal on Selected Areas in Communications, vol. 19(no. 7):1264-1275, Julio 2001.

[93] M. Patzöld, R. García, and F. Laue. Design of high-speed simulation models for mobile fading channels by using table look-up techniques. IEEE Transactions on Vehicular Technology, vol. 49(no. 4):11781190, Julio 2000.

[94] W.C. Jakes. Microwave Mobile Communications. Wiley-IEEE Press, 1993.

[95] S. Ruiz, M. García, and J. Olmos. Admission and cell congestion analysis through dynamic power allocation. In IEEE International Symposium on Personal, Indoor and Mobile Radio Communications, 2002.

[96] F. Adachi, M. Sawahashi, and K. Okawa. Tree-structured generation of orthogonal spreading codes with different lengths for forward link of DS-CDMA mobile radio. Electronics Letters, vol. 33(no. 1):27-28, Enero 1997.

[97] C. Botella, G. Piñero, A. González, P. Faus, and N. Cardona. Performance of joint power control and beamforming in WCDMA considering real multiple access interferences. In IEEE International Symposium on Wireless Communications Systems, 2005.

[98] C. Botella, G. Piñero, M. de Diego, A. González, and N. Cardona. Influencia de la interferencia de acceso múltiple en un entorno WCDMA de antenas distribuidas. In Simposium nacional de la unión científica internacional de radio, 2005.

[99] C. Botella, G. Piñero, A. González, and M. de Diego. Spreading sequence assignment in WCDMA for distributed antenna arrays based on interference model. In IEEE International Workshop on Signal Processing Advances in Wireless Communications, 2006. 
[100] C. Botella, G. Piñero, A. González, and M. de Diego. Downlink power control with hybrid channel knowledge for multi-cell multi-antenna WCDMA systems. In IEEE International Workshop on Signal Processing Advances in Wireless Communications, 2007.

[101] 3GPP. TS 25.213, Spreading and modulation (FDD).

[102] D.V. Sarwate and M.B. Pursley. Crosscorrelation properties of pseudorandom and related sequences. Proceedings of the IEEE, vol. 68(no. 5):593-619, Mayo 1980.

[103] M. Fong, V.K. Bhargava, and Q. Wang. Concatenated orthogonal/PN spreading sequences and their application to cellular DS-CDMA systems with integrated traffic. IEEE Journal on Selected Areas in Communications, vol. 14(no. 3):547-558, Abril 1996.

[104] Yan Zhou, Francois Chin, Ying-Chang Liang, and Chi-Chung Ko. Performance comparison of transmit diversity and beamforming for the downlink of DS-CDMA system. IEEE Transactions on Wireless Communications, vol. 2(no. 2):320-334, Marzo 2003.

[105] K.S. Kim, I. Song, C. Bang, and T. Kim. Performance analysis of forward link beamforming techniques for DS/CDMA systems using base station antenna arrays. IEEE Transactions on Signal Processing, vol. 48(no. 3):862-865, Marzo 2000.

[106] A.F. Naguib and A. Paulraj. Performance of wireless CDMA with $\mathrm{M}$-ary orthogonal modulation and cell site antenna arrays. IEEE Journal on Selected Areas in Communications, vol. 14(no. 9):17701783, Diciembre 1996.

[107] Y. Hara, D. Park, and Y. Kamio. Analysis of RAKE receiver in W-CDMA systems with downlink beamforming. In IEEE Vehicular Technology Conference, 2001.

[108] M. Schubert, D. Karadoulamas, H. Boche, and G. Lehmann. Joint downlink beamforming and power control for $3 \mathrm{G}$ WCDMA. In IEEE Vehicular Technology Conference, 2003.

[109] B.H. Khalaj, A. Paulraj, and T. Kailath. 2D RAKE receivers for CDMA cellular systems. In IEEE Global Telecommunications Conference, 1994. 
[110] N.B. Mehta, L.J. Greenstein, T.M. Willis, and Z. Kostic. Analysis and results for the orthogonality factor in WCDMA downlinks. IEEE Transactions on Wireless Communications, vol. 2(no. 6):1138-1149, Noviembre 2003.

[111] O. Awoniyi, N.B. Mehta, and L.J. Greenstein. Characterizing the orthogonality factor in WCDMA downlinks. IEEE Transactions on Wireless Communications, vol. 2(no. 4):621-625, Julio 2003.

[112] N.B. Mehta, A.F. Molisch, and L.J. Greenstein. Macrocell-wide behavior of the orthogonality factor in WCDMA downlinks. IEEE Transactions on Wireless Communications, vol. 5(no. 12):3394-3399, Diciembre 2006.

[113] J. Chen, C. Papadias, and G.J. Foschini. Space-time dynamic signature assignment for the reverse link of DS-CDMA systems. IEEE Transactions on Communications, vol. 52(no. 1):120-129, Enero 2004 .

[114] S. Ulukus and R.D. Yates. Iterative construction of optimum signature sequence sets in synchronous CDMA systems. IEEE Transactions on Information Theory, vol. 47(no. 5):1989-1998, Julio 2001.

[115] Li Gao and Tan F. Wong. Power control and spreading sequence allocation in a CDMA forward link. IEEE Transactions on Information Theory, vol. 50(no. 1):105-124, Enero 2004.

[116] M. Dell'Amico, F. Maffioli, and M.L. Merani. A tree partitioning dynamic policy for OVSF codes assignment in wideband CDMA. IEEE Transactions on Wireless Communications, vol. 3(no. 4):1013-1017, Julio 2004.

[117] ETSI. TR 101 112, Universal Mobile Telecommunications Systems (UMTS); selection procedures for the choice of radio transmission technologies of the UMTS.

[118] Ying-Chang Liang, F.P.S. Chin, and K.J.R. Liu. Downlink beamforming for DS-CDMA mobile radio with multimedia services. IEEE Transactions on Communications, vol. 49(no. 7):1288-1298, Julio 2001. 
[119] C. Botella, G. Piñero, A. González, and M. de Diego. Performance analysis of multi-user MIMO downlink with partial channel state information. In IEEE International Symposium on Wireless Communications Systems, 2006.

[120] M. Biguesh, S. Shahbazpanahi, and A.B. Gershman. Robust downlink power adjustment in cellular communication systems with antenna arrays at base stations. In IEEE International Workshop on Signal Processing Advances in Wireless Communications, 2003.

[121] C. Botella, G. Piñero, A. González, and M. de Diego. Coordination in a multi-cell multi-antenna multi-user W-CDMA system: a beamforming approach. IEEE Transactions on Wireless Communications. Aceptado para su publicación, Febrero 2008.

[122] Jinho Choi and S. Perreau. MMSE multiuser downlink multiple antenna transmission for CDMA systems. IEEE Transactions on Signal Processing, vol. 52(no. 6):1564-1573, Junio 2004.

[123] Zhu Han and K.J.R. Liu. Throughput maximization using adaptive modulation in wireless networks with fairness constraints. In IEEE Wireless Communications and Networking, 2003.

[124] H. Boche and M. Schubert. Convergence behavior of matrix-based iterative transceiver optimization. In IEEE International Workshop on Signal Processing Advances in Wireless Communications, 2006.

[125] A. Mercado and K.J.R. Liu. Adaptive QoS for wireless multimedia networks using power control and smart antennas. IEEE Transactions on Vehicular Technology, vol. 51(no. 5):1223-1233, Septiembre 2002.

[126] J. Zander. Performance of optimum transmitter power control in cellular radio systems. IEEE Transactions on Vehicular Technology, vol. 41(no. 1):57-62, Febrero 1992.

[127] R. Stridh, M. Bengtsson, and B. Ottersten. System evaluation of optimal downlink beamforming with congestion control in wireless communications. IEEE Transactions on Wireless Communications, vol. 5(no. 4):743-751, Abril 2006.

[128] D. Bartolomé and A.I. Pérez-Neira. Spatial scheduling in multiuser wireless systems: from power allocation to admission control. IEEE 
Transactions on Wireless Communications, vol. 5(no. 8):2082-2091, Agosto 2006.

[129] S.U. Pillai, T. Suel, and Seunghun Cha. The Perron-Frobenius theorem: some of its applications. IEEE Signal Processing Magazine, vol. 22(no. 2):62-75, Marzo 2005.

[130] C.D. Meyer. Matrix Analysis and Applied Linear Algebra. SIAM, 2000 .

[131] G.H. Golub and C.F. Van Loan. Matrix Computations. John Hopkins University Press, 1989.

[132] Zhi-Quan Luo and Wei Yu. An introduction to convex optimization for communications and signal processing. IEEE Journal on Selected Areas in Communications, vol. 24(no. 8):1426-1438, Agosto 2006. 


\section{Publicaciones relacionadas con la Tesis}

\section{Revistas Internacionales:}

- C. Botella, G. Piñero, A. González, M. de Diego, "Coordination in a Multi-Cell Multi-Antenna Multi-user W-CDMA system: a beamforming approach," IEEE Transactions on Wireless Communications, aceptada para su publicación, febrero 2008.

\section{Congresos Internacionales con Actas:}

- G. Piñero, C. Botella, M. de Diego, A. González, N. Cardona, "Cooperative transmission for wireless networks using power control and beamforming," Proc. European Signal Processing Conference (EUSIPCO), Viena, 2004.

- G. Piñero, C. Botella, A. González, M. de Diego, N. Cardona, "Downlink power control and beamforming for a cooperative wireless system," Proc. IEEE International Symposium on Personal, Indoor and Mobile Radio Communications (PIMRC), Barcelona, 2004.

- G. Piñero, A. González, C. Botella, M. de Diego, N. Cardona, "An efficient algorithm for downlink cooperative transmission using joint power control and beamforming," Proc. IEEE Sensor Array and Multichannel Signal Processing Workshop (SAM), Sitges, 2004.

- C. Botella, G. Piñero, M. de Diego, A. González, O. Lázaro, "An efficient joint power control and beamforming algorithm for distributed base stations," Proc. IEEE International Symposium on Wireless Communications Systems (ISWCS), Port Louis, 2004. 
- C. Botella, G. Piñero, A. González, P. Faus, N. Cardona, "Performance of joint power control and beamforming in WCDMA considering real multiple access interferences," Proc. IEEE International Symposium on Wireless Communications Systems (ISWCS), Siena, 2005.

- C. Botella, G. Piñero, A. González, M. de Diego, "Spreading sequence assignment in WCDMA for distributed antenna arrays based on interference model," IEEE Workshop on Signal Processing Advances in Wireless Communications (SPAWC), Cannes, 2006.

- C. Botella, G. Piñero, A. González, M. de Diego, "Performance analysis of multi-user MIMO downlink with partial channel state information," Proc. IEEE International Symposium on Wireless Communications Systems (ISWCS), Valencia, 2006.

- C. Botella, G. Piñero, A. González, M. de Diego, "Downlink power control with hybrid channel knowledge for multi-cell multi-antenna WCDMA systems," Proc. IEEE International Workshop on Signal Processing Advances in Wireless Communications (SPAWC), Helsinki, 2007.

\section{Congresos Nacionales con Actas:}

- C. Botella, G. Piñero, M. de Diego, A. González, "Algoritmos de procesado digital para una estructura Multi-nodo B," Jornadas de Telecom I+D, Madrid, 2004.

- C. Botella, G. Piñero, M. de Diego, A. González, N. Cardona, "Influencia de la interferencia de acceso múltiple en un entorno WCDMA de antenas distribuidas," Simposium Nacional de la Unión Científica Internacional de Radio (URSI), Gandía, 2005. 